\title{
Zur Analyse der Überlebensfähigkeit von Unternehmen
}

\author{
- methodisch-theoretische Grundlagen und \\ Simulationsergebnisse -
}

\author{
Dissertation \\ zur Erlangung des Doktorgrades \\ der Fakultät für Agrarwissenschaften \\ der Georg-August-Universität Göttingen
}

vorgelegt von

Ludger Hinners-Tobrägel

geboren in Lohne i.O.

Göttingen, im September 1998 


\section{7}

Referent: Professor Dr. W. Brandes, Göttingen

Korreferent: Professor Dr. T. Becker, Hohenheim

Tag der mündlichen Prüfung: 5. November 1998

Diese Arbeit ist unter gleichem Titel auch im Cuvillier-Verlag, Göttingen erschienen und im Buchhandel erhältlich. 
Für Jannis 



\section{Vorwort}

Insolvenzen besitzen in der westdeutschen Landwirtschaft kaum eine Bedeutung. In den neuen Ländern hingegen war in den vergangenen Jahren ein beträchtlicher Teil der landwirtschaftlichen Großbetriebe gezwungen zu schließen. Da die Datenbasis nicht ausreicht, um quantitative empirische Untersuchungen zu den Bestimmungsfaktoren des Scheiterns solcher Unternehmen durchzuführen, müssen hierfür andere Methoden herangezogen werden. In der vorliegenden Arbeit werden derartige Faktoren anhand von Unternehmensmodellen und einfachen dynamischen Systemen untersucht.

Zunächst werden stochastisch dynamische Systeme analytisch behandelt. Es zeigt sich, daß dieser Lösungsweg rasch an seine Grenzen stößt, da Überlebenswahrscheinlichkeiten eines stochastischen Prozesses sehr viel schwieriger zu berechnen sind als z.B. seine Erwartungswerte. Daher werden für analytisch nicht lösbare Probleme Monte-Carlo-Simulationen durchgeführt. Diese Technik wird auch zur Bestimmung der Überlebenswahrscheinlichkeit in einem stochastischen Betriebsentwicklungsmodell angewendet. Es basiert auf der linearen Programmierung, ist rekursiv dynamisch formuliert und optimiert simultan den Produktions-, Investitions- und Finanzbereich des Unternehmens. Entgegen der herrschenden Theorie zeigen die Simulationen, daß vorsichtige Strategien, wie einfache Diversifikationen oder Risikoabschläge, die Insolvenzhäufigkeit in bestimmten Situationen deutlich senken.

Die vorliegende Arbeit ist am Institut für Agrarökonomie der Universität Göttingen entstanden. Sie geht auf eine Idee meines Doktorvaters, Herrn Prof. Dr. Wilhelm Brandes, zurück. Für seine stete Gesprächsbereitschaft und seine freundliche Aufgeschlossenheit auch für Wege, die im ursprünglichen Konzept nicht vorgesehen waren, möchte ich mich sehr herzlich bedanken. Darüber hinaus bin ich den Kollegen und Kolleginnen im „Blauen Turm“ zu tiefem Dank verpflichtet. Die offene Diskussionsatmosphäre und die allgegenwärtige Hilfsbereitschaft haben nicht nur sehr zum Gelingen meiner Dissertation beigetragen, sie ließen die Arbeit auch meistens zu einem Vergnügen werden. Namentlich möchte ich den Koreferenten meiner Arbeit, Tilman Becker, nennen sowie Heinrich Hockmann, dem kein methodisches oder theoretisches Problem zu schwierig war, und Dagmar Paulik, die sich stets Zeit genommen hat, komplexe Programmierungsprobleme mit mir durchzusprechen. Nicht zuletzt gilt mein Dank Kristina, die mir neben fachlichem Austausch auch stets vor Augen geführt hat, daß es wichtigere Dinge im Leben gibt als eine Doktorarbeit. Dies war der kritischen Distanz zur Arbeit sehr förderlich. Und zum Schluß möchte ich auch meinen Eltern großen Dank aussprechen, die es schweren Herzens akzeptiert haben, daß der berufliche Weg ihres Sohnes nicht zurück auf den elterlichen Hof führt. 



\section{Verzeichnisse}

\subsection{Inhalt}

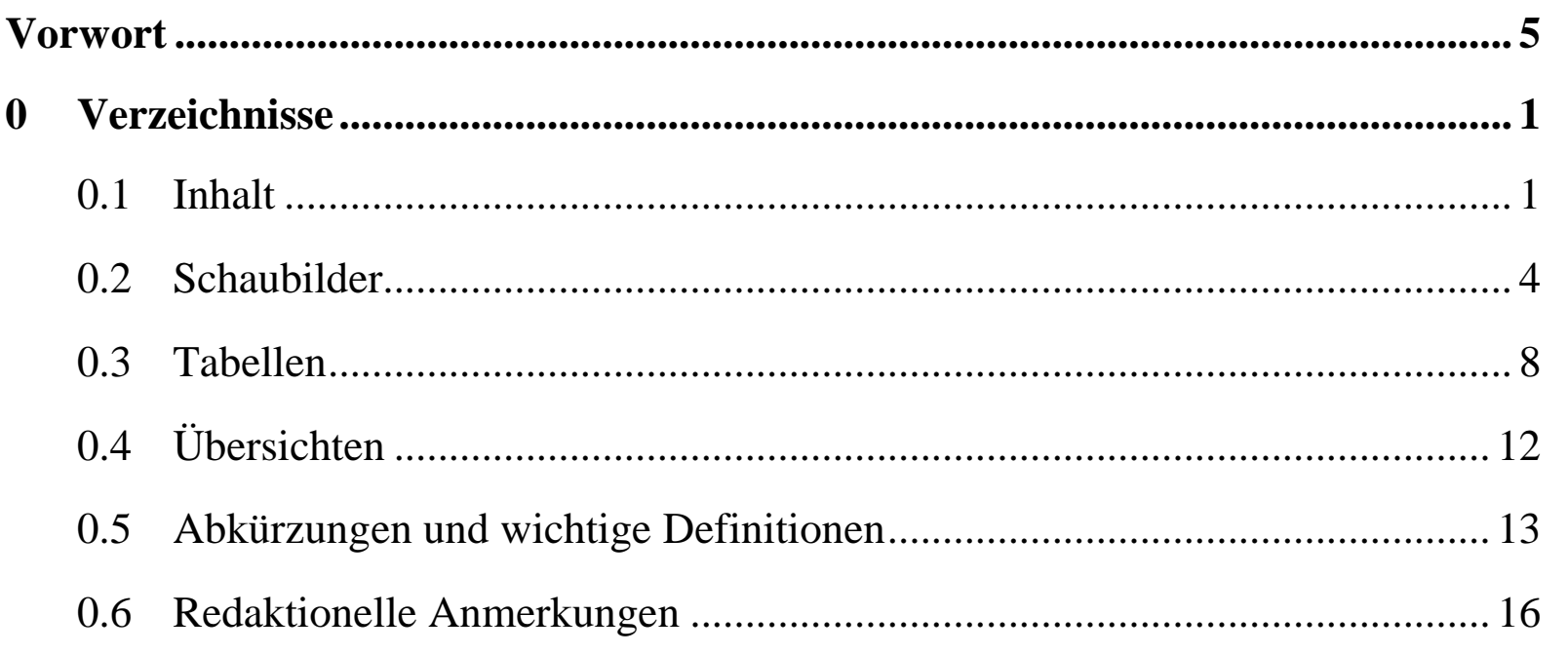

$1 \quad$ Einleitung......................................................................................................................17

2 Zur Analyse der Überlebensfähigkeit von Unternehmen.....................................21

$2.1 \quad$ Modelle als Analyseinstrument.................................................... 22

2.2 Exkurs: Zu Gewinnentwicklungen in Betriebsentwicklungsmodellen (LOHMANN-RUCHTI-Effekt)................................................................ 46

2.3 Theoretische Ansätze und empirische Methoden zur Erklärung der Uberlebensfähigkeit von Unternehmen ..................................................73

$2.4 \quad$ Studien zur Überlebenswahrscheinlichkeit von Unternehmen ..................... 84

3 Methodische Probleme in Entscheidungs- und Planungsmodellen ...................89

3.1 Entscheidungen unter Unsicherheit ...................................................... 89

$3.2 \quad$ Unsicherheit in der Betriebsplanung...................................................... 98

3.3 Signifikanztests in Monte-Carlo-Simulationen ........................................ 104

3.4 Berücksichtigung von Investitionen in Modellen der einperiodischen inearen Programmierung.................................................................... 110

4 Einige mathematische Eigenschaften einfacher stochastisch dynamischer Systeme ..................................................................................................................................... 129 
$4.1 \quad$ Vergleich zweier Zufallsveränderlicher.............................................. 130

4.2 Definition Uberlebenswahrscheinlichkeit (formal) .............................. 147

4.3 Deduzierbare Einflüsse auf die Uberlebenswahrscheinlichkeit................. 155

4.4 Stochastische Eigenschaften von Funktionen mehrerer

Zufallsveränderlicher

4.5 Exkurs: Probleme bei der Maximierung von Erwartungswerten

(Petersburger Paradoxon)................................................................. 178

5 Beispiele einfacher Überlebensmodelle ................................................................. 189

$5.1 \quad$ Allgemeine Systeme mit einer (stochastischen) Einzahlung ................... 192

5.2 Markovketten als Instrument zur Berechnung von

Überlebenswahrscheinlichkeiten bei diskreten Einzahlungen.................. 214

5.3 Konkrete Beispiele mit einer stochastischen Einzahlung .........................218

5.4 Modelle mit stochastischer und fester Einzahlung (Portfolio-Modelle)..... 258

5.5 Beispiele eines einfachen stochastischen Betriebsentwicklungsmodells .... 261

5.6 Überlebensaspekte im Planspiel „,Puten und Perlhühner“"...................... 265

6 Ein stochastisches Betriebsentwicklungs- und Überlebensmodell..................269

6.1 Strategien und Umweltszenarien in Betriebsentwicklungsmodellen .........269

6.2 Modellbeschreibung............................................................... 277

6.3 Ergebnisse ausgewählter Strategien im deterministischen Modell ............288

6.4 Ausgewählte Ergebnisse im stochastischen Modell ................................ 294

7 Zusammenfassung .......................................................................................305

$8 \quad$ Literaturverzeichnis ........................................................................................... 309

$9 \quad$ Anhang...................................................................................................................................329

9.1 Programm zum Petersburger Paradoxon ........................................... 330

9.2 Ableitung der Überlebenswahrscheinlichkeit für zwei Perioden für ein

Modell mit additiver stochastischer Einzahlung, Startkapital und fester

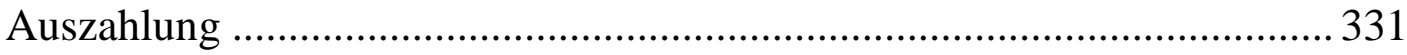

9.3 Berechnung der Überlebenswahrscheinlichkeit für die zweite Runde

nach dem Bayes-Ansatz für unterschiedliche Startkapitale..................... 331 
$9.4 \quad$ Uberlebenswahrscheinlichkeit und Planungshorizont ...............................333

9.5 Simulationsprogramm zur Bestimmung der

Uberlebenswahrscheinlichkeit für ein und zwei Runden ..........................334

$9.6 \quad$ Einzelheiten des Betriebsentwicklungsmodells aus Kapitel 6....................335

10 Index........................................................................................................................................... 393 


\subsection{Schaubilder}

chaubild 23.1: $\quad$ Typen mathematischer Modelle ................................................23

Schaubild 54.1: $\quad$ Entwicklung des Bestandes ......................................................5 54

Schaubild 55.1: $\quad$ Gewinnentwicklung für verschiedene Einzahlungen ......................55

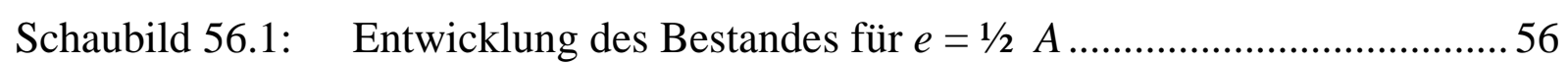

Schaubild 59.1: Bestandsentwicklung bei konstantem, steigendem und

fallendem Reparaturverlauf .............................................................59

Schaubild 60.1: $\quad$ Kritische Werte für Einzahlungskombinationen $(N=2$,

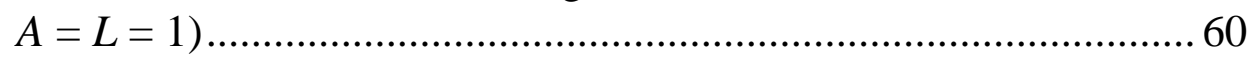

Schaubild 62.1: $\quad$ Investitionsentwicklung ......................................................62

Schaubild 62.2: $\quad$ Gewinnverlauf bei degressiver und linearer Abschreibung ...........62

Schaubild 66.1: $\quad$ Zeitpfade des Gewinns .............................................................66

Schaubild 67.1: $\quad$ Gewinnpfad bei minimalem Startkapital......................................67

Schaubild 67.2: $\quad$ Gewinnpfad bei minimalem Startkapital, Nicht-Ganzzahlig-

keit und Nutzungsdauern von zwei und acht Perioden ................... 67

Schaubild 68.1: Gewinnpfade bei ganzzahligen und stetigen

Investitionsmöglichkeiten............................................................. 68

Schaubild 71.1: Einzahlungsverlauf eines Wertpapiers (in TDM) mit

Zweijähriger Laufzeit ................................................................. 71

Schaubild 75.1: $\quad$ Liability of smallness ............................................................ 75

Schaubild 76.1: $\quad$ Liability of newness und liability of adolescence …........................ 76

Schaubild 86.1: Wahrscheinlichkeit einer Betriebsauflösung in der ,Münchner

Gründerstudie ‘....................................................................... 86

Schaubild 91.1: Entscheidungssituationen bei zwei Auswirkungen einer

Entscheidungsalternative ........................................................ 91

Schaubild 93.1: $\quad$ Beispiel einer Indifferenzkurve ..................................................93

Schaubild 93.2: $\quad$ Beispiel effizienter und ineffizienter Kombinationen aus

Erwartungswert und Streuung ..........................................................93

Schaubild 94.1: Entscheidungssituationen bei Berücksichtigung von

Erwartungswert und range ........................................................... 94

Schaubild 96.1: Vergleich von stochastischen Entscheidungssituationen bei

Berücksichtigung von Erwartungswert und range …...................... 96

Schaubild 97.1: $\quad$ Einzahlung mit kleiner Varianz ..................................................97

Schaubild 97.2: $\quad$ Einzahlung mit hoher Varianz......................................................... 97 
Schaubild 109.1: Dichtefunktionen der Verteilung des Kapitalwerts der Strategien I und II einer Simulationsstudie von BAILEY \& RICHARDSON (1985)....

Schaubild 141.1: Überlegenheit und Schiefe bei gleichem Median und gleicher Varianz............................................................................................ 141

Schaubild 142.1: Überlegenheit und Schiefe bei gleichem Erwartungswert und gleicher Varianz.................................................................... 142

Schaubild 143.1: Vergleich von Verteilungen gleichen Erwartungswertes mit Verteilungen gleichen Medians

Schaubild 145.1: Dichte- und Verteilungsfunktionen zweier Dreieckverteilungen............................................................ 145

Schaubild 146.1: Dichte- und Verteilungsfunktion der Dreieckverteilungen aus Tabelle 140.1, Szenario 10 ...................................................... 146

Schaubild 147.1: Entwicklungspfade für das Modell $K_{t}=K_{t-1}+d_{t}$, $d \sim \operatorname{uiid}\{-1,1\}, K_{0}=1$

Schaubild 148.1: Häufigkeitsverteilung der Kapitalbestände in der dritten Runde für das Modell in Schaubild 147.1 .............................. 148

Schaubild 154.1: Überlebenswahrscheinlichkeit im Zeitablauf ............................. 154 Schaubild 155.1: Survivor- und Hazardfunktion................................................. 155 Schaubild 156.1: $\quad$ Insolvenzwahrscheinlichkeit und Varianz................................. 156 Schaubild 157.1: Überlebenswahrscheinlichkeit und Varianz ............................. 157

Schaubild 161.1: Verteilungen mit gleichen ersten und zweiten Momenten ........... 161

Schaubild 164.1: Dichtefunktionen für Summen von 30 identischen Zufallsveränderlichen ...................................................... 164

Schaubild 165.1: $\quad$ Dichtefunktionen für Summen von 30 Zufallsveränderlichen ..... 165 Schaubild 168.1: $\quad$ Dichtefunktion einer quadrierten Standardnormalverteilung....... 168 Schaubild 171.1: $\quad$ Dichtefunktion einer quadrierten Gleichverteilung..................... 171 Schaubild 172.1: Quadrierte Gleichverteilung mit den Momenten $\mu=-1, \sigma=2 \ldots 172$

Schaubild 175.1: Dichtefunktion des Produkts zweier Standardnormalverteilungen im ersten Quadranten.

Schaubild 176.1: $\quad$ Dichtefunktion des Produktes zweier Standardnormalverteilungen im Monte-Carlo-Simulationsexperiment............... 176

Schaubild 176.2: Vergleich einer quadrierten Standardnormalverteilung mit dem Produkt zweier Standardnormalverteilungen im ersten Quadranten. 
Schaubild 183.1: Mittel- und Maximalwert in Abhängigkeit von der Zahl der Wiederholungen.............................................................. 183

Schaubild 184.1: Auszahlungen einer idealtypischen Stichprobe aus acht Wiederholungen........................................................................ 184

Schaubild 185.1: Mutmaßliche versus realisierte Auszahlungen bei 128 Wiederholungen........................................................... 185

Schaubild 194.1: Zeitliche Entwicklung der Überlebenswahrscheinlichkeit für symmetrisch verteilte Einzahlungen........................................... 194

Schaubild 199.1: Überlebenswahrscheinlichkeit für zwei Perioden ....................... 199

Schaubild 202.1: Kapitalverlauf bei verschiedener Varianz der Einzahlung ........... 202

Schaubild 205.1: Kapitalverlauf bei verschiedener Varianz der Einzahlung und positivem Startkapital ................................................................205

Schaubild 218.1: Übergangswahrscheinlichkeiten in einem einfachen diskreten Überlebensmodell in Abhängigkeit von der Zeit ........................ 218

Schaubild 224.1: Überlebenswahrscheinlichkeit für zwei Runden in Abhängigkeit vom Startkapital, diskretes Modell ..................... 224

Schaubild 227.1: Häufigkeit von Kapitalbeständen nach sechs bzw. zehn Runden ............................................................................... 227

Schaubild 229.1: Überlebenswahrscheinlichkeiten für diskrete Verteilungen unterschiedlicher Varianz. 229

Schaubild 232.1: Überlebenswahrscheinlichkeit für zwei Runden bei gleichverteilten stetigen Einzahlungen, untere Grenze $a$ konstant, obere Grenze $b$ zunehmend

Schaubild 232.2: $\quad$ Bestandteile des sigmoiden Kurvenverlaufs aus

Schaubild 232.1

Schaubild 233.1: Überlebenswahrscheinlichkeit für zwei Runden bei gleichverteilten stetigen Einzahlungen, Varianz konstant, Erwartungswert zunehmend 233

Schaubild 234.1: Überlebenswahrscheinlichkeit für zwei Runden bei gleichverteilten stetigen Einzahlungen in Abhängigkeit vom Startkapital .................................................................... 234

Schaubild 235.1: Überlebenswahrscheinlichkeit für zwei Runden bei standardnormalverteilten Einzahlungen in Abhängigkeit vom Startkapital ......................................................................... 235

Schaubild 239.1: Überlebensquoten für stetig und diskret gleichverteilte Einzahlungen, additives Modell 
Schaubild 241.1: Überlebensquote bei unterschiedlicher Varianz einer diskreten Verteilung im additiven Modell .............................................. 241

Schaubild 243.1: Überlebensquote bei unterschiedlicher Varianz und positivem Startkapital im additiven Modell ................................................. 243

Schaubild 243.2: Überlebensquote bei unterschiedlichem Startkapital im additiven Modell....

Schaubild 245.1: Überlebensquote bei normal-, gleich- oder symmetrisch dreieckverteilten Einzahlungen mit gleicher Varianz, additives

Modell.

Schaubild 246.1: Überlebensquote für variierte Auszahlungen im additiven Modell.......................................................................................... 246

Schaubild 247.1: Überlebensquote für variierte Varianzen und feste Auszahlung im additiven Modell................................................................. 247

Schaubild 248.1: Durchschnittlich erreichtes Alter und mittleres Kapital für variierte Varianzen und feste Auszahlung im additiven Modell...... 248

Schaubild 248.2: Lebensdauer und mittleres Kapital für variierte Varianzen, feste Auszahlung und Planungshorizont von zehn Perioden, additives Modell

Schaubild 250.1: Überlebensquote bei unterschiedlicher Varianz, multiplikatives Modell mit kleinem Startkapital

Schaubild 250.2: Überlebensquote bei unterschiedlicher Varianz für die Perioden fünf und zehn, multiplikatives Modell ....................... 250

Schaubild 251.1: Überlebensquote für stetig und diskret gleichverteilte Einzahlungen, multiplikatives Modell 251

Schaubild 252.1: Überlebensquote bei variierten Auszahlungen, multiplikatives Modell................................................................................................. 252

Schaubild 253.1: Beispiel einer Altersverteilung in einem multiplikativen Modell............................................................................ 253

Schaubild 255.1: Durchschnittliches Alter in einem multiplikativen Modell ......... 255

Schaubild 255.2: Lebenserwartung bei variierter Varianz in einem multiplikativen Modell .................................................. 255

Schaubild 256.1: Überlebensquote für eine vorgegebene Periode in Abhängigkeit von der Varianz der Verzinsung, multiplikatives Modell mit Auszahlung............................................................... 256

Schaubild 257.1: Überlebensquote bei variierter Varianz in einem multiplikativen Modell mit höherem Startkapital .....................257

Schaubild 260.1: $\quad$ Portfoliowahl und Überlebensquote für 10 Perioden ................... 260 
Schaubild 261.1: Portfoliowahl und Lebensdauer................................................ 261

Schaubild 264.1: Anzahl überlebender Betriebe nach vier Perioden und

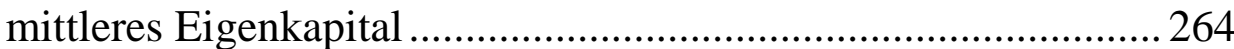

Schaubild 266.1: Vergleich expansiver und vorsichtiger Strategien beim Unternehmensplanspiel „Puten und Perlhühner“ .........................266

Schaubild 267.1: $\quad$ Expansion und zu erwartendes minimales Eigenkapital .............. 267

Schaubild 274.1: Einfaches Beispiel eines Modells mit rationalen Erwartungen .... 274

Schaubild 274.2: Bestimmung der Produktionsmenge eines Unternehmens mit rationalen Erwartungen..............................................................22

Schaubild 285.1: Ablaufschema des Simulationsprogramms ................................... 285

Schaubild 287.1: Entwicklung von Tier- und Pflanzenproduktion, Zupacht und AKh-Zukauf bei Gewinnmaximierung unter Sicherheit ..............287

Schaubild 288.1: Zeitliche Entwicklung der Finanzkennzahlen bei Gewinnmaximierung unter Sicherheit.........................................28

Schaubild 289.1: Produktionsverfahren bei Diversifikation im deterministischen

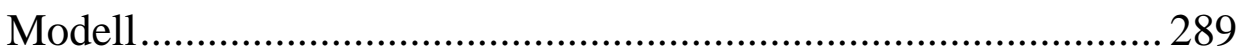

Schaubild 290.1: Finanzkennzahlen bei Diversifikation im deterministischen Modell ........................................................................................ 290

Schaubild 291.1: Produktionsverfahren mit Risikoabschlägen im deterministischen Modell

Schaubild 293.1: Finanzkennzahlen bei Risikoabschlägen im deterministischen Modell 293

Schaubild 299.1: Vermögensendwerte und Überlebenswahrscheinlichkeiten der Strategien im stochastischen Modell II

Schaubild 334.1: Anzahl überlebender Betriebe nach sechs Perioden und mittleres Eigenkapital

\subsection{Tabellen}

Tabelle 55.1: $\quad$ Voraussetzungen für lokale Minima................................................55

Tabelle 58.1: $\quad$ Bestandsentwicklung bei konstantem, fallendem und steigendem Reparaturverlauf ........................................................ 58

Tabelle 60.1: $\quad$ Kritische Werte für Einzahlungskombinationen ............................60

Tabelle 64.1: $\quad$ Parameter des ganzzahligen Referenzmodells ..............................64

Tabelle 65.1: $\quad$ Zeitpfade für ein ganzzahliges Modell ...........................................6 65 
Tabelle 69.1: $\quad$ Grenzwerte des Bestandes für stetige Investitionen .......................69

Tabelle 70.1: $\quad$ Grenzwerte des Bestandes für stetige Investitionen und zwei Startkapitale ............................................................. 70

Tabelle 71.1: $\quad$ Einzahlungsverlauf eines Wertpapiers ..................................... 71

Tabelle 96.1: $\quad$ Beispiele für Entscheidungssituationen bei Verwendung des Erwartungswert-range-Kriteriums ..................................... 96

Tabelle 100.1: $\quad$ Beispiel eines einfachen LP-Tableaus................................. 100

Tabelle 101.1: Zielfunktionswerte bei zwei stochastischen

Zielfunktionskoeffizienten................................................ 101

Tabelle 109.1: $\quad$ Signifikanztests von Überlebenswahrscheinlichkeiten ................. 109

Tabelle 110.1: $\quad$ Signifikanztests für die Verschiedenheit von Überlebenswahrscheinlichkeiten bei 100 Wiederholungen...........................110

Tabelle 113.1: $\quad$ Abkürzungen, die in Abschnitt 3.4 verwendet werden ................ 113

Tabelle 124.1: $\quad$ LP-Tableau nach BRANDES \& WOERMANN (1969, S. 112, ergänzt um Finanzanlagen) und Ergebnis ................................ 124

Tabelle 125.1: $\quad$ LP-Tableau nach Variante 7 und Ergebnis............................... 125

Tabelle 126.1: $\quad$ Vom Zielwert eines LPs zum Gewinn................................... 126

Tabelle 132.1: $\quad$ Wahrscheinlichkeiten bei zweimaligem Würfeln ...................... 132

Tabelle 134.1: $\quad$ Simulationsergebnisse für $\mathrm{p}(\xi \leq \eta), \xi$ und $\eta$ identisch und unabhängig verteilt ............................................................. 134

Tabelle 138.1: $\quad$ Simulationsergebnisse für $\mathrm{p}(\xi \leq \eta)$, 1. Teil: symmetrische Verteilung für $\xi$ und $\eta$, identische Erwartungswerte ................. 138

Tabelle 140.1: $\quad$ Simulationsergebnisse für $\mathrm{p}(\xi \leq \eta)$, 2. Teil: unsymmetrische Dreieckverteilungen für $\xi$ und $\eta$..................................... 140

Tabelle 141.1: $\quad$ Überlegenheit und Schiefe bei gleichem Median und gleicher

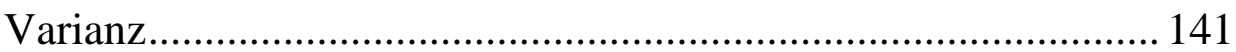

Tabelle 142.1: $\quad$ Wahrscheinlichkeit $\mathrm{p}(\xi \leq \eta)$ und Schiefe bei gleichem

Erwartungswert.......................................................... 142

Tabelle 143.1: $\quad$ Vergleich von Verteilungen gleichen Erwartungswertes mit Verteilungen gleichen Medians ....................................... 143

Tabelle 149.1: $\quad$ Überlebenspfade für das Modell in Schaubild 147.1 .................. 149

Tabelle 153.1: $\quad$ Überlebenswahrscheinlichkeit für das Beispiel aus Schaubild 147.1 ............................................................ 153

Tabelle 177.1: $\quad$ Simulierte Momente zusammengesetzter normalverteilter Zufallsveränderlicher 
Tabelle 180.1: $\quad$ Auszahlungsmöglichkeiten $\left(a_{n}\right)$ und ihre Wahrscheinlichkeiten $\left(p_{n}\right)$............................................ 180

Tabelle 182.1: $\quad$ Mittel- und Maximalwert von Auszahlungen in Abhängigkeit von der Zahl der Spielwiederholungen.................................. 182

Tabelle 183.1: $\quad$ Ein Ergebnis für einzelne und mittlere Auszahlungen bei ein bis zehn Spielwiederholungen ........................................... 183

Tabelle 184.1: $\quad$ Idealtypische Verteilung der Auszahlungen bei acht

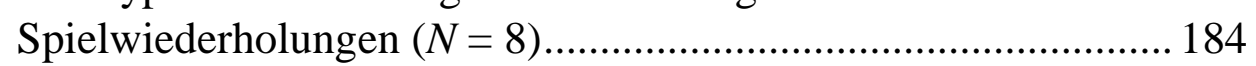

Tabelle 184.2: $\quad$ Verteilung der Auszahlungen bei 128 Wiederholungen

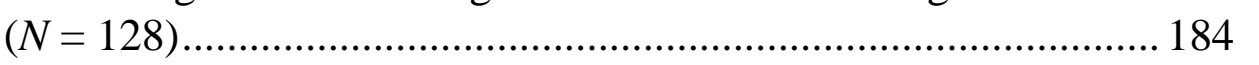

Tabelle 187.1: $\quad$ Empirische und theoretische Stichprobenmittel ......................... 187

Tabelle 196.1: $\quad$ Überlebenswahrscheinlichkeit in der ersten Runde für diskrete Gleichverteilung mit $k$ Elementen, $K_{0}=0 \ldots \ldots \ldots \ldots \ldots \ldots \ldots \ldots \ldots \ldots \ldots$

Tabelle 213.1: Zusammenfassung: analytische Ergebnisse zur Überlebenswahrscheinlichkeit..............................................213

Tabelle 215.1: $\quad$ Übergangswahrscheinlichkeiten in einem einfachen diskreten Überlebensmodell .............................................................. 215

Tabelle 216.1: $\quad$ Übergangswahrscheinlichkeiten von Periode 1 nach 3 ...............216

Tabelle 217.1: $\quad$ Bedingte Kapitalbestände in der dritten Runde, Bedingung:

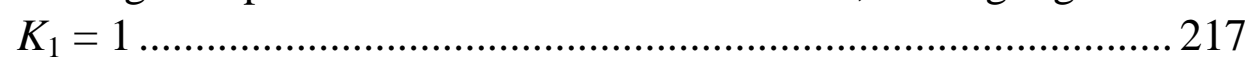

Tabelle 220.1: $\quad$ Kapital in der zweiten Runde in Abhängigkeit von den EinZahlungen und Auszählen der Überlebenswahrscheinlichkeit ..... 220

Tabelle 222.1: $\quad$ Berechnung der Überlebenswahrscheinlichkeit für die zweite Runde nach dem Bayes-Ansatz, ohne Startkapital...................222

Tabelle 223.1: $\quad$ Berechnung der Überlebenswahrscheinlichkeit für die zweite Runde nach dem Bayes-Ansatz, mit Startkapital ......................223

Tabelle 224.1: $\quad$ Konkrete Berechnung der Überlebenswahrscheinlichkeit für die zweite Runde nach dem Bayes-Ansatz für variierte Startkapitale ....................................................................... 224

Tabelle 225.1: $\quad$ Kapital in der zweiten Runde in Abhängigkeit von den Einzahlungen und Auszählen der Überlebenswahrscheinlichkeit, kleinere Varianz............................................................... 225

Tabelle 227.1: $\quad$ Zeitliche Entwicklung der Verteilung von Kapitalbeständen ......227

Tabelle 228.1: $\quad$ Realisationen für die zweite Periode eines Systems mit diskreten gleichverteilten Zufallsveränderlichen ...................... 228

Tabelle 238.1: $\quad$ Überlebenswahrscheinlichkeit für zwei Perioden ......................238 
Tabelle 240.1: $\quad$ Überlebenswahrscheinlichkeit bei unterschiedlicher Varianz im additiven Modell............................................................ 240

Tabelle 242.1: $\quad$ Vergleich von Überlebensquoten bei unterschiedlicher

Varianz und positivem Startkapital im additiven Modell........... 242

Tabelle 260.1: $\quad$ Parameter eines Portfoliomodells ............................................. 260

Tabelle 263.1: $\quad$ Parameter des Überlebensmodells ........................................... 263

Tabelle 263.2: Anzahl überlebender Unternehmen (keine Fremdkapitalaufnahme) .................................................................... 263

Tabelle 286.1: $\quad$ Parameter des Betriebsentwicklungsmodells (Auszug) ............... 286

Tabelle 294.1: $\quad$ Wichtige Finanzkennziffern von vier Strategien im deterministischen Modell ................................................... 294

Tabelle 296.1: $\quad$ Wichtige Produktionskennziffern der Strategien .......................296

Tabelle 297.1: $\quad$ Vermögensendwerte und Überlebenswahrscheinlichkeiten der

Strategien im stochastischen Modell ........................................ 297

Tabelle 301.1: $\quad$ Vermögensendwerte und Überlebenswahrscheinlichkeiten der

Strategien im stochastischen Modell: Variation des

Startkapitals ........................................................................ 301

Tabelle 302.1: $\quad$ Vermögensendwerte und Überlebenswahrscheinlichkeiten der Strategien im stochastischen Modell: Variation der Faktorausstattung...

Tabelle 303.1: $\quad$ Vermögensendwerte und Überlebenswahrscheinlichkeiten bei

Gewinnmaximierung und Variation der Getreidepreisstreuung .. 303

Tabelle 332.1: $\quad$ Berechnung der Überlebenswahrscheinlichkeit für die zweite

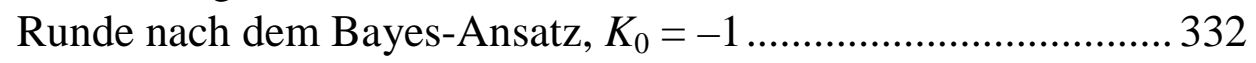

Tabelle 332.2: $\quad$ Berechnung der Überlebenswahrscheinlichkeit für die zweite

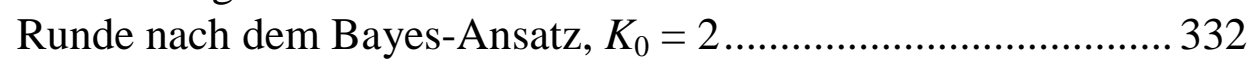

Tabelle 333.1: $\quad$ Berechnung der Überlebenswahrscheinlichkeit für die zweite

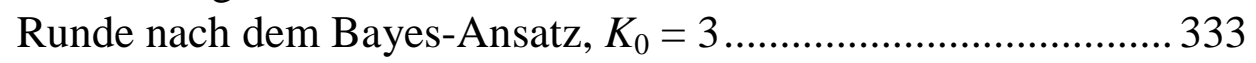

Tabelle 333.2: $\quad$ Berechnung der Überlebenswahrscheinlichkeit für die zweite

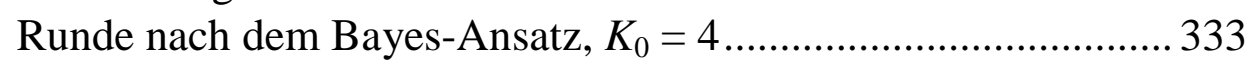

Tabelle 336.1: $\quad$ Arbeitswirtschaft .............................................................. 336

Tabelle 336.2: $\quad$ Produktionsverfahren Pflanzenproduktion .................................. 336

Tabelle 337.1: $\quad$ Produktionsverfahren Pflanzenproduktion bei 10\%iger

Preisreduzierung ............................................................. 337

Tabelle 337.2: $\quad$ Produktionsverfahren Tierproduktion ..........................................337 
Tabelle 338.1: $\quad$ Produktionsverfahren Tierproduktion bei 2.5\%iger

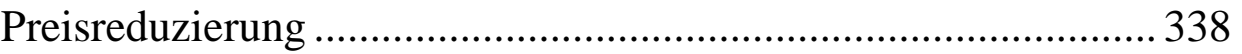

Tabelle 339.1: $\quad$ Zusammenfassung der im Tableau verwendeten Abkürzungen... 339

Tabelle 340.1: $\quad$ LP-Tableau des Grundmodells, erste Periode ............................ 340

Tabelle 359.1: $\quad$ Kennziffern zur Betriebsbeschreibung ................................... 359

Tabelle 381.1: $\quad$ Erläuterung der im Ergebnisausdruck verwendeten

Abkürzungen ....................................................................... 381

Tabelle 382.1: $\quad$ Ergebnisausdruck für das deterministische Grundmodell

(Tabelle 340.1)............................................................. 382

Tabelle 384.1: $\quad$ Ergebnisgrößen der Strategie „Risikoabschlag: 10\% bei

Pflanzenproduktion, $2.5 \%$ bei Tierproduktion“ im

deterministischen Modell ............................................................. 384

Tabelle 386.1: $\quad$ Ergebnisgrößen der Strategie „Risikoabschlag: 5\% bei

Pflanzenproduktion, $2.5 \%$ bei Tierproduktion" im

deterministischen Modell .................................................... 386

Tabelle 388.1: $\quad$ Subtraktion der Ergebnisgrößen der Strategie „Risikoabschlag: $10 \%$ bei Pflanzenproduktion, $2.5 \%$ bei Tierproduktion“ (Tabelle 382.1) von der Strategie ,Risikoabschlag: $5 \%$ bei Pflanzenproduktion, $2.5 \%$ bei Tierproduktion“" (Tabelle 384.1) ................................................ 388

\section{4 Übersichten}

Übersicht 50.1: $\quad$ Lösung einer Differenzengleichung zweiter Ordnung am Beispiel der Fibonacci-Zahlen ............................................. 50

Übersicht 51.1: $\quad$ Lösung der Differenzengleichung (49.1) .................................51

Übersicht 100.1: Vorschlag zur Begriffsbestimmung von Unsicherheit,

Ungewißheit und Risiko ................................................... 100

Übersicht 107.1: Ergebnisse einer Simulationsstudie von BAILEY \& RICHARDSON (1985) ............................................................ 107

Übersicht 136.1: Kennzahlen der Dreieckverteilung ........................................ 136

Übersicht 162.1: $\quad$ Dichtefunktionen für Summen von Zufallsveränderlichen .......... 162

Übersicht 166.1: Rechenregeln für Erwartungswerte und Varianzen zusammengesetzter Zufallsveränderlicher .............................. 166

Übersicht 172.1: Dichtefunktionen quadrierter Gleichverteilungen und ihrer Ausgangsfunktionen ........................................................ 172 
Übersicht 173.1: Dichtefunktionen quadrierter Verteilungen und ihrer Ausgangsfunktionen

Übersicht 190.1: $\quad$ Einteilung metrisch dynamischer Systeme..................................... 190

\subsection{Abkürzungen und wichtige Definitionen}

\subsubsection{Mathematisch-statistische Symbole}

$[a, b] \quad=\{\mathrm{x} \mid a \leq x \leq b)$, abgeschlossenes Intervall, die Grenzen des Intervalls gehören zur Menge

$(a, b)=\{\mathrm{x} \mid a<x<b)$, offenes Intervall, die Grenzen des Intervalls gehören nicht zur Menge

$\{-1,0,4\} \quad$ Elemente einer Menge

$u \quad$ untere Grenze einer Gleich- oder Dreieckverteilung

$o \quad$ untere Grenze einer Gleich- oder Dreieckverteilung

$\{u \ldots o\} \quad$ Menge aller ganzen Zahlen zwischen $u$ und $o$, auch gleichmäßige Menge genannt

$\sim \quad$ verteilt

$\sim \mathrm{u}\{\} \quad$ diskrete Gleichverteilung, z.B. bedeutet: $z \sim \mathrm{u}\{-1 ; 0 ; 1\}$, daß $z$ mit gleicher Wahrscheinlichkeit die Ausprägungen $-1,0$ und 1 annimmt.

$\sim \mathrm{u}\{u \ldots o\}$ gleichmäßige Verteilung im abgeschlossenen Intervall $[u, o]$ : Alle ganzen Zahlen zwischen $u$ und $o$ sind gleichwahrscheinliche Realisationsmöglichkeiten der Verteilung. BOSCH (1992) S. $168 \mathrm{f}$ definiert eine gleichmäßige Verteilung enger: alle Realisationsmöglichkeiten müssen natürliche Zahlen sein.

$\sim \mathrm{u}(a, b) \quad$ stetige Gleichverteilung mit den Parametern $a$ und $b$

Var Varianz

$\operatorname{Var}\{\} \quad$ Varianz der in den geschweiften Klammern näher bezeichneten Menge, jedes Element der Menge ist gleichwahrscheinlich.

$\operatorname{Var}(u, o) \quad$ Varianz einer stetigen Gleichverteilung mit den Parametern $u$ und $o$

$\operatorname{Var}(u \ldots o) \quad$ Varianz einer gleichmäßigen Verteilung zwischen $u$ und $o$

$s \quad$ Schiefe

E Erwartungswert

$\overline{\mathrm{x}} \quad$ arithmetisches Mittel

$m \quad$ Median 


\begin{tabular}{|c|c|}
\hline$h$ & Modalwert (häufigster Wert) \\
\hline$i i$ & identical and independent \\
\hline iid & identically independently distributed \\
\hline uiid & uniformly, identically and independently distributed \\
\hline niid & normally, identically and independently distributed \\
\hline $\mathrm{n}\left(\mu, \sigma^{2}\right)$ & Normalverteilung mit Erwartungswert $\mu$ und Varianz $\sigma^{2}$ \\
\hline $\mathrm{N}(0,1)$ & Standardnormalverteilung (Erwartungswert 0, Varianz 1) \\
\hline$N$ & Menge der natürlichen Zahlen \\
\hline $\mathbb{Z}$ & Menge der ganzen Zahlen \\
\hline $\mathbb{R}$ & Menge der reellen Zahlen \\
\hline $\mathrm{H}(x)$ & Heaviside-Funktion \\
\hline$\varnothing$ & durchschnittlich \\
\hline$\equiv$ & definitionsgemäß gleich \\
\hline$:=$ & definitionsgemäß gleich \\
\hline$\stackrel{?}{=}$ & vermutete Identität, die im folgenden überprüft wird, Hypothese \\
\hline $\mathrm{MC}$ & Monte-Carlo \\
\hline MCS & Monte-Carlo-Simulation \\
\hline ül $(t)$ & Ereignis, daß ein System bis zur Runde $t$ überlebt. \\
\hline $\mathrm{p}_{\mathrm{uil}}(t)$ & Wahrscheinlichkeit eines Systems, bis zur Runde $t$ zu überleben \\
\hline $\mathrm{p}_{\ddot{u ̈ l}}\left(x_{t}\right)>0$ & $\begin{array}{l}\text { Wahrscheinlichkeit, daß die Variable } x \text { sich in allen Runden } 1 \text { bis } t \text { grö- } \\
\text { ßer als Null realisiert. }\end{array}$ \\
\hline
\end{tabular}

Hazardrate Insolvenzrate. Anteil der Unternehmen, die in einer bestimmten Runde erstmals die Überlebensbedingung nicht erfüllen.

neutrale Einzahlungen: Einzahlungen, die den Erwartungswert des Kapitals nicht verändern

(1)(2)(4)(5)(6)(8)(9)(10) Numerierung für Spalten in Tabellen

123456889(10) Referenz auf einzelne Zellen in Tabellen

\subsubsection{Sonstige}

$A b$

Abschreibung

AK

Arbeitskraft-Einheit

$A K h$

Arbeitskraftstunde 
AW Agrarwirtschaft - Zeitschrift für Betriebswirtschaft, Marktforschung und Agrarpolitik

DB Deckungsbeitrag

Dreieckv. Dreieckverteilung

GeWiSoLa Gesellschaft für Wirtschafts- und Sozialwissenschaften des Landbaus e.V.

Gleichv. Gleichverteilung

ha Hektar

HdSW Handwörterbuch der Sozialwissenschaften, Stuttgart, Tübingen, Göttingen 1956 bis 1965 .

HdWW: Handwörterbuch der Wirtschaftswissenschaft

i in, im

Inv., Invest. Investition(en)

IRR interne Verzinsung (internal rate of return)

i.V. in Vorbereitung

Kap- Kapitel

kurzfr. kurzfristig

langfr. kurzfristig

LP lineare Programmierung

Normalv. Normalverteilung

o. oder

o.V. ohne Verfasser

PP Pflanzenproduktion

RW-DB Richtwert-Deckungsbeiträge der Landwirtschaftskammer Hannover

S. $\quad$ siehe

s.o. $\quad$ siehe oben

SAS Datenanalyseprogramm von SAS ${ }^{\circledR}$ Institute

Startkap. Startkapital

sog. Sogenannte

TP Tierproduktion

u. und

u.a. unter anderem 
Uelwa Überlebenswahrscheinlichkeit

vgl. vergleiche

vK variable Kosten

Wh., Whlg. Wiederholung

Whlgen Wiederholungen

WiSt Wirtschaftswissenschaftliches Studium

z.B. zum Beispiel

zfb Zeitschrift für Betriebswirtschaft

zfbf Zeitschrift für betriebswirtschaftliche Forschung

zit. n. zitiert nach

\subsection{Redaktionelle Anmerkungen}

- Tabellen, Schaubilder und Übersichten sind in dieser Arbeit seitenweise numeriert, um bei Verweisen das Auffinden zu Erleichtern. „Siehe Tabelle 17.2“ beispielsweise verweist auf die zweite Tabelle der Seite 17.

- In einigen Abschnitten wird teilweise Lehrbuchwissen referiert, da es die Basis für eigene Entwicklungen bildet. Ist dieses Lehrbuchwissen sehr grundlegend, verzichte ich wie üblich auf eine Quellenangabe. Um es dennoch von eigenen Gedanken unterscheidbar zu machen, ist es in Zweifelsfällen durch einen Zusatz wie ,bekanntlich“ oder ,wie bekannt“ gekennzeichnet. Auf der anderen Seite werden wichtige eigene Ergebnissen durch die Verwendung von Personalpronomen der ersten Person hervorgehoben.

- Die meisten Tabellen, Schaubilder und Übersichten in dieser Arbeit gehen nicht auf andere Veröffentlichungen zurück, sondern basieren auf eigenen Berechnungen und Überlegungen. Daher sind Quellen nur angegeben, wenn fremdes geistiges Eigentum verwendet wurde.

- Englischsprachige Fachtermini werden, sofern sie nicht sehr gängig sind, undekliniert übernommen und durch kursive Schreibweise gekennzeichnet.

- Den einzelnen Hauptkapiteln ist ein vollständiges Inhaltsverzeichnis vorangestellt. Daher beschränkt sich das Inhaltsverzeichnis zu Beginn der Arbeit auf zwei Ebene, um eine bessere Übersichtlichkeit zu ermöglichen, 


\section{Einleitung}

Die Zahl der Insolvenzen in Deutschland stieg in den vergangenen Jahren kontinuierlich an. Für 1998 wird ein neuer Höchststand erwartet, der achte Rekord in Folge (dpa, 28.8.1998). Seit 1990 hat sich die Anzahl der Unternehmensinsolvenzen mehr als verdreifacht und betrug im vergangenen Jahr 27500 (STATISTISCHES BUNDESAMT 1998). Die volkswirtschaftlichen Kosten von Unternehmensinsolvenzen sind erheblich. Allein im Jahr 1996 sind nach Angaben der größten deutschen Kreditauskunftei „Creditreform e.V." Firmenzusammenbrüchen rund 300000 Arbeitsplätze zum Opfer gefallen. Allerdings ist zu erwarten, daß der konkursbedingte Produktionsausfall zum Teil durch andere, expandierende Unternehmen ausgeglichen wird, so daß infolge einer damit verbundenen positiven Beschäftigungswirkung der Nettoverlust an Arbeitsplätzen geringer ausfällt.

Verluste entstehen einer Volkswirtschaft durch Insolvenzen insbesondere dann, wenn sie endgültige Betriebsschließungen nach sich ziehen. Die Höhe der im Rahmen von Insolvenzanträgen ermittelten Forderungen summierte sich im Bundesgebiet 1997 auf 38.5 Mrd. DM. Dies bedeutet im Vergleich zu 1990 eine Steigerung um $460 \%$. Der Großteil der Forderungen infolge von Insolvenzen wird nicht befriedigt. In den vergangenen Jahren lagen die Deckungsquoten nicht bevorrechtigter Forderungen in der Regel unter 5\%. Drei Viertel aller Konkursanträge wurden mangels Masse abgelehnt. Die Hauptursache der geringen Liquidationserlöse ist der drastische Wertverfall der in einem insolventen Unternehmen vorhandenen baulichen Anlagen und Maschinen. Es besteht daher ein wohlfahrtsökonomisches Interesse an einer Vermeidung von betrieblichen Investitionen, die mit einer Insolvenz enden.

Im landwirtschaftlichen Sektor spielen Insolvenzen eine untergeordnete Rolle. Die Zahl der landwirtschaftlichen Betriebe verringert sich zwar in Westdeutschland seit Jahrzehnten um $2 \%$ bis $3 \%$ per anno, doch erfolgen diese Schließungen meist freiwillig im Zuge des Generationenwechsels. Landwirtschaftliche Vollerwerbsbetriebe in Westdeutschland verfügen im allgemeinen wegen ihres Bodeneigentums über eine breite Eigenkapitalbasis, so daß die beiden Auslöser einer Insolvenz, Überschuldung und Zahlungsunfähigkeit, selten auftreten. Anders ist die Situation in Ostdeutschland, wo in den Jahren nach der Wende viele umgegründete Kapitalgesellschaften und Genossenschaften ohne Grundvermögen in Konkurs gerieten.

Angesichts dieser Entwicklung scheint eine wissenschaftliche Auseinandersetzung mit Insolvenzen in der Landwirtschaft geboten. Im Unterschied zu den meisten anderen Branchen ist jedoch aufgrund der relativ geringen Zahl echter Insolvenzen die Datenbasis für eine quantitative empirische Untersuchung der Ursachen für das Scheitern und möglicher Strategien zur Vermeidung von Insolvenzen nicht ausreichend. 
Daher versucht die vorliegende Arbeit an dieser Stelle einen anderen Beitrag zu leisten. Landwirtschaftliche Unternehmen werden als dynamisches System modelliert. Das Ziel besteht zunächst darin, aufzuzeigen, mit welchen Methoden Überlebenswahrscheinlichkeiten dynamischer Systeme generell analysiert werden können. Hierfür werden zunächst sehr einfache Systeme betrachtet, die einer analytischen Lösung zugänglich sind. Komplexere Systeme erfordern anspruchsvollere Verfahren zur Analyse der Überlebenswahrscheinlichkeit. Letztendlich wird in dieser Arbeit ein stilisierter landwirtschaftlicher Betrieb in Form eines komplexen dynamisch stochastischen Systems abgebildet und daraufhin untersucht, wie sich verschiedene Verhaltensweisen bzw. Strategien bei variierten Umweltbedingungen auf die Überlebensfähigkeit auswirken.

Der Schwerpunkt dieser Arbeit liegt dabei auf der methodischen Auseinandersetzung mit der Analyse von Überlebenswahrscheinlichkeiten. Daneben werden auch praktisch relevante Aussagen über die Auswirkungen unterschiedlicher Strategien in einem landwirtschaftlichen Unternehmen abgeleitet. Die Vorgehensweise für diese letztgenannte Aufgabenstellung ist in dieser Arbeit wie folgt: Es wird ein stilisierter landwirtschaftlicher Betrieb entworfen, der in einer hypothetischen, stochastisch modellierten Umwelt agiert. Durch dieses Vorgehen ist es möglich, wie in einem Experiment verschiedene Betriebstypen im Zeitablauf unter vorgegebenen Umweltszenarien zu studieren. Insbesondere können Auswirkungen von Unternehmensstrategien auf den Unternehmenserfolg beobachtet werden. Da die modellierte Umwelt durch Unsicherheit gekennzeichnet ist, wird eine stochastische Simulation durchgeführt. Das Hauptinteresse dieser Simulationsstudie gilt dabei nicht monetären Kennzahlen, wie Gewinn oder Umsatz, sondern der Überlebenswahrscheinlichkeit. Diese simpel anmutende Verschiebung der Fragestellung führt zu erheblichen methodischen Schwierigkeiten. Bei der Betrachtung von Finanz- oder Produktionskennziffern interessieren im allgemeinen ihre Erwartungswerte und Streuungen, die durch die Verteilungsmomente der Ergebnisgrößen ausgedrückt werden. Sie sind wahrscheinlichkeitstheoretisch weit einfacher herzuleiten als die Analyse von Überlebenswahrscheinlichkeiten. Um beispielsweise zu beantworten, mit welcher Wahrscheinlichkeit sich eine Zufallsvariable kleiner als Null realisiert, helfen die Momente der Zufallsvariablen allein nicht weiter, statt dessen muß ihre ganze Verteilung bekannt sein. Die Ableitung von Wahrscheinlichkeitsverteilungen dynamischer Variablen gelingt nur in Ausnahmefällen analytisch. Daher werden zur Bestimmung von Überlebenswahrscheinlichkeiten meist Monte-Carlo-Simulationen eingesetzt.

In dieser Arbeit wird entsprechend vorgegangen. Wo es möglich ist, werden analytische Lösungen abgeleitet. Die mathematischen Grundlagen hierfür werden anhand einfacher Modelle in Kapitel 4 dargelegt. Die Analyse der Überlebenswahrscheinlichkeit des erwähnten stilisierten landwirtschaftlichen Betriebes erfolgt indessen in einer Monte-Carlo-Simulation.

Die Arbeit gliedert sich in fünf inhaltliche Teile: Kapitel 2, 3 und 4 sind theoretischmethodischen Fragen gewidmet. Da die Verwendung von Modellen einen Schwerpunkt dieser Arbeit bildet, beginnt Kapitel 2 mit einer wissenschaftstheoretischen Auseinandersetzung mit Modellen als Analyseinstrument. Besonderes Gewicht erfah- 
ren dabei Simulationsmodelle. Im zweiten Abschnitt dieses Kapitels wird als ein Beispiel für eine Modellanalyse ein einfaches deterministisches Betriebsentwicklungsmodell vorgestellt, das zu überraschenden nicht monotonen Gewinnverläufen führt. Der dritte und vierte Abschnitt befassen sich mit der Insolvenzwahrscheinlichkeit von Unternehmen. Dabei liegt im dritten Abschnitt der Schwerpunkt auf theoretischen Ansätzen zur Erklärung von Insolvenzwahrscheinlichkeiten, die insbesondere den Forschungszweigen der Organisationstheorie und der Evolutorischen Ökonomik zuzuordnen sind. Im vierten Abschnitt folgt ein kurzer Überblick über empirische Ergebnisse und modellgestützte Analysen zur Überlebenswahrscheinlichkeit.

Ein Betriebsentwicklungsmodell dient dazu, die Entwicklung von Betrieben in einer ungewissen Zukunft abzubilden. Daher sollten Betriebsentwicklungsmodelle die Möglichkeit einer Expansion bzw. Veränderung der Produktion bieten und gleichzeitig die Unsicherheit zukünftiger Entwicklungen berücksichtigen. Die Bedeutung von Unsicherheit für Entscheidungsprobleme und Möglichkeiten ihrer Integration in die Betriebsplanung werden in Kapitel 3 erläutert. Da stochastische Betriebsentwicklungsmodelle meist als Monte-Carlo-Simulationen spezifiziert sind, schließt sich eine Diskussion von Möglichkeiten der Signifikanzprüfung für diese Modellart an. Dieses methodische Kapitel endet mit der Vorstellung eines Ansatzes zur Einführung von Investitionen in einperiodische LP-Modelle, die gleichzeitig einen Eigenkapital- und einen Fremdkapitalzinsfuß berücksichtigen.

Kapitel 4 rundet die theoretischen Betrachtungen ab, indem mathematische Eigenschaften einfacher dynamischer Systeme untersucht werden. Hier erfolgt auch eine genauere formale Definition des Begriffs Überlebenswahrscheinlichkeit. Anschließend werden Einflußfaktoren auf die Überlebenswahrscheinlichkeit abgeleitet, die einer analytischen oder logischen Herangehensweise zugänglich sind und daher keiner Untersuchung mit Hilfe einer Monte-Carlo-Studie bedürfen. Auf Probleme in MonteCarlo-Simulationen im Zusammenhang mit Ereignissen, die sehr kleine Wahrscheinlichkeiten besitzen, macht der abschließende Exkurs zum Petersburger Paradoxon aufmerksam.

Das Kapitel 4 dient zur Vorbereitung der Behandlung einfacher Überlebensmodelle, die in Kapitel 5 vorgestellt werden. Gemäß der in Kapitel 2 unterstützten Forderung, daß für eine bestimmte Fragestellung stets das einfachste Modell vorzuziehen ist, erfolgt im Kapitel 5 eine Auseinandersetzung mit Einflüssen des Datenkranzes auf die Überlebenswahrscheinlichkeit anhand sehr einfacher Modelle, die auf linearen Differenzengleichungen basieren. Die ersten Abschnitte dieses Kapitels zeigen die Grenzen analytischer Lösbarkeit dieser Modelle auf. Die nachfolgenden Abschnitte präsentieren Ergebnisse von Monte-Carlo-Simulationen. Untersucht werden insbesondere der Verteilungstyp und die Varianz der Einzahlungen. Seinen Abschluß findet das Kapitel mit einer Analyse der Erfolgsaussichten von Strategien in einem Unternehmensplanspiel, die auf empirische Ergebnisse dieses Spiels zurückgreift.

Kapitel 6 schließlich stellt ein stochastisches Betriebsentwicklungsmodell vor, das als Überlebensmodell konzipiert ist. Das Kapitel beginnt mit einer kurzen Einführung 
in mögliche Strategien und Umweltszenarien in Betriebsentwicklungsmodellen. Darauf folgt eine Beschreibung des gewählten Modells. Implikationen der Strategiewahl, der Umweltmodellierung und betrieblichen Kapazitäten werden für ein deterministisches und zwei stochastische Modelle dargestellt. Mit einer Zusammenfassung der wichtigsten Ergebnisse in Kapitel 7 schließt die Arbeit. 


\section{Zur Analyse der Überlebensfähigkeit von Unternehmen}

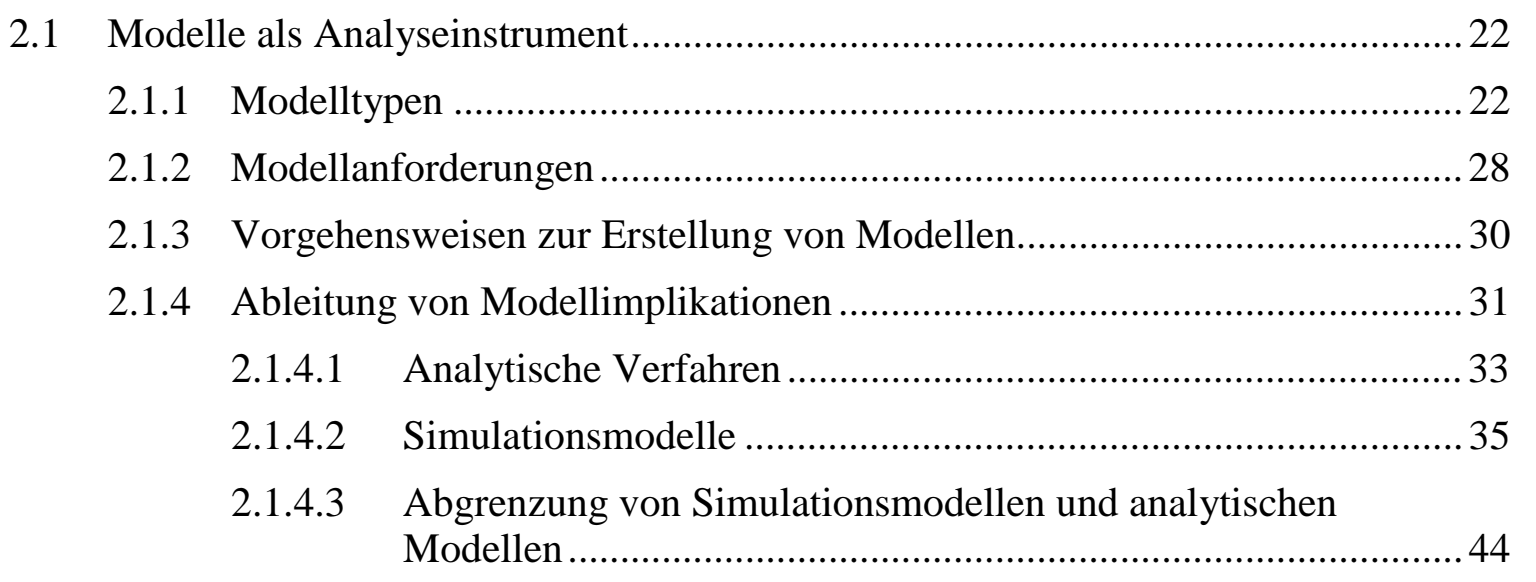

2.2 Exkurs: $\mathrm{Zu}$ Gewinnentwicklungen in Betriebsentwicklungsmodellen

(LOHMANN-RUCHTI-Effekt) ..................................................................46

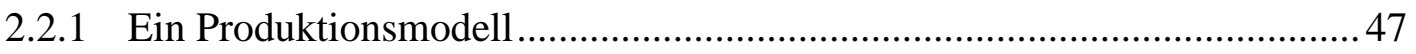

2.2.1.1 Berücksichtigung von Reparaturen....................................56

2.2.1.2 Einfluß der Abschreibungsmethode auf den LOHMANNRUCHTI-Effekt.......................................................................... 61

2.2.1.3 Besonderheiten für ganzzahlige Investitionen und längere

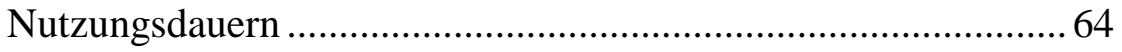

2.2.2 Der LOHMANN-RUCHTI-Effekt auf dem Finanzmarkt ............................. 70

2.2.3 Zur Bedeutung des Kapitalmarktes ............................................... 71

2.2 .4 Zusammenfassung ............................................................... 72

2.3 Theoretische Ansätze und empirische Methoden zur Erklärung der

Überlebensfähigkeit von Unternehmen............................................................. 73

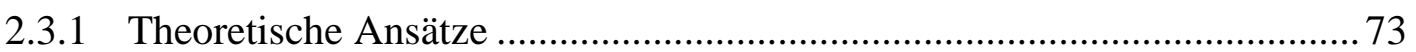

2.3.1.1 Organisationstheoretische Ansätze ....................................... 73

2.3.1.1.1 Organisationsökologie ............................................ 74

2.3.1.1.2 Random walk Modelle ........................................... 78

2.3.1.2 Industrieökonomische Ansätze ........................................... 79

2.3.1.3 Evolutionsökonomische Ansätze........................................ 80

2.3 .2 Empirische Methoden ...................................................................... 81

2.4 Studien zur Uberlebenswahrscheinlichkeit von Unternehmen ............................ 84

Dieses Kapitel dient der theoretischen Hinführung an die Thematik dieser Arbeit, in deren Zentrum analytische Ansätze und Simulationsmodelle zur Untersuchung von 
Überlebenswahrscheinlichkeiten stehen. ${ }^{1}$ Der erste Abschnitt gibt einen allgemeinen Überblick über die Bildung und Verwendung von mathematischen Modellen. Ein besonderer Schwerpunkt wird dabei auf Simulationsmodelle gelegt. Daran anknüpfend erfolgt in einem Exkurs die Anwendung eines Betriebsentwicklungsmodells, die überraschende Gewinnverläufe zum Ergebnis hat. Im dritten Abschnitt werden wichtige theoretische Ansätze zur Erklärung der Überlebensfähigkeit von Unternehmen beschrieben. Anwendungen und empirische Studien, die sich mit dieser Thematik befassen, werden im vierten Abschnitt vorgestellt.

\subsection{Modelle als Analyseinstrument}

"Answering questions is the sine qua non of a good model." (J. CASTI)

In diesem Abschnitt erfolgt eine Darstellung verschiedener Modelltypen, der Anforderungen, die an Modelle gestellt werden, und mögliche Verfahren für ihre Lösung. Anschließend werden zwei Hauptkategorien von Modellanalysen vorgestellt: analytische Verfahren und Simulationsmodelle.

Die Verwendung eines Modells ist im allgemeinen dann nötig, wenn die Durchführung der Problemlösung am Original nicht möglich ist oder zu aufwendig wäre, was in fast sämtlichen Wissenschaften der Fall ist. Über diesen Aspekt hinaus wird der Modellbegriff wenig einheitlich genutzt. Eine sehr weite Begriffsauslegung wählen MEADOWs et al. (1973, S. 14): "Jeder Mensch behandelt seine Probleme mit Hilfe von Modellen. Ein Modell ist nichts weiteres als eine möglichst systematische Reihe möglichst realer Annahmen über ein wirkendes System, das Ergebnis des Versuchs, durch Wahrnehmung und mit Hilfe vorhandener Erfahrung ... [ein Modell] auszuwählen, um so einen Ausschnitt aus der sinnverwirrend komplizierten Wirklichkeit zu verstehen."

\subsubsection{Modelltypen}

Üblicherweise wird zwischen folgenden Modellen nach Form ihrer Darstellung unterschieden (vgl. ORCUTT 1960, S. 857 und SCHANZ 1988):

- gegenständliche bzw. materielle Modelle (z.B. Globus),

- Verbalmodelle bzw. sprachlich-semantische Modelle (z.B. Modelle des Konsumentenverhaltens oder Kommunikationsmodelle),

- geometrische oder graphische Modelle (z.B. Landkarten, mikroökonomische Diagramme) und

- mathematische Modelle (z.B. in der Sprache der Algebra oder Kybernetik oder in einer Programmiersprache).

Im weiteren Verlauf dieser Arbeit werden nur noch mathematische Modelle betrachtet, auch wenn ganz allgemein von Modellen die Rede ist. Über diese Einteilung

1 Eine formale Definition wird in Abschnitt 4.2 gegeben. 
hinaus können Modelle in den Wirtschaftswissenschaften nach ihrer Zielsetzung in Beschreibungs-, Erklärungs- und Entscheidungsmodelle unterteilt werden. $\mathrm{Zu}$ den Erklärungsmodellen zählen auch Prognosemodelle und darunter die Simulationsmodelle (vgl. Unterabschnitt 2.1.4.2), mit deren Hilfe die Wirkungen alternativer Bedingungskonstellationen „durchgespielt“ werden können (SCHANZ 1988).

\section{Schaubild 23.1: Typen mathematischer Modelle}

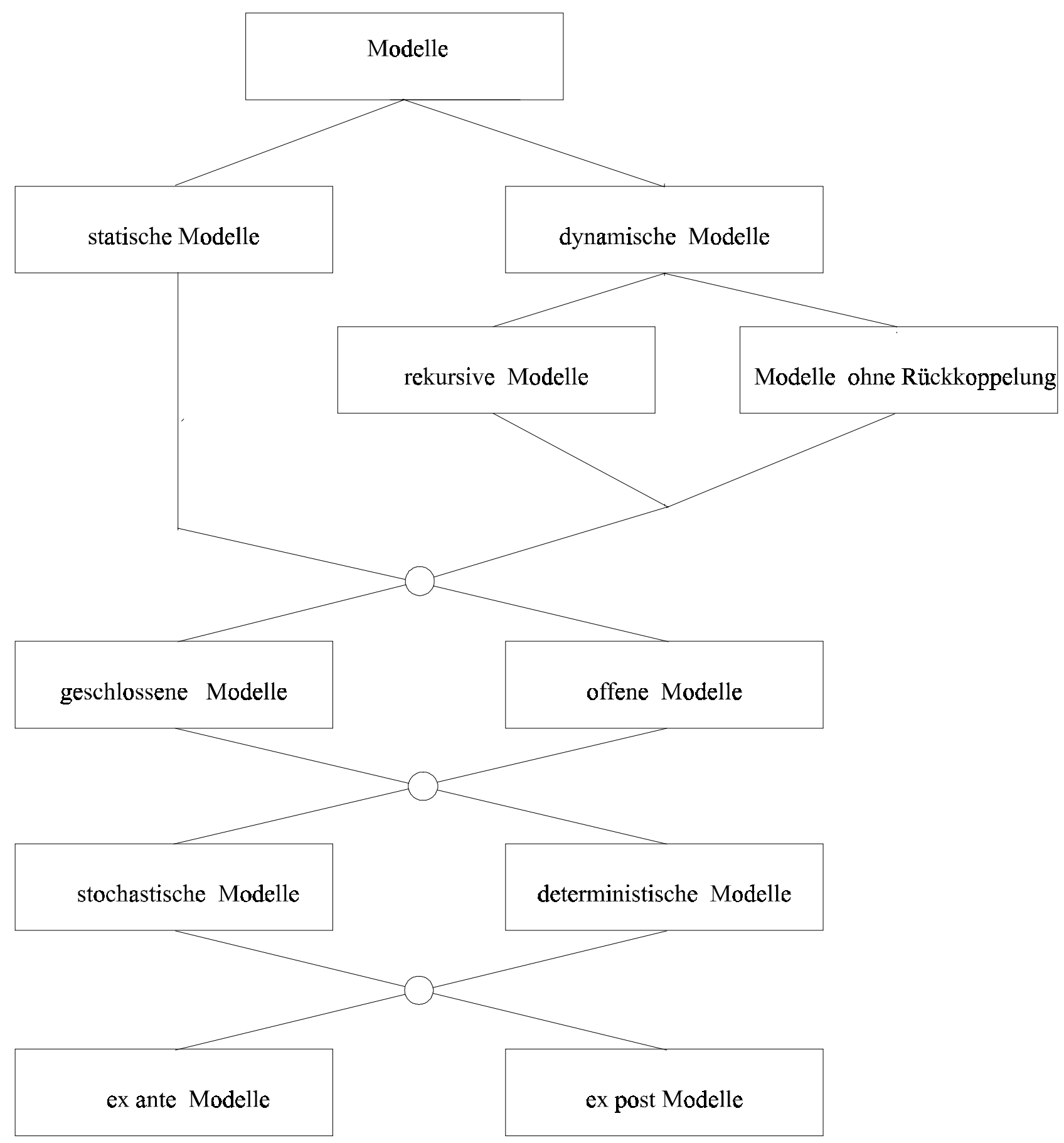

Quelle: WEINSCHENCK (1977, S. 60f), erweitert 
Die Konstruktion von Modellen und ihre Verwendung zu Gedankenexperimenten können als das wichtigste Verfahren zur Gewinnung neuer wirtschaftswissenschaftlicher Theorien angesehen werden (KROMPHARDT 1982, S. 902, zit. n. BRANDES 1985, S. 172).

Anhand von Begriffspaaren, die sich jeweils auf unterschiedliche Eigenschaften von Modellen beziehen, lassen sich innerhalb der mathematischen Modelle Unterscheidungen, wie in Schaubild 231 dargestellt, vornehmen. (Modelle, die sich in der gleichen Zeile befinden, schließen sich gegenseitig aus.)

\section{a) statische vs. dynamische Modelle}

Statische Modelle beziehen sich auf eine Periode, daher kommen sie zur Analyse von Betriebsentwicklungen nicht in Betracht. Dynamische Modelle zeichnen sich dadurch aus, daß zu den erklärenden Variablen prädeterminierte Variablen gehören, z.B. wenn der Umsatz eines Unternehmens durch den Werbeumfang in der Vorperiode erklärt wird. Enthält ein Modell neben den exogenen Variablen auch eine verzögert endogene Variable als Erklärende, das heißt, eine Variable, die die Ausprägung der endogenen Variablen in der Vorperiode zum Ausdruck bringt, so spricht man von einem rekursiven Modell. Ein einfaches Beispiel eines rekursiven Modells liegt vor, wenn angenommen wird, daß der aktuelle Umsatz $U$ außer von der Werbung $W$ auch vom Umsatz in der Vorperiode abhängt:

$$
U_{t}=\mathrm{f}\left(U_{t-1}, W_{t-1}\right) \text {. }
$$

Im Agrarbereich wurden rekursive Programmierungsmodelle zuerst von DAY eingesetzt (1963). In den Folgejahren verwendeten HEIDHUES (1966), DE HAEN (1971) und WossinK (1990) diese Technik zur Erforschung des agrarstrukturellen Wandels. MÜLLER wagte 1976 eine Vorausschätzung der landwirtschaftlichen Produktionsstruktur bis 1990. In den 80er Jahren veröffentlichte DAY eine ganze Reihe von Arbeiten zu einzelbetrieblichem (1982) und gesamtwirtschaftlichem Wachstum (1987a, 1987b, 1989).

\section{b) geschlossene vs. offene Modelle ${ }^{2}$}

Geschlossene Systeme sind vollständig, alle Einflußnahmen sind im Modell enthalten. Daher bestehen (idealtypisch) keine Wechselwirkungen mit der Umwelt. Obwohl daher streng genommen keine geschlossenen Systeme existieren können, ist es dennoch sinnvoll, geschlossene Modelle zu verwenden, wenn das Ausmaß der interessierenden Interaktion des Systems mit seiner Umwelt im Verhältnis zu den Interaktionen innerhalb des Systems vernachlässigbar gering ist (BERG \& KUHLMANN 1993, S. 2).

2 Neben den Bezeichnungen "offen-geschlossen" sind in diesem Zusammenhang auch andere Begriffspaare gebräuchlich: kausal - historisch, inhomogen - homogen, gezwungen - ungezwungen, nicht autonom - autonom, semi-endogen - endogen, absolut isoliert - relativ isoliert. Der Umfang der Kongruenz läßt sich nicht generell bestimmen, da die Autoren exemplarische, auf die konkrete Anwendung zugeschnittene Unterscheidungen vornehmen und nicht allgemeingültige Definitionen treffen (ZWICKER 1981, S. 276f). 
Eine besondere Form offener dynamischer Systeme stellen kybernetische Systeme dar. Kybernetik ist eine Theorie dynamischer Systeme, die sich insbesondere mit der Informationsverarbeitung sowie mit deren Regelung und Steuerung beschäftigt. Der Grundgedanke besteht in der Erforschung der wesentlichen Eigenschaften von dynamischen Systemen, mit dem Ziel, dynamische Systeme zielgerecht lenken zu können bzw. zu erreichen, daß sie sich selbst entsprechend lenken. Es wird unterschieden zwischen Steuerung und Kreisprozessen. Die Steuerung greift autonom in ein System ein. ${ }^{3}$ Die Wirkungen, die die Steuerung auf Variablen des System ausübt, beeinflussen nicht die weitere Steuerung, da die Steuerung systemexogen vorgegeben ist. ${ }^{4}$ In Kreisprozessen hingegen modifiziert das Ergebnis der Eingriffe die weitere Einflußnahme. Dies geschieht durch Rückkoppelung. Es wird zwischen positiver und negativer Rückkoppelung unterschieden. Positive Rückkoppelung kann zu Eskalation führen: Eine positive Wachstumsrate bei Größe $A$ induziere beispielsweise eine Zunahme bei Größe $B$, und deren Vergrößerung wirke beschleunigend auf den Zuwachs von $A$ zurück. Man spricht hier von einem Aufschaukelungskreis. Ein ökonomisches Beispiel sind verstärkte Werbeanstrengungen einer Firma, die die Konkurrenz zu gleichen Aktivitäten veranlassen. Oder die Einführungen technischer Neuerungen, die keine Nutzungskonkurrenz nach sich ziehen: Je mehr Menschen sich ein Faxgerät kaufen, desto wertvoller wird es für die Besitzer und desto größer wird der Anreiz für andere, sich ebenfalls ein solches Gerät anzuschaffen. Ein Anwendungsbereich für Abschaukelungsoder Konkurrenzkreise sind Nullsummenspiele, die ebenfalls auf positiver Rückkoppelung basieren: Bei unelastischer Nachfrage geht eine Umsatzsteigerung eines Unternehmens zu Lasten der Verkaufserlöse der Konkurrenz. Wirken im Gegensatz zu positiver Rückkoppelung eine gleichgerichtete und eine entgegengerichtete Beziehung zusammen, spricht man von einer negativen Rückkoppelung und einem stabilisierenden Rückkoppelungskreis oder einfach von einem Regelkreis. Negative Rückkoppelung kann als ein Gleichgewichts- oder Zielsuchverhalten aufgefaßt werden. Bei der Veränderung eines Elements, das sich vom Gleichgewichtswert entfernt, werden Kräfte angeregt, die zu einem neuen Gleichgewicht führen. Ein einfaches Beispiel für einen Regelkreis ist ein Heizungsthermostat, der die Heizung öffnet, wenn die Temperatur abgefallen ist, und sie wieder schließt, wenn die Normtemperatur erreicht ist.

Kybernetik ist in den 70er und 80er Jahren durch die Arbeiten des Club of Rome über Weltklimamodelle (MEADOWS et al. 1973) und durch VESTERs Veröffentlichungen über vernetzte Systeme bekannt geworden. VESTER weist auf die Gefahren hin, komplexe Systeme in isolierten Modellen zu betrachten, die die oft verborgenen Interdependenzen der Systemkomponenten nicht ausreichend berücksichtigen. ${ }^{5}$ Nach BERG \& KuHLMANN (1993, S. 2) lassen sich "sämtliche Organismen und Organisationen (Pflanzen, Tiere, Wirtschaftseinheiten) als offene, dynamische und in den meisten

Bestes Beispiel ist ein Wecker!

4 Ein anderes einfaches Beispiel ist eine Zeitschaltuhr.

5 Das achtbändige Werk "Systemstudie Ökoland" von VESTER et al. kam zwischen 1983 und 1989 heraus. Die siebte Auflage von "Unsere Welt, ein vernetztes System" erschien 1991. 
Fällen als kybernetische Systeme klassifizieren.” Dies beleuchtet die Bedeutung der Kybernetik. Ihre Methodik wird heute in der Kontrolltheorie weiterentwickelt (z.B. LENTZ 1993).

Eine wichtige Eigenschaft geschlossener Modelle ist ihre Zeitinvarianz (ZWICKER 1981, Kap. 2.2.). Ein geschlossenes statisches Modell behält seine Gültigkeit, wenn es auf eine andere Periode angewandt wird. Geschlossene dynamische Modelle können in der Zeit verschoben werden, ohne an Erklärungswert einzubüßen. Offene Modelle hingegen beziehen die Zeit ausdrücklich zur Erklärung mit ein.

In den Naturwissenschaften überwiegen geschlossene Modelle, da es ihr Ziel ist, zeitlose Gesetze festzustellen. Auch in den Sozialwissenschaften gibt es analoge Bestrebungen, zeitunabhängige empirische Gesetzmäßigkeiten zu finden. Die Forderung nach zeitinvarianten Beziehungen beinhaltet, daß die strukturellen Gleichungen nicht durch Größen verändert werden dürfen, die explizit von der Zeit abhängen. Ein geschlossenes Modell kann daher Strukturbrüche nicht erkennen. Wird die Zeit eigens in eine Modellformulierung aufgenommen, liegt ein offenes Modell vor, mit der Implikation, "daß es zeitveränderliche empirische Gesetze gibt. Eine solche Konsequenz ist zwar logisch denkbar, würde aber einem Grundpostulat der heutigen Naturwissenschaften widersprechen, welche behauptet, daß sich eine Ursache (hier repräsentiert durch den Wert einer exogenen Variablen) nicht allein deswegen ändern kann, weil die Zeit voranschreitet, sondern, weil sie zugleich die Wirkung einer anderen Ursache ist." (ZWICKER 1981, S. 277).

\section{c) stochastische vs. deterministische Modelle}

Werden für in die Zukunft gerichtete Fragestellungen deterministische Modelle verwendet, entsteht das Problem der Behandlung der Unsicherheit zukünftiger Größen. In der Regel beschränken sich Studien auf die Analyse der Umweltentwicklung, die für am wahrscheinlichsten angesehenen wird (AGRA-EUROPE 13/98, Sonderbeilage, S. 2). Methodisch etwas aufwendiger ist es, die als unsicher angesehenen exogenen Variablen im Modell als stochastische Größen aufzufassen, ihren Erwartungswert zu schätzen, und mit dieser Kennzahl die Berechnungen durchzuführen. Die auf diese Weise erzielten Ergebnisse können dennoch erheblich von Resultaten abweichen, die gewonnen werden, wenn der Rechenaufwand noch weiter gesteigert wird und neben dem Erwartungswert auch weitere Verteilungsparameter der unsicheren Größen in die Optimierung einbezogen werden (KÜPPER 1995, Kap 3.2.1).

Der Rechenaufwand für stochastische Modelle ist sehr groß und wird enorm, wenn mehrere stetige, stochastisch abhängige Variablen eingeführt werden. Daher beschränken sich viele Studien auf deterministische Modelle oder führen einfache Trendfortschreibungen durch. Die Unsicherheit der Ergebnisse wird herkömmlicherweise schlaglichtartig durch ergänzende Szenarioberechnungen für sehr schlechte und sehr 
gute Fälle beleuchtet ("worst case and best case scenarios", HENSCHE et al. 1998). ${ }^{6}$ Aufwendigere Ansätze fassen einzelne Variablen als diskret-stochastisch auf (z.B. HAZELL \& NORTON 1986, Kapitel 5) ${ }^{7}$ oder veranschaulichen das innewohnende Risiko durch Parametrisierung zufallsbehafteter Größen sowie durch Ausweisung eines geeigneten Risikomaßstabes (risikoeffiziente Pfade). Näheres hierzu wird in Abschnitt p.2 ausgeführt.

\section{d) ex-ante vs. ex-post-Modelle}

Bei ex-post-Analysen wird im allgemeinen der Zufallscharakter der in der Vergangenheit realisierten Werte vernachlässigt; es werden einfach die angefallenen historischen Werte eingesetzt. Dabei entsteht häufig ein Aggregationsproblem, das a priori nicht immer gelöst werden kann. Dazu ein einfaches Beispiel: Für einen vergangenen Zeitraum liege eine Zeitreihe mit Preisen und (physischen) Erträgen vor. Die mittleren jährlichen Einnahmen lassen sich nun auf zwei Wegen ermitteln: entweder durch das Produkt aus mittlerem Preis und mittlerem Ertrag oder durch die Berechnung der Einnahmen für jedes Jahr und die Bestimmung ihres Mittelwertes. Beide Verfahren führen im allgemeinen zu verschiedenen Ergebnissen. Steht eine Erfolgskontrolle im Vordergrund, wird man die zweite Methode wählen. Ist das Ziel hingegen, aus den Daten eine Einnahmeprognose abzuleiten, wird üblicherweise das erste Verfahren bevorzugt.

In ex-post-Kalkulationen ist es auch möglich, Erwartungswerte bei Fortschreibungen zu bilden, statt die angefallenen historischen Daten zu verwenden. Dabei kann es zweckdienlich sein, die Realisationen der inzwischen vergangenen Jahre bei der Erwartungswertbildung einzuschließen. Dies verändert im allgemeinen die Prognose und Prognosegüte, und es macht die Zufälligkeit auch der Ergebnisse von ex-postKalkulationen offenbar. Ein Beispiel hierfür ist die "MOTAD"-Erweiterung der linearen Programmierung. Sie wurde von HAZELL (1971) entwickelt und wird in Unterabschnitt 3.2.2 im Zusammenhang mit Methoden der Risikoberücksichtigung in der Betriebsplanung kurz angesprochen.

In Schaubild 231 nicht aufgeführt sind spieltheoretische Modelle. Sie können sich über eine oder mehrere Perioden erstrecken, daher also statisch oder dynamisch sein. Eine Zuordnung zu deterministischen oder stochastischen Modellen ist nicht zweckmäßig. Spieltheoretische Modelle befassen sich mit unsicheren Situationen. Die möglichen Situationen sind gegeben, ihnen werden oder können a priori keine Wahrscheinlichkeiten beigemessen werden. Statt dessen wird, ausgehend vom Konstrukt rational handelnder Co-Agenten, untersucht, welche Entscheidungen die anderen vernünftigerweise treffen werden, um darauf die eigene Wahl aufzubauen. Dieser Prozeß kann sequentiell gestaltet sein. Dann sind die Entscheidungen auf vorgelagerten Stufen

6 Besondere Bedeutung haben solche Extremszenarien für lange Planungshorizonte, um die mögliche Bandbreite der Entwicklung offenzulegen.

7 Zuordnungen von Wahrscheinlichkeiten können auch von Nachteil sein, weil "die Darstellung einer, wahrscheinlichen Situation “immer zu einer Beschränkung der Situation" führt und den Blick in die Zukunft verengt (AGRA-EUROPE 13/98, Sonderbeilage, S. 2). 
jeweils bekannt und die Beteiligten verfügen über sogenannte perfekte Information. Werden hingegen die Auswahlhandlungen gleichzeitig (simultan) getroffen, sind sie den jeweils anderen zum Zeitpunkt ihrer eigenen Entscheidung verborgen. Man spricht von Modellen mit imperfekter Information (ILLING 1995, S. 511, GIBBONS 1992, S. $55,121 \mathrm{f}){ }^{8}$

Nachdem nun die wichtigsten Unterscheidungen für Modelltypen vorgestellt sind, wird im nächsten Unterabschnitt besprochen, welche Anforderungen an Modelle zu stellen sind.

\subsubsection{Modellanforderungen}

Die wesentlichen Anforderungen an ökonomische Modelle sind:

- Einfachheit oder Realitätsnähe ${ }^{9}$

- Plausibilität und Konsistenz

- empirische Überprüfbarkeit

- Fähigkeit, den Ist-Zustand abzubilden.

Erkenntnis- oder Erklärungsmodelle sollten so einfach wie möglich gehalten sein, damit die entscheidenden Zusammenhänge nachzuvollziehen sind. Abstraktion von der Wirklichkeit ist daher notwendig und sinnvoll (WöHE 1996, S. 36f). Nur so kann ein Modell ein geeigneter Repräsentant eines ökonomischen Systems sein. Für CASTI (1997, S. 9) ist Einfachheit ein Unterscheidungsmerkmal für die Qualität eines Modells. Da sowohl Forscher wie Adressaten eines Modells über begrenzte kognitive Fähigkeiten verfügen, erleichtern einfache Modelle den Empfängern das Verständnis und verringern die Wahrscheinlichkeit von Konstruktions- und Verständnisfehlern. Sie erleichtern auch die Übernahme eines Modells durch andere Wissenschaftler. AXELROD (1997, S. 5) empfiehlt daher, das ,KISS'-Prinzip zu beachten: ,keep it simple, stupid‘. Wenn überraschende Ergebnisse aufträten, sei es sehr hilfreich, wenn alle Modellzusammenhänge leicht nachvollziehbar seien. Komplexe Ergebnisse sind um so wertvoller, je einfacher das Modell ist, das sie hervorgebracht hat. Modelle zu grundlegenden Fragestellungen sollten daher nach dem Erkenntnisgewinn, der durch ihren Einsatz möglich wird, beurteilt werden, und nicht nach ihrer Ähnlichkeit mit der Wirklichkeit. Detailgetreue Abbildungen sind unnötig und sogar schädlich.

Einfachheit ist eine Forderung an Erklärungsmodelle. Realitätsnähe oder diffiziler Aufbau hingegen sind Kennzeichen von Prognosemodellen. Hier sind alle Einflußfaktoren möglichst umfassend abzubilden, um zu guten Resultaten zu kommen. Gleiches gilt für technische Simulatoren, die für Trainingszwecke eingesetzt werden.

8 Von imperfekter Information ist unvollständige Information zu unterscheiden. Unvollständige (incomplete) Informationen liegen vor, wenn ein Mitspieler die payoffs nicht genau kennt (HARSANY 1995).

9 Zur Übereinstimmung von System und Modell in Abhängigkeit vom Erkenntnisinteresse vgl. GROOS (1994, Abschnitt 2.3). 
Der Umfang der Komplexreduktion beim Aufbau eines mathematischen Modells ist eine diffizile Aufgabe. Hochabstrakte Modelle sind zwar häufig elegant lösbar, büßen allerdings Realitätsnähe ein. Stark homo- oder gar isomorphe Abbildungen ${ }^{10}$ lassen sich andererseits nicht effizient berechnen. Diesen Zwiespalt nennen GROCHLA \& SZYPERSKI (1973, S. 26) das Bonini-Paradoxon: "Je realitätsnäher und damit komplexer ein Modell ist, desto weniger hilft es, die reale Welt zu verstehen und damit zu kontrollieren.” Und BÄUERLE (1987, S. 110f) vertritt zum in der Betriebswirtschaftslehre wichtigen Thema Programmplanung die Auffassung, daß finanzielle Programmplanungsprobleme, wie simultane Investitions-, Finanzierungs- und Produktionsplanungen, selbst bei Fortschritten in der Computertechnologie die Leistungsfähigkeit der Lösungsalgorithmen auch zukünftig übersteigen würden.

An Modelle sind generell einige Plausibilitätsanforderungen zu stellen, damit sie sinnvolle Abbilder eines empirischen Systems sein können. Der Modellaufbau soll frei von Widersprüchen sein und garantieren, daß die endogenen Variablen nur Werte annehmen, die nicht per definitionem ausgeschlossen sind. Zum Beispiel darf ein Modell, das zur Erklärung der Kinderzahl von Familien dienen soll, nicht zu negativen Werten der Anzahl führen. Ferner muß ein Modell für jede zulässige Kombination der exogenen Größen auch eindeutige Werte für die endogenen Variablen liefern. Darüber hinaus fordern beispielsweise ZWICKER (1981, S. 126ff) und ORIADE \& DILLON (1997, S. 46f) logische und definitorische Konsistenz. Sie bedeutet, daß die grundlegenden Annahmen unbestritten und insbesondere nicht selbst Gegenstand einer empirischen Überprüfung sein dürfen. Zu plausiblen und konsistenten Annahmen kann man durch Expertengespräche, Beobachten des Wirtschaftsablauf, Analyse früherer Modelle und der einschlägigen Theorie, aber auch durch Erfahrung und Intuition gelangen. ${ }^{11}$ Auch die Modellableitungen müssen logisch und formal korrekt sein. Die Ergebnisse eines Modells sollten ferner nicht anerkannten Theoremen widersprechen, beispielsweise im Vorzeichen bestimmter Koeffizienten.

Die Forderung nach empirischer Überprüfbarkeit soll sicherstellen, daß das Modell außer den "basic assumptions" keine weiteren Verhaltenshypothesen enthalten darf, die nicht empirisch überprüfbar sind (ORIADE \& DILLON 1997, S. 46f.). Die Überprüfung kann anhand der Anpassungsgüte des Modells sowie mit Hilfe von Sensitivitätsanalysen oder Signifikanztests der Parameter erfolgen.

Die Fähigkeit eines Modells, den Ist-Zustand abzubilden (positive economics, vgl. ORIADE \& DILLON 1997, S. 46f) ist eine notwendige Bedingung dafür, daß das Modell auch andere Szenarien zutreffend zu modellieren vermag. Nur an Modellen, die dieser Anforderung genügen und darüber hinaus auch ,,vergangene Entwicklungen

10 Eine Abbildung heißt isomorph oder eineindeutig, wenn jedes Element und jede Beziehung des Systems seine Entsprechung im Modell findet et vice versa. Bei homomorphen oder mehreindeutigen Abbildungen repräsentiert jede Modellkomponente ein reales Gegenstück, aber nicht alle Aspekte des Systems finden im Modell ihren Niederschlag (GroOs 1994, S. 20ff). Homomorphe Anwendungen überwiegen daher in der Ökonomik.

11 Vgl. spätere Ausführungen zur Kalibrierung (S. 30). 
mit befriedigender Genauigkeit“" (DE HAEN 1973, S. 166) nachzeichnen können, ist es statthaft, beispielsweise den Effekt zusätzlicher oder veränderter Verhaltenshypothesen zu untersuchen oder sie zu Prognosezwecken einzusetzen. Die Fähigkeit zur zuverlässigen Beantwortung von Fragen ist letztlich die conditio sine qua non eines Modells (CASTI 1997, S. 9). Inwieweit die erzielten Ergebnisse plausibel sind, kann oft nicht formal, sondern nur intuitiv durch Erfahrung beurteilt werden.

\subsubsection{Vorgehensweisen zur Erstellung von Modellen}

Modelle dienen, wie bereits erwähnt, dazu, an einem relativ einfachen gedanklichen Konstrukt die verborgenen inhärenten Zusammenhänge eines Systems zu erforschen. Dies geschieht in vier Phasen. ${ }^{12}$ Zunächst gilt es, die wesentlichen Kausalstrukturen eines Systems in einem vereinfachenden Modell abzubilden und seine Parameter zu schätzen (erste und zweite Phase). Anschließend ist das Modell auf logische und formale Korrektheit zu überprüfen. Diese dritte Phase wird Verifikation genannt. In der vierten Phase, als Validierung bezeichnet, sind die Ergebnisse eines Modells inhaltlich zu kontrollieren, ob sie in ausreichendem Einklang mit der Wirklichkeit stehen. ${ }^{13}$ Die genannten Schritte müssen häufig in einem Trial-and-Error-Prozeß wiederholt werden, bis Struktur und Parameter des Modells ausreichend angepaßt sind, das System adäquat wiederzugeben. Zur Anpassung der Parameter sind drei Verfahren gebräuchlich:

- Die Forscherin entnimmt "bewährte" Koeffizienten der Literatur oder vorgeschalteten Erhebungen und/oder führt Expertenbefragungen durch.

- Sie schätzt die Parameter regressionsanalytisch aus empirischen Daten. Dieser elegante Weg scheitert oft an der Datenlage (DE HAEN 1973, S. 167).

- Sie "kalibriert" die Koeffizienten: Plausible Parameter werden in das Modell eingesetzt, dann wird das Modellverhalten untersucht. Entspricht es den Vorgaben, die deduktiv (s. S. 30) aus anerkannten Theoremen abgeleitet, empirisch beispielsweise in einem Basisjahr beobachtet oder intuitiv einleuchtend sind, werden diese Werte verwendet, ansonsten wird die Forscherin sie mit viel Fingerspitzengefühl verändern. ${ }^{14}$

Für deterministische Modelle reichen diese drei Schritte aus. Im Falle stochastischer Modelle muß darüber hinaus die stochastische Komponente spezifiziert werden. Hierfür bestehen grundsätzlich die zwei Möglichkeiten, (a) Zufallszahlen zu ziehen oder (b) aus empirischen Daten zufällig einige auszuwählen.

Die Verteilungen der Parameter können mit den drei oben genannten Verfahren spezifiziert werden. Sollen in einem multivariaten Modell stochastische Beziehungen

12 Vgl. ORiade \& Dillon (1997, S. 46), WeinsChENCK (1977, S. 59).

13 Zur Abgrenzung von Validierung und Verifikation schreiben ORIADE \& DILLON (1997, S. 46): „The objective of validation is to ascertain the usefulness, rather than the truthfulness, of the model.“.

14 Hierfür können auch neuere Techniken wie Fuzzy-LP eingesetzt werden. TRIES (1998) macht hierzu einen Vorschlag. 
zwischen den Variablen berücksichtigt werden, müssen die Verteilungen im allgemeinen normal sein, wenn eine analytische Lösbarkeit des Systems angestrebt wird. Die Erzeugung stochastisch abhängiger Zufallszahlen, die für eine stochastische Simulation benötigt werden, ist mit neuerer Software (z.B. RISK ${ }^{\circledR}$ ) auch für andere als Normalverteilungen möglich.

Für stochastische Simulationen (vgl. Unterabschnitt 2.1.4.2) kann neben Methode (a) ein anderes Verfahren angewendet werden, um zufällige Daten zu erzeugen: Aus historischen Zeitreihen werden zufällig Beobachtungen ausgewählt. Diese Technik besitzt den Vorteil, daß keine Annahmen über die Verteilung nötig sind. Nachteilig ist, daß ohne eine Verteilungsannahme weder eine serielle Korrelation noch eine Kovarianz zwischen den Variablen abgebildet werden kann. Bestehen starke Beziehungen zwischen den Ausprägungen einzelner Variablen in einer Periode, kann den hiermit verbundenen Problemen durch eine Beschränkung der freien Zufallsauswahl Rechnung getragen werden: Es werden nicht einzelne Beobachtungen aus den Zeitreihen der Variablen gezogen, sondern Zeitpunkte. Dadurch erfolgt die Übernahme kompletter Sets von Variablenausprägungen eines Zeitpunktes in die Simulationsstichprobe.

Nach Modellspezifikation und möglicher Einbeziehung einer stochastischen Komponente besteht die letzte Phase darin, die Wenn-Dann-Beziehungen des Modells, z.B. die Reaktionen auf Veränderungen der exogenen Variablen, zu entschlüsseln. Enthält das Modell stochastische Komponenten, gilt es, die gefundenen Effekte gegen Zufallseinflüsse abzugrenzen. Abschließend sind die Ergebnisse der Modellanalyse auf das System rückzuübertragen. Dies ist keine leichte Aufgabe, bedarf sie doch ,unmittelbar der denkenden und wertenden Beurteilung" (WEINSCHENCK, 1977, S. 46). ${ }^{15}$

\subsubsection{Ableitung von Modellimplikationen}

Nachdem gemäß den oben erläuterten Grundsätzen ein Modell aufgestellt worden ist, liegen nicht nur seine expliziten Eigenschaften, auf denen die Modellspezifikation basiert, sondern zwangsläufig auch seine gesamten impliziten Eigenschaften fest; sie sind nur noch nicht bekannt. Ziel der Modellanalyse ist es daher, die impliziten Eigenschaften aufzudecken. Dies kann grundsätzlich entweder deduktiv oder induktiv erfolgen. Unter Deduktion versteht man allgemein ein logisches Verfahren der Ableitung von speziellen aus generellen Aussagen. Im allgemeinsten Fall wird eine Axiomatik aufgestellt (z.B. für die Wahrscheinlichkeitstheorie) auf deren Grundlage Sätze abgeleitet und geprüft werden. Ausgehend von bewiesenen Sätzen können wieder neue Sätze (oder Hypothesen) formuliert und geprüft werden. Die hierfür eingesetzten Methoden werden als analytische Verfahren bezeichnet. Ein Beispiel hierfür ist die Anwendung der Gesetze der Algebra und der Wahrscheinlichkeitstheorie, mit dem Ziel, das Verhalten von endogenen Variablen in einem dynamischen Modell offenzu-

15 HENSCHE et al. (1998, S. 2) verwenden in einer Szenarioanalyse der Zukunft der Schweinefleischproduktion eine differenziertere Vorgehensweise in acht Schritten. Sie scheint mir auch für andere ökonomische Fragestellungen geeignet. 
legen. Ein anderes Beispiel ist die Spieltheorie, die unter der Annahme rationalen Verhaltens Gleichgewichte in strategischen Situationen bestimmt. Auf der anderen Seite bestehen induktive Verfahren darin, aus empirischen Daten Regelmäßigkeiten herauszufiltern und systematische Zusammenhänge zu identifizieren. Diese Technik wird häufig in der Meinungsforschung oder in der Erforschung makroökonomischer Daten benutzt (AXELROD 1997, S. 3).

Oft können in einem Modell die Zusammenhänge und Abhängigkeiten zwar formal notiert, aber nur durch schrittweises Durchspielen der Abhängigkeiten imitierend untersucht ("gelöst") werden. Ein solches Durchspielen oder Experimentieren wird als Simulation bezeichnet. Simulation ist nach ORIADE \& DILLON (1997, S. 46) die Vorgabe von numerischen Variationen eines symbolischen Modells, mit dem Ziel, experimentelle Informationen über das Verhalten des modellierten Systems zu gewinnen.

Ein Anwendungsbereich für deterministische Simulationen sind die von AXELROD (1997) agent based modeling genannten komplexitätstheoretischen Ansätze. ${ }^{16} \mathrm{Im}$ deutschen Sprachraum spricht man von Multiagentensystemen. Betrachtet werden gleichzeitig eine Vielzahl von Elementen und ihre Interaktionen. Für die Methodik spielt es keine Rolle, ob es sich um Verbraucher, Unternehmen, Staaten oder Atome handelt. Werden vielschichtige oder gar dynamische Verknüpfungen zwischen den Akteuren modelliert oder wird adaptives statt rationales Verhalten unterstellt, kann das System nur mit Simulationen erforscht werden. Agent based modeling ist für AXELROD ein dritter Weg zur Ableitung von Modellimplikationen. Grundlage ist wie bei der Deduktion ein Set von Annahmen, doch lassen sich keine Sätze beweisen. Statt dessen erzeugt eine Simulation Daten, die mit induktiven Methoden untersucht werden können. Anders als bei der typischen induktiven Vorgehensweise entstammen die Daten aber nicht der Realität, sondern werden von einem Computer nach exakten Vorgaben erzeugt. Daher bezeichnet ZwICKER (1981, Kap. 1.6.2 u. S. 390) dieses Verfahren als pseudo-induktiv. Während es also das Streben der typischen Induktion ist, Regelmäßigkeiten in empirischen Daten aufzuspüren, und das Ziel der Deduktion, Konsequenzen von Annahmen logisch abzuleiten, ist es der Zweck von agent based modeling, die Intuition zu unterstützen.

Ein konkretes Interesse beim Einsatz von agent based modeling kann darin bestehen, sog. Emergenzen aufzudecken. Dies sind Eigenschaften eines Systems auf der Makroebene, die auf den Annahmen bezüglich der einzelnen Agenten und ihrer Beziehungen beruhen. Emergenzen sind häufig verblüffend, da schon die Auswirkungen relativ einfacher Annahmen nicht absehbar sind. In Abschnitt 2.2 wird eine Emergenz demonstriert, die auf einem Modell mit nur einem Agenten basiert.

Vor dem Aufstellen eines Modells ist es sinnvoll, zu berücksichtigen, auf welche Weise, also analytisch oder simulativ, es berechnet werden soll. In analytischen

16 Komplexitätsforschung ist ein multidisziplinärer Ansatz zur Untersuchung des Verhaltens komplexer Systeme. Sie hat ihre Wurzeln in der Chaostheorie und lehnt sich in ihrer Terminologie daher eng an sie an. 
Modellen erlaubt die mathematische Schreibweise beispielsweise, die Unsicherheit über zukünftige Umweltentwicklungen und Verschiedenheit betrieblicher Ausgangslagen sehr elegant aufzunehmen. Allerdings darf bei der Aufnahme zusätzlicher Hypothesen oder sonstiger Komplexitätssteigerungen die spätere Lösbarkeit des Gleichungssystems nicht aus den Augen verloren werden. Dies gilt besonders für dynamische und/oder stochastische Beziehungen, Rückkoppelungen, Nichtlinearitäten oder mehr der Wirklichkeit entsprechenden Verhaltenshypothesen (WEINSCHENCK 1977, S. 52). In Simulationsansätzen ist die Lösbarkeit weniger das Problem, da für jede einzelne Simulation nur deterministische Gleichungen berechnet werden. Engpässe sind eher die Implementierung des mathematischen Modells und insbesondere die stochastische Validierung der Ergebnisse. Im folgenden werden diese beiden wesentlichen Verfahren der Modellanalyse ausführlich erläutert.

\subsubsection{Analytische Verfahren}

Analytische Lösungstechniken finden nur für mathematische Modelle Anwendung. Sie führen zu allgemeingültigen Lösungen für den Zusammenhang zwischen unabhängigen und abhängigen Variablen. Beispiele sind auf der einzelbetrieblichen Ebene Maximierungsansätze zur Bestimmung optimaler Einsatzmengen von Inputs und in der Makroökonomie neoklassische Modelle, in denen rationale Agenten eine effiziente Allokation der Ressourcen herbeiführen. Ergebnisgrößen lassen sich in der Analysis direkt als Funktion der Eingangsgrößen darstellen. Analytische Modelle sind in der Regel stark reglementiert; sollen zufällige Einflußgrößen aufgenommen werden, sind häufig nur ganz bestimmte Verteilungsfunktionen analysierbar. Auf Modelle, in denen mehrere Zufallsgrößen gleichzeitig und interdependent wirken, ist meist keine analytische Methode mehr anwendbar, oft auch nicht, wenn Normalverteilungen eingesetzt werden.

Analytische Techniken zur Bearbeitung von Modellen sind u.a. die lineare oder die quadratische Programmierung. Für dynamische Fragestellungen können die mehrperiodische Programmierung oder die dynamische Programmierung von BELLMAN $(1967)^{17}$ sowie das Maximumprinzip von PONTRJAGIN eingesetzt werden. ${ }^{18}$ Dieses Verfahren ist besser unter der Bezeichnung Hamilton-Funktion bekannt, es wird besonders in der Ressourcenökonomik benutzt, z.B. um den optimalen Abbaupfad eines erschöpfbaren Bodenschatzes zu bestimmen.

Die Berücksichtigung von Stochastizität ist ein Beispiel dafür, daß die Forderung nach Realitätsbezogenheit zu Modellen führt, für die keine analytischen Lösungsverfahren existieren oder die nicht in mathematisch geschlossener Form dargestellt werden können. In solchen Fällen kann versucht werden, das Modell durch Vorgabe ein-

17 Die Originalausgabe erschien 1957 in Princeton, NJ. Beschrieben bzw. angewendet ist die Methode u.a. auch in: INTRILLIGATOR (1971), HANF \& SCHIEFER (1982) und in jüngerer Zeit MIRANDA \& GLAUBER (1993) sowie WIESEMANN (1994).

Weitere mathematische Lösungsalgorithmen beschreiben besonders AMMAN et al. (1996). 
zelner Werte für die exogenen Variablen "durchzurechnen". Zwei Techniken für diese Berechnungsweise stellen die Simplex-Methode ${ }^{19}$ und das Newton-Verfahren zur Nullstellenbestimmung dar (PAGENKOPF 1981). Diese numerische Lösungstechnik analytischer Modelle wird häufig als Simulation bezeichnet.

Besonders im dynamisch stochastischen Kontext sind den analytischen Verfahren enge Grenzen gesetzt. Werden z.B. für ein Betriebsentwicklungsmodell nicht sehr rigide Annahmen getroffen, ist eine Lösung der sich ergebenden meist nichtlinearen Gleichungssysteme nur schwer oder auch gar nicht durchführbar (ODENING 1991, WIESEMANN 1994, S. 367, HINNERS-TOBRÄGEL \& BRANDES 1997). Dies gilt insbesondere, wenn viele Optionen oder Handlungsmöglichkeiten zu berücksichtigen sind (LINSMEIER \& PEARSON 1996, S. 10ff). Schon einfache lineare Differenzengleichungen dritter und höherer Ordnung sind im allgemeinen nicht analytisch lösbar (näheres dazu im Exkurs über den LOHMANN-RUCHTI-Effekt in Abschnitt 2.4.

Auf ähnliche Schwierigkeiten stoßen Wahrscheinlichkeitsaussagen über die gefundenen Ergebnisse. In komplexen Modellen ist es in der Regel nicht möglich, die zeitliche Entwicklung der Parameter, die die Wahrscheinlichkeitsverteilung der endogenen Variablen beschreiben, zu quantifizieren. Beispielsweise läßt sich der Erwartungswert eines Produktes zweier stetiger Zufallsveränderlicher nicht immer ermitteln, wenn die beiden Zufallsveränderlichen voneinander abhängig sind (ROHATGI 1984) ${ }^{20}$ Gleiches gilt für die Varianz, so daß Aussagen der Art, in wieviel Prozent der Jahre das Produkt der Zufallsveränderlichen einen bestimmten Wert $a$ unterschreitet, auf analytischem Wege kaum abgeleitet werden können (BRANDES et al. 1980, BERG \& KUHLMANN $1993,268){ }^{21}$

Wo analytische Lösungen nicht erreichbar oder unverhältnismäßig aufwendig sind, kann man sich bei diskreten Modellen mit endlichem Zeithorizont einer enumerativen Methode bedienen: Per Simulation werden für alle Kombinationen der exogenen stochastischen Variablen die Werte der endogenen Variablen berechnet und so ihre Wahrscheinlichkeitsverteilung für jede Periode bestimmt. Bei stetigen Verteilungen oder unendlichem Zeithorizont scheidet ein enumeratives Verfahren naturgemäß aus. Hier behilft man sich damit, für möglichst repräsentative Sets von Kombinationen der stochastischen exogenen Variablen die endogenen Variablen zu berechnen. Dieses

19 Es gibt auch ein numerisches Optimierungsverfahren gleichen Namens, das vom Simplex-Algorithmus in dieser Verwendung zu unterscheiden ist, vgl. JUDGE et al. (1985, S. 967f).

Für Produkte unabhängiger Zufallsveränderlicher können Erwartungswert (und auch Varianz) bestimmt werden. Allerdings ist die Annahme, daß die Zufallsveränderlichen in keinem Zusammenhang zueinander stehen, häufig (etwa bei Erträgen und Preisen von Nicht-Marktordnungsprodukten) problematisch. Vgl Tabelle 166.1.

Solche Aussagen sind dann leicht möglich, wenn neben Unabhängigkeit auch Normalverteilung vorausgesetzt wird. Ist die Normalverteilungsannahme nicht gerechtfertigt $-z$. B. weisen empirische Untersuchungen von Ertragsreihen im Ackerbau auf eine deutliche Linksschiefe hin (KURZ 1991, S. 61ff; DAY 1965) - , lassen sich Quantile zum Teil nur mit erheblichem Aufwand berechnen. 
Verfahren wird Monte-Carlo-Simulation genannt und im folgenden Unterabschnitt neben anderen Simulationsansätzen beschrieben.

\subsubsection{Simulationsmodelle}

Simulation wird üblicherweise als Instrument der Szenarioanalyse und als mathematisches Lösungs- oder Näherungsverfahren verstanden. ${ }^{22}$ Daher wird häufig auch ein statisch deterministisches Modell, das analytisch gelöst wird, als Simulationsmodell bezeichnet, wenn es Szenarioanalysen oder diskrete Sensitivitätsanalysen enthält. Das Problem der Abgrenzung von Simulationsmodellen im Unterschied zu analytischen Modellen wird in Unterabschnitt 2.1.4.3 vertieft.

WEINSCHENCK (1977, S. 40) definiert ähnlich wie ORIADE \& DILLON (s.o., S. 32] Simulation als "systematische oder willkürliche Veränderung von exogenen Variablen oder Parametern von Systemmodellen". In diesem Sinne ist die Simulation ein wichtiges Hilfsmittel der Modellanalyse. Sie dient zunächst während der Aufbauphase des Modells dazu, zu überprüfen, ob das Modell in seiner gegenwärtigen Spezifikation geeignet ist, das System in der gewünschten Weise abzubilden, und anderenfalls die Richtung für eine Änderung der Modellspezifikation anzugeben. Nach Abschluß der Modellimplementierung kann die Simulation als Instrument zur Hypothesenprüfung und zur Gewinnung genereller Modellimplikationen eingesetzt werden. Neben dieser Verwendung des Begriffs bezeichnet Simulation numerische Techniken der Näherungslösung analytisch nicht lösbarer mathematischer Probleme, z.B. das bekannte AIDS-Modell in der Nachfrageanalyse.

In der Literatur werden unterschiedliche Einsatzgebiete für Simulationen genannt:

- als Algorithmus zur Bestimmung von Zeitpfaden endogener Variablen in dynamischen Systemen, d.h. zur Analyse des Systemverhaltens (insbesondere wenn keine analytischen Verfahren für die Lösung vorhanden sind, vgl. WEINSCHENCK 1977),

- zur Anpassung eines Systemmodells an die abzubildende Wirklichkeit,

- zur Ableitung von Wahrscheinlichkeitsaussagen für komplexe statische oder dynamische Systeme, auch Monte-Carlo-Methode genannt,

- zur Berechnung der numerischen Lösungen von Gleichungen und Gleichungssystemen, entweder anstelle unverhältnismäßig aufwendiger analytischer Verfahren oder für Probleme, für die analytische Lösungsverfahren nicht vorhanden sind. Beispiele sind höhergradige Polynome (BRANDES 1985, S. 174) oder numerische Suchverfahren zur iterativen Optimierung, z.B. zur Bestimmung der Parameter von logistischen Wachstumsfunktionen (LENTZ 1993, Abschnitt 1.3, bes. S. 277, und S. 313). Hierzu können auch allgemeine heuristische Lösungsverfahren gezählt werden. Ein aktuelles Beispiel ist die Optimierung mit dem Ameisensystem (SONDERGELD \& VOSS 1997).

22 Zu Simulationstechniken vgl. BERG \& KUHLMANN (1993), insbesondere Kapitel 5, und BRADLEY et al. (1987). Bei BERG \& KUHLMANN findet sich u. a. ein Algorithmus zur Erzeugung autokorrelierter Zufallszahlen (Unterabschnitt 5.1.2.5). 
- als Prognoseinstrument. Mit der Simulation ist es möglich, ,,sowohl quantitative als auch qualitative Aspekte vernetzter Zusammenhänge bei unterschiedlichen Entwicklungen in Zukunftsbildern darzustellen" (HENSCHE et al. 1998).

- Ein weiterer Einsatzbereich liegt in der Möglichkeit der Durchführung von Sensitivitätsanalysen. Reaktionen der endogenen Modellvariablen auf Veränderungen der Parameter oder der exogenen Variablen lassen sich nur in analytisch lösbaren Modellen allgemeingültig ableiten.

Einige dieser Einsatzgebiete werden später näher beschrieben. Gemeinsam ist diesen Anwendungen, daß der notwendige mathematische Aufwand deutlich geringer ist als bei entsprechenden analytischen Ansätzen, eine Lösung jedoch nicht mit Sicherheit nach einer endlichen Zahl von Schritten erreicht wird (LENTZ 1993, S. 276). Der aus der linearen Programmierung bekannte Simplex-Algorithmus, der auch allgemein zur Lösung linearer Gleichungssysteme eingesetzt werden kann, zählt zu den analytischen Methoden, da die optimale Lösung mit Sicherheit nach einer endlichen Zahl von Schritten erreicht wird.

Simulationsansätze werden vorgezogen, wenn keine analytischen Verfahren zur Verfügung stehen, allgemeingültige oder exakte Lösungen für verzichtbar erachtet werden oder der mit analytischen Verfahren verbundene Mehraufwand nicht gerechtfertigt erscheint.

ZWICKER (1981, S. 103ff) beschränkt den Einsatzbereich von Simulationen auf den ersten der oben in den Spiegelstrichen genannten Punkte, d.h. auf ,die Ermittlung des Zeitpfades einer oder mehrerer endogener Variablen eines parametrisch-singulären Modells." Gemein hat diese Definition mit der der Simulation als numerischer Lösungstechnik, daß die mathematischen Gleichungen nicht in eine geschlossene Form überführt werden müssen. Dies wäre genau die analytische Lösung einer Differenzen- oder Differentialgleichung. ${ }^{23}$ Der Unterschied zwischen beiden Verwendungen des Simulationsbegriffs besteht darin, daß die Simulation als Ermittlung des Zeitpfades einer Variablen zu einem eindeutigen und exakten Ergebnis führt, während eine Simulation als numerische Annäherung weder eine exakte noch eine eindeutige Lösung garantiert. Die Verwendung einer Simulation als Instrument zur Gewinnung genereller Modellimplikationen bezeichnet ZWICKER als "Modellexperiment". Diese stärker differenzierende Definition von Simulation hat sich nicht durchgesetzt.

Nach dieser ausführlichen Begriffsbestimmung werden nun einige Verwendungsbereiche von Simulationen näher beleuchtet und Vor- und Nachteile diskutiert.

\section{Einsatzgebiete}

Zur Ableitung von Wahrscheinlichkeitsaussagen kann die Simulation genutzt werden, um beispielsweise die oben aufgeworfene Frage zu beantworten, in wie viel Prozent der Jahre das Produkt der Zufallsveränderlichen einen bestimmten Wert $a$ unter-

23 Näheres zu der geschlossenen Form einer Differenzengleichung in Unterabschnitt 2.2.1. 
schreitet. Mit der Simulationstechnik ist eine approximative Lösung dieser Aufgabe kein Problem. Man erzeugt zwei Wertereihen mit den gewünschten Eigenschaften, bildet die Produkte und zählt aus, wieviele der Produkte kleiner als $a$ sind. Dieser Anteil im Simulationsergebnis ist in der Regel ein konsistenter Schätzer für den gesuchten Anteilswert. Der Mittelwert der Produkte schätzt gleichzeitig den Erwartungswert des Produktes der Zufallsveränderlichen im allgemeinen konsistent. ${ }^{24}$

Die Stichprobenziehung erfolgt auf EDV-Anlagen mit Hilfe von Pseudozufallszahlen. ${ }^{25}$ Der Zusatz ,Pseudo“ ist angebracht, da auf streng deterministischem Wege ein Strang von Zahlen produziert wird, der dennoch die Eigenschaften von Zufallszahlen aufweist (ZWICKER 1981, S. 390).

Differenzengleichungen sind Gleichungen oder Systeme von Gleichungen, die zeitverzögerte Variablen enthalten. Simulationsverfahren können zu ihrer Analyse benutzt werden: Ausgehend von der ersten Periode werden für jede Periode die aktuellen Werte der endogenen Variablen aus den vorangegangenen Variablenausprägungen berechnet. So entstehen Zeitpfade der endogenen Variablen. Simulation liefert also eine prozedurale Lösung von Differenzengleichungen. Sie ist im Unterschied zu numerischen Näherungsverfahren mathematisch exakt, zumindest theoretisch (näheres unter dem Unterpunkt Nachteile).

Gleichfalls exakte Lösungen liefert die Simulation, wenn sie zur deterministischen Szenario- oder Sensitivitätsanalyse, die in Abschnitt 3.2 beschrieben wird, eingesetzt wird. Hierbei wird für jeden zu untersuchenden Datenkranz bzw. für jede diskrete Parametervariation eine Berechnung ausgeführt. Lassen sich für einige Variablen sinnvollerweise keine festen Werte angeben, sondern nur Verteilungen, kann auch hier die Monte-Carlo-Methode weiterhelfen: Für die stochastischen Variablen werden nach vorgegebenen Bildungsgesetzen Sets von Zufallszahlen gezogen, anschließend erfolgt für jedes Set die Berechnung des Modells. ${ }^{26}$ Eine hohe Zahl an Wiederholungen ist notwendig, um zu statistisch stabilen und absicherbaren Ergebnissen zu kommen. Da also die endogene(n) Variable(n) nicht für alle Kombinationen der exogenen Variablen berechnet wird bzw. werden, sondern nur für eine Pseudozufallsauswahl, wird dieses Verfahren korrekt als pseudo-induktiv bezeichnet (vgl. S. 31). Für die hierfür verwendete Technik ist neben der Bezeichnung Monte-Carlo-Simulation auch der Begriff stochastische Simulation üblich. Sie unterscheidet sich deutlich von ihrer deterministischen Variante, wo für einige Sets von festen Parameterkombinationen die Werte der endogenen Variablen berechnet werden. $\mathrm{Zu}$ beachten ist, daß stochastische Simulationen grundsätzlich keine exakten Lösungen, sondern wegen der verwendeten Pseudozufallszahlen nur Annäherungen liefern können.

$24 \mathrm{Zu}$ einem Vergleich von analytischen Lösungsansätzen und Simulationen für die Portfolioanalyse vgl. LINSMEIER \& PEARSON (1996, S. 16-20).

25 Zur Entwicklung von Programmen, die Zufallszahlen bestimmter Verteilungen generieren, vgl. NAYLOR et al. (1966) und BRATLEY et al. (1987).

26 In der Literatur finden sich auch z.T. etwas abweichende Definitionen für den Begriff MonteCarlo-Simulation, z.B. bei WÖHE (1996, S. 177f, siehe dort auch S. 253f). 


\section{Vorteile}

Die leichte Implementierbarkeit von stochastischen Größen ist einer der Vorzüge von Simulationsansätzen. Ein weiterer Vorteil besteht in der größeren Anschaulichkeit im Vergleich zu analytischen Techniken (ORCUTT 1960, S. 905). Dies ist besonders wichtig, wenn die Adressaten nicht aus dem wissenschaftlichen Umfeld stammen (z.B. Wirtschaft, Politik). Ein wesentlicher Nutzen der Simulation liegt darüber hinaus in der Möglichkeit, neben Stochastizität auch andere komplexitätssteigernde Eigenschaften realer Systeme integrieren zu können. Brauchen die Grenzen analytischer Lösbarkeit nicht beachtet zu werden, kann beim Modellaufbau mehr Gewicht auf die adäquate Abbildung des Systems gelegt werden. Dies erlaubt es häufig erst, die Hypothesen in das Modell aufzunehmen und zu testen, die auf der einen Seite von besonderem Interesse sind und auf der anderen Seite das Modell analytisch unlösbar machen: "more adequate testing is one of the most serious problems facing model builders" (ORCUTT 1960, S. 900). MüLLER (1976, S. 28) nennt in diesem Kontext nichtlineare Zusammenhänge, Ganzzahligkeiten, Steuerungs- und Rückkoppelungsmechanismen und dynamische Verknüpfungen. Auch Modelle mit einer Vielzahl von Einheiten (Individuen, Firmen) sind hier zu nennen, z.B. die aus der Spieltheorie bekannten Invadierungsmodelle, das erwähnte agent based modelling (AXELROD 1997), demographische Modelle, ${ }^{27}$ oder die Agrarstrukturmodelle von BALMANN (1995, 1997, 1998) und BERGER (1999), die die Multi-Agenten-Technik nutzen. ORCUTT (1960, S. 905) betont insbesondere den Vorteil von Simulationen für dynamische Modelle: Simulation "is the only known approach to the satisfactory study and use of any of the existing dynamic models of economic systems for which pretense of realism can be claimed."

Weitere Vorteile von Simulationen sind, daß sie aufgrund der gestiegenen Leistungsfähigkeit von Computern auch Experimente ersetzen können, die zwar technisch möglich, aber sehr aufwendig und teuer sind, z.B. in der Atomindustrie, im Fahrzeugbau oder in der Logistik. Ausschließlich durch Simulationen können Systeme erforscht werden, die real (noch) nicht existieren (ex-ante-Simulation) oder die mit den heutigen technischen Mitteln nicht beobachtbar sind (z.B. in der Atomphysik).

\section{Nachteile $^{28}$}

Simulation als numerische Optimierungstechnik wird für Aufgaben eingesetzt, die mit anderen Verfahren nicht lösbar sind. Daher kann das erzielte Ergebnis auch nicht überprüft und die Wahrscheinlichkeit, statt eines globalen nur ein lokales Optimum gefunden zu haben, nicht völlig ausgeschaltet werden.

Ferner stellt ein Modell für Außenstehende meist eine black box dar; sie können die Ergebnisse des Simulationsmodell nicht nachvollziehen oder überprüfen. Im Zusammenspiel mit einer nicht auszuschließenden hohen Sensibilität des Modells auf Para- 
meteränderungen wiegt dieser Mangel besonders schwer. Diesem Manko kann nur durch eine ausführliche Dokumentation und Sensitivitätsanalyse für die wichtigsten Beziehungen entgegengewirkt werden. Ein Befolgen dieser Grundsätze führt zu dem Nachteil, daß sich Simulationsstudien nicht mehr in kompakter Form darstellen lassen. Um diesem Dilemma zu entgehen, schlägt AXELROD (1997, S. 19f) vor, eine der Simulation angepaßte neue Nomenklatur zu schaffen, ähnlich wie sie für die inferenzielle Statistik bestehe.

Aus der black-box-Eigenschaft der meisten Modelle ergibt sich auch, daß Außenstehende kaum Möglichkeiten besitzen, in praxi die Robustheit von Simulationsergebnissen zu überprüfen. Ein erzieltes Ergebnis einer deterministischen Simulation gilt streng genommen nur für den verwendeten Parameterkranz. Auf die Probleme der Kalibrierung eines Modells ist bereits hingewiesen worden. Im allgemeinen wird es nicht den plausiblen Parametersatz geben, sondern eine Reihe von stichhaltigen Parameterkombinationen. Differieren die Simulationsergebnisse erheblich in Abhängigkeit von den gewählten Koeffizienten, stellt sich die oft kaum zu beantwortende Frage, welches Modell auszuwählen ist. Eine Hilfestellung bietet die Vorgehensweise, solche Modelle vorzuziehen, die für eine breite Spanne gleich glaubwürdiger Annahmen zu ähnlichen Resultaten führen. In stochastischen Modellen kommt das Problem der Signifikanzprüfung hinzu. ${ }^{29}$ Sind die erhaltenen Ergebnisse systematisch oder ein Produkt des Zufalls? Diese Unsicherheit haben Monte-Carlo-Simulationen mit anderen inferentiellen Modellen gemein.

Ein weiterer Mangel der Simulation in ihrer Verwendung als numerische Optimierungstechnik besteht darin, daß die gefundenen Lösungen mathematisch nicht exakt sind. Er wiegt in einfachen statischen Gleichungssystemen weniger schwer, da die Genauigkeit durch Vergrößerung der Zahl der Iterationen beliebig gesteigert werden kann. In stärker verknüpften Modellen, in denen numerische Lösungen als Input für weitere nichtlineare Gleichungen dienen, können hingegen auch kleinere Ungenauigkeiten bereits große Wirkungen nach sich ziehen. Beispiele dafür ist der aus der Chaostheorie bekannte ,Schmetterlingseffekt': Die empfindliche Abhängigkeit in nichtlinearen Modellen kann so groß sein, daß der Flügelschlag eines Schmetterlings im brasilianischen Urwald ein Unwetter in der Karibik auslöst. „Man darf derartige Bilder aber nicht mißverstehen: der Flügelschlag bringt gleichsam das Faß zum Überlaufen. Genau so gut könnte irgendeine andere Bewegung, z.B. ein rutschendes Sandkorn, das Unwetter auslösen. “30 Der Name Schmetterlingseffekt soll ausdrücken, daß schon allerkleinste Veränderungen der Anfangsbedingungen oder die endliche Genauigkeit in Computerprogrammen in einem chaotischen System zu großen und auch qualitativen Änderungen des Systemverhaltens und damit der zukünftigen Entwicklung führen können. 
Auf dieses Problem ist in der Literatur vielfach hingewiesen worden (DAY 1982, S. 406, BRANDES 1985, S. 179). DE HAEN hält daher Betriebsentwicklungsmodelle mit Eigenschaften, die wir heute unter dem Begriff Chaos einordnen würden, für unbrauchbar, denn, seine Begründung in andere Worte kleidend, das Chaos ist ein Phänomen des Modells und nicht des abzubildenden Systems. ${ }^{31}$ In der Realität bleiben auch 100 Schmetterlinge ohne vorhersehbare Wirkung. ${ }^{32}$ Zwar kann nicht ausgeschlossen werden, daß real auch ein fallendes Reiskorn erhebliche Auswirkungen hat, doch können diese Folgen in keiner Weise prognostiziert werden. Daher ist ein Modell, das auf ein Reiskorn nicht reagiert, nützlicher als ein chaotisches.

Simulation als Analysewerkzeug für Differenzengleichungen liefert theoretisch exakte Lösungen, da auf jeder Stufe nur relativ einfache arithmetische Operationen ausgeführt werden müssen (im Vergleich zu einer analytischen Lösung). Allerdings können sich Rundungsfehler aufgrund des prozeduralen Charakters dieser Lösungstechnik erheblich kumulieren. Diese Gefahr potenziert sich, wenn die Differenzengleichungen nicht linear sind, sondern Produkte von endogenen verzögerten Variablen enthalten.

Ein grundsätzliches Problem des Simulationsansatzes liegt in der Generalisierbarkeit seiner Ergebnisse. Die Reaktionen der endogenen Modellvariablen auf Veränderungen der Parameter oder der exogenen Variablen lassen sich in Simulationen nicht allgemeingültig ableiten. Wie im Zusammenhang mit der Modellrobustheit schon angesprochen, sind Aussagen nur für die Umweltbedingungen und Parameterkombinationen, die in die Simulationen einbezogen wurden, möglich. Demgegenüber besteht eine analytische Lösung darin, den Wert der endogenen Variablen als Funktion der Startwerte und Parameter auszudrücken. Eine solche geschlossene Form erleichtert es, Auswirkungen von Parameter- oder Startwertvariationen zu beurteilen. Ist die geschlossene Form differenzierbar, können evtl. auch nicht auf die spezielle Datenkonstellation beschränkte Reaktionsfunktionen ermittelt werden. ${ }^{33}$ Eine analytische Lösung ist daher einer prozeduralen vorzuziehen. Aufgrund der Fortschritte in der Computeralgebra sind heute theoretisch mögliche geschlossene Formen zunehmend auch praktisch erreichbar. Dennoch wird die prozedurale Technik ihre Bedeutung nicht verlieren, da sie zum einen einfacher anwendbar ist und zum anderen ComputeralgebraProgramme auch auf absehbare Zeit mit Differenzengleichungen höherer Ordnung oder nichtlinearer Art überfordert bleiben werden.

31 Er schreibt: „Ein Modell [...], das unter bestimmten Bedingungen explodierende Entwicklungen einiger Variablen [...] ergeben würde, wird in der Regel auf Grund der vorliegenden Erfahrungen verworfen werden müssen. “(DE HAEN 1973, S. 167, Hervorhebungen durch den Verfasser).

Auch in rekursiven Modellen, die sich auf lineare Programmierung beschränken, sind chaotische optimale Pfade möglich, wenn das lineare Programm eine spezielle ,geschachtelte' Struktur aufweist. KOPEL \& FEICHTINGER (1995, S. 552f) referieren Beispiele aus der Literatur und demonstrieren einen eigenen kleinen LP-Ansatz:: Ein Unternehmer habe in jeder Periode zu entscheiden, wieviel der Ernte er verkaufen und wieviel er zur Wiederaussaat verwenden will. Sein Ziel ist die Maximierung der diskontierten Deckungsbeiträge. Zielfunktion und Nebenbedingungen sind linear und die generierte optimale Lösung kann (dennoch) chaotisch verlaufen.

Im Abschnitt 2.2 wird dies für ein einfaches Gewinnentwicklungsmodell demonstriert. 
Ein weiteres Problem ist grundsätzlicher Art. Wenn Simulationen zur Überprüfung von Hypothesen herangezogen werden, ist bei der Spezifikation des Modells darauf zu achten, daß es geeignet ist, die infrage stehenden Hypothesen zu testen. Insbesondere muß in einem Simulationsmodell grundsätzlich a priori die Möglichkeit bestehen, eine Hypothese sowohl anzunehmen als auch zu verwerfen. Kann eine Hypothese deduktiv aus den getroffenen Annahmen bestätigt oder verworfen werden, ist eine Simulation bestenfalls unnötig. Da eine stochastische Simulation immer auch das Risiko von Fehlschlüssen birgt, sollte die Anwendung von Simulationen auf Fragestellungen beschränkt werden, die nicht mit logischer Gedankenführung eindeutig beantwortet werden können. Daraus folgt umgekehrt, daß Resultate einer Simulation nur dann einen Erkenntnisgewinn darstellen, wenn das verwendete Modell a priori auch ein anderes Resultat hätte liefern können. ${ }^{34}$

Hierzu sei ein Beispiel angeführt. In einer vor kurzem erschienenen Dissertation ist zu lesen: "Eine große Preisschwankung erhöht in den meisten Fällen die Ruinwahrscheinlichkeit" (BURMESTER 1996, S. 406). Dieses Ergebnis leitet der Autor aus einem Simulationsmodell ab. Gleichwohl läßt sich dieses Ergebnis auch unmittelbar aus den getroffenen Annahmen ableiten: Sind die Preise kostendeckend, kann ein Betrieb bei festen Preisen nicht in Konkurs gehen, wenn man von Liquiditätsengpässen als andere Ursache für eine Insolvenz neben Überschuldung absieht. Eine (ausreichende) Variation der Preise ermöglicht c.p. erst eine Kostenunterdeckung und damit eine Insolvenz (s. Kap. 4.3. Ein gutes Simulationsmodell muß daher, ungeachtet seiner Berechtigung, diese deduktiv ableitbaren Implikationen ebenfalls zeigen, sonst wäre es schlecht spezifiziert. Das heißt, diese Aussage, bzw. deren Bestätigung durch ein Simulationsmodell bedeutet keinen Erkenntnisgewinn, sondern sollte nur dazu benutzt werden, das Modell zu validieren.

Abschließend seien zwei Nachteile von Simulationsmodellen genannt, die eher praktisch begründet sind und ihre Signifikanzprüfung und Weiterverwendung betreffen. In der Praxis wird der inferentiellen Beurteilung der erzielten Modellergebnisse oft nicht ausreichend Beachtung geschenkt. Eine der Ursachen dafür ist, daß die Entwicklung eines Modells und seine spätere Anwendung in Händen verschiedener Personengruppen liegen. Oft kennen die Modellbauer die spezifischen Anforderungen der späteren Anwendung nicht und sind mit den besonderen anwendungsbezogenen Vorstellungen der Nutzer nicht vertraut. Dies mag eine der Ursachen dafür sein, daß Modellergebnisse und Realität häufig auseinanderfallen. Eine weitere Schwäche liegt darin, daß Ergebnisse von Szenarioanalysen häufig keine optimale Lösung darstellen, da die meisten Simulationsmodelle keinen Maximierungsalgorithmus enthalten. Dies wird von Anwendern oft übersehen (ORIADE \& DILLON 1997 S. 48).

34 Dieser Satz darf nicht , auf die Goldwaage gelegt werden', da er in sich widersprüchlich ist: Besitzt ein Modell eine eindeutige Lösung, ist auch a priori keine andere Lösung möglich. Wichtig ist dennoch Folgendes: In ein Modell sollten keine Bedingungen eingebaut werden, die eine real für möglich gehaltene Entwicklung offensichtlich ausschließen oder offensichtlich erzwingen. 
Einer weiteren Verbreitung von Simulationsmodellen steht entgegen, daß viele im Wissenschaftsbereich entstandene Modelle von einer Einzelperson oder einer kleinen Gruppe für einen Spezialfall konzipiert werden. Die Übertragung auf verwandte Problemstellungen scheitert häufig daran, daß die Programme von "Fremden" nicht genutzt werden können. Die Dokumentation, sofern überhaupt vorhanden, ist auf den Auftraggeber der Studie und nicht auf spätere Nutzer zugeschnitten. Die Bedienerfreundlichkeit der Programme ist im allgemeinen gering. Schnittstellen zur leichten Implementierung anderer Parameter oder Fragestellungen sind nicht vorhanden. Diese Mängel ließen sich prinzipiell leicht beheben, doch besteht auf Seiten der Modellentwickler nur wenig Anreiz, hierin Energie und Zeit zu investieren. Ein Großteil der Modelle wird erstellt, um einen akademischen Grad zu erhalten, und nicht, um der Wissenschaftsgemeinde ein neues anwendbares Instrument für die weitere Forschung an die Hand zu geben. ${ }^{35}$ Aus diesem Grunde wandern viele Simulationsmodelle nach einmaligem Gebrauch in die Schublade. Positive Ausnahmen sind die Anstrengungen der Forschungsanstalt für Landwirtschaft in Braunschweig (ISERMEYER et al. 1996, KLEINHANSS 1998, JACOB 1998), die seit einigen Jahren Agrarmodelle sammeln und standardisieren, und einer Gruppe um HENRICHSMEYER, die die Vorteile vernetzter Modelle am Beispiel einer auf internationale Marktbeziehungen ausgerichteten Modellfamilie aufzeigt (EUROSTAT 1997). Wiederholt angewendet wurde das erwähnte FLIPSIM-Modell in den USA. ${ }^{36}$ Auch das Modell von BALMANN (1995) wird weiter genutzt. Es wird z.Z. von BERGER am Institut für Agrarökonomie in Göttingen um Verhaltensheterogenität und technischen Wandel erweitert.

\section{Anwendungen}

Die o.g. Vorteile und Einsatzmöglichkeiten von Simulationsansätzen haben sehr ihrer Verbreitung beigetragen. Große Beachtung haben die bereits erwähnten globalen Modelle des Club of Rome gefunden, welche die Gefahren ungebremsten Wachstums für die Umwelt und das Überleben der Menschheit erstmals einer breiteren Öffentlichkeit bekannt machten (MEADOWs et al. 1973). ${ }^{37}$ Ähnliche Aufmerksamkeit erregten Weltklimamodelle, die die Erwärmung der Erde und anthropogene Einflüsse auf das Klima untersuchen (z.B. FIROR 1993). In den Agrarwissenschaften wurden Simulationsmodelle unter anderem für pflanzenbauliche Fragen zur Analyse von Bewässerungsprojekten, Umsetzungs- und Verlagerungsprozessen in Kulturböden, Erosionsund Grundwasserschutz, Anbau- und Fruchtfolgesystemen und Düngungs- und Pflanzenschutzmanagement, auch unter Berücksichtigung von Witterungsrisiken (DILLON

35 Das in Kapitel 6 entwickelte Betriebsentwicklungsmodell bildet keine Ausnahme.

36 Einige Anwendungsbeispiele werden in den Abschnitten 2.1.4.2 und 3.3 vorgestellt. $\mathrm{Zu}$ weiteren Anwendungsbeispielen des Modells vgl: ORIADE \& DILLON (1997, S. 52).

37

Ihr Modell dient der Untersuchung von fünf wichtigen Trends mit weltweiter Wirkung: der beschleunigten Industrialisierung, dem rapiden Bevölkerungswachstum, der weltweiten Unterernährung, der Ausbeutung der Rohstoffreserven und der Zerstörung des Lebensraumes. Zwanzig Jahre später haben Donella und Dennis MEADOws „Die neuen Grenzen des Wachstums“ herausgebracht (1993, zusammen mit J. RANDERS). 
et al. 1989, King et al. 1993, MUCHOW \& Bellamy 1991, JOHNSON \& RAUSSER 1977, JOHNSON et al. 1991) angewandt. Die Erforschung naturwissenschaftlicher und technischer Aspekte und der Umweltwirkungen stand dabei meist im Vordergrund. Erst in jüngerer Zeit wurden auch ökonomische Implikationen integriert, z.B. Ernteterminstrategien für Produkte, deren Preise starken saisonalen Schwankungen unterliegen (KELLOG et al. 1988).

In der tierischen Produktion sind z.B. Fütterungstechniken und Beweidungssysteme untersucht worden. Dabei wurde auch das Wetterrisiko berücksichtigt. ${ }^{38}$ LITWIN (1986) analysiert den optimalen Informationsaufwand in der Milchviehhaltung und in der Schweinemast, während die Arbeiten von BUDDE (1974 ) und FUCHS (1988) optimale Fütterungsintensitäten und Vermarktungsstrategien in der Schweineproduktion zum Gegenstand haben.

Im agrarökonomischen Bereich schlug GROSSKOPF (1973) einen Simulationsansatz vor, um Unsicherheit in Taxationsfragen zu integrieren. Dies sei mit Simulationen besser möglich als mit Risikonutzenfunktionen, da Simulationen nicht die a-priori-Kenntnis expliziter Risikopräferenzen voraussetzen, die der Bernoulli-Ansatz erfordert. Zur Modellierung der Auswirkungen von amerikanischen Agrarpolitiken dienten ursprünglich die Modelle FLIPSIM von RICHARDSON \& NIXON (1986) und CEEPES (comprehensive environmental economic policy evaluation system) von BOUZAHER et al. (1995). CEEPES legt den Schwerpunkt auf eine Evaluierung konkurrierender Agrarumweltpolitiken. Insbesondere die Risiken von Agrarchemikalien für Natur und Gesundheit werden beleuchtet. Das FLIPSIM-Modell wird in Abschnitt 2.4 näher beleuchtet.

Simulationsmodelle werden häufig auch für Betriebsentwicklungsmodelle eingesetzt, die nicht nur den Verlauf interessierender Kenngrößen für realisierte (= vergangene) Umweltentwicklungen analysieren, sondern allgemeingültigere Ergebnisse erlauben sollen. Das Hauptaugenmerk der Autoren und Autorinnen solcher Untersuchungen gilt dabei nicht dem Thema der hier vorliegenden Arbeit, der Überlebenswahrscheinlichkeit, sondern anderen Kenngrößen, z.B. der durchschnittlichen Eigenkapitalentwicklung, dem Vermögensendwert u.ä. (CHIEN \& BRADFORD 1976, LiTWIN 1986, FuChS 1988, HARPAIN 1989, WOSSINK 1990, KÜHL 1992). Weitere Anwendungen von Simulationsmodellen werden in CSÁKI (1985) und ORIADE \& DILLON (1997) beschrieben.

Die große Bedeutung von Simulationsmodellen in der ökonomischen Forschung führt dazu, daß Simulationsmodelle auch verstärkt in der Lehre eingesetzt werden. Ein Beispiel ist die PC-Simulation eines 2-Sektoren-Gleichgewichts-Modells von WAGNER (1995). Sein Modell erlaubt Studierenden, mit Hilfe eines Rechners Simulationsmodelle zu erstellen, anhand derer sie sich die Wirkungsweise von mathematisch formulierten Modellen der Wirtschaftswissenschaften verdeutlichen können. Das behandelte Zwei-Sektoren-Gleichgewichtsmodell dient hierbei lediglich als Beispiel.

38 Vgl. hierzu die in ORIADE \& DILLON (1997, S. 54) und die dort angegebene Literatur. 
Ebenso ist die Verwendung der Standardsoftware SHAZAM exemplarisch für die Gruppe der Statistik- oder Simulationssoftware zu verstehen. Der Rechner wird also als Werkzeug benutzt, das dazu dienen kann, zu einem tieferen Verständnis von Modellen zu gelangen. Weitere Modelle wurden von LANGE \& LORENZ (1993, 1995), PAETOW (1997) und HILLMANN (1998) vorgestellt. Sie benutzen Standard-PC-Software zur Tabellenkalkulation, die weit verbreitet und einfach zu bedienen ist und sich daher zur Ergänzung von Lehrveranstaltungen besonders eignet.

\subsubsection{Abgrenzung von Simulationsmodellen und analytischen Modellen}

Häufig wird, wie auch in den vorangegangenen Abschnitten dieser Arbeit, zwischen Simulationsmodellen und mathematisch-analytischen Modellen (z.B. WEINSCHENCK 1977, S. 52) unterschieden. Diese Differenzierung ist problematisch. Beispielsweise definiert Axelrod (1997, S. 2) den Begriff Simulation folgendermaßen: „Simulation is driving a model of a system with suitable inputs and observing the outputs." Diese Begriffsbestimmung nimmt keinerlei Bezug auf die Lösungstechnik des Modells. In einem deterministischen Kontext berechnet ein Simulationsmodell die Werte der endogenen Variablen für vorgegebene Sets der Entscheidungsparameter. Die Auswahl der Parameter trifft die Modellbauerin oder die Entscheiderin in ihrer Szenariofestlegung. Folglich bezeichnen auch BANSE (1997) und MüNCH (BANSE \& MüNCH 1998) die von ihnen eingesetzten allgemeinen Gleichgewichtsmodelle (CGE-Modelle), die statisch-deterministisch sind und analytisch für vorgegebene Szenarien gelöst werden, als Simulationsmodelle. Dies kommt auch in der Namensgebung ihres Modells als European Simulation Model (ESIM) zum Ausdruck.

Der Gegenentwurf zu Szenarioanalysen ist ein Verfahren, das versucht, die einzelnen Szenarien in ein Zielfunktion einzubetten, die maximiert werden kann. Dies ist im allgemeinen nur in einfachen Modellen möglich (s.o). Die Alternativen müßten also Simulation oder Optimierung lauten und nicht Simulation oder analytische Verfahren. ${ }^{39}$ Ein Beispiel auf der Ebene der Betriebsplanung ist dafür die Unterscheidung in Programmplanung und linearer Optimierung. Aber auch diese Unterscheidung ist nicht aufrechtzuerhalten. Wenn ein LP-Modell für verschiedene Parameterwerte gerechnet wird, sind beide Elemente, Optimierung und Simulation, enthalten. Dies gilt im Grunde auch dann, wenn die Simulation durch Parametrisierung in das LP-Modell integriert wird. Formal ließe sich Simulation als Oberbegriff definieren, als Technik zur Analyse von Systemen mit Hilfe von Modellen. Denn auch wenn nur ein einziges Szenario betrachtet wird, ist die Auswahl dieses Szenarios ein willkürlicher Akt und damit eine degenerierte Simulation.

39 Vgl. zu einer ähnlichen Einschätzung ZWICKER (1981, S. 102). In Abhängigkeit vom Ergebnis werden die Entscheidungsparameter verändert, bis sich eine ausreichende Zielerreichung eingestellt hat. 
Bei der Behandlung dynamischer Systeme ist eine Unterscheidung zwischen analytischen und Simulationsmodellen zweckmäßiger. In einem Simulationsmodell können die endogenen Variablen ggf. rekursiv aus ihren zeitverzögerten Werten berechnet werden. Der analytische Ansatz ist im Gegensatz dazu ein Verfahren, das die strukturellen Gleichungen eines Modells so umformt, daß die endogenen Variablen als Funktion der Anfangswerte und Parameter ausgedrückt sind. Eine solche geschlossene Form könnte selbstverständlich auch in einem Simulationsmodell statt des rekursiven Vorgehens verwendet werden.

Ebenfalls zweckmäßig ist die Unterscheidung zwischen „,analytisch” und „Simulation” in stochastischen Modellen. Der entscheidende Punkt ist hier, wie der zufällige Charakter einiger Variablen in das Modell integriert wird. In der analytischen Herangehensweise wird versucht, die Wahrscheinlichkeitsverteilung der endogenen Variablen in jeder Periode zu berechnen oder, wenn dies nicht durchführbar ist, zumindest die wichtigsten Momente der Verteilung. Wie bereits angesprochen, ist dies in komplexeren, insbesondere dynamischen Modellen in der Regel nicht möglich. In Simulationsmodellen werden keine Verteilungen oder Momente der endogenen Variablen deduktiv abgeleitet. Vielmehr wird ihre Wahrscheinlichkeitsverteilung pseudoinduktiv ermittelt. ${ }^{40}$ Dies gestattet Inferenz-Aussagen, die bei analytischen Modellen schon bei geringer Komplexität unmöglich werden.

Die Einteilung in Simulationsmodelle und analytische Modelle ist trotz ihrer Schwächen verbreitet. Auch Betriebsentwicklungsmodelle werden nach diesem Schema differenziert. Allgemein können für das Arbeiten mit Betriebsentwicklungsmodellen zwei Pole unterschieden werden:

- Es werden relativ einfach strukturierte Unternehmen erforscht, die mit entsprechend einfachen Entscheidungkalkülen auskommen. Dies erlaubt, bezüglich der Umwelt relativ differenzierte Annahmen im Modell zu treffen und dennoch eine analytische Lösung zu erzielen (analytische Verfahren).

- Es werden stärker an die Realität angelehnte, komplexere Unternehmensorganisationen betrachtet, die entsprechend diffizilere Entscheidungsverfahren erfordern. Dafür werden bei den Umweltannahmen teils erhebliche Einschränkungen in Kauf genommen. Insbesondere wird, wegen der sehr aufwendigen Berechnung, der stochastische Charakter der Umwelt auf einfache diskrete Verteilungen reduziert (Simulationsmodelle).

Die Ermittlung der gewinnmaximierenden Lösung erfolgt in agrarökonomischen Modellen des zweiten Typs oft mit Methoden der linearen Programmierung. Dies setzt voraus, daß die Beziehungen im Modell linear (oder linearisiert) sind. LP als technisches Instrument der Entscheidungsfindung kompliziert Betriebsentwicklungsmodelle im Vergleich zu Modellen des ersten Typs bereits erheblich, ohne jedoch der komplexen Entscheidungsfindung in der Wirklichkeit gerecht werden zu können. Andererseits ist LP zur Entscheidungsvorbereitung in der wirtschaftlichen Praxis verbreitet. Lineare

40 Dies kann mit Hilfe der Risikoanalyse geschehen, auf die in Abschnitt 3.2 eingegangen wird. 
Programmierung stellt daher einen Kompromiß zwischen Handhabbarkeit und Realitätsnähe dar.

In der vorliegenden Arbeit wird in Kapitel 5.2 ein Mittelweg zwischen den genannten Polen entwickelt werden, um sowohl weniger einfache Entscheidungsregeln als auch ein umfangreicheres Spektrum an Umweltsituationen zuzulassen. Letzteres führt zu komplexeren Situationen, die sich nur mit Hilfe von Monte-Carlo-Simulationen untersuchen lassen (BRANDES \& ODENING 1992, Unterabschnitt 5.7.2, und BRANDES 1989). Dieses Verfahren wird daher für das Überlebensmodell in Kapitel 6 und für einfache dynamische Modelle in Kapitel 5 verwendet.

\subsection{Exkurs: Zu Gewinnentwicklungen in Betriebsentwicklungsmodellen (LOHMANN-RUCHTI-Effekt)}

Ist es möglich, daß in einem deterministischen Betriebsentwicklungsmodell mit Investitionen und konstanten Parametern die Gewinne in den ersten Jahren beständig ansteigen und dann zurückgehen? Zur Klärung dieser Frage wird ein möglichst einfaches Modell konstruiert: streng deterministisch, konstante Parameter, kein Kapitalmarkt. Das Modell repräsentiere ein Unternehmen, das in der ersten Periode einen positiven Gewinn erwirtschafte.

Von diesem Ansatz ausgehend, bietet sich folgender Gedankengang an: „Aufgrund des erzielten Gewinns in der ersten Runde ${ }^{41}$ befindet sich das Unternehmen zu Beginn der zweiten Periode in einer verbesserten Ausgangslage. Daher wird das Unternehmen in der zweiten Periode einen höheren oder zumindest einen gleichhohen, aber keinen geringeren Gewinn erreichen. Gleiches gilt für die Folgeperioden, so daß der Gewinnpfad monoton steigt.“

Diese Gedankenkette spiegelt eine nach eigener Erfahrung naheliegende und verbreitete Intuition wider, sie ist dennoch falsch. Für ein weites Feld von Anfangsbedingungen läßt sich nachweisen, daß die Gewinne in gewissen Perioden zwangsläufig einbrechen. Wann dies geschieht, ist von der Nutzungsdauer der Investitionen und der Gewinnrate abhängig. Die Ursache dieses Effekts liegt in einer ungleichmäßigen Entwicklung der Produktionskapazität: Aus dem Cash-Flow werden neue Anlagen erworben, die bis zum Ende der Nutzungsdauer der Erstausstattung den Bestand an Produktionsanlagen erhöhen. Dieser Effekt der Kapazitätserweiterung ist in seinen Implikationen auf die Produktionsmöglichkeiten erstmals in einem Briefwechsel von MARX und ENGELS beschrieben worden. Sie diskutieren die Möglichkeit für eine Spinnerei, aus den verdienten Abschreibungen weitere Spindeln zu finanzieren. ${ }^{42}$ In den 40er Jahren befaßten sich M. LOHMANN und H. RuCHTI mit den Möglichkeiten, eine Kapazitätsausdehnung ohne zusätzlichen Kapitaleinsatz zu finanzieren. Nach

\footnotetext{
41 Die Ausdrücke Runde und Periode werden synonym gebraucht.

42 Entnommen aus K. LoHMANN (1991, S. 182) und HAX (1958, S. 250f.)
} 
ihnen spricht man heute überwiegend vom LOHMANN-RUCHTI-Effekt und weniger vom MARX-ENGELS-Effekt. ${ }^{43}$

In der Literatur wird der Effekt überwiegend an Beispielen dargestellt. ${ }^{44}$ Einige Aufsätze, vor allen von BUCHNER \& WEINREICH (1971) und K. LOHMANN (1991) leiten auch allgemeine Voraussetzungen für das Auftreten des Effektes ab. In den Arbeiten wird angeführt, daß der Effekt nur auftrete, wenn unvollkommene Kapitalmärkte vorhanden und die Investitionen teilbar seien (HAX 1958, S. 256). Ferner werde die Kapazitätsentwicklung von der Abschreibungsmethode und der zeitlichen Entwicklung der Leistungsabgabe beeinflußt. ${ }^{45}$ In diesem Exkurs wird die Relevanz dieser Punkte für die Kapazitätsentwicklung diskutiert und der LOHMANN-RUCHTIEffekt um die Einflüsse auf den Gewinn und den Gewinnpfad erweitert. Ziel des Abschnitts ist es,

- den bekannten Kapazitätseffekt auf Unternehmen auszuweiten, die mit Gewinn arbeiten,

- nachzuweisen, daß in einem streng deterministischen dynamischen Modell lokale Gewinnminima in der Zeit auftreten können,

- die Voraussetzungen für das Auftreten dieser Minima aufzuzeigen und dabei insbesondere die Bedeutung unvollkommener Kapitalmärkte zu diskutieren und

- den Effekt von Reparaturen, der in der Literatur bisher nicht behandelt wird, und der Abschreibungsmethode zu erörtern.

Diese Fragen werden zunächst analytisch beleuchtet. Dafür ist eine Beschränkung auf ein Modell mit stetigen Investitionsmöglichkeiten und einer Nutzungsdauer von zwei Jahren notwendig. Besonderheiten ganzzahliger Investitionen und höherer Nutzungsdauern werden anschließend mit Hilfe einer Simulation studiert.

\subsubsection{Ein Produktionsmodell}

Gegeben sei ein einfaches deterministisches Modell: Ein Unternehmen maximiere seinen Gewinn in einer konstanten Umwelt ohne Kapitalmarkt, der Kalkulationszinsfuß sei Null. Weder Entnahmen noch Einlagen seien möglich. Die zu Beginn jeder Periode $t$ verfügbaren Mittel $L_{t}$ investiere das Unternehmen ausschließlich in eine Anlage $B_{t}$. Der Anschaffungspreis dieser Anlage betrage $A$. Ihr Betrieb der Anlage verursache keine variablen Kosten und erbringe pro Periode konstante Einzahlungen in Höhe von $e$. Die Anlage habe eine endliche Nutzungsdauer N. Die Abschreibung erfolge linear: $A b=A / N$ und sei kleiner als die Einzahlung $e$.

43 Vgl. K. LOHMANN (1991) insbesondere Kap. 3.1 und die dort angegeben Literatur, insbesondere HARDACH \& HAX (1958), BUCHNER \& WEINREICH (1971). Ferner SCHOPPE (1995) Kap. 1.4.3. K. LOHMANN \& RÜHMANN (1989) und GABLER (1988) und die dort angegebene Literatur.

45 Vgl. z.B. HARDACH \& HAX (1958).

45 Vgl. z.B. BUCHNER und WeINREICH (1971, S. 454), HAX (1958, S. 253, 256), HARDACH \& HAX (1958, S. 541ff), NEUBERT (1951). 
Aus diesen Annahmen folgt, daß der Gewinn pro Anlage stets positiv ist:

$$
G \quad=e-A b>0 \text {. }
$$

Der Gewinn pro Anlage hängt nicht von der Zeit ab, daher sind die Variablen auch nicht mit einem Zeitindex versehen. Der Gesamtgewinn $G_{t}$ ist wie die Gesamteinzahlung $E_{t}$ ein Vielfaches des Bestandes an Anlagen:

$$
G_{t} \quad=G \cdot B_{t}, E_{t}=e \cdot B_{t} .
$$

Das Investitionsvolumen in jeder Periode $t$ wird durch die Einzahlung, die in der Vorperiode erzielt wurde, bestimmt. Wenn nur ganzzahlige Investitionsumfänge möglich sind, beträgt das Investitionsvolumen (in Stück):

$$
I_{t}^{i} \quad=\text { floor }\left(\frac{E_{t-1}}{A}\right) .
$$

Der Operand "floor" rundet eine Zahl auf die nächste ganze Zahl ab. Kann in beliebiger Stückelung investiert werden, erhöht sich der Investitionsumfang auf:

$$
I_{t} \quad=\frac{E_{t-1}}{A} .
$$

Ersetzen der Einzahlungen in dieser Gleichung durch (48,2]:

$$
I_{t} \quad=\frac{B_{t-1} \cdot e}{A}
$$

zeigt, daß der Investitionsumfang von den Konstanten $e$ und $A$ und dem Anlagenbestand in der Vorperiode abhängt. Der Bestand in einer Periode $t$, betrachtet jeweils nach Durchführung der Investition, setzt sich zusammen aus dem Vorperiodenbestand, den Zugängen und den Abgängen. Abgängig sind in $t$ die in $t-N$ erworbenen Anlagen. Daher gilt für die Bestandsentwicklung:

$$
B_{t} \quad=B_{t-1}+I_{t}-I_{t-N} .
$$

Zweimaliges Einsetzen der Investitionsgleichung (48.3.

$$
\begin{aligned}
B_{t} & =B_{t-1}+B_{t-1} \cdot \frac{e}{A}-B_{t-N-1} \cdot \frac{e}{A} \\
& =\left(1+\frac{e}{A}\right) \cdot B_{t-1}+\frac{e}{A} \cdot B_{t-N-1}
\end{aligned}
$$

führt zu einer Differenzengleichung der Ordnung $N-1$, mit der die Bestandsentwicklung beschrieben werden kann. ${ }^{46}$ Für den Gewinn als affine Transformation des Bestandes ergibt sich entsprechend:

$$
G_{t} \quad=G \cdot\left[B_{t-1} \cdot\left(1+\frac{e}{A}\right)-B_{t-N-1} \cdot \frac{e}{A}\right] .
$$

46 Die Ordnung einer Differenzengleichung wird durch die größte in der Gleichung enthaltene Verzögerung bestimmt. 
Der Gewinn hängt in jeder Periode ausschließlich von den Startwerten $\left(B_{1}, B_{2}, \ldots, B_{N}\right)$ und Parametern $(A, e, N)$ ab. Daher wäre es praktisch, die obige Formel (48.5 so umzuformen, daß der Gewinn bzw. Bestand unmittelbar als Funktion der Zeit und der Startwerte/Parameter ausgedrückt wird. Diese Lösung einer Differenzengleichung wird auch als ,geschlossene Form” oder „Funktionslösung” bezeichnet, während die Berechnung des Verlauf einer endogenen Variablen über ihre Prädeterminierten ,periodische Regressionslösung" genannt wird. ${ }^{47}$

Zur Bestimmung der Funktionslösung einer Differenzengleichung ist ein System von Polynominalgleichungen zu lösen. Die höchste Ordnung der Polynome des Gleichungssystems stimmt mit der Ordnung der Differenzengleichung überein. Daher sind Differenzengleichungen höherer Ordnung (größer als zwei) häufig praktisch nicht zu lösen. Übersicht 501 demonstriert die Lösung einer Differenzengleichung zweiter Ordnung am Beispiel der Fibonacci-Zahlen.

Die Ordnung der Differenzengleichungen (48.5) bzw. (48.4 hängt von der Nutzungsdauer ab. Für $N=2$ ist bereits ein Gleichungssystem mit Polynomen dritter Ordnung zu lösen, um die geschlossene Form der Gleichung zu erhalten. Es böte daher Vorteile, (48.4) in eine Gleichung geringerer Ordnung zu überführen.

Dies ist möglich, wenn der Bestand $B_{t}$ nicht aus dem Saldo von $\mathrm{Zu}-$ und Abgängen berechnet wird wie in 484, sondern als Summe der aktuellen und der älteren, noch nutzbaren Investitionen. Bei einer zweijährigen Nutzungsdauer wird der Bestand durch Investitionen in der aktuellen und der Vorperiode aufgebaut:

$$
B_{t} \quad=I_{t}+I_{t-1} ;
$$

Einsetzen der Investitionsgleichung 48.3

$$
\begin{aligned}
B_{t} & =\frac{B_{t-1} \cdot e}{A}+\frac{B_{t-2} \cdot e}{A} \\
& =\left(B_{t-1}+B_{t-2}\right) \cdot \frac{e}{A}
\end{aligned}
$$

führt zu einer Differenzengleichung zweiter Ordnung, während 484 für $N=2$ eine Differenzengleichung dritter Ordnung ergibt. Für den Gewinn gilt entsprechend:

$$
G_{t} \quad=B_{t} \cdot G=G \cdot \frac{e}{A} \cdot\left(B_{t-1}+B_{t-2}\right) .
$$

$47 \quad$ Vgl. ZWICKER (1981, S. 72 f). 


\section{Übersicht 50.1: Lösung einer Differenzengleichung zweiter Ordnung am Beispiel der Fibonacci-Zahlen}

Gegeben sei die lineare Differenzengleichung zweiter Ordnung

$$
F_{n}=F_{\mathrm{n}-1}+F_{\mathrm{n}-2} \text {. }
$$

mit den Startwerten: $F_{0}=0, F_{1}=1$.

Um 501 in eine geschlossene Form zu überführen, ist ein Gleichungssystem zu lösen, das auf folgender Gleichung aufbaut:

$$
F_{n}=c_{1} \cdot r_{1}{ }^{\mathrm{n}}+c_{2} \cdot r_{2}{ }^{\mathrm{n}} \text {. }
$$

Die Gleichung enthält vier Unbekannte. Zur Bestimmung von $r_{1}$ und $r_{2}$ werden zunächst die Lags in 501 beseitigt:

$$
F_{n+2}-F_{\mathrm{n}+1}-F_{\mathrm{n}}=0 \text {, }
$$

damit das charakteristische Polynom abgeleitet werden kann:

$$
r^{2}-r-1=0 \text {. }
$$

Dieses quadratische Polynom kann leicht gelöst werden:

$$
r_{12}=1 / 2 \pm 1 / 2 \sqrt{5} \text {. }
$$

Zur Bestimmung von $c_{1}$ und $c_{2}$ in 502 2) wird diese Gleichung für $n=1$ und für $n=2$, d.h. auf die Startwerte von $F_{n}$, angewendet:

$$
\begin{aligned}
& F_{0}=0=c_{1} \cdot r_{1}^{0}+c_{2} \cdot r_{2}^{0} \\
& F_{1}=1=c_{1} \cdot r_{1}^{1}+c_{2} \cdot r_{2}^{1}
\end{aligned}
$$

Einsetzen der Lösung des charakteristischen Polynoms für $r_{12}$ führt zu:

$$
c_{12}= \pm 0.2 \sqrt{5}
$$

und

$$
F_{n}=0.2 \sqrt{5} \cdot\left[\left(\frac{1+\sqrt{5}}{2}\right)^{n}-\left(\frac{1-\sqrt{5}}{2}\right)^{n}\right] .
$$

Dieser Ausdruck ermöglicht es, den Wert der Funktion für beliebiges $n$ unmittelbar zu bestimmen.

Veränderte Startwerte wirken sich nicht auf das charakteristische Polynom aus, sondern nur auf die Werte für $c_{1}$ und $c_{2}$. Für beliebige Startwerte $s_{0}$ und $s_{1}$ gilt:

$$
\begin{array}{ll}
c_{1} & =\frac{5-\sqrt{5}}{10} \cdot s_{0}+\frac{2 \sqrt{5}}{10} \cdot s_{1} \\
c_{2} & =\frac{5+\sqrt{5}}{10} \cdot s_{0}-\frac{2 \sqrt{5}}{10} \cdot s_{1} \\
F_{n} & =\left(\frac{1+\sqrt{5}}{2}\right)^{n} \cdot c_{1}+\left(\frac{1-\sqrt{5}}{2}\right)^{n} \cdot c_{2} .
\end{array}
$$


Für eine zweijährige Nutzungsdauer können also Gewinn und Bestand als Differenzengleichungen zweiter Ordnung ausgedrückt werden. Diese Differenzengleichungen lassen sich nach dem in Übersicht 511 dargestellten Verfahren algebraisch lösen. Das Vorgehen ist in Übersicht 511 dargestellt.

\section{Übersicht 51.1: Lösung der Differenzengleichung 49.1}

Differenzengleichung:

$$
B_{t} \quad=\left(B_{t-1}+B_{t-2}\right) \cdot \frac{e}{A}
$$

Startwerte:

$$
\begin{array}{ll}
B_{1} & =L_{1} / A \\
B_{2} & =B_{1}+B_{1} \cdot e / A=B_{1} \cdot(1+e / A)=L_{1} / A \cdot(1+e / A)
\end{array}
$$

geschlossene Form:

$$
B_{t} \quad=c_{1} \cdot r_{1}{ }^{t}+c_{2} \cdot r_{2}{ }^{t} \text {. }
$$

Differenzengleichung ohne Lags $(d \equiv e / A)$ :

$$
B_{t+2}-d \cdot B_{t+1}-d \cdot B_{t}=0
$$

Charakteristisches Polynom:

$$
\begin{aligned}
r^{2}-d \cdot r-d & =0 \\
r_{12} & =1 / 2 d \pm \sqrt{1 / 4 d^{2}+d} \\
& =1 / 2[\mathrm{~d} \pm \sqrt{d \cdot(4+d)}]
\end{aligned}
$$

Startwerte:

$$
\begin{array}{ll}
B_{1} & =L_{1} / A \\
B_{2} & =L_{1} / A \cdot(1+e / A)
\end{array}
$$

Gleichungssystem:

$$
\begin{aligned}
& L_{1} / A \quad=c_{1} \cdot r_{1}{ }^{1}+c_{2} \cdot r_{2}{ }^{1} \\
& L_{1} / A \cdot(1+e / A)=c_{1} \cdot r_{1}{ }^{2}+c_{2} \cdot r_{2}{ }^{2}
\end{aligned}
$$

Einsetzen des charakteristischen Polynoms:

$$
\begin{aligned}
& L_{1} / A=c_{1} \cdot 1 / 2[\mathrm{~d}+\sqrt{d \cdot(4+d)}]+c_{2} \cdot 1 / 2[\mathrm{~d}-\sqrt{d \cdot(4+d)}] \\
& \wedge L_{1} / A \cdot(1+e / A)=c_{1} \cdot 1 / 4[\mathrm{~d}+\sqrt{d \cdot(4+d)}]^{2}+c_{2} \cdot 1 / 4[\mathrm{~d}-\sqrt{d \cdot(4+d)}]^{2} \\
& c_{1} \quad=\frac{2 L_{1}}{\mathrm{e}-A \sqrt{d \cdot(4+d)}} \\
& +\frac{[d+\sqrt{d \cdot(4+d)}] \cdot[2+d+\sqrt{d \cdot(4+d)}] \cdot L_{1}}{4 e \sqrt{d \cdot(4+d)}}
\end{aligned}
$$




$$
c_{2} \quad=-\frac{[d-\sqrt{d \cdot(4+d)}] \cdot[2+d+\sqrt{d \cdot(4+d)}] \cdot L_{1}}{4 e \sqrt{d \cdot(4+d)}} .
$$

Mit der Abkürzung $s \equiv \sqrt{d \cdot(4+d)}$ ergibt sich für die Koeffizienten $c_{1}$ und $c_{2}$ :

$$
\begin{array}{ll}
c_{1} & =\frac{2 L_{1}}{\mathrm{e}-A s}+\frac{[d+s] \cdot[2+d+s] \cdot L_{1}}{4 e s} \\
c_{2} & =-\frac{[d-s] \cdot[2+d+s] \cdot L_{1}}{4 e s} .
\end{array}
$$

Die geschlossene Form der Differenzengleichung:

$$
B_{t} \quad=c_{1} \cdot r_{1}{ }^{t}+c_{2} \cdot r_{2}{ }^{t}
$$

lautet nach Einsetzen der Terme für $c_{1}$ und $c_{2}$ :

$$
\begin{aligned}
B_{t} \quad & =\left[\frac{2 L_{1}}{e-A s}+\frac{(d+s) \cdot(2+d+s) \cdot L_{1}}{4 e s}\right] \cdot(d+s)^{t} \\
& -\frac{(d-s) \cdot(2+d+s) \cdot L_{1}}{4 e s} \cdot(d-s)^{t}
\end{aligned}
$$

Ohne die verwendeten Abkürzungen ergibt sich nach einigen Umformungen für den Bestand in Periode $t$ :

$$
\begin{aligned}
B_{t} & =\frac{4 \cdot(2 e)^{t-1} L_{1} A \sqrt{e \cdot(e+4 A)}}{e+4 A} \\
& \cdot\left[\frac{1}{[-e+\sqrt{e \cdot(e+4 A)}]^{t+1}}+\frac{(-1)^{t}}{[e+\sqrt{e \cdot(e+4 A)}]^{t+1}}\right]
\end{aligned}
$$

Diese Gleichung gibt den Bestand unter der Voraussetzung einer zweijährigen Nutzungsdauer zu einem beliebigen Zeitpunkt als Funktion der Parameter und des Startkapitals $L_{1}$ an. Trotz ihrer Komplexität ist unmittelbar zu erkennen, daß der Bestand in einer Periode ein Vielfaches des Startkapitals ist und die Beziehungen des Bestandes zu Einzahlung und Anschaffungspreis hochgradig nichtlinear sind.

Zur leichteren Beschreibung wird die Gleichung in drei symbolische Komponenten zerlegt:

$$
B_{t} \quad=X \cdot\left[\frac{1}{Y}+\frac{(-1)^{t}}{Z}\right] .
$$

Alle Konstanten in 521 sind per definitionem positiv, daher ist $X$ stets größer als Null. In $Y$ ist die Wurzel, $\sqrt{ }\left(e^{2}+\ldots\right)$, größer als $e$, damit sind $Y$ und $1 / Y$ positiv. Der zweite Summand in (52 2) alterniert wegen des Zählers $(-1)^{t}$. Sein Nenner, $Z$, ist immer größer als der Nenner des ersten Summanden, $Y$. Damit ist der Betrag des zweiten Summanden immer kleiner als der erste Summand und die Summe [•] damit nie negativ. Da beide Faktoren in $(522$ positiv sind, ist auch das Produkt $X \cdot[\bullet]$ und 
der Bestand immer positiv. Die Modellgleichung erfüllt also die Forderung einer positiven Bestandsentwicklung. Gleichzeitig sind durch den alternierenden zweiten Summanden $\frac{(-1)^{t}}{Z}$ prinzipiell lokale Minima möglich.

Der Gewinn ist nach 48,5 eine affine Transformation des Bestandes:

$G_{t} \quad=G \cdot B_{t}$

Einsetzen der Bestandsgleichung (52.1] führt zu:

$$
G_{t} \quad=G \frac{2^{t+1} e^{t-1} L_{1} A \sqrt{e \cdot(e+4 A)}}{e+4 A} \cdot\left[\frac{1}{[-e+\sqrt{e \cdot(e+4 A)}]^{t+1}}+\frac{(-1)^{t}}{[e+\sqrt{e \cdot(e+4 A)}]^{t+1}}\right] .
$$

Wie müssen die Parameter $\mathrm{G}, L_{1}, A$ und $e$ verknüpft sein, damit der Gewinnverlauf lokale Minima aufweist? In 531] sind $G$ und $L_{1}$ Konstanten, die die endogene Variable $G_{t}$ proportional verändern und daher nicht für lokale Extrema verantwortlich sein können. Damit eine Gewinnentwicklung möglich wird, müssen beide Konstanten von Null verschieden sein. Das Startkapital ist per definitionem positiv. Ein positiver Stückgewinn bedeutet nach 48,1] bei zweijähriger Nutzungsdauer, daß die Einzahlung pro Stück größer sein muß als der halbe Anschaffungswert. Unter diesen Voraussetzungen kann sich die Kurvendiskussion auf die Differenzengleichung des Bestandes (52.1) und darin auf die Konstanten $e$ und $A$ beschränken, die nicht linear mit $B_{t}$ verknüpft sind.

Üblicherweise wird eine Funktion abgeleitet, um ihre Extremstellen zu bestimmen. Dieser Ansatz ist für dieses Problem ungeeignet, da es sich zum einen nicht um eine stetige Funktion handelt. Zum anderen wird nicht ein Minimum in Abhängigkeit von $A$ oder $e$ gesucht, sondern es interessieren kritische Werte für $A$ und $e$, bei denen das Minimum der Bestandsfunktion in der Zeit verschwindet.

Der zweite Summand in 522 ist negativ, wenn und nur wenn $t$ ungerade ist. Die Funktion kann daher nur für ungerade $t$ lokale Minima besitzen. Schaubild 54,1, linke Hälfte, zeigt, daß für die Parameter $A=L_{1}=1, N=2, e=.51$ Bestandsminima in den Perioden drei und fünf auftreten. In den nachfolgenden Perioden sind keine Minima vorhanden, s. rechte Hälfte des Schaubilds. Der Bestand steigt monoton und nahezu linear an und übertrifft ab Periode 11 das lokale Maximum aus Periode 2.

Voraussetzung eines lokalen Minimums ist, daß der Bestand in $t$ kleiner ist als in der Vorperiode. Die Ungleichung $B_{t}=B_{t-1}$ kann nicht für beliebiges $t$ gelöst werden. Daher sei zunächst $t$ gleich drei gesetzt. 
Schaubild 54.1: $\quad$ Entwicklung des Bestandes

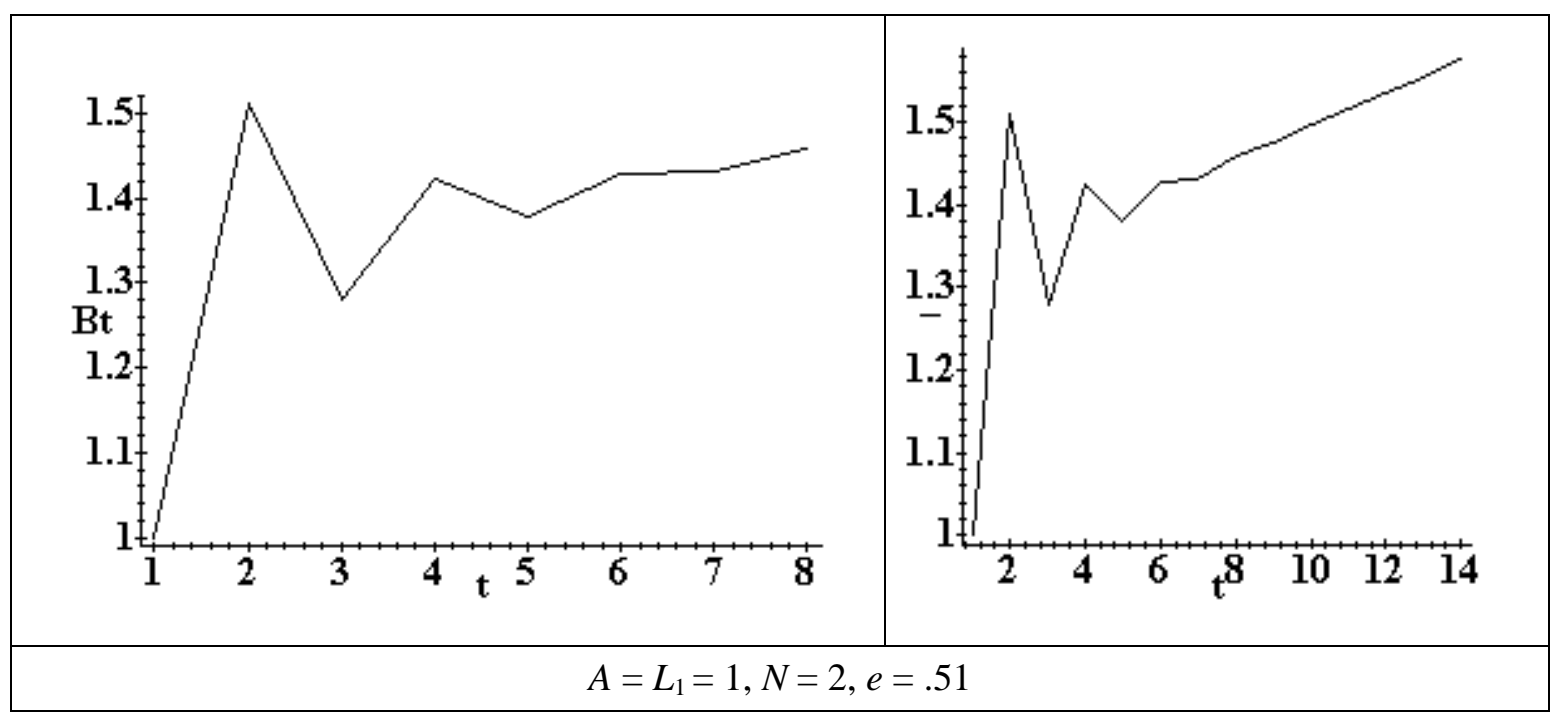

$$
\begin{array}{ll}
B_{3}<B_{2} & \\
\Leftrightarrow & \frac{2^{4} e^{2} A \sqrt{e \cdot(e+4 A)}}{e+4 A}\left[\frac{1}{[-e+\sqrt{e \cdot(e+4 A)}]^{4}}+\frac{(-1)^{3}}{[e+\sqrt{e \cdot(e+4 A)}]^{4}}\right] \\
< & \frac{2^{3} e A \sqrt{e \cdot(e+4 A)}}{e+4 A}\left[\frac{1}{[-e+\sqrt{e \cdot(e+4 A)}]^{3}}+\frac{(-1)^{2}}{[e+\sqrt{e \cdot(e+4 A)}]^{3}}\right] \\
\Leftrightarrow & \frac{2^{4} e^{2}}{[-e+\sqrt{e \cdot(e+4 A)}]^{4}}+\frac{2^{4} e^{2}(-1)^{3}}{[e+\sqrt{e \cdot(e+4 A)}]^{4}} \\
< & \frac{2^{3} e}{[-e+\sqrt{e \cdot(e+4 A)}]^{3}}+\frac{2^{3} e(-1)^{2}}{[e+\sqrt{e \cdot(e+4 A)}]^{3}} \\
\Leftrightarrow e & <( \pm 1 / 2 \sqrt{5}-1 / 2) A .
\end{array}
$$

Da alle Variablen definitionsgemäß größer als Null sind, kommt nur die positive Wurzel als Lösung für $e$ in Frage. Die Ungleichung $B_{3}<B_{2}$ wird erfüllt, wenn

$$
\frac{e}{A}<1 / 2 \sqrt{5}-1 / 2 \approx 0.618 .
$$

Die Einzahlung darf also einen bestimmten Prozentsatz der Investitionsauszahlung nicht übersteigen. Da der Gewinn nach 481 $G=e-A b$ ) proportional mit der Einzahlung steigt, gibt es auch für den Gewinn eine Obergrenze. Überschreitet der Gewinn diese Grenze, die ausschließlich vom Anschaffungspreis abhängt, verschwindet das Bestands- und Gewinnminimum bei $t=3$. Die Ursache liegt darin, daß bei höheren Gewinnen in einem Umfange Investitionen möglich werden, daß der kapazitätsreduzierende Effekt des Ausfalls der in der Startperiode erworbenen Anlagen überkompensiert wird. 
Schaubild 55.1 zeigt exemplarische Gewinnverläufe für $e=0.618 A+0.1, e=0.618$ $A$ und $e=0.618 A-0.1$. Wie erwartet, weist der mittlere Graph einen Sattelpunkt auf, während der obere monoton steigt und der untere ein lokales Minimum in $t=3$ besitzt.

\section{Schaubild 55.1: Gewinnentwicklung für verschiedene Einzahlungen}

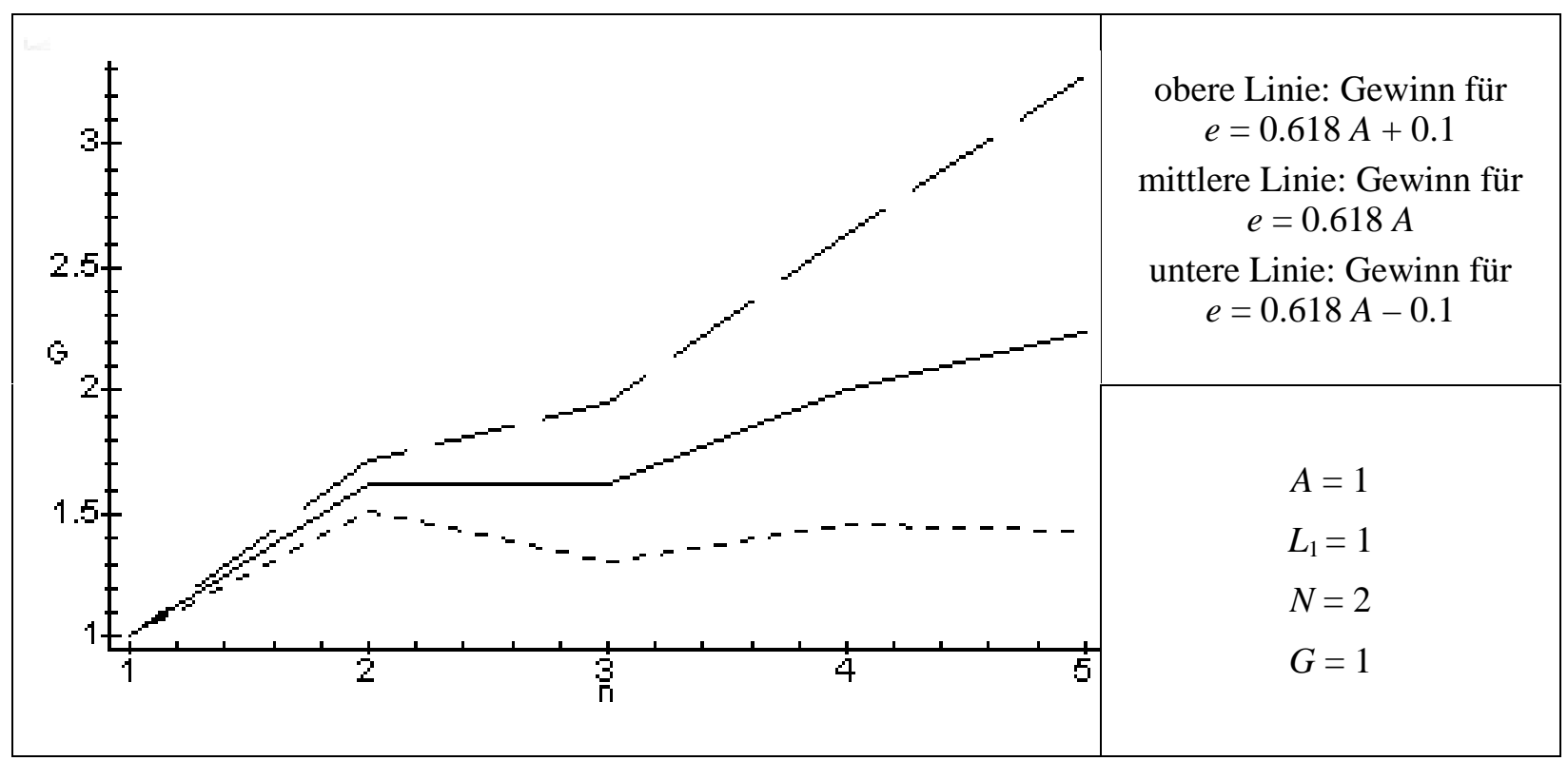

Kritische Werte für den Quotienten $e / A$ können für höhere Perioden mit dem gleichen Ansatz wie für $t=3$ bestimmt werden. Es ist jeweils die Ungleichung $B_{t+1}<B_{t} \mathrm{zu}$ lösen. Dafür sind die Nullstellen gebrochen-rationaler Polynome der Ordnung $t+1 \mathrm{zu}$ bestimmen. Die Lösung gelingt, weil alle Parameter als positive Zahlen definiert sind. Die Ergebnisse zeigt Tabelle 55 1. Bei größeren Verhältnissen als den in der Tabelle ausgewiesenen Werten für $e / A$ verschwinden die lokalen Minima ab der angegebenen Periode.

Tabelle 55.1: Voraussetzungen für lokale Minima

\begin{tabular}{|c|c|}
\hline Periode & Obergrenze für $e / A$ \\
\hline \hline 3 & .6180 \\
\hline 5 & .5321 \\
\hline 7 & .5084 \\
\hline 9 & .5022 \\
\hline
\end{tabular}

\begin{tabular}{|c|c|}
\hline Periode & Obergrenze für $e / A$ \\
\hline \hline 11 & .5005 \\
\hline 13 & .5001 \\
\hline 15 & .5000 \\
\hline \multicolumn{2}{|c|}{$N=2, L=1$} \\
\hline
\end{tabular}

Die kritischen Werte konvergieren schnell gegen $1 / 2 . e=1 / 2 A$ bedeutet, daß die Einzahlung gleich der Abschreibung (wegen $N=2$ ) und damit der Gewinn gleich Null ist. Für $e=1 / 2 A$ vereinfacht sich die Differenzengleichung für den Bestand (52,1] zu:

$$
B_{t} \mid\{N=2, e=1 / 2 \mathrm{~A}\}=\left[\frac{4}{3}+\frac{2^{1-t}}{3} \cdot(-1)^{t}\right] \cdot \frac{L_{1}}{A} .
$$


Der Bestand in 55,1) geht aufgrund des alternierenden Terms $(-1)^{t}$ in jeder ungeraden Periode zurück. Der Umfang des Rückganges strebt wegen des Faktors $2^{-t}$ gegen Null, wie in Schaubild 50.1 angedeutet. Der Grenzwert des Bestandes beträgt daher $\frac{4}{3} \frac{L_{1}}{A}$ oder das 1.j̄fache des Anfangsbestandes, da sich der Bestand in der ersten Periode aus dem Quotienten aus Startkapital $L_{1}$ und Anschaffungspreis $A$ ergibt. Trotz der Bestandsschwankungen ist der Gewinn annahmegemäß immer Null.

Schaubild 56.1: $\quad$ Entwicklung des Bestandes für $e=1 / 2 \quad A$

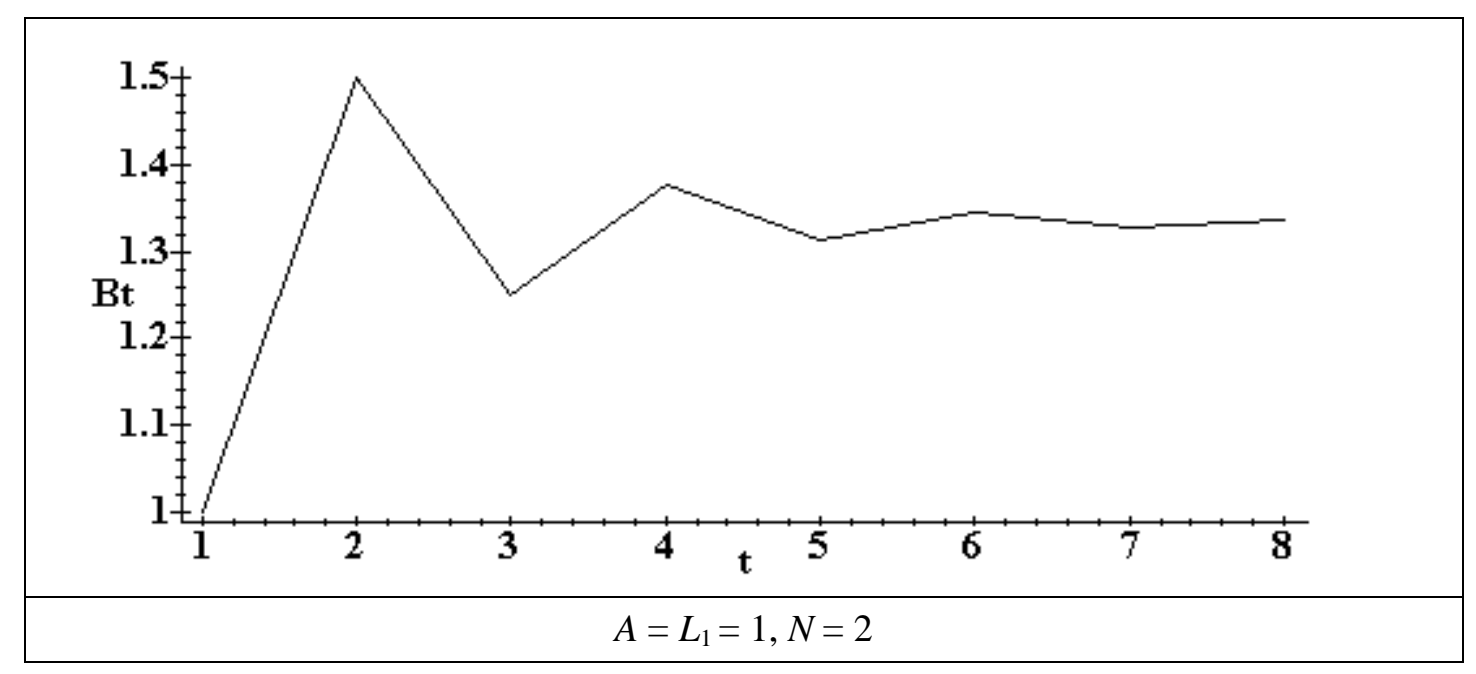

In einer gewinnlosen Situation vergrößert sich langfristig der Bestand nach Ende der ersten Nutzungsdauer auf das 1.5 fache des Ausgangswertes. Diese Produktionskapazität wird in der Folgezeit nicht wieder erreicht. Langfristig vergrößert sich das Produktionspotential pro Periode - gleichmäßige Leistungsabgabe vorausgesetzt -auf 4/3 des Ausgangswertes. Der Zeitwert des Anlagebestands verändert sich nicht, da in jeder Periode die Abschreibungsgegenwerte reinvestiert werden ${ }^{48}$ Die Anlagen sind im Durchschnitt älter und damit stärker abgeschrieben. Der durchschnittliche Zeitwert aller Anlagen beträgt $3 / 4$ des Anschaffungswertes, das mittlere Alter 13/4 Jahre.

\subsubsection{Berïcksichtigung von Reparaturen}

Das verwendete Modell ist bewußt einfach gehalten, um die Effekte leichter veranschaulichen zu können. Insbesondere sind keine expliziten Reparaturkosten berücksichtigt. Auch die bisher zum LOHMANN-RUCHTI-Effekt erschiene Literatur klammert Reparaturen aus. ${ }^{49}$ Wie ändern sich die Eigenschaften des Modells, wenn diese unrealistische Annahme aufgegeben wird?

$48 \quad$ Vgl. HAX (1958, S. 250f).

49 Vgl. K. LOHMANn (1991), HARDACH \& HAX (1958), K. LOHMANn \& RÜHMANN (1989), SCHOPPE (1995) Kap. 1.4.3. 
Zunächst sei der Fall zeitkonstanter Reparaturen betrachtet. Konstante Reparaturen pro Investitionsgut sind in diesem Modell äquivalent mit negativen Einzahlungen. Die Konstante $e$ in 48.1) kann daher auch als Saldo von Einzahlungen und Reparaturen aufgefaßt werden. Oben wurde gezeigt, daß eine hinreichend kleine Gewinnrate Voraussetzung für lokale Gewinnminima ist (vgl. Tabelle 55.1]. Konstante Reparaturen verringern die Gewinnrate und verschärfen daher den LOHMANN-RUCHTI-Effekt.

Empirisch von größerer Bedeutung als konstante Reparaturen sind Reparaturen, die mit dem Alter der Anlage zunehmen. Steigende Reparaturen können im Modell als sinkende Einzahlungen implementiert werden. Die Einzahlungen einer Investition sind daher nicht mehr über die Zeit konstant, sondern von ihrem Alter abhängig. Diese Erweiterung hat zur Folge, daß der Bestand einer Periode nicht mehr aus dem Bestand der Vorperiode erklärt werden kann, denn die Einzahlungen, die der Vorperiodenbestand erbringt, hängen von seiner Alterszusammensetzung ab. Beispielsweise ergeben sich für eine zweijährige Nutzungsdauer folgende Gleichungen:

für die Einzahlungen einer Investition:

$e_{1}=e-r_{1}$ Einzahlung einer Investition im ersten Jahr ihrer Nutzungsdauer

$e_{2}=e-r_{2}$ Einzahlung einer Investition im zweiten Jahr ihrer Nutzungsdauer

für die Einzahlungen in jeder Periode $t$ :

$E_{t} \quad=e_{1} \cdot I_{t}+e_{2} \cdot I_{t-1}$

für die Investitionen in jeder Periode $t$ :

$$
\begin{aligned}
I_{t} & =\frac{E_{t-1}}{A} \\
& =\frac{e_{1} \cdot I_{t-1}+e_{2} \cdot I_{t-2}}{A}
\end{aligned}
$$

für den Bestand in jeder Periode $t$ :

$$
\begin{aligned}
B_{t} & =I_{t}+I_{t-1} \\
& =\frac{E_{t-1}}{A}+\frac{E_{t-2}}{A} \\
& =\frac{e_{1} \cdot I_{t-1}+e_{2} \cdot I_{t-2}}{A}+\frac{e_{1} \cdot I_{t-2}+e_{2} \cdot I_{t-3}}{A}
\end{aligned}
$$

für den Bestand in den ersten beiden Perioden:

$$
\begin{array}{ll}
B_{1} & =\frac{L_{1}}{A} \\
B_{2} & =\frac{B_{1} \cdot e_{1}}{A} .
\end{array}
$$

Der Bestand in Periode $t$ wird durch die Investitionen der drei Perioden zuvor beeinflußt. Daher ist die Differenzengleichung dritter Ordnung. Dennoch kann das System gelöst werden, da die Investitionen durch eine Differenzengleichung zweiter Ordnung 
beschrieben werden können [siehe (57it)]. Die geschlossene Form für die Investitionsgleichung lautet:

$$
I_{t} \quad=\left(2 e_{2}\right)^{t} \cdot L_{1} \cdot\left(\frac{1}{\left(-e_{1}+\sqrt{e_{1}^{2}+4 e_{2} A}\right)^{t}}-\frac{(-1)^{t}}{\left(e_{1}+\sqrt{e_{1}^{2}+4 e_{2} A}\right)^{t}}\right)
$$

und für den Bestand in jeder Periode $t$ ergibt sich nach umfangreichen Umformungen:

$$
\begin{aligned}
B_{t} \quad= & I_{t}+I_{t-1} \\
= & \left(2 e_{2}\right)^{t} \cdot L_{1} \cdot \\
& {\left[\frac{1}{\left(-e_{1}+w\right)^{t}}-\frac{(-1)^{t}}{\left(e_{1}+w\right)^{t}}+\frac{1}{2 e_{2}} \cdot\left(\frac{1}{\left(-e_{1}+w\right)^{t-1}}-\frac{(-1)^{t}}{\left(e_{1}+w\right)^{t-1}}\right)\right] } \\
& \text { mit } w=\sqrt{e_{1}^{2}+4 e_{2} A} .
\end{aligned}
$$

In Tabelle 58.11 werden konstante mit steigenden und fallenden Reparaturverläufen verglichen. Wie Schaubild 59 Minima möglich. Durch stark steigende Reparaturen wird der Effekt bei den gegebenen Parametern insbesondere in höheren Perioden abgeschwächt und durch fallende verstärkt.

Tabelle 58.1: Bestandsentwicklung bei konstantem, fallendem und steigendem Reparaturverlauf

\begin{tabular}{|c||c|c|c|c|c|c|}
\hline \multicolumn{1}{|c||}{} & \multicolumn{2}{|c|}{ Konstante Reparaturen } & \multicolumn{2}{c|}{ fallende Reparaturen } & \multicolumn{2}{c|}{ steigende Reparaturen } \\
\cline { 2 - 7 } Periode & $\begin{array}{c}e_{1}=.51, \\
e_{2}=.51\end{array}$ & $\begin{array}{c}\Delta B_{t} \\
(\text { in \%) }\end{array}$ & $\begin{array}{c}e_{1}=.41, \\
e_{2}=.61\end{array}$ & $\begin{array}{c}\Delta B_{t} \\
\text { (in \%) }\end{array}$ & $\begin{array}{c}e_{1}=.61, \\
e_{2}=.41\end{array}$ & $\begin{array}{c}\Delta B_{t} \\
\text { (in \%) }\end{array}$ \\
\hline \hline 1 & 1.000 & & 1.000 & & 1.000 & \\
\hline 2 & 1.510 & 0.510 & 1.410 & 0.410 & 1.610 & 0.610 \\
\hline 3 & 1.280 & -0.152 & 1.188 & -0.157 & 1.392 & -0.135 \\
\hline 4 & 1.423 & 0.112 & 1.347 & 0.134 & 1.509 & 0.084 \\
\hline 5 & 1.379 & -0.031 & 1.277 & -0.052 & 1.491 & -0.012 \\
\hline 6 & 1.429 & 0.036 & 1.345 & 0.053 & 1.529 & 0.025 \\
\hline 7 & 1.432 & 0.002 & 1.331 & -0.011 & 1.544 & 0.010 \\
\hline 8 & 1.459 & 0.019 & 1.366 & 0.027 & 1.569 & 0.016 \\
\hline 9 & 1.474 & 0.011 & 1.372 & 0.004 & 1.590 & 0.014 \\
\hline \multicolumn{7}{|c|}{$\left(A=1, L_{1}=1, N=2\right)$} \\
\hline
\end{tabular}




\section{Schaubild 59.1: Bestandsentwicklung bei konstantem, steigendem und fallendem Reparaturverlauf}

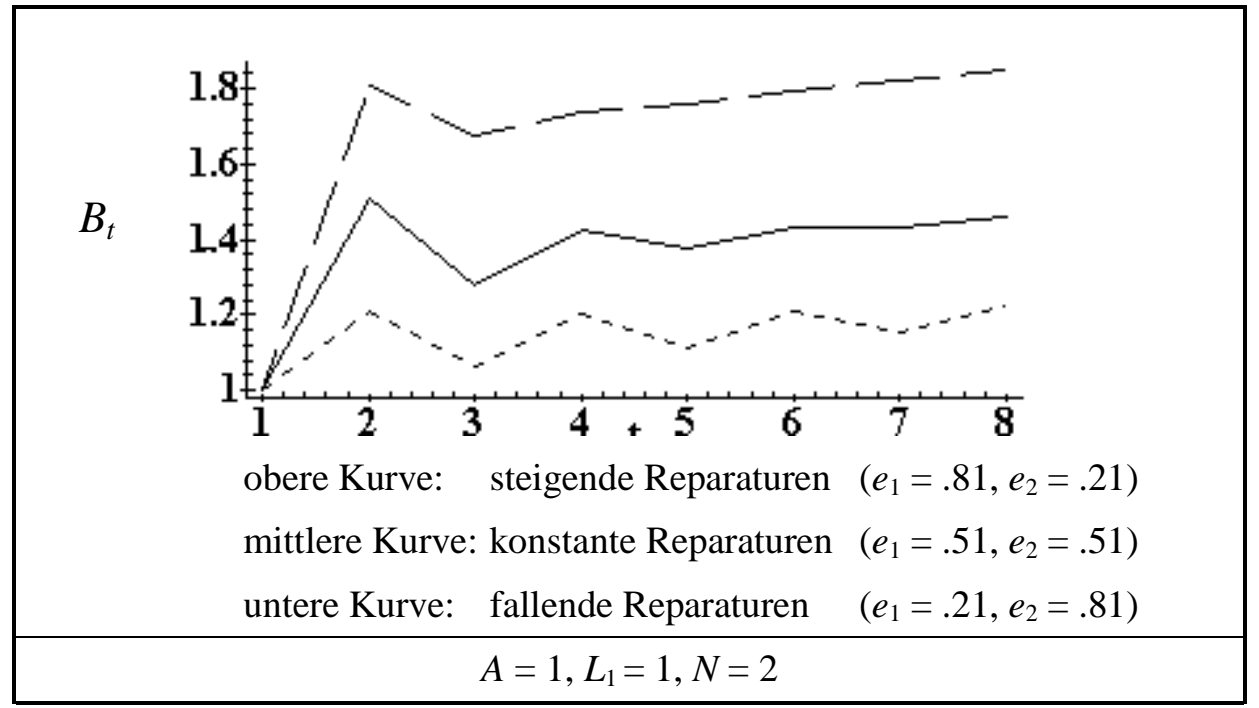

Um zu generellen Aussagen über den Einfluß der Reparaturverteilung auf die Bestandsentwicklung zu kommen, wird der gleiche Ansatz wie bei konstanten Reparaturen verwendet. Voraussetzung eines lokalen Minimums ist, da $\beta$ der Bestand in $t$ kleiner ist als in der Vorperiode. Die Gleichung $B_{t}=B_{t-1}$ kann wiederum nicht für beliebiges $t$ gelöst werden. Daher sei zunächst $t$ gleich drei gesetzt.

Aus $B_{3}=B_{2}$ folgt

$$
\text { A } \quad=1 / 2 \cdot\left(e_{2}+\sqrt{e_{2}^{2}+4 e_{1}^{2}}\right) \text {. }
$$

Die Ungleichung $B_{3}<B_{2}$ wird erfüllt, wenn

$$
A \quad>1 / 2 \cdot\left(e_{2}+\sqrt{e_{2}^{2}+4 e_{1}^{2}}\right)
$$

oder

$$
e_{1} \quad<\sqrt{e_{2} \cdot A+A^{2}} .
$$

Beträgt z.B. der Anschaffungspreis 1 und die Einzahlung einer ein Jahr alten Investitionseinheit $1 / 2$, dann darf die Einzahlung einer zwei Jahre alten Investitionseinheit $3 / 4$ betragen. Weitere Kombinationen für gemeinsame Obergrenzen von $e_{1}$ und $e_{2}$ sind in Tabelle 601 berechnet.

Bei konstanten Einzahlungen durfte die Einzahlung maximal 0.618 pro Periode betragen (s. Tabelle 551). Überschritten die über die zweijährige Nutzungsdauer aggregierten Einzahlungen die Grenze von 1.236, verschwanden die Bestandsminima. Diese Summe sei "kritische Einzahlungssumme" genannt. Eine Konzentration der Reparaturen auf das erste oder zweite Nutzungsjahr verringert die kritische Einzahlungssumme auf 1, vgl. Schaubild 601 Die höchste Summe wird erreicht, wenn die Einzahlung im ersten Jahr der Nutzung $1 / 2$ und im zweiten Jahr $3 / 4$ beträgt, also bei 
leicht fallenden Reparaturen. Dies bedeutet umgekehrt, daß bei leicht fallenden Reparaturen die Gewinnminima besonders ausgeprägt sind. Dieses allgemeine Ergebnis stimmt mit dem Resultat aus Tabelle p 8.1 für spezielle Parameter überein.

Tabelle 60.1: Kritische Werte für Einzahlungskombinationen

\begin{tabular}{|c|c|c|}
\hline $\boldsymbol{e}_{\mathbf{1}}$ & $\boldsymbol{e}_{\mathbf{2}}$ & $\boldsymbol{e}_{\boldsymbol{1}}+\boldsymbol{e}_{\boldsymbol{2}}$ \\
\hline \hline 0 & 1 & 1 \\
\hline .1 & .99 & 1.09 \\
\hline .2 & .96 & 1.16 \\
\hline .3 & .91 & 1.21 \\
\hline .4 & .84 & 1.24 \\
\hline .5 & .75 & 1.25 \\
\hline .6 & .64 & 1.24 \\
\hline .7 & .51 & 1.21 \\
\hline .8 & .36 & 1.16 \\
\hline .9 & .19 & 1.09 \\
\hline 1.0 & 0 & 1.0 \\
\hline \multicolumn{3}{|c|}{$N=2, A=L=1$} \\
\hline
\end{tabular}

Schaubild 60.1: Kritische Werte für Einzahlungskombinationen $(N=\mathbf{2}, A=L=1)$

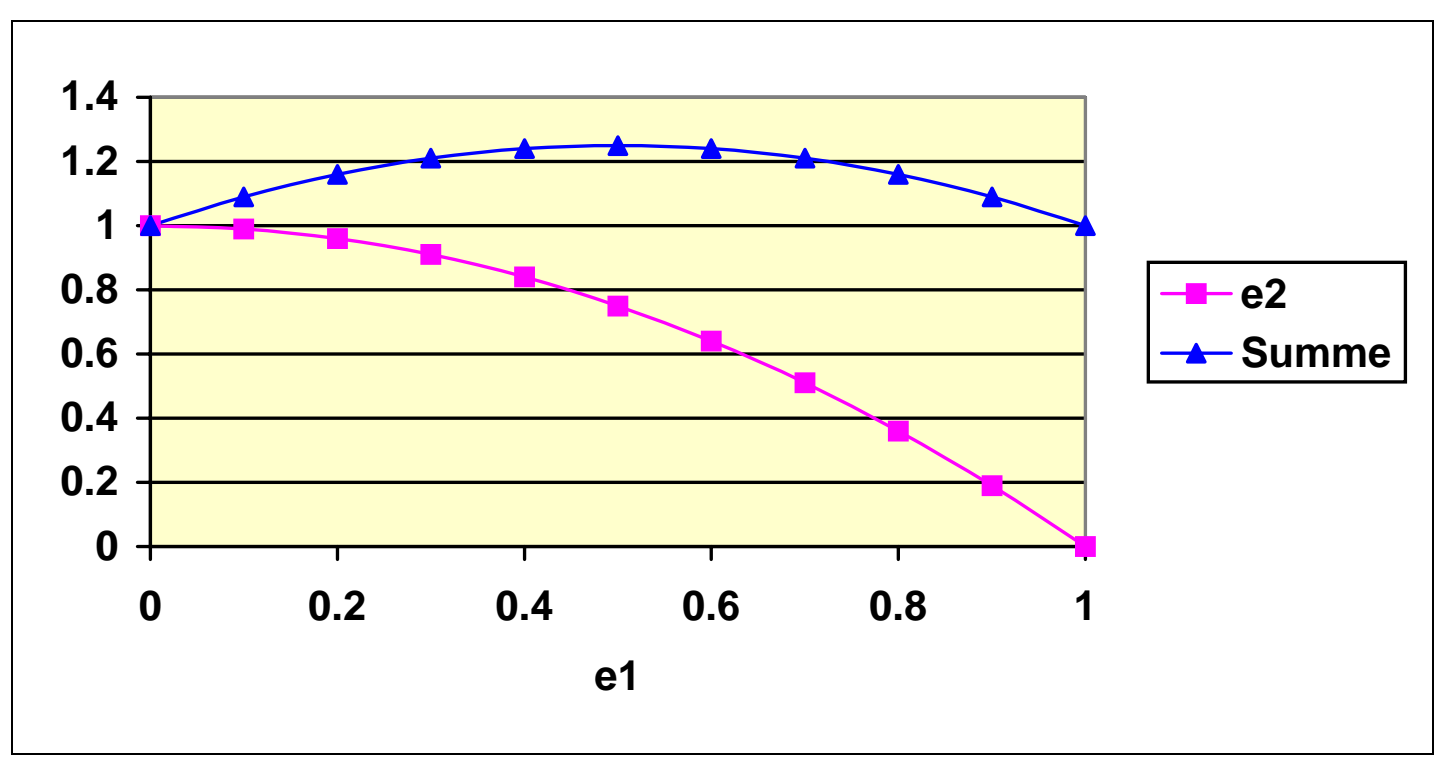




\subsubsection{Einfluß der Abschreibungsmethode auf den LOHMANN-RUCHTI- Effekt}

Bei steigenden Reparaturen ist eine lineare Abschreibung nicht adäquat, wenn die Leistungsabgabe konstant ist, da wachsende Reparaturauszahlungen und konstante Abschreibungen nicht zu gleichbleibenden Kosten pro Periode führen. Stattdessen ist eine Abschreibungsmethode mit jährlich abnehmenden Beträgen zu verwenden. Wie wirkt sich eine solche degressive Abschreibung auf den LOHMANN-RUCHTI-Effekt aus?

Eine degressive Abschreibung ist so zu bemessen, daß die Summe aus Reparaturen und Abschreibung in jedem Jahr gleich ist, die Gewinne also, ohne Berücksichtigung von Erweiterungsinvestitionen, konstant verlaufen. Auf die Bestandsentwicklung hat die zeitliche Verteilung der Abschreibung keinen Einfluß, wenn in jeder Periode in Höhe der freien Mittel investiert wird. Diese reale Größe wird durch die Wahl der Bemessungsmethode für die (kalkulatorischen) Abschreibungen nicht beeinflußt. Dennoch wird in der Literatur häufig ein Zusammenhang zwischen Abschreibungsverlauf und LOHMANN-RUCHTI-Effekt gesehen (K. LOHMANN 1991, Kap. 3.1; HAX 1958, S. 253ff, ). Z.B. schreiben BUCHNER \& WEINREICH (1971, S. 466): „Das erzielbare Kapazitätswachstum beim »Marx-Engels-Effekt « ist nicht nur der Höhe, sondern auch der Schnelligkeit nach abhängig von der Verteilung der Abschreibungskoeffizienten."

Voraussetzung dieser Beziehung ist, daß sich die Investitionen an der Höhe der Abschreibungen bemessen. Bei degressiver Abschreibungsmethode sind diese kalkulatorischen Kosten c.p. höher als die Mittelzuflüsse, die durch den Verkauf der Produktion hereinkommen. Die im Vergleich zu linearer Abschreibung zusätzlichen Investitionen müssen daher c.p. außenfinanziert werden. K. LOHMANN und HAX nennen eine andere Möglichkeit: Die degressive Abschreibung wird bei der Preisgestaltung berücksichtigt und der Preis entsprechend heraufgesetzt, so daß ausreichend Mittel für die verstärkte Investitionstätigkeit zur Verfügung stehen.

Ohne Fremdfinanzierungs- und/oder Preissetzungsmöglichkeit verändert die Abschreibungsmethode nicht die Bestandsentwicklung, da die Abschreibung als kalkulatorische Größe nicht den realen Mittelzufluß verändert. Daher ist in 521, der Bestimmungsgleichung für den Bestand, die Abschreibung nicht enthalten. Beeinflußt wird durch die Abschreibungsmethode nur der Gewinnverlauf. Im Jahr 3 stehen die im Jahr 1 aus dem Startkapital erworbenen Anlagen zum Ersatz an. Daher ist das Investitionsvolumen in dieser Periode höher (s. Schaubild 621 und der Anlagebestand im Durchschnitt jünger als im Jahr davor. Bei degressiver Abschreibung werden neuere Anlagen stärker abgeschrieben als ältere. Folglich ist die Abschreibungssumme im dritten Jahr größer als im zweiten. Dieser Effekt verstärkt den Gewinneinbruch in der dritten Periode. Die Summe der Gewinne über den Planungszeitraum bleibt hingegen gleich, da die Kapazitätsentwicklung nicht verändert wird. 


\section{Schaubild 62.1: Investitionsentwicklung}

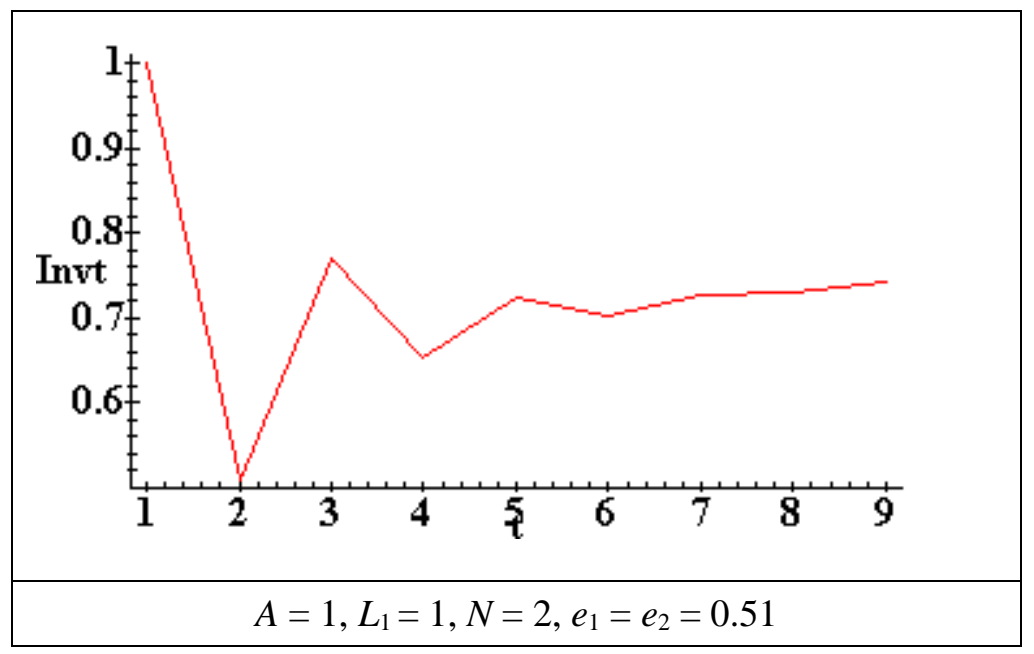

$\mathrm{Zu}$ deutlich größeren Gewinnausschlägen führt es in den ersten Perioden, wenn trotz konstanter Reparaturen degressiv abgeschrieben wird, z.B. aus steuerlichen Gründen. Auf lange Sicht unterscheiden sich die Gewinnverläufe nicht. Bleibt der Preis für die Produktion unverändert und wird nicht fremdfinanziert, verändert sich die Bestandsentwicklung im Vergleich zu linearer Abschreibung nicht (vgl. Schaubild 62.2 und 54.1.

\section{Schaubild 62.2: Gewinnverlauf bei degressiver und linearer Abschreibung}

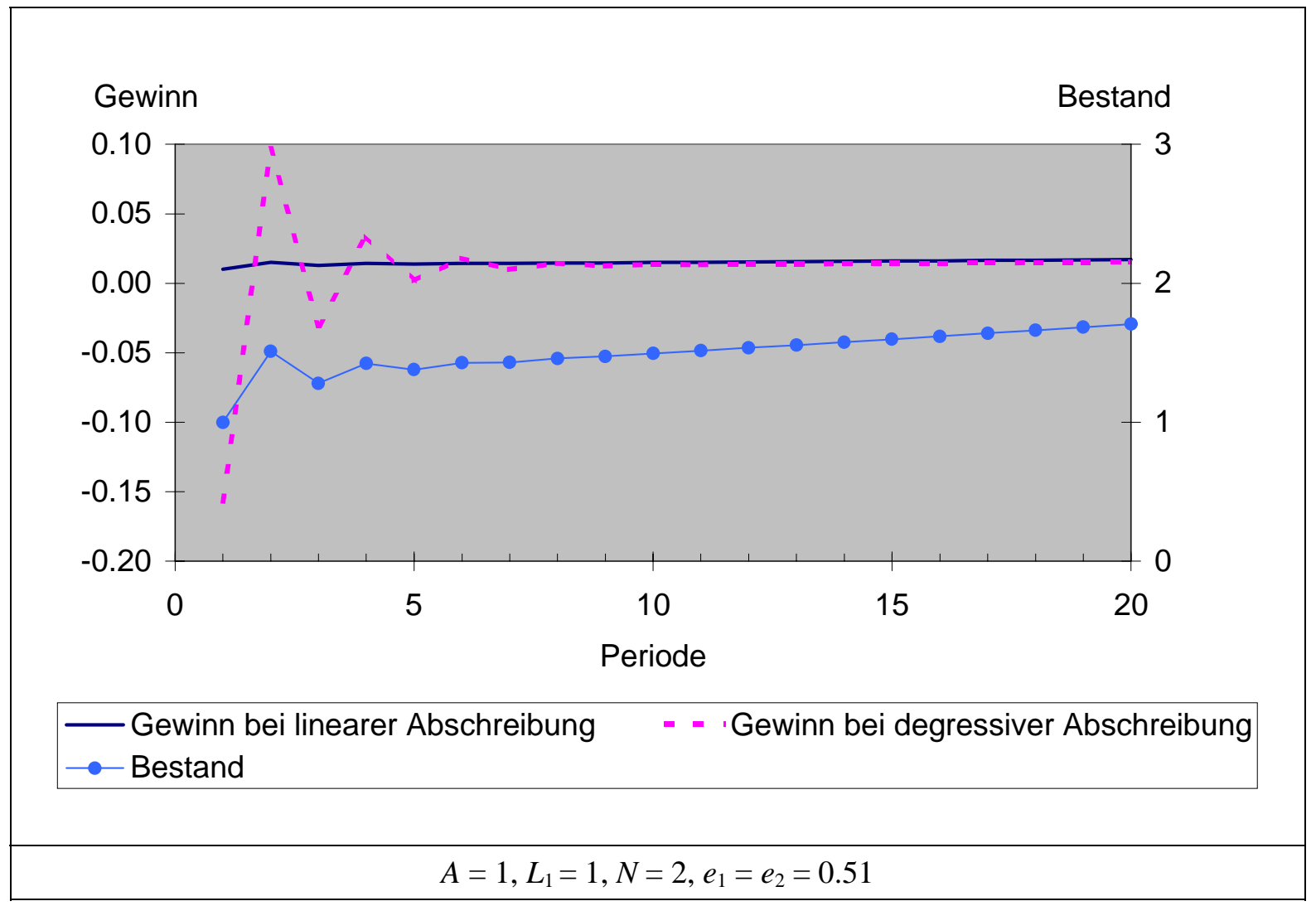


Der Kapazitätseffekt ist in seinem Kern, als Kapazitätserweiterung ohne zusätzlichen Kapitaleinsatz, wird also nicht durch die Abschreibungsmethode beeinflußt, da die von HAX und K. LOHMANN angeführten Veränderungen voraussetzen, daß den Unternehmen zusätzliches Kapital zufließt, als Fremdkapital oder über höhere Preise.

Die Einzahlungs-Auszahlungs-Differenzen können nicht nur durch steigende Reparaturen, sondern auch durch abnehmende Leistungsabgabe des Investitionsgutes geschmälert werden. Diesem Umstand ist in einer verfeinerten ex-ante-Analyse durch eine stärker degressive Abschreibung Rechnung zu tragen. Daher wirkt sich degressive Leistungsabgabe prinzipiell nicht anders als steigende Reparaturen aus (HAX 1958, S. 536) und wird daher nicht separat behandelt. Auch in der Praxis wird oft durch besonders intensive Wartung und vorsorgliche Reparaturen einem Leistungsabfall vorgebeugt.

Gibt man allerdings den Grundsatz konstanter Kosten während der Nutzungsdauer auf, wird am wirtschaftlichen Nutzungsende einer Anlage kaum noch ein Grenzgewinn erzielt, so daß bei Wegfall dieser Anlage auch kein Gewinneinbruch erfolgen kann. Aber ist es wenig sinnvoll, von im Laufe der Nutzungsdauer steigenden Kosten auszugehen:

Wenn eine Anlage im letzten Jahr ihrer Nutzungsdauer nur noch einen unterdurchschnittlichen Gewinn erbringt, dann wäre sie besser vorher ersetzt worden; die wirtschaftlich optimale Nutzungsdauer wurde also in diesem Fall falsch berechnet.

Konstante Kosten implizieren, daß bei einem Verkauf der Anlage zu einem beliebigen Zeitpunkt erster und zweiter Eigentümer mit gleichen Kosten arbeiten. Ein ansteigender Kostenverlauf hingegen bedeutet selbstverständlich, daß der zweite Eigentümer höhere Kosten zu tragen hat, mithin durch den Kauf einer neuen Maschine seine Kosten verringern könnte. Daher sind Zeitwerte, die sich auf der Grundlage steigender Kosten berechnet werden, für einen Zweitnutzer als Kaufpreis im allgemeinen uninteressant.

Die bisherigen Ergebnisse für eine Nutzungsdauer von zwei Perioden lassen sich wie folgt zusammenfassen:

- In einem streng deterministischen Modell können lokale Gewinnminima auftreten. Sie werden durch entsprechende Minima in der Kapazitätsentwicklung verursacht und kommen nur in ungeraden Perioden vor.

- Vorkommen und Umfang der Gewinnminima sind nicht vom Startkapital abhängig.

- Reparaturen, insbesondere fallende, und degressive Abschreibung verstärken die Gewinnminima.

- Die Gewinnminima nehmen mit der Zeit ab und verschwinden ab einem bestimmten Zeitpunkt vollständig. 
- Mit steigendem Stückgewinn werden die Minima kleiner und verschwinden im Zeitablauf früher. Ab einem kritischen Wert tritt auch in der dritten Periode kein Gewinneinbruch mehr auf.

Für eine Nutzungsdauer von mehr als zwei potenziert sich der mathematische Aufwand, Differenzengleichungen in eine geschlossene Form zu überführen. Wie 48.4 gezeigt hat, führt eine verlängerte Nutzungsdauer zu einem Gleichungssystem höherer Ordnung, das analytisch kaum zu handhaben ist. Aus diesem Grund werden im folgenden die Eigenschaften des Modells für längere Nutzungsdauern mit Hilfe von periodischen Regressionslösungen untersucht, die mit Hilfe von Simulation sehr einfach bestimmt werden können. Diese Technik bietet den zusätzlichen Vorteil, auch für ganzzahlige Investitionen geeignet zu sein.

\subsubsection{Besonderheiten für ganzzahlige Investitionen und längere Nutzungsdauern}

Die für das Schaubild 541 verwendeten Parameter $A=1, L_{1}=1, N=2, e=0.51$ führen zu Anlagebeständen, die in ersten Perioden immer unter 2 bleiben. Daher wird in diesen Jahren stets weniger als eine Einheit investiert. Wenn Investitionsgüter nur in ganzen Einheiten zugekauft werden können, sind daher auf mittlere Sicht keine Erweiterungsinvestitionen durchführbar und der Bestand bliebe konstant. Daher wird das Startkapital auf vier erhöht. Gleichzeitig wird die Nutzungsdauer auf vier Perioden verlängert, weil sich bei diesen Parametern die Effekte am einfachsten zeigen lassen. Die Parameter sind in Tabelle 641 dargestellt. Sie werden später die Parameter variiert.

Tabelle 64.1: Parameter des ganzzahligen Referenzmodells

\begin{tabular}{|c|c|c|}
\hline Variable & Kürzel & Wert \\
\hline liquide Mittel am Periodenanfang & $L_{1}$ & 4 \\
Anschaffungspreis & $A$ & 1 \\
Nutzungsdauer & $N$ & 4 \\
Einzahlung & $e$ & 0.26 \\
\hline
\end{tabular}

Die Entwicklung der wichtigsten Variablen für die ersten 9 Perioden ist in Tabelle 65.1 berechnet. Der Zeitpfad des Gewinns verläuft, wie im stetigen Beispiel, nicht monoton steigend, sondern weist lokale Minima auf. Die Einzahlung der Investition(en) reicht aus, um in jeder Periode eine neue Anlage zu erwerben. Dadurch steigen bis einschließlich Periode 4 Kapazität und Gewinn kontinuierlich an. In Periode 5 müssen die zu Beginn erworbenen vier Anlagen ersetzt werden. Die liquiden Mittel reichen aber nur für den Ankauf von zwei Anlagen. Zusammen mit den in den Perioden 2 bis 3 angeschafften beträgt die Kapazität nun fünf Anlagen. Dies sind zwei weniger als in der Vorperiode; folgerichtig sinkt der Gewinn von 0.07 auf 0.05. 
Tabelle 65.1: Zeitpfade für ein ganzzahliges Modell

\begin{tabular}{|c|c|c|c|c|c|c|c|}
\hline $\begin{array}{c}\text { Perio- } \\
\text { de }\end{array}$ & $\begin{array}{c}\text { liquide Mittel } \\
\text { am Anfang } \\
L_{t}\end{array}$ & $\begin{array}{c}\text { Investi- } \\
\text { tionen } \\
I_{t}\end{array}$ & $\begin{array}{c}\text { Bestand nach } \\
\text { Investitionen } \\
B_{t}\end{array}$ & $\begin{array}{c}\text { liquide Mittel } \\
\text { nach Invest. } \\
L_{t}-I_{t} \cdot A\end{array}$ & $\begin{array}{c}\text { Einzah- } \\
\text { lungen } \\
E_{t}\end{array}$ & $\begin{array}{c}\text { Abschrei- } \\
\text { bung } \\
A b_{t}\end{array}$ & $\begin{array}{c}\text { Gewinn } \\
G_{t}\end{array}$ \\
\hline 1 & 4.00 & 4 & 4 & 0.00 & 1.04 & 1.00 & 0.04 \\
2 & 1.04 & 1 & 5 & 0.04 & 1.30 & 1.25 & 0.05 \\
3 & 1.34 & 1 & 6 & 0.34 & 1.56 & 1.50 & 0.06 \\
4 & 1.90 & 1 & 7 & 0.90 & 1.82 & 1.75 & 0.07 \\
5 & 2.72 & 2 & 5 & 0.72 & 1.30 & 1.25 & $\mathbf{0 . 0 5}$ \\
6 & 2.02 & 2 & 6 & 0.02 & 1.56 & 1.50 & 0.06 \\
7 & 1.58 & 1 & 6 & 0.58 & 1.56 & 1.50 & 0.06 \\
8 & 2.14 & 2 & 7 & 0.14 & 1.82 & 1.75 & 0.07 \\
9 & 1.96 & 1 & 6 & 0.96 & 1.56 & 1.50 & 0.06 \\
\hline \multicolumn{7}{|l}{} \\
\hline
\end{tabular}

Dieser Gewinneinbruch nach Ende der vierjährigen Nutzungsdauer ist in Periode 9 erneut zu beobachten. Auch in dieser Periode stehen mehr Anlagen zum Ersatz an als liquide Mittel vorhanden sind. Der Kapazitäts- und damit Gewinnrückgang ist aber geringer als nach Ende der ersten Nutzungsdauer. Kann daher vermutet werden, daß auf lange Sicht eine monotone Gewinnentwicklung eintritt? Schaubild 66, 1 zeigt den Zeitpfad des Gewinns über einen längeren und einen sehr langen Horizont.

Es wird deutlich, daß der Gewinnrückgang in der fünften Periode zwar der größte, aber keineswegs der einzige ist. Die Gewinne verlaufen auch auf lange Sicht sehr unregelmäßig und im Trend steigend. Der Gewinn aus der vierten Periode z.B. wird erst in der zwanzigsten (!) Periode überschritten. Das letzte lokale Minimum tritt in Periode 78 auf. Später sind die über die Nutzungsdauer von vier Perioden kumulierten Gewinne ausreichend hoch, so daß die aus Gewinnen finanzierten Investitionen den unregelmäßigen Ersatzinvestitionsbedarf in jeder Periode zumindest ausgleichen.

Der besonders starke Gewinnrückgang in der fünften Periode (s.o.) ist darauf zurückzuführen, daß in dieser Periode die mit dem vorhandenen Startkapital erworbenen Anlagen nicht mehr zur Verfügung stehen. Kann daher vielleicht eine Minimierung des Startkapitals auf $L_{1}=A$ eine nicht-monotone Gewinnentwicklung ausschlieBen?

Es wurde bereits oben darauf hingewiesen, daß bei einem Startkapital von Eins der Bestand über lange Zeit konstant bleibt. Die kumulierten Einzahlungen reichen nicht aus, um aus ihnen vor Ablauf der Nutzungsdauer eine zweite Anlage zu finanzieren. Auf lange Sicht alternieren die Gewinne, wie Schaubild 671 zeigt. In Periode 28 übersteigen die kumulierten Gewinne zusammen mit der Abschreibung aus den vergangenen drei Perioden erstmals den Anschaffungspreis einer Anlage, so daß in dieser Periode die Kapazität auf zwei gesteigert werden kann. Am Ende der Periode fällt die 
schon vorhandene Anlage wieder aus und damit die Kapazität auf 1 zurück. Ab Periode 46 reicht das Vermögen aus, um dauerhaft zwei Anlagen finanzieren zu können.

\section{Schaubild 66.1: Zeitpfade des Gewinns}

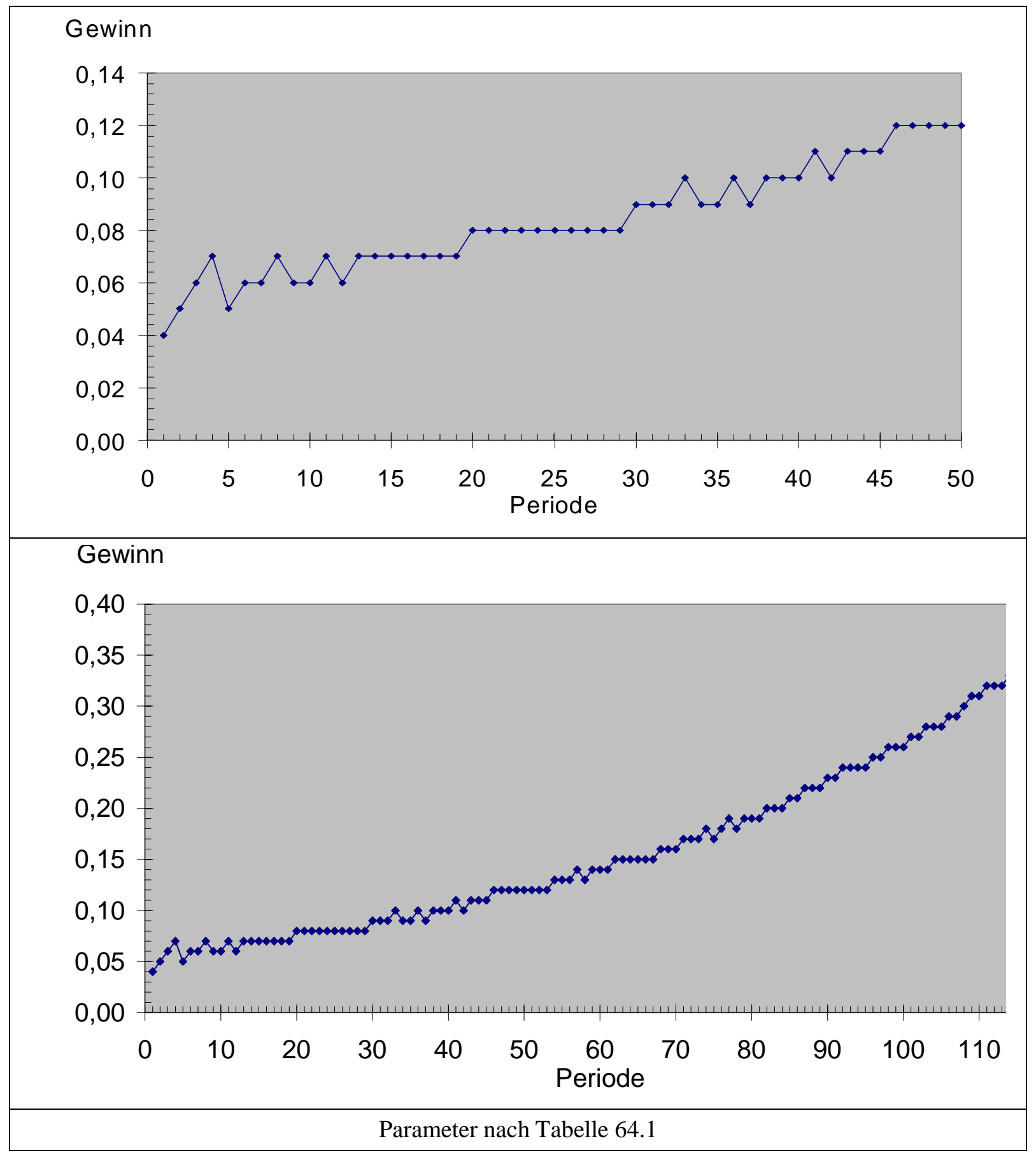

Als nächstes wird die Nutzungsdauer variiert. Für die bisherigen Szenarien betrug sie einheitlich 4. Es liegt auf der Hand, daß bei einer einperiodischen Nutzungsdauer keine Gewinneinbrüche auftreten können. Bei höheren Nutzungsdauern nimmt der Gewinn stets in der Periode $N+1$ ab, wie Schaubild 672 für zwei- und acht Jahre illustriert. Das höhere langfristige Gewinniveau erklärt sich durch die geringeren $\mathrm{Ab}$ - 
schreibungen bei verlängerter Nutzungsdauer. Umgekehrt erlauben die geringeren Abschreibungen in den ersten Perioden nur einen langsameren Bestandsaufbau.

Schaubild 67.1: Gewinnpfad bei minimalem Startkapital

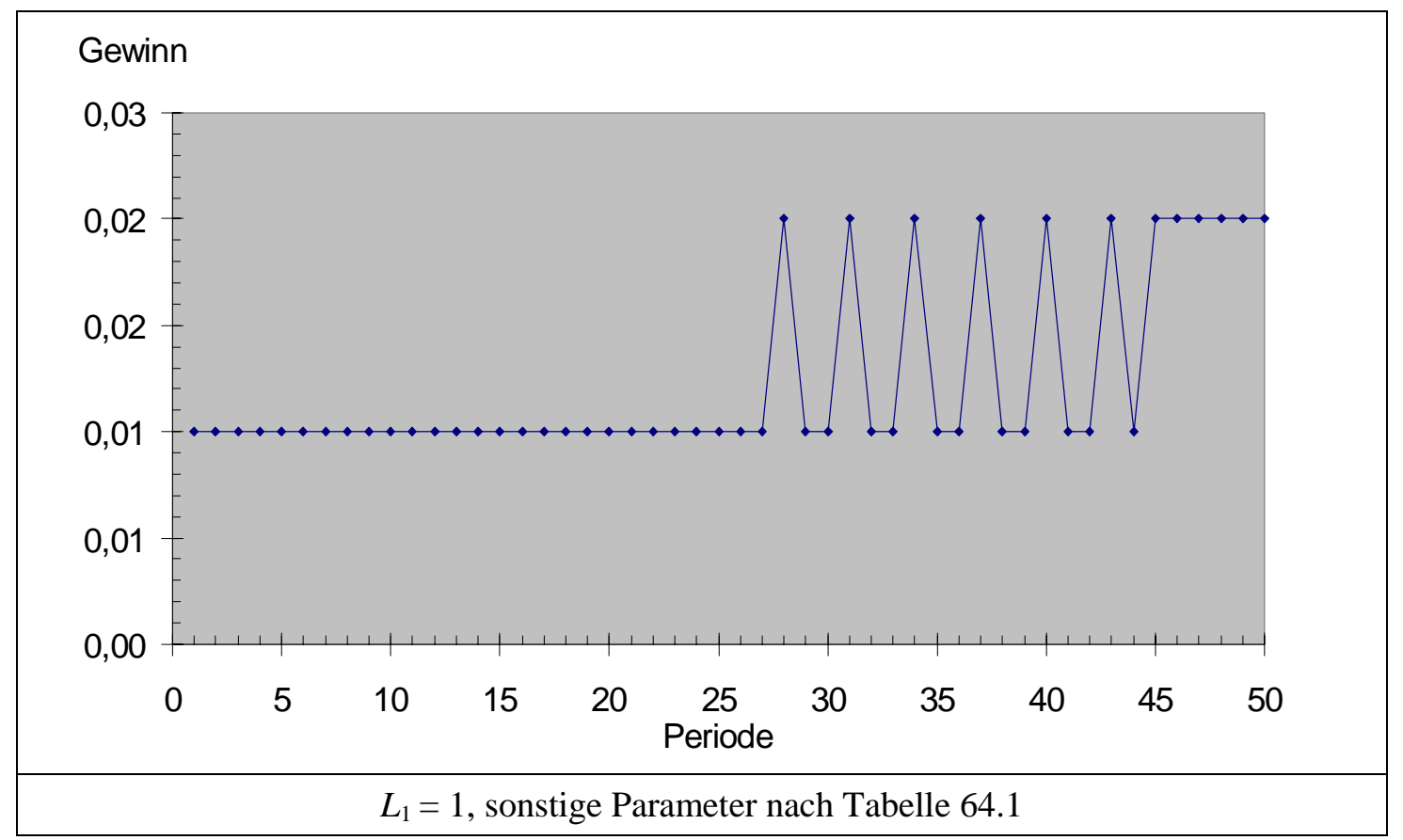

Schaubild 67.2: Gewinnpfad bei minimalem Startkapital, Nicht-Ganzzahligkeit und Nutzungsdauern von zwei und acht Perioden

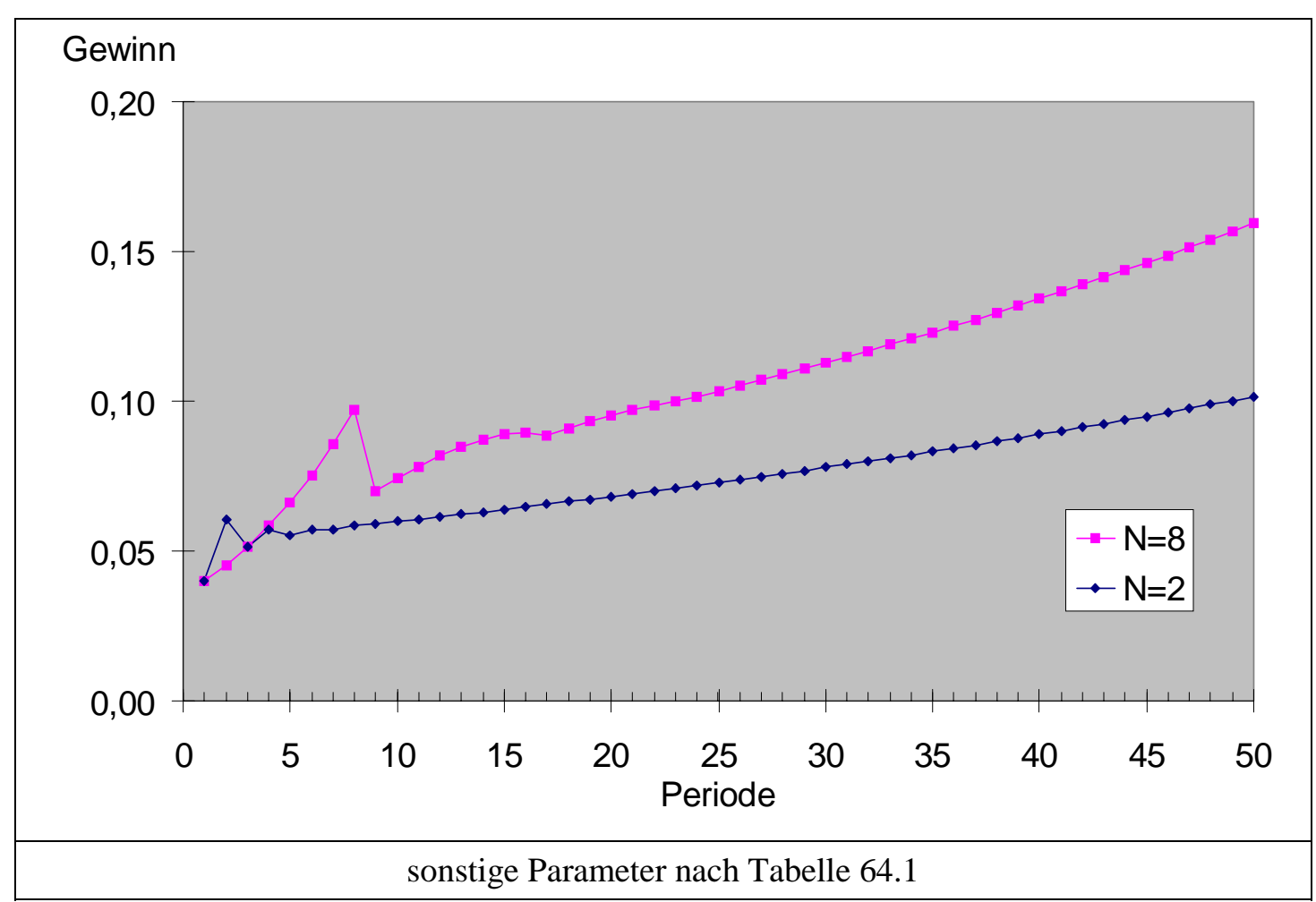


Von den Parametern der Tabelle 64.1 haben wir bislang das Startkapital, den Anschaffungspreis und die Nutzungsdauer variiert und gesehen, daß stets nach $N$ Perioden der Gewinn unter seinen Vorperiodenwert fällt. Erhöhen wir nun c.p. die Einzahlung und damit verbunden den Gewinn, so ist qualitativ klar, daß bei sehr hohen Gewinnen in einem Umfange Investitionen möglich werden, daß der kapazitätsreduzierende Effekt des Ausfalls der in der Startperiode erworbenen Anlagen überkompensiert wird. Für $N=2, L=A=1$ konnte gezeigt werden, daß ab einem Stückgewinn von 0.118 keine lokalen Gewinnminima mehr vorhanden sind (vgl. Tabelle 55.1] ein kritischer Wert für $e / A$ von 0.668 bedeutet bei einem Anschaffungspreis von Eins und Abschreibungen von $1 / 2$ einen Gewinn von $G=e-A=0.668-0.5=0.118$ ).

Bei $N=8$ sind ab 0.107 keine Gewinneinbußen feststellbar und bei $N=16$ liegt der kritische Gewinnwert bei 0.0775 . Je größer die Nutzungsdauer ist, desto kleiner müssen also die Gewinne sein, damit nach Ende der ersten Nutzungsdauer ein Gewinnrückgang zu beobachten ist. Entscheidend ist, daß die kumulierten Gewinne nicht ausreichen dürfen, um die in Periode $N+1$ fällig werdenden Ersatzinvestitionen auszugleichen. Da bei einer höheren Nutzungsdauer mehr Summanden addiert werden, müssen diese kleiner sein als bei einer niedrigeren Nutzungsdauer.

\section{Schaubild 68.1: Gewinnpfade bei ganzzahligen und stetigen Investitionsmöglichkeiten}

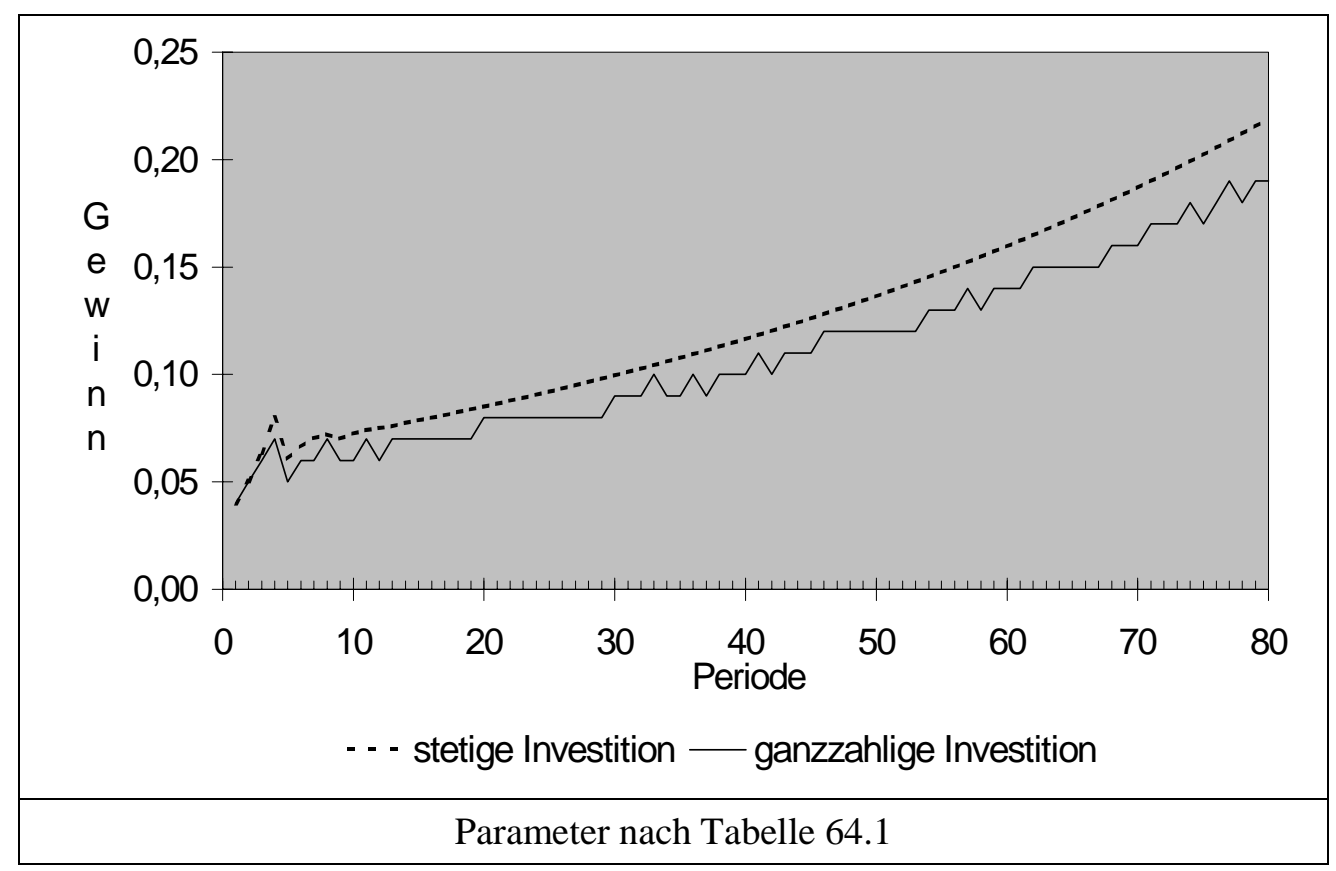

Abschließend seien ganzzahliges und stetiges Investitionsmodell verglichen. Das Ergebnis für den Gewinn ist in Schaubild 68,1 dargestellt. Die Beschränkung auf ganzzahlige Investitionsvolumina ist eine zusätzliche Restriktion und verringert daher in jeder Periode den Gewinn. Der ganzzahlige Pfad ist sehr viel unregelmäßiger als der stetige. Das letzte lokale Minimum tritt, wie erwähnt, in Periode 78 auf, während bei 
unbeschränkten Investitionsmöglichkeiten nur zwei Minima in den Perioden 5 und 9 $\mathrm{zu}$ verzeichnen sind.

Die Bestandsentwicklung in einer Konstellation ohne Gewinne wird durch Ganzzahligkeit sehr gedämpft, wenn das Startkapital verglichen mit dem Anschaffungspreis gering ist. Für die Parameter $e=1 / 2 A, A=L_{1}=1, N=2$ konvergiert der Bestand bei unbeschränkten Investitionsmöglichkeiten gegen 4/3. Da ein Bestand in dieser Größe bei nur ganzzahligen Investitionen nicht möglich ist, verharrt der Bestand in diesem Fall bei Eins. Ist das Startkapital hingegen ausreichend groß, stellt sich auch bei Ganzzahligkeit ein langfristiger Bestand von $\frac{4}{3} \frac{L_{1}}{A}$ ein, wie periodische Regressionslösungen mit verschiedenen Werten für das Startkapital zeigen. Daher ist für eine gewinnlose Situation und zweijährige Nutzungsdauer der Zusammenhang

$$
\lim _{t \rightarrow \infty} B_{t} \quad=\text { floor }\left(\frac{4}{3} \frac{L_{1}}{A}\right)
$$

zu vermuten. Diese Beziehung wird analytisch nicht bewiesen, sondern akzeptiert, da ihr kein Ergebnis einer durchgeführten periodischen Regression für vorgegebene Parameterwerte widersprach. (Da die Anzahl möglicher Parameterkombinationen unendlich ist, liefert dieses Vorgehen keinen allgemeingültigen Beweis.)

Mit dem Hilfsmittel der periodischen Regression kann auch der Grenzwert des Bestandes für größere Nutzungsdauern $(N)$ bestimmt werden. Die Ergebnisse der Tabelle 69.1 lassen vermuten, daß zwischen Nutzungsdauer und Grenzwert des Bestandes die Beziehung

$$
B(N) \quad=2-\frac{2}{N+1}
$$

für $A=L_{1}=1$ besteht, da zwischen den Ergebnissen dieser Formel und dem Bestand in der 100. bzw. 500. Periode kein Unterschied besteht.

Tabelle 69.1: Grenzwerte des Bestandes für stetige Investitionen

\begin{tabular}{|c||c|c|c|c|c|c|c|c|c|}
\hline $\boldsymbol{N}$ & 2 & 3 & 4 & 5 & 6 & 7 & 8 & 9 & 10 \\
\hline $\boldsymbol{B}_{\mathbf{1 0 0}}$ & 1.33 & 1.5 & 1.6 & $12 / 3$ & 1.71 & $13 / 4$ & 1.78 & 1.8 & 1.82 \\
\hline $\mathbf{2}-\frac{\mathbf{2}}{\boldsymbol{N + 1}}$ & 1.33 & 1.50 & 1.60 & 1.67 & 1.71 & 1.75 & 1.78 & 1.80 & 1.82 \\
\hline $\boldsymbol{N}$ & 12 & 15 & 20 & 25 & 35 & 50 & 75 & 100 & 200 \\
\hline $\boldsymbol{B}_{\mathbf{5 0 0}}$ & 1.846 & 1.875 & 1.905 & 1.923 & 1.944 & 1.961 & 1.974 & 1.980 & 1.999 \\
\hline $\mathbf{2}-\frac{\mathbf{2}}{\boldsymbol{N}+\mathbf{1}}$ & 1.846 & 1.875 & 1.905 & 1.923 & 1.944 & 1.961 & 1.974 & 1.980 & 1.990 \\
\hline \multicolumn{8}{|c|}{$e=1 / 2 A, A=L_{1}=1$, Bestand in der 100. bzw. 500. Periode } \\
\hline
\end{tabular}

Weitere Regressionsrechnungen, die in Tabelle 701 dargestellt sind, unterstützen die Vermutung, daß 69.1 für beliebige Werte von Startkapital und Anschaffungspreis auf 


$$
B(N) \quad=\left(2-\frac{2}{N+1}\right) \frac{L_{1}}{A}
$$

erweitert werden kann.

Tabelle 70.1: Grenzwerte des Bestandes für stetige Investitionen und zwei Startkapitale

\begin{tabular}{|c||c|c|c|c|c|c|c|c|c|}
\hline $\boldsymbol{N}$ & 2 & 3 & 4 & 5 & 10 & 20 & 50 & 100 & 200 \\
\hline $\boldsymbol{L}_{\mathbf{1}}$ & 2 & 2 & 2 & 2 & 2 & 2 & 2 & 2 & 2 \\
\hline $\boldsymbol{B}_{\mathbf{5 0 0}}$ & 2.667 & 3 & 3.2 & $31 / 3$ & 3.636 & 3.810 & 3.922 & 3.961 & 3.997 \\
\hline$\left(\mathbf{2}-\frac{\mathbf{2}}{\boldsymbol{N}+\mathbf{1}}\right) \frac{\boldsymbol{L}_{\mathbf{1}}}{\boldsymbol{A}}$ & $2 \frac{2 / 3}{3}$ & 3 & 3.2 & $31 / 3$ & 3.636 & 3.810 & 3.922 & 3.960 & 3.980 \\
\hline \hline $\boldsymbol{N}$ & 2 & 3 & 4 & 5 & 10 & 20 & 50 & 100 & 200 \\
\hline $\boldsymbol{L}_{\mathbf{1}}$ & 4 & 4 & 4 & 4 & 4 & 4 & 4 & 4 & 4 \\
\hline $\boldsymbol{B}_{\mathbf{5 0 0}}$ & 5.333 & 6 & 6.4 & 6.667 & 7.273 & 7.619 & 7.843 & 7.921 & 7.995 \\
\hline$\left(\mathbf{2}-\frac{\mathbf{2}}{\boldsymbol{N + 1}}\right) \frac{\boldsymbol{L}_{\mathbf{1}}}{\boldsymbol{A}}$ & $51 / 3$ & 6 & 6.4 & 6.667 & 7.273 & 7.619 & 7.843 & 7.921 & 7.960 \\
\hline & \multicolumn{7}{|c|}{$e=1 / 2 A, A=1$} \\
\hline
\end{tabular}

\subsubsection{Der LOHMANN-RUCHTI-Effekt auf dem Finanzmarkt}

Der Kapazitätserweiterungseffekt läßt sich auch an Finanzpapieren zeigen, obwohl der zentrale Mechanismus, die Finanzierung zusätzlicher Kapazitäten aus zurückgelegten Abschreibungen, hier per se nicht möglich ist. Nehmen wir als einfaches Beispiel ein zweijähriges Wertpapier zu einem Preis $A$, das in jeder Periode eine Einzahlung von $e$ $=0.54 A$ erbringt. ${ }^{50}$ Das Papier sei nur in einer Stückelung von $A=1$ TDM erhältlich. Wir unterstellen weiterhin, daß die Investorin alle Rückflüsse unverzüglich wieder in diesem Papier anlegt, da der Opportunitätszinsfuß Null betrage. Dann ergeben sich die in Tabelle T.T. und Schaubild TI.Tdargestellten Zahlungsströme.

Auch hier tritt ein deutlicher Einzahlungsrückgang nach Ende der ersten "Nutzungsdauer" der Wertpapiere auf. Allerdings bleibt dieses lokale Minimum das einzige. In der Folge sind nur noch Stagnationsphasen erkennbar; ab Periode 43 steigen die Einzahlungen streng monoton an.

50 Die Summe der "Rückzahlungen" beträgt also 1,08 A. 
Tabelle 71.1: Einzahlungsverlauf eines Wertpapiers

\begin{tabular}{|c|c|c|c|c|c|}
\hline $\begin{array}{c}\text { Peri- } \\
\text { ode }\end{array}$ & $\begin{array}{c}\text { liquide Mittel } \\
\text { am Anfang } \\
L_{t}\end{array}$ & $\begin{array}{c}\text { Anzahl zuge- } \\
\text { kaufter Papiere } \\
I_{t}\end{array}$ & $\begin{array}{c}\text { Bestand } \\
\text { nach Zukauf } \\
B_{t}\end{array}$ & $\begin{array}{c}\text { liquide Mittel } \\
\text { nach Zukauf } \\
L_{t}-I_{t} \cdot A\end{array}$ & $\begin{array}{c}\text { Einzah- } \\
\text { lungen } \\
E_{t}\end{array}$ \\
\hline \hline 1 & 2.00 & 2 & 2 & 0.00 & 1.08 \\
2 & 1.08 & 1 & 3 & 0.08 & 1.62 \\
3 & 1.70 & 1 & 2 & 0.70 & 1.08 \\
4 & 1.78 & 1 & 2 & 0.78 & 1.08 \\
5 & 1.86 & 1 & 2 & 0.86 & 1.08 \\
6 & 1.94 & 1 & 2 & 0.94 & 1.08 \\
7 & 2.02 & 2 & 3 & 0.02 & 1.62 \\
\hline
\end{tabular}

\section{Schaubild 71.1: Einzahlungsverlauf eines Wertpapiers (in TDM) mit} zweijähriger Laufzeit

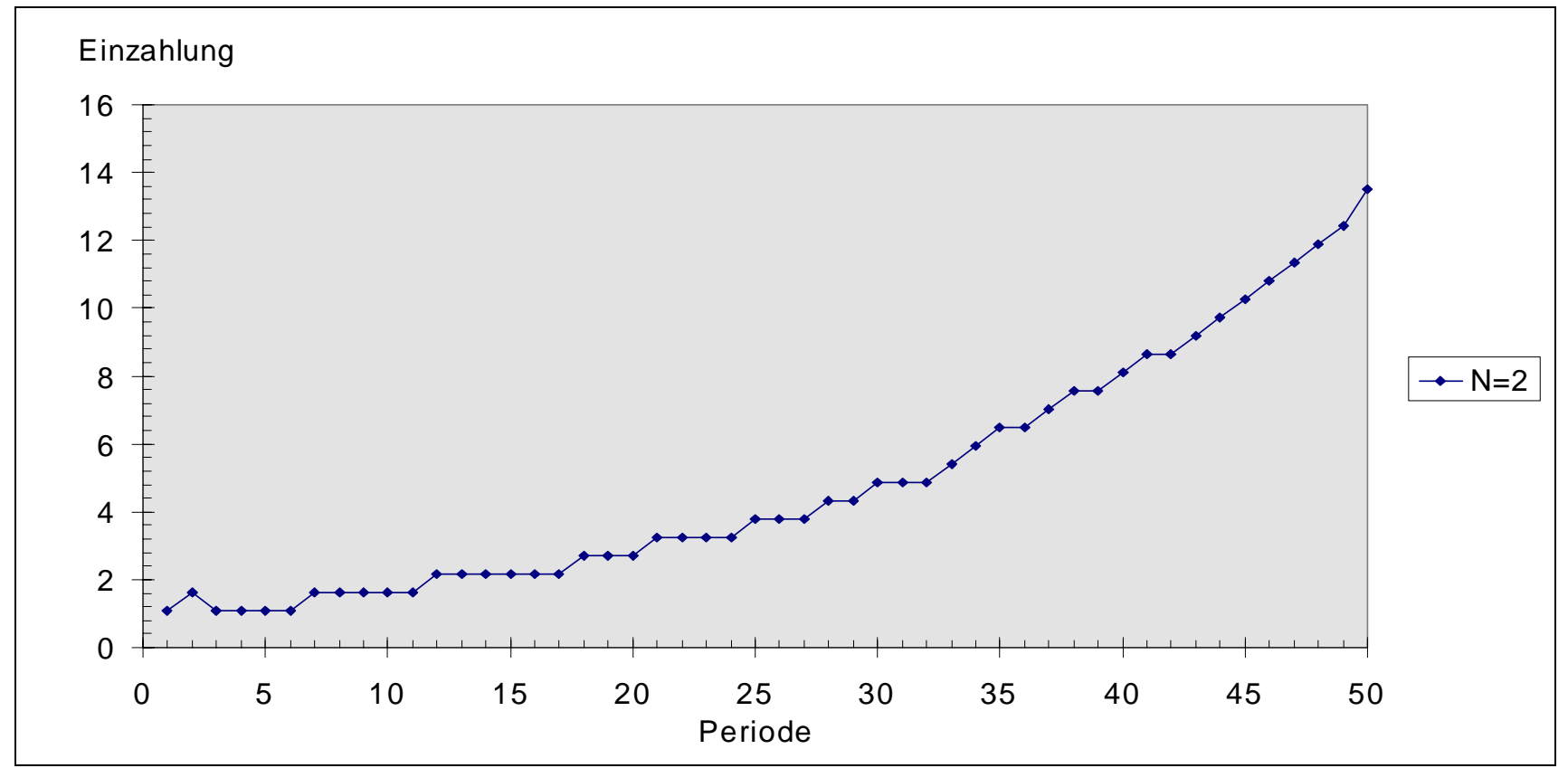

\subsubsection{Zur Bedeutung des Kapitalmarktes}

Der LOHMANN-RUCHTI-Effekt wird besonders in Zeiten mit unvollkommenen Kapitalmärkten als bedeutsam angesehen (K. LOHMANN 1991, S. 222). Daher stammen viele Veröffentlichungen aus den Jahren um den Zweiten Weltkrieg. ${ }^{51}$ In dem vorgestellten

51 Vgl. RUCHTI (1942), M. LOHMANN (1949). 
Modell wurde ebenfalls das Fehlen eines Kapitalmarktes unterstellt. Diese Voraussetzung ist jedoch nicht zwingend, sie wurde nur der Einfachheit halber eingeführt.

Ist ein Kapitalmarkt vorhanden, dann können freiwerdende Mittel zinsbringend angelegt werden, anstatt sie unmittelbar zu reinvestieren. Dies ist dann sinnvoll, wenn der Habenzins größer ist, als die erzielbare interne Verzinsung der Investition (IRR). Ist diese Bedingung erfüllt, dann war jedoch bereits die erste Investition im Vergleich zu einer Kapitalanlage ökonomisch falsch. Es wird daher nur investiert, wenn die interne Verzinsung der Investition die Opportunitätskosten des Kapitals übersteigt.

Ist eine Investition darüber hinaus so rentabel, daß der IRR oberhalb des Sollzinses liegt, besteht kein Anlaß, die Investition auf die Höhe der Abschreibung zu begrenzen, vielmehr gibt es theoretisch bereits in der ersten Periode keine obere Grenze für die erreichbare Kapazität. In der Praxis führen begrenzte Kreditverfügbarkeit und/oder abnehmende Skalenerträge zu einer endlichen Anfangsinvestition und damit zu einem effektiven Sollzinsfuß oberhalb des IRR der Investition. In den Folgeperioden sind daher nur Investitionen im Umfang der zugeflossenen Mittel möglich.

Abschließend kann festgehalten werden, daß die Existenz des LOHMANN-RUCHTIEffektes nicht von den Bedingungen auf dem Kapitalmarkt und der Finanzierung der Investition abhängt. ${ }^{52}$

\subsubsection{Zusammenfassung}

An einem kleinen mikroökonomischen Modell wurde gezeigt, daß in einer konstanten Umwelt mit vollständiger Information eine positive monotone Gewinnentwicklung über mehrere Perioden nicht ausschließt, daß dieses Gewinniveau in einer oder mehreren Folgeperioden verfehlt wird. Dies gilt sowohl für Anlage- wie für Finanzinvestitionen. Auch auf lange Sicht braucht kein monoton steigender Gewinnverlauf einzutreten. Ursache dieses Phänomens ist das Reinvestieren von Abschreibungen bzw. Rückflüssen im Verbund mit einer ausreichend kleinen Gewinnrate. Die Reinvestitionen führen zu einer unregelmäßigen Bestandsentwicklung. Diese ist abhängig von der (realen) Einzahlungs-Auszahlungs-Differenz und nicht von den (kalkulatorischen) Abschreibungen. Der Effekt ist nicht von der Abschreibungsmethode oder der Form der Finanzierung der Investition abhängig. Durch steigende Reparaturen wird er leicht abgeschwächt. Langfristig kann der Effekt vermieden werden, wenn auch beliebig teilbare Investitionen möglich sind. Ein Gewinneinbruch nach Ende der ersten Nutzungsdauer tritt aber in jedem Falle auf. Wenn keine Gewinne erzielt werden, konvergiert der Bestand. Der Grenzwert ist abhängig von der Nutzungsdauer. Bei sehr langer Nutzungsdauer wird der Bestand langfristig verdoppelt.

Der LOHMANN-RUCHTI-Effekt ist nicht nur von Interesse für Modellbauer. In der ostdeutschen Landwirtschaft ist in den vergangenen Jahren in kurzer Zeit sehr viel investiert worden. Daher ist es nicht unwahrscheinlich, daß diese Betriebe, auch bei

52 Zu dieser Auffassung kommen auch HARDACH \& HAX (1958, S. 533). 
gleichbleibenden Preisen, Gewinneinbrüche hinnehmen müssen. Diese wäre dann c.p. nicht auf Managementfehler zurückzuführen, im Gegenteil, die hohen Gewinne vor dem Einbruch wären das Verdienst guter Betriebsführung.

\subsection{Theoretische Ansätze und empirische Methoden zur Erklärung der Überlebensfähigkeit von Unternehmen}

Da sich diese Arbeit in erster Linie mit der Überlebenswahrscheinlichkeit von Unternehmen befaßt, werden in diesem Abschnitt zunächst die wichtigsten theoretischen Ansätze zur Erklärung des Überlebens von Unternehmen behandelt. Sie lassen sich einteilen in:

- organisationstheoretische Ansätze,

- industrieökonomische Ansätze und die wohl bedeutendsten

- evolutionsökonomischen Ansätze.

In jüngster Zeit werden vermehrt Elemente der Komplexitätsforschung (KOPEL \& FEICHTINGER 1995, NeumaIR 1998), der Chaostheorie (FeICHTINGER \& KoPEL 1994, KOPEL 1996) und der Katastrophentheorie (PINKWART 1993) auf Probleme der Unternehmensforschung übertragen. Dabei geht es in erster Linie um das Verhalten komplexer Systeme, die sich durch die Eigenschaften des Lebens, Sterbens und Entstehens auszeichnen. In diesem Kontext werden auch die Ursachen für das Scheitern von Unternehmen untersucht. ${ }^{53}$ Weitere theoretische Ansätze zur Analyse der Überlebensfähigkeit von Unternehmen stammen aus der Arbeitsmarkttheorie, bei der im wesentlichen das optimale Investitionsverhalten in Humankapital während eines Lebenszyklus den Gegenstand der Untersuchung bildet (BRÜDERL et al. 1996, S. 45f), und aus der Transaktionskostentheorie, die auf den Einfluß von Hierarchien und Vertragsbeziehungen abhebt (BRÜDERL et al. 1996, S. 55f). Hierauf wird im folgenden nicht weiter eingegangen.

Nach der Diskussion theoretische Erklärungsansätze schließt der Unterabschnitt 2.3 mit der Darstellung empirischer Methoden zur Beurteilung und Prognose der Existenzgefährdung von Unternehmen.

\subsubsection{Theoretische Ansätze}

\subsubsection{Organisationstheoretische Ansätze}

Die Organisationstheorie ist durch eine besonders große methodische Bandbreite charakterisiert und umfaßt sowohl Mikro- (entscheidungslogische und entscheidungsverhaltensorientierte Ansätze) als auch Makroansätze (systemtheoretische, situative und evolutionstheoretische Ansätze). Im Zusammenhang mit der Überlebensfähigkeit von

53 NeUMAIR (1998) untersucht mit Hilfe der Komplexitätsforschung die Ursachen für das Scheitern von Unternehmen. 
Organisationen wird deren strukturellen Merkmalen, wie beispielsweise dem Alter oder der Größe, ein wesentlicher Einfluß auf die Überlebenswahrscheinlichkeit beigemessen. Wichtige Determinante ist gemäß der Organisationstheorie auch das Ausmaß der Übereinstimmung zwischen Eigenschaften von Organisationen und den 'relevanten' Umweltbedingungen (vgl. WOYWODE 1998, S. 38ff und die dort angegebene Literatur). Die wichtigsten Ansätze der Organisationstheorie, die sich mit dem Überleben von Unternehmen befassen, sind die Organisationsökologie und random walk Modelle.

\subsection{Organisationsökologie}

Ausgangspunkt dieses Ansatzes ist die Annahme, daß die Prozesse des Wandels von Industrien den Prozessen des Wandels biologischer Populationen ähneln. BRÜDERL et al. (1996, S. 59) beschreiben den Begriff und die Zielsetzung der Organisationsökologie folgendermaßen: „,...Organisationsökologien [stellen] die Anpassungsfähigkeit bestehender Organisationen in Frage und betonen, daß der durch die Umwelt gesteuerte Wandel organisationaler Strukturen vor allem durch Mechanismen der Variation und Selektion vorangetrieben wird. Dies bedeutet, daß Prozesse der Neugründung von Organisationen, die für Variation sorgen, und Prozesse des Absterbens von Organisationen, die Selektionskräfte widerspiegeln, zu den beiden Basisprozessen der Analyse des Wandels organisationaler Strukturen avancieren. Das Ziel der Organisationsökologen besteht dabei nicht in erster Linie darin, das Schicksal einzelner Organisationen und deren Veränderungen nachzuvollziehen. Betrachtet werden vielmehr Populationen von Organisationen, deren Dynamik über die Zeit analysiert werden soll.“"

Aufgrund seiner Analogie zur Entwicklungsbiologie bezeichnet BURMESTER (1996, S. 83) diesen Ansatz als einen Spezialfall der evolutorischen Ökonomik. Aus der Vielzahl der organisationsökologischen Konzepte werden vier Theorien ausgewählt und erläutert, die sich mit der Überlebenswahrscheinlichkeit von Unternehmen befassen: (1) liability of smallness, (2) liability of newness, (3) theory of founding characteristics und (4) density dependence theory. Auf diese Konzepte wird in Abschnitt 6.4 dieser Arbeit im Zusammenhang mit den Ergebnissen einer Simulationsstudie Bezug genommen.

\section{(1) Liability of smallness ${ }^{54}$}

Im Mittelpunkt des Konzeptes der liability of smallness steht die Beziehung zwischen der Größe von Unternehmen zum Zeitpunkt ihrer Gründung und ihrer Überlebenswahrscheinlichkeit. Die These besagt, daß kleinere Unternehmen ceteris paribus eine geringere Überlebenswahrscheinlichkeit besitzen als größere Unternehmen gleichen Alters. Untermauert wird diese These durch die Auffassung, daß größere Unternehmen über eine stärkere Marktmacht und Größenvorteile verfügen, beispielsweise bei Kredit- oder Preisverhandlungen.

54 Näheres vgl. BRÜDERL 1996, S. 62ff. 
In internationalen empirischen Untersuchungen wird die liability of smallness in der Regel bestätigt (BRÜDERL et al. 1996, S. 118f). Arbeiten, die sich differenzierter mit dem Zusammenhang zwischen Unternehmensgröße und Überlebenswahrscheinlichkeit befassen (WHOLEY et al. 1992 und AMBURGEY et al. 1994, zit. n. WOYWODE 1998, S. 40) ergeben, daß die Insolvenzrate nicht von vornherein mit der Größe abnimmt, sondern zunächst ansteigt, ein Maximum erreicht und für große Unternehmen wieder monoton sinkt, wie dies in Schaubild 75.1 skizziert ist.

\section{Schaubild 75.1: $\quad$ Liability of smallness}

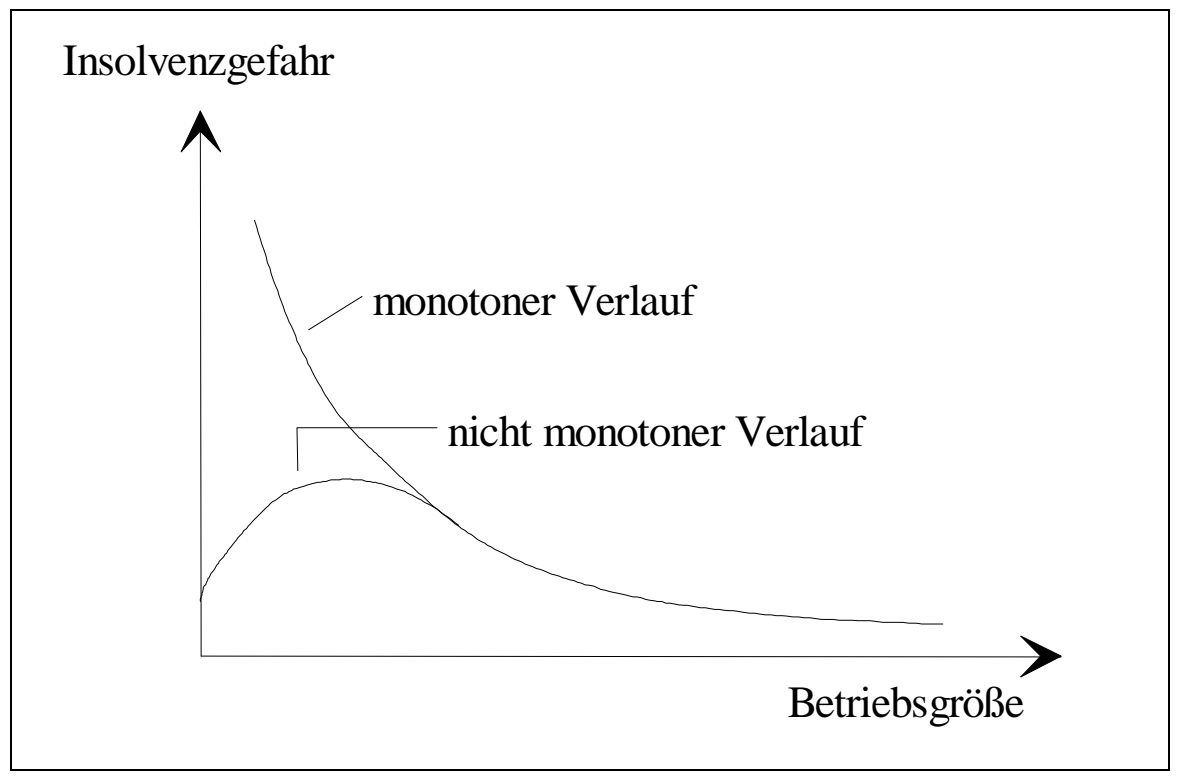

Die Autoren geben keine Begründung für diesen empirischen Befund. Vorstellbar ist, daß die geringe Ausscheiderate sehr kleiner Unternehmen aus der Fähigkeit, wirtschaftliche Schwierigkeiten mit Hilfe des Privatvermögens auszugleichen, resultiert. Bei mittleren Unternehmen ist dies nicht mehr möglich, daher steigt die Insolvenzrate an. Für große Betriebe treffen dann wieder die Argumente liability of smallness zu, so daß die Insolvenzgefahr zurückgeht.

\section{(2) Liability of newness}

Das auf STINCHCOMBE (1965, zit. in WoYwODE 1998, S. 40) zurückgehende Konzept der liability of newness befaßt sich mit der beobachtbaren Tendenz junger Unternehmen, häufiger aus dem Wettbewerb auszuscheiden als alte Unternehmen. Als Ursachen werden üblicherweise genannt (vgl. WOYWODE 1998, S. 40f und BURMESTER 1996, S. 85f):

- Junge Unternehmen sind aufgrund mangelnder Erfahrungen fehleranfälliger als alte Unternehmen.

- Junge Unternehmen müssen Beziehungen zu Marktpartnern aufbauen und können dabei auf verfestigte Strukturen stoßen. Dieses Problem wird bei BURMESTER (1996, S. 85) als 'Eintrittsschranken' bezeichnet. 
- Junge Unternehmen verfügen nicht über eine nachweisbare Zuverlässigkeit und Rechenschaftsfähigkeit.

- Der geringere Bekanntheitsgrad junger Unternehmen und ihrer Produkte erschwert den Absatz.

Die beiden letztgenannten Argumente können auf eine Risikoaversion der Marktpartner zurückgeführt werden. Lieferanten vermögen die Zahlungsfähigkeit junger Unternehmen weniger gut zu beurteilen und verlangen daher größere Sicherheiten. Abnehmer können die Qualität der Produkte schlechter einschätzen und besitzen daher eine geringere Zahlungsbereitschaft. Dies trifft insbesondere auf Produkte zu, deren relevante Eigenschaften nicht ohne weiteres zu beurteilen sind. Solche Eigenschaften werden in der informationsökonomischen Literatur als Erfahrungs- bzw. Vertrauenseigenschaften bezeichnet (WEIBER \& ADLER 1995 und die dort angegebene Literatur).

Ähnlich wie bei dem Konzept der liability of smallness ist auch in der liability of newness eine nicht-monotone Beziehung zwischen dem Alter eines Unternehmens und seiner Überlebenswahrscheinlichkeit möglich (vgl. Schaubild 761). WOYWODE (1998, S. 41f) gibt einen Überblick über jüngere Studien, die zeigen, daß in der unmittelbaren Anfangsphase eines Betriebes das Risiko des Scheiterns zunächst ansteigt und später wieder abfällt. Dieses Phänomen wird als liability of adolescence bezeichnet (BRÜDERL et al. 1996, S. 61). Die Hauptursache hierfür liegt im sogenannten Organisationskapital, über das Unternehmen nur in ihrer Anfangsphase verfügen. Dieses Kapital besteht einerseits aus finanziellen Ressourcen, andererseits aus sozialem Kapital in Form eines Vertrauensvorschusses interessierter Kunden und nahestehender Personen. Erfolglose Unternehmen scheitern, wenn dieses Kapital aufgebraucht ist.

Schaubild 76.1: Liability of newness und liability of adolescence

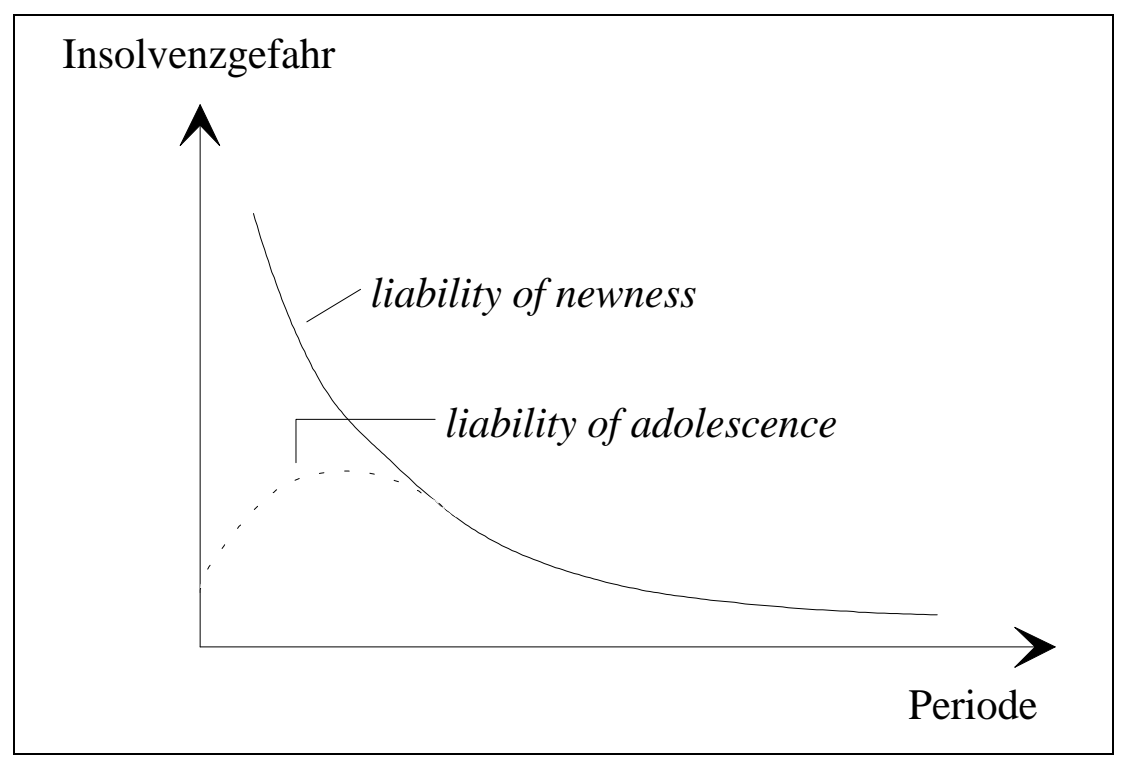




\section{(3) Theory of founding characteristics}

Das dritte Konzept der Organisationsökologie, die theory of founding characteristics befaßt sich mit dem Einfluß der Charakteristika einer Unternehmensgründung auf die Überlebenswahrscheinlichkeit des Unternehmens. Dieses Konzept geht ebenfalls auf STINCHCOMBE (1965, zit. nach WoYWODE 1998, S. 44f) zurück. Als Gründungscharakteristika werden sowohl interne Faktoren, wie z.B. Eigentümerstruktur, Kapitalstruktur und Diversifikationsgrad, als auch externe Einflüsse, wie z.B. Marktkonzentration oder Konjunktur zur Gründungszeit, betrachtet.

Die Theorie der founding characteristics basiert auf der Prämisse, daß die genannten internen und externen Faktoren einen Einfluß auf die Überlebenswahrscheinlichkeit besitzen, den es empirisch zu verifizieren gilt. Allerdings wird diesem Konzept vorgeworfen, nicht ausreichend theoretisch fundiert zu sein und lediglich ad-hoc Erklärungen für beobachtbare empirische Phänomene zu liefern (WOYwODE 1998, S. 45f).

\section{(4) Density dependence theory}

Die liability of newness läßt sich auch auf ganze Branchen übertragen. Eine neue Produktgattung wird von potentiellen Abnehmern kritisch betrachtet, da ihr Nutzen schwer eingeschätzt werden kann. Dieser Vorbehalt gilt verstärkt, wenn die Produkte nur von sehr wenigen Anbietern offeriert werden. Dadurch kann eine geringere Anzahl von Konkurrenten in einem neuen Marktsegment die Wirtschaftlichkeit und damit die Überlebensfähigkeit senken. Bestehen in der Kundschaft keine Vorbehalte gegen das neue Produkt, erhöht umgekehrt eine geringe Wettbewerbsintensität die betriebswirtschaftliche Rentabilität (Pioniergewinne) Die Abhängigkeit des wirtschaftlichen Erfolges von der Dichte vorhandener Mitbewerber führte zu der Bezeichnung dieses Ansatzes als density dependence theory (WOYWODE 1998, S. 46ff).

Ein aktuelles Beispiel sind die Anstrengungen der Firma Claas, einen neuartigen Ackerschlepper, einen Traktor mit Raupenfahrwerk, am Markt zu plazieren. Claas ist zur Zeit der einzige Anbieter dieser Innovation, und die Landwirte sind skeptisch, ob diese Maschine herkömmlicher Technik überlegen ist. Würde dieses Produkt auch von anderen Unternehmen hergestellt, könnte dies von Landwirten als Indiz gewertet werden, daß die neue Technik von anderen (kompetenten) Firmen ebenfalls für zukunftsträchtig gehalten wird, und ihre Vorbehalte verringern.

Nimmt die Anzahl der Anbieter zu, kann dies also die Absatzaussichten einer Branche verbessern. Gleichzeitig steigt die Wettbewerbsintensität und wirkt dem positiven Effekt eines höheren Bekanntheits- und Glaubwürdigkeitsgrades entgegen. Die Beziehungen zwischen Industriedichte ${ }^{55}$ und Scheiterrate sind daher wie bei den anderen liabilities nicht monoton. Am einfachsten läßt sich die Scheiterrate durch eine loglineare quadratische Funktion der Industriedichte darstellen. Vielversprechender

55 Als Industriedichte wird die Anzahl der Unternehmen verstanden, die in einer Industrie zu einem bestimmten Zeitpunkt existieren (WOYWODE 1998, S. 47). 
erscheint mir jedoch ein Ansatz zu sein, der neben der Dichte auch ihre Veränderung berücksichtigt. In schnell wachsenden Sektoren ist die Insolvenzgefahr vermutlich deutlich geringer als in schrumpfenden Wirtschaftsbereichen. ${ }^{56}$

Die empirischen Befunde zur density dependence theory sind uneinheitlich. WoYwode (1998, S. 47f) berichtet sowohl über Studien, die den Zusammenhang bestätigen (z.B. Untersuchungen zu deutschen und amerikanischen Brauereien, zu amerikanischen Telefongesellschaften und Gewerkschaften, sowie zu der amerikanischen Halbleiterindustrie) als auch von einer Analyse kalifornischer Weingüter, wo keine Beziehung festzustellen war.

\subsection{Random walk Modelle}

Zur Erklärung der Überlebenswahrscheinlichkeit von Unternehmen werden in der Literatur auch random walk Modelle herangezogen. In diesen dynamischen Modellen wird das Unternehmen auf einen Kapitalstock reduziert. Der Kapitalbestand wächst zum einen um einen konstanten Betrag $(w)$ und wird zum anderen von einem stochastischen Term $\left(z_{t}\right)$ mit Erwartungswert Null beeinflußt:

$$
K_{t} \quad=K_{t-1}+w+z_{t} .
$$

Ein solcher Prozeß wird als random walk mit Drift bezeichnet. ${ }^{57}$ Der Erwartungswert der Veränderung des Kapitalstocks zwischen zwei Perioden ist gleich dem Wachstumsparameter $w$, und die Varianz des Kapitalstocks wächst linear mit der Zeit. Ein Unternehmen wird geschlossen, wenn der Kapitalstock den Wert Null erreicht oder unterschreitet.

Beträgt die Wachstumsrate Null, liegt ein reiner random walk vor (CHATFIELD 1996, Unterabschnitt 3.4.2). Die Überlebenswahrscheinlichkeit nimmt mit jeder Runde ab und nähert sich asymptotisch Null. Die erreichte Lebensdauer wird als, hitting time oder ,passage time ' bezeichnet. Bei einer positiven Wachstumsrate wird ein Unternehmen mit einer positiven Wahrscheinlichkeit unendlich lange überleben.

Random walk Modelle stellen eine interessante Methode dar, um die Auswirkungen von Verhaltensweisen und unterschiedlichen Umwelt- und Unternehmenscharakteristika auf die Entwicklung eines Kapitalstocks zu studieren. So kommt WoywODE (1998, Unterabschnitt 4.1.2) in einer Simulationsstudie zu dem Ergebnis, daß die Überlebenswahrscheinlichkeit mit dem Startkapital und der mittleren Verzinsung ansteigt und mit der Varianz der Einzahlung zurückgeht. In Kapitel 5] wird von dieser Technik umfangreich Gebrauch gemacht, um die Implikationen von Varianz- und Eigenkapitalunterschieden sowie der Höhe der Fremdkapitalaufnahme auf die Über-

\footnotetext{
56 Ein Gegenbeispiel ist allerdings die Landwirtschaft, wo trotz eines nachhaltigen Rückgangs der Zahl der Unternehmen sehr wenig Insolvenzen zu beobachten sind.

57 Vgl. GREEN (1993, S. 562). Der Unterschied zwischen random walk Modellen und Wiener-Prozessen besteht in der Skalierung der Zeit. Während random walks zur Analyse von Zeitreihen dienen und Veränderungen zu diskreten Zeitpunkten beschreiben, gehen Wiener-Prozesse von einem kontinuierlichen Zeitverlauf aus (KRUSCHWITZ 1995, S. 296f). Näheres in Kapitel 5.
} 
lebenswahrscheinlichkeit zu untersuchen. Eine Theorie für das Scheitern liefern Random walk Modelle allerdings weniger.

\subsubsection{Industrieökonomische Ansätze}

$\mathrm{Zu}$ diesen Ansätzen werden lerntheoretische und marktstrukturelle Begründungen für den Marktaustritt von Unternehmen gezählt. ${ }^{58}$

Lerntheoretisch motivierte Sichtweisen gehen von dem Gedanken aus, daß ein Unternehmensgründer vor dem Markteintritt keine exakten und zutreffenden Vorstellungen über die künftige Rentabilität seines Unternehmens bilden kann. Erst mit der praktischen Umsetzung der Gründungsidee wächst auch der Informationsstand über die Wirtschaftlichkeit des Unternehmens. In jeder Periode hat der Unternehmer zu entscheiden, ob er sein Unternehmen fortführt oder einstellt.

Diese Theorie führt zu der gleichen Hazardrate wie die liabilities of adolescence. In den ersten Perioden gehen die Unternehmen auch unter widrigen Bedingungen davon aus, daß ihre a priori Erwartungen zutreffend sind. Sie werden daher trotz evtl. Anfangsverluste ihre Produktion aufrechterhalten. Verdichten sich in mittleren Jahren die Informationen, daß die eigene Wirtschaftlichkeit falsch eingeschätzt wurde, entscheiden sich in diesem Zeitraum verstärkt Unternehmen zur Aufgabe. Unternehmen in einem höheren Alter hingegen haben sich davon überzeugen können, daß ihre Erwartungen zutreffend waren und können rentabel im Markt verbleiben.

Der lerntheoretische Ansatz läßt sich leicht in ökonomischen Modellen nachvollziehen (z.B. JAVANOVIC 1982). Sein theoretischer Gehalt ist insbesondere für die ersten Perioden von Bedeutung. Daß Unternehmen desto eher aus einem Markt ausscheiden, je ineffizienter sie arbeiten, und daß auf lange Sicht nur effiziente Unternehmen überleben, ergibt sich auch, wenn kein bayesianisches Lernen unterstellt wird. Erklären kann der lerntheoretische Ansatz aber, warum Unternehmen in den ersten Jahren trotz objektiv gegebener Unwirtschaftlichkeit fortgeführt werden: Auch rationale Unternehmer wissen noch nicht hinreichend um ihre Ineffizienz und hoffen weiterhin, daß ihre a priori Erwartungen zutreffen.

Ältere lerntheoretische Ansätze haben mit der organisationsökologischen Sichtweise, vor allem der theory of founding characteristics, gemein, daß sie von nicht wandelbaren Unternehmen ausgehen, die insbesondere nach ihrer Gründung ihre Effizienz nicht verbessern können. Erst eine neuere lerntheoretische Arbeit gibt diese Prämisse auf (ERICSCON \& PAKES 1995) und erlaubt effizienzsteigernde Investitionen.

Marktstrukturelle Ansätze sehen nicht nur in der Wettbewerbsintensität einen Einflußfaktor auf die Überlebenswahrscheinlichkeit von Unternehmen. Auch die Wirksamkeit von Markteintrittsbarrieren und die Rate des technischen Fortschritts werden von WoYWODE (1998, S. 62) genannt. Hohe Eintrittsbarrieren schirmen die

58 WOYWODE (1998, Abschnitt 4.2) ordnet darunter auch spieltheoretische Ansätze ein, auf die hier nicht eingegangen wird. 
agierenden Unternehmen gegen neue Konkurrenten ab. Sie ermöglichen den etablierten Unternehmen höhere Gewinne und senken daher die Ruinwahrscheinlichkeit.

Hinzugefügt sei noch, daß auch Austrittsbarrieren das Ausscheiden von Unternehmen beeinflussen. Ist der größte Teil des Investitionskapitals versunken und kann weder veräußert noch für andere Zwecke genutzt werden, ist es auch bei nicht erreichbarer Vollkostendeckung sinnvoll, die Produktion fortzuführen, solange die variablen Kosten erwirtschaftet werden. Versunken können nicht nur materielle Investitionen sein, sondern auch Investitionen in Humankapital. Daher widersetzen sich sowohl Manager als auch Einzelunternehmer oft einer betriebswirtschaftlich gebotenen Unternehmensschließung, weil sie nur eingeschränkte Möglichkeiten sehen, ihr erworbenes spezifisches Humankapital anderweitig gewinnbringend einzusetzen (WoYwODE 1998, S. 74).

Sind die hohen Ausstiegskosten potentiellen Neueinsteigern bekannt, erhöht dies die Überlebenswahrscheinlichkeit der etablierten Unternehmen auf zweierlei Weise: Zum einen senken hohe Ausstiegskosten c.p. die a priori Gewinnerwartungen eines Neueinsteigers. Zum anderen müssen Neulinge damit rechnen, daß es ihnen auch bei deutlich effizienterer Produktion nicht gelingen wird, alteingesessene Unternehmen kurzfristig zu verdrängen, da diese auf lange Zeit von den Abschreibungen leben können. ${ }^{59}$

\subsubsection{Evolutionsökonomische Ansätze}

Die evolutorische Ökonomik stellt einen neuen theoretischen Ansatz in den Wirtschafts- und Sozialwissenschaften dar, der versucht, an die Tradition der institutionellen Ökonomik anzuknüpfen. Während die neoklassische Ökonomik für dynamische Prozesse bis auf die Beschreibung von Gleichgewichten keine Erklärungen bietet, geht es in der evolutorischen Ökonomik gerade darum, die Dynamik von Systemen, wie z.B. das Entstehen technischer Neuerungen oder das Wachstum oder Sterben von Unternehmen, zu erklären (BERGER \& BRANDES 1998). Dabei steht die Unternehmerrolle meist im Vordergrund. Das Besondere dieses Ansatzes ist die Integration der Erkenntnisse der modernen Evolutionstheorie, wodurch die Modellierung komplexer Systemdynamiken als Variation und Selektion von Regeln möglich wird. Der Prozeß der ökonomischen Evolution verläuft über die Kette:

- Wettbewerb um Ressourcen (Rohstoffe, Arbeitskräfte, Absatzmärkte, Technologien)

- Variation von Verhaltensweisen (Regeln) und Entwicklung von Innovationen (ana$\log \mathrm{zu}$ Mutationen und Modifikationen in der Biologie)

- Wettbewerbsvorteile von Unternehmen mit erfolgreichen Innovationen und Adaption erfolgreicher Neuerungen (Diffusion der Innovation)

59 Dies kann als wichtige Ursache für die (langsame) Geschwindigkeit des Strukturwandels in der Landwirtschaft angesehen werden (vgl. hierzu u.a. BALMANN 1995 und HANF 1996) und führt gleichzeitig zu der erwähnten geringen Insolvenzrate. 


\section{- Ausscheiden nicht angepaßter Unternehmen. ${ }^{60}$}

Die wichtigen evolutionsökonomischen Impulse basieren auf der Kontroverse darüber, ob sich die neoklassische Gewinnmaximierungshypothese mit dem Selektionsvorteil optimierenden Verhaltens gegenüber anderer Verhaltensformen bzw. Zielsetzungen (z.B. satisfizierendes Verhalten) begründen läßt (WITT 1987, S. 34) ${ }^{61}$ Die Einflüsse gewinnmaximierenden Verhaltens auf die Überlebenswahrscheinlichkeit werden in Abschnitt 4.3 ausführlich behandelt. Angestoßen durch diese Kontroverse wurden zwei Aspekte diskutiert: mögliche Analogien zur biologischen Evolutionstheorie und die Relevanz behavioristischer Modelle in der ökonomischen Theorie. Während weitgehend Einigkeit darüber besteht, daß Analogien zur biologischen Evolutionstheorie nur in loser Form gebildet werden können (FOSTER 1998, S.20), da in sozioökonomischen Prozessen weitaus weniger konstante Größen vorliegen als in der Biologie, wurde das Argument der ökonomischen natürlichen Auslese weiterentwikkelt und für die Erklärung der Dynamik von Märkten insbesondere von NELSON \& WINTER (1982), GERYBADZE (1982) sowie WITT (1987, S. 93 ff.) eingesetzt. In dieser Ausrichtung überschneiden sich organisationsökologische und evolutorische Ansätze weitgehend. Ein Unterschied zwischen beiden Ansätzen besteht darin, daß die Organisationsökologie die Wettbewerbskraft von im Zeitablauf nicht wandelbaren Unternehmen untersucht, während die Evolutionsökonomik gerade den Wandel von Unternehmen zum Forschungsgegenstand hat.

Erklärungsansätze liefert die evolutorische Ökonomik z.B. für die Koexistenz von Generalisten und Spezialisten auf dem gleichen Markt. Wegen der Größenvorteile von Generalisten werden diese vor allem das Zentrum des Marktes dominieren. Kleinbetriebe werden an den Rand gedrängt. Während es im Marktzentrum wegen zunehmender Konkurrenz und Überproduktion zu Marktaustritten kommt, sind die Spezialisten in ihren Nischen weniger gefährdet (CARROL 1983).

Nach der Vorstellung theoretischer Erklärungsansätze folgt nun eine Beschreibung empirischer Methoden. Sie werden in der Praxis sehr häufig angewandt, um frühzeitig Hinweise auf eine Unternehmenskrise zu erhalten.

\subsubsection{Empirische Methoden}

„What is good today need not to be good tomorrow. “ (K. SIGMUND)

In einfachen empirischen Methoden der Analyse betrieblicher Entwicklungen, wie z.B. horizontalen oder vertikalen Betriebsvergleichen sowie der Kennzahlenanalyse, wird die Geschichte des eigenen Betriebes oder ähnlich gelagerter Unternehmen für aktuelle Entscheidungsprobleme herangezogen. Ein starkes Interesse an der Identifikation von Frühwarnindikatoren für eine Insolvenz ist ursprünglich aus der Befürchtung der

60 Vgl. zu einer ähnlichen Abfolge BURMESTER 1996 S. 81.

61 In der erwähnten Münchner Gründerstudie nannten rund 40\% der befragten knapp 2000 Unternehmen als ihr Ziel Einkommensmaximierung und 60\% Einkommensatisfizierung. 
Banken entstanden, daß gewährte Kredite von den Schuldnern nicht zurückgezahlt werden können. Methoden, die hierfür eingesetzt werden, sind z.B. Expertenurteile (meist durch Kreditvergeber), univariate Analysen anhand von Liquiditätskennzahlen, probabilistische Verfahren (z.B. logit-Modelle) und die Diskriminanzanalyse, ein multivariates Verfahren. Aufgrund der großen wirtschaftlichen Bedeutung ist auch die Anzahl der Veröffentlichungen zu diesem Thema entsprechend hoch, z.B. HAUSER 1989, MÜLLER 1992, FÖRSCHLE \& KROPP 1995, HÜLS $1995 .{ }^{62}$ Neben den Frühwarnindikatoren ist für das Kreditgewerbe die Insolvenzwahrscheinlichkeit von großem Interesse. Je höher Banken das Hazardrisiko einschätzen, desto größer wird auch die geforderte Risikoprämie (in Form höherer Zinssätze) sein (SCHELlBERG 1994, BALMANN et al. 1998).

Für eine Analyse von Überlebenswahrscheinlichkeiten sind reine Querschnittsdaten, wie sie beispielsweise für horizontale Betriebsvergleiche verwendet werden, nicht geeignet, da ein Querschnitt zu einem bestimmten Zeitpunkt nur aktive Unternehmen enthält (Survivor-Bias). Günstiger sind Panelldaten, also Daten möglichst identischer Betriebe über mehrere Jahre. Durch ihre Analyse kann die Entwicklung, die zum Ausscheiden geführt hat, nachvollzogen werden. Ein Beispiel hierfür ist die ,Münchner Gründerstudie“ (BRÜDERL et al. 1996), die zum Abschluß des Abschnitts 2.4 beschrieben wird.

Im allgemeinen greifen empirische Methoden auf das Rechnungswesen zurück, indem ex post Einflüsse konkreter Entscheidungen auf die Eigenkapital- und Liquiditätsentwicklung und damit auch auf die Überlebenswahrscheinlichkeit evaluiert werden. Im Nachhinein kann auf diese Weise beurteilt werden, ob eine Entscheidung richtig oder falsch war, oder möglicherweise sogar, ob eine andere Entscheidung mehr Erfolg gebracht hätte.

Eine Extrapolation der vergangenen Entwicklung auf die Zukunft birgt einige Gefahren in sich. Welge \& AL-LAHAM (1997, S. 759f) bewerten diese Vorgehensweise insbesondere aufgrund der reinen Vergangenheitsorientierung der Daten als "für die strategische Planung nur beschränkt geeignet". Denn aus dem Erfolg einer Unternehmung kann nicht zwingend geschlossen werden, daß die verfolgte Strategie gut $w{ }^{63}{ }^{63}$ oder daß das Unternehmen für künftige Herausforderungen gut gerüstet ist (ODENING 1996, S. 32). Ein vorliegendes Betriebsergebnis ist nicht nur die Folge einer Entscheidung, sondern auch durch stochastische Umweltzustände in der Vergangenheit beeinflußt. Das heißt, die gleiche Entscheidung hätte auch zu einem anderen Ergebnis führen können. ALCHIAN (1950, S. 215) formuliert: "Should one rule out luck and chance as the essence of the factors producing long-term survival of the enterprise?" Auch in der neueren Literatur wird weiterhin eine Isolierung der Faktoren "Glück oder Können?" (REICHLING 1986) versucht.

62 Mit der Abwehr von Kreditbetrug setzt sich EvERDING (1996) auseinander.

63 Vgl. zum Zwiespalt zwischen rationalen und erfolgreichen Entscheidungen EISENFÜHR \& WEBER (1993, S. 4). 
Bei der Analyse von Erfolgsursachen von Unternehmen kann nicht einmal unterstellt werden, daß prosperierende Unternehmen überhaupt eine wohldefinierte Strategie, z.B. mit den Komponenten Erwartungsbildung, Zielbestimmung usw., verfolgt haben. Bei einer hinreichend großen Zahl von Akteuren, die alle nur rein zufällig aus den zur Verfügung stehenden Handlungsmöglichkeiten auswählen, wird immer eine Unternehmung dabei sein, die sich im Nachhinein bestmöglich verhalten hat (ex-postOptimalität) und eine, die eine Alternative wählte, die der optimalen Nutzung der zum Entscheidungszeitpunkt zur Verfügung stehenden Informationen entspricht (ex-anteOptimalität, vgl. ALCHIAN 1950, S. 216). Ein sich rational verhaltendes Unternehmen muß keinesfalls auch das erfolgreichste sein. Sind auf einem Markt viele Akteure und unter ihnen einige Hasardeure vorhanden, kann ein rationales Unternehmen in der Regel nicht einmal am erfolgreichsten sein: Wer bei einer Wette auf das richtige Pferd gesetzt hat, entschied sich ex post richtig. Dennoch kann diese Strategie zur Kapitalanlage kaum rationalen, risikoneutralen Entscheidern zur Nachahmung empfohlen werden.

Eine bessere Entscheidungshilfe ist zu erwarten, wenn statt der vorgenommenen Entscheidungen der dahinterstehende Entscheidungsprozeß, also z.B. die Art und Weise der Informationsbeschaffung und -nutzung, betrachtet wird. Aber auch dann ist nicht auszuschließen, daß z.B. eine Wiederholung einer erfolgreichen Entscheidungsfindung trotz gleicher rationaler Zukunftserwartungen wie vor zehn Jahren jetzt den Ruin des Unternehmens zur Folge hat.

Fazit: Die empirische Herangehensweise ist problematisch, weil sowohl Unsicherheit als auch zwischenzeitlich veränderte Rahmenbedingungen nicht oder nur wenig berücksichtigt werden. Auswirkungen von Entscheidungen müssen aber - vom Entscheidungszeitpunkt aus (ex ante) betrachtet - nicht nur für die am wahrscheinlichsten angesehene Entwicklung, sondern für eine Vielzahl infrage kommender Umweltzustände untersucht werden. Dies kann besser mit Hilfe von Modellen geschehen, die die Auswirkungen einer Entscheidung für unterschiedliche Zukunftsszenarien analytisch ableiten oder simulieren.

Nach diesen kritischen Bemerkungen zur empirischen Methodik sei abschließend ein Beispiel angeführt: die anhand empirischer Befunde und deduktiver Ableitungen geführte Kontroverse über die optimale Betriebsgröße. Schon STIGLER (1958) wies auf den seit langem teilweise festzustellenden Zusammenhang zwischen Betriebsgröße und Erfolgsaussichten hin und begründete ihn mit economies of scale. Er verwendete dieses Argument in umgekehrter kausaler Richtung wie die Theorie der liability of smallness. Während diese These besagt (s.o.), daß kleinere Betriebe aufgrund ihrer Größe geringere Überlebenschancen besitzen, beobachtete STIGLER, welche Unternehmen überlebt haben und schloß daraus auf die optimale Betriebsgröße ${ }^{64} \mathrm{Ob}$ und in

64 Dazu sortiert er die Unternehmen eines Wirtschaftszweiges nach ihrer Größe, teilt sie in Gruppen ein und beobachtet, wie sich die Anzahl der Unternehmen in den einzelnen Unternehmen entwikkelt. Andere Herangehensweisen, die Größe eines Unternehmens zu bestimmen, hält er aufgrund von methodischen Schwierigkeiten für wenig erfolgversprechend (STIGLER 1958, S. 54f). 
welchem Umfang economies of scale auch in der westdeutschen Landwirtschaft möglich sind, ist unter Agrarökonomen heftig diskutiert worden. Insbesondere SCHMITT (1992, 1993) vertritt die Auffassung, daß die Spezifika der Landwirtschaft ein wirtschaftliches Betreiben von Großbetrieben unmöglich machen. Er begründet dies deduktiv insbesondere mit den Kontrollkosten von nicht familienzugehörigen Arbeitskräften und höheren Kommunikations- und Informationskosten außerhalb einer Familienwirtschaft. Zur empirischen Begründung verwendet er die Argumentation von STIGLER: da der Familienbetrieb die vorherrschende Organisationsform in Westdeutschland bilde, sei er auch effizient. Dieser Ansicht wird beispielsweise von HANF (1996) unter Hinweis auf die Betriebsgrößen in der Veredlungsproduktion und der Agrarstruktur in den ostdeutschen Bundesländern widersprochen. PETER (1994) errechnet mit Hilfe eines economic engineering Ansatzes, daß die kostenminimale Betriebsgröße weit oberhalb der Durchschnittsgröße in Westdeutschland erreicht wird. BALMANN (1995) zeigt in einem Agrarstrukturmodell, daß klein- und großbetriebliche Strukturen über mehrere Jahrzehnte nebeneinander existieren können und daher aus dem Vorherrschen von Familienbetrieben nicht zwingend auf die Ineffizienz von Großbetrieben geschlossen werden kann.

Nach der Diskussion von empirischen Methoden zur Analyse von Überlebenswahrscheinlichkeiten schließt dieses Kapitel mit der Vorstellung einiger empirischer Studien, in denen die vorgenannten Methoden angewandt wurden.

\subsection{Studien zur Überlebenswahrscheinlichkeit von Unternehmen}

Eine Literaturrecherche im Internet nach dem Stichwort ,Überlebensmodell‘ und seiner englischen Entsprechung zeigt, daß bei weitem die meisten Anwendungen im Bereich der Medizin, und dort vor allem in der Krebsforschung, zu finden sind. ${ }^{65}$ Deshalb wird Überlebenswahrscheinlichkeit üblicherweise definiert als "Wahrscheinlichkeit, mit der eine Person im Alter $x$ das Alter $x+n$ Jahre erreicht (z.B. die Wahrscheinlichkeit, mit der ein Neugeborenes 20 Jahre alt wird). Es gilt für die Überlebenswahrscheinlichkeit: ${ }_{n} p_{x}=1-{ }_{n} q_{x}$ mit ${ }_{n} q_{x}$ für die Wahrscheinlichkeit der Personen im Alter $x$, bis zum Alter von $x+n$ Jahren zu sterben. Die Werte der Überlebenswahrscheinlichkeit sind Bestandteile der Sterbetafel" (GABLER Wirtschaftslexikon 1988, S. 2014). Überlebenswahrscheinlichkeit nach dieser Definition ist also eine bedingte Wahrscheinlichkeit. ${ }_{n} q_{x}$ entspricht der Hazardrate oder Risikofunktion.

Überlebensmodelle werden auch auf ökologische Fragestellungen (z.B. die Überlebenswahrscheinlichkeit von Ökosystemen) und, wie im folgenden, auf Unternehmen übertragen. Ökonomische Überlebensmodelle sind ein Spezialfall von Betriebsent-

65 Vgl. hierzu auch WOYWODE 1998 S. 158. 
wicklungsmodellen und befassen sich im wesentlichen mit der Untersuchung von Überlebenswahrscheinlichkeiten. ${ }^{66}$

In der Literatur, die sich dem Scheitern von Unternehmen widmet, stehen im wesentlichen drei Fragestellungen im Vordergrund (NEUMAIR 1998, S. 11f.):

(1) Analyse der Vorhersagbarkeit und der Frühindikatoren des wirtschaftlichen Ruins,

(2) Untersuchung des Verlaufs des Prozesses hin zum Ruin eines Unternehmens (d.h. ex-post Analyse der Unternehmensgeschichte) und

(3) Identifikation der Faktoren, die das Scheitern eines Unternehmens beeinflussen bzw. charakterisieren.

Insolvenzprognosen sind bereits in Unterabschnitt 2.3.2 angesprochen worden. Die Untersuchung des Prozesses hin zur Insolvenz eines Unternehmens (2) ist rein deskriptiv und verfolgt das Ziel, Muster bzw. typische Verlaufsformen herauszuarbeiten. Der Zweck einer Identifikation der Determinanten für den Erfolg oder das Scheitern eines Unternehmens (3) besteht darin, Verhaltensvorschriften darüber abzuleiten, wie eine Insolvenz vermieden werden kann. Hierbei entsteht das Problem, daß häufig keine eindeutige Abgrenzung zwischen Ursachen und Symptomen einer Insolvenz vorgenommen werden kann.

Überlebensmodelle im engeren Sinne sind folglich in Punkt 3 einzuordnen. Methodisch-theoretische Vorgehensweisen zur Erklärung des Ruins von Unternehmen sind zu Beginn dieses Abschnitts vorgestellt worden. Im folgenden werden einige Untersuchungen beschrieben, die aufgrund ihres Anwendungsbezugs sich einer unmittelbaren Zuordnung zu einem einzelnen theoretischen Konzept entziehen.

Eine umfangreiche empirische Untersuchung zu den Determinanten der Überlebenswahrscheinlichkeit legen BRÜDERL et al. (1996) vor. Sie basiert auf der ,Münchner Gründerstudie', einer im Frühjahr 1990 durchgeführten mündlichen Befragung von knapp 2000 Unternehmen. Diese Stichprobe bildete eine geschichtete Zufallsauswahl aus allen Gewerbeanmeldungen der Jahre 1985 und $1986 \mathrm{im}$ der Region München und Oberbayern. Durch diese Art der Erhebung konnte der sonst üblich ,Survivor-Bias' vermieden werden. Mit diesem Terminus ist das Problem gemeint, daß in einer einmaligen Querschnittserhebung nur existierende und keine ausgeschiedenen Unternehmen befragt werden können. Hinzu kommt die naheliegenden Gefahr, daß Unternehmer, die sich in wirtschaftlichen Schwierigkeiten befinden, weniger Interesse daran haben, über ihren Betrieb und ihre (Miß-)Erfolgsgeschichte

66 Überlebensmodelle können auch in die Klasse der Verweildauermodelle eingeordnet werden, die synonym als Übergangsratenmodelle, Durationsmodelle oder failure time models bezeichnet werden (WOYWODE 1998, S. 158). Ein Einsatzgebiet dieser statistischen Modellklasse ist neben der Medizin die Qualitätskontrolle, z.B. die Frage nach der Haltbarkeit von Komponenten eines Systems. Auch in der Arbeitsmarktforschung wird dieses Modell eingesetzt, um die Wahrscheinlichkeit eines Andauern der Arbeitslosigkeit abzuschätzen (BODE i.V.). 
Auskunft zu geben. Insbesondere die Rücklaufquote in schriftlichen Befragungen wird daher verzerrt sein und den Survivor-Bias verstärken.

Ihre Ergebnisse bestätigen die aus der Literatur bekannte besondere Insolvenzgefahr kleiner und junger Unternehmen. Schaubild 861 zeigt den Verlauf der Sterberate seit Gründung des Unternehmens, also die liability of smallness und die liability of newness (s. S. $74 \mathrm{f}$ ).

\section{Schaubild 86.1: Wahrscheinlichkeit einer Betriebsauflösung in der ,Münchner Gründerstudie 6}

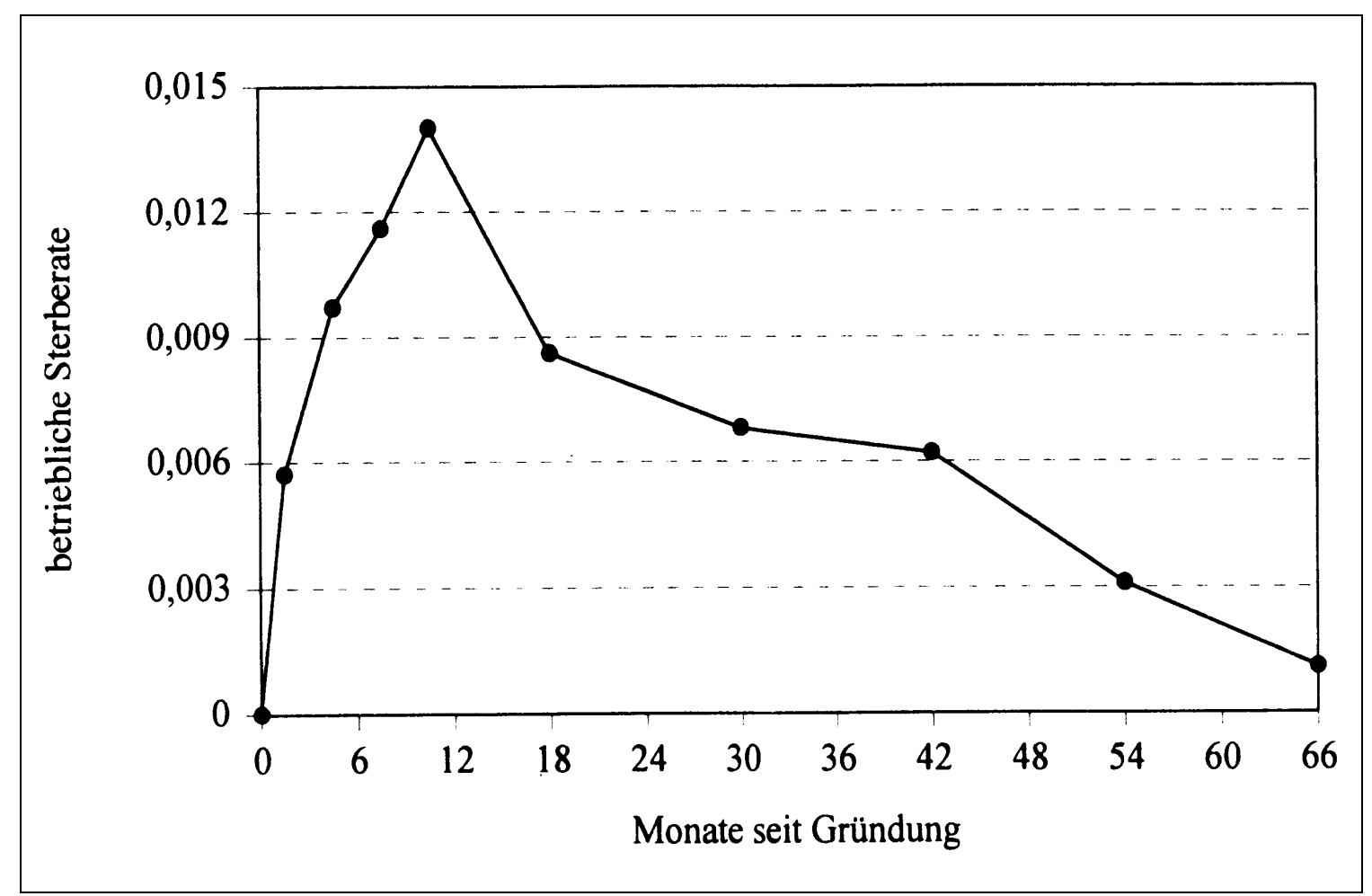

Quelle: BRÜDERL et al. (1996, S. 95)

Weiter bestätigt diese umfangreiche empirische Studie eine Vermutung der Humankapitaltheorie, daß eine bessere Ausbildung die Ruinwahrscheinlichkeit senkt, und eine These der Organisationsökologie, daß ein größeres Startkapital in die gleiche Richtung wirkt (BRÜDERL et al. 1996. S. 116, 118). Ebenfalls erweist sich eine Aussage der theory of founding characteristics als zutreffend: Der Gründungserfolg ist branchenabhängig. Als überdurchschnittlich in ihrem Bestand gefährdet erweisen sich Gründungen in den drei Bereichen Verkehr/Spedition, Versicherungen und Gastgewerbe. Betriebe im Handel liegen im Mittelbereich und Unternehmen des Bausektors haben, vielleicht überraschend, eine hohe Erfolgsaussicht, ebenso wie Dienstleister im Bereich Bildung/Verlage.

Im internationalen Bereich ist der Modellansatz FLIPSIM (RICHARDSON \& NIXON 1986, zit. n. ORIADE \& DILLON 1997, S. 49f) häufig angewendet worden. Auf ihm basiert z.B. das international farm comparison network, mit dem die Bundesanstalt für 
Landwirtschaft (FAL) unter Leitung von ISERMEYER international vergleichend die Wettbewerbsfähigkeit von Unternehmen untersucht, die für eine Region und eine Produktionsrichtung typisch sind. Ursprünglich wurde FLIPSIM entwickelt, um die wahrscheinlichen Konsequenzen alternativer Agrarpolitiken auf betrieblicher Ebene für einen Zeitraum von 10 Jahren zu simulieren, z.B. die Entwicklung des Produktionsprogramms, des Eigenkapitals, der Verschuldung und der Einkommensteuer und damit des sektoralen Einkommensteueraufkommens. Ein optimaler Wachstumspfad kann enumerativ berechnet werden. Spätere Anwendungen dieses Modells erforschten auch die ökonomische Stabilität und Überlebensfähigkeit bestimmter Gruppen landwirtschaftlicher Betriebe, z.B. von Baumwollfarmern in Texas oder Milchviehhaltern in Ostafrika (ORIADE \& DILLON 1997). Eine Untersuchung von Reisbauern im Mississippi-Delta ergab, daß durch Produktdiversifikation eine nachhaltig höhere Produktion gesichert werden kann. Diese Studie verwendet ein deterministisches Überlebensmodell (SALASSI et al. 1987).

Ebenfalls mit FLIPSIM nehmen BAILEY \& RICHARDSON (1985) Vermarktungsstrategien unter die Lupe. Es wird ein gesamter Betrieb simuliert, der seine Produktion zu Tagespreisen verkaufen oder über verschiedene Formen von Warentermingeschäften absichern kann. Der Einfluß der Vermarktungsstrategie wird untersucht hinsichtlich:

- der Überlebenswahrscheinlichkeit. Ausscheiden wird definiert als Unterschreiten eines minimalen Eigenkapitalanteils, der von den Kreditgebern fixiert worden ist.

- der Wahrscheinlichkeit wirtschaftlichen Erfolgs. Als erfolgreich wird ein Unternehmen angesehen, wenn die erreichte Eigenkapitalverzinsung 5\% per anno nicht unterschreitet.

- des Kapitalwerts nach Steuern

- des Vermögensendwerts

- des Betriebsgrößenwachstums.

Der Planungshorizont beträgt 10 Jahre. Als Ergebnis wird festgehalten, daß es zwischen erzielbaren Gewinnen auf der einen Seite und der Überlebenswahrscheinlichkeit einen Trade-Off gibt. Als Kriterium für die Vorzüglichkeit der Strategien in Abhängigkeit von der Risikoaversion der Entscheider wird das Konzept der stochastischen Dominanz angewandt. ${ }^{67}$

In einer französischen Studie verwenden COLSON et al. (1993) einen Mehr-Variablen-Ansatz zur Vorhersage von Insolvenzen. Singuläre Kenngrößen, wie Betriebsgröße, Einkommen oder cash flow, seien üblicherweise verwendete Indikatoren für eine finanziellen Schieflage; durch das gleichzeitige Betrachten dieser Variablen könne jedoch die Prognosequaltiät verbessert werden. Die Autoren kombinieren einige wenige buchhalterische Kenngrößen zu einer score function, die in einem einzigen

67 Vgl. Abschnitt 4.1. Dort wird das Konzept der stochastischen Dominanz in einem etwas anderen Zusammenhang diskutiert. 
Wert die Insolvenzgefahr zum Ausdruck bringt, und wenden diese Funktion auf landwirtschaftliche Betriebe in fünf Regionen Frankreichs an. Die score function erweist sich als ein guter Indikator für die Früherkennung von Unternehmenskrisen ${ }^{68}$ und die Analyse ihres Verlaufs, unabhängig von der Betriebsgröße oder dem Produktionsprogramm.

BRANDES (1989) hat die FRIEDMANsche Gewinnmaximierungsthese eingehend mit Hilfe von Monte-Carlo-Simulationen untersucht. Er vergleicht zwei Strategien, $A$ und $B$, in einem Szenario mit festen Auszahlungen. Strategie $A$ beinhaltet eine feste Einzahlung größer als die Auszahlung und Strategie $B$ eine stochastische Einzahlung mit höheren Erwartungswert als die feste Einzahlung in Strategie $A$ und Minimum kleiner als die für beide Strategien gleiche und zeitlich konstante Auszahlung. Die gewinnmaximierende Strategie ist also $B$. Auch ohne Simulation geht aus dieser Modellspezifikation bereits hervor, daß Alternative $A$ mit Sicherheit überlebt, während die Überlebenswahrscheinlichkeit bei Alternative $B$ kleiner als $100 \%$ ist.

Weitere Simulationen von BRANDES $(1989,1990)$ zu stochastischen Kapitalstockentwicklungen zeigen, daß es zwischen dem Umfang des eingesetzten Fremdkapitals und der Flexibilität der Produktion eine nicht monotone Beziehung besteht. Danach gibt es eine optimale Fremdkapitalintensität und einen optimalen Grad an Flexibilität (BRANDES unterstellt in seinen Modellen, daß das Bereithalten von Flexibilität zu höheren minimalen Durchschnittskosten führt.).

Während BRANDES einen polypolistischen Markt untersucht, analysiert FISCHER (1988) Strategien in einem Oligopol. Bemerkenswertes Ergebnis seiner Simulationsstudien ist, daß der relative Erfolg maßgeblich vom Verhalten der Konkurrenten abhängig ist: „Vielmehr ist es in dieser Markkonstellation für alle Strategien möglich, auf beliebige Plätze der Rangfolge zu gelangen.“ (FISCHER 1988, S. 133).

BURMESTER (1996) untersucht Überlebenswahrscheinlichkeiten von Verhaltensweisen in einem nichtoptimierenden Modell. Gegenstand der Modellierung sind landwirtschaftliche Veredlungsbetriebe. Seine Ergebnisse decken sich mit den erwähnten von BRANDES: Vermeidet ein Unternehmen die Fremdkapitalaufnahme absolut, kann dies seine Überlebensfähigkeit mindern. Ferner weist er die Existenz ruinminimierender Wachstumspfade nach.

Zum Einfluß der Obergrenze der Verschuldung auf die Überlebenswahrscheinlichkeit führten LEATHAM et al. (1986) ebenfalls eine Simulationsstudie durch. Für texanische Reisfarmer kommen sie zu dem Ergebnis, daß ein Mindest-Eigenkapitalanteil für Investitionen nicht unterschritten werden sollte. Dieser ist für Pächter größer als für Bewirtschafter von Eigentumsflächen. ${ }^{69}$

68 Hierzu gibt es eine umfangreiche Literatur, z.B. im landwirtschaftlichen Bereich Odening (1994), im Gartenbau BOKELMANN (1993) und in der allgemeinen Betriebswirtschaftslehre KÜHNBERGER et al. (1996).

$69 \mathrm{Zu}$ einer analytischen Herangehensweise an den optimalen Verschuldungsgrades vgl. ODENING 1991. Allerdings lassen sich nur unter restriktiven Annahmen Lösungen ableiten. 


\section{Methodische Probleme in Entscheidungs- und Planungsmodellen}

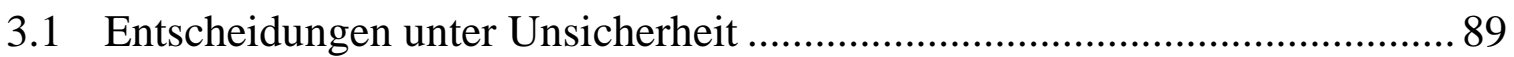

$3.2 \quad$ Unsicherheit in der Betriebsplanung...................................................... 98

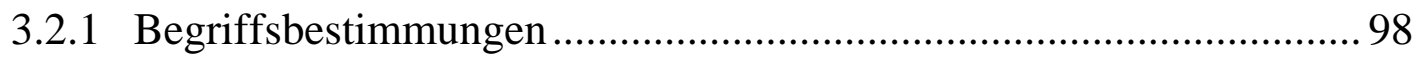

3.2.2 Behandlung von Unsicherheit in der linearen Programmierung ...... 100

3.3 $\quad$ Signifikanztests in Monte-Carlo-Simulationen ..................................... 104

3.4 Berücksichtigung von Investitionen in Modellen der einperiodischen

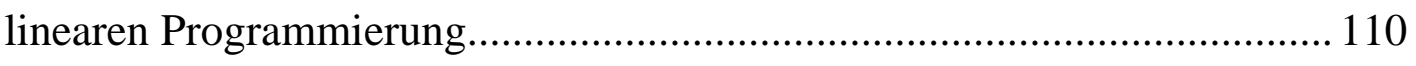

3.4.1 LP-Varianten zur Berücksichtigung von Investitionen ................... 112

3.4.2 Zusammenfassung und Erweiterungen ........................................ 126

Eine Aufgabe von Betriebsentwicklungsmodellen besteht in der Entscheidungsunterstützung. Mit ihrer Hilfe können sowohl kurz- als auch langfristige Implikationen alternativen Handelns aufgezeigt werden. Zwei Aspekte sind bei der Modellierung betrieblicher Entwicklungen von besonderer Bedeutung. Beide hängen mit der Längerfristigkeit des Planungshorizonts zusammen. Zum einen muß, da die Zukunft nicht sicher vorhergesagt werden kann, der Unsicherheit im Planungsansatz Rechnung getragen werden. Zum anderen wäre es eine erhebliche Einschränkung, vorauszusetzen, daß die Ausstattung eines Betriebes mit Investitionsgütern über einen längeren Planungszeitraum unverändert blieb. Vielmehr sollte ein Betriebsentwicklungsmodell die Möglichkeit von Investitionen vorsehen. Wie beide Anforderungen in Betriebsentwicklungsmodelle, die auf linearer Programmierung (LP), integriert werden können, basieren, wird in diesem Kapitel dargelegt. Darüber hinaus werden Möglichkeiten diskutiert, die Ergebnisse von Monte-Carlo-Studien statistisch abzusichern.

Das Kapitel beginnt mit grundsätzlichen Ausführungen zur Entscheidungsproblematik. Dieser Abschnitt schließt mit der Entwicklung eines Ansatzes, der den Unbestimmtheitsbereich bei Entscheidungen zwischen unsicheren Alternativen einschränken kann.

\subsection{Entscheidungen unter Unsicherheit}

Die Wahl zwischen Alternativen ist abhängig von den Zielen und Einschätzungen der Akteure. Dabei bedeutet Entscheiden, unter mehreren Handlungsmöglichkeiten die- 
jenige auszuwählen, deren Wirkungen dem Entscheider am meisten zusagen. Im Grundsatz sind folgende Fragen für ein Entscheidungsproblem von Bedeutung:

(a) Hat die jeweilige Handlungsmöglichkeit eine oder mehrere Wirkungen?

(b) In welchen Wirkungen unterscheiden sich die Handlungsmöglichkeiten ${ }^{70}$

(c) Welche Wirkungen von Handlungsmöglichkeiten sind für den Entscheider von Belang?

Entscheidungsprobleme treten nur auf, wenn mehr als eine Handlungsmöglichkeit besteht und die aus (a) bis (c) leicht ableitbaren Voraussetzungen erfüllt sind. Das heißt, eine Handlungsmöglichkeit muß mehrere Wirkungen besitzen (a), die Handlungsmöglichkeiten müssen sich in ihren Wirkungen unterscheiden (b), und diese Unterschiede müssen für den Entscheider relevant sind (c). Anderenfalls ergibt sich eine eindeutige Lösung, die zwangsläufig für jeden Entscheider gleich ist; das Problem ist entscheidungstheoretisch trivial:

- Ist dem Entscheider nur eine Wirkung der Handlungsmöglichkeiten bekannt, gleichgültig, für wieviele Wirkungen er sich interessiert, oder

- ist für ihn nur eine Wirkung relevant, unabhängig davon, in wieviel anderen Wirkungen sich die Handlungsmöglichkeiten unterscheiden mögen,

so lassen sich die Handlungsmöglichkeiten leicht in eine Rangfolge bringen und eine Entscheidung herbeiführen. Gleiches gilt, wenn sich der Entscheider zwar für mehrere vorhandene Wirkungen interessiert, aber nur in einer Wirkung Unterschiede zwischen den Handlungsmöglichkeiten bestehen.

Ebenfalls trivial ist das Entscheidungsproblem, wenn eine Handlungsalternative mit zwei Wirkungen verknüpft ist und beide besser als die Referenzsituation beurteilt werden. Eine Entscheidung ist nicht möglich, wenn sich zwei Handlungsalternativen in bezug auf die entscheidungsrelevanten Eigenschaften nicht unterscheiden.

Zur Beurteilung der Auswirkungen von Handlungsmöglichkeiten wird oft das Konzept der Nutzenfunktion angewendet. Eine Nutzenfunktion ordnet einem Güterbündel einen Nutzenindex zu. Das Skalierungsniveau des Nutzenindex muß zumindest ordinal sein. Denn dann ist es möglich, die Nutzenindizes der Güterbündel zu vergleichen und damit die Güterbündel gemäß den Präferenzen der Entscheiderin in eine Rangfolge zu bringen. Indifferenz zwischen zwei Güterbündeln ist dabei nicht ausgeschlossen.

Entscheidungsprobleme können selbstverständlich nur dann auftreten und modelliert werden, wenn zwei Bedingungen gleichzeitig erfüllt sind. Zum einen müssen mehrdimensionale Nutzenfunktionen, also Nutzenfunktionen mit mindestens zwei

70 Ausschlaggebend ist nur, was der Entscheider von den Handlungsmöglichkeiten und ihren Wirkungen weiß oder zu wissen meint, nicht ihre wirklichen Eigenschaften. Wenn in dieser Arbeit von entscheidungsrelevanten Merkmalen die Rede ist, sind stets Eigenschaften in diesem subjektiv wahrgenommenen Sinne gemeint. 
Argumenten ${ }^{71}$, benutzt werden und zum anderen ist erforderlich, daß diese Argumente auch durch die Wirkungen der Handlungsalternativen berührt werden. Dabei dürfen die Argumente für sich den Nutzen nicht alle in die gleiche Richtung verändern (s.o.). Entscheiden bedeutet also ein Abwägen zwischen Teilnutzen von Handlungsmöglichkeiten. Dieses Abwägen ist unproblematisch, wenn Verschlechterungen bei einer Auswirkung auch durch noch so große Verbesserungen bei der oder den anderen Auswirkungen nicht kompensiert werden können. In diesem Fall liegt eine lexikographische Präferenzordnung vor. Können hingegen Nutzenverluste (Nachteile) bei einer Wirkung durch Vorteile bei einer anderen Wirkung ausgeglichen oder sogar überkompensiert werden, dann weist die Nutzenfunktion einen substitutionalen Charakter auf, und es sind indifferente Kombinationen aus den beiden Wirkungen möglich. Wird eine unendliche Anzahl von Handlungsmöglichkeiten unterstellt, die sich infinitesimal in ihren Wirkungen unterscheiden, dann lassen sich die Punkte gleichen Nutzens graphisch verbinden, man spricht von einer Indifferenzkurve.

\section{Schaubild 91.1: Entscheidungssituationen bei zwei Auswirkungen einer Entscheidungsalternative}

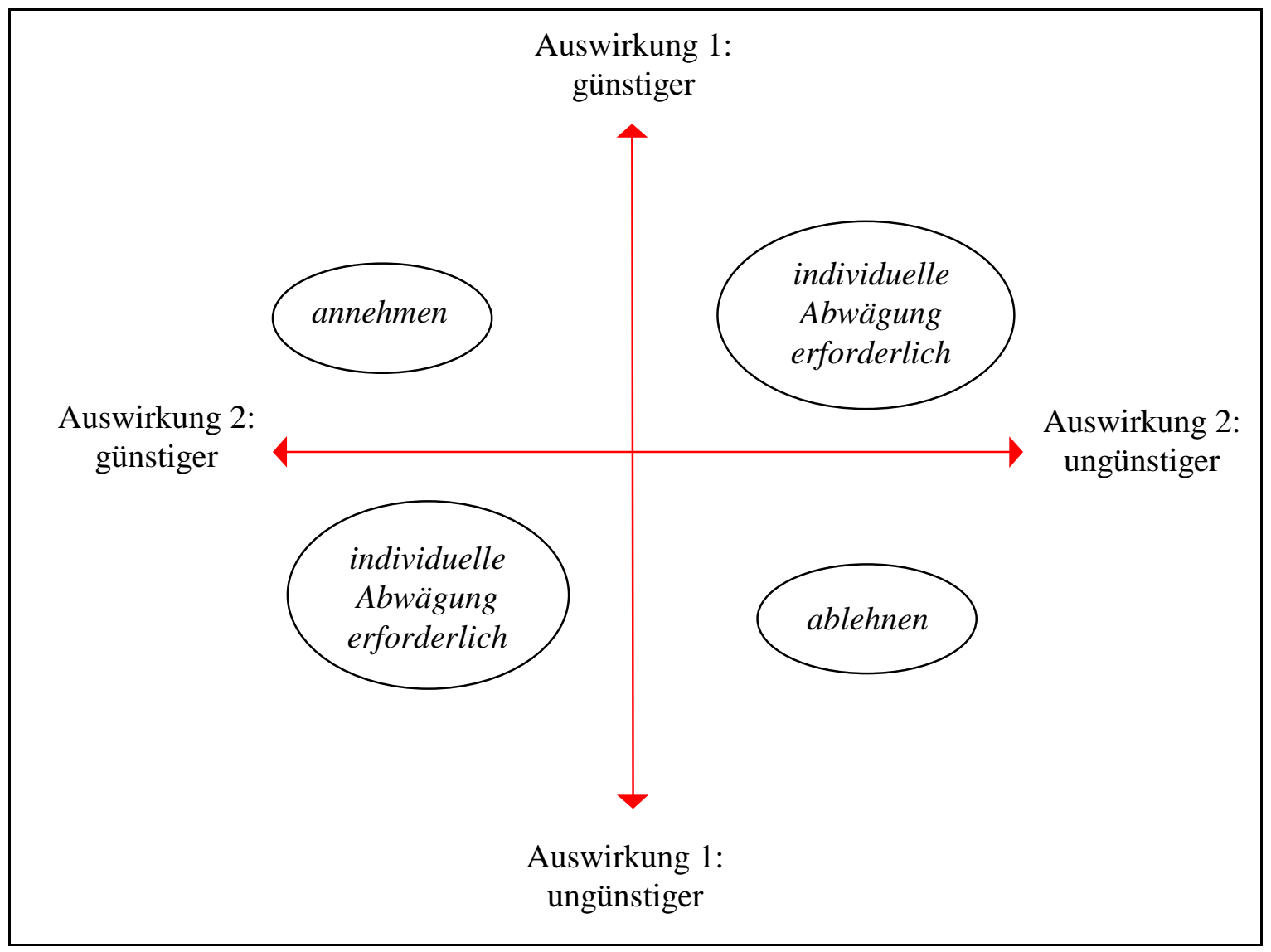

71 In der Literatur gelegentliche auch multiattributive Nutzenfunktion genannt: mehrere Attribute einer Entscheidung tragen zum Nutzen des Entscheiders bei. Vgl. EISENFÜHR \& WEBER (1993). 
Schaubild 91.1 illustriert diese Zusammenhänge für eine zweidimensionale Nutzenfunktion. Führt eine Alternativhandlung $A$ bezüglich beider Argumente entweder zu einem besseren oder zu einem schlechteren Ergebnis als die Referenzsituation, ist eine eindeutige Entscheidung möglich. Hingegen ist eine Abwägung erforderlich, wenn durch $A$ ein Argument positiv und das andere negativ beeinflußt wird. In Schaubild 91.1 ist dies in den Quadranten I und III der Fall, in den Quadranten II und IV ist die Entscheidung eindeutig. ${ }^{72}$

Bisher wurden nur solche Handlungsmöglichkeiten betrachtet, die mehr als eine entscheidungsrelevante Auswirkung besitzen. Aber auch bei ausschließlicher Berücksichtigung von nur einer Wirkung können Abwägungen erforderlich werden, und zwar dann, wenn das Resultat einer Entscheidung stochastisch ist. In der Regel besitzt die Verteilung des zugehörigen Ereignisses einen Erwartungswert und kann durch weitere statistische Kennziffern, wie Varianz und Spanne der Verteilung (range), beschrieben werden. ${ }^{73}$ Entscheidungsprobleme treten nur dann auf, wenn eine Handlungsalternative sich in mehr als einer statistischen Kennziffer der Ergebnisverteilung von einer Referenzsituation unterscheidet und diese Abweichungen den Nutzen jeweils in unterschiedliche Richtungen beeinflussen und somit nutzendivergierende Wirkungen vorliegen.

In der Ökonomik werden vor allem Handlungsalternativen betrachtet, die mit Zahlungen in nicht genau bekannter Höhe verbunden, also zufallsbehaftet sind. Die Entscheidung zwischen diesen unsicheren Alternativen hängt von Zielen und Einschätzungen der Akteure ab. Unterscheiden sich Auszahlungen in ihrem Erwartungswert, so wird ein Entscheider, der nur auf diese Kennzahl achtet, selbstverständlich stets die Alternative mit dem höheren Erwartungswert wählen. Interessiert sich der Entscheider außerdem noch für ein anderes Merkmal der Verteilung und sind diese beiden Charakteristika nutzendivergierend, dann liegt ein (nichttriviales) Entscheidungsproblem mit Zielkonkurrenz vor. In der Regel wird das mit einer Entscheidung verbundene Risiko als zweite Eigenschaft herangezogen. ${ }^{74}$

Unter der Annahme, daß ein erhöhtes Risiko negativ bewertet wird, könnte daher im obigen Schaubild 911 der Erwartungswert nach oben ("günstiger") und das Risiko

72 Das Schaubild für sich ist etwas trivial. Es ist aufgenommen worden, weil auf seinem Grundgedanken im weiteren mehrfach Bezug genommen und die Darstellung modifiziert wird.

73 Eine Entscheidungsmöglichkeit, oder synonym, eine Alternative hat strenggenommen keine Varianz; stochastisch ist das mit dieser Entscheidungsmöglichkeit verbundene mögliche Ergebnis. Wird im folgenden dennoch von der Varianz oder dem Erwartungswert einer Alternative gesprochen, so geschieht dies aus Gründen sprachlicher Vereinfachung.

Eine Verteilung ist durch mehr Eigenschaften als durch Erwartungswert und Streuung gekennzeichnet, die u.a. in Form der Varianz, der mittleren absoluten Abweichung, der Spanne oder des Quantilsabstandes gemessen wird. Die Beschränkung auf Erwartungswert und z.B. Varianz ist eine bewußte Konzentration auf für den Entscheider relevante Eigenschaften. Das Ignorieren darüber hinausgehender Eigenschaften ist keine Besonderheit des Erwartungswert-Varianz-Ansatzes, sondern unvermeidlich für fast jede Art von Entscheidung, wie zu Beginn des Abschnittes ausgeführt wurde. 
nach rechts ("ungünstiger") abgetragen werden. Die höchstens noch hinnehmbare Erhöhung des Risikos bei vorgegebener Steigerung des Erwartungswertes gilt als Ausdruck der individuellen Risikoaversion des Entscheiders. Kombinationen gleicher Wertschätzung können $\mathrm{zu}$ einer Indifferenzkurve verbunden werden, wie in Schaubild 901 beispielhaft dargestellt. Hier wirà die Varianz als Maß für das Risiko verwendet. Der Verlauf der Kurve zeigt, daß für kleine Varianzsteigerungen nur sehr kleine Erhöhungen im Erwartungswert erforderlich sind, um das Nutzenniveau konstant

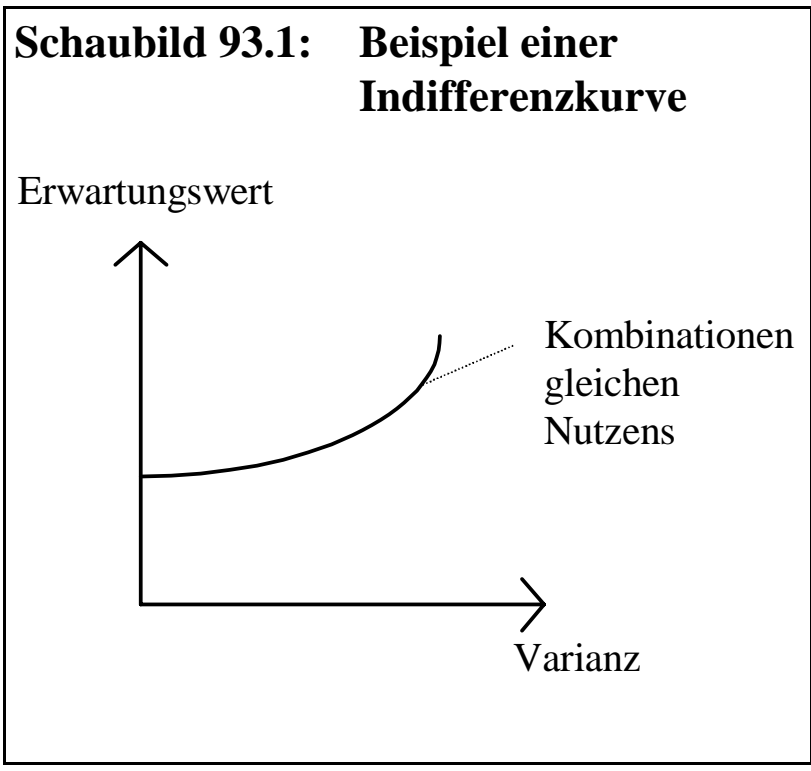
zu halten. Sehr hohe Varianzzunahmen erfordern hingegen überproportional große Kompensationen. Punkte oberhalb der Indifferenzkurve bedeuten ein höheres, Punkte unterhalb ein niedrigeres Nutzenniveau.

Mit Hilfe einer Nutzenfunktion können alle Erwartungswert-Varianz-Kombinationen in eine Rangfolge gebracht werden, so daß für jede Entscheidungsalternative eine Aussage möglich ist, ob sie einer Referenzsituation vorgezogen wird. Häufig jedoch sind die Indifferenzkurven von Entscheidern nicht bekannt. Kann auch ohne ihre Kenntnis der Unbestimmtheitsbereich in Schaubild 911 verkleinert werden? In diesen Fällen wird häufig das Konzept der stochastischen Dominanz verwendet, das in vielen Lehrbüchern zur Entscheidungstheorie beschrieben ist (z.B. ANDERSEN et al. 1977, Kapitel 9, HANF 1986, Abschnitt 5.5). Auf diese Methode wird hier nicht eingegangen, ${ }^{75}$ da ein anderer Ansatz zur Reduzierung des Unsicherheitsbereichs entwickelt wird.

Dazu muß etwas ausgeholt werden. Risikoaverse Entscheider ziehen, ausgehend von einem Referenzpunkt, alle Punkte vor, die einen höheren Erwartungswert und/oder eine geringere Vari-

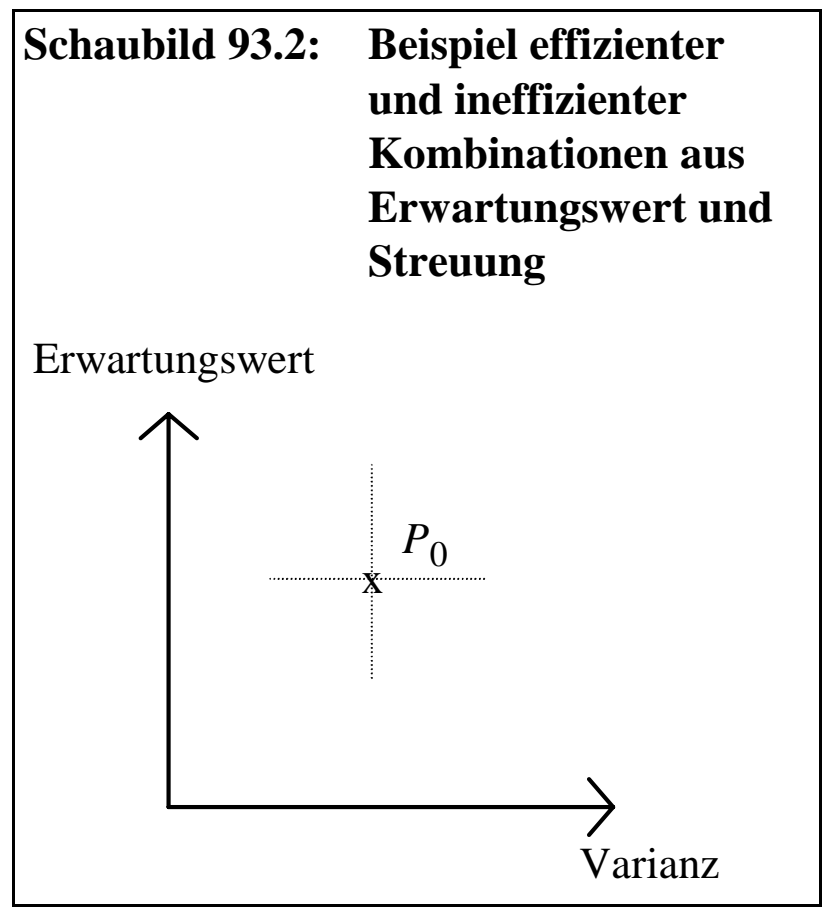

75 In Abschnitt 4.1 wird auf die stochastische Dominanz zweiten Grades kurz Bezug genommen. Dort wird geprüft, ob sie als Indikator für die ,Überlegenheit ‘ von Verteilungen, ein Konzept, das in diesem Abschnitt eingeführt wird, dienen kann. 
anz repräsentieren. Dieser Punktraum entspricht in Schaubild Pिमि dem nordwestlichen Quadranten (der Ursprung des Koordinatenkreuzes liege in $P_{0}$ ). Alle Punkte im südöstlichen Quadranten hingegen sind als nachteilig zu bewerten, da sie durch eine höhere Varianz und/oder einen geringeren Erwartungswert gekennzeichnet sind. Für die beiden verbleibenden Quadranten lassen sich zunächst wie in Schaubild f111 keine Aussagen über die Vorteilhaftigkeit bestimmter Erwartungswert-Varianz-Kombinationen treffen. $\mathrm{Da} ß$ es für Teile dieser Bereiche bei Vorliegen beschränkter Verteilungen dennoch möglich ist, Aussagen über die Vorteilhaftigkeit abzuleiten, sei an folgenden Überlegungen veranschaulicht:

Für viele risikoaverse Entscheider ist insbesondere der schlechtest mögliche Ausgang, also das Minimum der Verteilung des möglichen Ergebnisses, von Interesse. Nehmen wir an, es stünden eine Referenz-Handlungsmöglichkeit mit sicherem Ausgang, die wiederum mit $P_{0}$ benannt sei (aber im Unterschied zu oben ein sicheres Ereignis und keine Zufallsvariable bezeichne), und eine Reihe von unsicheren Alternativen mit den Ergebnissen $P_{i}, i \in \mathrm{M}$, zur Verfügung. Der Einfachheit halber seien die Handlungsalternativen mit ihren zugehörigen Ergebnissen bezeichnet. Die Verteilungen der Alternativen $P_{i}$ seien (zumindest nach unten) beschränkt und ihre Erwartungswerte sowie Varianzen größer als $P_{0}$. Damit liegen sie alle im NordostQuadranten von $P_{0}$ (s. Schaubild 932). Unter diesen Annahmen wird selbstverständlich jeder Entscheider, über den zumindest bekannt ist, daß für ihn 'mehr besser ist als weniger', alle Handlungsmöglichkeiten $P_{k}, k \in \mathrm{M}$, der Referenzsituation $P_{0}$ vorziehen, für die gilt, daß ihr Minimum größer ist als $P_{0}$. Damit ist der Unbestimmtheitsbereich, in dem alle Punkte $P_{i}$ liegen, erheblich verkleinert.

\section{Schaubild 94.1: Entscheidungssituationen bei Berücksichtigung von} Erwartungswert und range

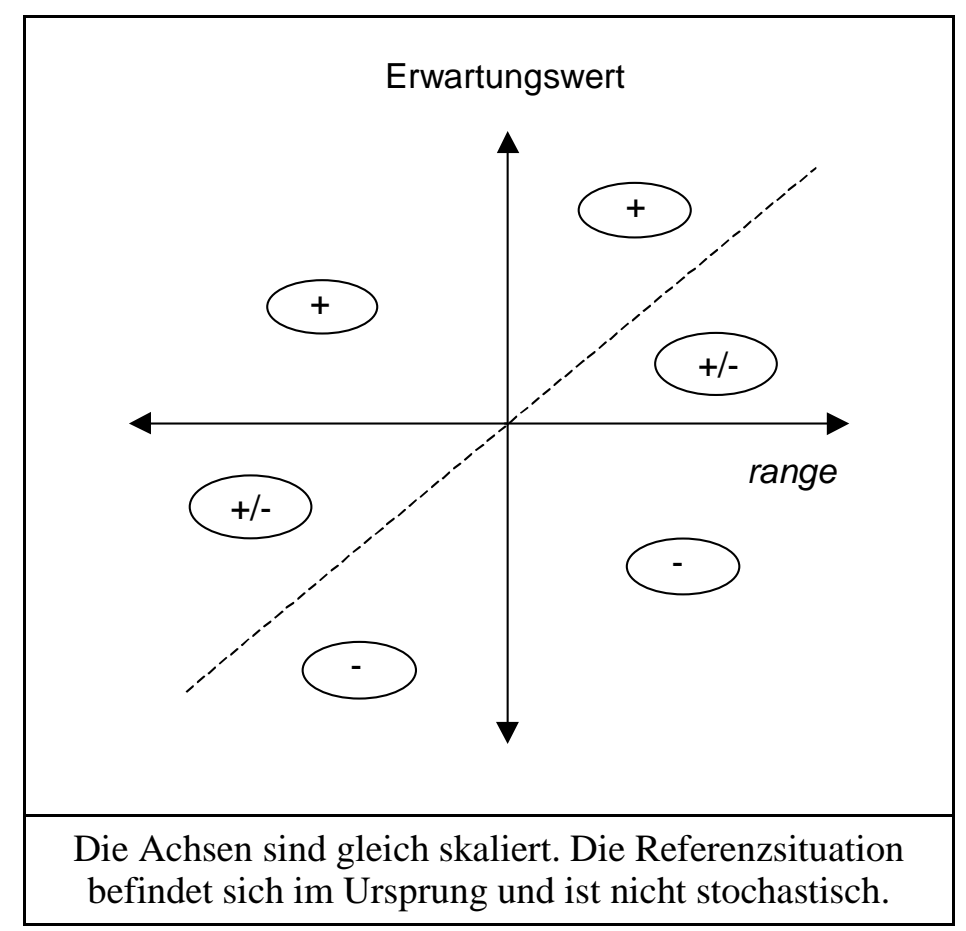


Für die formale Herangehensweise an diesen Sachverhalt wird im folgenden statt der Varianz die negative, range , also die maximal mögliche negative Abweichung der Realisation einer Zufallsvariablen von ihrem Erwartungswert herangezogen. Sie bilde neben dem Erwartungswert das zweite Argument der Nutzenfunktion.

In Schaubild 941 ist im Vergleich zum vorigen Diagramm die Varianz durch die range ersetzt. Da die Skalierung auf Abszisse und Ordinate gleich sei, weisen alle Punkte des ersten Quadranten, die oberhalb der Winkelhalbierenden liegen, ein größeres Minimum auf als der Referenzpunkt im Ursprung. Sie werden daher vorgezogen, so daß mit Hilfe der range als Argument der Nutzenfunktion der Unbestimmheitsbereich halbiert werden kann. Entsprechendes gilt für den dritten Quadranten.

Die bisherigen Überlegungen bezogen sich auf den Vergleich stochastischer Alternativen mit einem sicheren Referenzergebnis. Im folgenden sei auch die Referenzsituation stochastisch.

Die Punkte in Tabelle 961 stehen beispielhaft für alle wichtigen Vorzeichenkombinationen, die im Vergleich zwischen Referenz- und Alternativerteilung in bezug auf Erwartungswert und Streuung auftreten können. Bedeutsam ist, daß alle Punkte im Unbestimmtheitsbereich (vgl. Schaubild 911) liegen. Sie sind in Schaubild 96,1 dargestellt. Der Referenzpunkt $P_{0}$ befindet sich im Ursprung des separaten Koordinatenkreuzes. Unter der gleichen Annahme wie zuvor, daß die Nutzenfunktion, well behaved' ist und insbesondere beinhaltet, daß größere Beträge kleineren vorgezogen werden, gilt für die Punkte bezüglich der Referenzverteilung:

- $P_{11}$ besitzt einen höheren Erwartungswert und eine größere Streuung. Aufgrund des höheren Minimums wird er präferiert.

- $P_{12}$ besitzt ebenfalls einen höheren Erwartungswert und eine größere Streuung als die Referenzverteilung. Aufgrund des geringeren Minimums seiner Verteilung fällt er in den Unbestimmtheitsbereich.

- $P_{21}$ ist durch einen geringeren Erwartungswert und eine kleinere Streuung gekennzeichnet. Da auch das Minimum kleiner ausfällt, kann er abgelehnt werden.

- $P_{22}$ besitzt wie $P_{21}$ einen geringeren Erwartungswert und eine kleinere Streuung. Da hier jedoch das Minimum größer ist, kann ohne konkretere Kenntnis der Nutzenfunktion keine Entscheidung getroffen werden.

Werden diese Überlegungen verallgemeinert, folgt daraus eine generelle Präferenz für alle Punkte im ersten Quadranten, die oberhalb der Winkelhalbierenden liegen. Im dritten Quadranten folgt eine Ablehnung für alle Punkte, die sich unterhalb der $45^{\circ}$ Linie befinden. Zusammenfassend kann festgehalten werden, daß die Verwendung von Erwartungswert und range als Argumente einer Risikonutzenfunktion den Unbestimmtheitsbereich halbiert. Denn wenn die Varianz herangezogen wird, kann über die Vorteilhaftigkeit aller Punkte im ersten und dritten Quadranten ohne Kenntnis der spezifischen Indifferenzkurve keine Aussage getroffen werden. Bei Verwendung der range hingegen verringert sich dieser Bereich auf die der Abszisse benachbarten Hälften der genannten Quadranten, so daß in den Quadranten II und IV und den der 
Ordinate benachbarten Hälften der Quadranten I und III für risikoaverse Entscheider eindeutige Entscheidungen möglich sind. Dieser Ansatz wird in Abschnitt 5.o zur Entscheidungsunterstützung auf das Planspiel "Puten und Perlhühner" übertragen.

Tabelle 96.1: Beispiele für Entscheidungssituationen bei Verwendung des Erwartungswert-range-Kriteriums

\begin{tabular}{|c|c|c|c|c|c|}
\hline Punkt & Referenz & $P_{11}$ & $P_{12}$ & $P_{21}$ & $P_{22}$ \\
\hline $\bar{x}$ & 100 & 120 & 120 & 80 & 80 \\
\hline Range & 100 & 110 & 130 & 90 & 70 \\
\hline Minimum & 0 & 10 & -10 & -10 & 10 \\
\hline Präferenz & & + & $+1-$ & - & $+1-$ \\
\hline \multicolumn{6}{|c|}{$U=U(\bar{x}$, range $), U=$ Nutzen. } \\
\hline
\end{tabular}

Schaubild 96.1: Vergleich von stochastischen Entscheidungssituationen bei Berücksichtigung von Erwartungswert und range

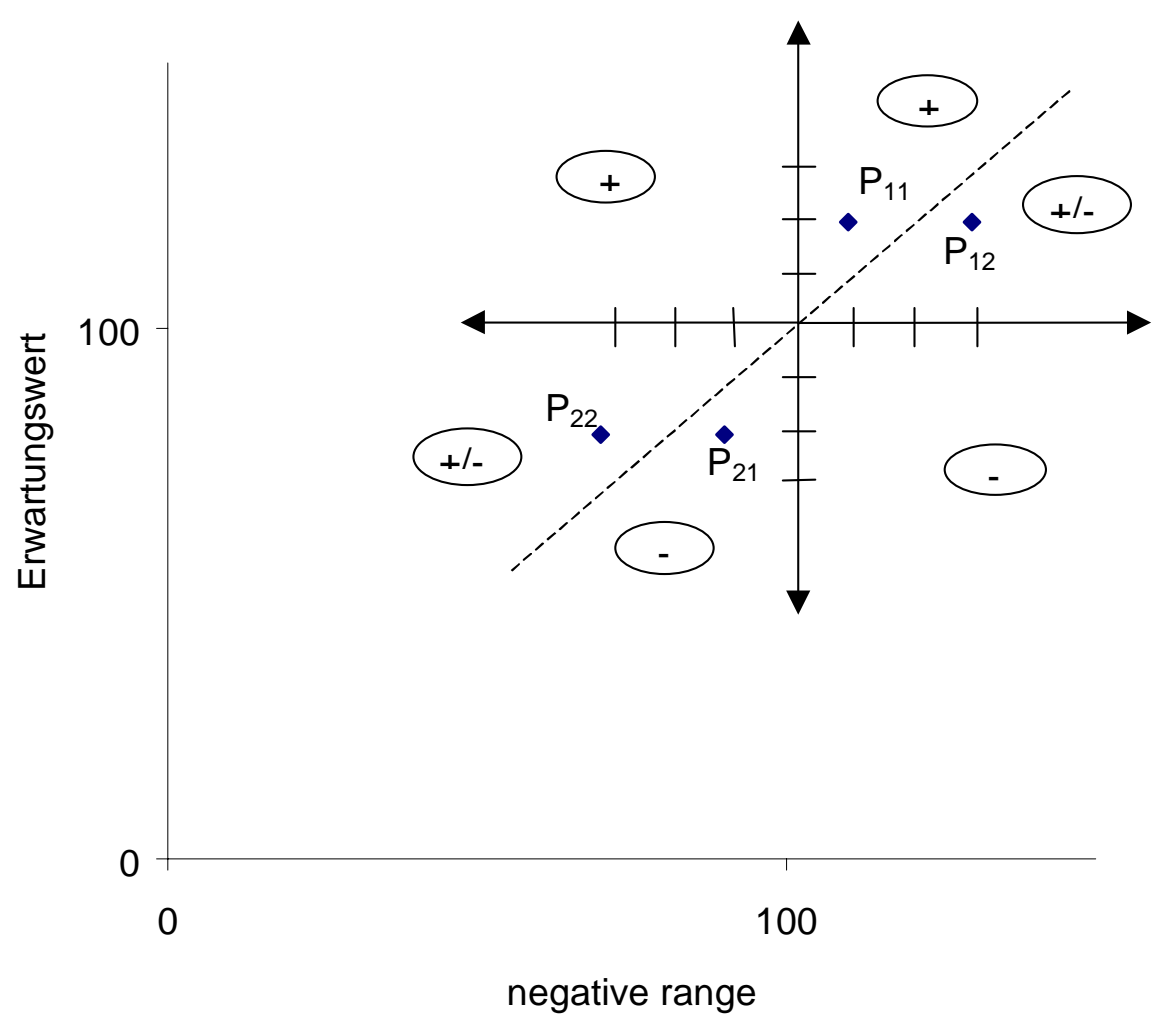

Die Koordinaten der Punkte sind Tabelle 961 entnommen. 
Die Reduzierung des Abwägungsbereichs in Schaubild 961 basiert auf der Überlegung, daß eine Alternative präferiert wird, die neben einem höheren Erwartungswert auch ein höheres Minimum aufweist. Schaubild 97.1 illustriert diesen Zusammenhang am Beispiel einer Dreieckverteilung. Dargestellt ist eine feste Einzahlung $e_{1}$ und eine stochastische $e_{2}$, deren Minimum größer als $e_{1}$ ist und die daher vorgezogen wird. Das Schaubild enthält zusätzlich eine feste Auszahlung $a$, die die Entscheidung in dieser Konstellation nicht beeinflußt, da sie kleiner als die sichere Einzahlung $e_{1}$ ist.

In Schaubild 97.2 ist nun die Verteilung von $e_{2}$ gestreckt worden. Ihr Minimum befindet sich jetzt zwischen $a$ und $e_{1}$. Dies bedeutet, daß mit einer sehr kleinen, aber positiven Wahrscheinlichkeit die Einzahlung von $e_{2}$ kleiner als $e_{1}$ ist. In jeden Fall aber ist sie größer als $a$. Ohne Kenntnis der Nutzenfunktion kann keine Präferenz eines Entscheiders vorausgesagt werden.

Es ist denkbar, daß sich die Risikoaversion von Individuen in einer lexikographischen Präferenz-

Schaubild 97.1: Einzahlung mit kleiner Varianz
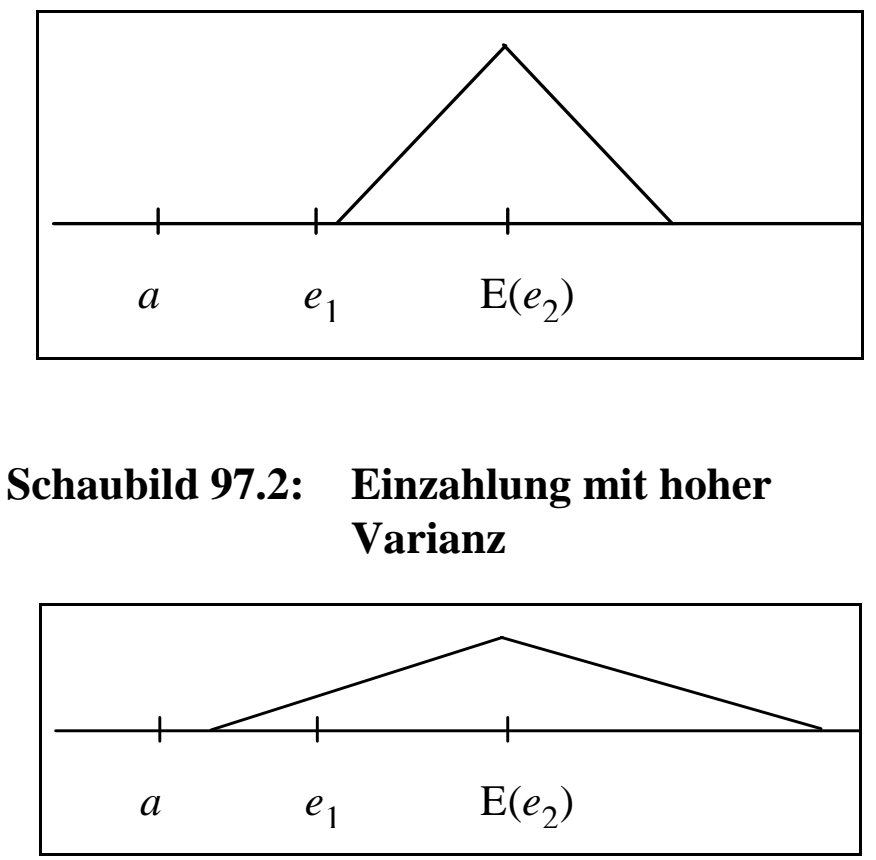
ordnung äußert: Zunächst muß die Auszahlung gedeckt sein. Von den Handlungsmöglichkeiten, die diese Bedingung erfüllen, wird die mit dem höchsten Erwartungswert ausgewählt. Entscheider, für die diese Annahmen gelten, werden sich für $\mathrm{e}_{2}$ entscheiden, die Entscheidung anderer Individuen kann nicht vorhergesagt werden.

Zum Abschluß dieses Exkurses sei folgendes zur Rationalität von Entscheidungen in stochastischen Situationen angemerkt. Individuen treffen ihre Entscheidungen in der Absicht, ihre Ziele bestmöglich zu erreichen. Sie berücksichtigen dabei, daß sie über eine begrenzte Informationsverarbeitungskapazität verfügen ${ }^{76}$ und daß Informationsbeschaffung Kosten verursacht. In der Literatur werden ökonomische Verhaltensweisen, die nicht alle erreichbaren Informationen nutzen, häufig als begrenzt rational bezeichnet. $^{77}$ Diese Bezeichnung fußt auf der althergebrachten neoklassischen Annahme vollkommener Märkte und vollständiger Information und Voraussicht. Da diese Voraussetzungen in der Praxis nur selten erfüllt sind, weil u.a. jede weitere Annäherung an Gewißheit steigende Kosten verursacht, ist es für Unternehmen nur sinnvoll, auf eine Gewinnmaximierung unter vollständiger Information zu verzichten

\footnotetext{
76 Vgl. AlchiAn (1950, S. 212).

77 Vgl. SELTEN (1990) und die dort angegebene Literatur.
} 
und statt dessen die Kosten der Informationsgewinnung und -verarbeitung in der Gewinnfunktion explizit zu berücksichtigen. Eine solche Verhaltensweise ist daher eigentlich rational. Um sie sprachlich von der neoklassischen Rationalität ohne Informationskosten abzugrenzen, empfiehlt sich m.E. eher der Begriff ,erweiterte Rationalität' als , begrenzte Rationalität‘.

\subsection{Unsicherheit in der Betriebsplanung}

\subsubsection{Begriffsbestimmungen}

Die Begriffe Unsicherheit, Ungewißheit und Risiko werden in der Literatur unterschiedlich verwendet. Die Differenzierung zwischen Unsicherheit und Risiko geht auf KNIGHT zurück, wonach "im Falle von, risk' objektiv bekannte Wahrscheinlichkeiten oder subjektive Vorstellungen darüber vorliegen, während der Entscheider im Falle von ,uncertainty ' die Wahrscheinlichkeitsverteilung der Zufallsvariablen nicht kennt" (BRANDES et al. 1997, S. 287). Im deutschen Sprachgebrauch sind außer Risiko die Termini Unsicherheit und Ungewißheit verbreitet. Die inhaltliche Füllung ist allerdings zum Teil diametral verschieden. Eine Informationslage, die der KNIGHTschen 'uncertainty' entspricht, beschreibt HANF (1986, S. 3) als Ungewißheit, nämlich eine Situation, ,in der man keine Vorstellungen über das entwickeln kann, was möglicherweise passiert. Genau diese Situation ist im Wirtschaftslexikon GABLER (1988, S. 2114) hingegen mit dem Begriff Unsicherheit ("Ungewißheitsgrad, der auf der Unvorhersehbarkeit zukünftiger Ereignisse basiert”) gekennzeichnet. Entsprechend 'über Kreuz' definieren HANF und GABLER Unsicherheit resp. Ungewißheit. Während HANF den Begriff Unsicherheit für eine Situation verwendet, ,in der man zwar abschätzen kann, was passieren kann, nicht jedoch, welche Wahrscheinlichkeit den einzelnen möglichen Situationen zukommt," wird bei GABLER (1988, S. 2101) Ungewißheit folgendermaßen definiert: "Ungewißheitsgrad, bei dem für das Eintreten zukünftiger Ereignisse zwar keine objektiven Wahrscheinlichkeiten vorliegen $(\rightarrow$ Risiko), aber subjektive Wahrscheinlichkeiten gebildet werden können.”

Der letzte Satz beinhaltet gleichzeitig eine Definition des Begriffs Risiko. Risiko wird also bei GABLER im Gegensatz zu KNIGHT auf Situationen beschränkt, in denen objektive Wahrscheinlichkeiten vorliegen. ${ }^{78}$

Objektive Wahrscheinlichkeiten, etwa die Wahrscheinlichkeitsverteilung eines fairen Würfels, werden intersubjektiv von allen Individuen geteilt. Im Wirtschaftsalltag können objektive Wahrscheinlichkeiten gebildet werden, wenn in der Vergangenheit eine Vielzahl gleichartiger Entscheidungen getroffen wurde, so daß eine empirische Häufigkeitsverteilung der Ergebnisse abgeleitet werden kann. Sind objektive Wahrscheinlichkeiten hingegen nicht bekannt, müssen auf der Grundlage persönlicher

$78 \mathrm{Zu}$ einer wissenschaftstheoretischen Diskussion des Risikos, des Nicht-Gewußten und des NichtWißbaren vgl. HUBIG 1994. 
Erfahrungen und Überlegungen subjektive Erwartungen gebildet werden, sogenannte "Glaubwürdigkeitsziffern" (BLOHM \& LÜDER 1995, S. 247). Bei Investitionsentscheidungen sind in der Regel nur subjektive Wahrscheinlichkeiten möglich (KRUSCHWITZ 1995, S. 88). Daher ziehen HANF (1986, S. 3f) und BRANDES et al. (1997, S. 287f) die Zweckmäßigkeit der Unterscheidung zwischen objektiven und subjektiven Wahrscheinlichkeiten Zweifel. Zum einen hafte im Wirtschaftsablauf allen Wahrscheinlichkeitseinschätzungen ein subjektives Element an. "Zum anderen, und das ist wichtiger, gibt es in der Wirtschaftswirklichkeit nur wenige Ereignisse, die als Realisationen von bekannten Zufallsverteilungen aufgefaßt werden können. Für Naturalerträge [...] oder ähnliche durch die Natur ausgelöste Schwankungen existieren zwar Zeitreihen; es ist allerdings zu vermuten, daß sich die diesen Realisationen zugrunde liegenden Wahrscheinlichkeitsverteilungen im Zeitablauf, und zwar in schwer vorhersagbarer Weise, ändern.” Und weiter, ,... [für Marktpreise] läßt sich aber noch mit weit weniger Berechtigung als bei den in erster Linie naturbestimmten Ereignissen sagen, daß eine Wahrscheinlichkeitsverteilung (wenn es hier so etwas überhaupt gibt) konstant bleibt." (BRANDES et al. 1997, S. 287).

Für das individuelle Entscheidungskalkül spielt es nur eine untergeordnete Rolle, ob ein objektives Risiko oder eine subjektive Ungewißheit vorliegt. ${ }^{79}$ Denn die Verfahren der Entscheidungsunterstützung, die im folgenden skizziert und von JASPERSEN (1997) als „ungewißheitsbedingte Ergänzungsverfahren” bezeichnet werden, lassen sich m.E. bei Vorliegen subjektiver wie objektiver Wahrscheinlichkeiten anwenden, auch wenn sie nach JASPERSEN (1997, S. 63) "ihrem Denkansatz nach in solche gegliedert werden [können], die sich an den subjektiven und in solche, die sich an den objektiven Wahrscheinlichkeiten orientieren."

Aus semantischen und mnemotechnischen ${ }^{80}$ Gründen scheint es mir sinnvoll, die Abwesenheit von Sicherheit generell als Unsicherheit zu bezeichnen. Diese Definition steht im Einklang mit dem üblichen Begriffspaar "Entscheidungen unter Sicherheit" vs. "Entscheidungen unter Unsicherheit". Unsicherheit könnte dann nach dem Charakter der Wahrscheinlichkeiten weiter unterteilt werden in Risiko, wenn objektive Wahrscheinlichkeit vorliegen, und Ungewißheit, wenn subjektive Erwartungen gebildet werden müssen. Für eine Situation, in der keine Vorstellungen über das Mögliche bestehen, wird bei dieser Terminologie kein Begriff reserviert. Dies erweist sich kaum als Mangel, da für solche Situationen ohnehin keine ökonomische Entscheidungsunterstützung möglich ist. In Schaubild 1001 ist diese Begriffsbestimmung zusammengefaßt.

Nach diesen Begriffsklärungen werden nun Möglichkeiten skizziert, Unsicherheit in Betriebsentwicklungsmodelle, die auf LP basieren, zu integrieren.

79 Zu den Unterschieden zwischen Entscheidungen bei Risiko und Ungewißheit vgl. SINN (1980) und BAMBERG \& COENENBERG (1989, Kapitel 5 und 6).

80 Mnemotechnik: Kunst, das Gedächtnis durch Merk- oder Lernhilfen zu unterstützen (KIENLE o.J., Fremdwörterlexikon). 


\section{Übersicht 100.1: Vorschlag zur Begriffsbestimmung von Unsicherheit, Ungewißheit und Risiko}

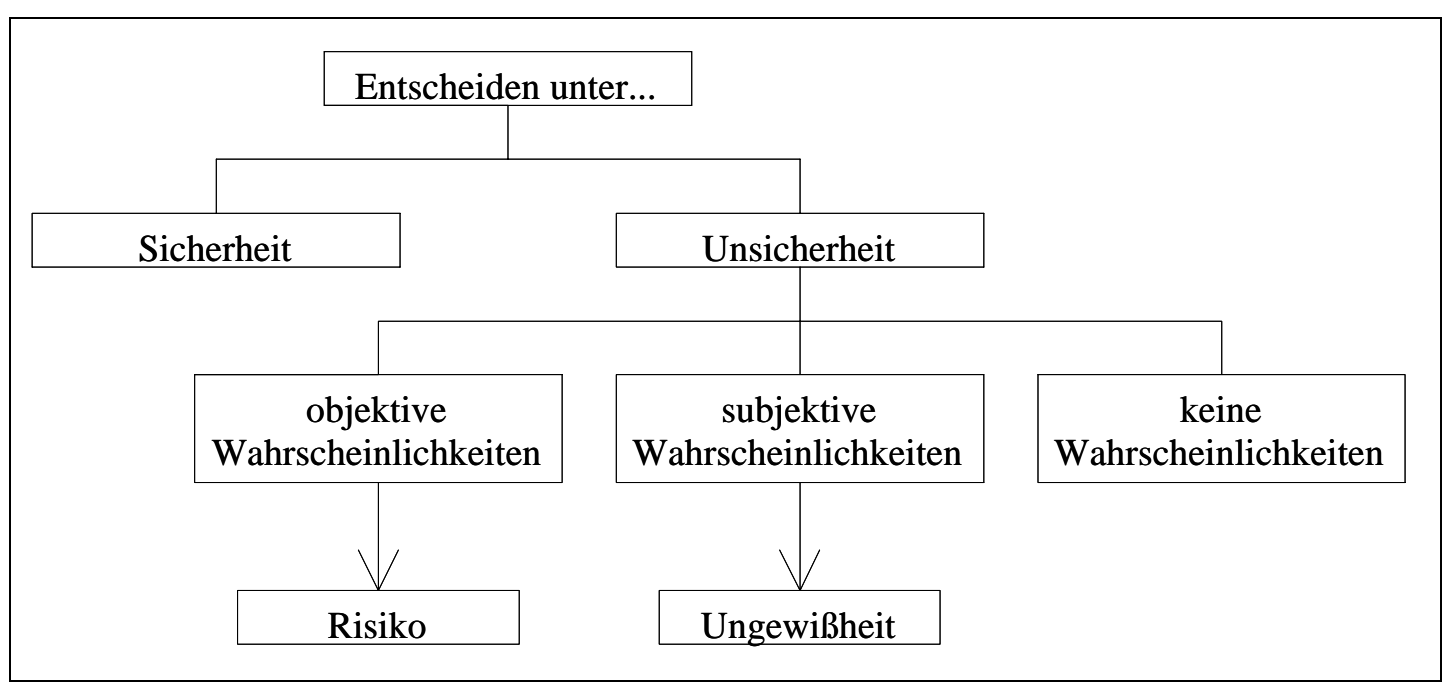

\subsubsection{Behandlung von Unsicherheit in der linearen Programmierung}

Bei der linearen Programmierung handelt es sich um die Optimierung einer linearen Zielfunktion unter ebenfalls linearen Nebenbedingungen, die überwiegend in Form von Ungleichungen vorliegen. Die Zielfunktion kann als

$$
c^{\prime} \boldsymbol{x} \rightarrow \max ,
$$

und die Nebenbedingungen können als

$$
\boldsymbol{A} \boldsymbol{x} \leq \boldsymbol{b}
$$

Tabelle 100.1: Beispiel eines einfachen LPTableaus

$$
\boldsymbol{x} \geq \mathbf{0}
$$

geschrieben werden. Dabei bedeuten

c: Vektor der Zielbeitragskoeffizienten

$\boldsymbol{x}$ : Vektor der Umfänge der Aktivitäten

A: $\quad$ Matrix der technischen Koeffizienten

$\boldsymbol{b}$ : Vektor der Kapazitätsgrenzen, auch RHS genannt

0: Nullvektor.

\begin{tabular}{|c||c|c|c|c|c|}
\hline ID & $x_{1}$ & $x_{2}$ & $x_{3}$ & Typ & $b$ \\
\hline \hline Ziel & $c_{1}$ & $c_{2}$ & $c_{3}$ & $\max$ & \\
\hline$R_{1}$ & $a_{11}$ & $a_{12}$ & $a_{13}$ & $\leq$ & $b_{1}$ \\
\hline$R_{2}$ & $a_{21}$ & $a_{22}$ & $a_{23}$ & $\leq$ & $b_{2}$ \\
\hline$R_{3}$ & $a_{31}$ & $a_{32}$ & $a_{33}$ & $\leq$ & $b_{3}$ \\
\hline$R_{4}$ & $a_{41}$ & $a_{42}$ & $a_{43}$ & $\leq$ & $b_{4}$ \\
\hline \multicolumn{6}{|c|}{$R_{i}:$ Restriktion $i$} \\
ID: Identifikationsspalte \\
\hline \multicolumn{7}{c}{}
\end{tabular}

Tabelle 100,1 zeigt die Grundzüge eines LPTableaus mit drei Aktivitäten und vier Restriktionen.

Das obige Tableau ist für sich allein rein deterministisch. Die Koeffizienten können allerdings auch als Erwartungswerte unsicherer Größen aufgefaßt werden. Das Tableau maximiert in einer solchen Interpretation den Erwartungswert des Zielwertes (Bayes- oder $\mu$-Prinzip). Für stochastische Betriebsentwicklungsmodelle ist es zweckmäßiger, die im Tableau stehenden Zahlen jeweils als eine Realisation einer Zufallsveränderlichen aufzufassen. Für jede weitere mögliche Kombination der stochasti- 
schen Koeffizienten ist ein weiteres Tableau separat zu lösen. Daher kann diese Technik nur auf diskrete Zufallsvariablen angewandt werden. Durch dieses Vorgehen läßt sich enumerativ eine vollständige Verteilung des Zielwertes gewinnen, wie nachfolgend an einem kleinen Beispiel demonstriert wird:

Der Koeffizient $c_{3}$ im obigen Tableau sei diskret stochastisch mit den drei Ausprägungen $c_{3}^{+}, c_{3}^{0}$ und $c_{3}^{-}$. In Abhängigkeit von der Realisation von $c_{3}$ ergeben sich als mögliche Zielwerte $z^{+}, z^{0}$ und $z^{-}$. Sind die drei Ausprägungen von $c_{3}$ gleichwahrscheinlich, so trifft dies auch auf die drei Zielwerte zu. Der Erwartungswert des Zielwertes ist dann $\left(z^{+}+z^{0}+z^{-}\right) / 3$. Bei zwei stochastischen Zielfunktionskoeffizienten $c_{2}$ und $c_{3}$ mit ebenfalls jeweils drei Ausprägungen ergeben sich schon $3^{2}$ mögliche Zielbeitragswerte. Sie sind in Tabelle 101.1 aufgeführt.

Die Tabelle verdeutlicht auch bereits die Tabelle 101.1: Zielfunktionswerte Nachteile dieser Art der Berücksichtigung von Unsicherheit:

- Es können nur diskrete Zufallsveränderliche aufgenommen werden.

- Die Anzahl der Zufallsveränderlichen und/ oder ihrer Ausprägungen muß aus Gründen der Übersichtlichkeit gering gehalten werden. ${ }^{81}$ Bei $n$ stochastischen Zielfunktionskoeffizienten und $r$ möglichen Realisatiobei zwei stochastischen Zielfunktionskoeffizienten nen pro Koeffizient ergeben sich $r^{n}$ mögliche Zielfunktionswerte. Neben den Koeffizienten der Zielfunktion können auch die Kapazitäten eines Betriebes $(b)$ und die technischen Koeffizienten $\left(a_{i j}\right)$ stochastisch sein. Sind in der obigen, sehr kleinen Matrix alle 19 Koeffizienten stochastisch mit jeweils drei möglichen Ausprägungen, ergeben sich bereits $3^{19} \approx 1.16$ Mrd. Zielwerte. ${ }^{82}$

Die gerade beschriebene Vorgehensweise stellt eine Risikoanalyse dar. WÖHE (1996) und JASPERSEN (1997) unterscheiden neben diesem Verfahren der Entscheidungsunterstützung bei Unsicherheit vier weitere: ${ }^{83}$ Korrekturverfahren, Sensitivitätsanalysen, Entscheidungsbaumverfahren und portfoliotheoretische Ansätze ${ }^{84}$. Diese

81 Vgl. LITWIN (1986), FISCHER (1988).

82 HENSCHE et al. (1998, S. 3) berechnen in ihrer in Kapitel 0 erwähnten Szenarioanalyse über die Zukunft der Schweinefleischproduktion mehr als 500 Mill. Szenarien. Durch Plausibilitäts- und Konsistenzprüfung gelingt es ihnen, die Anzahl der relevanten Kombinationen auf 800 zu verringern. Da auch diese Anzahl noch nicht handhabbar ist, setzen sie eine Clusteranalyse ein, um die Szenarien weiter zu Gruppen zusammenzufassen, die in sich möglichst homogen und untereinander möglichst heterogen sind. 
Ansätze, außer den Entscheidungsbaumverfahren ${ }^{85}$, finden in dieser Arbeit Verwendung und werden kurz erläutert.

Die älteste Technik sind vermutlich die Korrekturverfahren. Nach Faustregeln werden für als besonders bedeutsam angesehene Koeffizienten Abschläge vorgenommen, beispielsweise für den Kalkulationszinsfuß, die Lebensdauer von Investitionen, für Erträge und Preise. Damit soll erreicht werden, daß nur solche Investitionen durchgeführt werden, die auch bei Eintreten ungünstiger Umweltlagen noch Gewinne abwerfen. Die Entscheiderin trägt der Unsicherheit also dadurch Rechnung, daß sie ihre Planung nicht auf den eigentlich erwarteten Werten aufbaut, sondern statt dessen vorsichtigere Ansätze verwendet. Der erreichbare Zielwert wird dadurch stark nach unten korrigiert, ohne daß eine Ursachenanalyse der Unsicherheit erfolgt. Dies birgt die Gefahr, daß “unter Umständen lukrative Investitionen 'totgerechnet' [werden]. Der Einsatzbereich der Korrekturverfahren beschränkt sich [daher] auf kleinere Investitionsvorhaben, bei denen sich ein höherer Planungsaufwand nicht lohnt" (WÖHE 1996, S. 781). Unsicherheitsabschläge sind in LPs besonders einfach anwendbar.

Sensitivitätsanalysen sind eine Weiterentwicklung der Korrekturverfahren. In Simulationen werden mit Hilfe eines Rechners kritische Parameter unter ceteris-paribus-Bedingungen variiert, um ihren Einfluß auf das System zu analysieren. Ziel von Sensitivitätsanalysen ist es herauszufinden, welche Variablen einzeln oder in Kombination $^{86}$ das Ergebnis besonders stark beeinflussen, z.B. Preise oder bestimmte betriebliche Kapazitäten. Während bei den Risikoabschlägen singuläre Rechnungen durchgeführt werden, ,bilden die Analysen der Sensibilität das systematische Aufrastern der Eingangswerte ... [und die] Verfolgung der Konsequenz auf das Ergebnis der Investitionsrechnung wie beispielsweise den Kapitalwert." (JASPERSEN 1997, S. 67). Dabei ist von besonderem Interesse, innerhalb welcher Schwankungsbreiten Veränderungen der Inputvariablen ohne Einfluß auf den optimalen Plan bleiben. Diese kritischen Werte der Variablen werden heute von vielen PC-Programmen zur linearen Programmierung (z.B. SAS ${ }^{\circledR}$ OR) mit Hilfe der parametrischen Programmierung identifiziert und ausgewiesen.

Bei Sensitivitätsanalysen liegt der Schwerpunkt auf den Auswirkungen von Veränderungen der Inputvariablen. Diese Technik der systematischen Variation von Inputvariablen wird auch in der Risikoanalyse genutzt, jetzt mit dem Ziel, eine Wahrscheinlichkeitsverteilung der Outputvariablen $\mathrm{zu}$ generieren. Im Anfangsbeispiel wurde hierzu eine Berechnung aller Kombinationen der (diskreten) Inputvariablen skizziert, um die Wahrscheinlichkeitsverteilung des Zielwertes zu ermitteln. Eine Risikoanalyse wird also nicht innerhalb eines LPs durchgeführt, sondern durch Wiederholen der LPBerechnung mit entsprechend veränderten Koeffizienten. Dieses Verfahren der Vollenumeration kann nur für diskrete Zufallsveränderliche durchgeführt werden. Bei

85 Vgl. hierzu u.a. JASPERSEN (1997, Unterabschnitt 2.3.4).

86 Zur gleichzeitigen Veränderung mehrerer Variablen vgl. KRUSCHWITZ (1995a, S. 275ff). 
stetigen Variablen ist eine Monte-Carlo-Simulation durchzuführen(vgl. zur Methodik Unterabschnitt 2.1.4.2 diese Technik wird in Kapitel foangewandt).

Risikoanalysen, die die lineare Programmierung benutzen, finden sich selten in der Literatur. Meistens werden andere Modelle verwendet. Dann lassen sich unter bestimmten Annahmen die Verteilungen der Outputgrößen auch analytisch ermitteln. Für den landwirtschaftlichen Bereich haben BRANDES \& BUDDE (1980) eine Simulationssoftware unter dem Namen "Compri - eine computergestützte Planung risikobehafteter Investitionen” vorgestellt.

Das Ergebnis von Risikoanalysen, gleich wie sie gelöst sind, ergibt ein Risikoprofil der Zielgröße, das als Hilfsmittel zur Entscheidungsunterstützung verwandt werden könnte. Allerdings äußert sich OLFERT (1992, S. 92) wenig optimistisch: "Die Risikoanalyse stellt ein interessantes Modell dar, das in der betrieblichen Praxis keine nennenswerte Bedeutung erlangt hat." Durch (populär-)wissenschaftliche Artikel (beispielsweise von HIRSCHAUER \& GIEREND in der Neuen Landwirtschaft 7/98 oder GÜNTHER \& GRONALT 1994 in WiSt) und neue EDV-Programme kann dieser Bedeutungslosigkeit vielleicht entgegengewirkt werden. Vielversprechend erscheint zum Beispiel die Software RISK ${ }^{\circledR}$, da sie es in Standard-Tabellenkalkulationsprogrammen, die weit verbreitetet sind, ermöglicht, sehr elegant und einfach Risikoanalysen durchzuführen.

Portfoliotheoretische Ansätze zielen darauf ab, das Risiko des Gesamtunternehmens durch gezielte Diversifikation zu senken. Mit Hilfe der Technik der Risikoanalysen lassen sich Mischungen eines Portfolios ermitteln, die bei gleichem Erwartungswert eine höhere Varianz aufweisen und daher für eine risikoaverse Entscheiderin ineffizient sind. Da die Varianz quadratisch mit dem Umfang der Aktivität wächst (vgl. Übersicht 166.1, ergibt sich ein quadratisches Optimierungsproblem. ${ }^{87}$ Die technische Durchführung erfordert leistungsfähige Hard- und Software. Daher hat die theoretisch seit langem bekannte Portfolioanalyse vor allem zu Beginn dieses Jahrzehnts einen starken Aufschwung erfahren. ${ }^{88}$

Einfache ad- hoc Diversifikationsauflagen ohne Optimierung der Diversifikationsanteile lassen sich sehr leicht in LPs einbauen. Beispielsweise erzwingt die Nebenbedingung $x_{1}-x_{2}=0$ gleiche Umfänge der Aktivitäten $x_{1}$ und $x_{2}$. Durch das Unglei-

87 Durch entsprechende Linearisierung können diese Aufgaben auch mit Hilfe der linearen Programmierung gelöst werden. Dazu entwickelte HAZELL (1971) das LP-Modell MOTAD. Die Abkürzung steht für "Minimization Of the Total Absolute Deviations". MOTAD wird besonders in der agrarökonomischen Forschung verbreitet eingesetzt (z.B. MACCARL 1989, KURZ 1991, MALEKA 1993, GAJANANA 1994).

88 Als Begründer der Portfolioanalyse gilt MARKOwITZ (1959, 1977). Er erhielt dafür 1990 den Nobelpreis für Wirtschaftswissenschaften. Sie ist heute in den meisten Lehrbüchern zur Finanzwirtschaft enthalten, z.B. PERRIDON \& STEINER (1995). 
chungssystem $\left\{x_{1}-1 / 2 x_{2} \geq 0 \wedge x_{1}-1.5 x_{2} \leq 0\right\}$ wird eine feste Bandbreite des Verhältnisses der Umfänge beider Aktivitäten $x_{1}$ und $x_{2}$ festgeschrieben. ${ }^{89}$

Weitere Ansätze zur Berücksichtigung des Risikos zeichnen sich dadurch aus, daß die Nebenbedingungen nicht zu 100\% eingehalten werden müssen. Sie seien nur kurz genannt:

- Beim Strafkostenkonzept (HANF 1986, S. 162f) sind die Kapazitätsgrenzen zwar fest, sie können jedoch durch "Zukauf” erweitert werden. Dieser Zukauf von Kapazitäten verursacht überproportionale Kosten in der Zielfunktion, so daß Kapazitätsüberschreitungen "bestraft" werden.

- Das Chance Constrained Programming (CCP) ist dadurch charakterisiert, daß die Nebenbedingungen nicht absolut bindend sind, sondern nur mit einer vorgegebenen Wahrscheinlichkeit erfüllt sein müssen. ${ }^{90} \mathrm{CCP}$ führt wie die portfoliotheoretischen Ansätze zu quadratischen Zielfunktionen. Die stochastischen Restriktionen können aber unter bestimmten Bedingungen in deterministische Ersatzprobleme transformiert und nach Linearisierung durch LP gelöst werden. ${ }^{91}$

- Auch in der $\boldsymbol{F u z z y - S e t - T h e o r i e ~ w e r d e n ~ k e i n e ~ s c h a r f e n ~ K a p a z i t a ̈ t s g r e n z e n ~ v o r g e g e - ~}$ ben. In Fuzzy-LPs wird vom Entscheider nicht die Angabe fester betrieblicher Ausstattungen oder Produktionsbeziehungen verlangt. Vielmehr hat er lediglich Bereiche anzugeben, in denen sich die betrieblichen Ressourcen "mutmaßlich" bewegen (KOFLER 1989, BUSCHER \& ROLAND 1992, BERGER 1996, HOLZAPFEL 1997). Durch diese weichen Grenzen können $F u z z y$-LPs die betriebliche Realität oft besser abbilden als LPs mit harten Grenzen, wie TRIES (1998) an einem kleinen Beispiel demonstriert.

Diese und weitere Möglichkeiten, Unsicherheit in Entscheidungsmodelle einzubeziehen, finden sich bei HANF (1986). Die beiden folgenden Abschnitte dieses Kapitels sind den Möglichkeiten von Signifikanztests in Monte-Carlo-Simulationen und der Entwicklung eines Ansatzes zur gleichzeitigen Berücksichtigung von Investitionen und Finanzanlagen in LPs gewidmet.

\subsection{Signifikanztests in Monte-Carlo-Simulationen}

Signifikanztests sind bei Ergebnissen von Monte-Carlo-Simulationen relativ einfach ${ }^{92}$, da die zugrundeliegenden Verteilungen bekannt sind. Werden normalverteilte Zufallszahlen verwendet, bieten sich die üblichen t- und F-Tests an, um Mittelwert- und Vari-

89 Portfoliooptimierung unter Überlebensgesichtspunkten wird in Abschnitt 5.4 näher beschrieben. Diversifikation ist eine der Strategien, die in Kapitel 6 in einem Betriebsentwicklungsmodell untersucht werden.

Näheres zu Chance-Constrained Programming s. BLOHM \& LÜDER (1995, Abschnitt 6.3) und HANF (1986, Unterabschnitt 7.43.42).

91 Zu den Voraussetzungen vgl. HAEGERT (1970 Kapitel II).

92 Vgl. hierzu auch BøLVIKEN \& SKOVLUND (1996) und die dort angegebene Literatur. 
anzunterschiede auf Signifikanz zu überprüfen. Wenn andere Verteilungen zum Einsatz kommen, haben parametrische Tests bekanntlich Schwächen. Insbesondere kann zur Untersuchung auf Gleichheit von Varianzen (Varianzhomogenität) nicht auf den F-Test zurückgegriffen werden. Der Test ist nur für normalverteilte Grundgesamtheiten zulässig und reagiert auf Abweichungen von der Normalverteilung sehr empfindlich (SACHS 1984, S. 206). Eine Nicht-Normalverteilung kann nicht durch eine Erhöhung des Stichprobenumfanges ausgeglichen werden, wie es beim t-Test möglich ist.

Als Alternative zu parametrischen Signifikanztests kann das Grundprinzip der Randomisation verwendet werden. Die Randomisation ist ein Testverfahren, das keine Verteilungsannahmen benötigt und den Einsatz eines Computer erfordert. Da dieses Verfahren in der Agrarökonomie bisher wenig verbreitet ist, sei es an einem kleinen Beispiel erläutert. Gegeben seien zwei Stichproben, und es interessiere die übliche Frage, ob sie sich systematisch in ihren Mittelwerten unterscheiden.

Zur Beantwortung dieser Frage werden bildlich gesprochen die Beobachtungen beider Stichproben in eine Urne geworfen und anschließend zwei neue Stichproben gezogen. Ihre Mittelwertdifferenz wird ermittelt und festgehalten. Nun werden wieder alle Beobachtungen in die Urne geworfen, erneut zwei Stichproben gezogen und ihre Mittelwertdifferenz festgestellt. Durch häufiges Wiederholen dieses Verfahrens entsteht eine Verteilung der Differenzen der Stichprobenmittelwerte. Sie erfüllt offensichtlich durch die verwendete Technik ihrer Genese die Annahme, daß beide Stichproben aus der gleichen Grundgesamtheit stammen, mithin zwischen ihnen kein systematischer Unterschied besteht. Die Verteilung genügt also der Anforderung einer Nullhypothese $\left(\mathrm{H}_{0}\right.$ : „,kein systematischer Unterschied”). Daher kann mit ihr die Mittelwertdifferenz der beiden Ausgangsstichproben verglichen werden. Ist z.B. die empirische Mittelwertdifferenz 7 und trat bei den 100 Randomisationen nur 5mal eine Mittelwertdifferenz von größer als 7 auf, dann unterscheiden sich die Stichproben mit einer Irrtumswahrscheinlichkeit von 5\% systematisch in ihrem Mittelwert. ${ }^{93}$

Durch den Einsatz von Randomisation können neben Mittelwertdifferenzen auch andere statistische Kennziffern wie Varianz und Quantile auf systematische Abweichungen getestet werden. Nicht verwenden kann man dieses Verfahren, wenn das Ergebnis einer Simulation eine Wahrscheinlichkeit ist, die keine Varianz besitzt. Wird z.B. in einem Simulationsmodell die Überlebenswahrscheinlichkeit untersucht, indem die "überlebenden Wiederholungen" abgezählt werden, ist das Ergebnis eine bestimmte feste Zahl. Für jedes Szenario ergibt sich genau eine Überlebenswahrscheinlichkeit, ohne jegliche Streuung. Daher sind mit dieser Technik (wie auch mit tTests) keine inferentiellen Aussagen über Unterschiede in der Überlebenswahrscheinlichkeit zwischen den Szenarien möglich.

$93 \mathrm{Zu}$ einer ausführlichen Darstellung der Randomisation vgl. COX \& HiNKLEY (1974, Kap 6.4) und zu einer Anwendung in Monte-Carlo-Studien HINNERS-TOBRÄGEL (1993, S. 144ff). 
Häufigkeiten eines dichotomen Ereignisses in Monte-Carlo-Simulationen, z.B. die Anzahl ausgeschiedener Unternehmen bei 100 Wiederholungen, können als Anteilswerte interpretiert werden. Ein Anteilswert ist beispielsweise der Anteil roter Kugeln in einer Urne, die nur rote und schwarze Kugeln enthält. Diese Interpretation legt nahe, statistische Testverfahren, die für Anteilswerte zur Verfügung stehen, auch auf Häufigkeitsunterschiede in Monte-Carlo-Simulationen anzuwenden.

Die Prüfgröße, die die Anteilswerte zweier Stichproben auf Signifikanz ihrer Unterschiedlichkeit testet, lautet (vgl. BLEYMÜLLER et al. 1998, Abschnitt 17.4)

$$
Z:=\frac{\frac{x_{1}}{n_{1}}-\frac{x_{2}}{n_{2}}}{\sqrt{\frac{\left(x_{1}+x_{2}\right)\left(1-\frac{x_{1}+x_{2}}{n_{1}+n_{2}}\right)}{n_{1}+n_{2}}}} \sqrt{\frac{n_{1}+n_{2}}{n_{1} n_{2}}}
$$

mit: $x_{1} \quad$ absolute Häufigkeit des Ereignisses in Stichprobe 1

$x_{2} \quad$ absolute Häufigkeit des Ereignisses in Stichprobe 2

$n_{1}, n_{2} \quad$ Anzahl Wiederholungen in Stichprobe 1 bzw. Stichprobe 2.

Sie ist standardnormalverteilt. Daher muß bei einem Signifikanzniveau von $5 \%$ der Betrag von $Z$ größer als 1.96 sein. Bezogen auf die Überlebenswahrscheinlichkeiten in Monte-Carlo-Simulationen entsprechen $x_{1}$ und $x_{2}$ den Überlebensfällen in den Szenarien 1 bzw. 2 sowie $n_{i}$ der Anzahl der Wiederholungen in den beiden Szenarien.

Die Häufigkeit eines Ereignisses in mehr als zwei Szenarien kann mit Hilfe von $\chi^{2}$ Anpassungstests auf signifikante Abweichung von einer Gleichverteilung ${ }^{94}$ untersucht werden (vgl. BORZ 1993, Abschnitte 5.3.1 und 5.3.2). Die Trennschärfe dieser Tests, angewandt auf das Problem, ob in einzelnen Szenarien systematisch höhere Überlebenswahrscheinlichkeiten vorliegen, ist nicht sehr groß.

Um solche Ergebnisse dennoch inferentiell beurteilen zu können, wurde von TUKEY (1958) das Jackknife- und von EFRON (1979) das Bootstrap-Verfahren entwickelt. ${ }^{95}$ Kurz gesagt werden aus der gegebenen Stichprobe neue Stichproben gezogen, aus denen der interessierende Parameter berechnet wird. Nach ausreichend vielen Wiederholungen steht eine Verteilung des Parameters zur Verfügung, die für inferentielle Aussagen genutzt werden kann.

94 Es kann auch Abweichungen von der Normalverteilung oder jeder anderen Verteilung getestet werden. Da in Monte-Carlo-Simulationen normalerweise interessiert, ob ein Ereignis in einem Szenario häufiger auftritt als in den anderen, ist hierfür ein Test auf Gleichverteilung anzuwenden. Das Prinzip des Jackknife wurde zuerst von QUENOUILLE (1949) vorgestellt. TUKEY $(1958,1972)$ entwickelte die Technik weiter. Er wandte es zur Schätzung von Standardfehlern an und gab der Methode seinen eingängigen Namen. 
Der Unterschied der beiden Ansätze liegt in der Technik der Generierung der Stichproben. Im Jackknife-Verfahren entstehen neue Stichproben, indem aus der ursprünglichen jeweils eine andere Beobachtung weglassen wird. Daher ist die Anzahl möglicher Jackknife-Stichproben endlich. Dagegen ergibt das Prinzip des Bootstrapping, aus der ursprünglichen Stichprobe neue Stichproben mit Wiederholung $\mathrm{zu}$ ziehen (resampling), eine potentiell unendliche Menge an neuen Stichproben. ${ }^{96}$

Eine bereits in Abschnitt 2.4 angesprochene Studie von BAILEY \& RICHARDSON (1985) ist ein gutes Beispiel dafür, wie schwierig Interpretationen von Simulationsergebnissen sein können. BAILEY \& RICHARDSON präsentieren ihre Resultate in einer sehr übersichtlichen Tabelle, die als Fotokopie in Übersicht 107,1 wiedergegeben ist.

\section{Übersicht 107.1: Ergebnisse einer Simulationsstudie von BAILEY \& RICHARDSON} (1985)

818 November 1985

Amer. J. Agr. Econ.

Table 3. Impacts of Selected Marketing Strategies on the Probability of Survival, Success, and Economic Characteristics of a 2,019-Acre Typical Southern High Plains Cotton Farm, 1983-92

\begin{tabular}{|c|c|c|c|c|c|c|c|c|c|}
\hline \multirow[b]{2}{*}{ Variable } & \multicolumn{9}{|c|}{ Strategy } \\
\hline & 1 & II & III & IV & V & VI & VII & VIII & IX \\
\hline Survival $(\%)^{a}$ & 98 & 100 & 100 & 88 & 42 & 80 & 80 & 94 & 100 \\
\hline Success $(\%)^{\mathrm{b}}$ & 60 & 66 & 70 & 38 & 38 & 56 & 54 & 62 & 52 \\
\hline \multicolumn{10}{|c|}{$\begin{array}{l}\text { After-tax net present } \\
\text { value }\end{array}$} \\
\hline $\bar{X}(\$ 1,000 \mathrm{~s})^{\mathrm{c}}$ & 284.1 & 325.5 & 354.8 & 106.5 & 208.8 & 285.1 & 307.1 & 348.7 & 178.3 \\
\hline$\sigma(\$ 1,000 s)^{d}$ & 549.7 & 540.3 & 554.5 & 434.6 & 841.7 & 668.0 & 743.0 & 643.8 & 455.9 \\
\hline C.V. $(\%)^{\circ}$ & 193.5 & 166.0 & 156.3 & 408.1 & 403.1 & 234.3 & 241.9 & 184.6 & 255.6 \\
\hline $\max .(\$ 1,000 \mathrm{~s})$ & 2160.4 & 2226.3 & 2437.4 & 1441.9 & 2512.8 & 2299.4 & 2673.3 & 2316.4 & 1970.2 \\
\hline $\min .(\$ 1,000 s)$ & -304.0 & -213.5 & -196.3 & -326.7 & -481.0 & -515.3 & -403.3 & -381.0 & -252.2 \\
\hline \multicolumn{10}{|l|}{$\begin{array}{l}\text { Present value of } \\
\text { ending net worth }\end{array}$} \\
\hline $\bar{X}(\$ 1,000 \mathrm{~s})$ & 737.0 & 777.6 & 800.4 & 561.1 & 651.9 & 727.5 & 748.9 & 789.4 & 632.7 \\
\hline$\sigma(\$ 1,000 \mathrm{~s})$ & 531.1 & 520.4 & 534.8 & 409.9 & 812.0 & 647.6 & 718.0 & 621.3 & 435.2 \\
\hline $\max .(\$ 1,000 \mathrm{~s})$ & 2567.8 & 2633.7 & 2831.6 & 1822.2 & 2907.0 & 2706.8 & 3067.5 & 2696.7 & 2364.4 \\
\hline $\min .(\$ 1,000 \mathrm{~s})$ & 170.0 & 260.6 & 277.8 & 147.3 & -0.2 & -34.5 & 81.3 & 96.2 & 221.9 \\
\hline \multicolumn{10}{|l|}{$\begin{array}{l}\text { Acres of cropland } \\
\text { farmed last } \\
\text { solvent year }\end{array}$} \\
\hline $\bar{X}$ & 3414.0 & 3478.0 & 3503.0 & 3183.0 & 4003.0 & 3782.0 & 3772.0 & 3849.0 & 3311.0 \\
\hline$\sigma$ & 1341.0 & 1410.0 & 1289.0 & 1354.0 & 1978.0 & 1543.0 & 1709.0 & 1625.0 & 1383.0 \\
\hline $\max$ & 6499.0 & 6499.0 & 7139.0 & 6499.0 & 7779.0 & 7139.0 & 7779.0 & 7779.0 & 7139.0 \\
\hline $\min$ & 2019.0 & 2019.0 & 2019.0 & 2019.0 & 2019.0 & 2019.0 & 2019.0 & 2019.0 & 2019.0 \\
\hline \multicolumn{10}{|l|}{ Leverage ratio } \\
\hline $\bar{X}$ & 0.40 & 0.28 & 0.27 & 0.59 & 2.18 & 1.04 & 0.82 & 0.66 & 0.38 \\
\hline$\sigma$ & 0.33 & 0.19 & 0.15 & 0.50 & 2.13 & 1.27 & 0.90 & 0.73 & 0.27 \\
\hline $\max$ & 1.90 & 0.89 & 0.72 & 1.82 & 6.00 & 6.00 & 3.68 & 3.87 & 1.38 \\
\hline $\min$ & 0.06 & 0.06 & 0.10 & 0.05 & 0.06 & 0.10 & 0.06 & 0.06 & 0.11 \\
\hline
\end{tabular}

a Probability of surviving is the probability the farm will remain solvent through 1992.

'Probability of success is the probability the farm operator will receive at least a $5 \%$ return on beginning owner's equity.

c Mean.

d Standard deviation.

- Coefficient of variation.

Quelle: BAILEY \& RICHARDSON (1985, S. 818).

$96 \mathrm{Zu}$ einer anschaulichen Darstellung der beiden Verfahren mit Beispielen und einem Vergleich siehe auch EFRON (1982) und EFRON \& TIBSHIRANI (1993). 
Für die getesteten Strategien (I bis IX), die hier nicht näher erläutert werden, sind jeweils die Ergebnisse aus 50 Wiederholungen dargestellt. Konzentrieren wir uns auf den Kapitalwert nach Steuern ("after tax net present value"). Der Mittelwert der Wiederholungen differiert zwischen den Strategien deutlich. Dennoch können den einzelnen Strategien keine unterschiedlichen Kapitalwerte zugeordnet werden, weil die Differenzen nicht systematisch sind. Der Variationskoeffizient beträgt im Schnitt über 200\%, keiner der Mittelwertunterschiede ist signifikant, wie man mit Hilfe eines tTests aus Mittelwert $\bar{X}$ und Standardfehler $\sigma$ der Parameter leicht nachrechnen kann. ${ }^{97}$ Streuungen ähnlicher Größenordnung weisen auch die anderen Ergebnisse in der Tabelle (Vermögensendwert, Betriebsgröße, Eigenkapitalanteil) auf.

Um dieses Problem zu verdeutlichen, seien die Ergebnisse der Strategien I und II von BAILEY \& RICHARDSON herausgegriffen. Unter Annahme einer Normalverteilung sind die beiden Parameter Erwartungswert und Varianz hinreichend zur Bestimmung der Dichtefunktion des Kapitalwertes. Die Parameter sind in Schaubild 109.1 angegeben. Die Graphik macht offensichtlich, daß aufgrund der hohen Streuung der Ergebnisse, die bei den einzelnen Iterationen erzielt wurden, ein signifikanter Unterschied zwischen den Strategien nicht abgeleitet werden kann.

In der Tabelle von BAILEY \& RICHARDSON bezeichnen die ersten beiden Zeilen die Überlebens- und Erfolgswahrscheinlichkeit der neun untersuchten Strategien. Es sind keine Streuungsmaße beigefügt. Dies ist, wie bereits begründet, auch nicht möglich, da die angegebenen Prozentzahlen keine Streuung besitzen. Eine Überlebenswahrscheinlichkeit von $98 \%$ beispielsweise ergibt sich, wenn sich bei den 50 Iterationen, die die beiden Autoren durchführten, genau eine Insolvenz auftritt, zwei Insolvenzen führen zu einer Überlebenswahrscheinlichkeit von 96\%. Wegen der Wiederholungsanzahl von 50 sind alle angegebenen Überlebenswahrscheinlichkeiten gerade Zahlen. In wieweit die Unterschiede in den Überlebenswahrscheinlichkeit der Strategien signifikant sind, kann aus der Tabelle nicht entnommen werden. Ein Test mit dem oben vorgestellten Verfahren auf systematische Häufigkeitsunterschiede [s. (106.1)] führt zu den in Tabelle 109.1 dargestellten Irrtumswahrscheinlichkeiten.

Die Tests ergeben, daß die Strategie V im Vergleich zu allen anderen Verhaltensweisen zu signifikant mehr Insolvenzen führt. Ferner sind sechs Ausfälle wie ins Strategie IV signifikant mehr als einer (Strategie I). Damit sind auch die Verhältnisse 6:0 sowie 10:1 und 10:0 (Strategien IV zu II, VI zu I bzw. VI zu II) signifikant.

In der abschließenden Tabelle 110.1 sind signifikante Häufigkeitsunterschiede bei 100 Wiederholungen dargestellt. Die Irrtumswahrscheinlichkeiten $\alpha$ sind für einen zweiseitigen Test berechnet, da a priori nicht bekannt ist, welche Häufigkeit größer ist. $\alpha$ ergibt sich daher als das Doppelte des Wertes der Verteilungsfunktion einer Standardnormalverteilung an der Stelle $z_{0}$, wobei $z_{0}$ nach 106.1 bestimmt wird:

97 Als Daumenregel für Signifikanz sollte der Quotient aus geschätztem Parameter und seinem Standardfehler größer als zwei sein. 
$\alpha \quad=2 \cdot \mathrm{F}_{\mathrm{N}}\left(z_{0}\right)$.

Schaubild 109.1: Dichtefunktionen der Verteilung des Kapitalwerts der Strategien I und II einer Simulationsstudie von BAILEY \& RICHARDSON (1985)

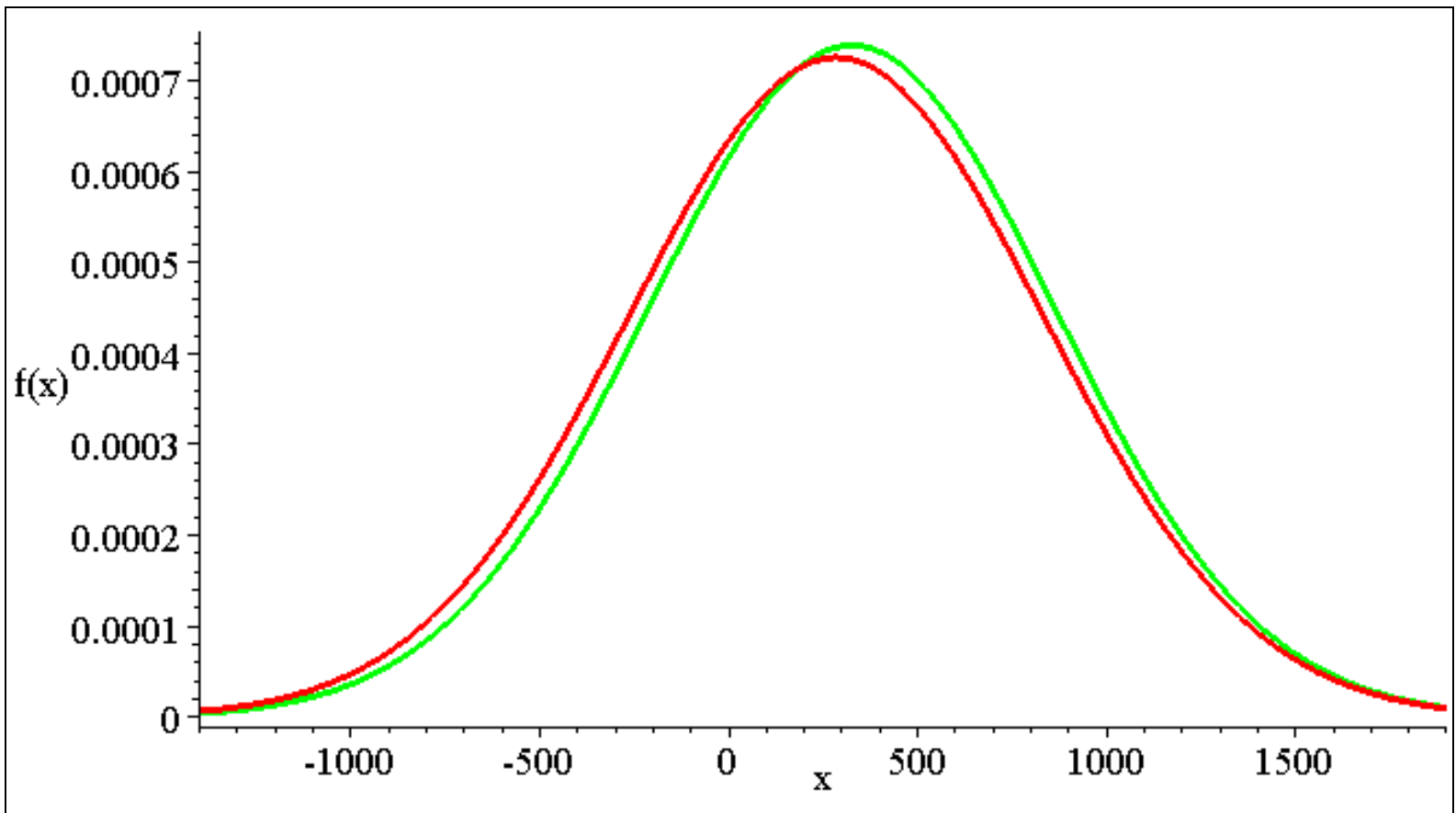

Tabelle 109.1: Signifikanztests von Überlebenswahrscheinlichkeiten

\begin{tabular}{|c|c|c|c|c|c|c|c|}
\hline \multirow{2}{*}{ 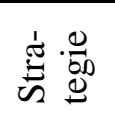 } & \multirow{2}{*}{ 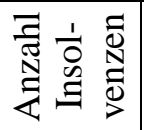 } & I & II & IV & V & VI & Strategie \\
\hline & & 1 & 0 & 6 & 29 & 10 & Anzahl Insolvenzen \\
\hline II & 0 & 0.314 & & & & & \\
\hline IV & 6 & $\begin{array}{l}0.050 \\
*\end{array}$ & $\begin{array}{l}0.012 \\
*\end{array}$ & & & & \\
\hline V & 29 & $\begin{array}{c}0.000 \\
* * *\end{array}$ & $\begin{array}{c}0.000 \\
* * *\end{array}$ & $\begin{array}{c}0.000 \\
* * *\end{array}$ & & & \\
\hline VI & 10 & $\begin{array}{c}0.004 \\
* *\end{array}$ & $\begin{array}{c}0.000 \\
* * *\end{array}$ & 0.276 & $\begin{array}{c}0.000 \\
* * *\end{array}$ & & \\
\hline VIII & 3 & 0.308 & 0.078 & 0.294 & $\begin{array}{c}0.000 \\
* * *\end{array}$ & $\begin{array}{c}0.038 \\
*\end{array}$ & \\
\hline \multicolumn{8}{|c|}{$\begin{array}{c}\text { Im Inneren der Tabelle befinden sich die Irrtumswahrscheinlichkeiten }(a) \text { der Nullhypothese: } \\
\text { Die Anzahl der Insolvenzen ist in beiden Strategien gleich. }\end{array}$} \\
\hline \multicolumn{8}{|c|}{$*: a<5 \%, * *: a<1 \% * * *: a<0.1 \%$} \\
\hline
\end{tabular}

Quelle: Eigene Berechnungen auf Grundlage der Überlebenswahrscheinlichkeiten einer Simulationsstudie von BAILEY \& RICHARDSON (1985, S. 818) 
Die Signifikanzniveaus der Tabelle gelten für beliebige Häufigkeitsunterschiede, seien es Ergebnisse aus Monte-Carlo-Simulationen oder Häufigkeiten von roten und schwarzen Kugeln beim Ziehen mit Zurücklegen aus zwei Urnen. Für den Test werden keinerlei Verteilungsannahmen benötigt.

Tabelle 110.1: Signifikanztests für die Verschiedenheit von Überlebenswahrscheinlichkeiten bei 100 Wiederholungen

\begin{tabular}{|c|c|c|c|c|c|c|c|c|c|c|c|c|}
\hline & \multicolumn{11}{|c|}{ Häufigkeit 1} \\
\hline & & 0 & 1 & 2 & 3 & 4 & 5 & 6 & 7 & 8 & 9 & 10 \\
\hline \multirow{20}{*}{ 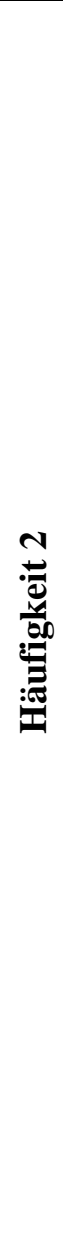 } & 1 & .316 & & & & & & & & & & \\
\hline & 2 & .155 & .561 & & & & & & & & & \\
\hline & 3 & .081 & .312 & .651 & & & & & & & & \\
\hline & 4 & .043 & .174 & .407 & .700 & & & & & & & \\
\hline & 5 & .024 & .097 & .248 & .470 & .733 & & & & & & \\
\hline & 6 & .013 & .054 & .149 & .306 & .516 & .756 & & & & & \\
\hline & 7 & .007 & .030 & .088 & .194 & .352 & .552 & .774 & & & & \\
\hline & 8 & .004 & .017 & .052 & .121 & .234 & .390 & .579 & .788 & & & \\
\hline & 9 & .002 & .009 & .030 & .074 & .152 & .268 & .421 & .602 & .800 & & \\
\hline & 10 & .001 & .005 & .017 & .045 & .096 & .179 & .297 & .447 & .621 & .809 & \\
\hline & 11 & .001 & .003 & .010 & .027 & .060 & .118 & .205 & .323 & .469 & .637 & .818 \\
\hline & 12 & .000 & .002 & .006 & .016 & .037 & .076 & .138 & .228 & .346 & .489 & .651 \\
\hline & 13 & .000 & .001 & .003 & .009 & .023 & .048 & .091 & .157 & .249 & .366 & .506 \\
\hline & 14 & .000 & .000 & .002 & .005 & .014 & .030 & .059 & .106 & .175 & .268 & .384 \\
\hline & 15 & .000 & .000 & .001 & .003 & .008 & .018 & .038 & .071 & .121 & .192 & .285 \\
\hline & 16 & .000 & .000 & .001 & .002 & .005 & .011 & .024 & .046 & .082 & .134 & .207 \\
\hline & 17 & .000 & .000 & .000 & .001 & .003 & .007 & .015 & .030 & .054 & .093 & .147 \\
\hline & 18 & .000 & .000 & .000 & .001 & .002 & .004 & .009 & .019 & .036 & .063 & .103 \\
\hline & 19 & .000 & .000 & .000 & .000 & .001 & .002 & .005 & .012 & .023 & .042 & .071 \\
\hline & 20 & .000 & .000 & .000 & .000 & .000 & .001 & .003 & .007 & .015 & .027 & .048 \\
\hline \multicolumn{13}{|c|}{$\begin{array}{l}\text { Irrtumswahrscheinlichkeit für die Ablehnung der Nullhypothese, daß die Häufigkeiten in Wirklichkeit } \\
\text { gleich sind. }\end{array}$} \\
\hline
\end{tabular}

\subsection{Berücksichtigung von Investitionen in Modellen der einperiodischen linearen Programmierung}

Werden LP-Modelle zur langfristigen Betriebsplanung eingesetzt, sollten sie die Möglichkeit von Investitionen enthalten. Dabei interessiert oft nicht nur die Frage, ob eine bestimmte Investition sinnvoll ist, sondern auch ihr optimaler Umfang. Solche Frage- 
stellungen können relativ leicht in mehrperiodische LP-Tableaus integriert werden ${ }^{98}$, da hier alle Zahlungsströme erfaßt sind und folglich nicht mit kalkulatorischen Kosten gearbeitet werden muß. Der Nachteil mehrperiodischer Tableaus liegt in ihrer rasch wachsenden Größe, die sie unübersichtlich und für Außenstehende schwer verständlich macht. Obwohl mit ihnen auch der optimale Zeitpunkt von Investitionen bestimmt werden kann (HAZELL \& NORTON 1986, S. 54f), konnten sie sich in der Praxis bisher nicht durchsetzen. Darüber hinaus ist es auch in methodischer Sicht aus Gründen der Komplexreduktion ${ }^{99}$ nützlich, Wege aufzuzeigen, wie Probleme einfacher und dennoch exakt gelöst werden können, für die sonst aufwendigere Modellen (nämlich mehrperiodische) angewendet werden.

In der Standardliteratur zu Operations Research (LP ist ein Teilgebiet von OR) findet sich nur sehr wenig zur Einbeziehung von Investitionen in Modelle der linearen Programmierung. Viele allgemeine Lehrbücher zu OR, wie NEUMANN (1975), WiTTE et al. (1975), EISELT \& FRAJER (1977), GAL (1991), DOMSCHKE \& DREXL (1991) und NEUMANN \& MORLOCK (1993), behandeln hauptsächlich Lösungsalgorithmen. Konkrete Formulierungsprobleme sind kaum und Investitionen überhaupt nicht erwähnt. In KREKó (1974, S. 310) werden Investitionen als Kapazitätsproblem betrachtet, die Kosten der Investition bleiben ausgeklammert. HILLIER \& LIEBERMANN (1988, Kap. 3) geben die Kosten einer Investition vor, eine Herleitung unterbleibt. In STEINHAUSER et al. (1989) werden Investitionen im Zusammenhang mit LP zwar behandelt, die Kapitalkosten jedoch nur für einen Zinssatz bestimmt und die Investition selbst nicht in das LP aufgenommen (s. S. 255f).

HAZELL \& NORTON (1986, Abschnitt 4.1) betonen ausdrücklich die Schwierigkeiten, die mit der Implementierung von Investitionen in die lineare Programmierung verbunden sind. Sie verweisen insbesondere darauf, daß Ein- und Auszahlungen nicht gleichmäßig auf die Nutzungsdauer verteilt seien ${ }^{100}$ und betonen die Vorteile mehrperiodischer LPs. Für eine einperiodische Formulierung stellen sie in Abschnitt 12.8 ein Tableau vor. Es basiert auf Annuitäten, berücksichtigt allerdings nur einen Zinssatz.

BRANDES \& WOERMANN (1969) beschäftigen sich ausführlich mit Investitionen und ihren Finanzierungen in der Linearen Programmierung. Bei unterschiedlichen Zinssätzen schlagen sie die Bildung eines Kalkulationszinsfußes vor. Der Kalkulationszinsfuß bestimmt sich aus den Zinssätzen und Anteilen der herangezogenen Finanzierungsquellen (gewogenes Mittel). Daher ist bei Aufstellung des Tableaus für jede Investition festzulegen, zu welchen Anteilen sie aus welchen Quellen finanziert wird. Dieses Verfahren ist zweckmäßig, wenn wenige diskrete Investitionsvorhaben berechnet werden. Steht hingegen auch der optimale Umfang von Investitionen im Blickpunkt des Interesses, ist diese Technik nicht anwendbar, weil a priori beispielsweise

98 Vgl. KÖHNE 1968.

99 Vgl. ODENING 1994. Er geht allerdings nicht auf Investitionen in LP-Modellen ein.

100 Im Original heißt es ,costs and returns“ (S. 54). Kosten werden im allgemeinen aber als periodisierte Auszahlungen verstanden (vgl. Unterabschnitt 3.4.1). Deswegen wird ,costs” mit „Auszahlungen“ übersetzt. 
nicht bekannt ist, ob für jedes Investitionsvolumen auch genügend Eigenkapital für den beabsichtigten Finanzierungsmix vorhanden ist, oder ob das vorhandene Eigenkapital nicht besser für andere Zwecke verwendet wird. Mit anderen Worten: Kann ein Finanzierungsanteil nicht vorgegeben werden, sondern ist dieser vielmehr selbst Gegenstand der Optimierung, ist es zum Zeitpunkt der Aufstellung des Tableaus unmöglich, einen Kalkulationszinsfuß und damit auch eine Annuität zu berechnen. Auch für dieses Problem schlagen BRANDES \& WOERMANN, ausgehend von Überlegungen von KÖHNE (1966), eine Formulierung vor, die im Anschluß an die Darstellung meiner Lösungsmethode in Unterabschnitt P.4.1.4 diskutiert wird.

Im Folgenden wird ein Ansatz entwickelt, der in einperiodischen LPs ohne Kenntnis des Kalkulationszinsfußes eine Investition bei Vorhandensein mehrerer Zinssätze und Opportunitätskosten des Eigenkapitals zuläßt, um eine simultane Optimierung von Produktion, Investition und Finanzierung zu ermöglichen. Dabei geht es nur um die korrekte Implementierung der Kosten in den LP-Ansatz, die Leistungen der Investition werden nur am Rande berücksichtigt.

\subsubsection{LP-Varianten zur Berücksichtigung von Investitionen}

In der linearen Programmierung müssen sich Investitionen als eine betriebliche Ausgabe c.p. im Prinzip negativ auf den Zielwert auswirken. Der Zielbeitrag einer Investition stimmt im allgemeinen nicht mit der Anschaffungsauszahlung überein, er hängt vielmehr von den Kosten der Investition ab. Denn in Planungsrechnungen für Investitionen mit mehrjähriger Nutzungsdauer berücksichtigt man in der Regel nicht die Anschaffungsauszahlung, da diese nur in einem Jahr fällig wird, die Leistungen der Investition hingegen dem Betrieb über viele Jahre zugute kommen. Statt dessen wird die Anschaffungsauszahlung in einen über die Jahre konstanten Kostenstrom transformiert, unter der Maßgabe, daß diese Annuität den Betrieb finanziell gleichstellt wie die einmalige Anschaffungsauszahlung. Die Annuität wird als Kostenansatz der Investition interpretiert und ihren Leistungen gegenübergestellt.

Die Übernahme dieses Annuitätenansatzes in Modelle der linearen Programmierung ist unproblematisch, wenn zur Finanzierung nur ein Zinssatz in Betracht kommt. Stehen aber z.B. Eigenkapital und Fremdkapital zur Verfügung oder zwei Kredite mit unterschiedlichem Zinssatz, sind die Kapitalkosten a priori nicht mehr bestimmbar. Es kann vor Beginn der Optimierung nicht gesagt werden, mit welchem Kredit eine bestimmte Investition finanziert werden wird, welcher Zinsfuß also zur Bestimmung der Kapitalkosten heranzuziehen ist. Dies gilt insbesondere, wenn mehrere begrenzte Kreditrahmen zur Verfügung stehen, der Umfang der Investition selbst Gegenstand der Optimierung ist und es sich nicht nur um eine Ja/Nein-Entscheidung handelt.

Wie kann nun eine Investition in einem einperiodischen LP formuliert werden? Es soll nicht nur entschieden werden, ob eine bestimmte Investition in vorgegebener Größenordnung getätigt, sondern auch, in welchem Umfang investiert. Gleichzeitig ist das optimale Produktions- und Finanzierungsprogramm zu bestimmen. Linearität und (zunächst) beliebige Teilbarkeit der Investition werden vorausgesetzt. Im folgenden 
werden einige Verfahren entwickelt und ihre Schwachpunkte diskutiert. Dies geschieht an einem kleinen Beispiel. Die dabei benutzten Abkürzungen sind in Tabelle 113.1 aufgeführt.

\section{Tabelle 113.1: Abkürzungen, die in Abschnitt 3.4 verwendet werden}

\begin{tabular}{|c|c|}
\hline$A$ & Anschaffungspreis \\
\hline$A b$ & Abschreibung \\
\hline$A n n_{E K}$ & Annuität bei Finanzierung mit Eigenkapital \\
\hline$A n n_{K r 1}$ & Annuität bei Finanzierung mit Kredit $1, A n n_{E K}<A n n_{K r 1}$ \\
\hline$A n n_{K r 2}$ & Annuität bei Finanzierung mit Kredit 2, $A n n_{K r 1}<A n n_{K r 2}$ \\
\hline$b$ & betriebliche Kapazität, auch Right Hand Side (RHS) genannt \\
\hline$D B$ & Deckungsbeitrag ohne Zinskosten \\
\hline$E K$ & Eigenkapital \\
\hline EntgZi & Entgangene Zinsen, Näheres in der Beschreibung zu Tableau 7. \\
\hline FinAnl & Finanzanlagen, z.B. Wertpapiere \\
\hline $\begin{array}{l}\text { Fin } E K, \\
K r 1, K r 2\end{array}$ & Finanzierung des Kapitalbedarfs mit Eigenkapital, Kredit 1 oder Kredit 2 \\
\hline$i_{1}, i_{2}$ & Kreditzinsen \\
\hline $\operatorname{Inv} E K$ & Investitionen, die mit Eigenkapital finanziert werden \\
\hline $\operatorname{Inv} \mathrm{Kr} 1$ & Investitionen, die mit Kredit 1 finanziert werden \\
\hline $\operatorname{Inv} K r 2$ & Investitionen, die mit Kredit 2 finanziert werden \\
\hline$K O G$ & Kreditobergrenze \\
\hline KOG-Korr. & korrigierte Kreditobergrenze \\
\hline$K_{\text {Umlauf }}$ & notwendiges Umlaufkapital für die Produktion \\
\hline lower & $\begin{array}{l}\text { Restriktionstyp in LP-Modellen. Die Restriktion schreibt vor, daß jede Aktivität für sich } \\
\text { allein kleiner als die Kapazität sein muß: } x_{i}<b_{j} \forall i \text {. }\end{array}$ \\
\hline LP & lineare Programmierung \\
\hline$N$ & Nutzungsdauer \\
\hline $\operatorname{Prdn}$ & Produktion \\
\hline $\operatorname{PrdnInv}$ & Produktion und Investition \\
\hline$Z A$ & Zinsanspruch \\
\hline
\end{tabular}

\subsubsection{Das Beispieltableau}

Als Beispiel dient ein Auszug aus einem LP-Tableau. Der Auszug ist so klein wie möglich gewählt. Er enthält nur eine Produktionsmöglichkeit. Sie erhöht über ihren Deckungsbeitrag den Zielwert. Der Deckungsbeitrag enthält keinen Zinsanspruch, da im Modell mehrere Finanzierungsmöglichkeiten mit unterschiedlichen Zinssätzen zur Verfügung stehen und die Auswahl der Finanzierungsform und damit des Zinssatzes 
Gegenstand der Optimierung ist. Zur Finanzierung des Umlaufkapitals ist eine Kreditaufnahme notwendig, wenn kein (ausreichendes) Startkapital vorhanden ist (dann ist die betriebliche Kapazität $b_{1}$ Null). Die maximalen Kredithöhen sind über eine Restriktion begrenzt. Dies kann im verwendeten LP-Programm (SAS ${ }^{\circledR}$ OR) durch eine sog. „lower“-Restriktion erfolgen (s. letzte Zeile des Tableaus). Sie erlaubt es, für beliebig viele Variablen in nur einer Zeile eine Obergrenze festzulegen.

Die Produktion erfordert eine spezifische Investition. Sie kann in diesem Grundmodell nur über Kredit finanziert werden. Laufzeit des Kredites und Nutzungsdauer der Investition sind aufeinander abgestimmt (Fristenkongruenz). Es stehen zwei Kreditformen mit unterschiedlichen Zinsfüßen zur Verfügung. Die Kredite können nicht nur zur Investitionsfinanzierung aufgenommen werden, sondern auch zur Bereitstellung von Liquidität, die u.a. für das Umlaufkapital der Produktion benötigt wird (s. Liquiditätsrestriktion im Tableau-Ausschnitt. Da Kreditaufnahme die Liquidität verbessert, also eine Lieferung darstellt, ist der Koeffizient dieser Aktivität in der Zeile „Liquidität" mit einem negativen Vorzeichen versehen.) Die Leistung der Investition wird über die Produktion berücksichtigt, die durch sie ermöglicht wird.

Tableau-Ausschnitt, Grundmodell

\begin{tabular}{|c||cccc|c|c|}
\hline ID & Produktion & Investition & Kredit 1 & Kredit 2 & Typ & $b$ \\
\hline \hline Ziel & $+D B$ & - Kosten $?$ & $-i_{1}$ & $-i_{2}$ & $\max$ & \\
\hline Liquidität & $\begin{array}{c}\text { Umlaufkapital } \\
\text { der Produktion }\end{array}$ & $\cdot$ & -1 & -1 & $\leq$ & $b_{1}$ \\
Kapazität & 1 & -1 & $\cdot$ & $\cdot$ & $\leq$ & 0 \\
Kreditgrenze & $\cdot$ &. & Grenze 1 & Grenze 2 & lower & 0 \\
\hline
\end{tabular}

Es gelten folgende Annahmen:

- Ziel ist Gewinnmaximierung. Dazu gehört, den gewinnmaximierenden Investitionsumfang zu bestimmen.

- Zu Beginn sind keine Produktionsanlagen vorhanden.

- Zur Produktion einer Einheit ist eine Investition in Höhe von ebenfalls einer Einheit erforderlich. (Die Investition kann selbstverständlich auch in den Folgejahren zur Produktion benutzt werden, solange die Nutzungsdauer nicht überschritten ist.)

- Das Investitionsobjekt ist beliebig teilbar und der Anschaffungspreis pro Einheit konstant.

- Die Reparaturen hängen linear vom Produktionsumfang ab, es gibt keine zeitabhängigen Reparaturen. Sie werden daher bereits im Deckungsbeitrag berücksichtigt.

- Zur Finanzierung von Investitionen stehen mehrere fristenkongruente Kredite zur Verfügung.

- Die Zinsen der Kredite sind unterschiedlich: $i_{2}>i_{1}$. 
- Alle Auszahlungen außer den Zinsen sind zu Periodenbeginn fällig, Deckungsbeitrag und Zinsen zu Periodenende.

- Die Abschreibung erfolgt nach der Zeit und zwar linear: $A b=A / N$.

- Investitionsentscheidungen werden nach kalkulatorischen Kapitalkosten getroffen.

Kalkulatorische Jahreskosten von Investitionen bestehen aus Abschreibung und Zinsanspruch. Es ist das Ziel der Kostenrechnung, jährlich wechselnde Auszahlungen in einen über die Zeit gleichbleibenden Kostenstrom (Annuität) zu verwandeln. Um den Wertverlust während der Nutzungsdauer zu berücksichtigen, wird die kalkulatorische Verzinsung nicht auf den vollen, sondern auf einen Teil des Neuwertes bezogen. Bei Maschinen beträgt dieser Teil, f-Wert oder durchschnittlicher Anlagewert genannt, rund die Hälfte, ${ }^{101}$ so daß gilt: $Z A=A \cdot i / 2$. Äquivalent dazu ist die Formulierung, daß für den Zinsanspruch der (volle) Anschaffungspreis mit dem halben Zinssatz verzinst wird. Bei Gebäuden liegt der durchschnittliche Anlagewert in der Regel über 0.5, denn er steigt mit der Nutzungsdauer (und mit dem Zinssatz) an. ${ }^{102}$ Obwohl die Näherung $\mathrm{f}=0.5$ insbesondere bei Gebäuden problematisch ist, wird sie im folgenden aus Gründen der Vereinfachung zunächst beibehalten.

\subsubsection{Entwicklung eines Tableaus für eine ausschließlich fremdfinanzierte Investition}

\section{Ein erster Ansatz}

In der Literatur ist vorgeschlagen worden, die Kosten einer Investition in das zugehörige Produktionsverfahren $\mathrm{zu}$ integrieren, sofern die Investition nur von einem Produktionsverfahren genutzt werden kann. ${ }^{103}$ Stehen für dieses Produktionsverfahren bereits betriebliche Kapazitäten bereit, so ist das Verfahren in zwei Aktivitäten aufzuteilen: einmal mit vorhandenen Maschinen und einmal mit anzuschaffenden Maschinen. Dieses ,implizit“ genannte Verfahren führt also bei einem gewachsenen Betrieb leicht $\mathrm{zu}$ einer teilweisen Verdoppelung der Zahl der Produktionsaktivitäten. ${ }^{104}$ Die Frage nach der korrekten Spezifikation der Kosten einer Investition wird nicht erleichtert. Daher wird im folgenden nur der Weg einer expliziten Investitionsformulierung verfolgt.

Investitionen müssen als Kosten in der Zielfunktion berücksichtigt werden. In einem ersten Versuch (Variante 1) wird das Tableau so formuliert, daß die Investition mit einer Annuität negativ in die Zielfunktion eingeht.

\footnotetext{
101 Der Restwert wird hier mit Null angenommen.

102 Vgl. BRANDES \& WOERMANN (1969, S. 105).

103 Vgl. KöHne (1966, S. 70), BRANDES \& WOERMANN (1969, S. 121).
}

104 Dies wird durch den Wegfall der expliziten Investitionsaktivitäten nur z.T. wieder ausgeglichen, so daß das implizite Verfahren nicht unbedingt übersichtlicher ist, wie BRANDES \& WOERMANN (1969, S. 121) meinen. 
Tableau-Ausschnitt, Variante 1: Jahreskosten

\begin{tabular}{|c||cccc|c|c|}
\hline ID & Produktion & Investition & Kredit 1 & Kredit 2 & Typ & $b$ \\
\hline \hline Ziel & $+D B$ & - Jahreskosten & $-i_{1}$ & $-i_{2}$ & $\max$ & \\
\hline \multirow{2}{*}{$\begin{array}{c}\text { Liquidität } \\
\text { Kapazität }\end{array}$} & $\begin{array}{c}\text { Umlaufkapital } \\
\text { der Produktion }\end{array}$ & $\cdot$ & -1 & -1 & $\leq$ & $b_{1}$ \\
Kreditgrenze & 1 & -1 &. &. & $\leq$ & 0 \\
\hline
\end{tabular}

Bei diesem Verfahren entsteht die Schwierigkeit, die Höhe der Jahreskosten festzulegen. Die Zinskosten einer Investition sind davon abhängig, ob mit dem preisgünstigen Kredit 1 finanziert werden kann, oder ob der Kreditspielraum ausgeschöpft ist und auf den teueren Kredit 2 zurückgegriffen werden muß. Es kann also nicht a priori entschieden werden, aus welcher Finanzierungsquelle die Anschaffung bestritten wird und damit, mit welchem Kalkulationszins die Investition zu belasten ist.

Die Abschreibung als zweiter Bestandteil der Kapitalkosten wird von dieser Frage nicht berührt. Daher scheint es angebracht, die Jahreskosten einer Investition in Abschreibung und Zinskosten aufzuteilen.

\section{Aufteilung der Annuität in Abschreibung und Zinsanspruch}

Die Abschreibung ist von der Finanzierungsform unabhängig. In die Zielfunktion der Variante 2 geht daher in der Spalte Investition nur die Abschreibung ein, während die Zinskosten über die Liquiditätsrestriktion berücksichtigt werden: Die Investition beansprucht Liquidität in Höhe der Anschaffungsauszahlung. Über welchen Kredit die Liquidität refinanziert wird, bestimmt das LP-Tableau intern.

Tableau-Ausschnitt, Variante 2: Abschreibung und Zinsen getrennt

\begin{tabular}{|c||cccc|c|c|}
\hline ID & Produktion & Investition & Kredit 1 & Kredit 2 & Typ & $b$ \\
\hline \hline Ziel & $+D B$ & $-A b$ & $-i_{1}$ & $-i_{2}$ & $\max$ & \\
\hline Liquidität & $\begin{array}{c}\text { Umlaufkapital } \\
\text { der Produktion }\end{array}$ & $A$ & -1 & -1 & $\leq$ & $b_{1}$ \\
Kapazität & 1 & -1 &. &. & $\leq$ & 0 \\
Kreditgrenze &. &. & Grenze 1 & Grenze 2 & lower & 0 \\
\hline
\end{tabular}

Durch diese Formulierung erzwingt eine Investition eine Kreditaufnahme in Höhe des Anschaffungspreises (sofern kein Eigenkapital vorhanden ist). Die Zinsen für die Kreditaufnahme gehen als Kosten in die Zielfunktion ein, ebenso die verlorenen Zinsen, wenn die Investition aus Eigenkapital finanziert würde (s. nächster Unterabschnitt). Die Kredite mindern den Zielwert in Höhe ihrer Zinsen. Dieses Verfahren berücksichtigt die Abschreibung korrekt, hat aber einen entscheidenden Nachteil: Die Zinsen für die Anschaffung eines Investitionsgutes gehen in voller Höhe in die Ziel- 
funktion des einperiodischen LPs ein. Damit wird der optimale Investitionsumfang unterschätzt.

\section{Halbe Zinssätze bzw. halber Anschaffungswert}

Die Überschätzung der Zinskosten ließe sich vermeiden, indem die Zinssätze in der Zielfunktion $\left(i_{1}\right.$ und $i_{2}$ ) halbiert würden (Variante 3 ). Nun wird eine Investition mit den gewünschten Zinskosten belastet und nahezu im optimalen Umfange investiert.

Tableau-Ausschnitt, Variante 3: Abschreibung und Zinsen getrennt, halbe Zinssätze

\begin{tabular}{|c||cccc|c|c|}
\hline ID & Produktion & Investition & Kredit 1 & Kredit 2 & Typ & $b$ \\
\hline \hline Ziel & $+D B$ & $-A b$ & $-i_{1} / 2$ & $-i_{2} / 2$ & $\max$ & \\
\hline Liquidität & $\begin{array}{c}\text { Umlaufkapital } \\
\text { der Produktion }\end{array}$ & $A$ & -1 & -1 & $\leq$ & $b_{1}$ \\
Kapazität & 1 & -1 &. &. & $\leq$ & 0 \\
Kreditgrenze &. &. & Grenze 1 & Grenze 2 & lower & 0 \\
\hline
\end{tabular}

Allerdings verursachen jetzt auch alle anderen kapitalbeanspruchenden Aktivitäten nur halbe Zinskosten, der Zielwert wird damit systematisch überhöht und kapitalintensive Produktionsverfahren werden begünstigt.

Ein anderer Ansatz könnte sein, nicht die Zinssätze, sondern den Anschaffungspreis zu halbieren, also statt $A$ nur $A / 2$ in der Liquiditätsrestriktion zu verwenden (Variante 4). Auch in dieser Variante werden Investitionen mit den gewünschten Kosten belastet, und es wird daher nahezu im optimalen Umfange investiert.

Tableau-Ausschnitt, Variante 4: Abschreibung und Zinsen getrennt, halber Anschaffungswert

\begin{tabular}{|c||cccc|c|c|}
\hline ID & Produktion & Investition & Kredit 1 & Kredit 2 & Typ & $b$ \\
\hline \hline Ziel & $+D B$ & $-A b$ & $-i_{1}$ & $-i_{2}$ & $\max$ & \\
\hline \multirow{2}{*}{$\begin{array}{c}\text { Liquidität } \\
\text { Kapazität }\end{array}$} & $\begin{array}{c}\text { Umlaufkapital } \\
\text { der Produktion }\end{array}$ & $A / 2$ & -1 & -1 & $\leq$ & $b_{1}$ \\
Kreditgrenze & 1 & -1 & $\cdot$ & $\cdot$ & $\leq$ & 0 \\
& $\cdot$ &. & Grenze 1 & Grenze 2 & lower & 0 \\
\hline
\end{tabular}

Diesem Vorteil steht gegenüber, daß eine Investition eine Kreditaufnahme nur in Höhe des halben Anschaffungspreises verursacht, die vom Programm ausgewiesenen Kreditumfänge wären also nach unten verzerrt. Es könnte daher evtl. mehr Kredit aufgenommen werden, als es die Kreditobergrenzen eigentlich zulassen. Ferner werden evtl. Aktivitäten, die nur mit dem billigen Kredit wirtschaftlich sind, durchgeführt, 
weil wegen des angenommenen halben Anschaffungswertes dieser Kreditrahmen scheinbar noch nicht ausgeschöpft ist.

\section{Anpassung der Kreditobergrenzen}

Diesem Problem ließe sich begegnen, indem die Kreditobergrenzen in Abhängigkeit von den fremdfinanzierten Investitionen gesenkt würden. Dies geschieht in Variante 5 durch die beiden zusätzlichen Aktivitäten KOG-Korr 1 und 2 in Verbindung mit der Restriktion KOG-Korrekt: Investitionen beanspruchen Liquidität und damit den Kreditspielraum in Höhe von $A / 2$. Zusätzlich verringern sie den Kreditspielraum um weitere $A / 2$, insgesamt also um den vollen Anschaffungspreis. Diese Reduzierung kann allerdings beliebig auf die beiden Kreditarten aufgeteilt werden. Der LP-Algorithmus wird stets die weniger ,schmerzhafte“ Kreditobergrenze, also die des teueren Kredits 2, zurücknehmen. Auf der anderen Seite werden Investitionen so weit als möglich mit dem billigeren Kredit 1 finanziert. Daher wird die Aufteilung der Kreditrahmenverringerung auf die beiden Kreditarten im allgemeinen nicht mit der Aufteilung der Finanzierung der Investitionen auf die Kredite übereinstimmen.

Tableau-Ausschnitt, Variante 5: Abschreibung und Zinsen getrennt, Korrektur der Kreditobergrenzen

\begin{tabular}{|c|c|c|c|c|c|c|c|c|}
\hline ID & Produktion I & ivestition & $\begin{array}{l}\text { KOG- } \\
\text { Korr.1 }\end{array}$ & $\begin{array}{l}\text { KOG- } \\
\text { Korr. } 2\end{array}$ & Kredit 1 & Kredit 2 & Тур & $b$ \\
\hline Ziel & $+D B$ & $-A b$ & . & . & $-i_{1}$ & $-i_{2}$ & $\max$ & \\
\hline Liquidität & $\begin{array}{l}\text { Umlaufkap. } \\
\text { der Prdn. }\end{array}$ & $A / 2$ & . & . & -1 & -1 & $\leq$ & $b_{1}$ \\
\hline Kapazität & 1 & -1 & . & . & . & . & $\leq$ & 0 \\
\hline Kreditgrenze1 & . & $\cdot$ & 1 & & 1 & . & $\leq$ & $K O G_{1}$ \\
\hline Kreditgrenze2 & . & . & & 1 & . & 1 & $\leq$ & $K O G_{2}$ \\
\hline KOG-Korrekt & . & $-A / 2$ & 1 & 1 & & . & $=$ & 0 \\
\hline
\end{tabular}

Dieses Verfahren führt daher nur annähernd zu korrekten Investitionsumfängen. Für $n$ Finanzierungsarten sind $n$ zusätzliche Aktivitäten und $n+1$ zusätzliche Restriktionen erforderlich. Die Höhe der aufgenommenen Kredite kann wie beim vorigen Tableau aus dem LP-Ergebnis nicht direkt abgelesen werden. Zusätzliche Formulierungsprobleme ergeben sich, wenn neben Krediten auch Eigenmittel zur Investitionsfinanzierung zugelassen werden. Aus diesen Gründen wird dieser Ansatz nicht weiter verfolgt.

\subsubsection{Entwicklung eines Tableaus für eigen- oder fremdfinanzierte Investitionen}

Der Betrieb verfüge im Gegensatz zum vorigen Unterabschnitt über Eigenmittel. Mit ihnen kann er eine Investition finanzieren, die Mittel können aber auch zinsbringend 
angelegt werden. Das Tableau enthält daher eine zusätzliche Aktivität „Finanzanlagen“(FinAnl). Der Habenzinsfuß $i_{0}$ liege unter dem niedrigsten Sollzins.

\section{Aufteilung der Investitionsaktivitäten}

Die im vorigen Unterabschnitt vorgestellte Idee, eine Investition im Tableau durch mehrere Aktivitäten, abhängig von der Finanzierung, auszudrücken, wird wieder aufgegriffen. Für eine Investition wird für jede mögliche Form der Finanzierung eine eigene Aktivität eingeführt (Inv $E K$, Inv $K r 1$, Inv $K r 2$, s. Variante 6). ${ }^{105}$

Jede dieser „Investitionsaktivitäten“ wirkt sich in Höhe ihrer spezifischen Annuität (, die Abschreibung und Zinsanspruch einschließt,) negativ auf den Zielwert aus. Die Annuität ist abhängig von der Nutzungsdauer (für alle Finanzierungsformen gleich) und dem Zinssatz für die Finanzierungsform.

Die fremdfinanzierten Investitionsaktivitäten haben im Tableau keinen Einfluß auf die Liquidität. Wären sie liquiditätswirksam, müßte die in Anspruch genommene Liquidität durch Kreditaufnahme refinanziert werden, und eine Investition würde den Zielwert neben der Annuität zusätzlich um $A \cdot i$ verringern. Diese Doppelzählung der Zinsen wird durch die Formulierung der fremdfinanzierten Investitionen als nicht liquiditätswirksam vermieden.

Dennoch verringert eine Investition, die mit Fremdkapital finanziert wird, den Kreditspielraum in Höhe ihres Anschaffungspreises. Dies wird durch die Zeilen „Kreditgrenze $1^{\text {“ }}$ und „Kreditgrenze 2“ gewährleistet. ${ }^{106}$ Die Kapazität „KOG ${ }^{\text {“ }}$ (Kreditobergrenze 1) wird z.B. durch Investitionen, die mit billigem Kredit finanziert werden, und durch sonstige Kreditaufnahme 1 (z.B. für das Umlaufkapital) in Anspruch genommen. $^{107}$

Tableau-Ausschnitt, Variante 6: Eigen- und Fremdfinanzierung I

\begin{tabular}{|c|c|c|c|c|c|c|c|c|c|}
\hline ID & $\operatorname{Prdn}$ & Inv EK & $\operatorname{Inv} K r 1$ & $\operatorname{Inv} K r 2$ & FinAnl & Kredit 1 & Kredit 2 & Тур & $b$ \\
\hline Ziel & $+D B$ & $-A n n_{E K}$ & $-A n n_{K r 1}$ & $-A n n_{K r 2}$ & $i_{0}$ & $-i_{1}$ & $-i_{2}$ & $\max$ & \\
\hline Liquidität & $K_{\text {Umlauf }}$ & $?$ & . & . & 1 & -1 & -1 & $\leq$ & $b_{1}$ \\
\hline Kapazität & 1 & -1 & -1 & -1 & . & . & . & $\leq$ & 0 \\
\hline Kreditgrenze 1 & . & . & $A$ & . & . & 1 & . & $\leq$ & $K O G_{1}$ \\
\hline Kreditgrenze 2 & . & . & . & $A$ & . & . & 1 & $\leq$ & $K O G_{2}$ \\
\hline Kreditsumme & . & . & -1 & -1 & . & -1 & -1 & free & . \\
\hline
\end{tabular}

105 Vgl. BRANDES \& WOERMANN (1969, S. 118).

106 Da die Investitionen im Vergleich zur Variante 5 in Abhängigkeit von der Finanzierung in verschiedene Aktivitäten aufgeteilt ist, erfolgt in dieser Variante eine korrekte Reduzierung der Kreditobergrenzen.

107 Da nun zwei Aktivitäten auf die gleiche Obergrenze einwirken, kann diese Obergrenze nicht mehr durch eine Zeile mit Hilfe einer lower-Restriktion formuliert werden. 
Dieses Verfahren garantiert eine richtige Erfassung der Kosten fremdfinanzierter Investitionen und damit insofern optimale Investitionsumfänge. Die Kreditspielräume werden durch die Investitionen korrekt in Höhe des Anschaffungspreises vermindert. Die letzte Zeile im Tableau, „Kreditsumme“, ist eine Restriktion vom Typ ,free“. Restriktionen dieses Typs sind nicht in jedem LP-Programm vorhanden. Im ORModul des Programmpakets SAS summieren ,free" -Restriktionen über eine Zeile. Das heißt z.B., alle Aktivitäten, die in dieser Zeile mit einer Eins vertreten sind, werden aufaddiert. Hier wird also die Summe der Kreditbelastung des Betriebes saldiert. Die technischen Koeffizienten in dieser Restriktion müssen negative Vorzeichen tragen, damit die Summe im LP-Ergebnis positiv ist, ähnlich wie "liefernde" Aktivitäten im sonstigen Tableau mit negativen Vorzeichen eingehen.

Ein Problem bleibt die Implementierung einer Investition aus Eigenkapital. Sie vermindert den Zielwert in Höhe ihrer Annuität. Die Annuität beinhaltet die Opportunitätskosten des eingesetzten eigenen Kapitals. Da nur so viel eigenfinanziert werden kann, wie Mittel vorhanden sind, ist es erforderlich, die Investition aus Eigenmitteln an die verfügbare Liquidität zu binden (Liquidität im Sinne von baren Mitteln). Geld, das für Investitionen verwendet wurde, kann selbstverständlich nicht noch ein zweites Mal für andere Zwecke ausgegeben werden, z.B. kann es nicht zinsbringend angelegt werden. Dies kann berücksichtigt werden, indem in Variante 6 das Fragezeichen (,,?“) durch die Anschaffungsauszahlung ersetzt wird, siehe Variante 6a. Doch diese Formulierung führt zu einem Problem. Vergleichen wir zwei Szenarien: Den Betrieben $X$ und $Y$ stehen jeweils 100 TDM zur Verfügung. $X$ legt das Geld zu $10 \%$ an und erzielt einen Gewinn von 10 TDM. $Y$ erwirbt eine Maschine für 100 TDM. Im Vergleich zu $X$ verliert er durch die Anschaffung die Zinseinnahmen in Höhe von 10 TDM und wird zusätzlich bei diesem Verfahren durch die Annuität der Investition belastet. Investitionen mit Eigenkapital wirken sich also bei dieser Vorgehensweise doppelt auf den Zielwert aus, einmal über die Annuität und einmal über den Verlust von Zinseinnahmen.

Tableau-Ausschnitt, Variante 6a: Eigen- und Fremdfinanzierung II

\begin{tabular}{|c||ccccccc|c|c|}
\hline ID & $P r d n$ & Inv EK & Inv Kr 1 & Inv Kr 2 & FinAnl & Kredit 1 & Kredit 2 & Typ & $b$ \\
\hline \hline Ziel & $+D B$ & $-A n n_{E K}$ & $-A n n_{K r 1}$ & $-A n n_{K r 2}$ & $i_{0}$ & $-i_{1}$ & $-i_{2}$ & $\max$ & \\
\hline Liquidität & $K_{\text {Umlauf }}$ & $A$ & $\cdot$ & $\cdot$ & 1 & -1 & -1 & $\leq$ & $b_{1}$ \\
Kapazität & 1 & -1 & -1 & -1 &. &. &. & $\leq$ & 0 \\
Kreditgrenze 1 &. &. & $A$ &. &. & 1 & $\cdot$ & $\leq$ & $K O G_{1}$ \\
Kreditgrenze 2 &. &. &. & $A$ &. &. & 1 & $\leq$ & $K O G_{2}$ \\
Kreditsumme &. &. & -1 & -1 &. & -1 & -1 & free &. \\
\hline
\end{tabular}

Bei Investitionen aus Eigenkapital daher den Zinsanteil in der Annuität wegzulassen, brächte zwar eine Verbesserung; sie würde aber nicht ausreichen: Ist eine eigenfinanzierte Investition in Höhe der Anschaffungsauszahlung liquiditätswirksam, ver- 
ringert sich durch die Investition der Zielwert um das Produkt aus Anschaffungswert und Zinssatz für Eigenmittel, also doppelt so stark, wie es den gewünschten Kosten entspricht. Diesem Problem kann begegnet werden, indem eine Hilfsvariable eingefügt wird.

\section{Hilfsvariable für Eigenkapital-Investitionen}

In Tableauvariante 6 a bewirken Investitionen aus Eigenkapital Opportunitätskosten von $A \cdot i$, da eigenfinanzierte Investitionen die Liquidität verringern sollen. ${ }^{108}$ Durch eine Hilfsvariable können diese Opportunitätskosten ausgeglichen werden, um eine Doppelzählung der Zinsen zu vermeiden. Im Zusammenspiel mit der Zeile „EK-Korrektur" erhöht die Hilfsvariable den Zielwert bei Investitionen aus Eigenkapital genau um die Höhe der entgangenen Zinseinnahmen (vgl. Variante 7).

Tableau-Ausschnitt, Variante 7: Hilfsvariable für Eigenkapital-Investitionen

\begin{tabular}{|c|c|c|c|c|c|c|c|c|c|c|}
\hline ID & $\operatorname{Prdn}$ & $\operatorname{Inv} E K$ & $\operatorname{Inv} K r I$ & $\operatorname{Inv} K r 1$ & EntgZi & FinAr & Kredit & redit 2 & Тур & $b$ \\
\hline Ziel & $+D B$ & $-A n n_{E K}$ & $-A n n_{K r 1}$ & $-A n n_{K r 2}$ & $i_{0}$ & $i_{0}$ & $-i_{1}$ & $-i_{2}$ & $\max$ & \\
\hline Liquidität & $K_{\text {Umlauf }}$ & $A$ & . & . & & 1 & -1 & -1 & $\leq$ & $b_{1}$ \\
\hline EK-Korrektur & . & $-A$ & . & . & 1 & . & . & . & $\leq$ & 0 \\
\hline Kapazität & 1 & -1 & -1 & -1 & . & . & . & . & $\leq$ & 0 \\
\hline Kreditgrenze 1 & . & . & $A$ & . & . & . & 1 & . & $\leq$ & $K O G_{1}$ \\
\hline Kreditgrenze 2 & . & . & . & $A$ & . & . & . & 1 & $\leq$ & $K O G_{2}$ \\
\hline Kreditsumme & . & . & $-A$ & $-A$ & . & . & -1 & -1 & free & . \\
\hline Inv.volumen & . & -1 & -1 & -1 & . & . & . & . & free & . \\
\hline Integer & . & 1 & 1 & 1 & . & . & . & . & integer & . \\
\hline
\end{tabular}

Die Hilfsvariable „Entgangene Zinsen“ („EntgZi“) liefert einen positiven Zielbeitrag, ihr Zielfunktionskoeffizient entspricht dem Habenzins. Die Variable kann über die Restriktion „EK-Korrektur“ genau im Umfang der Investitionen aus Eigenkapital realisiert werden. Abstrahierend von anderen Verknüpfungen erhöhen Investitionen aus Eigenkapital also den Zielwert um $A$. Habenzins und gleichen damit exakt den Zinsverlust aus, der durch die Liquiditätswirksamkeit der EK-Investition entsteht, weil z.B. weniger Finanzanlagen getätigt werden können. Summa summarum belastet eine Investition aus Eigenkapital den Zielwert also nur in Höhe der Annuität.

In der vorletzten Zeile wird über eine weitere Free-Restriktion die Summe der Investitionen gebildet (eigen- und fremdfinanzierte). Dies ist z.B. für die Bestimmung der Produktionskapazität in der nächsten Periode nützlich. Fügt man dem Tableau die letzte Zeile, „Integer“ genannt, hinzu, ist sichergestellt, daß die Investition nur ganz-

108 Gleichzeitig reduzieren sie den Zielwert in Höhe ihrer Annuität (s.o.). 
zahlig erfolgt. Dies wird im Programmpaket SAS® einfach durch eine Restriktion vom Typ ,integer" erreicht. ${ }^{109}$

Die Variante 7 erfüllt endlich für jegliche fristenkongruente Finanzierung mit ausschließlich variablen Reparaturkosten, die damit im DB berücksichtigt werden können, die gestellten Anforderungen:

- Investitionen werden mit den kalkulatorischen Jahreskosten belastet.

- Investitionen aus Eigenkapital verringern die baren Mittel in Höhe ihres Anschaffungspreises.

- Die Kreditbelastung wird richtig ausgewiesen.

- Der Kreditspielraum kann nicht überschritten werden.

Mit diesem Tableau kann simultan das Produktions-, Investition- und Finanzierungsprogramm optimiert werden. Sein Prinzip wird in Kapitel 6 für das dort entwikkelte Betriebsentwicklungsmodell verwendet. Auf eine Einschränkung der Aussagefähigkeit der Tableaus sei hingewiesen. Der in ihnen bestimmte Zielwert ist nicht dekkungsgleich mit dem Gewinn. Dazu sind einige Anschlußrechnungen erforderlich (s. übernächsten Unterabschnitt).

\subsubsection{Diskussion des Ansatzes von BRANDES \& WOERMANN}

KÖHNE (1966) und BRANDES \& WOERMANN (1969) haben sich bereits sehr früh mit der Problematik der Einbeziehung von Investitionen in Modelle der linearen Programmierung auseinandergesetzt. Die von ihnen entwickelten Ansätze sind weitgehend deckungsgleich. Verglichen mit den anderen $\mathrm{zu}$ Beginn dieses Abschnitts erwähnten Arbeiten integrieren sie Investitionen am umfassendsten in die einperiodische lineare Programmierung. Mit Hilfe ihrer Tableaus kann der optimale Umfang fremdfinanzierter Investitionen berechnet werden, für die mehrere Kreditformen zur Auswahl stehen. Nachteilig ist, daß eine Anlagemöglichkeit für Eigenmittel nicht vorgesehen werden darf: Werden Finanzanlagen ermöglicht, verfehlen diese Tableaus den optimalen Investitionsumfang. Dies wird in diesem Unterabschnitt stellvertretend für beide Arbeiten anhand der Formulierung von BRANDES \& WOERMANN gezeigt, da deren Notation der dieser Arbeit verwandten Schreibweise ähnlicher ist.

Auf Seite 121 von BRANDES \& WOERMANN ist ein Tableau-Ausschnitt dargestellt. Er entspricht, verkürzt auf die hier interessierende Fragestellung und der bisher verwendeten Notation angepaßt, dem als Variante 8 wiedergegebenen Tableau.

Investition und Produktion sind in einer Aktivität (,PrdnInv“) zusammengefaßt. Zur Finanzierung stehen drei Möglichkeiten zur Verfügung, Eigenkapital („Fin EK“) und

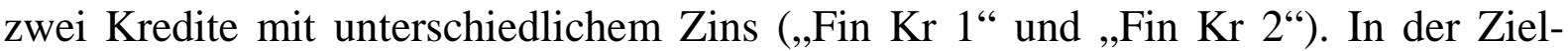
funktion sind die Finanzierungskosten, die für eine Kapitalbereitstellung von 1000 DM

109 Zusätzlich ist eine hier nicht dargestellte ,upper“-Restriktion erforderlich, die in einer Zeile ähnlich wie die erwähnte „lower“-Restriktion eine obere Grenze für alle Aktivitäten, die ganzzahlig sein sollen, festlegt. 
anfallen, ${ }^{110}$ aufgeführt und nicht wie bisher die Annuität der Anschaffungsauszahlung. Die Finanzierung über Eigenkapital darf das vorhandene Eigenkapital nicht übersteigen (Nebenbedingung „Eigenkapital“). Die Restriktionen „Kapazität“ und „Kreditsumme" sind im Original nicht enthalten.

Tableau-Ausschnitt, Variante 8: LP-Tableau von BRANDES \& WOERMANN (1969, S. 121) für Investitionen bei unterschiedlicher Finanzierung

\begin{tabular}{|c||cccc|c|c|}
\hline ID & PrdnInv & Fin EK & Fin Kr 1 & Fin Kr 2 & Typ & $b$ \\
\hline \hline Ziel & $+D B$ & $-A n n_{E K}$ & $-A n n_{K r 1}$ & $-A n n_{K r 2}$ & $\max$ & \\
\hline Liquidität $^{111}$ & $A$ & -1000 & -1000 & -1000 & $\leq$ & 0 \\
Kapazität & 1 & -1 & -1 & -1 & $\leq$ & 0 \\
Eigenkapital &. & 1000 &. &. & $\leq$ & $b_{1}$ \\
Kreditgrenze &. &. & 1000 & 1000 & $\leq$ & $K O G$ \\
Kreditsumme &. &. & -1 & -1 & free &. \\
\hline
\end{tabular}

Bei Produktion wird automatisch eine Anschaffungsauszahlung $A$ fällig, die über die drei Finanzierungsaktivitäten „Fin EK“, „Fin Kr 1“ und „Fin Kr 2“ aufgebracht werden kann. Daher ist keine separate Kapazitätsrestriktion erforderlich. Sie ist dennoch eingetragen, um die Vergleichbarkeit mit den vorangegangenen Tableaus zu verbessern. Die Kopplung von Produktion und Investition birgt zwei Nachteile. Zum einen kann keine Produktion mit Altanlagen ermöglicht werden. Zum anderen ist stets eine volle Kapazitätsauslastung notwendig.

Die Formulierung von BRANDES \& WOERMANN berücksichtigt die Leistungen der Produktion und die Kosten der Investition korrekt. Probleme treten auf, wenn das Eigenkapital nicht nur für Investitionen verwendet, sondern z.B. auch zinsbringend auf dem Kapitalmarkt angelegt werden kann. Im folgenden Ausschnitt ist zusätzlich eine Aktivität ,Finanzanlagen“ eingetragen.

Die Finanzanlage bringt Zinserträge. Sie konkurriert mit der Produktion um das knappe Eigenkapital. In dem Umfange, wie Investitionen mit Eigenkapital finanziert werden, müssen die Finanzanlagen eingeschränkt werden. Eigenfinanzierung verursacht daher neben der Annuität noch Opportunitätskosten, die Gesamtkosten der Eigenfinanzierung sind dadurch größer als die einer Fremdfinanzierung (unter der Voraussetzung eines nicht zu großen Zinsunterschieds zwischen Eigen- und Fremdkapital)! Es ergeben sich also ähnliche Probleme, wie sie zu Beginn dieses Abschnitts erörtert wurden. In den Ansatz von BRANDES \& WOERMANN dürfen daher entweder

\footnotetext{
110 Entspricht der Annuität für ein langfristiges Darlehn von 1000 DM.

111 Im Original „Kapitalbedarf“ genannt.
} 
keine Finanzanlagen aufgenommen werden, oder er führt zu überhöhten Investitionskosten und damit zu zu geringen Investitionsvolumina.

Tableau-Ausschnitt, Variante 9: LP-Tableau von BRANDES \& WOERMANN (1969,

S. 112), ergänzt um Finanzanlagen

\begin{tabular}{|c||ccccc|c|c|}
\hline ID & PrdnInv & Fin EK & Fin Kr 1 & Fin Kr 2 & FinAnl & Typ & $b$ \\
\hline \hline Ziel & $+D B$ & $-A n n_{E K}$ & $-A n n_{K r 1}$ & $-A n n_{K r 2}$ & $i_{0}$ & $\max$ & \\
\hline Liquidität & $A$ & -1000 & -1000 & -1000 & $\cdot$ & $\leq$ & 0 \\
Kapazität & 1 & -1 & -1 & -1 & $\cdot$ & $\leq$ & 0 \\
Eigenkapital &. & $A$ & & & 1 & $\leq$ & $b_{1}$ \\
Kreditgrenze &. &. & $A$ & $A$ & $\cdot$ & $\leq$ & KOG \\
Kreditsumme &. &. & -1 & -1 & $\cdot$ & free &. \\
\hline
\end{tabular}

Die nachfolgenden Tabellen zeigen das von BRANDES \& WOERMANN verwendete Tableau (in verkürzter Form; der Zielbeitrag der Produktion ist auf 29 gesenkt) und das Ergebnis in Original-SAS-Notation.

Wie erwähnt, ist Eigenfinanzierung teurer als Fremdfinanzierung. Um dies deutlich zu machen, ist der Zielbeitrag der Produktion auf 29 gesenkt worden: Die Investitionen werden jetzt ausschließlich mit Kredit finanziert und die Eigenmittel angelegt! Eine Formulierung nach Variante 7 hingegen führt $\mathrm{zu}$ einer ökonomisch sinnvollen Finanzierung (siehe Tabelle 125.1). Gleichzeitig zeigen sie auf, daß durch eine verbesserte Optimierung Investitionsumfang $(+25 \%)$ und Gewinn $(+8 \%)$ deutlich ansteigen.

Tabelle 124.1: LP-Tableau nach BRANDES \& WOERMANN (1969, S. 112, ergänzt um Finanzanlagen) und Ergebnis

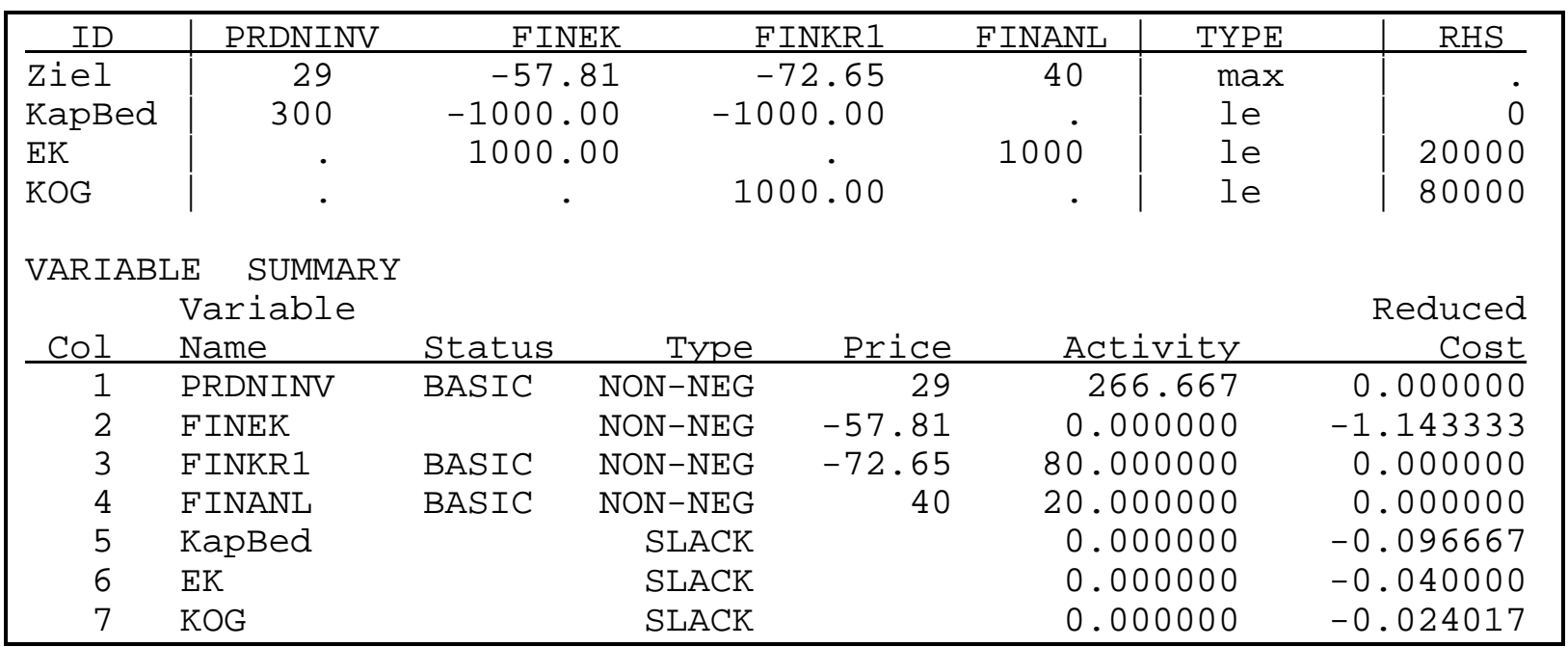

Zielwert: 2 721.333, Output von SAS ${ }^{\circledR}$ OR 
Tabelle 125.1: LP-Tableau nach Variante 7 und Ergebnis

\begin{tabular}{|c|c|c|c|c|c|c|c|}
\hline ID & PRDNINV & FINEK & FINKR1 & ENTGZIN & FINANL & TYPE & RHS \\
\hline Ziel & 29 & -57.81 & -72.65 & 40 & 40 & $\max$ & \\
\hline KapBed & 300 & -1000.00 & -1000.00 & & & le & 0 \\
\hline $\mathrm{EK}$ & . & 1000.00 & & & 1000 & le & 20000 \\
\hline KOG & . & & 1000.00 & & & le & 80000 \\
\hline EK_Korr & - & -300.00 & • & 1000 & . & le & 0 \\
\hline \multicolumn{8}{|c|}{ VARIABLE SUMMARY } \\
\hline & Variable & & & & & & Reduced \\
\hline $\mathrm{Col}$ & Name & Status & Type & Price & Activity & & Cost \\
\hline 1 & PRDNINV & BASIC & $\mathrm{NON}-\mathrm{NEG}$ & 29 & 333.333 & & .000000 \\
\hline 2 & FINEK & BASIC & $\mathrm{NON}-\mathrm{NEG}$ & -57.81 & 20.000000 & & .000000 \\
\hline 3 & FINKRI & BASIC & $\mathrm{NON}-\mathrm{NEG}$ & -72.65 & 80.000000 & & .000000 \\
\hline 4 & ENTGZIN & BASIC & NON-NEG & 40 & 6.000000 & & .000000 \\
\hline 5 & FINANL & & NON-NEG & 40 & 0.000000 & -10 & .856667 \\
\hline 6 & KapBed & & SLACK & & 0.000000 & & .096667 \\
\hline 7 & $\mathrm{EK}$ & & SLACK & & 0.000000 & & .050857 \\
\hline 8 & KOG & & SLACK & & 0.000000 & & .024017 \\
\hline 9 & EK_Korr & & SLACK & & 0.000000 & & .040000 \\
\hline
\end{tabular}

Zielwert: 2 938.467, Output von $\mathrm{SAS}^{\circledR}$ OR

\subsubsection{Vom Zielwert zum Gewinn}

Das LP-Tableau dient zur Optimierung des Produktions- und Investitionsprogramms in der aktuellen Periode. Der Zielwert dieser Planungsrechnung weicht daher vom Gewinn als einem Begriff aus der Erfolgsrechnung in der Regel ab. Um vom Zielwert des Tableaus zum Periodengewinn zu gelangen, sind folgende Modifikationen erforderlich:

Der Zielwert berücksichtigt nur die Annuitäten der Investitionen in der aktuellen Periode. Für fremdfinanzierte Altinvestitionen sind deren Annuitäten abzuziehen, sofern ihre Nutzungsdauer noch nicht überschritten ist (siehe in Tabelle 126.1 die Zeile, die am Ende mit ,a“" gekennzeichnet ist). Eigenfinanzierte Investitionen aus Vorperioden verringern den Gewinn in Höhe ihrer Abschreibungen (siehe Tabelle 126. 1. Zeile b). Ebenfalls ist der Zielwert um weitere, nicht im Tableau enthaltene Fest- und Gemeinkosten zu reduzieren (d).

Bei Investitionen aus Eigenmitteln sind nur die Abschreibungen gewinnwirksam. In der Zielfunktion haben solche Investitionen jedoch abweichende Auswirkungen. Sie verursachen in der Zielfunktion Kosten durch ihre Annuität, also Abschreibung und Zinsanspruch, und verringern die freie Liquidität und dadurch die Höhe der Finanzanlagen. Deswegen ermöglichen Investitionen mit Eigenkapital im gleichen Umfange die Aktivität „entgangene Zinsen“. Investitionen mit Eigenkapital beeinflussen die Zielfunktion also dreifach: durch die Annuität, durch nichtmögliche Finanzanlagen (Zinsverlust: $A \cdot i$ ) und durch die Aktivität ,entgangene Zinsen“ (Zielbeitrag: $A \cdot i$ ): 
Der Zielbeitrag von Investitionen aus Eigenkapital bestimmt sich also zu:

\begin{tabular}{l|l} 
- Annuität & $-A n n_{E K}$ \\
- Reduzierung der Finanzanlagen & $-A \cdot i_{0}$ \\
+ Aktivität „entgangene Zinsen“ & $+A \cdot i_{0}$
\end{tabular}

Die beiden letzten Effekte gleichen sich aus. Damit reduzieren Investitionen mit Eigenkapital den Zielwert lediglich um die Annuität. Für den Gewinn wird aber nur die Abschreibung benötigt. Daher ist der Zinsanspruch zuviel abgezogen worden, so daß er zur Bestimmung des Gewinns dem Zielwert zuzuschlagen ist (siehe Tabelle 126. 1, Zeile c). ${ }^{112}$

\section{Tabelle 126.1: Vom Zielwert eines LPs zum Gewinn}

\begin{tabular}{|l|l|l|}
\hline Gewinn & $=$ & \\
& + & Zielwert \\
& - & Annuität der Investitionen mit Fremdkapital in den Vorperioden (a) \\
& - & Abschreibung von Investitionen mit Eigenkapital in den Vorperioden (b) \\
& + & Zinsanspruch von Investitionen mit Eigenkapital in der aktuellen Periode (c) \\
& - & im LP nicht enthaltene Fest- und Gemeinkosten (d) \\
\hline
\end{tabular}

\subsubsection{Zusammenfassung und Erweiterungen}

Es wurde ein Verfahren vorgestellt, das es in einperiodischen LPs ermöglicht, den optimalen Umfang von eigen- und fremdfinanzierten Investitionen zu bestimmen. Das Verfahren wird durch folgende Punkte charakterisiert:

- Eine Investition verringert den Zielwert genau in Höhe ihrer Annuität (Kapitalkosten).

- Für jede Finanzierungsform wird eine eigene Investitionsaktivität geschaffen.

- Vollständig fremdfinanzierte Investitionen wirken sich nicht unmittelbar auf die Liquidität aus,

- sie verringern aber die verfügbaren Kreditspielräume.

Zur Berücksichtigung von eigenfinanzierten Investition ist die Implementierung einer zusätzlichen Restriktion und einer Hilfsvariablen erforderlich. Diese Hilfsvaria-

112 Es ist auch möglich, ein Tableau ohne die Aktivität „entgangene Zinsen“ zu konstruieren. Dann ist in der Zielfunktion statt $-A n n_{E K}$ die Größe $\left(-A n n_{E K}+A \cdot i\right)$ zu verwenden.

$-\left(A n n_{E K}-A \cdot i\right)$ läßt sich umformen zu:

$-(A b+A \cdot f \cdot i-A \cdot i)$

$-(A b+(f-1) \cdot A \cdot i)$

und ist daher Abschreibung plus der Verzinsung des durchschnittlichen Komplementärwertes. Damit einheitlich Annuitäten in der Zielfunktion stehen, wird dieser Weg allerdings nicht gewählt. 
ble gleicht die Opportunitätskosten des Eigenkapitals aus, das zur Finanzierung von Investitionen eingesetzt wird.

Die zu Beginn genannten (einschränkenden) Annahmen waren überwiegend didaktisch motiviert und können erweitert werden:

- Zu Beginn können beliebig viele Produktionsanlagen vorhanden sein.

- Ganzzahligkeit der Investitionen kann in geeigneten Programmen wie SAS ${ }^{\circledR}$ OR durch eine geeignete Restriktion leicht sichergestellt werden.

- Kostendegression mit zunehmendem Investitionsumfang ist grundsätzlich, wie in LPs ohne Investition, möglich (durch abschnittsweise Linearisierung nichtlineare Funktionen).

- Der Abschreibungsverlauf kann beliebig gewählt werden. Wichtig ist nur, daß die jährlichen Kosten konstant sind.

- Die Rangfolge der Zinssätze kann beliebig sein. 



\section{Einige mathematische Eigenschaften einfacher stochastisch dynamischer Systeme}

$4.1 \quad$ Vergleich zweier Zufallsveränderlicher............................................. 130

$4.2 \quad$ Definition Uberlebenswahrscheinlichkeit (formal) ................................ 147

4.3 Deduzierbare Einflüsse auf die Überlebenswahrscheinlichkeit.................. 155

4.4 Stochastische Eigenschaften von Funktionen mehrerer

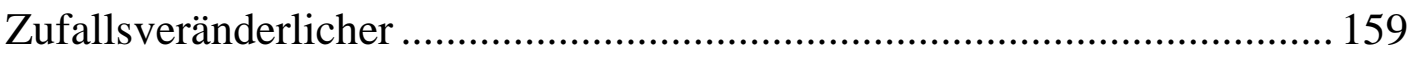

4.5 Exkurs: Probleme bei der Maximierung von Erwartungswerten

(Petersburger Paradoxon).............................................................. 178

Überleben in einem stochastischen Kontext steht immer im Zusammenhang mit Zufallsveränderlichen und ihren Eigenschaften. Überleben bedeutet, tautologisch definiert, nicht mehr als nicht auszuscheiden. Diese Definition ist für den ersten Abschnitt ausreichend, eine genauere folgt im Abschnitt 4.2 Im ersten Abschnitt werden zwei Würfelspielerinnen betrachtet, die in einem Ausscheidungswettkampf gegeneinander antreten. Sie dienen als ein sehr einfaches Beispiel für Überlebenswahrscheinlichkeiten. Wer häufiger eine größere Zahl geworfen hat als die Konkurrentin, erreicht die nächste Runde. Handelt es sich bei den „Würfeln” nicht um gegenständliche Quader, sondern um Zufallszahlengeneratoren, die gemäß einer ausgewählten Verteilung Zahlen erzeugen, fällt die Wahl der Verteilung oft nicht leicht: Ist, gleicher Erwartungswert vorausgesetzt, eine Gleichverteilung einer Normalverteilung überlegen? Oder sollte einer Dreieckverteilung der Vorzug gegeben werden? Wenn ja, einer symmetrischen oder einer schiefen? Schief in welche Richtung? Bereits bei dieser simplen Fragestellung entstehen eine Reihe mathematisch anspruchsvoller Probleme. Ein Teil dieser Fragen wird in diesem Abschnitt näher beleuchtet. Dabei beschränke ich mich zunächst im wesentlichen auf zwei Zufallsveränderliche in einem statischen System und die Frage, welche der beiden Zufallsvariablen realisiert sich öfter größer als die andere, oder mit anderen Worten, welche ist überlegen?

In Abschnitt 4.2 wird, wie bereits angekündigt, der Begriff der Überlebenswahrscheinlichkeit, der schon in Kapitel 2 in einem intuitiven Sinne gebraucht wurde, formal präziser gefaßt. Nachfolgend stehen sachlogische Überlegungen im Vordergrund, die es ermöglichen, die Wirkungsrichtung bestimmter Einflußfaktoren auf die Überlebenswahrscheinlichkeit abzuleiten, so daß diese Fragen nicht mehr analytisch oder in einer Simulationsstudie untersucht zu werden brauchen. Im nächsten Abschnitt erfolgt eine Analyse der Wahrscheinlichkeitsverteilung von Funktionen von Zufallsvariablen. 
Dieses Thema wird deshalb behandelt, weil Überlebensmodelle oft Systeme abbilden, die Funktionen von Zufallsveränderlichen enthalten. Daher erleichtern Kenntnisse der wahrscheinlichkeitstheoretischen Eigenschaften von zusammengesetzten Zufallsveränderlichen die Modellanalyse. Die Untersuchung beschränkt sich nicht auf die Betrachtung von Momenten, da diese zum Beurteilen von Überlebenswahrscheinlichkeiten nicht ausreichen. Das Kapitel schließt mit mathematischen Problemen in Monte-Carlo-Simulationen, die aus der Endlichkeit des Stichprobenumfangs resultieren. Die Schwierigkeiten werden am Beispiel eines einfachen mehrperiodischen Spiels, des bekannten Petersburger Paradoxons, demonstriert.

Die Analyse des Petersburger Paradoxons ist nach einer Systematik von WEINSCHENCK (1977) der Objektforschung zuzuordnen. Sie bildet zusammen mit der theoretischen Systemforschung, die sich mit der Theorie und den Eigenschaften mathematischer Systemmodelle beschäftigt, die empirische Systemforschung, auch Systemanalyse genannt. Zur Objektforschung gehören auch die einfachen dynamischen Systeme, die im nächsten Kapitel vorgestellt werden, sowie Betriebsentwicklungsmodelle, die den zweiten Hauptteil dieser Arbeit ausmachen. Die anderen genannten Teile dieses Kapitels hingegen können der theoretischen Systemforschung zugeordnet werden.

\subsection{Vergleich zweier Zufallsveränderlicher}

Dieser Abschnitt beschäftigt sich mit Wahrscheinlichkeiten: Den Wahrscheinlichkeiten, daß sich eine Zufallsvariable ${ }^{113} \xi$ größer als eine Konstante, größer als eine andere Zufallsvariable und größer als eine Summe von Zufallsvariablen realisiert.

Zunächst sei die Wahrscheinlichkeit betrachtet, daß eine Zufallsveränderliche $\xi$ kleiner bzw. größer als eine Konstante $a$ ist (s. nebenstehende Grafik $\left.^{114}\right)$. Die Wahrscheinlichkeit für $\xi \leq a$ wird bekanntlich durch die Verteilungsfunktion ausgedrückt: Die Verteilungsfunktion der Zufallsvariablen $\xi$ an der Stelle $a$, notiert als $\mathrm{F}_{\xi}(a)$, ist das Integral der Dichtefunktion von $\xi$ im Intervall $[-\infty, a]$.

Daher gilt für die Wahrscheinlichkeit $\xi \leq a$ :

113 In diesem Abschnitt werden Zufallsvariablen mit den kleinen griechischen Buchstaben $\xi$ und $\eta$ und ihre konkreten Realisationen mit den lateinischen Buchstaben $x$ und $y$ bezeichnet.

114 Die abgebildete Dichtefunktion steht stellvertretend für eine beliebige Verteilung. Eine Dreiecksverteilung bietet den Vorteil, sich relativ leicht grafisch darstellen zu lassen. 


$$
\mathrm{p}(\xi \leq a)=\mathrm{F}_{\xi}(a)=\int_{-\infty}^{a} \mathrm{f}_{x}(x) \cdot \mathrm{d} x
$$

und für das Gegenereignis $\xi>a$ :

$$
\begin{aligned}
\mathrm{p}(\xi>a) \quad & =1-\mathrm{p}(\xi \leq a)=1-\mathrm{F}_{\xi}(a) \\
& =1-\int_{-\infty}^{a} \mathrm{f}_{x}(x) \cdot \mathrm{d} x=\int_{a}^{\infty} \mathrm{f}_{x}(x) \cdot \mathrm{d} x .
\end{aligned}
$$

Wir betrachten nun die Zufallsveränderliche $\xi$ nicht im Vergleich zu einer Konstanten, sondern statt dessen die Wahrscheinlichkeit, daß $\xi$ sich kleiner oder gleich bzw. größer als eine andere Zufallsveränderliche $\eta$ realisiert. Der Graf zeigt beispielhaft zwei Dichtefunktionen:

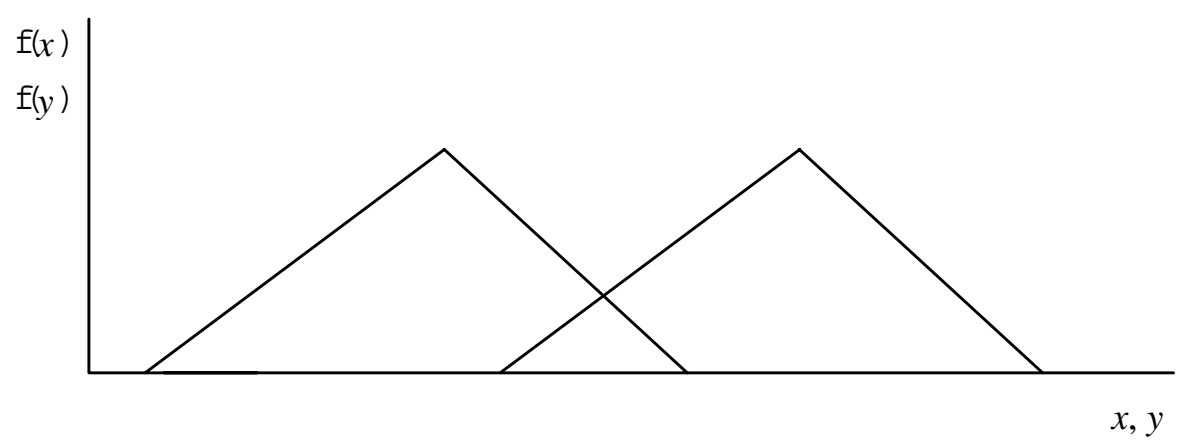

Um die Wahrscheinlichkeit $\xi>\eta$ zu berechnen, konzentriere ich mich zunächst auf die Wahrscheinlichkeit, daß sich die Zufallsveränderliche $\xi$ größer als ein beliebig herausgegriffenes, aber festes $y$ realisiert, und notiere diese Wahrscheinlichkeit mit

$$
\mathrm{p}(\xi>\eta \mid y){ }^{115}
$$

Diese Wahrscheinlichkeit läßt sich als bedingte Wahrscheinlichkeit im Sinne von Bayes, daß das Ereignis $x$ eintritt, wenn das Ereignis $y$ schon eingetreten ist, auffassen. Wenn die Zufallsvariable $\eta$ diskret verteilt ist, kann für jede ihrer Realisationsmöglichkeiten die Wahrscheinlichkeit $\mathrm{p}(\xi>\eta \mid y)$ bestimmt werden. Diese Wahrscheinlichkeiten sind anschließend gewichtet aufzusummieren, wobei als Gewichte die Einzelwahrscheinlichkeiten der Ausprägungen von $\eta$ verwendet werden:

$$
\mathrm{p}(\xi>\eta)=\sum_{y} \mathrm{p}(x>h \mid y) \cdot \mathrm{p}(y)
$$

Diese Formel läßt sich gut am Beispiel des Würfelns illustrieren. Die Augenzahl des ersten Würfelns werde durch die Zufallsvariable $\eta$ repräsentiert, die des zweiten durch $\xi$. $y$ sei die erzielte Augenzahl beim ersten Wurfs, $x$ die beim zweiten. $\mathrm{p}(\xi>\eta)$

115 Vgl. Fußnote 113. 
ist damit die Wahrscheinlichkeit, mit dem zweiten Wurf eine höhere Zahl zu werfen als mit dem ersten.

Tabelle 132.1: Wahrscheinlichkeiten bei zweimaligem Würfeln

\begin{tabular}{|c|c|c|c|c|}
\hline $\boldsymbol{y}$ & $\mathbf{p}(\boldsymbol{y})$ & $\mathbf{p}(\boldsymbol{x}>\boldsymbol{y})$ & $\mathbf{p}(\boldsymbol{x}>\boldsymbol{y}) \cdot \mathbf{p}(\boldsymbol{y})$ & $\sum \mathbf{p}(\boldsymbol{x}>\boldsymbol{y}) \cdot \mathbf{p}(\boldsymbol{y})$ \\
\hline \hline 1 & $1 / 6$ & $5 / 6$ & $5 / 36$ & $5 / 36$ \\
\hline 2 & $1 / 6$ & $4 / 6$ & $4 / 36$ & $9 / 36$ \\
\hline 3 & $1 / 6$ & $3 / 6$ & $3 / 36$ & $12 / 36$ \\
\hline 4 & $1 / 6$ & $2 / 6$ & $2 / 36$ & $14 / 36$ \\
\hline 5 & $1 / 6$ & $1 / 6$ & $1 / 36$ & $15 / 36$ \\
\hline 6 & $1 / 6$ & 0 & 0 & $15 / 36$ \\
\hline
\end{tabular}

Tabelle 132.1 zeigt für alle Realisationsmöglichkeiten des ersten Wurfes (1. Spalte) die Wahrscheinlichkeit, beim zweiten Wurf eine höhere Augenzahl zu erzielen (3. Spalte). Das gewichtete Mittel dieser Wahrscheinlichkeiten ist in der unteren rechten Ecke der Tabelle ausgewiesen: $15 / 36=5 / 12$. Sie ist, vielleicht anfänglich, überraschend, kleiner als 1/2, da von den insgesamt 36 (gleichwahrscheinlichen) Kombinationen der beiden Würfel nur in 15 Fällen der zweite Würfel größer ist als der erste.

Im Falle einer stetigen Verteilung ist der Summenoperator in 131,3 durch ein Integral und die (diskreten) Wahrscheinlichkeiten $\mathrm{p}$ durch die Werte der Dichtefunktion von $\eta$ zu ersetzen: ${ }^{116}$

$$
\mathrm{p}(\xi>\eta) \quad=\int_{y} \mathrm{p}(x>h \mid y) \cdot \mathrm{f}(y) \cdot \mathrm{d} y .
$$

Mit der für ein festes $y$ gültigen Gleichung (131,2] wird daraus:

$$
\begin{aligned}
\mathrm{p}(\xi>\eta) \quad & =\int_{y}\left[1-\mathrm{F}_{x}(y)\right] \cdot \mathrm{f}(y) \cdot \mathrm{d} y \\
& =\int_{y} \int_{y}^{\infty} \mathrm{f}_{x}(x) \mathrm{d} x \cdot \mathrm{f}_{h}(y) \cdot \mathrm{d} y .
\end{aligned}
$$

Diese Formel gibt also die Wahrscheinlichkeit, daß sich die Zufallsveränderliche $\xi$ größer als die Zufallsvariable $\eta$ realisiert, mit Hilfe der Dichtefunktionen von $\xi$ und $\eta$ an. Ist die Wahrscheinlichkeit größer als $1 / 2$, realisiert sich $\xi$ öfter größer als $\eta$ und wird daher im weiteren als $\eta$ überlegen bezeichnet:

116 Vgl. GibBONS (1992, S. 80). 
Die Verteilung $\xi$ heißt der Verteilung $\eta$ überlegen, wenn gilt: $\mathrm{p}(\xi>\eta)>1 / 2$.

Für spezielle stetige Verteilungen läßt sich die Wahrscheinlichkeit $\mathrm{p}(\xi \leq \eta)$ analytisch angeben: Die Zufallsvariablen müssen identisch und unabhängig verteilt sein. Diese Bedingung wird im Englischen mit iid (identically and independently distributed) abgekürzt. Sie bedeutet, daß die Verteilungs- und Dichtefunktionen der Zufallsveränderlichen jeweils übereinstimmen:

$$
\mathrm{F}_{\xi}=\mathrm{F}_{\eta} \Rightarrow \mathrm{f}_{\xi}=\mathrm{f}_{\eta} .
$$

Daher erhalte ich in diesem Spezialfall für die Gegenwahrscheinlichkeit von 132, 2, vgl. (131.1) und 1312:

$$
\begin{aligned}
\mathrm{p}(\xi \leq \eta) \quad & =\int_{y} \mathrm{~F}_{x}(y) \cdot \mathrm{f}_{h}(y) \cdot \mathrm{d} y \\
& =\int_{-\infty}^{+\infty} \mathrm{F}(y) \cdot \mathrm{f}(y) \cdot \mathrm{d} y \\
& =\int_{-\infty}^{+\infty} \mathrm{F}(y) \cdot \mathrm{F}^{\prime}(y) \cdot \mathrm{d} y .
\end{aligned}
$$

Einsetzen der Integrationsregel:

$$
\int_{-\infty}^{+\infty} \mathrm{y} \mathrm{y}^{\prime} \cdot \mathrm{d} x=1 / 2\left|y^{2}\right|_{-\infty}^{+\infty}
$$

führt zu:

$$
\begin{aligned}
\mathrm{p}(\xi \leq \eta) & =\int_{-\infty}^{+\infty} \mathrm{F}(y) \cdot \mathrm{F}^{\prime}(y) \cdot \mathrm{d} y \\
& =1 / 2\left|[\mathrm{~F}(y)]^{2}\right|_{-\infty}^{+\infty} \\
& =1 / 2\left[[\mathrm{~F}(\infty)]^{2}-[\mathrm{F}(-\infty)]^{2}\right] \\
& =1 / 2\left(1^{2}-0^{2}\right)=1 / 2 .
\end{aligned}
$$

Die Wahrscheinlichkeit, daß sich eine stetige Zufallsveränderliche kleiner als eine andere, identisch und unabhängig verteilte Zufallsveränderliche realisiert, ist also im Unterschied zu diskreten Zufallsveränderlichen 1/2. Dieses Ergebnis gilt für jeden Verteilungstyp und beliebige Parameter der Verteilungen. 
Ein kleines Simulationsexperiment, dessen Ergebnisse in Tabelle 134.1 zusammengefaßt sind, bestätigt diese deduktiven Ergebnisse. ${ }^{117}$ Für Gleich-, Normal- und (auch unsymmetrische) Dreieckverteilung beträgt die Wahrscheinlichkeit 1/2, unabhängig von Erwartungswert, Varianz und Symmetrie der Verteilung.

\section{Tabelle 134.1: Simulationsergebnisse für $\mathrm{p}(\xi \leq \eta), \xi$ und $\eta$ identisch und} unabhängig verteilt

\begin{tabular}{|c|c|c|c|c|}
\hline $\begin{array}{c}\text { Verteilungs- } \\
\text { form }\end{array}$ & \multicolumn{2}{|c|}{ Parameter } & $\mathbf{p}(\boldsymbol{\xi} \leq \eta)$ & Besonderheit \\
\hline \hline Gleich & $u=-1, o=1$ & dito & 0.50225 & Gleichverteilung \\
\hline Gleich & $u=-2, o=2$ & dito & 0.50225 & $\sigma^{2} \uparrow$ \\
\hline Gleich & $u=-1, o=3$ & dito & 0.50225 & $\mu \neq 0$ \\
\hline Normal & $\mu=0, \sigma^{2}=1$ & dito & 0.49786 & Normalverteilung \\
\hline Normal & $\mu=0, \sigma^{2}=4$ & dito & 0.49786 & $\sigma^{2} \uparrow$ \\
\hline Normal & $\mu=1, \sigma^{2}=4$ & dito & 0.49786 & $\mu \neq 0$ \\
\hline Dreieck & $u=-1, h=0, o=1$ & dito & 0.5019 & Dreieckverteilung \\
\hline Dreieck & $u=-2, h=0, o=2$ & dito & 0.5019 & $\sigma^{2} \uparrow$ \\
\hline Dreieck & $u=-2, h=1, o=2$ & dito & 0.5019 & $\mu \neq 0$, schief \\
\hline
\end{tabular}

Mit Hilfe der Simulationstechnik läßt sich leicht überprüfen, ob die iid-Annahme gelockert werden kann. Naheliegend ist z.B. die Vermutung, daß die Wahrscheinlichkeit $\mathrm{p}(\xi \leq \eta)$ auch dann $1 / 2$ beträgt, wenn symmetrische Verteilungen nur im Erwartungswert übereinstimmen, sich in den anderen Momenten aber unterscheiden. Bei unsymmetrischen Verteilungen fallen Erwartungswert $(\mu)$ und Median $(m)$ auseinander. Da sich eine unsymmetrische Zufallsvariable in der Hälfte der Fälle kleiner als ihr Median (und nicht als ihr Erwartungswert) realisiert, scheint es sinnvoller, eine Übereinstimmung der Mediane zu fordern. In einem Simulationsexperiment, dessen Ergebnisse in Tabelle 138 1f wiedergegeben sind, wird die Überlegenheit zweier Zufallsveränderlicher für folgende Szenarien geprüft:

- symmetrische Verteilungen (Gleich-, Normal- und Dreieckverteilungen)

$\diamond$ gleicher Erwartungswert, ungleiche Varianz

$\diamond$ gleicher Median, ungleiche Varianz

117 Die Simulationen dienen darüber hinaus zur Verifikation eines Monte-Carlo-Simulationsprogramms, das für die Untersuchung der Überlegenheit bei nicht identischen Verteilungen benutzt und später beschrieben wird. 
- unsymmetrische Dreieckverteilungen

$\diamond$ gleicher Erwartungswert, ungleiche Varianz

$\diamond$ gleicher Median, ungleiche Varianz

$\diamond$ gleicher Erwartungswert und gleiche Varianz, aber ungleiche Schiefe

$\diamond$ gleicher Erwartungswert und gleiche Schiefe, aber ungleiche Varianz

$\checkmark$ gleicher Median und gleiche Varianz, aber ungleiche Schiefe

$\diamond$ gleicher Median und gleiche Schiefe, aber ungleiche Varianz.

Zuvor informiert Übersicht 136.1 über wichtige Kennzahlen von Dreieckverteilungen. Die abgeleiteten Kennzahlen werden benötigt, da mit ihrer Hilfe für vorgegebene Eigenschaften von Dreieckverteilungen ihre zugehörigen Parameter $(u, h, o)$ berechnet werden können (s. Tabelle 140 1]. In einigen Formeln wird die HeavisideFunktion benutzt. Diese Funktion erleichtert es, Fallunterscheidungen auszudrücken. Die Funktion liefert den Wert +1 zurück, wenn ihr Argument positiv ist, und sonst Null. Sie wird abgekürzt mit $\mathrm{H}(x)$. Beispielsweise lautet die Vorschrift:

$$
a=\left\{\begin{array}{l}
2, \text { wenn } x<y \\
0 \text { sonst }
\end{array}\right.
$$

in Heaviside-Schreibweise:

$$
a=2 \cdot \mathrm{H}(x-y) .
$$

Zunächst wird der Median einer rechtssteilen Verteilung abgeleitet. Bei einer rechtssteilen Verteilung, die auch linksschief genannt wird (RINNE 1997, S. 58), sind große Merkmalswerte häufiger als kleine Werte. Die betragsmäßige Steigung der Dichtefunktion ist daher rechts des Modalwerts größer als links davon. Median und Erwartungswert liegen links des Modalwertes, wobei der Erwartungswert stets kleiner als der Median ist.

Die Herleitung erfolgt über die Definitionsgleichung des Medians:

$$
m \quad \equiv\{x \mid \mathrm{F}(x)=1 / 2\} .
$$

Die Verteilungsfunktion wird bekanntlich durch Integration der Dichtefunktion bestimmt. Für den Spezialfall einer rechtssteilen Verteilung erhalte ich eine Formel, ist sehr viel einfacher als eine allgemeine Medianformel ist, weil auf Fallunterscheidungen verzichtet werden kann. Sie basiert auf einer Flächenbestimmung unterhalb der Dichtefunktion dieser Dreieckverteilung. (Von links $(u)$ kommend, wird die Fläche durch Erhöhen von $x$ solange vergrößert, bis sie $1 / 2$ ist.) Die einfache Formel wird soweit als möglich für die weiteren Rechnungen benutzt. Da sie nicht für alle verwendeten Szenarien ausreicht, leite ich im zweiten Teil der Übersicht die Medianformel für eine beliebige Dreieckverteilung ab. Dazu werden die Dichte- und die Verteilungsfunktion zunächst auf allgemeine Dreieckverteilungen im Intervall $[u, o]$ erweitert. Im nächsten Schritt erweitere ich das Intervall auf $[-\infty,+\infty]$. Nun kann der gleiche Ansatz 
wie bei der rechtssteilen Verteilung verwandt werden. Neben den bekannten Formeln für Erwartungswert und Varianz ist zusätzlich eine Bestimmungsgleichung für das dritte Moment, die absolute Schiefe, angegeben. Ich habe sie über die Definition der absoluten Schiefe ${ }^{118}$

$$
s \quad \equiv \int[x-\mathrm{E}(x)]^{3} \mathrm{~d} x
$$

gewonnen.

\section{Übersicht 136.1: $\quad$ Kennzahlen der Dreieckverteilung}

\begin{tabular}{|c|c|}
\hline Parameter: & $\begin{array}{l}\text { Unterster Wert: } u \text {, häufigster Wert: } h \text {, oberster Wert: } o \\
\qquad u \leq h \leq o\end{array}$ \\
\hline \multicolumn{2}{|c|}{$\begin{array}{l}\text { für rechtssteile Verteilung (d.h. Modus ist größer als Mitte von } u \text { und } o: h>\frac{u+o}{2} \text { ), } \\
\text { Dichte- und Verteilungsfunktion nur für den Bereich } \boldsymbol{u} \leq \boldsymbol{x} \leq \boldsymbol{h} \text { gültig. }\end{array}$} \\
\hline Dichtefunktion & $\mathrm{f}(x)=2 \cdot \frac{x-u}{(o-u)(h-u)}$ \\
\hline $\begin{array}{l}\text { Verteilungs- } \\
\text { funktion }\end{array}$ & $\begin{array}{c}\mathrm{F}(x)=\int_{u}^{x} \mathrm{f}(y) d y \\
=\int_{u}^{x} 2 \frac{y-u}{(o-u)(h-u)} d y \\
=\frac{(x-u)^{2}}{(o-u)(h-u)}\end{array}$ \\
\hline Median & $\begin{array}{c}m \equiv\{x \mid \mathrm{F}(x)=1 / 2\} \\
=\{x \mid(\mathrm{x}-\mathrm{u}) \cdot \mathrm{f}(x) \cdot 1 / 2=1 / 2\} \text { (Flächenbetrachtung) } \\
=u+\frac{1}{2} \sqrt{2 o h-2 o u-2 u h+2 u^{2}} \\
u+\sqrt{2 \cdot(u-o) \cdot(u-h)}\end{array}$ \\
\hline für beliebig & ilung, aber weiterhin nur für den Bereich $u \leq x \leq h$ \\
\hline Dichtefunktion & $\mathrm{f}(x)=2 \frac{(x-u) \mathrm{H}(h-x)}{(o-u)(h-u)}+2 \frac{(o-x) \mathrm{H}(x-h)}{(o-u)(o-h)}$ \\
\hline
\end{tabular}

118 Die relative Schiefe ist der Quotient aus absoluter Schiefe und Varianz, vgl. RINNE (1997, S. 58). 


\begin{tabular}{|c|c|}
\hline $\begin{array}{l}\text { Verteilungs- } \\
\text { funktion }\end{array}$ & $\begin{array}{c}\mathrm{F}(x)=\int_{u} \mathrm{f}(y) d y \\
=\int_{u}^{x} 2 \frac{(y-u) \mathrm{H}(h-y)}{(o-u)(h-u)}+2 \frac{(o-y) \mathrm{H}(y-h)}{(o-u)(o-h)} d y \\
=\frac{(x-u)^{2} \mathrm{H}(h-x)}{(o-u)(h-u)}+\left(1-\frac{(o-x)^{2}}{(o-u)(o-h)}\right) \mathrm{H}(-h+x)\end{array}$ \\
\hline \multicolumn{2}{|c|}{$\begin{array}{c}\text { für beliebige Verteilung, Dichte- und Verteilungsfunktion auch gültig, wenn ihr } \\
\text { Argument } x \text { außerhalb des Intervalls }[u, o] \text { liegt }\end{array}$} \\
\hline Dichtefunktion & $\mathrm{f}(x)=2 \frac{(x-u) \mathrm{H}(x-u) \mathrm{H}(-x+h)}{(o-u)(h-u)}+2 \frac{(o-x) \mathrm{H}(x-h) \mathrm{H}(o-x)}{(o-u)(o-h)}$ \\
\hline $\begin{array}{l}\text { Verteilungs- } \\
\text { funktion }\end{array}$ & $\begin{array}{c}\mathrm{F}(x)=\frac{(x-u)^{2} \mathrm{H}(x-u) \mathrm{H}(-x+h)}{(o-u)(h-u)} \\
+\left(1-\frac{(o-x)^{2}}{(o-u)(o-h)}\right) \mathrm{H}(x-h) \mathrm{H}(o-x)+\mathrm{H}(x-o)\end{array}$ \\
\hline Mittelwert & $\mu:=\frac{u+h+o}{3}$ \\
\hline Varianz & $\operatorname{Var}:=\frac{(o-u)^{2}-(o-u) \cdot(h-u)+(h-u)^{2}}{18}$ \\
\hline Schiefe & $s:=\frac{2}{5} \frac{(h+o-2 u)(-2 h+o+u)(2 o-h-u)}{\left(o^{2}-o u+u^{2}-o h-h u+h^{2}\right) \sqrt{2 o^{2}-2 o u+2 u^{2}-2 o h-2 h u+2 h^{2}}}$ \\
\hline Median & $\begin{array}{l}m=u+\sqrt{2 \cdot(u-o) \cdot(u-h)} \cdot \mathrm{H}\left(h-\frac{u+o}{2}\right) \\
+o-\sqrt{2 \cdot(u-o) \cdot(h-o)} \cdot \mathrm{H}\left(-h+\frac{u+o}{2}\right)\end{array}$ \\
\hline
\end{tabular}

Tabelle 138.1 gibt Simulationsergebnisse für die Überlegenheit zwischen $\xi$ und $\eta$, wenn zwei Voraussetzungen gleichzeitig erfüllt sind: a) $\xi$ und $\eta$ sind symmetrisch verteilt und b) sie besitzen den gleichen Erwartungswert. Die vorletzte Spalte der Tabelle zeigt, daß unter diesen Annahmen keine Überlegenheit besteht, wenn die Zufallsveränderlichen sich in den Varianzen unterscheiden (Szenarien 1 bis 6), $\eta$ sich also als Funktion von $\xi$ darstellen läßt: 
$\eta$

$$
=\sqrt{\frac{\operatorname{Var}(h)}{\operatorname{Var}(x)}} \cdot[\xi-\mathrm{E}(\xi)]+\mathrm{E}(\eta) .
$$

Eine Überlegenheit ist ebenfalls nicht festzustellen, wenn die Verteilungen sich außer in der Varianz auch im Verteilungstyp unterscheiden (Szenarien 7 bis 9).

Tabelle 138.1: Simulationsergebnisse für $\mathbf{p}(\xi \leq \eta)$,

1. Teil: symmetrische Verteilung für $\xi$ und $\eta$, identische

Erwartungswerte

\begin{tabular}{|c|c|c|c|c|c|c|c|}
\hline $\begin{array}{c}\text { Sze- } \\
\text { nario }\end{array}$ & $\begin{array}{c}\text { Vertei- } \\
\text { lungsform }\end{array}$ & Parameter & Momente & Parameter & Momente & $\mathbf{p}(\xi \leq \eta)$ & $\begin{array}{l}\text { Beson- } \\
\text { derheit }\end{array}$ \\
\hline 1 & Gleich & $u=-1, o=1$ & $\mathrm{E}=0, \sigma^{2}=1 / 3$ & $u=-2, o=2$ & $\mathrm{E}=0, \sigma^{2}=4 / 3$ & 0.5012 & Normalv. \\
\hline 2 & Gleich & $u=0, o=2$ & $\mathrm{E}=1, \sigma^{2}=1 / 3$ & $u=-1, o=3$ & $\mathrm{E}=1, \sigma^{2}=4 / 3$ & 0.5012 & $\mu \neq 0$ \\
\hline 3 & Normal & $\mu=0, \sigma^{2}=1$ & & $\mu=0, \sigma^{2}=4$ & & 0.4984 & Gleichv. \\
\hline 4 & Normal & $\mu=1, \sigma^{2}=1$ & & $\mu=1, \sigma^{2}=4$ & & 0.4984 & $\mu \neq 0$ \\
\hline 5 & Dreieck & $u=-1, h=0, o=1$ & $\mathrm{E}=0, \sigma^{2}=1 / 6$ & $u=-2, h=0, o=2$ & $\mathrm{E}=0, \sigma^{2}=4 / 6$ & 0.4998 & Dreieckv. \\
\hline 6 & Dreieck & $u=1, h=2, o=3$ & $\mathrm{E}=2, \sigma^{2}=1 / 6$ & $u=0, h=2, o=4$ & $\mathrm{E}=2, \sigma^{2}=4 / 6$ & 0.5018 & $\mu \neq 0$ \\
\hline 7 & $\begin{array}{l}\text { Gleich/ } \\
\text { Normal }\end{array}$ & $u=-1, o=5$ & $\mathrm{E}=2, \sigma^{2}=3$ & $\mu=2, \sigma^{2}=9$ & & 0.5016 & \\
\hline 8 & $\begin{array}{l}\text { Gleich/ } \\
\text { Dreieck }\end{array}$ & $u=-1, o=5$ & $\mathrm{E}=2, \sigma^{2}=3$ & $u=-3, h=2, o=7$ & $\mathrm{E}=4, \sigma^{2}=25 / 6$ & 0.5030 & 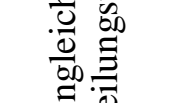 \\
\hline 9 & $\begin{array}{l}\text { Normal/ } \\
\text { Dreieck }\end{array}$ & $\mu=2, \sigma^{2}=1$ & & $u=-3, h=2, o=7$ & $\mathrm{E}=4, \sigma^{2}=25 / 6$ & 0.5004 & $\stackrel{\frac{0}{0}}{>}$ \\
\hline \multicolumn{8}{|c|}{10000 Wiederholungen } \\
\hline
\end{tabular}

Die Simulationsergebnisse in obiger Tabelle (2. Spalte von rechts) sind in der Schriftart Courier dargestellt. Diese Schriftart wird generell zur Kenntlichmachung von Simulationsergebnissen verwandt.

Die Frage, ob eine Verteilung einer anderen mit gleichem Erwartungswert, aber verschiedener Varianz überlegen sein kann, läßt sich für eine Normalverteilung auch deduktiv beantworten. Die Wahrscheinlichkeit $\mathrm{p}(\xi \leq \eta)$ ist äquivalent zu $\mathrm{p}(\xi-\eta \leq 0)$. Wegen der Reproduktionseigenschaft der Normalverteilung ${ }^{119}$ ist $\xi-\eta$ ebenfalls eine Normalverteilung mit Erwartungswert $\mu_{\xi}-\mu_{\eta}$. Da annahmegemäß die Erwartungswerte von $\xi$ und $\eta$ gleich sind, ist die Differenz $\xi-\eta$ normal um Null verteilt. Die Frage nach der Überlegenheit von Normalverteilungen mit gleichem Erwartungswert

119 Sie besagt: Wenn $x \sim \mathrm{N}\left(\mu, \sigma^{2}\right)$, dann $(a+b x) \sim \mathrm{N}\left(a+b \mu, b^{2} \sigma^{2}\right)$, vgl. GREEN (1993, S. 58). Auch die $\chi^{2}$-Verteilung besitzt die Reproduktionseigenschaft, vgl. SPRINGER (1979, S. 63). Daher gilt das abgeleitete Ergebnis auch für die $\chi^{2}$-Verteilung. 
ist gleichbedeutend mit der Wahrscheinlichkeit, daß sich eine Zufallsveränderliche kleiner als ihr Erwartungswert realisiert. Diese Wahrscheinlichkeit ist bekanntlich für alle symmetrischen Verteilungen $1 / 2$, da für sie Median und Erwartungswert zusammenfallen, und ist damit unabhängig von ihrer Varianz.

In Tabelle 140.1 sind Ergebnisse für unsymmetrische Verteilungen aufbereitet. Die Simulationen beschränken sich auf Dreieckverteilungen als einem einfachen Beispiel für eine unsymmetrische Verteilung. In Szenario 10 wird eine leicht linksschiefe Verteilung $\xi$ mit einer symmetrischen Verteilung $\eta$ verglichen. Beide Verteilungen sind durch den gleichen Erwartungswert gekennzeichnet, sie unterscheiden sich in Varianz und Median. Die Wahrscheinlichkeit $\mathrm{p}(\xi \leq \eta)=0.480$ weicht deutlich von $1 / 2 \mathrm{ab}$, die symmetrische Verteilung $\xi$ ist überlegen. Die Überlegenheit verstärkt sich, wenn die Varianz der symmetrischen Zufallsveränderlichen verringert wird. Im Extrem einer Einpunktverteilung (Szenario 11) beträgt die Überlegenheit 55:45.

Wie erklärt sich diese ausgeprägte Überlegenheit? Praktisch wird in Experiment 11 die Wahrscheinlichkeit $\mathrm{p}\left(\xi \leq \mu_{\xi}\right)$ simuliert. Aufgrund der Linksschiefe von $\xi$ übertrifft ihr Median ihren Erwartungswert. Die Wahrscheinlichkeit $\mathrm{p}\left(\xi \leq m_{\xi}\right)$ beträgt $1 / 2$, folglich muß die Wahrscheinlichkeit $\mathrm{p}\left(\xi \leq \mu_{\xi}\right)$ kleiner als $1 / 2$ sein, da der Erwartungswert kleiner als der Median ist:

$$
\mathrm{p}\left(\xi \leq \mu_{\xi} \mid \mu_{\xi}<m_{\xi}\right)<1 / 2 .
$$

Oben war die Vermutung geäußert worden, daß zwischen unsymmetrischen Verteilungen, die im Median übereinstimmen, keine Überlegenheit bestünde. Diese Hypothese wird durch die Simulationen nicht gestützt (s. Szenario 12). Die Abweichung der Wahrscheinlichkeit $\mathrm{p}(\xi \leq \eta)$ von $1 / 2$ ist ähnlich groß wie bei gleichen Erwartungswerten (10). Wird wiederum die symmetrische Verteilung $\eta$ degeneriert, nähert sich dieses Mal selbstverständlich die Wahrscheinlichkeit $1 / 2$ an, da $\eta$ über dem Median von $\xi$ kollabiert (13).

Stimmt neben dem Erwartungswert noch ein weiteres Moment der beiden Verteilungen überein, führt dies ebenfalls nicht zu Wahrscheinlichkeiten von 1/2. Die Simulationsergebnisse für gleiche Varianzen $(14,14 a)$ oder gleiche Schiefen (15) liegen deutlich unter 0.5. Gleiches gilt, wenn die Verteilungen im Median und in einem höheren Moment übereinstimmen (16, 16a, 17). Wird eine der Verteilungen symmetrisch gewählt, verringern sich die Abweichungen von 1/2 (14a, 16a).

Die Simulationen zeigen also, daß im Gegensatz zu symmetrischen Verteilungen bei schiefen Verteilungen Über- bzw. Unterlegenheit möglich sind und die höheren Momente die Überlegenheit beeinflussen. In Tabelle 1411 sind Dreieckverteilungen unterschiedlicher Schiefe dargestellt, die jedoch durch Wahl entsprechender Parameter in Median und Varianz übereinstimmen. Schaubild 141.1 zeigt diese Verteilungen im Vergleich zu einer symmetrischen Verteilung gleicher Varianz und gleichen Medians. Es wird deutlich, daß rechtssteile Verteilungen $\left(s_{\eta}>0\right)$ der symmetrischen Referenzverteilung $\xi$ überlegen und linkssteile unterlegen sind. Zwischen der Schiefe von $\eta$ und der Wahrscheinlichkeit $\mathrm{p}(\xi \leq \eta)$ besteht annähernd ein linearer Zusammenhang. 
Tabelle 140.1: Simulationsergebnisse für $p(\xi \leq \eta)$,

2. Teil: unsymmetrische Dreieckverteilungen für $\xi$ und $\eta$

\begin{tabular}{|c|c|c|c|c|c|c|c|}
\hline \multirow{2}{*}{$\begin{array}{l}\text { Sze- } \\
\text { nario }\end{array}$} & \multicolumn{2}{|r|}{$\xi$} & \multicolumn{2}{|r|}{$\eta$} & \multirow{2}{*}{$\mathbf{p}(\xi \leq \eta)$} & \multirow{2}{*}{$\begin{array}{l}\text { Dichte- } \\
\text { funktion }\end{array}$} & \multirow{2}{*}{$\begin{array}{l}\text { Besonder- } \\
\text { heit }\end{array}$} \\
\hline & Param. & Momente & Param. & Momente, $m$ & & & \\
\hline 10 & $\begin{aligned} u & =-3 \\
h & =1 \\
o & =2\end{aligned}$ & $\begin{array}{c}\mathrm{E}=0, \sigma^{2}=7 / 6 \\
m=-3+\sqrt{ } 10 \approx 0.162 \\
s=-18 / 245 \cdot \sqrt{ } 42 \\
\approx-.476\end{array}$ & $\begin{aligned} u & =-2 \\
h & =0 \\
o & =2\end{aligned}$ & $\begin{array}{c}\mathrm{E}=0 \\
\sigma^{2}=2 / 3 \\
m=\mathrm{E} \\
s=0\end{array}$ & 0.480 & & $\begin{array}{c}\xi \\
\text { unsymm. }\end{array}$ \\
\hline 11 & $\begin{array}{l}\text { wie } \\
\text { oben }\end{array}$ & wie oben & $\begin{array}{c}u=h= \\
o=0\end{array}$ & $\begin{array}{c}\mathrm{E}=m=0, \\
\sigma^{2}=s=0\end{array}$ & 0.450 & & $\sigma_{\eta}=0$ \\
\hline 12 & $\begin{array}{c}u=-1 \\
h=-2 / 3 \\
o=2\end{array}$ & $\begin{array}{c}\mathrm{E}=1 / 9, m=0, \\
\sigma^{2}=73 / 162 \approx 0.451 \\
s=238 / 5329 \cdot \sqrt{ } 146 \\
\approx 0.540\end{array}$ & $\begin{aligned} u & =-1 \\
h & =0 \\
o & =1\end{aligned}$ & $\begin{array}{c}\mathrm{E}=m=0 \\
\sigma^{2}=1 / 6 \\
s=0\end{array}$ & 0.482 & & $\begin{array}{c}\mu_{\xi} \neq \mu_{\eta} \\
m_{\xi}=m_{\eta}=0\end{array}$ \\
\hline 13 & $\begin{array}{l}\text { wie } \\
\text { oben }\end{array}$ & wie oben & $\begin{array}{c}u=h= \\
o=0\end{array}$ & $\begin{array}{c}\mathrm{E}=m=0 \\
\sigma^{2}=s=0\end{array}$ & 0.500 & & $\sigma_{\eta}=0$ \\
\hline 14 & $\begin{aligned} u & =-3 \\
h & =1 \\
o & =2\end{aligned}$ & $\begin{array}{c}\mathrm{E}=0, \sigma^{2}=7 / 6, \\
m \approx 0.162, \\
s \approx-.476\end{array}$ & $\begin{array}{c}u=-2 \\
h=-1 \\
o=3\end{array}$ & $\begin{array}{c}\mathrm{E}=0, \sigma^{2}=7 / 6 \\
m \approx-0.162 \\
s \approx 0.476\end{array}$ & 0.480 & & $\begin{array}{c}\mu_{\xi}=\mu_{\eta}, \\
\text { gleiche } \\
\text { Varianz }\end{array}$ \\
\hline $14 \mathrm{a}$ & $\begin{array}{l}\text { wie } \\
\text { oben }\end{array}$ & wie oben & $\begin{array}{c}u=-\sqrt{ } 7 \\
h=0 \\
o=-\mathrm{u}\end{array}$ & $\begin{array}{c}\mathrm{E}=m=0 \\
\sigma^{2}=7 / 6 \\
s=0\end{array}$ & 0.490 & & $\begin{array}{l}\text { wie oben, } \\
\text { zusätzlich } \\
\eta \text { sym- } \\
\text { metrisch }\end{array}$ \\
\hline 15 & $\begin{array}{l}\text { wie } \\
\text { oben }\end{array}$ & wie oben & $\begin{array}{c}u=-2 \\
h=2 / 3 \\
o=4 / 3\end{array}$ & $\begin{array}{c}\mathrm{E}=0, \sigma^{2}=14 / 27 \\
m \approx 0.108 \\
s \approx-0.476\end{array}$ & 0.478 & & $\begin{array}{c}\mu_{\xi}=\mu_{\eta} \\
\text { gleiche } \\
\text { Schiefe }\end{array}$ \\
\hline 16 & $\begin{array}{c}u=-1 \\
h=-2 / 3 \\
o=2\end{array}$ & $\begin{array}{c}\mathrm{E}=1 / 9, m=0, \\
\sigma^{2} \approx 0.451, \\
s \approx 0.540\end{array}$ & $\begin{array}{c}u=-2 \\
h=2 / 3 \\
o=1\end{array}$ & $\begin{array}{c}\mathrm{E}=-1 / 9, m=0, \\
\sigma^{2} \approx 0.451 \\
s \approx-0.540\end{array}$ & 0.443 & & $\begin{array}{c}m_{\xi}=m_{\eta} \\
\text { gleiche } \\
\text { Varianz }\end{array}$ \\
\hline $16 \mathrm{a}$ & $\begin{array}{l}\text { wie } \\
\text { oben }\end{array}$ & wie oben & $\begin{array}{c}u=-1 / 9 \\
\sqrt{ } 219 \\
\approx-1.644 \\
h=0 \\
o=-\mathrm{u}\end{array}$ & $\begin{array}{l}\mathrm{E}=m=0 \\
\sigma^{2} \approx 0.451 \\
\quad s=0\end{array}$ & 0.473 & & $\begin{array}{l}\text { wie oben, } \\
\text { zusätzlich } \\
\eta \text { sym- } \\
\text { metrisch }\end{array}$ \\
\hline 17 & $\begin{array}{l}\text { wie } \\
\text { oben }\end{array}$ & wie oben & $\begin{array}{c}u=-2 \\
h=-4 / 3 \\
o=4\end{array}$ & $\begin{array}{c}\mathrm{E}=2 / 9, m=0 \\
\sigma^{2} \approx 1.802, \\
s \approx 0.540\end{array}$ & 0.488 & & $\begin{array}{c}m_{\xi}=m_{\eta} \\
\text { gleiche } \\
\text { Schiefe }\end{array}$ \\
\hline
\end{tabular}


Tabelle 141.1: Überlegenheit und Schiefe bei gleichem Median und gleicher Varianz

\begin{tabular}{|c|c|c|c|c|c|c|}
\hline \multicolumn{5}{|c|}{ Parameter und Kennzahlen der Verteilung $\eta$} & \multirow{2}{*}{$\mathbf{p}(\xi \leq \eta)$} & \\
\hline$u$ & $\boldsymbol{h}$ & $\boldsymbol{o}$ & $\mu$ & $s$ & & \\
\hline-3.6 & 9980013905 & 2.037231875 & -.19 & -.49 & .474 & \multirow{6}{*}{ 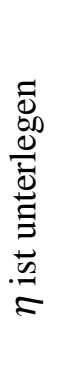 } \\
\hline-3.5 & .7219149989 & 2.303053829 & -.16 & -.39 & .478 & \\
\hline-3.4 & .5232706965 & 2.493042257 & -.13 & -.30 & .482 & \\
\hline-3.3 & .3623192475 & 2.647051179 & -.10 & -.20 & .487 & \\
\hline-3.2 & .2254126447 & 2.778841712 & -.07 & -.14 & .492 & \\
\hline-3.1 & .1059108781 & 2.895176014 & -.03 & -.07 & .497 & \\
\hline-3.0 & 0 & 3.0 & 0 & 0 & .5 & \\
\hline-2.8 & -.2035614723 & 3.182648816 & .06 & .13 & .512 & \multirow{7}{*}{ 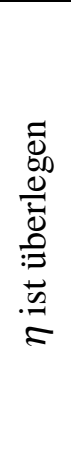 } \\
\hline-2.6 & -.4114272704 & 3.332427749 & .11 & .25 & .519 & \\
\hline-2.4 & -.6206144178 & 3.452085381 & .14 & .35 & .524 & \\
\hline-2.2 & -.8293023646 & 3.544092831 & .17 & .44 & .528 & \\
\hline-2.0 & -1.036400763 & 3.610503878 & .19 & .50 & .531 & \\
\hline-1.8 & -1.241284169 & 3.652932837 & .20 & .54 & .532 & \\
\hline-1.6 & -1.443633938 & 3.672570602 & .21 & .56 & .533 & \\
\hline \multicolumn{7}{|c|}{$\begin{array}{c}\xi \text { ist eine symmetrische Verteilung mit }-u=o=3, h=\mu=0 \text {. } \\
\text { Beide Verteilungen besitzen eine Varianz von } 1.5 \text { und einen Median von } 0 . \\
\text { Die Parameter } h \text { und } o \text { dienen als Inputs für das Simulationsprogramm. Daher sind sie mit } \\
\text { einer großen Anzahl von Dezimalstellen angegeben. }\end{array}$} \\
\hline
\end{tabular}

\section{Schaubild 141.1: Überlegenheit und Schiefe bei gleichem Median und gleicher Varianz}

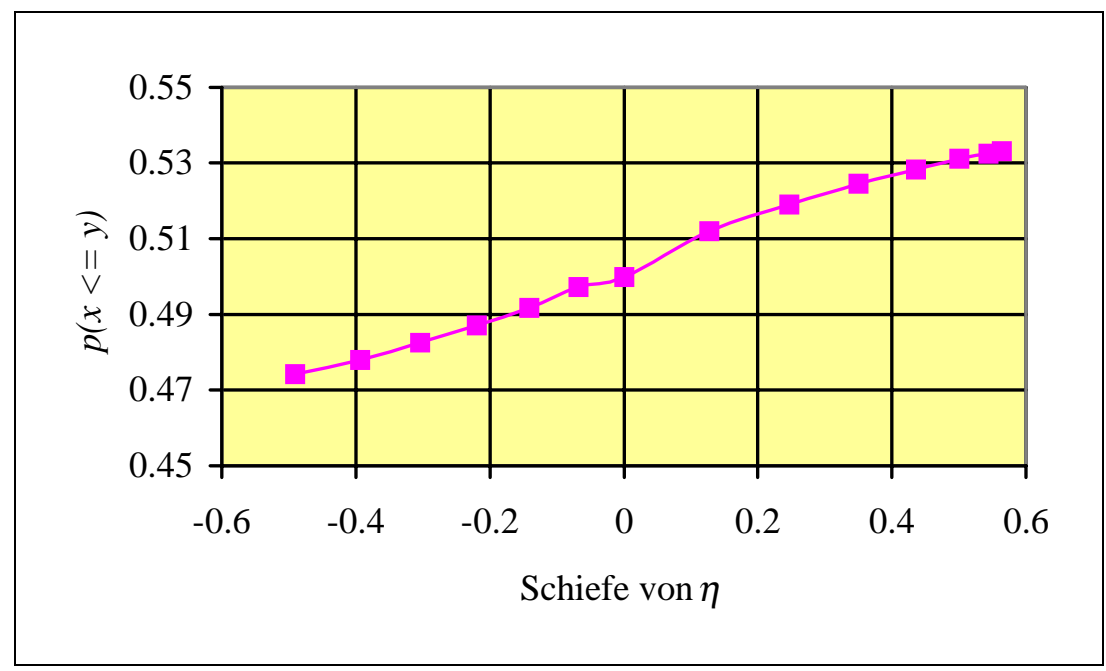


In Tabelle 142.1 und Schaubild 142.1 wird die gleiche Fragestellung noch einmal untersucht, nun für gleiche Erwartungswerte.

Tabelle 142.1: Wahrscheinlichkeit $\mathrm{p}(\xi \leq \eta)$ und Schiefe bei gleichem Erwartungswert

\begin{tabular}{|c|c|c|c|c|c|c|}
\hline \multicolumn{5}{|c|}{ Parameter und Kennzahlen der Verteilung $\eta$} & \multirow{2}{*}{$\mathbf{p}(\xi \leq \eta)$} & \\
\hline$u$ & $\boldsymbol{h}$ & $\boldsymbol{o}$ & $m$ & $s$ & & \\
\hline-3.4 & 1.125543733 & 2.274456266 & .18 & -.47 & .515 & \multirow{3}{*}{ 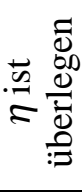 } \\
\hline-3.2 & .4510874719 & 2.748912529 & .10 & -.22 & .509 & \\
\hline-3.0 & 0 & 3.0 & 0 & 0 & .5 & \\
\hline-2.8 & -.3663251736 & 3.166352173 & -.08 & .18 & .497 & \multirow{6}{*}{ 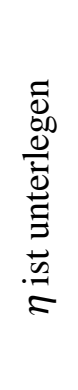 } \\
\hline-2.6 & -.6824227603 & 3.282422760 & -.13 & .32 & .494 & \\
\hline-2.4 & -.9633307655 & 3.363330765 & -.17 & .42 & .491 & \\
\hline-2.2 & -1.217326045 & 3.417326045 & -.19 & .50 & .489 & \\
\hline-2.0 & -1.449489743 & 3.449489743 & -.20 & .54 & .488 & \\
\hline-1.8 & -1.663201124 & 3.463201123 & -.21 & .56 & .487 & \\
\hline
\end{tabular}

Der Vergleich der Ergebnisse ist überraschend. Die Graphen für gleichen Erwartungswert bzw. gleichen Median haben entgegengesetzte Steigungen, sie scheinen nahezu an der Ordinate gespiegelt. Zur Veranschaulichung greifen wir die Referenzverteilung $\xi$ und je eine weitere Verteilung für $\eta$ aus den beiden obigen Tabellen heraus. Der kleinste Wert der beiden letztgenannten ist $u=-3.4$, alle drei haben eine Varianz von 1.5, vgl. Tabelle 143.1.

\section{Schaubild 142.1: Überlegenheit und Schiefe bei gleichem Erwartungswert und gleicher Varianz}

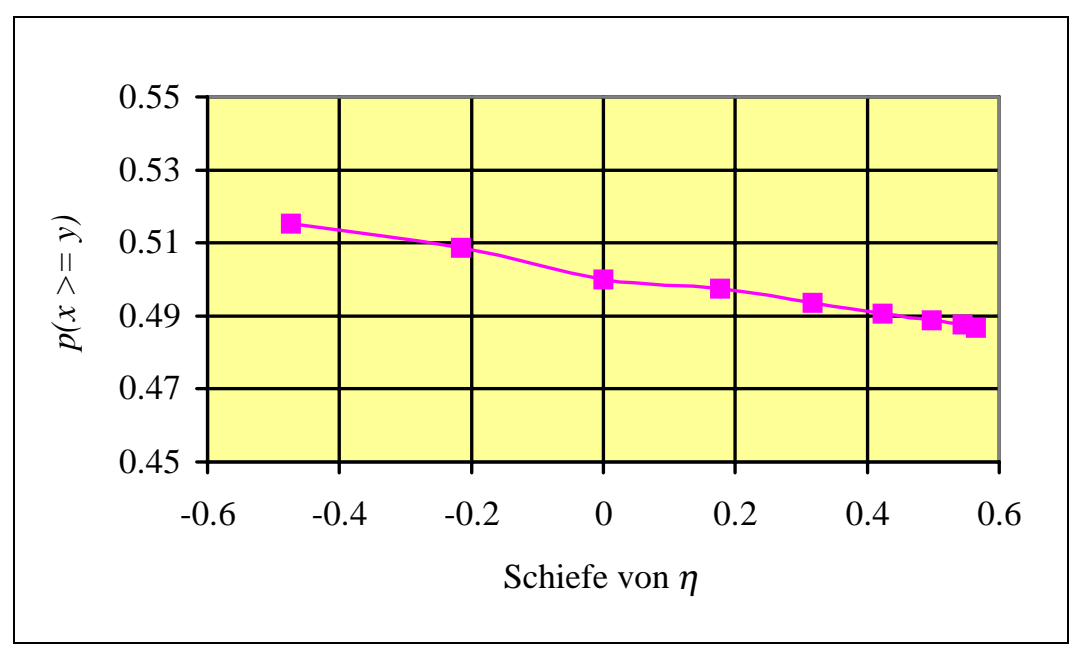


Tabelle 143.1: Vergleich von Verteilungen gleichen Erwartungswertes mit Verteilungen gleichen Medians

\begin{tabular}{|c|c|c|c|c|c|c|c|}
\hline $\boldsymbol{u}$ & $\boldsymbol{h}$ & $\boldsymbol{o}$ & $\boldsymbol{V a r}$ & $\boldsymbol{\mu}$ & $\boldsymbol{m}$ & $\boldsymbol{s}$ & $\mathbf{p}(\boldsymbol{\xi} \leq \boldsymbol{\eta})$ \\
\hline \hline-3.0 & 0 & 3.0 & 1.5 & 0 & 0 & 0 & 0.5 \\
\hline-3.4 & 0.523 & 2.493 & 1.49 & $\mathbf{- 0 . 1 2 8}$ & 0 & -0.304 & $\mathbf{0 . 4 8 3}$ \\
\hline-3.4 & 1.126 & 2.274 & 1.5 & 0 & $\mathbf{0 . 1 8 3}$ & -0.474 & $\mathbf{0 . 5 1 5}$ \\
\hline
\end{tabular}

\section{Schaubild 143.1: Vergleich von Verteilungen gleichen Erwartungswertes mit} Verteilungen gleichen Medians

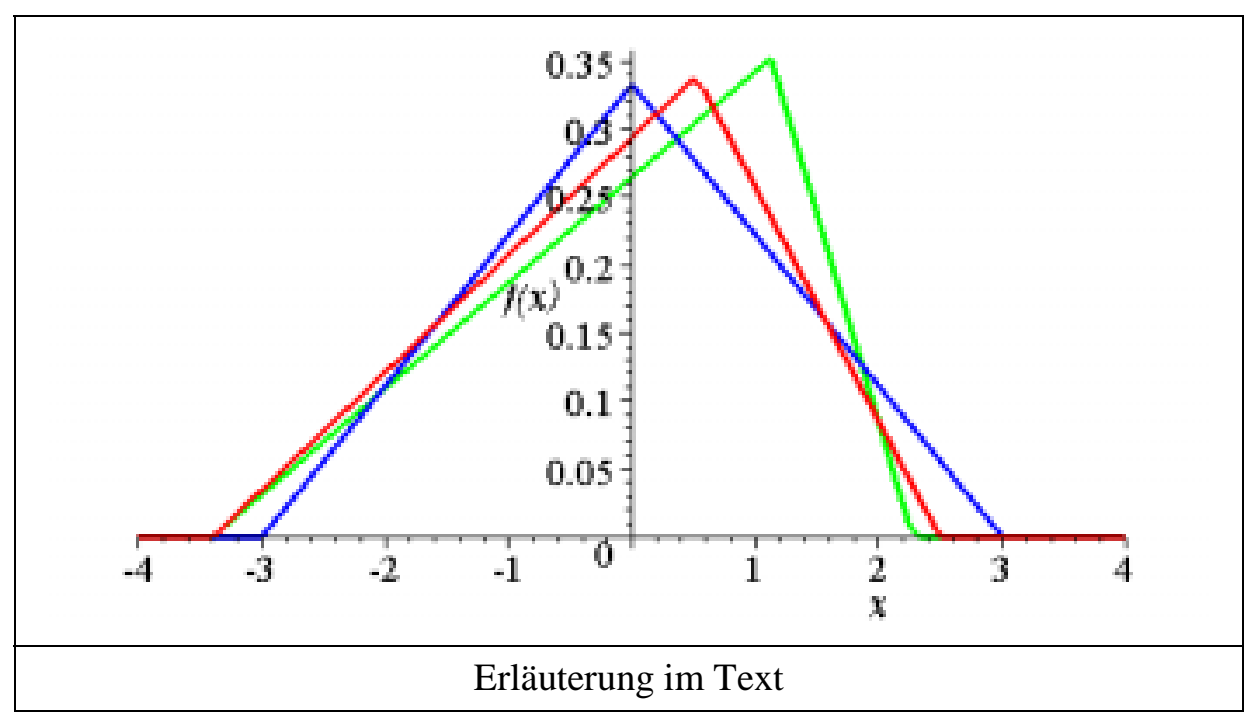

Schaubild 1431 zeigt die Dichtefunktionen dieser drei Verteilungen mit gleicher Varianz in einem Diagramm. Die linke (bezogen auf die Spitze) ist die symmetrische Referenzverteilung $\xi$ mit Erwartungswert und Median gleich Null; die mittlere besitzt einen Median von Null und die rechte einen Erwartungswert von Null. Die mittlere ist der Referenzverteilung unterlegen, die rechte ist ihr überlegen. Addiert man die relativen "Überlegenheitswerte", also die Überlegenheit der Referenzverteilung über die mittlere und die Überlegenheit der rechten über die Referenzverteilung:

$$
1 / 2-0.48261+0.51537-1 / 2=0.53276 \text {, }
$$

so kann die Summe als die Überlegenheit der rechten über die mittlere Zufallsveränderliche aufgefaßt werden. Ein Vergleich per Simulation zwischen den beiden Verteilungen unterstützt diese Interpretation. Das Experiment ergibt eine Überlegenheit von 0.53628 zugunsten der rechten Verteilung.

Zusammenfassend legen die Ergebnisse der Tabelle 143.1 folgenden Schluß nahe:

Stimmen zwei Zufallsveränderliche in ihrer Varianz und in einem Zentralmaß (Median oder Erwartungswert) überein, dann ist die Verteilung überlegen, deren anderes Zentralmaß größer ist. 
Diese These wird durch die beiden vorangegangenen Tabellen, in denen der Zusammenhang zwischen Vorzeichen der Schiefe und Überlegenheit dokumentiert ist, bestätigt. In schiefen Verteilungen fallen Median und Erwartungswert auseinander. In beiden Tabellen stimmen die beiden Zufallsvariablen $\xi$ und $\eta$ in einem Zentralmaß überein; diejenige Verteilung ist überlegen, deren anderes Zentralmaß größer ist.

Abschließend vergleichen wir die Zufallsvariable $\xi$ mit einer Summe aus zwei Zufallsvariablen. Die Wahrscheinlichkeit, daß $\xi$ größer als diese Summe ist, beträgt bei diskreten Verteilungen:

$$
\mathrm{p}\left(\xi>\eta_{1}+\eta_{2}\right)=\sum_{h 1+h 2} \mathrm{p}\left(x>h_{1}+h_{2} \mid y_{1}+y_{2}\right) \cdot \mathrm{p}\left(y_{1}+y_{2}\right) .
$$

Die Wahrscheinlichkeit kann durch Abzählen bestimmt werden. Bei stetigen Verteilungen ist die gemeinsame Verteilungsfunktion von $\eta_{1}$ und $\eta_{2}$ zu verwenden:

$$
\mathrm{p}\left(\xi>\eta_{1}+\eta_{2}\right)=\int_{h 1+h 2} \mathrm{p}\left(x>h_{1}+h_{2} \mid y_{1}+y_{2}\right) \cdot \mathrm{f}_{h 1} h_{h 2}\left(y_{1}+y_{2}\right) \cdot \mathrm{d}\left(y_{1}+y_{2}\right) .
$$

Dieser Ausdruck kann nicht generell analytisch bestimmt werden. Daher ist es nur mit Monte-Carlo-Simulationen möglich, für jede Verteilung pseudoinduktiv die Wahrscheinlichkeit zu bestimmen, daß eine Zufallsvariable sich größer als die Summe zweier anderer Zufallsvariabler realisiert.

Schaubild 143,1 zeigte drei sich überschneidende Dichtefunktionen. Kann aus der Größe der Schnittflächen der Dichtefunktion oder der zugehörigen Verteilungsfunktionen vielleicht auf die Überlegenheit geschlossen werden?

\section{Grafische Veranschaulichung (stochastische Dominanz)}

Um diese Frage zu beantworten, sind in Schaubild 145 1 zwei Dreieckverteilungen in Form ihrer Dichte- und Verteilungsfunktionen dargestellt. Ein Simulationsexperiment ergibt für Wahrscheinlichkeit $\xi \leq \eta$ den Wert $46.967 \%$. Die Grafiken legen die Vermutung nahe, daß zwischen der Überlegenheit und dem Verhältnis der Schnittflächen ein Zusammenhang besteht. Die Schnittflächen von Dichtefunktionen lassen sich unmittelbar nur schwer bestimmen, wenn es sich nicht um Gleichverteilungen handelt. Auch bei den abgebildeten Funktionen macht es unverhältnismäßige Mühe, die Dreiecke zu berechnen. Einfach läßt sich diese Aufgabe über die Verteilungsfunktionen lösen.

Im rechten nachfolgenden Schaubild ist die obere Schnittfläche zwischen den Verteilungsfunktionen kleiner als die untere Schnittfläche, damit ist die Fläche unter der Verteilungsfunktion $\eta$ kleiner als die Fläche unter $\xi$ oder in Form von Integralen ausgedrückt: 


$$
\int_{-\infty}^{+M} \mathrm{~F}_{h}<\int_{-\infty}^{+M} \mathrm{~F}_{x}
$$

$M$ ist in dieser Formel eine große endliche Zahl, die zwei Bedingungen erfüllt:

- die Dichte von $\xi$ und $\eta$ ist für $M$ Null,

- die Dichte von $\xi$ und $\eta$ ist für alle Zahlen größer als $M$ ebenfalls Null.

Die Einführung dieser oberen Integrationsgrenze erleichtert es, die Flächendifferenzen $\mathrm{zu}$ berechnen, da ansonsten die Integrale unendlich werden. ${ }^{120}$

Schaubild 145.1: Dichte- und Verteilungsfunktionen zweier Dreieckverteilungen

\begin{tabular}{|c|c|c|c|c|c|c|c|}
\hline & \\
2
\end{tabular}

Besteht zwischen dieser Flächendifferenz und der Wahrscheinlichkeit für $\xi \leq \eta$ ein Zusammenhang? Der Unterschied in der Größe der Flächen könnte als Maß für die Differenz zwischen $\mathrm{p}(\xi \leq \eta)$ und der Wahrscheinlichkeit seines Gegenereignisses aufgefaßt werden oder als das Zweifache der Abweichung von $\mathrm{p}(\xi \leq \eta)$ von $1 / 2$. Für die gegebenen Parameter ergibt sich für $\int F_{h}-\int F_{x}$ der Wert -0.0666667 . Teilen wir ihn durch zwei, entspricht er annähernd der Abweichung von $\mathrm{p}(\xi<\eta)$ von $1 / 2$ :

$$
0.5-\frac{\int \mathrm{F}_{h}-\int \mathrm{F}_{x}}{2}=0.466665 \approx 46.967 \% \text {. }
$$

120 Flächendifferenzen könnten evtl. auch bei unendlicher oberer Integrationsgrenze berechnet werden, vgl. BRANDES et al. (1997, S. 275) und die dort angegebene Literatur. 
Der Ausdruck (1451) entspricht genau der Definition stochastischer Dominanz zweiten Grades. ${ }^{121}$ Es liegt also die Vermutung nahe, daß stochastisch dominante Zufallsvariablen sich häufiger größer realisieren als dominierte Zufallsvariable und daß sich dieser Häufigkeitsunterschied mit Hilfe von $\int \mathrm{F}_{h}-\int \mathrm{F}_{x}$ berechnen läßt:

$$
\mathrm{p}(\xi \leq \eta) \stackrel{?}{=} 1 / 2-\frac{\int \mathrm{F}_{h}-\int \mathrm{F}_{x}}{2} .
$$

Eine ,vermutete“ Gleichheitsrelation wird durch das Symbol $\stackrel{?}{=}$ gekennzeichnet. Die Allgemeingültigkeit der Vermutung (146.1) läßt sich bereits mit den bisherigen Ergebnissen leicht widerlegen. Bei den erwartungswertgleichen Verteilungen des Szenarios 10 aus Tabelle 1401 ergibt eine Integration der zugehörigen Verteilungsfunktion, daß die Fläche unter der Verteilungsfunktion von $\xi$ von $-\infty$ bis 3 genau Drei ist. Gleiches ergibt sich für die Verteilungsfunktion $\eta$. Obere und untere Schnittflächen in Schaubild 146. L sind damit gleich groß, so daß die Flächendifferenz $\int F_{h}-\int F_{x}$ Null ergibt und keine stochastische Dominanz besteht.. Die Wahrscheinlichkeit für $\xi \leq$ $\eta$ unterscheidet sich dennoch mit $\mathrm{p}=0.47998$ deutlich von 1/2.

\section{Schaubild 146.1: Dichte- und Verteilungsfunktion der Dreieckverteilungen aus Tabelle 140.1, Szenario 10}

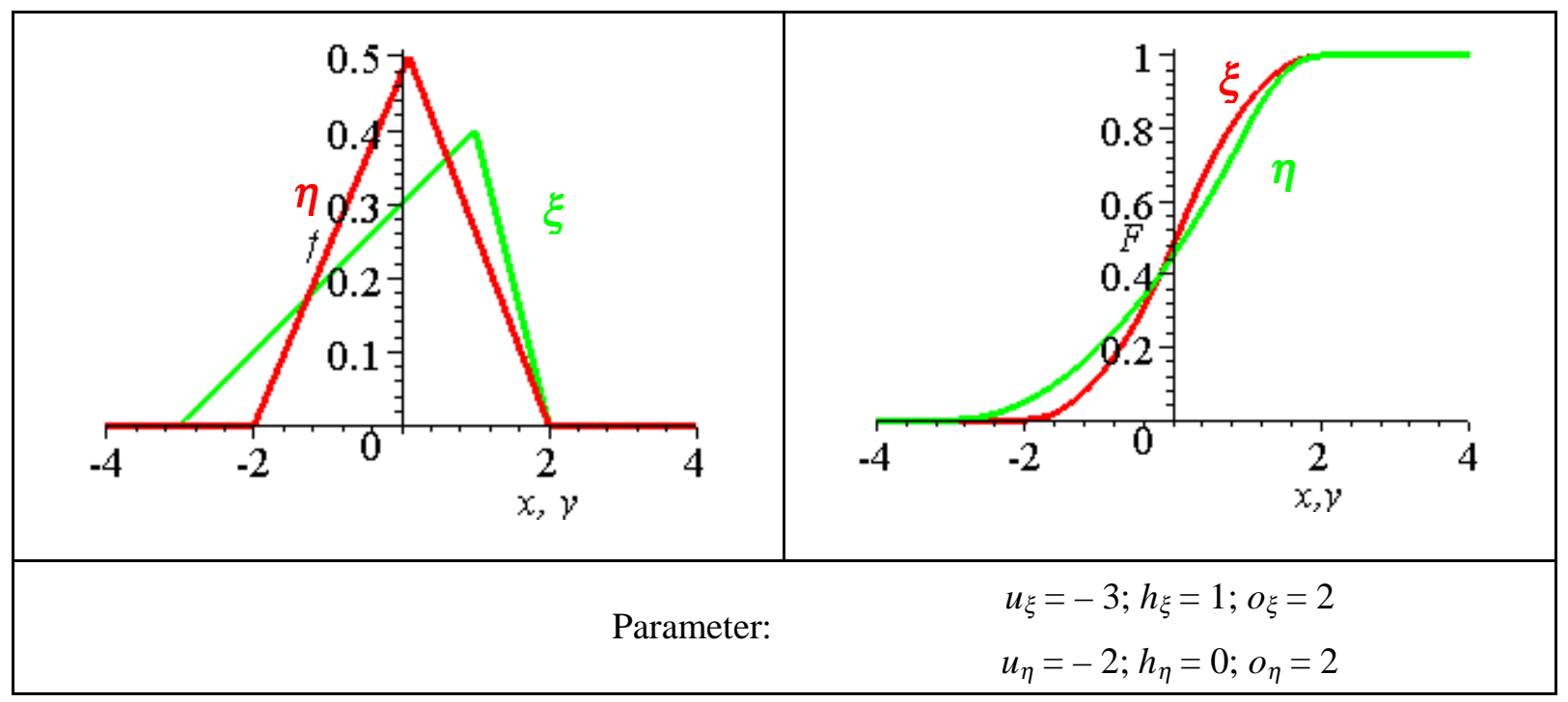

Es bleibt noch die Frage zu untersuchen, ob aus dem Vorliegen stochastischer Dominanz auf das Vorzeichen der Abweichung der Wahrscheinlichkeit von 1/2 geschlossen werden kann. Dazu wird der Modalwert von $\eta$ in Szenario 10 geringfügig nach rechts verschoben, so daß die Variable $\eta$ nun $\xi$ dominiert. Dennoch erweist sie sich im Simulationsexperiment als weiterhin unterlegen: $\mathrm{p}(\xi \leq \eta)=0.48977 \Rightarrow$ $\mathrm{p}(\eta \leq \xi)=1-0.48977=0.51023$.

121 Vgl. ANDERSON et al. (1977, S. 285), BRANDES \& ODENING (1992, S. 206). 
Abschließend bleibt festzuhalten, daß mit Hilfe der stochastischen Dominanz weder der Betrag der Wahrscheinlichkeit $\mathrm{p}(\xi \leq \eta)$ unmittelbar berechnet noch das Vorzeichen der Abweichung der Wahrscheinlichkeit von $1 / 2$ bestimmt werden können.

\subsection{Definition Überlebenswahrscheinlichkeit (formal)}

Zur Vorbereitung der Definition betrachten wir ein stochastisches, dynamisches System mit einer endogenen Variablen $K_{t}$, zu der in jeder Periode eine Zufallsvariable $d_{t}$ addiert werde:

$$
K_{t} \quad=K_{t-1}+d_{t} .
$$

$K$ repräsentiere einen Kapitalbestand, und $d_{t}$ seien dichotome Einzahlungen mit den gleichwahrscheinlichen Ausprägungen -1 und +1 . Der Erwartungswert der Einzahlungen ist somit Null. Einzahlungen mit dieser Eigenschaft werden künftig als neutrale Einzahlungen bezeichnet, da sie den Erwartungswert des Kapitals nicht verändern. Schaubild 147,1 zeigt alle möglichen Entwicklungspfade für die endogene Variable Kapital in den ersten drei Perioden unter der Annahme, daß das Startkapital Eins betrage.

Schaubild 147.1: Entwicklungspfade für das Modell $K_{t}=K_{t-1}+d_{t}, d_{t} \sim u i i d\{-1,1\}$, $K_{0}=1$

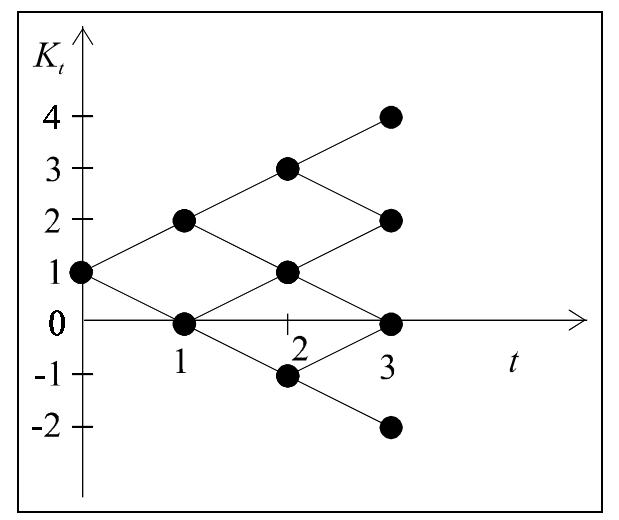

Die Wahrscheinlichkeit, daß das Kapital in der dritten Runde größer als Null ist, kann selbstverständlich nicht dadurch bestimmt werden, daß in der Grafik für Runde drei die Kapitalbestände größer Null gezählt und zu der Anzahl aller möglichen Kapitalbestände in dieser Runde ins Verhältnis gesetzt werden, da die Endkapitalbestände nicht gleichwahrscheinlich sind. Auch wenn dieses Vorgehen im vorliegenden Fall mit $2 / 4=1 / 2$ zum richtigen Ergebnis führt, so ist generell dennoch die Häufigkeitsverteilung der Kapitalbestände zu berücksichtigen, wie sie in Schaubild 148.1 dargestellt ist. 


\section{Schaubild 148.1: Häufigkeitsverteilung der Kapitalbestände in der dritten Runde für das Modell in Schaubild 147.1]}

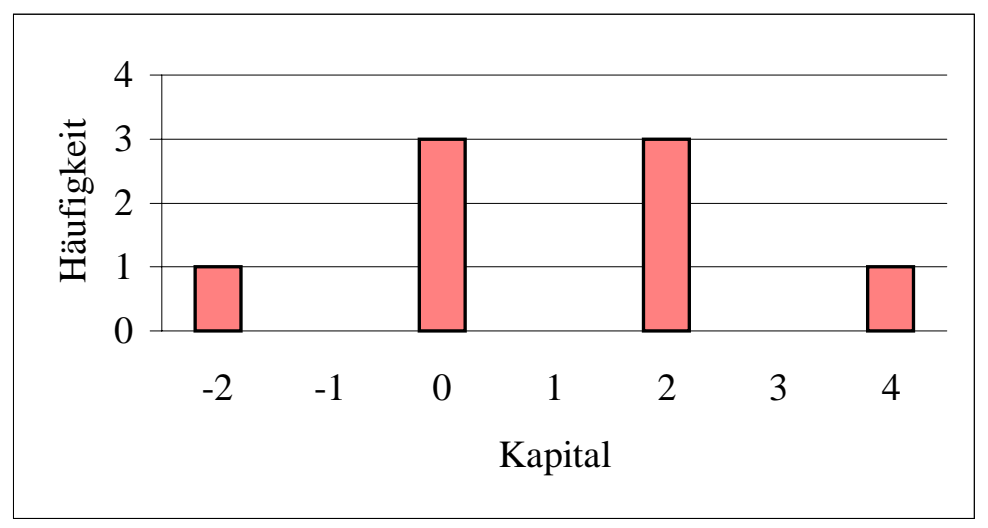

Das Schaubild zeigt, daß in vier von acht Fällen, also mit einer Wahrscheinlichkeit von $50 \%$, das Kapital in der dritten Runde größer als Null ausfällt. Auf diese Weise kann eine Form der Überlebenswahrscheinlichkeit definiert werden:

Überlebenswahrscheinlichkeit $=\mathrm{p}\left(K_{t}>0\right)$,

die ich als ergebnisorientiert bezeichnen möchte. Die Formulierung ,größer als Null“ anstelle von ,größer als oder gleich Null“" ist gewählt worden, weil $\mathrm{p}\left(K_{t}>0\right)$ sich leichter mit Hilfe der Verteilungsfunktion von $K$ ausdrücken läßt als $\mathrm{p}\left(K_{t} \geq 0\right)$. Daher möchte ich die Menge der positiven Zahlen als alle reellen Zahlen, die größer als Null sind, definieren. Die Menge der positiven Zahlen schließt also die Null nicht ein.

In der Definition wird ausschließlich das Resultat in der letzten Periode betrachtet und die zeitliche Entstehungsgeschichte des Kapitalbestandes außer acht gelassen. Für ökonomische Zusammenhänge sinnvoller erscheint mir eine Definition, die zusätzlich fordert, daß das Kapital nicht nur in der letzten Runde, sondern auch in allen Runden zuvor die Überlebensbedingung erfüllt. Konkret heißt das:

Ein System $K_{t}=f\left(K_{t-1}\right)$ überlebt, wenn die endogene Variable $K_{t}$ sich in allen Zeitstufen $(1,2, \ldots, T)$ größer als Null realisiert:

$K_{1}>0 \wedge K_{2}>0 \wedge \ldots \wedge K_{T}>0$.

Diese Definition möchte ich als verlaufsorientierte Version der Überlebenswahrscheinlichkeit bezeichnen. Sie ist nie größer als die ergebnisorientierte Überlebenswahrscheinlichkeit. Wenn in dieser Arbeit der Terminus Überlebenswahrscheinlichkeit ohne weiteres Attribut verwendet wird, ist stets die verlaufsorientierte Definition gemeint. Sie wird auch als Überlebenswahrscheinlichkeit für $t$ Runden oder als Überlebenswahrscheinlichkeit bis zur $t$. Runde bezeichnet, während die ergebnisorientierte Version synonym mit der Überlebenswahrscheinlichkeit in einer bestimmten Runde ist.

Um die verlaufsorientierte Überlebenswahrscheinlichkeit zu ermitteln, ist zunächst die Anzahl aller möglichen Zeitpfade der endogenen Variablen zu bestimmen. Dabei handelt es sich in der Terminologie der Kombinatorik um die Anzahl der Kombinatio- 
nen mit Wiederholung unter Berücksichtigung der Anordnung. ${ }^{122}$ Für das betrachtete Beispiel gibt es für die dritte Runde $2^{3}=8$ verschiedene Pfade. In Tabelle 149.1 sind alle Zeitpfade aus Schaubild 1471 stilisiert, die die Überlebensbedingung befrieägen. Auf diesen drei (von acht) Prađen ist das Kapital in allen drei Runden größer als Null. Daher beträgt die Überlebenswahrscheinlichkeit in diesem Modell für die dritte Runde $3 / 8$.

Tabelle 149.1: Überlebenspfade für das Modell in Schaubild 147,1

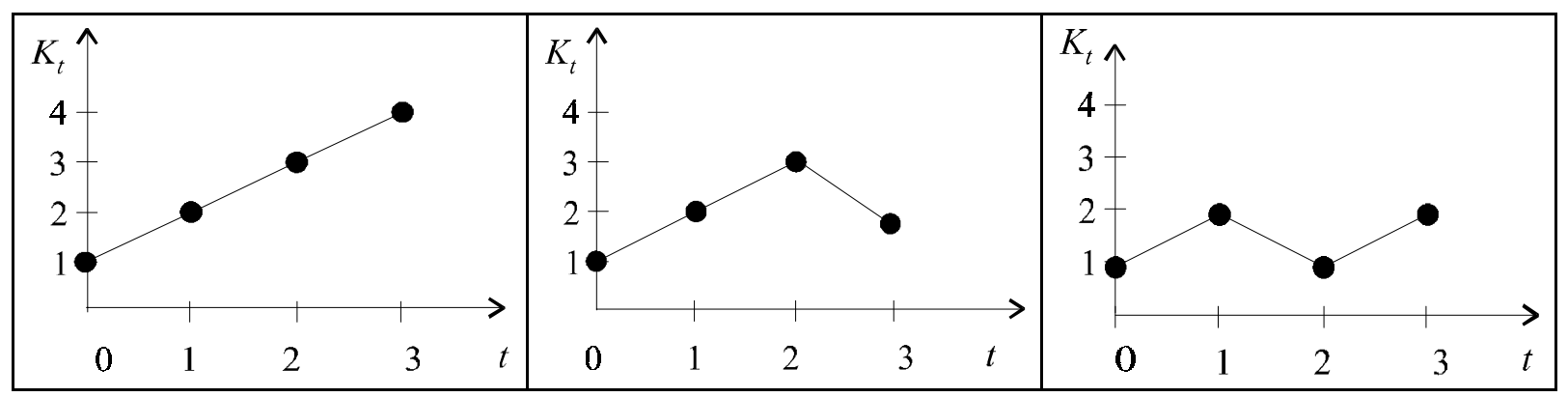

Nach diesem einführenden Beispiel wollen wir uns nun allgemein der Bestimmung der Überlebenswahrscheinlichkeit zuwenden. Für die erste Periode stimmen ergebnisund verlaufsorientierte Definitionen naturgemäß überein:

$$
\mathrm{p}_{\mathrm{uil}}(1) \quad \equiv \mathrm{p}_{\mathrm{ül}}\left(K_{1}>0\right) \equiv \mathrm{p}\left(K_{1}>0\right) .
$$

$\mathrm{p}_{\text {ül }}(t)$ sei die allgemeinste Form einer (verlaufsorientierten) Definition der Überlebenswahrscheinlichkeit und bedeute die Wahrscheinlichkeit, daß das System die Runde $t$ und alle Vorrunden überlebt hat. Das Ereignis, daß das System die Runde $t$ und alle Vorrunden überlebt hat, wird mit ül $(t)$ bezeichnet. Für das vorliegende Modell beinhaltet Überleben zum einen, daß sich die Variable $K$ in der Runde $t$ größer als Null realisiert, notiert als $\mathrm{p}\left(K_{t}>0\right)$. Und zum anderen, daß $K_{t}$ auch in allen Vorperioden größer als Null ausgefallen ist. Die Wahrscheinlichkeit für das Vorliegen beider Voraussetzungen wird durch den Index ül gekennzeichnet: $\mathrm{p}_{\ddot{u ̈ l}}\left(K_{t}>0\right)$. Es gilt daher:

$$
\mathrm{p}_{\ddot{\mathrm{uil}}}\left(K_{t}>0\right) \leq \mathrm{p}\left(K_{t}>0\right) \text {. }
$$

Die Überlebenswahrscheinlichkeit ist also die Wahrscheinlichkeit für das gemeinsame Eintreten von $t$ Ereignissen. Solche Zusammenhänge können als $t$-dimensionale Zufallsvariable aufgefaßt werden, für die mehrere Schreibweisen gebräuchlich sind: ${ }^{123}$

$$
\begin{aligned}
& \mathrm{p}\left(X_{1}=x_{1} \wedge X_{2}=x_{2} \wedge \ldots \wedge X_{t}=x_{t}\right) \\
& =\mathrm{p}\left(\left\{X_{1}=x_{1}\right\} \cap\left\{X_{2}=x_{2}\right\} \cap \ldots \cap\left\{X_{t}=x_{t}\right\}\right)
\end{aligned}
$$

122 Vgl. zu „Kombination mit Wiederholung mit Berücksichtigung der Anordnung“ BLEYMÜLLER et al. 1991, Kap. 9.2. (2) (a). BRONSTEIN, SEMENDJAJEW (1991), Kap. 22.5.2 und RASCH (1976, S. 16) bezeichnen diesen Teil der Kombinatorik als „Variation mit Wiederholung“.

123 Vgl. hierzu z.B. BlEYMÜLLER et al. (1994), Kap. 8.1 und BRONSTEIN \& SEMENDJAJEW (1991), Kap. 5.1.4. 


$$
\begin{aligned}
& =\mathrm{p}\left(X_{1}=x_{1}, X_{2}=x_{2}, \ldots, X_{t}=x_{t}\right) \\
& =\mathrm{f}\left(x_{1}, x_{2}, \ldots, x_{t}\right) .
\end{aligned}
$$

Die isolierte Wahrscheinlichkeit einer Zufallsveränderlichen, unabhängig von den Realisationen der übrigen, wird als Randwahrscheinlichkeit bezeichnet. Bei einer diskreten zweidimensionalen Wahrscheinlichkeitsfunktion $\mathrm{f}(x, y)$ beträgt die Randwahrscheinlichkeit von $X$ :

$$
\mathrm{p}(X=x) \quad=\sum_{y_{i}} \mathrm{f}\left(x ; y_{i}\right)
$$

Für gemeinsame Zufallsveränderliche der Dimension $t+1$ gilt entsprechend:

$$
\mathrm{p}(X=x) \quad=\sum_{y_{1}} \sum_{y_{2}} \ldots \sum_{y_{t}} \mathrm{f}\left(x, y_{i}, y_{2}, \ldots, y_{t}\right) .
$$

Liegt keine diskrete, sondern eine stetige Verteilung vor, sind die Summenzeichen durch Integrale zu ersetzen:

$$
\mathrm{p}(X=x) \quad=\iint_{y_{1}} \int_{y_{2}} \ldots \int_{y_{t}} \mathrm{f}\left(x, y_{1}, y_{2}, \ldots, y_{t}\right) \cdot \mathrm{d} y_{1} \cdot \mathrm{d} y_{1} \cdot \ldots \cdot \mathrm{d} y_{n} .
$$

Randwahrscheinlichkeiten können als ein Baustein zur Bestimmung der Überlebenswahrscheinlichkeit benutzt werden. Beispielsweise gebe es in der ersten Runde $n$ mögliche Kapitalbestände. Um die Wahrscheinlichkeit auszudrücken, daß das Kapital in der zweiten Runde größer als Null ausfällt, losgelöst vom Ausgang in der ersten Runde, ist in (150 1 ) für $K_{2}$ die Gleichheitsrelation durch eine Ungleichheitsrelation zu ersetzen:

$$
\mathrm{p}\left(K_{2}>0\right) \quad=\sum_{i=1}^{n} \mathrm{p}\left(K_{1 i} ; K_{2}>0\right)
$$

Diese Gleichung besagt, daß für jede Ausprägung von $K_{1}$ die Wahrscheinlichkeit $K_{2}>0 \mathrm{zu}$ bestimmen und diese Wahrscheinlichkeiten, gewichtet mit der Wahrscheinlichkeit von $K_{1}$, aufzusummieren sind. Diese Vorschrift kann auch mit Hilfe von bedingten Wahrscheinlichkeiten formuliert werden als:

$$
\mathrm{p}\left(K_{2}>0\right) \quad=\sum_{i=1}^{n} \mathrm{p}\left(K_{2}>0 \mid K_{1 i}\right) \cdot \mathrm{p}\left(K_{1 i}\right)
$$

Wenn die möglichen Kapitalbestände der ersten Runde nicht diskret, sondern stetig sind, gilt analog:

$$
\mathrm{p}\left(K_{2}>0\right) \quad=\int_{K_{1}} \mathrm{p}\left(K_{1} ; K_{2}>0\right) \cdot \mathrm{d} K_{1}
$$




$$
=\int_{K_{1}} \mathrm{p}\left(K_{2}>0 \mid K_{1}\right) \cdot \mathrm{p}\left(K_{1}\right) \cdot \mathrm{d} K_{1}
$$

Die weitere Ableitung von Überlebenswahrscheinlichkeiten mit Hilfe von gemeinsamen Verteilungsfunktionen führt zu relativ aufwendigen algebraischen Ausdrücken. Sie werden in Abschnitt 5.1 für bestimmte Beispiele wieder aufgegriffen. Für die weitere Analyse ist zunächst eine andere Herangehensweise an die Berechnung der Überlebenswahrscheinlichkeit ausreichend. Sie macht es sich zu Nutze, daß die $t$ Ereignisse, die notwendig eintreten müssen, damit das System bis zur Runde $t$ überlebt, nicht simultan geschehen, sondern sich sequentiell ereignen. Die Gleichzeitigkeit ist eine implizite Annahme der gemeinsamen Wahrscheinlichkeitsfunktion. Für die Modellierung dynamischen Überlebens ist sie nicht notwendig. Daher kann die Überlebenswahrscheinlichkeit einfacher über folgende Gedankenkette abgeleitet werden:

Betrachten wir als Beispiel die Überlebensbedingungen für zwei Runden. Sie beinhalten zum einen, daß das Kapital in der zweiten Runde größer als Null ausfällt, und zum anderen, diese Runde überhaupt zu erreichen, sprich die erste Runde überlebt zu haben. Die Überlebenswahrscheinlichkeit für zwei Runden ist daher die Wahrscheinlichkeit, in der zweiten Runde nicht $\mathrm{zu}$ fallieren ${ }^{124}$, vorausgesetzt, diese Runde wurde erreicht, multipliziert mit der Wahrscheinlichkeit, diese zweite Runde zu erreichen:

$$
\mathrm{p}_{\ddot{u ̈ l}}\left(K_{2}>0\right) \equiv \mathrm{p}_{\ddot{u ̈ l}}(2)=\mathrm{p}\left(K_{2}>0 \mid K_{1}>0\right) \cdot \mathrm{p}\left(K_{1}>0\right) .
$$

Für die dritte und $t$. Runde gilt entsprechend:

$$
\begin{aligned}
\mathrm{p}_{\text {ül }}\left(K_{3}>0\right) & =\mathrm{p}\left[K_{3}>0 \mid \mathrm{ül}(2)\right] \cdot \mathrm{p}_{\text {ül }}(2) \\
& =\mathrm{p}\left(K_{3}>0 \mid K_{2}>0 \wedge K_{1}>0\right) \cdot \mathrm{p}_{\mathrm{ül}}(2) \\
& =\mathrm{p}\left(K_{3}>0 \mid K_{2}>0 \wedge K_{1}>0\right) \cdot \mathrm{p}\left(K_{2}>0 \mid K_{1}>0\right) \cdot \mathrm{p}\left(K_{1}>0\right) \\
& =\mathrm{p}\left[K_{t}>0 \mid \ddot{\mathrm{ul}}(t-1)\right] \cdot \mathrm{p}_{\mathrm{ül}}(t-1) \\
& \left.=\mathrm{p}\left(K_{t}>0 \mid K_{t-1}>0 \wedge K_{t-2}>0 \wedge \ldots \wedge K_{1}>0\right) \cdot \mathrm{p}_{\text {ül }}(t)=1\right) .
\end{aligned}
$$

Die bedingte Wahrscheinlichkeit $\mathrm{p}\left(K_{2}>0 \mid K_{1}>0\right)$ aus 151,2 läßt sich nach dem Satz von Bayes umformen:

$$
\mathrm{p}\left(K_{2}>0 \mid K_{1}>0\right)=\frac{\mathrm{p}\left(K_{2}>0 ; K_{1}>0\right)}{\mathrm{p}\left(K_{1}>0\right)} \cdot{ }^{125}
$$

\footnotetext{
124 fallieren [lateinisch-italienisch]: in Konkurs gehen. Duden Fremdwörterbuch, 5. Auflage, Mannheim u.a. 1990.

125 Die übliche Schreibweise der Bayes-Regel:

$\mathrm{p}\left(K_{2}>0 \mid K_{1}>0\right)=\frac{\mathrm{p}\left(K_{1}>0 \mid K_{2}>0\right) \cdot \mathrm{p}\left(K_{2}>0\right)}{\mathrm{p}\left(K_{1}>0 \mid K_{2}>0\right) \cdot \mathrm{p}\left(K_{2}>0\right)+\mathrm{p}\left(K_{1}>0 \mid K_{2} \leq 0\right) \cdot \mathrm{p}\left(K_{2} \leq 0\right)}$

führt hier nicht weiter, da die bedingte Wahrscheinlichkeit $\mathrm{p}\left(K_{1}>0 \mid K_{2}>0\right)$ stets Eins ist und die bedingte Wahrscheinlichkeit $\mathrm{p}\left(K_{1}>0 \mid K_{2} \leq 0\right)$ nicht sinnvoll definiert werden kann.
} 
Der Zähler gibt die Wahrscheinlichkeit des gemeinsamen Auftretens von $K_{1}$ und $K_{2}$ an und wird daher auch als $\mathrm{p}\left(K_{2}>0 \wedge K_{1}>0\right)$ oder in Mengenschreibweise als $\mathrm{p}\left(\left\{K_{2}>\right.\right.$ $\left.0\} \cap\left\{K_{1}>0\right\}\right)$ dargestellt, vgl. (149.1). Einsetzen von (151.5 in (151.2 ergibt:

$$
\begin{aligned}
\mathrm{p}_{\text {ül }}(2) & =\frac{\mathrm{p}\left(K_{2}>0 ; K_{1}>0\right)}{\mathrm{p}\left(K_{1}>0\right)} \cdot \mathrm{p}\left(K_{1}>0\right) \\
& =\mathrm{p}\left(K_{2}>0, K_{1}>0\right) \\
& =\mathrm{p}\left(K_{2}>0 \wedge K_{1}>0\right) .
\end{aligned}
$$

Für die dritte und $t$. Runde gilt entsprechend:

$$
\begin{aligned}
\mathrm{p}_{\text {ül }}(3) \quad & =\frac{\mathrm{p}\left(K_{3}>0 ; K_{2}>0 \mid K_{1}>0\right)}{\mathrm{p}\left(K_{2}>0 \mid K_{1}>0\right)} \cdot \mathrm{p}\left(K_{2}>0 \mid K_{1}>0\right) \cdot \mathrm{p}\left(K_{1}>0\right) \\
& =\mathrm{p}\left(K_{3}>0, K_{2}>0 \mid K_{1}>0\right) \cdot \mathrm{p}\left(K_{1}>0\right) \mid \mathrm{vgl}(151.5) \\
& =\frac{\mathrm{p}\left(K_{3}>0 ; K_{2}>0 ; K_{1}>0\right)}{\mathrm{p}\left(K_{1}>0\right)} \cdot \mathrm{p}\left(K_{1}>0\right) \\
& =\mathrm{p}\left(K_{3}>0, K_{2}>0, K_{1}>0\right) \\
& =\mathrm{p}\left(K_{3}>0 \wedge K_{2}>0 \wedge K_{1}>0\right) \\
& =\mathrm{p}\left(K_{t}>0 \wedge K_{t-1}>0 \wedge \ldots \wedge K_{1}>0\right) .
\end{aligned}
$$

Die Verwendung der Bayes-Regel führt also zu der gleichen Form einer Definition der Überlebenswahrscheinlichkeit, wie sie zu Anfang dieses Abschnittes in (148.1] intuitiv eingeführt wurde. Da die einzelnen Ereignisse, von denen $\mathrm{p}_{\ddot{u ̈ l}}(t)$ abhängt, nicht stochastisch unabhängig sind - das Kapital ist eine Funktion des Vorperiodenkapitals -, kann 1522 im allgemeinen nicht als Produkt von Einzelwahrscheinlichkeiten $\mathrm{p}\left(K_{t}>0\right) \cdot \mathrm{p}\left(K_{t-1}>0\right) \cdot \ldots \cdot \mathrm{p}\left(K_{1}>0\right)$ geschrieben werden.

Die Formel der Überlebenswahrscheinlichkeit sei abschließend auf das Eingangsbeispiel aus Schaubild 147.1 angewendet. Tabelle 153,1 zeigt die Entwicklung der Überlebenswahrscheinlichkeit nach (151.3) und ihre Komponenten. Die Wahrscheinlichkeit einer positiven Einzahlung beträgt in jeder Periode 1/2 (zweite Spalte, (2)). Aus den Einzahlungen resultieren die in den nächsten Spalten angegebenen potentiellen Kapitalbestände (3) und die unbedingte Wahrscheinlichkeit eines positiven Kapitalbestandes in Spalte (4). Die Wahrscheinlichkeit eines positiven Kapitalbestandes ist natürlich größer, wenn das Vorperiodenkapital größer als Null war. Infolgedessen ist die bedingte Wahrscheinlichkeit für $K_{t}>0$ in Spalte (5) stets größer als die unbedingte in Spalte (4). Wie erklärt sich die bedingte Wahrscheinlichkeit in Höhe von $3 / 4$ in Periode 3? In Periode 2 gibt es vier Ausgänge für das Kapital in dieser Runde. Von ihnen liegen zwei auf Überlebenspfaden und zwei auf Insolvenzpfaden: Die Ausgänge 3 und 1 folgen auf das Ereignis 2 in der ersten Runde und befinden sich daher auf Überlebenspfaden, während die Ausgänge 1 und -1 auf Null in der ersten Runde folgen und daher auf Insolvenzpfaden liegen. Für die bedingte Wahrscheinlichkeit in Periode 3 interessieren nur die Ausgänge der Runde 2, die auf Überlebenspfaden liegen, also 3 und 1. Für den Ausgang $K_{2}=3$ besteht in der nachfolgenden Runde keine Insolvenz- 
gefahr und für $K_{2}=1$ beträgt sie 1/2. Da beide Kapitalbestände gleichwahrscheinlich sind, beträgt die bedingte Wahrscheinlichkeit für $\left[K_{3}>0 \mid \ddot{u ̈ l}(2)\right]$ in der folgenden Runde $(1+1 / 2) / 2=3 / 4$.

In Spalte 6 ist die Überlebenswahrscheinlichkeit für die Vorperiode wiederholt, um in der letzten Spalte die Überlebenswahrscheinlichkeit bis zur aktuellen Periode einfach als Produkt der Spalten (4) und (5) berechnen zu können.

Folgende Ergebnisse seien für dieses Beispiel ausdrücklich festgehalten, auch wenn sich die Ergebnisse bei näherem Hinsehen als trivial herausstellen:

- Die Überlebenswahrscheinlichkeit sinkt monoton, aber nicht streng monoton mit der Zeit:

$$
\mathrm{p}_{\mathrm{ül}}(t) \leq \mathrm{p}_{\mathrm{ül}}(t-1)
$$

- Ist die Bedingung erfüllt, daß das Vorperiodenkapital (a) oder alle bisherigen Kapitalbestände (b) größer als Null sind, erhöht dies die Wahrscheinlichkeit eines positiven Kapitalbestands in der aktuellen Runde:

(a) $\mathrm{p}\left(K_{t}>0 \mid K_{t-1}>0\right) \geq \mathrm{p}\left(K_{t}>0\right)$

(b) $\mathrm{p}\left[K_{t}>0 \mid \ddot{u ̈ l}(t-1)\right] \geq \mathrm{p}\left(K_{t}>0\right)$.

- Die Wahrscheinlichkeit eines positiven Kapitalbestands in einer Runde ist größer als die Überlebenswahrscheinlichkeit bis zu dieser Runde einschließlich (sofern die Ausscheidewahrscheinlichkeit größer als Null ist):

$$
\mathrm{p}\left(K_{t}>0\right) \geq \mathrm{p}_{\mathrm{ül}}\left(K_{t}\right)=\mathrm{p}\left[K_{t}>0 \mid \ddot{\mathrm{ul}}(t-1)\right] \cdot \mathrm{p}_{\mathrm{ül}}(t-1) .
$$

Tabelle 153.1: Überlebenswahrscheinlichkeit für das Beispiel aus Schaubild 147.1.

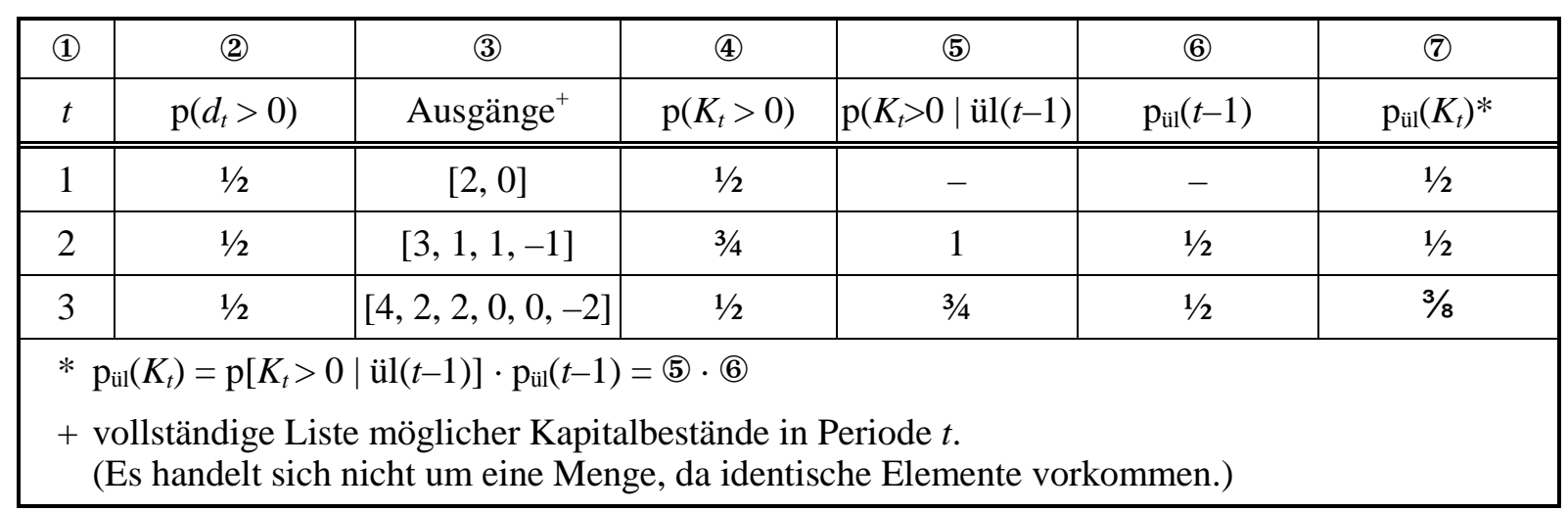

In der ökonomischen Literatur zu Überlebenswahrscheinlichkeiten ist eine Reihe von weiteren Begriffen gebräuchlich, die nun kurz vorgestellt werden.

Es liege zum Zeitpunkt $t=0$ eine empirische Stichprobe vor, die, allgemein gesprochen, aus $n$ Elementen bestehe. Statt tatsächlicher Objekte kann es sich auch um die Anfangsmenge einer Simulation handeln. Ist die Stichprobe geschlossen, das heißt, es können keine neuen Elemente hinzukommen, sinkt die Anzahl der Elemente in jeder 
Periode monoton $n(t)>n(t+1)$, sofern die Ausscheidewahrscheinlichkeit positiv ist. Schaubil i 1.4 1 skizziert diesen Zusammenhang qualitativ.

Wird die Anzahl der in einer Periode noch verbliebenen Elemente auf den Umfang der Startpopulation bezogen: $n(t) / n(0)$, spricht man von einer Überlebensquote. Die Überlebensquote in Periode $t$ bezeichnet also den Anteil der Startpopulation, der die Runde $t$ erreicht hat. Ist die Stichprobe unendlich groß, konvergiert die Überlebensquote gegen die Überlebenswahrscheinlichkeit.

Dieser Terminus wird auch in analy-

\section{Schaubild 154.1: Überlebenswahrschein- lichkeit im Zeitablauf}

Überlebenswahrscheinlichkeit

Periode tischen Ableitungen (vgl. Spalte (7) in Tabelle 153.1 verwendet. Ein Synonym für Überlebenswahrscheinlichkeit ist der Ausdruck Survivorfunktion (WOYWODE 1998, S. 160). Eine Survivorfunktion kann im Prinzip sowohl für analytische Ansätze als auch zur Beschreibung von empirischen Daten sowie von Simulationsergebnissen eingesetzt werden. Alle drei genannten Terme, Anzahl Überlebender, Überlebensquote und Überlebenswahrscheinlichkeit, sind monoton fallende Funktionen und können durch den Graphen in Schaubild 154,1 qualitativ beschrieben werden (wenn die Ordinatenbezeichnung entsprechend angepaßt wird).

Eine Insolvenz, oder synonym ein Ruin, ist das Gegenereignis zum Überleben. Daher kann Insolvenzwahrscheinlichkeit als Eins minus Überlebenswahrscheinlichkeit definiert werden. Die Insolvenzwahrscheinlichkeit für eine Runde $t$ bezeichnet in dieser Festlegung die Wahrscheinlichkeit, in der Runde $t$ oder zuvor auszuscheiden. Im Gegensatz dazu drückt die Hazardrate die Wahrscheinlichkeit aus, in der nächsten Runde insolvent zu werden. Da das Ereignis, ,Insolvenz in der nächsten Runde ' nur möglich ist, wenn die aktuelle Periode erreicht wurde, handelt es sich bei der Hazardrate um eine bedingte Wahrscheinlichkeit. In Tabelle 1531 ist die Hazardrate, die auch als Risikofunktion bezeichnet wird, nicht eingetragen. Sie entspricht Eins minus der bedingten Überlebenswahrscheinlichkeit $\mathrm{p}\left[K_{t}>0 \mid \ddot{\mathrm{l}}(t-1]\right.$, die in Spalte (5) berechnet ist.

Die Hazardrate ergibt sich mathematisch aus der Survivorfunktion. In Schaubild 155.1 ist die Überlebensfunktion $\mathrm{p}_{\text {ül }}(t)=0.5^{t}$ für eine diskrete Zeitachse dargestellt. Die Häufigkeit von Insolvenzen verringert sich mit der Zeit, erkennbar am flacher werdenden Kurvenverlauf. Die Wahrscheinlichkeit, daß ein Element, das bisher nicht 
ausgeschieden ist, die nächste Runde erreicht, erweist sich als konstant. Mathematisch kann ich die Hazardfunktion $\lambda(t)$ ausdrücken als

$$
\lambda(t) \quad=\frac{\mathrm{p}_{\mathrm{ül}}(t)-\mathrm{p}_{\mathrm{ull}}(t-1)}{\mathrm{p}_{\mathrm{ül}}(t)} .
$$

Dieser Ausdruck ergibt für die gewählte Überlebensfunktion in allen Perioden 0.3.

Schaubild 155.1: Survivor- und Hazardfunktion

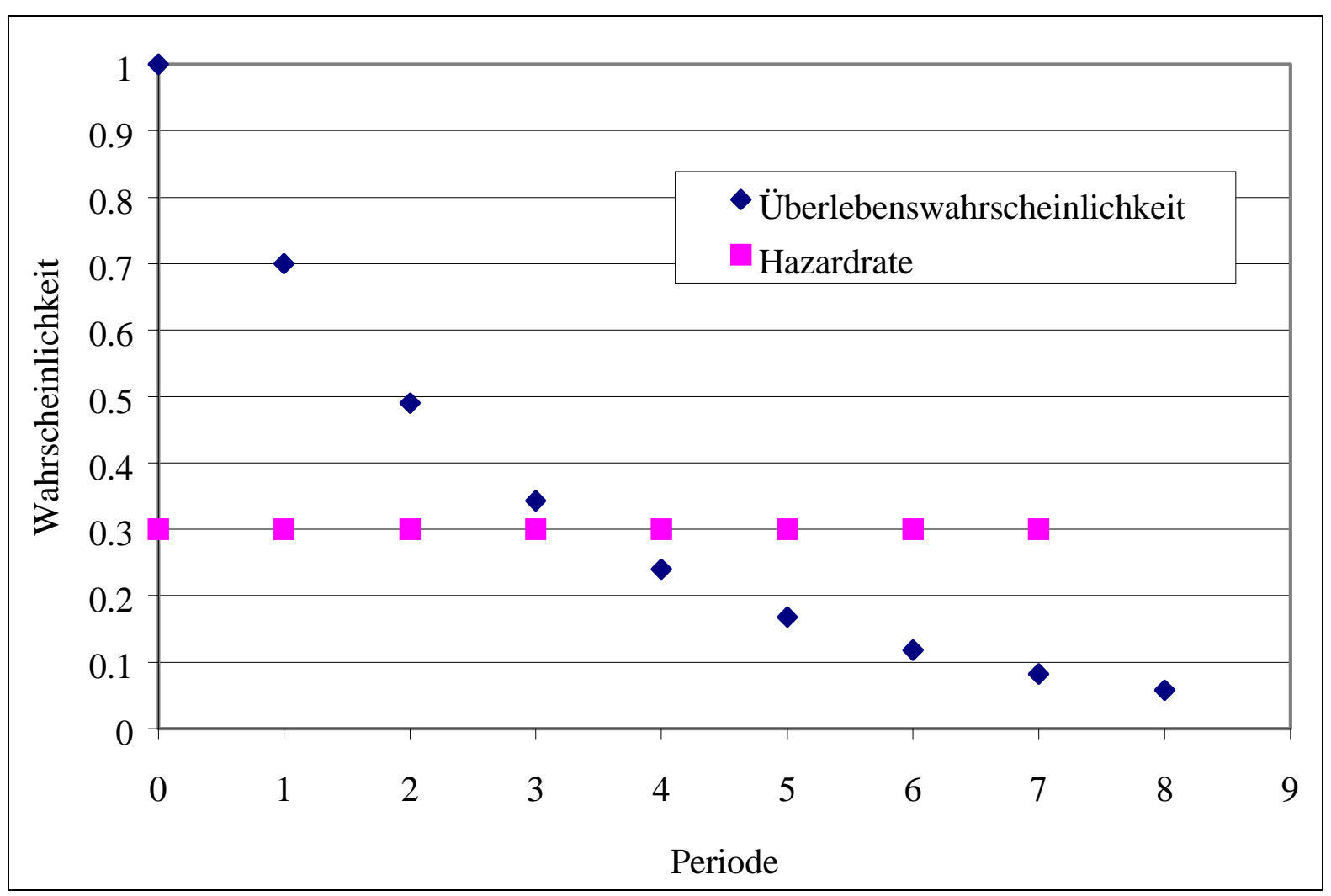

\subsection{Deduzierbare Einflüsse auf die Überlebenswahrscheinlichkeit}

Mit Hilfe sachlogischer Überlegungen lassen sich einige Determinanten der Ruinwahrscheinlichkeit ableiten. Diese Einflußfaktoren brauchen daher nicht in Modellanalysen geprüft zu werden, wie in Abschnitt 2.1 dargestellt wurde.

\section{Varianz}

Wenn ein Unternehmen in einem deterministischen Modell mit konstanten Parametern erfolgreich arbeitet, kann es per definitionem nicht fallieren (in Konkurs gehen). Erst die Einführung eines stochastischen Terms eröffnet die Gefahr von Verlusten. Die Insolvenzwahrscheinlichkeit steigt in einem statischen Modell monoton mit Zunahme der Varianz an. 
In Schaubild 156,1 sind einer festen Auszahlung $a$ verschiedene Alternativen von Einzahlungen $e$ gegenübergestellt. Die Höhe der Säulen gibt den Erwartungswert der Einzahlungen an. Bei der festen Einzahlung $A$ ist keine Insolvenz möglich. Die stochastische Einzahlung $B$ hat zwar einen höheren Erwartungswert als $A$; aufgrund ihrer Streuung, dargestellt durch Quartile, kann dennoch Insolvenz eintreten. Diese Gefahr verstärkt sich bei Einzahlung $C$. Sie realisiert sich im Mittel noch größer, aufgrund des kleineren unteren Quartils steigt dennoch die Insolvenzgefahr. Werden die Erwartungswerte konstant gehalten, ist die Insolvenzwahrscheinlichkeit eine direkte Funktion der Varianz der Einzahlung.

Übertrifft die Auszahlung die Einzahlung, kann ein Unternehmen in einem deterministischen Modell selbstverständlich nicht überleben. Nun schafft erst die Einführung einer Preisvariation eine, wenn auch kleine, Überlebensmöglichkeit (s. Einzahlung $D$ ).

\section{Schaubild 156.1: Insolvenzwahrscheinlichkeit und Varianz}

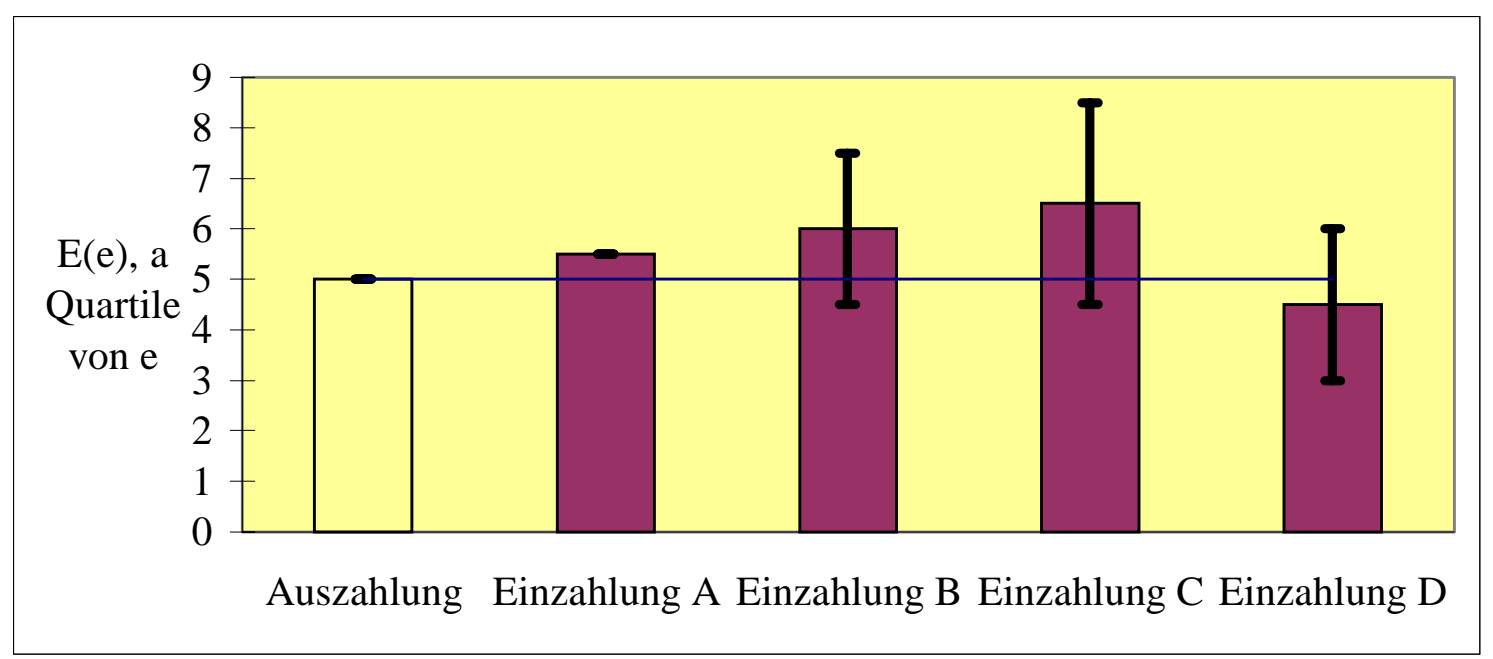

In einem dynamischen Modell besteht keine monotone Beziehung zwischen Varianz und Ausscheidewahrscheinlichkeit. Sehr kleine Varianzen sind hier wie in einem statischen Modell solange unschädlich, wie die Deckung der Auszahlungen auch im ungünstigsten Fall gegeben ist. Bei steigender Einzahlungsvarianz werden die ersten Verluste und damit auch Konkurse möglich. Weiter zunehmende Einzahlungsvariation erhöht zunächst die Ruinwahrscheinlichkeit.

Dieser Trend dreht sich um, wenn die negativen Einzahlungsausschläge ein Ausmaß übersteigen, das ausreicht, den Betrieb binnen einer Periode insolvent zu machen. Eine noch höhere Einzahlungsamplitude führt zwar auf der einen Seite zu höheren Verlusten, aber nicht zu weiter zunehmenden Insolvenzen. Auf der anderen Seite steigen die Gewinne stark an und machen den Betrieb für künftige Einzahlungseinbrüche unempfindlicher. Insgesamt ist also ein u-förmiger Verlauf der Überlebenswahrscheinlichkeit in Abhängigkeit von der Varianz zu erwarten, wie es die folgende Skizze qualitativ zeigt. 


\section{Schaubild 157.1: Überlebenswahrscheinlichkeit und Varianz}

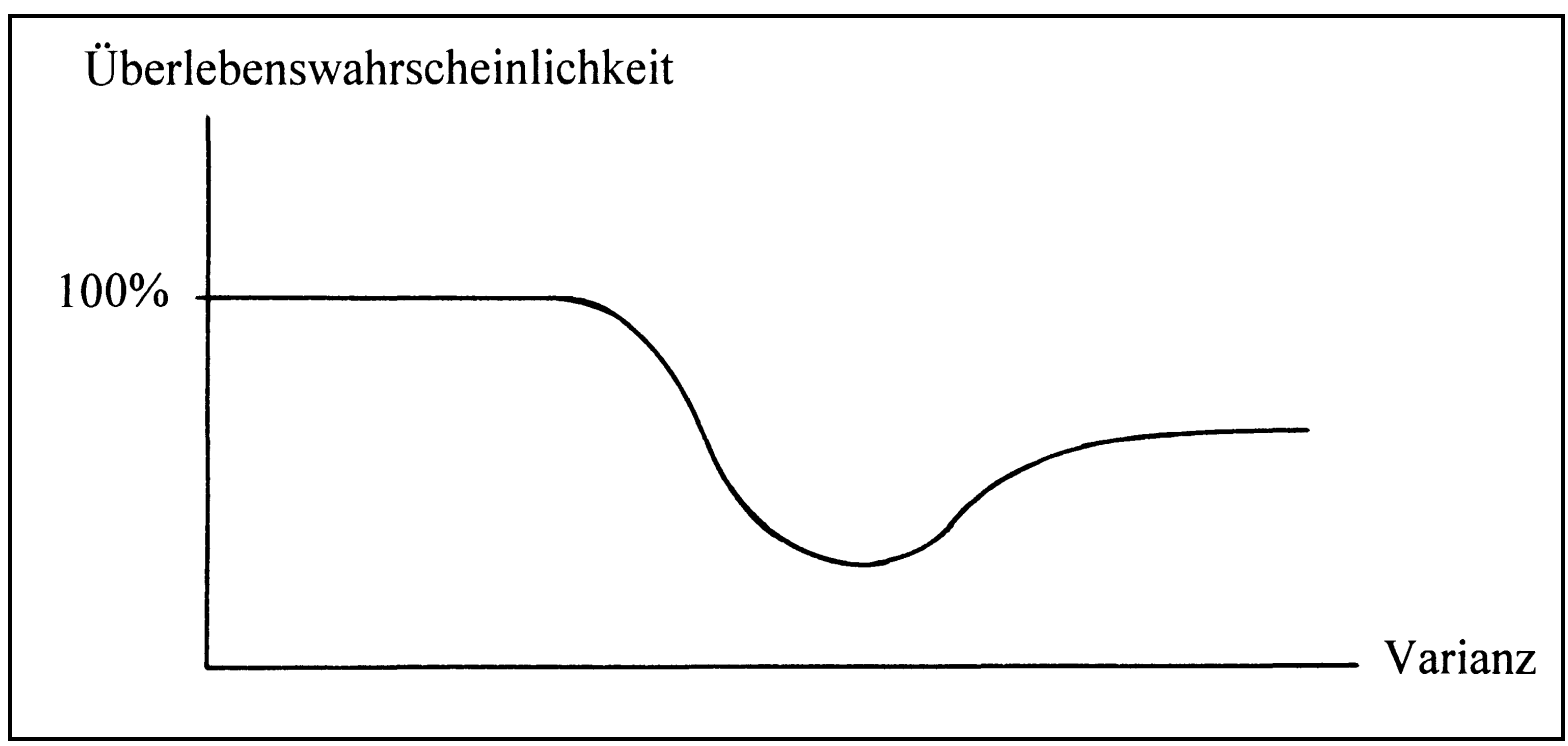

\section{Fremdkapitalaufnahme}

Maximierung der Überlebenswahrscheinlichkeit kann nicht bedeuten, auf Fremdkapital zu verzichten. Extremes Beispiel: Wenn die ohne Kreditaufnahme erzielbaren Einnahmen nicht genügen, kann das Überleben nur durch Investitionen (mit positivem Kapitalwert) erreicht werden.

\section{Verhalten der Konkurrenz in einem Marktmodell}

In einem Markt mit mehreren konkurrierenden Unternehmen ist die eigene Überlebensfähigkeit auch vom Verhalten der anderen abhängig. Eine expansive Strategie ist um so erfolgreicher, je zurückhaltender die anderen produzieren. Umgekehrt ist es bei hohen Produktionsmengen der anderen sinnvoll, selbst wenig zu investieren (steigende Grenzkosten vorausgesetzt). Dies bestätigt sich seit Jahren beim Unternehmensplanspiel Puten \& Perlhühner (vgl. Abschnitt 5.6 oder beim Schweinezyklus. ${ }^{126}$

\section{Gewinnmaximierung}

Kann durch gewinnmaximierendes Verhalten die Gefahr eines Ruins gemindert werden? Diese Frage ist in der Literatur breit diskutiert worden (WINTER 1964 1987, 1987a, IWAI 1984, WITT 1987, FISCHER 1988, WALDMAN 1994, BRANDES et al. 1997). Sie geht zurück auf Aufsätze von AlCHIAN (1950) und FRIEDMAN (1953). Da das Argument von ALCHIAN bereits in Kapitel 2 diskutiert wird, erfolgt hier eine Auseinandersetzung mit den Ausführungen von FRIEDMAN.

FRIEDMAN leitet seine Überlegungen mit dem Beispiel eines Billardspielers ein. Wenn dieser einen Stoß ausführe, werde er nicht die komplizierten Gesetze der

126 BRANDES (1989) kommt in einer Simulationsstudie zu gleichen Ergebnissen. 
mechanischen Physik explizit anwenden. Dennoch stimme der Stoß eines geübten Spielers mit den Berechnungen eines Physikers überein.

Gleiches läßt sich nach FRIEDMAN auch für Unternehmen vermuten. Erfolgreich Firmen verhielten sich so, als ob sie ihre erwarteten Gewinne maximierten. Die Ursache hierfür sei nicht, daß das Management ex ante aufwendige Optimierungskalküle löse, sondern nur, daß ex post erfolgreiche Unternehmen die gleichen Entscheidungen getroffen haben, wie sie ein theoretischer Ökonom zum Zwecke der Gewinnmaximierung empfohlen hätte. FRIEDMAN begründet seine Verhaltensannahme empirisch mit dem Natural-Selection -Argument: Unternehmen, die sich faktisch so verhalten, als ob sie ihre Gewinne maximierten, wüchsen schneller und verdrängten auf Dauer andere aus dem Markt. Faktische Gewinnmaximierung sei daher Voraussetzung zum langfristigen Überleben.

Bei der Diskussion dieses Arguments ist eine Unterscheidung zwischen Ex-Anteund Ex-Post-Optimalität notwendig. Maximale Gewinne anzustreben garantiert unter Unsicherheit nicht, auch ex post die größtmöglichen Gewinne erzielt zu haben. Daher braucht eine Strategie, die den erwarteten Gewinn eines Unternehmens maximiert, nicht unbedingt auch gleichzeitig seiner Überlebensfähigkeit förderlich sein. Dies zeigen die Beispiele aus Schaubild 156.1 Auch empirisch kann aus dem Umstand, daß ein Unternehmen überlebt hat, nicht zwingend geschlossen werden, daß es stets seine Gewinne maximiert hat. ${ }^{127}$

Neben dem Auseinanderfallen von erwartetem und realisiertem Gewinn gibt es noch einen zweiten Einwand gegen das Natural-Selection-Argument. Er betrifft die Fristigkeit des Ziels der Gewinnmaximierung. Wenn es einem Unternehmen, das seine erwarteten Gewinne maximiert, gelingt, auch die realisierten Gewinn zu maximieren, bedeutet dies nicht zwingend, die größtmögliche Überlebensfähigkeit erlangt zu haben. Durch die Entscheidungen in der Vorperiode hat sich das Unternehmen in einem gewissen Umfange festgelegt und damit an Flexibilität verloren. Diese Pfadabhängigkeit kann mittelfristig seine Überlebensfähigkeit vermindern (BRANDES 1989, S. 17).

Einzelbetrieblich ist also Gewinnmaximierung nicht immer die beste Strategie zur Überlebenssicherung. Wie sieht es auf aggregierter Ebene aus? Werden sich auf einem Markt maximierende Verhaltensweisen gegen andere durchsetzen? Diese zentrale Annahme des Natural-Selection-Arguments trifft ebenfalls nicht immmer zu. Bei hoher Varianz und hohen Entnahmen kann es zu soviel Insolvenzen kommen, daß die mittlere Wachstumsrate geriner ausfällt als bei Wahl einer sicheren Strategie mit geringerem "Erwartungswert". Wie Schaubild 1561 veranschaulicht, ist Gewinnmaximierung offensichtlich nicht der sicherste Weg zum Wachstum. Vermutlich

127 Vgl. AlCHIAN (1950). Für ALCHIAN sind die Gründe, die dazu geführt haben, daß ein Unternehmen den maximal möglichen Gewinn erzielt hat, unerheblich. Entscheidend sei, (hohe) Gewinne zu erzielen, um nicht aus dem Markt gedrängt zu werden. Gewinnmaximierung sei zum Erreichen dieses Zieles nur bedingt geeignet, da sie aufgrund der herrschenden Unsicherheit nicht durchführbar sei oder ihr Ziel nicht erreiche. 
werden maximierende Unternehmen auch nicht im Mittel (bezogen auf alle Unternehmen zu Beginn des Betrachtungszeitraumes) einen größeren Marktanteil erreichen als andere Strategien.

Ein Zusammenhang zwischen Überlebenswahrscheinlichkeit und Gewinn oder Marktanteil kann auf Märkten vielmehr in umgekehrter kausaler Richtung vermutet werden. Je höher die Insolvenzrate ist, desto stärker nimmt die Marktkonzentration zu - wenn keine Markteintritte möglich sind. Damit können die verbleibenden Unternehmen c.p. zunächst größere Gewinne erzielen.

In Simulationsmodellen besteht die Gefahr, daß sich dieser Effekt auch einstellt, wenn er ökonomisch nicht beabsichtigt ist, beispielsweise in einem statischen Modell, in dem eine hohe Preisvarianz bei einem Teil der simulierten Unternehmen zu hohen Verlusten führt. Damit verbunden sind als andere Seite der gleichen Medaille hohe Gewinnen des anderen, überlebenden Teils der Unternehmen. Aus diesem Grund führt auch in der Simulation eines Polypolmodells eine Steigerung der Preisvarianz zu einer Erhöhung der Gewinne der nicht-ausscheidenden Unternehmen. Dies läßt sich auch sehr leicht wahrscheinlichkeitstheoretisch belegen. Wenn von einer Verteilung ein bestimmter unterer Teil ausgeblendet wird, dann steigt der Erwartungswert des verbleibenden Teils der Verteilung an (vgl. auch die Ausführungen zu bedingten Wahrscheinlichkeiten in Erläuterung zu Tabelle 153.11.

Dieser Effekt tritt auch in dynamischen Modellen auf. Daher wird eine exogene Erhöhung der Preisvarianz die Gewinne der überlebenden Unternehmen erhöhen, auch wenn der Markt ausreichend groß gewählt ist, so daß die ausscheidenden Unternehmen keinen Einfluß auf den Preis ausüben. Ein so gestaltetes Simulationsmodell kann nicht die Einflüsse der Marktkonzentration auf die Gewinnentwicklung beschreiben, da die Gewinne bereits durch die exogene Preisvariation maßgeblich verändert werden.

\subsection{Stochastische Eigenschaften von Funktionen mehrerer Zufallsveränderlicher}

Stochastisch dynamische Systeme enthalten häufig neben einfachen auch zusammengesetzte Zufallsveränderliche. Addition, Multiplikation und Quadrierung sind dabei die wichtigsten Verknüpfungen der Zufallsveränderlichen. In Betriebsmodellen wird z.B. der Gewinn oft als unternehmerische Erfolgskennzahl verwendet und als Erlös abzüglich Kosten definiert. Sind Erlöse und/oder Kosten mit Unsicherheit behaftet, ist der Gewinn ebenfalls eine stochastische Größe. Ein Beispiel für die Multiplikation zweier Zufallsveränderlicher ist ein Erlös, der als Produkt von unsicheren Preisen und Mengen aufgefaßt wird. Quadrate von Zufallsvariablen gehen in die Berechnung der Streuung von Zufallsveränderlichen ein. ${ }^{128}$ Ferner sind quadrierte Zufallsvariablen ein Bestandteil der Zielfunktion in der quadratischen (Risiko-) Programmierung, die in Unterabschnitt 3.2 näher vorgestellt wurde. Besondere Bedeutung haben quadrierte

${ }^{128} \operatorname{Var}(X)=\mathrm{E}\left(X^{2}\right)-[\mathrm{E}(X)]^{2}$ 
Normalverteilungen. Sie werden eingesetzt, um Häufigkeitsunterschiede mittels Kontingenztafeln auf Signifikanz zu prüfen (s. Abschnitt p.2.

In diesem Abschnitt werden kurz die Momente Erwartungswert und Varianz von Summen und Produkten von Zufallsveränderlichen referiert. Wie gezeigt werden wird, reicht die Kenntnis dieser Momente zur Analyse von Überlebenswahrscheinlichkeiten nicht aus. Daher wird versucht, auch die Verteilungsgesetze zusammengesetzter Zufallsveränderlicher abzuleiten.

Zufallsveränderliche werden im allgemeinen mit großen lateinischen Buchstaben gekennzeichnet und ihre Realisationen mit entsprechenden kleinen Buchstaben. Von dieser Konvention wird für die Zufallsvariable $z$ abgewichen. $z$ wird in den angewandteren Teilen dieser Arbeit als Zufluß oder Zahlung interpretiert und im Gegensatz zur Bestandsgröße Kapital klein geschrieben.

Betrachten wir zunächst Summen von Zufallsveränderlichen. In einem System werde in jeder Periode zu einer endogenen Variablen $X$ eine Zufallszahl $z$ addiert. Der Wert von $X$ betrage in der zweiten Periode

$$
x_{2} \quad=x_{1}+z_{2}=z_{1}+z_{2}
$$

und in der $t$-ten Periode

$$
x_{t}=x_{t-1}+z_{t}=\sum_{i=1}^{t} z_{i} .
$$

Der Erwartungswert der Zufallsveränderlichen $X_{t}$ beträgt:

$$
\mathrm{E}\left(X_{t}\right) \quad=\sum_{i=1}^{t} \mathrm{E}\left(z_{i}\right)
$$

und ihre Varianz, sofern die $z_{i}$ unabhängig verteilt sind:

$$
\operatorname{Var}\left(X_{t}\right) \quad=\sum_{i=1}^{t} \operatorname{Var}\left(z_{i}\right)
$$

Werden die Zufallsveränderlichen $z_{i}$ mit den Koeffizienten $a_{i}$ gewichtet, ergibt sich bekanntlich für Erwartungswert und Varianz von $X_{t}=\sum_{i=1}^{t} a_{i} \cdot z_{i}$ :

$$
\mathrm{E}\left(X_{t}\right) \quad=\sum_{i=1}^{t} a_{i} \mathrm{E}\left(z_{i}\right)
$$




$$
\operatorname{Var}\left(X_{t}\right) \quad=\sum_{i=1}^{t} a_{i}^{2} \operatorname{Var}\left(z_{i}\right), \text { sofern die } z_{i} \text { unabhängig verteilt sind. }{ }^{129}
$$

Für die Analyse von Überlebenswahrscheinlichkeiten genügt es nicht, die ersten Momente einer Verteilung zu kennen. Wie zu Beginn dieses Kapitels demonstriert wurde, können sich Zufallsveränderliche, die in Erwartungswert und Varianz übereinstimmen, dennoch in ihrer Überlegenheit unterscheiden. Auch Differenzen in der für die Überlebensanalyse wichtigen Wahrscheinlichkeit $\mathrm{p}\left(Y_{i}>0\right)$ sind bei gleichen Momenten möglich, wie in Schaubild 1611 angedeutet ist. Die Verteilung $A$ sei eine dichotome Gleichverteilung mit den Ausprägungen Eins und Drei und den Momenten $\mu=2$ und $\sigma^{2}=1 / 3$. Die Verteilung $B$ ist so gewählt, daß sie die gleichen Momente aufweist und die Einzelwahrscheinlichkeiten sich dennoch erheblich unterscheiden. Insbesondere realisiert sich die Verteilung $A$ immer größer als Null, während hierfür bei Verteilung $B$ die Wahrscheinlichkeit nur $1-24 / 25=96 \%$ beträgt. Verteilungen mit größeren Unterschieden in der Überlebenswahrscheinlichkeit trotz gleicher Momente lassen sich leicht konstruieren.

\section{Schaubild 161.1: Verteilungen mit gleichen ersten und zweiten Momenten}

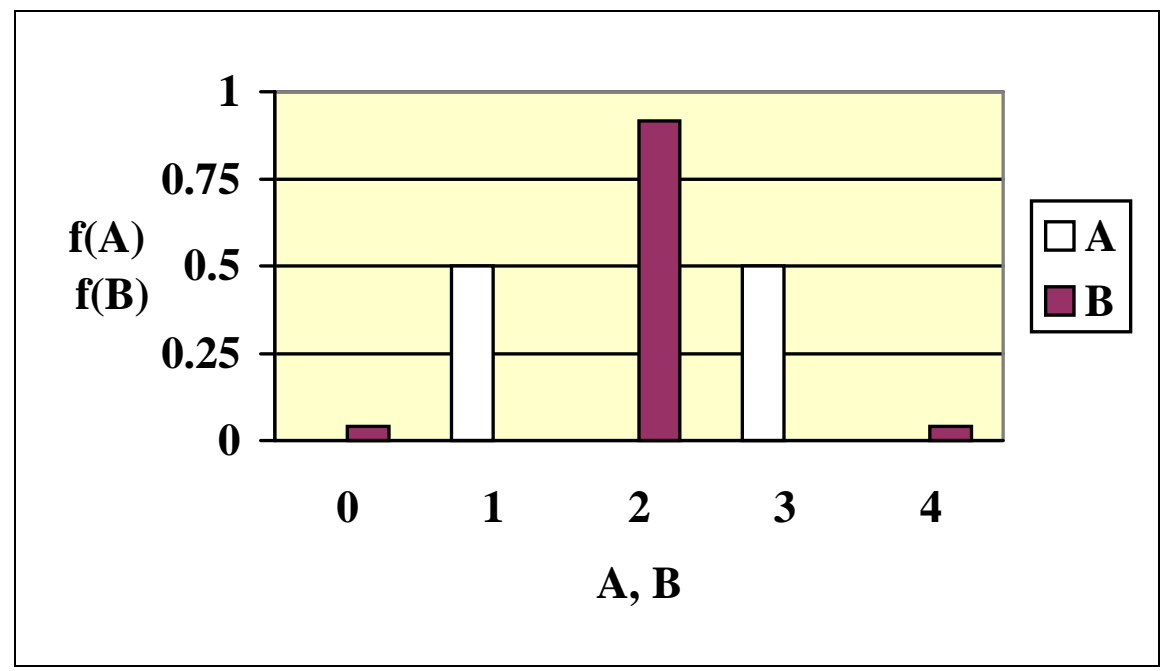

Daher ist es entscheidend, außer den Momenten auch die Verteilung einer zusammengesetzten Zufallsvariablen anzugeben. Sind ihre Bestandteile diskret, kann im Prinzip immer die gemeinsame Verteilungsfunktion abgeleitet werden, da der Ereignisraum und die Wahrscheinlichkeit für jedes Element dieses Raumes bestimmt werden können. Bei stetigen Verteilungen scheidet ein enumeratives Verfahren hingegen naturgemäß aus. In Übersicht 16211 sind beispielhaft die Dichtefunktionen einer Summe aus zwei oder drei Zufallsveränderlichen dargestellt.

129 Vgl. RASCH (1976, S. 170), ROHATGI (1984, S. 258). 
Die durchgezogenen Linien kennzeichnen die Verteilung der identischen Summanden, die gestrichelte die Summe $X$ aus der linken Spalte. Wie die linken Grafiken in der Übersicht beispielhaft zeigen, nimmt die Spanne der Verteilung bei diskreten Verteilungen mit der Anzahl der Summanden zu, und die Verteilungsform verändert sich. Die Summe aus zwei stetigen Gleichverteilungen ergibt eine Dreieckverteilung mit doppelter Spanne (mittlere Grafiken oben). ${ }^{130}$ Darunter ist die Summe aus drei identischen Gleichverteilungen (im Vergleich dazu eine Normalverteilung, durchgezogene Linie) abgebildet. Sie entspricht keinem bekannten Verteilungsgesetz. Die Dichtefunktion ist daher mit Hilfe einer Monte-Carlo-Simulationen erstellt worden. Diese Technik bietet sich für die grafische Darstellung von Funktionen von Zufallsveränderlichen an, wenn die zusammengesetzte Zufallsvariable keine Reproduktionseigenschaft besitzt. Die Unterschiede zur Normalverteilung, die den gleichen Erwartungswert und die gleiche Varianz besitzt, sind in der Mitte der Verteilung erheblich, aber ansonsten erstaunlich gering.

\section{Übersicht 162.1: Dichtefunktionen für Summen von Zufallsveränderlichen}

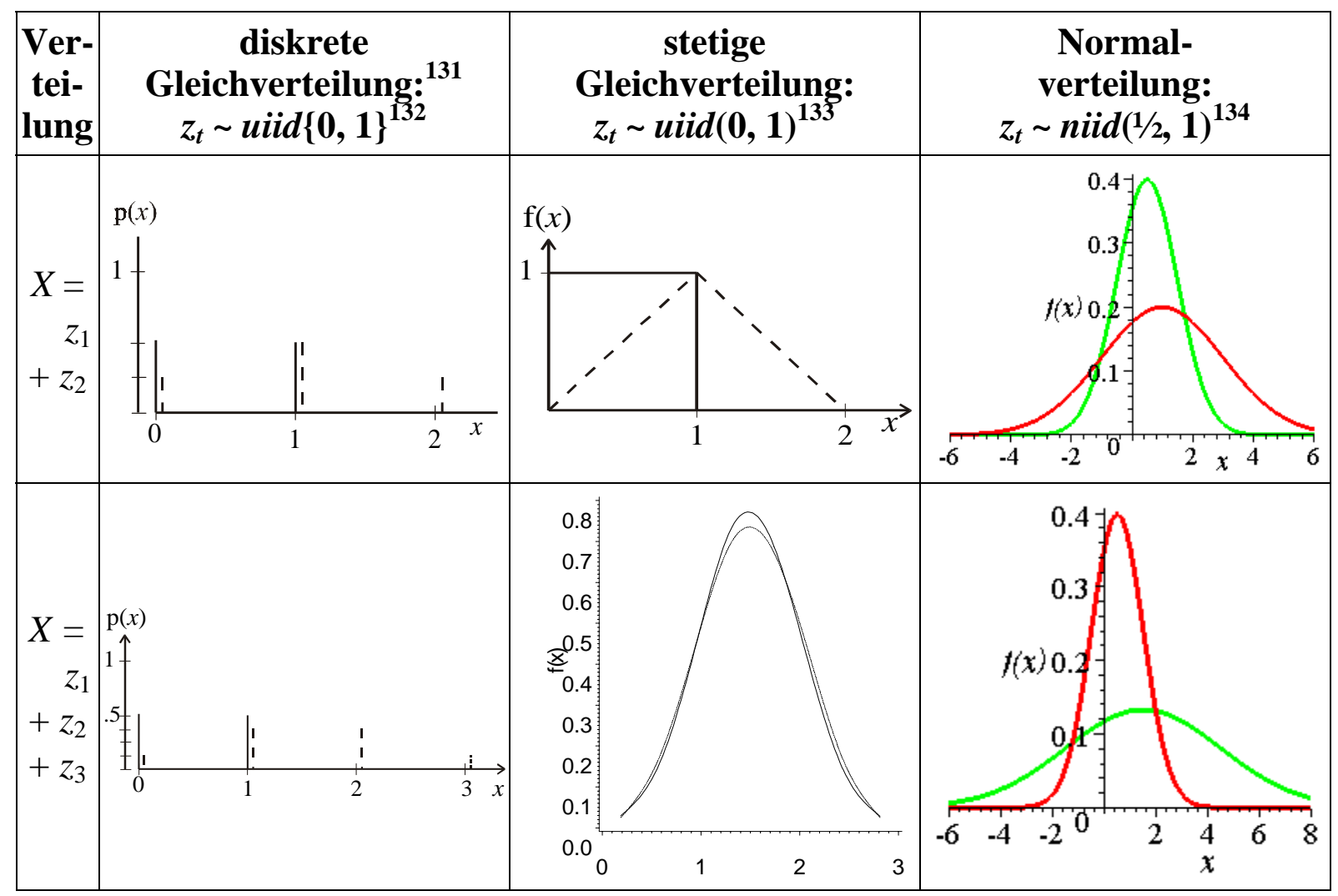

130 Vgl. SPRINGER (1979, S. 50).

131 Auch ,gleichmäßige Verteilung“ genannt, z.B. von BoscH (1992, S. 168f).

$132 \mathrm{p}(0)=\mathrm{p}(1)=1 / 2$.

133 Die Notation $z_{t} \sim \operatorname{uiid}(0,1)$ bedeutet, daß die Zufallsveränderlichen $z_{t}$ gleichverteilt (uniform) im Intervall $[0,1]$ sind und die Verteilungen identisch und stochastisch unabhängig sind (iid). Siehe hierzu auch das Abkürzungsverzeichnis zu Beginn der Arbeit.

134 Diese Notation steht für eine Normalverteilung mit einem Erwartungswert von 1/2 und einer Varianz von 1 sowie zusätzlich der iid-Eigenschaft. 
In der rechten Spalte der Übersicht 1621 sind Normalverteilungen dargestellt. Die Summe aus zwei oder mehr Normalverteilungen ist bekanntlich wieder normalverteilt. ${ }^{135}$ Die Parameter einer Summe aus $t$ Normalverteilungen lauten nach 160.1] und (161.1): ${ }^{136}$

$$
\mu_{t}=\sum_{i=1}^{t} \mu_{i} \text { und } \sigma_{t}^{2}=\sum_{i=1}^{t} \sigma_{i}^{2}
$$

Daher erhöhen sich die Varianzen und im vorliegenden Fall auch die Erwartungswerte der zusammengesetzten Verteilungen.

Allgemein gilt für die Verteilungsfunktion der Summe aus zwei unabhängigen und stetigen Zufallsvariablen beliebiger Verteilungsform, mit Dichtefunktionen $f_{1}\left(x_{1}\right)$ und $f_{2}\left(x_{2}\right)$ und Verteilungsfunktionen $F_{1}\left(x_{1}\right)$ und $F_{2}\left(x_{2}\right)$, nach RASCH $(1976$, S. 165)

$$
G(y) \quad=\int_{-\infty}^{\infty} f_{1}\left(x_{1}\right) \cdot F_{2}\left(y-x_{1}\right) d x_{1}=\int_{-\infty}^{\infty} f_{2}\left(x_{2}\right) \cdot F_{1}\left(y-x_{2}\right) d x_{2} .
$$

$G(y)$ wird auch als Faltung der Verteilungen mit den Verteilungsfunktionen $F_{1}\left(x_{1}\right)$ und $F_{2}\left(x_{2}\right)$ bezeichnet.

Für drei und mehr Summanden läßt sich ebenfalls eine parametrische Verteilung angeben: ${ }^{137} W$ sei die Summe aus $n$ stetigen unabhängigen Zufallsvariablen:

$$
W \quad=\sum_{j=1}^{n} X_{j}
$$

Dann gilt für die Dichtefunktion von $W$ :

$$
\mathrm{g}(W) \quad=\frac{1}{2 p} \int_{-\infty}^{\infty} e^{i t w} F_{t}\left(f_{j}\left(x_{j}\right)\right) d t \quad \text { mit } i=V_{-} 1
$$

Allerdings ist meines Wissens dieser Ausdruck im allgemeinen nicht handhabbar. Ab 30 Summanden jedoch läßt sich nach dem zentralen Grenzwertsatz jede Summe aus identisch und unabhängig verteilten Zufallsvariablen gut durch eine Normalverteilung annähern. Wie Schaubild 16411 im rechten Teil zeigt, kann sogar eine Summe aus 30 Zufallsveränderlichen mit extrem schiefer Verteilung relativ gut durch eine Normalverteilung approximieren werden.

Die in der Literatur verbreitete Einschränkung, daß der zentrale Grenzwertsatz nur auf die Summe aus identisch verteilten Zufallsveränderlichen anwendbar sei ${ }^{138}$, braucht nicht unbedingt sehr eng ausgelegt zu werden. Schaubild I65.I zeigt Dichte-

\footnotetext{
135 Vgl. Fußnote 119.

136 Vgl. BLEYMÜLLER et al. (1994, S. 49).

137 Vgl. SPRINGER (1979, S. 57 ff).

138 Vgl. z.B. BLEYMÜLLER et al. (1994 S. 78), BOSCH (1992, S. 333).
} 
funktionen von Summen, die aus zwei Typen von Summanden bestehen. Im linken Teil sind alle 30 Summanden gleichverteilt, 15 von ihnen im Intervall [0,1] und 15 im Intervall $[-2,0]$. Die beiden Typen unterscheiden sich also sowohl im Erwartungswert als auch in der Varianz. Dennoch wird die Dichtefunktion der Summe ebenso gut durch eine Normalverteilung approximiert wie bei einer Summe aus identischen Gleichverteilungen in Schaubild 104.1.

\section{Schaubild 164.1: Dichtefunktionen für Summen von 30 identischen Zufallsveränderlichen}

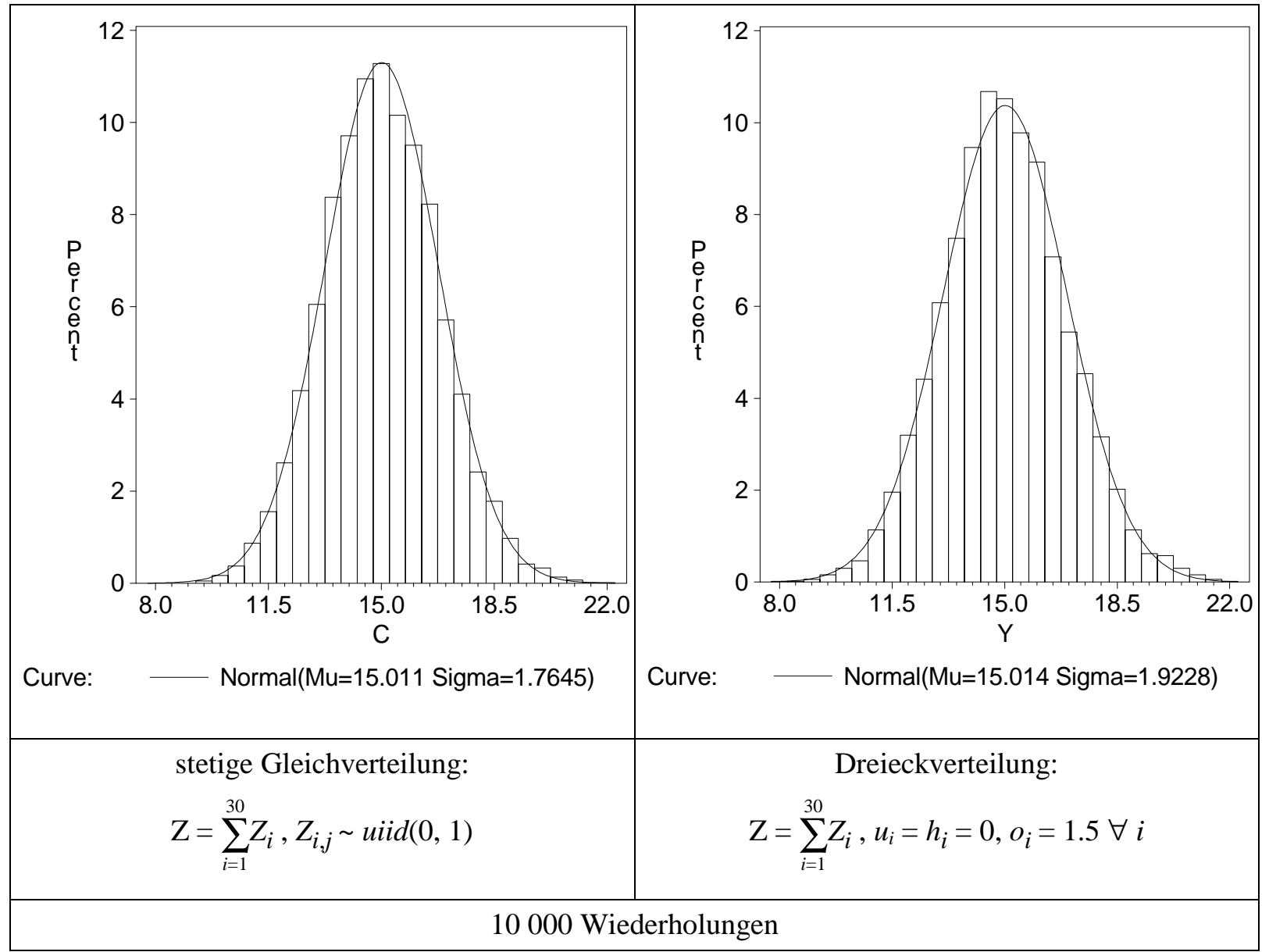

Auch eine Mischung aus gleich- und dreieckverteilten Zufallsveränderlichen kann gut durch eine Normalverteilung angenähert werden. Im rechten Teil von Schaubild 165.1 besteht die Summe aus 15 Summanden, die gleichverteilt zwischen Null und Eins sind, und 15 dreieckverteilten Zufallsvariablen mit den Parametern $u=h=0, o=$ 1.5. Die Approximation ist dem Augenschein nach nicht schlechter als bei den anderen zusammengesetzten Zufallsveränderlichen. Weitere Simulationsexperimente könnten klären, wie weit die Forderung nach identischer Verteilung der Summanden außer acht gelassen werden darf, ohne daß die Approximationsgüte der Normalverteilung merklich nachläßt. 
Schaubild 165.1: Dichtefunktionen für Summen von 30 Zufallsveränderlichen

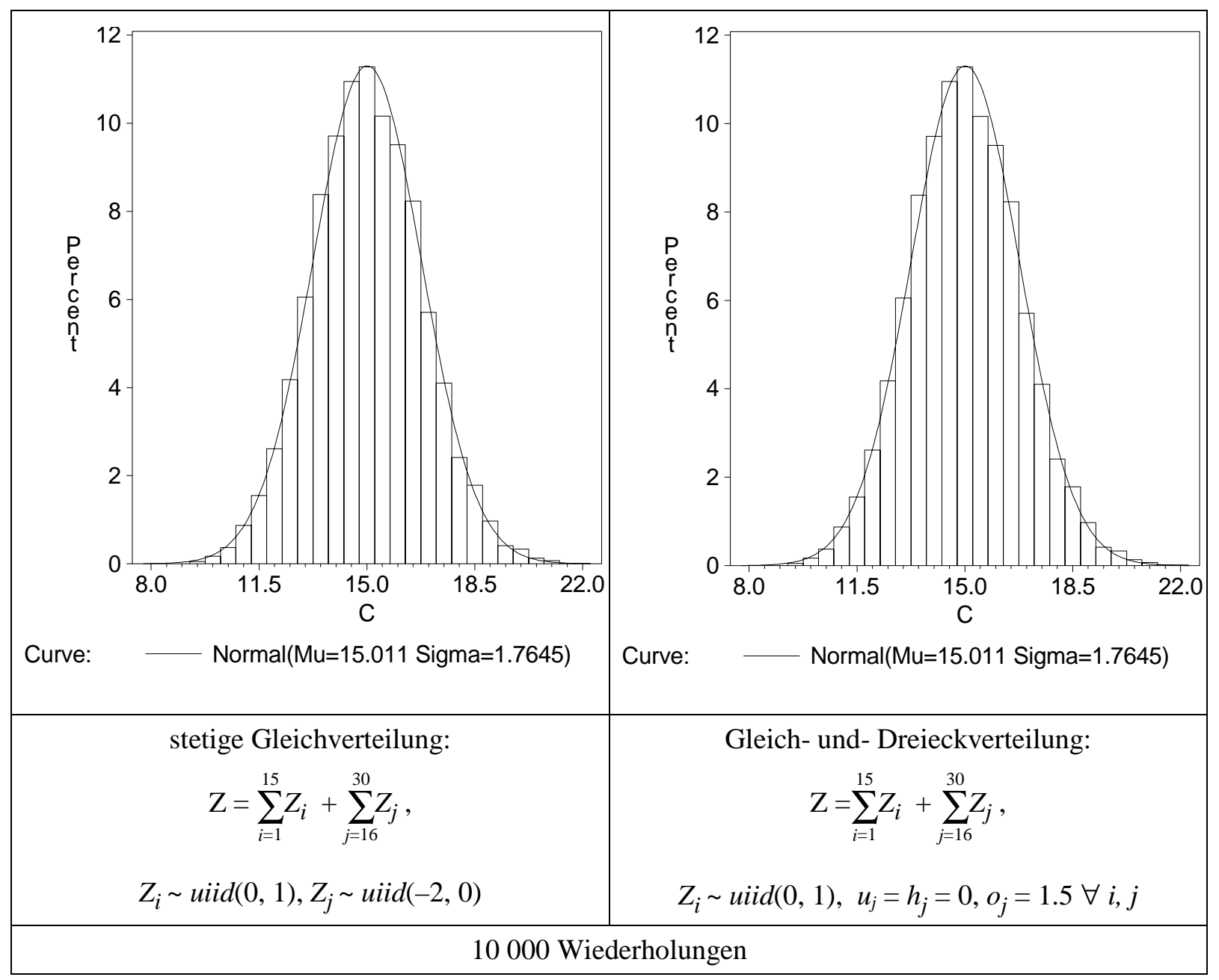

Einen höheren mathematischen Aufwand als Summen erfordern Produkte von Zufallsveränderlichen, insbesondere wenn die Variablen nicht stochastisch unabhängig sind. Beispielsweise kann bei Preisen und Mengen in der Regel nicht von Unabhängigkeit ausgegangen werden. Übersicht 166,1 gibt hierzu einen Überblick zu den wichtigsten Kennzahlen zusammengesetzter Zufallsvariablen.

Ist Unabhängigkeit gegeben, entspricht der Erwartungswert des Produktes dem Produkt der jeweiligen Erwartungswerte. Diese Formel gilt auch, wenn das Produkt aus mehr als zwei unabhängigen Faktoren besteht. Bei der Multiplikation von zwei stochastisch abhängigen Zufallsvariablen, ist zu dem Produkt der Erwartungswerte die gemeinsame Kovarianz zu addieren:

$$
\mathrm{E}(X \cdot Y) \quad=\mathrm{E}(X) \cdot \mathrm{E}(Y)+\operatorname{cov}(X, Y) .
$$

Für den Erwartungswert des Produktes aus mehr als zwei stochastisch abhängigen Zufallsvariablen, finden sich in den in Übersicht 1661 genannten Quellen keine Angaben. Allerdings kann durch entsprechende Substitution jedes Produkt als ein Produkt aus zwei Faktoren dargestellt werden, auf welches anschließend 165:1] mehrfach angewendet werden kann. 


\section{Übersicht 166.1: Rechenregeln für Erwartungswerte und Varianzen zusammengesetzter Zufallsveränderlicher}

\begin{tabular}{|c|c|c|c|}
\hline$Z=$ & $E(Z)=$ & $\operatorname{Var}(Z)=\sigma<_{Z}^{2}$ & Quellen \\
\hline$a X \pm b Y$ & $a \mathrm{E}(X) \pm b \mathrm{E}(Y)$ & $\begin{array}{l}a^{2} \operatorname{Var}(X)+b^{2} \operatorname{Var}(Y) \\
\quad \pm 2 a b \operatorname{Cov}(X, Y)\end{array}$ & $\begin{array}{l}\text { BLEYMÜLLER (1998) } \\
\text { S. 49; JUDGE et al. } \\
\text { (1988) S. 39f; } \\
\text { BRONSTEIN \& } \\
\text { SEMENDJAJEW (1991) } \\
\text { S. } 665 .\end{array}$ \\
\hline \multirow[t]{2}{*}{$\sum_{i=1}^{n} a_{i} X_{i}$} & $\sum_{i=1}^{n} a_{i} \mathrm{E}\left(X_{i}\right)$ & $\begin{array}{l}\text { Bei Unabhängigkeit: } \\
\qquad \sum_{i=1}^{n} a_{i}^{2} \operatorname{Var}\left(X_{i}\right)\end{array}$ & $\begin{array}{c}\text { RASCH (1976) S. 170, } \\
\text { ROHATGI (1984) } \\
\text { S. } 258 \\
\text { BAMBERG \& BAUR } \\
\text { (1987) S. 120ff }\end{array}$ \\
\hline & & $\begin{array}{c}\text { allgemein: } \\
\sum_{i=1}^{n} a_{i}^{2} \operatorname{Var}\left(X_{i}\right)+ \\
2 \sum_{i<j} \sum a_{i} a_{j} \operatorname{cov}\left(X_{i} ; X_{j}\right)\end{array}$ & $\begin{array}{c}\text { ROHATGI (1984) } \\
\text { S. } 258 \\
\text { KENDALL \& STUART } \\
\text { (1977) S. } 53\end{array}$ \\
\hline$X \cdot Y$ & $\begin{array}{c}\text { Bei Unabhängigkeit: } \\
\mathrm{E}(X) \cdot \mathrm{E}(Y)\end{array}$ & $\begin{array}{c}\text { Bei Unabhängigkeit: } \\
\begin{array}{c}(\mathrm{E} X)^{2} \cdot \operatorname{Var}(Y)+(\mathrm{E} Y)^{2} \cdot \operatorname{Var}(X) \\
+\operatorname{Var}(Y) \cdot \operatorname{Var}(X)\end{array}\end{array}$ & $\begin{array}{c}\text { BENJAMIN \& } \\
\text { CORNELL (1970) } \\
\text { S. } 170\end{array}$ \\
\hline & $\begin{array}{l}\text { allgemein: } \\
\mathrm{E}(X) \cdot \mathrm{E}(Y)+ \\
\quad \operatorname{cov}(X, Y)\end{array}$ & $\begin{array}{c}\text { allgemein: } \\
\text { keine Angaben } \\
\text { bei den genannten Arbeiten }\end{array}$ & $\begin{array}{l}\text { BENJAMIN \& } \\
\text { CORNELL } 1970 \text {, } \\
\text { S. } 169\end{array}$ \\
\hline$\prod_{i=1}^{n} a_{i} X$ & $\prod_{i=1}^{n} a_{i} \mathrm{E}\left(X_{i}\right)$ & $\begin{array}{l}\text { keine Angaben } \\
\text { bei den genannten Arbeiten }\end{array}$ & \\
\hline \multicolumn{2}{|c|}{ Normalverteilung: } & \multicolumn{2}{|c|}{ Anteil der Werte in $[-\sigma+\mu, \mu+\sigma]=0.68$} \\
\hline
\end{tabular}

Für die Varianz eines Produktes gilt, wenn Unabhängigkeit vorausgesetzt werden kann: ${ }^{139}$

$$
\operatorname{Var}(X \cdot Y)=(\mathrm{E} X)^{2} \cdot \operatorname{Var}(Y)+(\mathrm{E} Y)^{2} \cdot \operatorname{Var}(X)+\operatorname{Var}(Y) \cdot \operatorname{Var}(X) .^{140}
$$

139 Für die Varianz des Produktes stochastisch abhängiger Zufallsvariablen ist in den in Übersicht 166.1 genannten Quellen keine Formel angegeben. 
Über die Varianz von mehr als zwei Faktoren oder zwei stochastisch abhängigen Zufallsvariablen finden sich in der durchgesehenen Literatur ebenfalls keine Angaben. Daher beschränken sich die folgenden Ausführungen auf Produkte aus zwei Faktoren. $\mathrm{Zu}$ Anfang wird ein etwas einfacherer Spezialfall betrachtet, das Quadrat einer stetigen Zufallsvariablen. Daran anknüpfend erfolgt die Behandlung des allgemeinen Verteilungsgesetzes eines Produktes.

Die Zufallsvariable $X$ werde durch die Verteilungsfunktion $\mathrm{F}_{X}(x)$ beschrieben, und die Zufallsvariable $Y$ sei definiert als $Y=a X^{2}$. Dann gilt nach LARSON (1969, S. 102f) für die Verteilung von $Y$ :

$$
\begin{aligned}
\mathrm{F}_{Y}(x) & =\mathrm{H}(x) \cdot \mathrm{F}_{X}\left(\sqrt{\frac{x}{a}}\right)-\mathrm{F}_{X}\left(-\sqrt{\frac{x}{a}}\right), \mathrm{H}(0) \equiv 1 \\
\mathrm{f}_{Y}(x) & =\mathrm{H}(x) \cdot \frac{1}{2 \sqrt{a x}}\left[\mathrm{f}_{X}\left(\sqrt{\frac{x}{a}}\right)+\mathrm{f}_{X}\left(-\sqrt{\frac{x}{a}}\right)\right], \mathrm{x} \neq 0 .
\end{aligned}
$$

Wende ich die letzte Formel auf die Normalverteilung an, erhalte ich für die Dichtefunktion des Quadrates einer normalverteilten Zufallsvariablen oder, kurz gesagt, für die Dichte einer quadrierten Normalverteilung:

$$
\mathrm{f}(x)=\frac{1}{4} \frac{\mathrm{H}(x) \sqrt{2}\left(\mathbf{e}^{\left(-\frac{1}{2} \frac{(\mu+\sqrt{x})^{2}}{\sigma^{2}}\right)}+\mathbf{e}^{\left.\left(-\frac{1}{2} \frac{(-\mu+\sqrt{x})^{2}}{\sigma^{2}}\right)\right)}\right.}{\sqrt{x} \sigma \sqrt{\pi}}
$$

Für eine Standardnormalverteilung vereinfacht sich die Dichte für $\mathrm{x} \neq 0 \mathrm{zu}$ :

$$
\mathrm{f}(x)=\frac{1}{2} \frac{\mathrm{H}(x) \mathbf{e}^{\left(-\frac{1}{2} x\right)} \sqrt{2}}{\sqrt{x} \sqrt{\pi}}
$$

Diese Funktion ist äquivalent zu einer zentralen $\chi^{2}$-Verteilung mit einem Freiheitsgrad. ${ }^{141}$ Ihr Erwartungswert ist Eins und ihre Varianz Zwei. Sie ist in Schaubild 108 im Vergleich zu einer nichtquadrierten Standardnormalverteilung (nichtgestrichelte Linie) dargestellt. Die quadrierte Normalverteilung: realisiert nur positive Werte, daher ist die Dichte im zweiten Quadranten stets Null. Eine durchgeführte Grenzwertbetrachtung ergibt, daß die Verteilung für $x \rightarrow 0$ gegen $\infty$ konvergiert.

140 Rohatgi (1984, S. 268f, 273) gibt hierfür eine Näherung an: $\operatorname{Var}(X \cdot Y)=(\mathrm{E} X)^{2} \cdot \operatorname{Var}(Y)+(\mathrm{E} Y)^{2}$ - $\operatorname{Var}(X)$, die sich von der exakten Formel nur durch Fehlen des letzten Summanden unterscheidet. Da die für die Bildung dieses Terms benötigten Informationen auch in andere Teile der Gleichung einfließen, ist die Näherung nur wenig einfacher zu berechnen. Auch ist der Näherungsfehler nicht unbeträchtlich, wie in Tabelle 177.1 gezeigt wird.

Vgl. zu einer Formulierung der $\chi^{2}$-Verteilung BENJAMIN \& CORNELL (1970, S. 292). 
Das Quadrat einer beliebigen Normalverteilung, dividiert durch ihre Varianz, wird dezentrale $\chi^{2}$-Verteilung, ebenfalls mit einem Freiheitsgrad, genannt. Erwartungswert und Varianz entsprechen der zentralen $\chi^{2}$-Verteilung. Der zweite Parameter einer dezentralen $\chi^{2}$-Verteilung neben den Freiheitsgraden ist der sog. Nicht- oder Dezentralitäts-Parameter $\lambda$. Er ist eine Funktion von Varianz und Erwartungswert der Normalverteilung (DOBSON 1983, S. 6):

$$
\lambda \quad=\frac{\mu^{2}}{2 \cdot \sigma^{2}} \cdot{ }^{142}
$$

Aus der Formel ist offensichtlich, daß für Normalverteilungen mit Erwartungswert Null der Nichtzentralitäts-Parameter ebenfalls Null beträgt. ${ }^{143}$

\section{Schaubild 168.1: Dichtefunktion einer quadrierten Standardnormalverteilung}

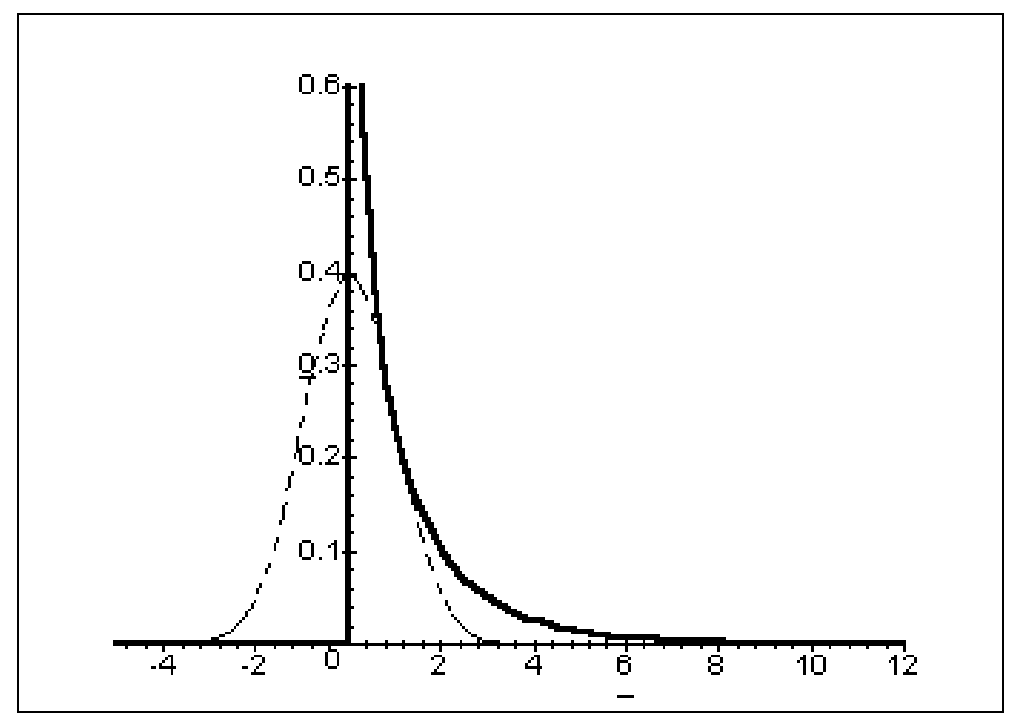

Die Momente einer quadrierten Normalverteilung, die nicht durch ihre Varianz dividiert ist und z.B. in der Quadratischen Programmierung Verwendung findet, kann ich mit Hilfe einer Umkehr der Z-Transformation ableiten. ${ }^{144} X$ sei eine normalverteilte Zufallsveränderliche mit den Parametern $\mu$ und $\sigma^{2}$ und $Z$ eine Standardnormalverteilung. Dann lassen sich $X$ und $X^{2}$ mit Hilfe von $Z$ ausdrücken:

142 Für $\lambda$ sind auch leicht abweichenden Definitionen üblich, z.B. $\lambda=\frac{\mu^{2}}{\sigma^{2}}$ (RINNE, 1997, S. 377) oder $\lambda=\frac{\mu}{\sigma}$ in der Programmiersprache Gauss.

$143 \mathrm{Zu}$ weiteren Eigenschaften der dezentralen $\chi^{2}$-Verteilung siehe ABRAMOWITZ \& STEGUN (1970, 144 S. 943) und RINNE (1997), Unterabschnitt 3.10.4.2.

Die Z-Transformation besagt bekanntlich: Wenn $X$ normalverteilt ist mit den Parametern $\mu$ und $\sigma^{2}$, dann ist $Z=\frac{X-\mu}{\sigma}$ standardnormalverteilt. 


$$
\begin{array}{ll}
X & =\sigma \cdot Z+\mu \\
X^{2} & =(\sigma \cdot Z+\mu)^{2} \\
& =\sigma^{2} \cdot Z^{2}+2 \cdot \sigma \cdot Z \cdot \mu+\mu^{2} .
\end{array}
$$

Die Momente von $Z^{2}$ sind (s.o.): $\mathrm{E}\left(Z^{2}\right)=1$ und $\operatorname{Var}\left(Z^{2}\right)=2$. Daher erhalte ich für die Momente von $X^{2}$ :

$$
\begin{aligned}
\mathrm{E}\left(X^{2}\right) & =\mathrm{E}\left(\sigma^{2} \cdot Z^{2}+2 \cdot \sigma \cdot \mu \cdot Z+\mu^{2}\right) \\
& =\sigma^{2} \cdot 1+2 \cdot \sigma \cdot \mu \cdot 0+\mu^{2} \\
& =\sigma^{2}+\mu^{2} \\
\operatorname{Var}\left(X^{2}\right) \quad & =\operatorname{Var}\left(\sigma^{2} \cdot Z^{2}+2 \cdot \sigma \cdot \mu \cdot Z+\mu^{2}\right) \\
& =\sigma^{4} \cdot 2+2^{2} \cdot \sigma^{2} \cdot \mu^{2} \cdot 1+2 \cdot \sigma^{2} \cdot 2 \sigma \mu \cdot \operatorname{Cov}\left(Z^{2}, Z\right)+0 .
\end{aligned}
$$

Für die Kovarianz zwischen einer standardnormalverteilten Zufallsgröße und ihrem Quadrat gilt:

$$
\operatorname{Cov}\left(Z^{2}, Z\right)=\mathrm{E}\left(Z^{2} \cdot Z\right)-\mathrm{E}\left(Z^{2}\right) \cdot \mathrm{E}(Z) .
$$

Da der Erwartungswert von $Z$ Null ist, vereinfacht sich die Kovarianz zwischen $Z$ und $Z^{2} \mathrm{zu}$ :

$$
\operatorname{Cov}\left(Z^{2}, Z\right)=\mathrm{E}\left(Z^{3}\right)
$$

Für eine Normalverteilung mit Erwartungswert Null ist $\mathrm{E}\left(Z^{3}\right)$ äquivalent mit ihrer Schiefe. Da die Normalverteilung symmetrisch ist und daher eine Schiefe von Null besitzt, ist auch die Kovarianz zwischen $Z$ und $Z^{2}$ gleich Null. Damit vereinfacht sich die Varianz von $X^{2} \mathrm{zu}$ :

$$
\operatorname{Var}\left(X^{2}\right) \quad=2 \cdot \sigma^{2} \cdot\left(\sigma^{2}+2 \cdot \mu^{2}\right) .
$$

Verglichen mit den bekannten Momenten eines Produktes von zwei iid-Normalverteilungen mit den Parametern $\mu$ und $\sigma^{2}$ (s. S. 165:

$$
\begin{aligned}
\mathrm{E}(X \cdot Y) & =\mathrm{E}(X) \cdot \mathrm{E}(Y)=\mu^{2} \\
\operatorname{Var}(X \cdot Y) & =(\mathrm{E} X)^{2} \cdot \operatorname{Var}(Y)+(\mathrm{E} Y)^{2} \cdot \operatorname{Var}(X)+\operatorname{Var}(Y) \cdot \operatorname{Var}(X) \\
& =\mu^{2} \cdot \sigma^{2}+\mu^{2} \cdot \sigma^{2}+\sigma^{2 \cdot 2} \\
& =\sigma^{2} \cdot\left(2 \cdot \mu^{2}+\sigma^{2}\right)
\end{aligned}
$$

ergeben sich folgende Beziehungen:

$$
\begin{array}{ll}
\mathrm{E}\left(X^{2}\right) & =\mu^{2}+\sigma^{2}=\mathrm{E}(X \cdot Y)+\sigma^{2} \\
\operatorname{Var}\left(X^{2}\right) & =2 \cdot \sigma^{2} \cdot\left(2 \cdot \mu^{2}+\sigma^{2}\right)=2 \cdot \operatorname{Var}(X \cdot Y) .
\end{array}
$$

Der Erwartungswert eines Quadrates ist also um die Varianz größer als der eines Produktes aus zwei identischen und unabhängig normalverteilten Zufallsveränderlichen. Für die Varianz des Quadrates gilt, daß sie das Doppelte der Varianz des entsprechenden Produktes beträgt. 
Findet sich in der Literatur schon wenig zu quadrierten Normalverteilungen, die nicht standardnormalverteilt sind, so fehlen Angaben über Quadrate von gleichverteilten Zufallsveränderlichen in den zitierten Lehrbüchern völlig, obwohl deren Eigenschaften durchaus Überraschendes bergen. Daher leite ich im folgenden einige Merkmale quadrierter Gleichverteilungen ab.

Die Dichtefunktion einer Gleichverteilung lautet unter Verwendung der HeavisideFunktion mit $b>a$ und $\mathrm{H}(0) \equiv 1$

$$
\mathrm{f}(x)=\frac{\mathrm{H}(x-a) \mathrm{H}(b-x)}{b-a}
$$

Für die Dichte einer quadrierten Gleichverteilung erhalte ich gemäß (167.1):

$$
\mathrm{f}\left(x^{2}\right)=\frac{1}{2} \frac{\mathrm{H}(x)(\mathrm{H}(\sqrt{x}-a) \mathrm{H}(b-\sqrt{x})+\mathrm{H}(-\sqrt{x}-a) \mathrm{H}(b+\sqrt{x}))}{\sqrt{x}(b-a)}, \mathrm{x} \neq 0
$$

Naheliegende Intuition läßt vermuten, daß für eine Gleichverteilung ebenso wie für eine Normalverteilung gilt, daß sie durch die Angabe ihrer ersten beiden Momente eindeutig spezifiziert ist. In der Tat läßt sich durch wenige Umformungen zeigen, daß durch die Vorgabe von Momenten $\left(\mu\right.$ und $\left.\sigma^{2}\right)$ die Parameter der Gleichverteilung $a$ und $b$ exakt bestimmbar sind:

$$
a=\mu-\sqrt{3 \cdot \sigma} \text { und } b=\mu+\sqrt{3 \cdot \sigma} \text {. }
$$

In Schaubild 171 1 stelle ich eine Gleichverteilung mit den Momenten einer Standardnormalverteilung (gestrichelte Linie) und ihr Quadrat gemäß 170.1 dar. Die quadrierte Verteilung ist auf den ersten Quadranten beschränkt. Sie erstreckt sich von Null bis Drei. Es läßt sich zeigen, daß diese rechte Grenze des quadrierten Verteilung, $b q$ genannt, eine lineare Funktion der Varianz ist: $(b q \mid \mu=0)=3 \cdot \sigma^{2}$.

Allgemein ist die Dichte einer quadrierte Gleichverteilung: gleich Null, wenn gilt [s. (170.1)]:

- $x<0$ oder

- $\mathrm{H}(\sqrt{ } x-a) \cdot \mathrm{H}(b-\sqrt{ } x)+\mathrm{H}(-\sqrt{ } x-a) \cdot \mathrm{H}(b+\sqrt{ } x)=0$.

Die letzte Bedingung ist nur erfüllt, wenn beide Summanden jeweils Null sind, da keiner der Summanden negativ sein kann. Dies ist gegeben, wenn mindestens einer der Faktoren in beiden Summanden Null ergibt, wenn also:

$$
\begin{array}{ll} 
& {[(\sqrt{ } x-a<0) \vee(b-\sqrt{ } x<0)] \wedge[(-\sqrt{ } x-a<0) \vee(b+\sqrt{ } x<0)]} \\
\Leftrightarrow \quad & {\left[\left(x<a^{2}\right) \vee\left(x>b^{2}\right)\right] \wedge\left[\left(x>a^{2}\right) \vee\left(x<b^{2}\right)\right] .}
\end{array}
$$




\section{Schaubild 171.1: Dichtefunktion einer quadrierten Gleichverteilung}

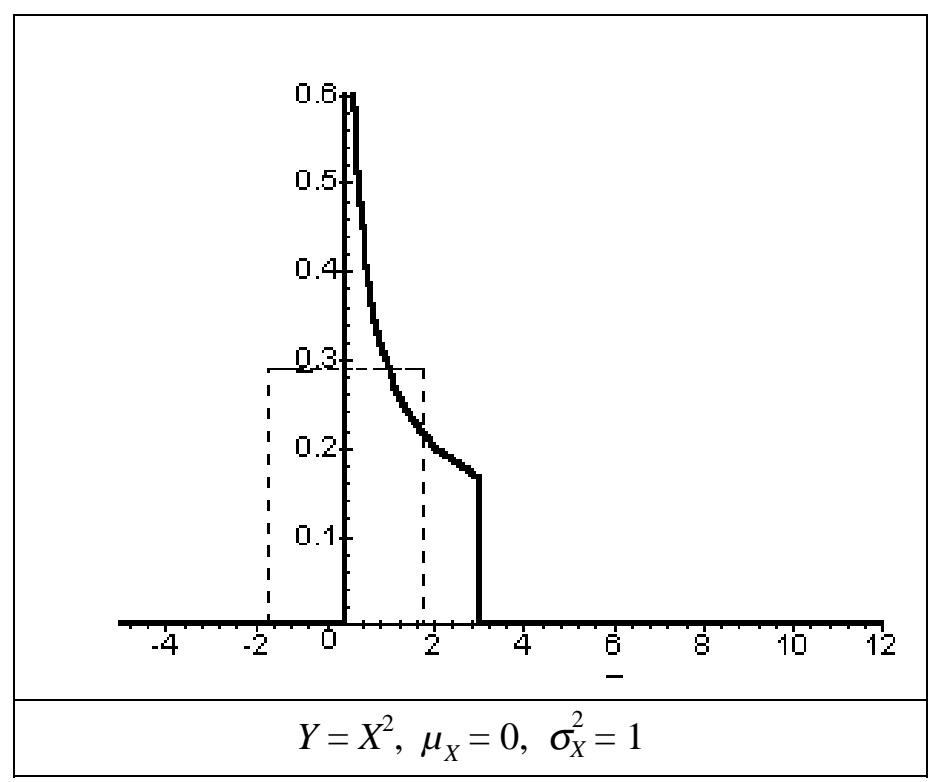

Der oben genannte logische Ausdruck wird wahr, wenn eine (oder mehrere) von vier Konstellationen zutrifft, die sich am einfachsten in einer Kreuztabelle veranschaulichen und zusammenfassen lassen:

\begin{tabular}{c|cc} 
& $x<a^{2}$ & $x>b^{2}$ \\
\hline$x>a^{2}$ & - & $x>\max \left(a^{2}, b^{2}\right)$ \\
$x<b^{2}$ & $x<\min \left(a^{2}, b^{2}\right)$ & -
\end{tabular}

Die Bedingungen auf der Hauptdiagonalen können nicht gleichzeitig erfüllt sein, sie schließen sich gegenseitig aus. Die Bedingungen der Nebendiagonalen vereinfachen wegen der Definition $a<b$ für positive Parameter zu $\left(x<a^{2} \vee x>b^{2}\right)$ und für negative Parameter zu $\left(x<b^{2} \vee x>a^{2}\right)$. Überdeckt die Gleichverteilung den Nullpunkt, erstreckt sich der Bereich positiver Dichte von Null bis $b^{2}$. In Übersicht 172.1 sind beispielhaft die Dichtefunktionen von drei Gleichverteilungen und ihren Quadraten dargestellt. Die mittlere quadrierte Verteilung weist einen Pol auf, sie konvergiert für $x$ $=0$ gegen $\infty$.

In Übersicht 173,1 sind quadrierte Gleich- und Normalverteilungen für verschiedene Momente der Ausgangsverteilung und die Ausgangsverteilung selbst abgetragen. 


\section{Übersicht 172.1: Dichtefunktionen quadrierter Gleichverteilungen und ihrer Ausgangsfunktionen}

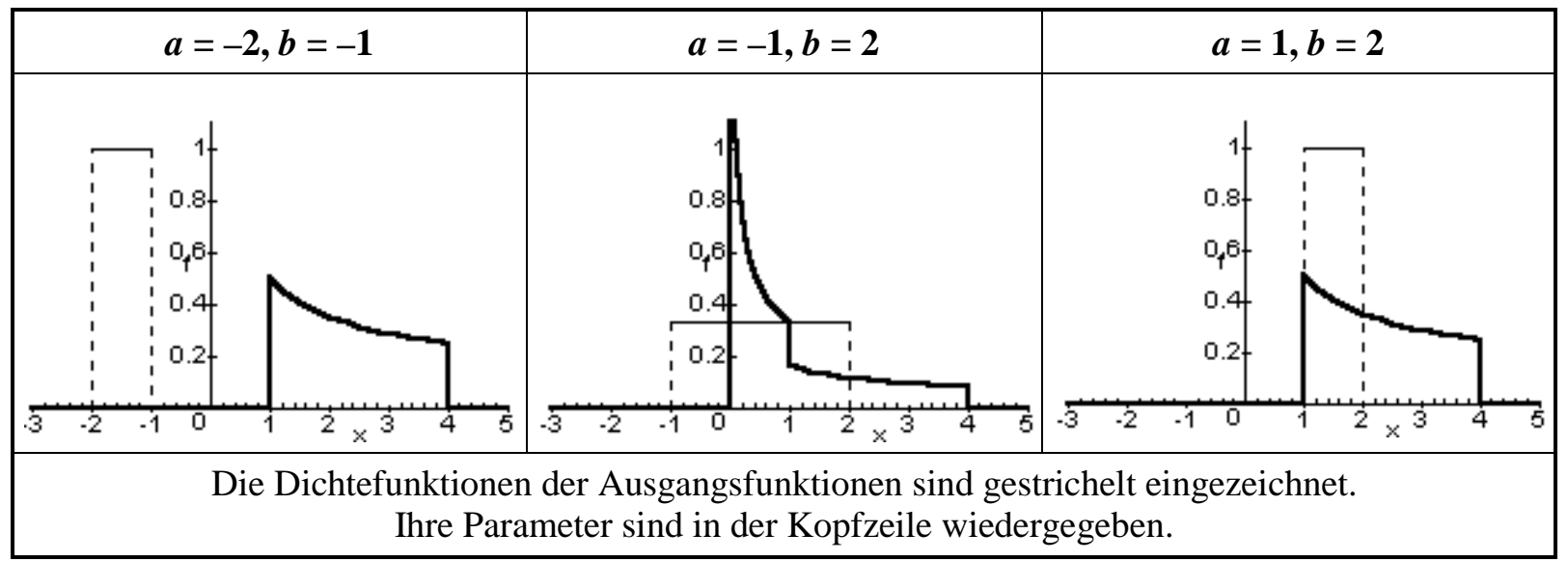

Die Dichtefunktion der quadrierten Normalverteilung verändert sich mit zunehmendem Erwartungswert von einer Hyperbel zu einer sehr flachen Glockenkurve. Dieser Prozeß wird durch eine größere Varianz verlangsamt. Die Dichtefunktion der quadrierten Gleichverteilung reagiert weniger stark als die Normalverteilung auf eine Verdoppelung der Varianz. Die Dichtefunktion weist für alle dargestellten Parameterkombinationen die Gestalt einer abgestuften Hyperbel auf, die sowohl nach oben wie nach rechts begrenzt ist. Schaubild 1721 zeigt diese Stufen und die rechte Grenze der Dichtefunktion im Detail für $\mu=-1$ und $\sigma=2$. Die rechte Grenze beträgt $a^{2}=(\mu-$ $\sqrt{3 \cdot \sigma})^{2} \approx 19.2$, vgl. (1702

Schaubild 172.1: Quadrierte Gleichverteilung mit den Momenten $\mu=-1, \sigma=2$

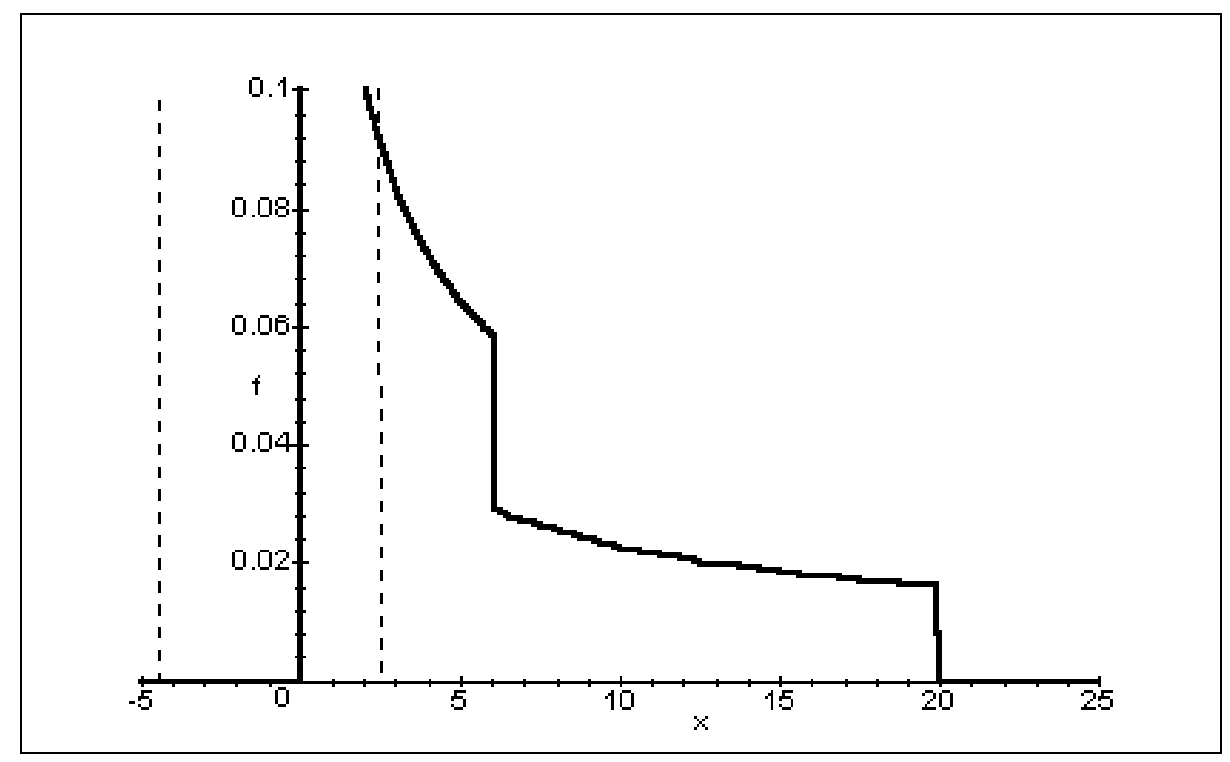




\section{Übersicht 173.1: Dichtefunktionen quadrierter Verteilungen und ihrer Ausgangsfunktionen}

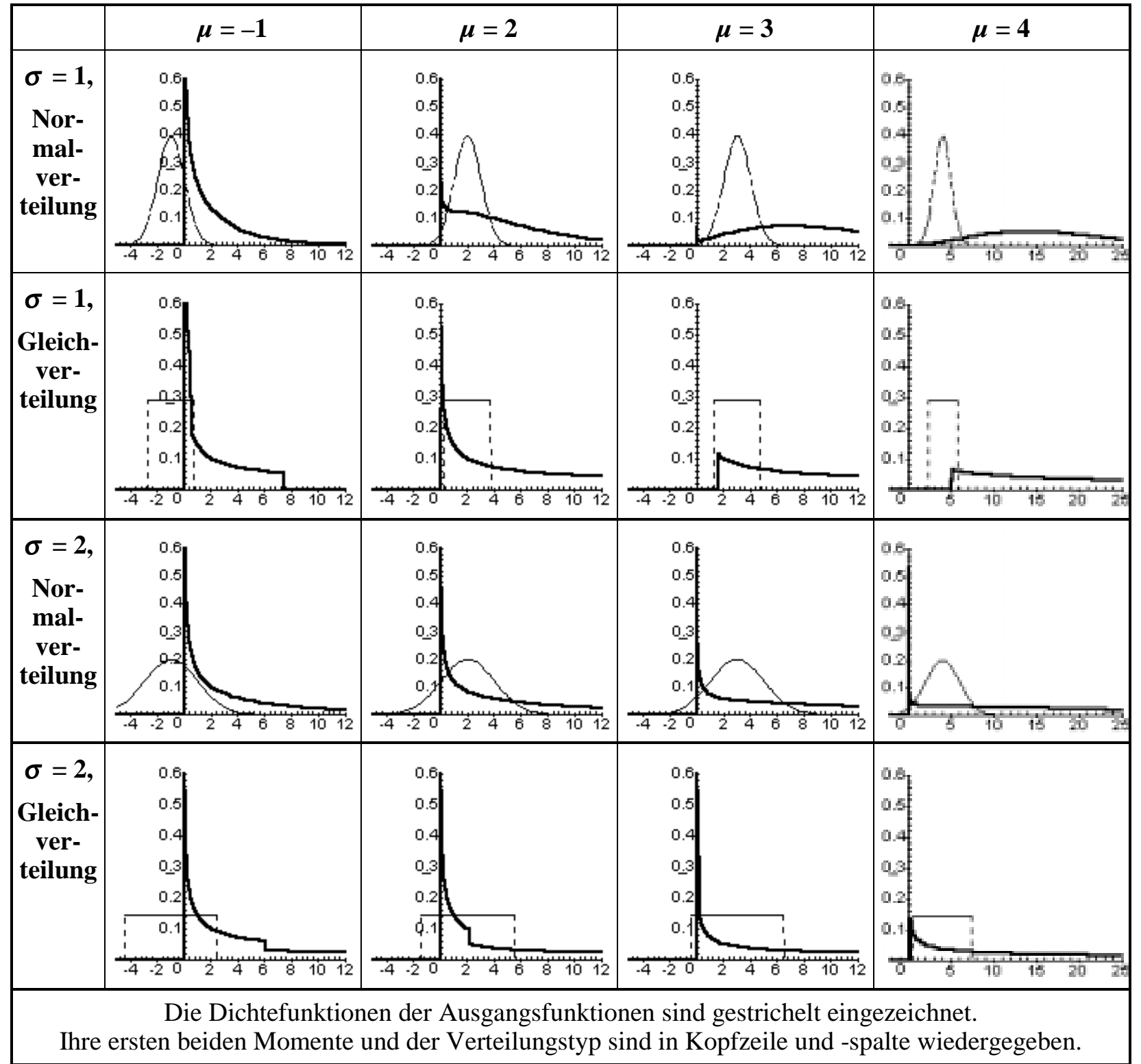

Durch Quadrierung ändert sich also die Verteilungsform drastisch. Auch das Produkt von zwei Zufallsveränderlichen, die identisch und unabhängig verteilt sind, ist in der Regel anders verteilt als die Ausgangsfaktoren. ${ }^{145}$

145 Dies wird in der Literatur gelegentlich übersehen, beispielsweise wenn für einen Erlös, der als Produkt der normalverteilten Zufallsvariablen Preis und Ertrag eingeführt wird, eine Normalverteilung unterstellt wird (NOELL 1996). 
Das Produkt zweier Zufallsveränderlicher, die nur positive Werte annehmen können, läßt sich durch die sog. „Melin convolution“ (SPRINGER 1979, S. 91, 97) angeben. Es sei:

$$
Z \quad \equiv X \cdot Y .
$$

Dann gilt für die Dichtefunktion von $Z$ :

$$
\begin{aligned}
\mathrm{h}_{2}(z) \quad & =\int_{0}^{\infty} \frac{1}{y} \cdot \mathrm{f}_{X}\left(\frac{z}{y}\right) \cdot \mathrm{f}_{Y}(y) \cdot \mathrm{d} y \\
& =\int_{0}^{\infty} \frac{1}{x} \cdot \mathrm{f}_{X}(x) \cdot \mathrm{f}_{Y}\left(\frac{z}{x}\right) \cdot \mathrm{d} x, x, y \geq 0 .
\end{aligned}
$$

Das Subscript 2 kennzeichnet die Anzahl Faktoren in $Z$. Für drei nichtnegative Faktoren $X_{1}, X_{2}, X_{3}$ gilt entsprechend (SPRINGER 1979, S. 97):

$$
\mathrm{h}_{3}(z) \quad=\int_{0}^{\infty} \frac{1}{x_{3}} \cdot \mathrm{h}_{2}\left(\frac{z}{x_{3}}\right) \cdot \mathrm{f}_{X_{3}}\left(x_{3}\right) \cdot \mathrm{d} x_{3}
$$

und für $n$ nichtnegative Faktoren ergibt sich ebenfalls nach SPRINGER die rekursive Formel:

$$
\mathrm{h}_{n}(z) \quad=\int_{0}^{\infty} \frac{1}{x_{n}} \cdot \mathrm{h}_{n-1}\left(\frac{z}{x_{n}}\right) \cdot \mathrm{f}_{X n}\left(x_{n}\right) \cdot \mathrm{d} x_{n} .
$$

Setze ich in Formel ( 174.1 , die, wie erwähnt, nur für nichtnegative Zufallsvariablen gilt, die Dichte der Normalverteilung ein, erhalte ich für das Produkt zweier ii-Normalverteilungen $Z=\mathrm{N}_{X}\left(\mu, \sigma^{2}\right) \cdot \mathrm{N}_{Y}\left(\mu, \sigma^{2}\right)$ unter der Bedingung $x, y, z \geq 0$

$$
\mathrm{f}(z)=\int_{0}^{\infty} \frac{1}{2} \frac{\mathrm{e}^{\left(-\frac{1}{2} \frac{z^{2}-2 z \mu y+2 \mu^{2} y^{2}+y^{4}-2 y^{3} \mu}{y^{2} \sigma^{2}}\right)}}{y \sigma^{2} \pi} d y .
$$

Für Standardnormalverteilungen vereinfacht sich die Formel zu: 


$$
\mathrm{f}(z)=\int_{0}^{\infty} \frac{1}{2} \frac{\mathbf{e}^{\left(-\frac{1}{2} \frac{z^{2}}{y^{2}}\right)} \mathbf{e}^{\left(-\frac{1}{2} y^{2}\right)}}{y \pi} d y
$$

In Schaubild 175.1 ist das Produkt zweier unabhängiger Standardnormalverteilungen $Z=\mathrm{N}_{X}(0,1) \cdot \mathrm{N}_{Y}(0,1)$ für $x, y, z \geq 0$ im Vergleich zu einer einfachen Standardnormalverteilung abgetragen. Die Momente des Produktes stimmen mit den Momenten der Ausgangsverteilung überein, da stochastische Unabhängigkeit vorliegt (vgl. Übersicht 166.1]; dennoch verändert sich die Verteilungsform sehr deutlich. Die Verteilung des Produktes erscheint zum Ursprung hin eingedrückt. Sie konvergiert für $z \rightarrow 0$ gegen $\infty$.

\section{Schaubild 175.1: Dichtefunktion des Produkts zweier Standardnormal- verteilungen im ersten Quadranten}

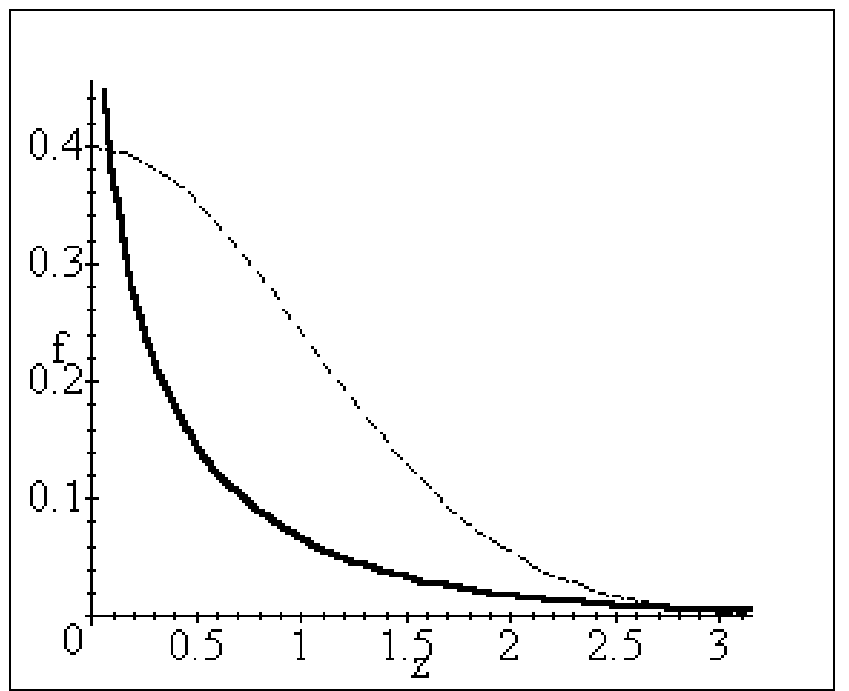

Da (174.1] nur für positive Argumente gilt, wird ein Monte-Carlo-Simulationsexperiment durchgeführt. Schaubild 1761 zeigt die Dichtefunktionen des Produktes zweier Standardnormalverteilungen (gestrichelte Linie). Der Graph ähnelt stark einer Laplace-Verteilung; zum Vergleich ist die Standardnormalverteilung eingezeichnet (durchgezogene Linie). 


\section{Schaubild 176.1: Dichtefunktion des Produktes zweier Standardnormal- verteilungen im Monte-Carlo-Simulationsexperiment}

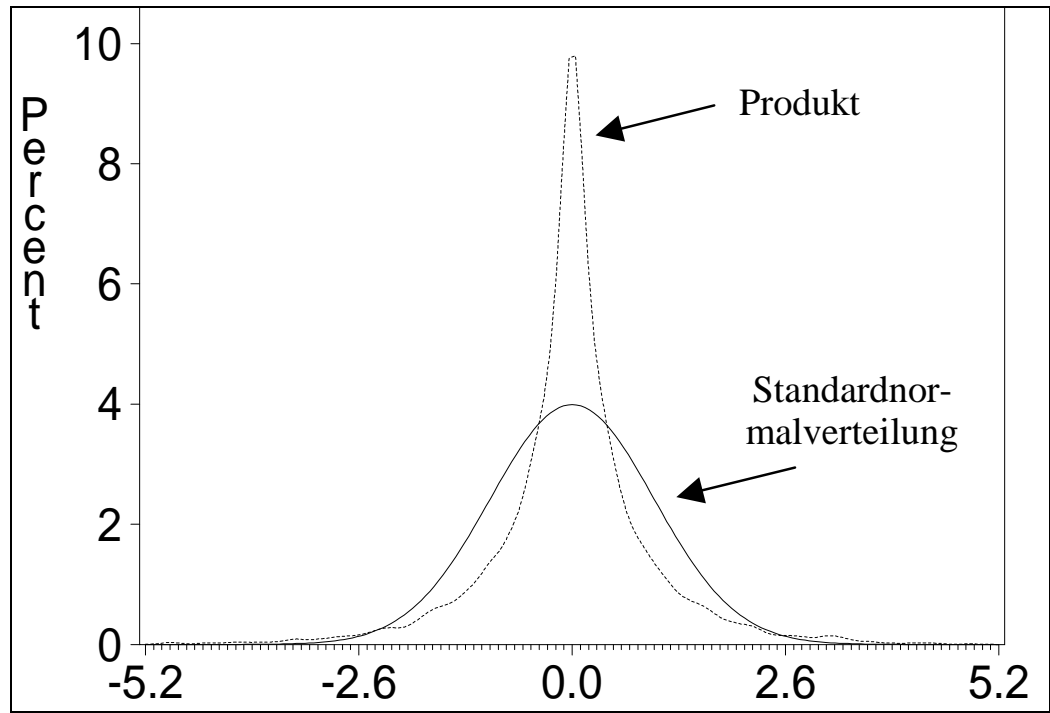

Wie unterscheidet sich das Produkt zweier standardnormalverteilter Zufallsveränderlicher von ihrem Quadrat? In Schaubild 1762 sind beide Verknüpfungen dargestellt. Beide Funktionen weisen einen Pol über dem Ursprung auf und konvergieren hier gegen $\infty$. Die Dichtefunktion des Produktes (durchgezogene Linie) verläuft sehr viel flacher als die der quadrierten Verteilung, da die quadrierte Verteilung ihre gesamte Wahrscheinlichkeitsdichte auf den ersten Quadranten konzentriert.

\section{Schaubild 176.2: Vergleich einer quadrierten Standardnormalverteilung mit dem Produkt zweier Standardnormalverteilungen im ersten Quadranten}

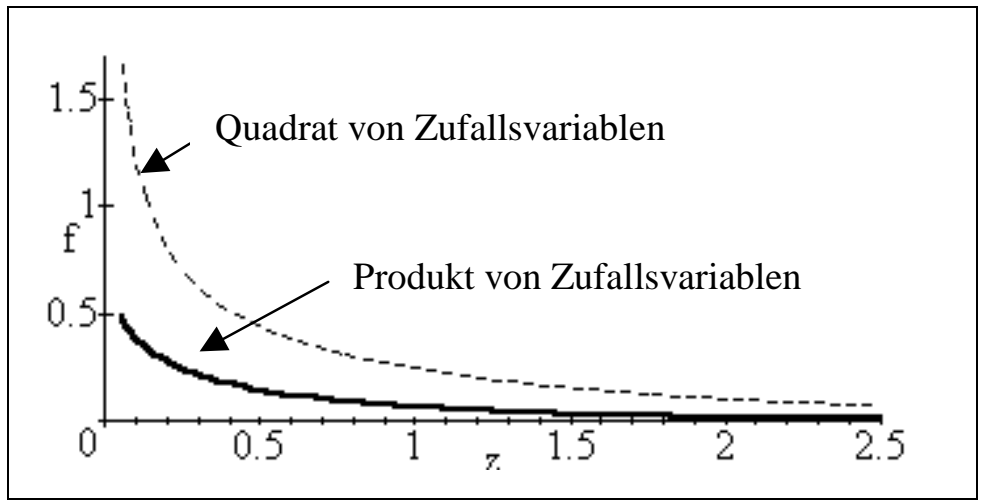

Die Graphen zeigen sehr deutlich, daß die Multiplikation einer Zufallsveränderlichen mit sich selbst zu einer anderen Zufallsgröße führt als die Multiplikation der gleichen Zufallsveränderlichen mit einer identisch und unabhängig verteilten Zufallsveränderlichen: 


$$
\mathrm{F}\left(x^{2}\right) \quad \neq \mathrm{F}(x \cdot y) \text { auch wenn } \mathrm{F}(x)=\mathrm{F}(y) .
$$

In Tabelle 177.1 sind einige Simulationsergebnisse für Funktionen von normalverteilten Zufallsvariablen mit $n_{1,2} \sim \mathrm{n}\left(2,2^{2}\right)$ zusammengestellt, die die analytischen Ergebnisse verdeutlichen. Für die Parameter der Normalverteilung werden bewußt nicht 0 und 1 verwendet, da diese Zahlen die neutralen Elemente bezüglich der Verknüpfungen Addition bzw. Multiplikation bilden.

Durch Summation zweier iid-Normalverteilungen addieren sich, entsprechend (160.1) und (1611), Erwartungswerte und Varianzen (Zeile 3). Der Erwartungswert der Summe und des Zweifachen einer Normalverteilung (4) stimmen überein, während die Varianz der Summe (3) halb so groß ist wie die Varianz des Zweifachen (4). Dieser Unterschied ergibt sich ebenfalls unmittelbar aus (161,1). Daher weisen das $\sqrt{ } 2$ fache $(5)$ und die Summe (4) die gleiche Varianz auf, allerdings nicht den gleichen Erwartungswert.

Der Erwartungswert eines Produktes ergibt sich einfach durch Multiplikation der Erwartungswerte der unabhängigen Faktoren. ${ }^{146}$ Der Erwartungswert der quadrierten Verteilung (Zeile 7) ist um die Varianz größer als der Erwartungswert des Produktes [s. (1691]], während ihre Varianz das Doppelte beträgt [s. (169.2]. Auffällig ist auch das Ergebnis für $n_{1} / n_{2}$. Der Erwartungswert ist deutlich größer als $2 / 2=1$. Die Varianz steigt durch die Quotientenbildung drastisch an, da durch eine Division mit Werten nahe Null extreme Werte entstehen.

\section{Tabelle 177.1: Simulierte Momente zusammengesetzter normalverteilter Zufallsveränderlicher}

\begin{tabular}{|lllcccc|}
\hline \multicolumn{2}{l}{ Variable } & $\mathbf{N}$ & Mean & Variance & Skewness & Kurtosis \\
1 & $\mathrm{n}_{1}$ & 10000 & 2.004 & 2.011 & -0.009 & 0.006 \\
2 & $\mathrm{n}_{2}$ & 10000 & 1.997 & 2.016 & -0.010 & -0.036 \\
3 & $\mathrm{n}_{1}+\mathrm{n}_{2}$ & 10000 & 4.000 & 4.135 & -0.023 & 0.069 \\
4 & $2 \cdot \mathrm{n}$ & 10000 & 4.007 & 8.043 & -0.009 & 0.006 \\
5 & $\sqrt{2} \cdot \mathrm{n}_{1}$ & 10000 & 2.834 & 4.022 & -0.009 & 0.006 \\
6 & $\mathrm{n}_{1} \cdot \mathrm{n}_{2}$ & 10000 & 4.055 & 20.432 & 1.131 & 2.356 \\
7 & $\mathrm{n}_{1}{ }^{2}$ & 10000 & 6.025 & 40.192 & 1.753 & 4.040 \\
8 & $\mathrm{n}_{1} / \mathrm{n}_{2}$ & 10000 & 1.334 & 1679.224 & 36.837 & 3038.884 \\
\hline
\end{tabular}

Folgendes sei für die spätere Analyse von dynamischen Modellen festgehalten:

- Verteilungsform und Momente eines Quadrates von Zufallsvariablen unterscheiden sich deutlich von einem Produkt von zwei Zufallsveränderlichen, auch wenn beide Zufallsvariablen $i i$-verteilt sind.

146 Die Näherung für die Varianz des Produktes, die in Fußnote 140 beschrieben ist, beträgt 16 und weicht daher um etwa $25 \%$ vom exakten Wert 20 ab [s. (166.1)]. 
- Die Varianz einer Summe zweier iid-Zufallsveränderlicher ist nicht gleich der Varianz des Zweifachen einer der Summanden. ${ }^{147}$

Abschließend sei die Frage behandelt, ob die Wahrscheinlichkeit eines Produktes auch dann nicht mit dem Produkt der Wahrscheinlichkeiten übereinstimmt, wenn die Zufallsvariablen $i i$-verteilt sind:

$$
\mathrm{p}(X \cdot Y) \stackrel{\stackrel{?}{=}}{\mathrm{p}}(X) \cdot \mathrm{p}(Y) \text {. }
$$

$X$ und $Y$ seien normalverteilte Zufallsvariablen mit Erwartungswert und Varianz von Eins. Eine Monte-Carlo-Simulation ergibt folgende Wahrscheinlichkeiten:

$$
\begin{aligned}
& \mathrm{p}(X>0)=0.8370 ; \quad \mathrm{p}(Y>0)=0.8392 \\
& \mathrm{p}(X \cdot Y>0)=0.7354 .
\end{aligned}
$$

Das Produkt aus $\mathrm{p}(X>0)$ und $\mathrm{p}(Y>0)$ ergibt 0.7024 und weicht daher deutlich von $\mathrm{p}(X \cdot Y>0)=0.7354 \mathrm{ab}$.

Zum gleichen Ergebnis gelangt man auch durch ein Gedankenexperiment. $N$ sei eine Zufallsvariable, die sich nur kleiner als Null realisiere, und $P$ eine mit nur positiven Werten. Dann ist die Wahrscheinlichkeit $\mathrm{p}(N \cdot P<0)$ gleich Eins, während das Produkt der Wahrscheinlichkeiten $\mathrm{p}(N<0) \cdot \mathrm{p}(P<0)$ Null ergibt.

\subsection{Exkurs: Probleme bei der Maximierung von Erwartungswerten (Petersburger Paradoxon)}

Das Petersburger Paradox ist eine Lotterie, deren Auszahlung einen unendlichen Erwartungswert aufweist. Nichtsdestotrotz bewegt sich die Zahlungsbereitschaft potentieller Mitspieler auf einem sehr geringen Niveau. ${ }^{148}$ JERGER (1992) zitiert empirische Untersuchungen, die eine Zahlungsbereitschaft um zwei DM ermittelt haben, und erklärt diese Differenz mit Risikoaversion der Befragten. ${ }^{149}$ BERNOULLI (1738)

147 Dennoch gilt selbstverständlich: $\operatorname{Var}(X+X)=\operatorname{Var}(2 \cdot X)$. Wichtig ist, daß aus einer Übereinstimmung von Varianzen: $\operatorname{Var}(X)=\operatorname{Var}(Y)$ nicht folgt: $\operatorname{Var}(X+Y)=\operatorname{Var}(2 \cdot X)$. Im Gegensatz dazu folgt aus einer Übereinstimmung der Erwartungswerte, $\mathrm{E}(X)=\mathrm{E}(Y)$, daß gilt $\mathrm{E}(X+Y)=\mathrm{E}(2 \cdot X)$, sofern $X$ und $Y$ stochastisch unabhängig sind.

148

Dieses Spiel wird im allgemeinen Daniel BERNOULLI (1738) zugeschrieben. BERNOULLI selbst hingegen (in der englischen Fassung von 1956 auf S. 31) gibt als Quelle dieses Spiels seinen Cousin Nicolas BERNOULLI an, der es an Pierre Rémond de MONTMORT geschickt habe. MONTMORT veröffentlichte als erster das Spiel 1713 in dem Werk "L'analyse sur les jeux de hazard". Das Paradoxon hat in der Folge viele Mathematiker und Ökonomen inspiriert, z.B. hat MENGER (1934) das Spiel verallgemeinert. $\mathrm{Zu}$ weiteren Arbeiten siehe die Bibliographie von SOMMER (1954) am Ende der Übersetzung von BERNOULLIs Aufsatz und danach z.B. SCHUMPETER (1965, S. 386).

$149 \mathrm{Zu}$ anderen Erklärungsansätzen für das Petersburger Paradoxon vgl. MENGER (1967). SAMUELSEN (1977) beleuchtet die geschichtliche Bedeutung des Paradox für die Weiterentwicklung der Wahrscheinlichkeits- und Nutzentheorie. SIGMUND (1993) verwendet den Gedanken ei- 
illustrierte mit diesem Spiel seine Risikonutzentheorie. Dieser Ansatz wird seitdem in der Literatur immer wieder diskutiert. SENNETTI (1976) zeigt, daß das Paradox auch ohne Nutzenfunktion mit Hilfe der Portfolio-Theorie aufgelöst werden kann. Allerdings ist dieser Ansatz nach EPPS (1978) nicht sehr hilfreich, da er dazu führt, daß risikoaverse Spieler nicht einmal zu einem Preis von Null an dem Spiel teilnähmen. Streit entzündete sich ferner an der Frage, ob die Nutzenfunktion beschränkt sein müsse, um nicht das Paradox mit einem neuen zu erklären (SHAPLEY 1977, AUMANN 1977, SHAPLEY 1977a). NIELSON (1992) modifiziert die Von-Neumann-Morgenstern-Axiomatik, um auch unbeschränkte Nutzenfunktionen, beispielsweise logarithmische, mit dem Petersburger Paradox in Einklang zu bringen. Auch in nicht auf ökonomisch interessierte Leser zielenden Publikationen wird das Petersburger Paradox gern als Beispiel für die Wirkungen des Zufalls verwendet (SCHEID 1992, FLUSSER 1992). Dieser Exkurs handelt davon, daß es auch ohne Risikoscheu oder abnehmenden Grenznutzen gute Gründe gibt, die Einsätze bei diesem Spiel nicht in den Himmel wachsen zu lassen.

Beim Petersburger Paradox handelt es sich um ein Münzwurf-Spiel. Ein Geldstück wird solange geworfen, bis das erste Mal „Zahl“ fällt. Dann erfolgt die Auszahlung: Fiel schon beim ersten Mal "Zahl", beträgt der Gewinn 1. Er verdoppelt sich mit jedem Wurf, der nicht „Zahl“" ergibt. Die folgenden Tabelle zeigt die Auszahlungen $a_{n}$ in Abhängigkeit von der Anzahl der Würfe $n$ (bis zum Eintreten des Ereignisses „Zahl“). $n$ ist also mit anderen Worten die Dauer eines Spiels.

Wie aus Tabelle 1801. Spalte 1 und 2, hervorgeht, beträgt allgemein die Auszahlung:

$$
a_{n} \quad=2^{(n-1)} .
$$

Die Wahrscheinlichkeit für die kleinste Gewinnmöglichkeit $a_{1}=1$ beträgt $1 / 2$, für $a_{2}=2$ lautet sie $1 / 4$. Sie halbiert sich jeweils von einer Auszahlungsmöglichkeit zur nächsten.

Allgemein (s. Tabelle 180.1, Spalte 3) gilt:

$$
p\left(a_{\mathrm{n}}\right) \quad \equiv p_{n}=\left(\frac{1}{2}\right)^{n} \text {. }
$$

Die fünfte Spalte der Tabelle 180.1 gibt die Wahrscheinlichkeit an, daß die Spieldauer $n$ erreicht wurde. Die durchschnittliche Spieldauer:

$$
\sum_{n=1}^{\infty} n \cdot p_{n}=\sum_{n=1}^{\infty} n \cdot\left(\frac{1}{2}\right)^{n}=\sum_{n=1}^{\infty} \frac{n}{2^{n}}
$$

nes unendlichen Erwartungswertes für die Analyse der Überlebenswahrscheinlichkeit von Bakterienkulturen. In seinem Beispiel ist der Erwartungswert der Lebenserwartung der Mikrobenpopulation unendlich, dennoch wird sie mit Sicherheit aussterben. 
beträgt 2. ${ }^{150}$ Damit ist der mittlere Gewinn aber keineswegs $a_{2}=2^{(2-1)}=2$. Vielmehr errechnet sich als Erwartungswert des Spiels:

$$
\text { E } \quad=\sum_{n=1}^{\infty} a_{n} \cdot p_{n} .
$$

\section{Tabelle 180.1: Auszahlungsmöglichkeiten $\left(a_{n}\right)$ und ihre} Wahrscheinlichkeiten $\left(p_{n}\right)$

\begin{tabular}{|c|c|c|c|c|c|}
\hline$n$ & $a_{n}$ & $P_{n}$ & $a_{n} \cdot p_{n}$ & $n \cdot p_{n}$ & $\begin{array}{c}n \cdot p_{n} \\
\text { (kumuliert) }\end{array}$ \\
\hline 1 & 1 & $1 / 2$ & $1 / 2$ & $1 / 2$ & 0.500 \\
2 & 2 & $1 / 4$ & $1 / 2$ & $1 / 2$ & 1.000 \\
3 & 4 & $1 / 8$ & $1 / 2$ & $3 / 8$ & 1.375 \\
4 & 8 & $1 / 16$ & $1 / 2$ & $1 / 4$ & 1.625 \\
5 & 16 & $1 / 32$ & $1 / 2$ & $5 / 32$ & 1.781 \\
6 & 32 & $1 / 64$ & $1 / 2$ & $3 / 32$ & 1.875 \\
$\vdots$ & $\vdots$ & $\vdots$ & $\vdots$ & $\vdots$ & $\vdots$ \\
$\infty$ & $\infty$ & 0 & $1 / 2$ & 0 & 2 \\
\hline
\end{tabular}

Die obere Summationsgrenze in 1801 resultiert aus dem Umstand, daß die Anzahl theoretisch möglicher Gewinne unendlich ist. Der Erwartungswert ist also eine unendliche Summe. Jeder Summand ist ein Produkt, das jeweils 0.5 ergibt, da der Auszahlungsbetrag mit der gleichen Rate wächst, wie seine Wahrscheinlichkeit abnimmt: $a_{n} \cdot p_{n}=2^{(n-1)} \cdot(1 / 2)^{n}=\frac{2^{n}}{2} \cdot \frac{1}{2^{n}}=0.5($ vgl. Tabelle 100.1, Spalte 4). Damit läßt sich (180.1) auch schreiben als

$$
\mathrm{E} \quad=\sum_{n=1}^{\infty} 0,5 .
$$

Der Erwartungswert ist also unendlich. Wie anfangs erwähnt, ist dennoch kaum jemand bereit, für eine Teilnahme an diesem Spiel einen beträchtlichen Einsatz zu leisten. Aufgelöst werden kann dieses Paradox, wie JERGER (1992) beschreibt, wenn Risikoaversion der Spielteilnehmerinnen und -teilnehmer berücksichtigt wird.

Diese Risikoaspekte können jedoch im allgemeinen vernachlässigt werden, wenn Quasi-Sicherheit vorliegt. ${ }^{151}$ Damit ist gemeint, daß bei einer ausreichenden Anzahl von Wiederholungen davon ausgegangen werden kann, daß der Mittelwert aller Spiele

\footnotetext{
150 Vgl. SCHEID 1992, S. 1141.

151 Vgl. BRANDES \& WOERMANN 1982, S. 167; 176.
} 
nicht wesentlich vom Erwartungswert eines Spiels abweicht. Übertragen auf unsere Situation heißt dies, daß, wenn nicht nur einmal, sondern häufiger gespielt wird, ein höherer Einsatz pro Spiel lohnend erscheint. Reale Experimente mit diesem Spiel sind schwer durchführbar ("Wer soll das bezahlen, wer hat soviel Geld?"), mit einem Computer läßt sich die Spielsituation jedoch sehr leicht simulieren:

- Eine Münze wird solange geworfen, bis "Zahl" fällt;

- in Computersprache: es wird solange aus einer binären $(0,1)$-Gleichverteilung eine Zahl gezogen, bis die Ziehung eine Eins ergibt.

- Dann erfolgt die Auszahlung nach der Formel $a=2^{n-1}$ mit $n$ als der Anzahl der erfolgten Ziehungen.

- Die Auszahlung wird festgehalten und das Spiel wiederholt. Nach dem letzten Durchgang wird die Gesamtsumme der Auszahlungen durch die Anzahl der Wiederholungen geteilt.

- Das Ergebnis ergibt die durchschnittliche Auszahlung des Spiels. Dieser Betrag bildet bei Quasi-Sicherheit die Obergrenze eines Einsatzes.

In Tabelle 183,1 sind die Ergebnisse einer Simulation aufbereitet. Die durchschnittliche Auszahlung gibt risikoaversen Entscheidern recht, mit ihrem Einsatz zurückhaltend zu sein. Dem Gesetz der Quasi-Sicherheit folgend, wäre anzunehmen, daß mit der Anzahl der Wiederholungen die mittleren Auszahlungen ansteigen. Dies ist für dieses Beispiel nicht der Fall. Im Gegenteil, sie fallen sogar. Nun sind zehn Wiederholungen noch nicht sehr aussagekräftig. Daher wird das Programm jetzt mit steigenden Iterationen gestartet. Die Ergebnisse sind in Tabelle 182.1 zusammengefaßt (siehe auch Schaubild 183.1.

Aus ihnen geht hervor, daß mit zunehmender Anzahl der Wiederholungen die mittlere Auszahlung keinesfalls in dem Maße ansteigt, das den Regeln der Quasi-Sicherheit entspräche. Wie ist dieser Widerspruch zu erklären?

Stellen wir uns dazu der Einfachheit halber vor, dieses Spiel werde 8mal gespielt. Dann ist folgendes Ergebnis das mutmaßlichste [vgl. 179.2] und Tabelle 184.1]:

- 4mal fällt schon beim ersten Mal "Zahl"; die Auszahlung beträgt also jeweils eins.

- 2mal fällt erst beim zweiten Mal "Zahl"; die Auszahlung beträgt also jeweils zwei.

- 1 mal fällt erst beim dritten Mal "Zahl"; die Auszahlung beträgt also vier.

Im noch verbleibenden Fall ist die wahrscheinlichste Auszahlung acht. Jeder gröBere Betrag ist selbstverständlich möglich, aber weniger wahrscheinlich. Tabelle 184.1 und Schaubild 184.1 zeigen diese Realisation einer idealtypischen Stichprobe. ${ }^{152}$

152 Die idealtypische Verteilung von Stichproben, die nicht vom Umfang $2^{n}, n \in N$, sind, ist komplexer und wird hier nicht dargestellt. 
Diese theoretischen Überlegungen zur Verteilung der Auszahlungen werden durch Simulationen mit einer höheren Anzahl von Wiederholungen bestätigt, wie Tabelle 184.2 und Schaubild 1851 mit 128 Wiederholungen demonstrieren.

Tabelle 182.1: Mittel- und Maximalwert von Auszahlungen in Abhängigkeit von der Zahl der Spielwiederholungen

\begin{tabular}{|r|r|r|r|}
\hline $\begin{array}{c}\text { Zahl der } \\
\text { Wiederholungen }\end{array}$ & Mittelwert & \multicolumn{2}{|c|}{ Maximalwert } \\
als log. dualis*
\end{tabular}

* log. dualis: Logarithmus zur Basis 2 (ld) 
Tabelle 183.1: Ein Ergebnis für einzelne und mittlere Auszahlungen bei ein bis zehn Spielwiederholungen

\begin{tabular}{|c|c|c|c|}
\hline $\begin{array}{c}\text { Nummer der } \\
\text { Wiederholung }\end{array}$ & $\begin{array}{c}\text { Einzel- } \\
\text { auszahlung }\end{array}$ & $\begin{array}{c}\text { aggregierte } \\
\text { Auszahlung }\end{array}$ & $\begin{array}{c}\text { mittlere } \\
\text { Auszahlung }\end{array}$ \\
\hline 1 & 4 & 4 & 4.0 \\
2 & 1 & 5 & 2.5 \\
3 & 8 & 13 & 4.3 \\
4 & 1 & 14 & 3.5 \\
5 & 1 & 15 & 3.0 \\
6 & 1 & 16 & 2.7 \\
7 & 1 & 17 & 2.4 \\
8 & 1 & 18 & 2.3 \\
9 & 1 & 19 & 2.1 \\
10 & 2 & 21 & 2.1 \\
\hline \multicolumn{2}{|l|}{ durchschnittliche Auszahlung: } \\
\hline
\end{tabular}

Schaubild 183.1: Mittel- und Maximalwert in Abhängigkeit von der Zahl der Wiederholungen

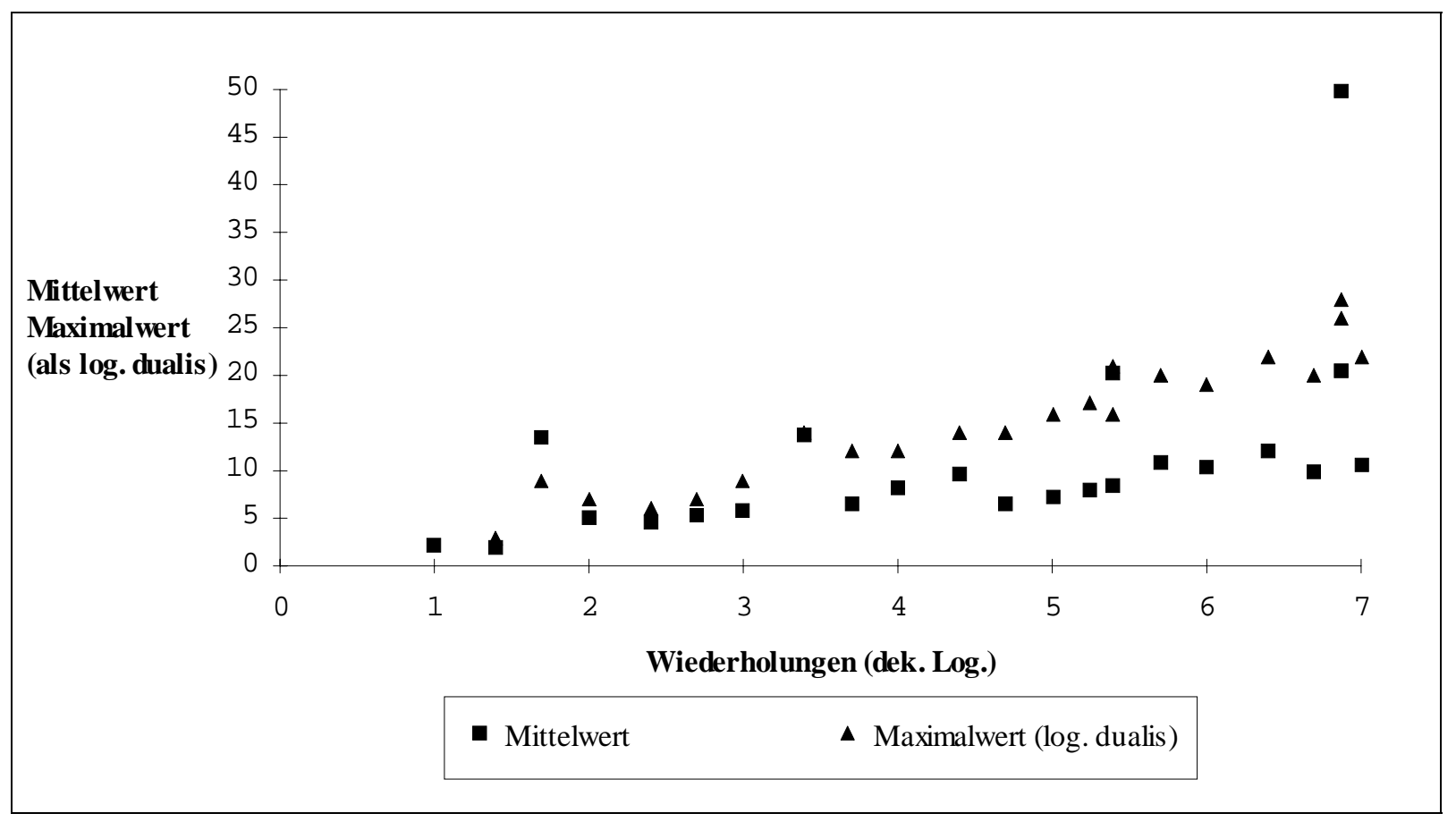


Tabelle 184.1: Idealtypische Verteilung der Auszahlungen bei acht Spielwiederholungen $(N=8)$

\begin{tabular}{|c|c|c|}
\hline$a_{n}$ & $p_{n}$ & $h_{N}^{*}$ \\
\hline 1 & $1 / 2$ & 4 \\
2 & $1 / 4$ & 2 \\
4 & $1 / 8$ & 1 \\
$?$ & $1 / 8$ & 1 \\
\hline
\end{tabular}

$h_{N}^{*}$ drückt aus, wie oft idealtypisch die Auszahlung $a_{n}$ bei der angegebenen Anzahl von Spielwiederholungen, hier: $N=8$, zu erwarten ist.

Schaubild 184.1: Auszahlungen einer idealtypischen Stichprobe aus acht Wiederholungen

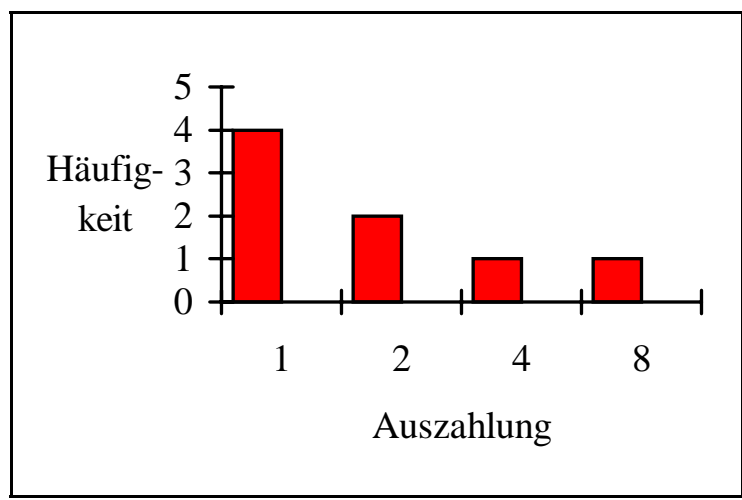

Tabelle 184.2: Verteilung der Auszahlungen bei 128 Wiederholungen $(N=128)$

\begin{tabular}{|c|c|c|c|c|c|}
\hline \multirow{2}{*}{$\begin{array}{l}\text { Einzel- } \\
\text { auszahlung }\end{array}$} & \multicolumn{2}{|c|}{ absolute Häufigkeit } & \multirow{2}{*}{$\begin{array}{l}\text { relative } \\
\text { Häufigkeit }\end{array}$} & \multicolumn{2}{|c|}{ kumulierte Häufigkeit } \\
\hline & tatsächliche & theoretische & & absolut & relativ \\
\hline 1 & 58 & 64 & 45.3 & 58 & 45.3 \\
\hline 2 & 34 & 32 & 26.6 & 92 & 71.9 \\
\hline 4 & 14 & 16 & 10.9 & 106 & 82.8 \\
\hline 8 & 10 & 8 & 7.8 & 116 & 90.6 \\
\hline 16 & 6 & 4 & 4.7 & 122 & 95.3 \\
\hline 32 & 4 & 2 & 3.1 & 126 & 98.4 \\
\hline 64 & 1 & 1 & 0.8 & 127 & 99.2 \\
\hline 128 & 0 & $1^{153}$ & $0^{-}$ & 127 & 99.2 \\
\hline 256 & 1 & 013 & 0.8 & 128 & 100 \\
\hline
\end{tabular}

153 Theoretisch ist genau eine Auszahlung größer als 64 zu erwarten, die wahrscheinlichste Auszahlung ist 128 und entspricht damit exakt dem Stichprobenumfang $N$. 


\section{Schaubild 185.1: Mutmaßliche versus realisierte Auszahlungen bei 128 Wiederholungen}

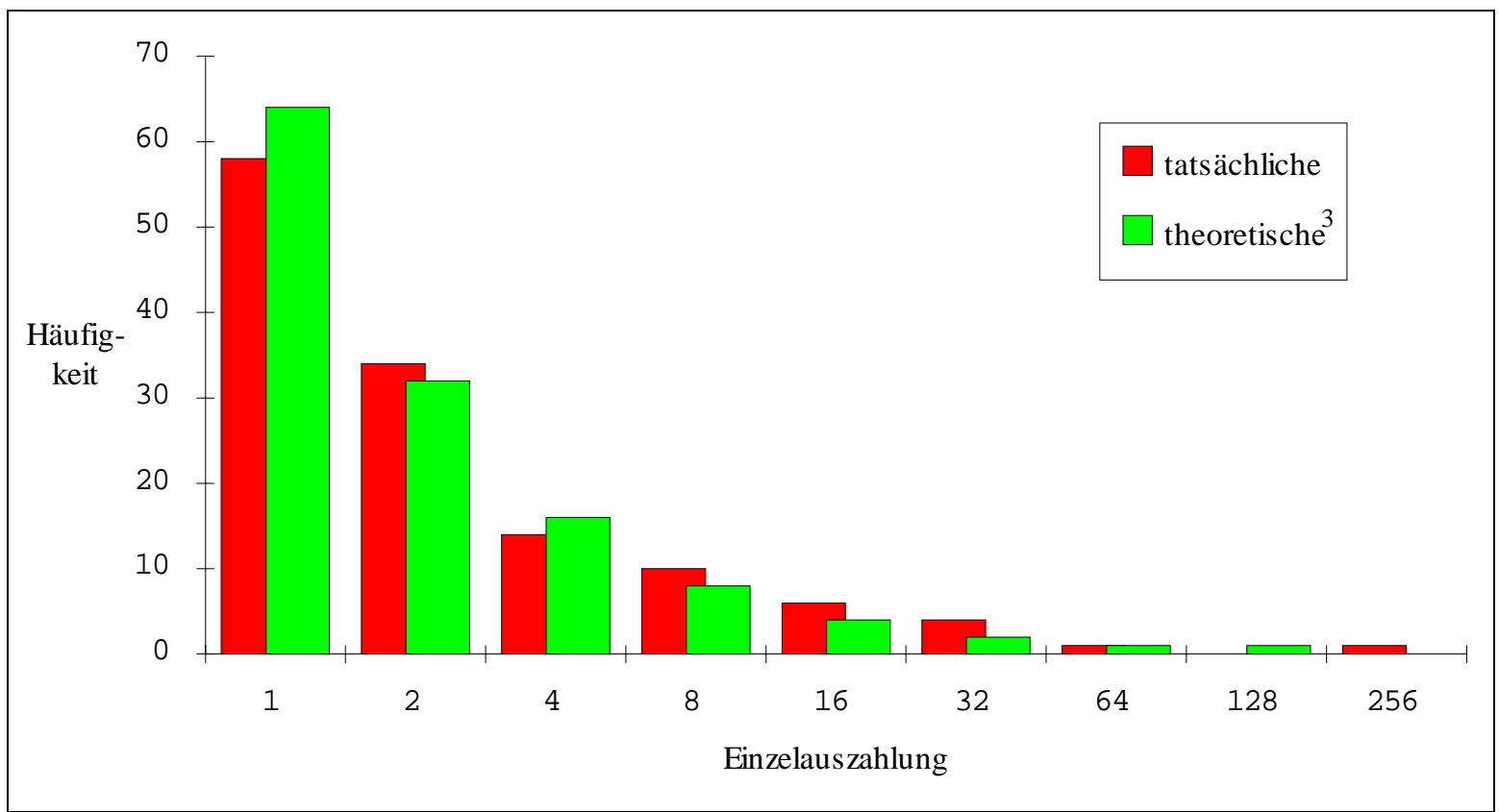

Die theoretisch zu erwartende Anzahl $h_{N}^{*}$ für jede Auszahlung, die den Wert 64 (oder allgemein die Hälfte des Stichprobenumfanges $N$ übersteigt) ist jeweils kleiner als 1. Präziser gesagt, aus der Gruppe der unendlich vielen Auszahlungsmöglichkeiten größer als N/2 ist bei einer (endlichen) Stichprobe insgesamt genau eine Realisation zu erwarten. ${ }^{154}$ Das wahrscheinlichste Ergebnis aus dieser Gruppe ist $N$. Damit ist der mutmaßliche Mittelwert einer Stichprobe nicht unendlich, sondern eine Funktion des Stichprobenumfanges $N$ : Die der Größe nach sortierten Auszahlungsmöglichkeiten werden, gewichtet mit ihren Eintrittswahrscheinlichkeiten, solange aufsummiert, wie ihre theoretische Anzahl in der Stichprobe mindestens eins beträgt. Jetzt fehlt noch genau eine Auszahlung. Diese ist die eben erwähnte unbestimmte Auszahlung größer als N/2. Sie werde als $a_{\text {last }}$ bezeichnet. Ihr Anteil ist 1/N. Für den Mittelwert einer idealtypischen Stichprobe ergibt sich damit:

$$
\overline{\mathrm{X}}_{\text {Stichprobe }}(N)=\left(\sum_{n=1}^{p\left(a_{n}\right) \cdot N<1} a_{n} \cdot p\left(a_{n}\right)\right)+\frac{a_{\text {last }}}{N} .
$$

Der Erwartungswert des ersten Summanden aus (185.1) ist endlich, für $a_{\text {last }}$ und $a_{\text {last }} / N$ hingegen ist er ebenso wie für das gesamte Spiel unendlich. Damit ist auch der Erwartungswert des Stichprobenmittels unendlich, wobei diese Eigenschaft einzig durch den Erwartungswert einer einzigen Auszahlungsmöglichkeit, nämlich der Auszahlungsmöglichkeit $a_{\text {last }}$, hervorgerufen wird. Da aber bei einer endlichen Stichprobe

154 Exakt gilt diese Aussage nur für Stichprobenumfänge, die eine natürliche Potenz von zwei sind (vgl. Fußnote 152). 
aus der unendlichen Menge an Möglichkeiten für $a_{\text {last }}$, hier: $\{128,256,512, \ldots\}=\{N$. $\left.2^{0}, N \cdot 2^{1}, N \cdot 2^{2}, \ldots\right\}$, im Mittel nur eine einzige Auszahlung realisiert wird und sich die Wahrscheinlichkeiten von einer Realisationsmöglichkeit zur nächst größeren jeweils halbieren, bleibt der Mittelwert der simulierten Stichproben stets in einem sehr kleinen Bereich, auch wenn mehr als eine Million Spielwiederholungen durchgeführt werden (vgl. Tabelle 183.1).

Unter Verwendung von (180.1) und 180.2 ergibt sich aus 185.1):

$$
\bar{x}_{\text {Stichprobe }}^{*}(N)=\left(\sum_{n=1}^{0,5^{n} \cdot N<1} 0.5\right)+\frac{a_{\text {last }}}{N} .
$$

Die obere Summationsgrenze kann nach $n$ aufgelöst werden:

$$
\begin{aligned}
& 0.5^{n} \cdot N<1< \\
& n<\frac{\log (N)}{\log (2)} .
\end{aligned}
$$

Es wird also solange aufsummiert, wie $n$ kleiner ist als $\frac{\log (N)}{\log (2)}$. Die Summationsobergrenze lautet daher

$$
\operatorname{int}\left(\frac{\log (N)}{\log (2)}\right) \text {. }
$$

Für $N=128$ ergibt sich für $n$ der Wert 7. Mit Hilfe von 1862 vereinfacht sich (186.1) $\mathrm{zu}$

$$
\bar{x}_{\text {Stichprobe }}^{*}(N)=\operatorname{int}\left(\frac{\log (N)}{\log (2)}\right) \cdot 0.5+\frac{a_{\text {last }}}{N} \text {. }
$$

Diese Formel soll nun auf die Stichprobenmittel der Tabelle 182.1 angewendet werden, um eine Vorstellung von der Größenordnung von $\mathrm{a}_{\text {last }} \mathrm{zu}$ erhalten. Der Schätzwert $\widetilde{a}_{\text {last }} / N$ wird einfach als Differenz von empirischen Stichprobenmittel und der ersten Summanden aus (186.3) bestimmt.

In Tabelle 187.1 ist zu beachten, daß der jeweils in der vierten Spalte notierte Wert nicht allein die idealtypisch eine Auszahlung größer als N/2 repräsentiert, also $a_{\text {last }}$, sondern zusätzlich auch die Abweichungen in den Häufigkeiten aller anderen Auszahlungen von ihren idealtypischen Werten beinhaltet. Daher ist die Bezeichnung um eine Tilde ergänzt worden: $\widetilde{a}_{\text {last }} / N$. Die Tabelle zeigt, daß mit $\widetilde{a}_{\text {last }}=3.9 \cdot N$ eine gute Übereinstimmung zwischen den hier empirisch gefundenen und nach (186.4) berechneten Werten erzielt wird. Bleiben jedoch die aus dem Rahmen fallenden Szenarien mit Mittelwerten größer als 20 unberücksichtigt, wird die beste Übereinstimmung mit $\tilde{a}_{\text {last }}=1.5 \cdot N$ erreicht. 
Tabelle 187.1: Empirische und theoretische Stichprobenmittel

\begin{tabular}{|c|c|c|c|}
\hline $\begin{array}{c}\text { Anzahl } \\
\text { Wiederholungen } \\
N\end{array}$ & $\begin{array}{l}\text { empirisches } \\
\text { Stichproben- } \\
\text { mittel }\end{array}$ & $\begin{array}{c}\text { erster } \\
\text { Summand aus } \\
11863\end{array}$ & $\tilde{a}_{\text {last }} / N$ \\
\hline 10 & 2.1 & 1.5 & 0.6 \\
\hline 25 & 2.0 & 2.0 & 0.0 \\
\hline 50 & 13.5 & 2.5 & 11.0 \\
\hline 100 & 5.1 & 3.0 & 2.1 \\
\hline 250 & 4.5 & 3.5 & 1.0 \\
\hline 500 & 5.3 & 4.0 & 1.3 \\
\hline 1000 & 5.8 & 4.5 & 1.3 \\
\hline 2500 & 13.7 & 5.5 & 8.2 \\
\hline 5000 & 6.5 & 6.0 & 0.5 \\
\hline 10000 & 8.1 & 6.5 & 1.6 \\
\hline 25000 & 9.6 & 7.0 & 2.6 \\
\hline 50000 & 6.6 & 7.5 & -0.9 \\
\hline 100000 & 7.3 & 8.0 & -0.7 \\
\hline 175000 & 8.0 & 8.5 & -0.5 \\
\hline 250000 & 8.0 & 8.5 & -0.5 \\
\hline 250000 & 20.2 & 8.5 & 11.7 \\
\hline 500000 & 10.8 & 9.0 & 1.8 \\
\hline 1000000 & 10.4 & 9.5 & 0.9 \\
\hline 2500000 & 12.1 & 10.5 & 1.6 \\
\hline 5000000 & 9.9 & 11.0 & -1.1 \\
\hline 7500000 & 49.8 & 11.0 & 38.8 \\
\hline 7500000 & 20.6 & 11.0 & 9.6 \\
\hline \multirow[t]{2}{*}{10000000} & 10.7 & 11.5 & -0.8 \\
\hline & & Mittelwert & 3.9 \\
\hline
\end{tabular}

Die Ergebnisse der Tabellen 1831 und 1871 geben noch einen weiteren interessanten Aufschluß. Sie zeigen, daß für Verteilungen, wie sie in den Schaubildern 184,1 und 1851 dargestellt sind, das arithmetische Mittel x ein sehr schlechter Schätzer des Erwartungswerts der Grundgesamtheit ist. Es ist zum einem in hohem Maße verzerrt. Zum zweiten ist seine Variabilität sehr hoch, wie in der Tabelle exemplarisch für die Stichprobenumfänge 250000 und 7500000 dargestellt. Die Abweichung der beiden Stichprobenmittel voneinander um mehr als $100 \%$ wiegt um so schwerer, als in praktischen ökonometrischen Fragestellungen ein so hoher Stichprobenumfang selten zur Verfügung steht. 


\section{Zusammenfassung}

Das dem bekannten Petersburger Paradoxon zugrunde liegende Spiel ist durch einen unendlichen Erwartungswert gekennzeichnet. Die Annahme, daß den Regeln der Quasi-Sicherheit folgend bei einer großen Anzahl von Wiederholungen des Spiels deren Mittelwert sich in die Nähe des theoretischen Erwartungswertes bewegt, konnte mit Hilfe von einfachen Computersimulationen nicht bestätigt werden. Beispielsweise liegt die mittlere Auszahlung bei 20 Spielwiederholungen in einem Bereich, der auch der empirisch gefundenen Zahlungsbereitschaft von risikoaversen Entscheidern entspricht. Auch bei millionenfacher Wiederholung sind Einsätze größer als 20 pro Spiel nicht rentabel. Den Ergebnissen der Simulationen zufolge sind geringe (einstellige) Einsätze beim Petersburger Paradox also auch für risikoneutrale Entscheider rational.

Als zweites ist festzuhalten, daß das arithmetische Mittel als Schätzer für den Erwartungswert für Verteilungen wie der, der die Auszahlungen bei diesem Spiel gehorchen, sehr ungeeignet ist, da es sowohl einen hohen Bias wie eine große Varianz aufweist. 


\section{Beispiele einfacher Überlebensmodelle}

5.1 Allgemeine Systeme mit einer (stochastischen) Einzahlung ....................... 192

5.2 Markovketten als Instrument zur Berechnung von Uberlebenswahrscheinlichkeiten bei diskreten Einzahlungen...................214

$5.3 \quad$ Konkrete Beispiele mit einer stochastischen Einzahlung ..........................218

5.3.1 Additive Verknüpfung zwischen Kapital und Einzahlung ...............219

5.3.1.1 Ganzzahlige Einzahlungen zwischen -2 und $+2 \ldots \ldots \ldots \ldots \ldots .219$

5.3.1.2 Ganzzahlige gleichverteilte Einzahlungen zwischen -1

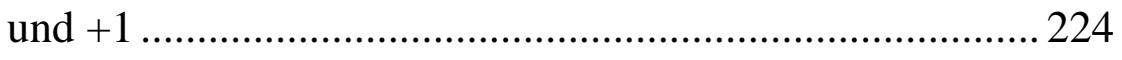

5.3.1.3 Diskrete Gleichverteilung allgemein .............................228

5.3.1.4 Stetige Verteilungen ................................................ 230

5.3.1.5 Monte-Carlo-Simulationsergebnisse für die Überlebenswahrscheinlichkeit bis zur zweiten Runde ......236

5.3.1.6 Überlebenswahrscheinlichkeit für mehr als zwei

Perioden ........................................................ 239

5.3.2 Multiplikative Verknüpfung zwischen Kapital und Einzahlung ......249

5.4 Modelle mit stochastischer und fester Einzahlung (Portfolio-Modelle).....258

5.5 Beispiele eines einfachen stochastischen Betriebsentwicklungsmodells ....261

5.6 Uberlebensaspekte im Planspiel „Puten und Perlhühner“...........................265

In diesem Kapitel werden einfache dynamische, mathematisch-ökonomische Systeme betrachtet. Sie sind durch einen Kapitalbestand und periodische Einzahlungen gekennzeichnet und unterscheiden sich darin, ob bzw. wie

- das Kapital in die nächste Periode transferiert wird,

- der Erwartungswert der Einzahlung über die Zeit konstant ist,

- die Höhe der Einzahlung vom Kapitalbestand in der Vorperiode abhängt,

- neben der stochastischen Einzahlung eine feste Einzahlungsalternative besteht,

- die Einzahlung in beliebiger Stückelung erfolgen kann,

- Startkapital vorhanden ist, 
- Auszahlungen erfolgen und ob

- ein kontinuierlicher oder ein diskreter Zeitverlauf betrachtet wird.

In Übersicht 1901 sind einige dieser Klassen formal näher beschrieben. Nach der Begriffsbestimmung von ZWICKER (1981, Kap. 1.2.1) handelt es sich dabei ausschließlich um metrisch dynamische Funktionsmodelle, da alle Variablen metrisch skaliert sind und sich ihre Beziehungen untereinander in Form von Gleichungen mit zeitverzögerten Variablen darstellen lassen.

Übersicht 190.1: Einteilung metrisch dynamischer Systeme

\begin{tabular}{|c|c|c|c|c|}
\hline Charakteristikum & formale & Ausp & ungen & $*$ \\
\hline $\begin{array}{l}\text { Einfluß der } \\
\text { Vorperiode }\end{array}$ & $\mathrm{E} \frac{\mathrm{d}\left[K_{t}-K_{t-1}\right]}{\mathrm{d} K_{t-1}}$ & 0 & $\neq 0$ & $\mathrm{a}, \mathrm{c}$ \\
\hline Verknüpfung & $K_{t-1} \circ z_{t}$ & additiv & multiplikativ & $\mathrm{c}$ \\
\hline Startkapital & $K_{0}$ & 0 & $\neq 0$ & $\mathrm{f}$ \\
\hline $\begin{array}{l}\text { Verteilungstyp der } \\
\text { Einzahlungen }\end{array}$ & $\mathrm{F}(z)$ & $\begin{array}{c}\text { diskret } \\
\text { (Markovkette) }\end{array}$ & $\begin{array}{c}\text { stetig } \\
\text { (Markovprozeß) }\end{array}$ & $\mathrm{e}$ \\
\hline $\begin{array}{l}\text { Trend in den } \\
\text { Einzahlungen }\end{array}$ & $\mathrm{E}\left(z_{t}\right)$ & neutral & $\neq$ neutral & $\mathrm{b}$ \\
\hline $\begin{array}{l}\text { Autokorrelation } \\
\text { der Einzahlungen }\end{array}$ & $\mathrm{E}\left(z_{i} z_{j}\right)$ & 0 & $\neq 0$ & - \\
\hline Zeitverlauf & $t$ & diskret & stetig & $\mathrm{h}$ \\
\hline $\begin{array}{l}\text { Für alle Systeme gilt: } \\
\left(K_{t-1}\right) \text {, der stochastische } \\
\text { * Die Buchstaben die } \\
\text { ökonomischer Mode }\end{array}$ & $\begin{array}{l}\text { Kapital in der Pel } \\
\text { inzahlung in } t\left(z_{t}\right) \\
\text { Spalte beziehen si }\end{array}$ & $\begin{array}{l}t\left(K_{t}\right) \text { ist eine Funk } \\
\text { er konstanten Paran } \\
\text { fie oberhalb im T }\end{array}$ & $\begin{array}{l}\text { des Kapitals in der } \\
(\theta): K_{t}=\mathrm{f}\left(K_{t-1}, z_{t},\right. \\
\text { etroffene Untersche }\end{array}$ & rstufe \\
\hline
\end{tabular}

Die Eigenschaften der Systeme werden insbesondere durch die Verteilung der stochastischen Einzahlungen $z_{t}$ bestimmt. Wird ein diskreter Zeitablauf betrachtet, bilden die Einzahlungen einen stochastischen Prozeß. Einzahlungen, die den Erwartungswert des Kapitals nicht verändern, werden als neutrale Einzahlungen bezeichnet. In einem additiven Modell besitzen neutrale Einzahlung daher einen Erwartungswert von Null, während ihr Erwartungswert im Falle einer multiplikativen Verknüpfung Eins betragen muß. Folgen alle Einzahlungen in der Zeit dem selben Verteilungsgesetz und sind sie zusätzlich stochastisch unabhängig, bezeichnet man diese Eigenschaft als iid. ${ }^{155}$ Ein stochastischer Prozeß, der identisch und unabhängig um Null verteilt ist, wird ,weißes Rauschen' genannt (white noise, CHATFIELD 1996, S. 32).

155 iid-Eigenschaft und neutrale Einzahlungen wurden in Abschnitt 4.1 bereits kurz angesprochen. 
Systeme mit stetiger Zeitachse sind unter den Bezeichnungen Wiener-Prozesse und Black/Scholes-Formel bekannt ${ }^{156}$ und können als Differentialgleichungen abgebildet werden. Da reale sozioökonomische Systeme sich in der Regel besser und einfacher durch zeitdiskrete äquidistante Modelle repräsentieren lassen, werden Differentialgleichungen in dieser Arbeit nicht untersucht. ${ }^{157}$ Ein diskreter Zeitverlauf bedeutet, daß die dynamischen Variablen nur zu Beginn (oder Ende) einer Periode eine Veränderung erfahren und zwischen Anfang und Ende einer Periode konstant sind. Dies entspricht der ökonomischen Realität, denn Preise und Löhne z.B. werden auch in Zeiten von Hyperinflation nicht kontinuierlich verändert. Diese Arbeit beschäftigt sich ebenfalls nicht mit Modellen, in die nichtstationäre oder autokorrelierte Einzahlungen eingehen, oder mit Modellen mit mehr als einer endogenen Variablen.

In den folgenden fünf Abschnitten werden Modelle beschrieben, die sich als einfache Differenzengleichungen ersten Grades darstellen lassen, deren prädeterminierte Variablen also nur eine Periode zurückreichen. In der Regel reicht eine Gleichung, wenn man von der Definition der Startwerte absieht, aus, das System hinreichend zu beschreiben. Die Modelle sind daher eindimensional. Die endogene Variable wird als Kapitalbestand interpretiert. Er wird von bis zu drei Zahlungen beeinflußt, von denen genau eine Einzahlung stochastisch ist. Diese Zahlung kann entweder nur eine endliche Anzahl von Zuständen annehmen (diskretes Modell), oder die Zustandsstufen sind stetig.

Die Betrachtung der genannten Modelle fokussiert auf die Überlebenswahrscheinlichkeit. Darin unterscheidet sie sich von anderen Arbeiten, in denen vorrangig Erwartungswerte und Varianzen von Differenzengleichungen untersucht werden. Aus diesem Grund kann für die vorgestellten Ableitungen nur selten auf vergleichbaren Ergebnissen aus der Literatur aufgebaut werden.

Im ersten Abschnitt erfolgt eine wahrscheinlichkeitstheoretische Analyse von Modellen, die sich als Differenzengleichungen erster Ordung darstellen lassen. Sie sind durch einen Kapitalbestand gekennzeichnet, der durch eine stochastische Ein- und eine feste Auszahlung verändert wird. Daher ist auch das Kapital eine Zufallsgröße. Die Parameter sind so gewählt, daß für das System in der Regel keine Überlebensgarantie besteht. Der zweite Abschnitt beschreibt, wie für Modelle dieser Art die Technik der Markovketten eingesetzt werden kann, um Überlebenswahrscheinlichkeiten numerisch zu berechnen. Die Methode setzt diskret verteilte Einzahlungen voraus. Im dritten Abschnitt schließt sich eine Erörterung konkreter Beispiele an. In den folgenden Abschnitten wird das Modell schrittweise erweitert. Das Modell in Abschnitt 5.4 besitzt neben der stochastischen auch eine feste Einzahlungsmöglichkeit, so daß sich dem Investor die Frage nach dem optimalen Portfoliomix stellt. Im dann folgenden Abschnitt schließlich wird ein einfaches Betriebsentwicklungsmodell

156 Vgl. KRUSCHWITZ 1995 Kap. 8.4.

157 ZWICKER (1981) nennt in Kapitel 1.2.2 noch weitere Vorzüge zeitdiskreter äquidistanter Modelle, wie Vorteile bei der stochastischen Simulation (S. 31) und der graphischen Darstellung mit Hilfe von Pfeildiagrammen (S. 30). 
vorgestellt und die Auswirkungen unterschiedlicher Fremdkapitalaufnahme auf die Überlebenswahrscheinlichkeit untersucht. Den Abschluß des Kapitels bildet eine Diskussion eines Unternehmensplanspiels unter Überlebensgesichtspunkten.

\subsection{Allgemeine Systeme mit einer (stochastischen) Einzahlung}

In diesem und den folgenden drei Abschnitten werden dynamische Systeme erster Ordnung mit einer endogenen Variablen und zwei exogenen Variablen vorgestellt. Die endogene Variable wird mit $K$ bezeichnet und die exogenen Variablen mit $z$ und $a$, wobei $z$ stochastischer Natur und $a$ eine Konstante ist. $K_{t}$ wird von ihrem Vorperiodenwert, der Zufallsvariablen $z_{t}$ und $a$ bestimmt

$$
K_{t} \quad=f\left(K_{t-1}, z_{t}, a\right),
$$

und ist daher ebenfalls eine stochastische Variable. Die Variable $K$ kann als Kapitalbestand, die Zufallsveränderliche $z$ als stochastische Ein- und die Konstante $a$ als feste Auszahlung interpretiert werden. Im Zuge der analytischen Behandlung des Systems interessiert z.B. die Frage, mit welcher Wahrscheinlichkeit sich das Kapital in einer bestimmten Runde größer als Null realisiert. Das Problem ist mathematisch relativ einfach handhabbar, solange die Einzahlungen stochastisch unabhängig sind.

Die Untersuchung erfolgt in drei Schritten. Zunächst werden Modelle analysiert, in denen das Vorperiodenkapital den aktuellen Kapitalbestand nicht beeinflußt. Anschließend werden Modelle vorgestellt, in denen Vorperiodenkapital und stochastische Einzahlung additiv oder multiplikativ verknüpft sind. Additiv bedeutet, daß zum Vorperiodenkapital eine stochastische Einzahlung addiert wird, und multiplikativ, daß das Vorperiodenkapital mit einer Zufallszahl multipliziert wird. Letzteres ist als stochastische ökonomische Verzinsung interpretierbar. Jedes System gliedert sich in zwei Teile, in Modelle ohne und mit fester Auszahlung.

Wir beginnen mit einem Modell, das keine Auszahlung enthalte. Das Kapital könne nicht in die nächste Runde transferiert werden. Der Kapitalbestand einer Runde sei ausschließlich von der Einzahlung in der selben Runde abhängig. Daher ist ein eventuelles Startkapital ohne Belang. Der Erwartungswert der Einzahlung sei zeitpunktinvariant für alle Runden gleich Null:

$$
K_{t}=z_{t}, \mathrm{E}\left(z_{t}\right)=0 \quad \forall t .
$$

Der Ruinwahrscheinlichkeit in der ersten Runde entspricht in diesem einfachen Modell der Wert der Verteilungsfunktion von $z_{1}$ an der Stelle Null:

$$
\mathrm{p}\left(K_{1} \leq 0\right) \quad=\mathrm{p}\left(z_{1} \leq 0\right)=F_{z_{1}}(0)
$$

und der Überlebenswahrscheinlichkeit das Gegenereignis:

$$
\mathrm{p}\left(K_{1}>0\right) \quad=1-F_{z_{1}}(0) .
$$


Außer der ersten auch die zweite Runde zu überleben, bedeutet zum einen, daß die Einzahlung in der zweiten Runde größer als Null ausfällt und zum anderen, daß diese Runde überhaupt erreicht wurde. Diese Wahrscheinlichkeit wird durch den Index „ül““, gefolgt von der Anzahl der überlebten Perioden in Klammern ausgedrückt:

$$
\begin{aligned}
\mathrm{p}_{\text {ül }}(2) & =\mathrm{p}\left(K_{1}>0 \wedge K_{2}>0\right) \\
& =\mathrm{p}\left(z_{1}>0 \wedge z_{2}>0\right) .
\end{aligned}
$$

Die Höhe der Einzahlung in der zweiten Periode hänge in keiner Weise von der realisierten Einzahlung der ersten Runde ab, die beiden Einzahlungen seien stochastisch unabhängig. Daher kann die gemeinsame Wahrscheinlichkeit durch Multiplikation der Einzelwahrscheinlichkeiten bestimmt werden:

$$
\begin{aligned}
\mathrm{p}_{\text {ül }}(2) & =\mathrm{p}\left(z_{1}>0\right) \cdot \mathrm{p}\left(z_{2}>0\right) \\
& =\left[1-F_{z_{1}}(0)\right] \cdot\left[1-F_{z_{2}}(0)\right] .
\end{aligned}
$$

Für die $t$. Runde gilt entsprechend:

$$
\mathrm{p}_{\mathrm{uil}}(t) \quad=\prod_{i=1}^{t} \mathrm{p}\left(z_{i}>0\right)=\prod_{i=1}^{t}\left[1-F_{z_{i}}(0)\right] \text {. }
$$

Wenn die stochastischen Einzahlungen in den einzelnen Perioden nicht nur unabhängig, sondern auch identisch verteilt sind (iid), also $\mathrm{F}\left(\mathrm{z}_{i}\right)=\mathrm{F}\left(\mathrm{z}_{j}\right)=\mathrm{F}(\mathrm{z}) \forall i, j$, dann vereinfacht sich obige Gleichung zu: $\mathrm{p}_{\mathrm{uul}}(t)=\left[1-F_{z}(0)\right]^{t}$ oder zu pül $(t)=\left[\mathrm{p}_{\mathrm{uul}}(1)\right]^{t}$, da $1-$ $F_{z}(0)$ die Überlebenswahrscheinlichkeit der ersten Periode beschreibt. Diese Exponentialfunktion \#\# ist monoton fallend, da $F_{z}(0)$ konstant ist und zwischen Null und 1 liegt. Die Funktion konvergiert gegen Null. Die Überlebenswahrscheinlichkeit nimmt daher mit jeder zusätzlichen Runde ab und nähert sich Null.

Für symmetrische Verteilungsfunktionen (mit Erwartungswert Null) ist $F_{z}(0)$ gleich $1 / 2$, die Überlebenswahrscheinlichkeit in der i-ten Periode somit $1 / 2^{t}$. Schaubild 194,1 stilisiert diesen Zusammenhang.

Wir ergänzen das Modell nun um eine feste, zeitinvariante Auszahlung:

$$
K_{t}=z_{t}-a, \mathrm{E}\left(z_{t}\right)=0 \quad \forall t ; a>0 .
$$

Überleben in der ersten Runde bedeutet, daß die stochastische Einzahlung größer ausfällt als die Auszahlung.

$$
\begin{aligned}
\mathrm{p}\left(K_{1}>0\right) & =\mathrm{p}\left(z_{1}-a>0\right)=\mathrm{p}\left(z_{1}>a\right) . \\
& =1-F_{z_{1}}(a) .
\end{aligned}
$$

Die Überlebensbedingung für zwei Runden:

$$
\mathrm{p}_{\text {ül }}(2) \quad=\mathrm{p}\left(K_{1}>0 \wedge K_{2}>0\right)
$$

ist erfüllt durch:

$$
\mathrm{p}_{\text {ül }}(2) \quad=\mathrm{p}\left(z_{1}>a \wedge z_{2}>a\right) .
$$




\section{Schaubild 194.1: Zeitliche Entwicklung der Überlebenswahrscheinlichkeit für symmetrisch verteilte Einzahlungen}

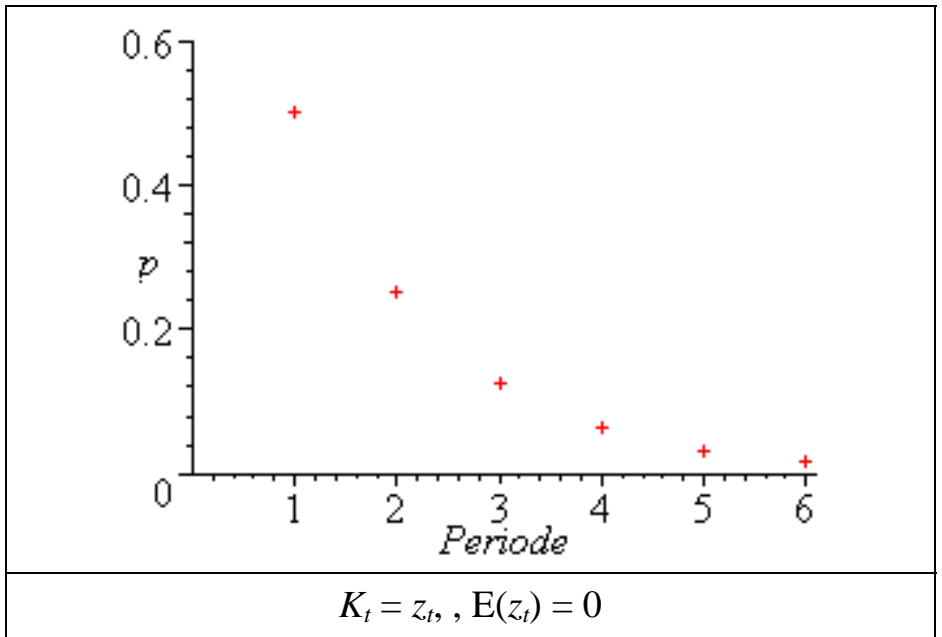

Beide Ereignisse sind annahmegemäß stochastisch unabhängig. Daher kann die gemeinsame Wahrscheinlichkeit wie in den vorigen Beispielen durch Multiplikation der Einzelwahrscheinlichkeiten bestimmt werden:

$$
\begin{aligned}
\mathrm{p}_{\text {ül }}(2) & =\mathrm{p}\left(z_{1}>a\right) \cdot \mathrm{p}\left(z_{2}>a\right) \\
& =\left[1-F_{z_{1}}(a)\right] \cdot\left[1-F_{z_{2}}(a)\right] .
\end{aligned}
$$

Für die $t$. Runde gilt entsprechend:

$$
\mathrm{p}_{\mathrm{uil}}(t) \quad=\prod_{i=1}^{t} \mathrm{p}\left(z_{i}>a\right)=\prod_{i=1}^{t}\left[1-F_{z_{i}}(a)\right]
$$

Für iid-Einzahlungen vereinfacht sich die Gleichung zu: $\mathrm{p}_{\mathrm{ül}}(t)=\left[1-F_{z}(a)\right]^{t}=$ $\left[\mathrm{p}_{\mathrm{uil}}(1)\right]^{t}$. Da die Basis dieser Exponentialfunktion \#\# kleiner ist als in dem Modell ohne Auszahlung, ist die Überlebenswahrscheinlichkeit, wie erwartet, in jeder Runde kleiner und konvergiert rascher gegen Null.

Wir gehen nun zu Modellen über, in denen das Kapital in die nächste Runde übertragen werden kann. Diese Systeme sind realitätsnäher und mathematisch anspruchsvoller. Zwei Fälle werden unterschieden:

- Die Einzahlung ist wie im vorigen Modell autonom, wird also von keinen Vorereignissen beeinflußt.

- die Einzahlung hängt vom Kapital der Vorperiode ab. ${ }^{158}$

158 Die Realisation der Zufallsvariable „Einzahlung in $t^{“}$ wird also von der Ausprägung einer anderen Zufallsvariablen, hier „Einzahlung in $t-1$ “ beeinflußt. Aufgrund der zeitlichen Struktur ist nur eine Beeinflussung in eine Richtung möglich. Wegen der fehlenden Rückkoppelung liegt daher keine 
Zum ersten Fall. Das Kapital in Runde $t$ sei definiert als

$$
K_{t} \quad \equiv K_{t-1}+z_{t}-a .
$$

Das Kapital ist also additiv mit dem Vorperiodenkapital verknüpft. Bilden die Zufallsvariablen $z_{t}$ einen stochastischen Prozeß, der die Anforderungen eines weißen Rauschens erfüllt, liegt ein random walk Modell vor. Der Wachstumsparameter ist hier negativ und entspricht dem Negativum der Auszahlung (vgl. Unterabschnitt 2.3.1.1.2].

Zunächst betrage sowohl das Anfangskapital $K_{0}$ als auch die Auszahlung Null. Dann ist die Wahrscheinlichkeit, schon in der ersten Runde zu fallieren, wie im ersten Modell (s. S. 192 gleichbedeutend mit der Wahrscheinlichkeit einer negativen Einzahlung:

$$
\begin{array}{ll}
K_{1} & =K_{0}+z_{1}=z_{1} \wedge K_{0}=a=0 \\
\mathrm{p}\left(K_{1} \leq 0\right) & =\mathrm{p}\left(z_{1} \leq 0\right)=\mathrm{F}_{z 1}(0),
\end{array}
$$

und für die Überlebenswahrscheinlichkeit gilt entsprechend:

$$
\mathrm{p}_{\text {ül }}(1) \quad=\mathrm{p}\left(K_{1}>0\right)=1-\mathrm{F}_{z 1}(0) .
$$

Sind die Einzahlungen symmetrisch, stetig und neutral verteilt, gilt $\mathrm{F}(0)=\mathrm{F}(\mu)=1 / 2$, so daß die Überlebenswahrscheinlichkeit in der ersten Runde unter diesen drei Bedingungen $50 \%$ beträgt.

Für gleichverteilte, ganzzahlige Einzahlungen zwischen -1 und 1 lautet die Wahrscheinlichkeit einer positiven Einzahlung, d.h. einer Einzahlung größer als Null, in der ersten Runde $1 / 3$. Wenn die Spanne der möglichen Einzahlungen größer ist, z.B. $z_{1}$ uiid $\{-2,-1,0,1,2\}$, erhöht sich auch die Überlebenswahrscheinlichkeit, wie in Tabelle 1961 dargestellt. Bezeichnen wir die Anzahl der positiven Realisationsmöglichkeiten von $z_{1}$ mit $k$, so gilt:

$$
\mathrm{p}_{\mathrm{ül}, k}(1) \quad=\mathrm{p}\left(z_{1}>0\right)=\frac{k}{2 k+1} \text {. }
$$

Der Ausdruck konvergiert für eine zunehmende Spanne der Verteilung der Einzahlung gegen 1/2 (s. Schaubild 2291]. Der Verlauf der Graphik beruht auf der gewählten Formulierung für die Überlebenswahrscheinlichkeit: das Kapital muß größer als Null sein. Gleichung 195,1] ist mathematisch interessanter als ökonomisch. Wäre Überleben so definiert, daß ein Kapital von Null zum Überleben ausreichte, betrüge die Wahrscheinlichkeit hierfür in der ersten Runde statt $1 / 3$ das Doppelte davon und würde sich mit zunehmendem $k$ von oben statt von unten dem Wert $1 / 2$ nähern.

Kovarianz vor, sondern in der Notation von FöRSTNER, BAMBERG \& HENN „ein zusammengesetztes Zufallsexperiment“"(1973, S. 135). 
Tabelle 196.1: Überlebenswahrscheinlichkeit in der ersten Runde für diskrete Gleichverteilung mit $\boldsymbol{k}$ Elementen, $K_{0}=0$

\begin{tabular}{|c|c|c|}
\hline Z & $\boldsymbol{k}$ & $\begin{array}{c}\text { Überlebens- } \\
\text { wahrscheinlichkeit }^{159}\end{array}$ \\
\hline$\{-1,0,1\}$ & 2 & $1 / 3$ \\
\hline$\{-2,-1,0,1,2\}$ & 3 & $2 / 5$ \\
\hline$\{-3,-2,-1,0,1,2,3\}$ & 4 & $3 / 7$ \\
\hline$\{-k-1,-k-2, \ldots, k-2, k-1\}$ & $k$ & $\frac{k}{2 k+1}$ \\
\hline$\{-\infty, \ldots, \infty\}$ & $\infty$ & $1 / 2$ \\
\hline \multicolumn{3}{|c|}{$K_{1}=z_{1}, z_{i} \in \mathbf{Z}$} \\
\hline
\end{tabular}

In der zweiten Runde besteht das Kapital aus den beiden bisherigen Einzahlungen:

$$
K_{2}=K_{1}+z_{2}=z_{1}+z_{2} \text {. }
$$

Für die Überlebenswahrscheinlichkeit gilt nach (152.1):

$$
\begin{aligned}
\mathrm{p}_{\mathrm{uil}}\left(K_{2}>0\right) & \equiv \mathrm{p}\left[\left(K_{1}>0\right) \wedge\left(K_{2}>0\right)\right] \\
& =\mathrm{p}\left[\left(z_{1}>0\right) \wedge\left(z_{1}+z_{2}>0\right)\right] \\
& =\mathrm{p}\left[\left(z_{1}>0\right) \wedge\left(z_{1}>-z_{2}\right)\right] .
\end{aligned}
$$

In diesem Ausdruck sind die beiden Einzelereignisse offensichtlich nicht unabhängig von einander ( $z_{1}$ ist Bestandteil beider Ereignisse). Daher kann der Ausdruck nicht wie beim vorigen Beispiel als Produkt der Einzelwahrscheinlichkeiten berechnet werden. Statt dessen sind die mit Hilfe der Bayes-Regel abgeleiteten Formeln (151,2] und (151.5) zu verwenden.

Wir beschränken uns zunächst darauf, die Überlebenswahrscheinlichkeit in der zweiten Runde, also ohne Berücksichtigung des Ausgangs der ersten Runde, zu formulieren:

$$
\mathrm{p}\left(K_{2}>0\right) \quad=\mathrm{p}\left(z_{1}+z_{2}>0\right)=\mathrm{p}\left(z_{2}>-z_{1}\right)
$$

Die Wahrscheinlichkeit, daß eine Zufallsvariable größer ist als eine andere, kann nach den Ausführungen in Abschnitt 4.1 zum Vergleich zweier Zufallsveränderlicher als

$$
\begin{aligned}
\mathrm{p}(\xi>\eta) & =\int_{y} \mathrm{p}(x>h \mid y) \cdot \mathrm{f}(y) \cdot \mathrm{d} y \\
& =\int_{y}\left[1-\mathrm{F}_{x}(y)\right] \cdot \mathrm{f}(y) \cdot \mathrm{d} y
\end{aligned}
$$

159 Einheitliche Fonts zur Darstellung von Brüchen waren leider nicht verfügbar. 
geschrieben werden. Daher gilt für die Wahrscheinlichkeit, daß $z_{2}$ sich größer als $-z_{1}$ realisiert:

$$
\begin{aligned}
\mathrm{p}\left(z_{2}>-z_{1}\right) & =\int_{z_{1}} \mathrm{p}\left(z_{2}>-z_{1} \mid z_{1}\right) \cdot \mathrm{f}\left(z_{1}\right) \cdot \mathrm{d} z_{1} \\
& =\int_{z_{1}}\left[1-\mathrm{F}_{z_{2}}\left(-z_{1}\right)\right] \cdot \mathrm{f}\left(z_{1}\right) \cdot \mathrm{d} z_{1} .
\end{aligned}
$$

Diese Formel entspricht der in Abschnitt 4.2 abgeleiteten Randwahrscheinlichkeit:

$$
\begin{aligned}
& \mathrm{p}\left(K_{2}>0\right)=\int_{K_{1}} \mathrm{p}\left(K_{2}>0 \mid K_{1}\right) \cdot \mathrm{p}\left(K_{1}\right) \cdot \mathrm{d} K_{1} \\
& \mathrm{p}\left(z_{2}+z_{1}>0\right)=\int_{z_{1}} \mathrm{p}\left(z_{2}>-z_{1} \mid z_{1}\right) \cdot \mathrm{p}\left(z_{1}\right) \cdot \mathrm{d} z_{1} .
\end{aligned}
$$

(197.1) beschreibt nur die Wahrscheinlichkeit, daß ein System, das die zweite Runde erreicht hat, diese auch überlebt. Es berücksichtigt nicht die Frage, ob das System in der ersten Runde fallierte. Zur Bestimmung der Überlebenswahrscheinlichkeit für zwei Runden können nur solche "Realisationsmöglichkeiten" von $K_{1}$ in das Integral aufgenommen werden, die nicht kleiner als Null sind:

$$
\mathrm{p}_{\mathrm{uil}}\left(K_{2}>0\right)=\int_{\mathrm{z}_{1}>0}\left[1-\mathrm{F}_{z 2}\left(-z_{1}\right)\right] \cdot \mathrm{f}\left(z_{1}\right) \cdot \mathrm{d} z_{1} .
$$

Wenn $z_{1}$ und $z_{2}$ identisch und unabhängig verteilt sind, gilt $\mathrm{F}_{z_{1}}=\mathrm{F}_{z_{2}}$ und $\mathrm{f}_{z_{1}}=\mathrm{f}_{z_{2}}$. Dadurch vereinfacht sich 197.2 zu

$$
\mathrm{p}_{\mathrm{ül}}\left(K_{2}>0\right)=\int_{z>0}\left[1-\mathrm{F}_{z}(-z)\right] \cdot \mathrm{f}(z) \cdot \mathrm{d} z .
$$

Es ist ggf. nicht sinnvoll, eine Identität der Verteilungen schon früher zu verwenden, da dies zu irreführenden Formulierungen führt:

$$
\begin{array}{lll}
K_{1} & =\mathrm{z} \\
K_{2} & & =K_{1}+\mathrm{z}=2 z \\
\mathrm{p}_{\text {uil }}(2) & =\mathrm{p}\left[K_{1}>0 \wedge K_{2}>0\right] \\
& =\mathrm{p}(z>0 \wedge 2 z>0) \\
& =\mathrm{p}(z>0 \wedge z>0) .
\end{array}
$$

Aus dieser Gleichung folgt nicht: $\mathrm{p}_{\mathrm{ül}}(2)=\mathrm{p}(z>0)$, sondern analog zum vorigen Vorgehen:

$$
\mathrm{p}_{\mathrm{ull}}(2) \quad=\int_{z>0}\left[1-\mathrm{F}_{z}(0)\right] \cdot \mathrm{f}(z) \cdot \mathrm{d} z .
$$


Betrachten wir nun den Ausdruck für die Überlebenswahrscheinlichkeit bis zur zweiten Runde für iid-Einzahlungen (197.3 näher:

$$
\begin{aligned}
\mathrm{p}_{\mathrm{uil}}\left(K_{2}>0\right) & =\int_{z>0}\left[1-\mathrm{F}_{z}(-z)\right] \cdot \mathrm{f}(z) \cdot \mathrm{d} z \\
& =\int_{z>0}\left[\mathrm{f}(z)-\mathrm{F}_{z}(-z) \cdot \mathrm{f}(z)\right] \cdot \mathrm{d} z \\
& =\int_{z>0} \mathrm{f}(z) \mathrm{d} z-\int_{z>0} \mathrm{~F}_{z}(-z) \cdot \mathrm{f}(z) \cdot \mathrm{d} z .
\end{aligned}
$$

Für eine symmetrisch um Null verteilte, stetige Zufallsveränderliche gilt:

$$
\begin{array}{ll}
\text { für die Dichtefunktion: } & \int_{x>0} \mathrm{f}(x) \cdot \mathrm{d} x=1 / 2 \\
& \mathrm{f}(x)=\mathrm{f}(-x) \\
\text { für die Verteilungsfunktion: } & \mathrm{F}(0)=1 / 2 \\
& \mathrm{~F}(-x)=1-\mathrm{F}(x) \\
& \mathrm{F}(-x-\mathrm{a})=1-\mathrm{F}(x+\mathrm{a}) .
\end{array}
$$

Für symmetrisch um Null verteilte, stetige Zufallsveränderliche ergibt sich daher aus (198.1):

$$
\begin{aligned}
\mathrm{p}_{\mathrm{uil}}\left(K_{2}>0\right) & =\int_{z>0} \mathrm{f}(z) \mathrm{d} z-\int_{z>0}\left[1-\mathrm{F}_{z}(z)\right] \cdot \mathrm{f}(z) \cdot \mathrm{d} z \\
& =\int_{z>0} \mathrm{~F}_{z}(z) \cdot \mathrm{f}(z) \mathrm{d} z-\int_{z>0} \mathrm{~F}_{z}(z) \cdot \mathrm{f}(z) \mathrm{d} z+\int_{z>0} \mathrm{~F}_{z}(z) \cdot \mathrm{f}(z) \mathrm{d} z \\
& =\int_{z>0} \mathrm{~F}_{z}(z) \cdot \mathrm{f}(z) \mathrm{d} z \infty \\
& =1 / 2 \cdot\left|[\mathrm{F}(z)]^{2}\right|_{0}^{+\infty} \\
& =1 / 2 \cdot\left[[\mathrm{F}(\infty)]^{2}-[\mathrm{F}(0)]^{2}\right] \\
& =1 / 2 \cdot\left[[1]^{2}-[1 / 2]^{2}\right] \\
& =1 / 2 \cdot[1-1 / 4] \\
& =3 / 8 .
\end{aligned}
$$

Die Überlebenswahrscheinlichkeit bis zur zweiten Runde beträgt also $3 / 8$. Dieses Ergebnis wurde für beliebige stetige, symmetrisch um Null und identisch verteile

160 Die Vereinfachung (198.3) $\int_{x>0} \mathrm{f}(x) \cdot \mathrm{d} x=1 / 2$ an dieser Stelle zu verwenden, ist nicht sinnvoll, wie im Weiteren offensichtlich wird. 
Zufallsveränderliche erzielt. Die Varianz oder die Wahl des Verteilungstyps innerhalb der Klasse der stetigen, symmetrisch um Null und identisch verteilten Zufallsvariablen beeinflußt also nicht die Überlebenswahrscheinlichkeit bis zur zweiten Runde. Für diskrete Verteilungen trifft (1907) nicht zu, da hierfür die Identität (190.): $F(-x)=1$ $-\mathrm{F}(\mathrm{x})$ nicht erfüllt ist.

Das Ergebnis 1987 kann für eine beschränkte Gleichverteilung auch graphisch veranschaulicht werden. In Schaubild 199.1 zeige das Quadrat den Realisationsraum der um Null gleichverteilten Zufallsveränderlichen $z_{1}$ und $z_{2}$. Seine Größe sei ohne Beschränkung der Allgemeinheit mit Eins angenommen.

\section{Schaubild 199.1: Überlebenswahrscheinlichkeit für zwei Perioden}

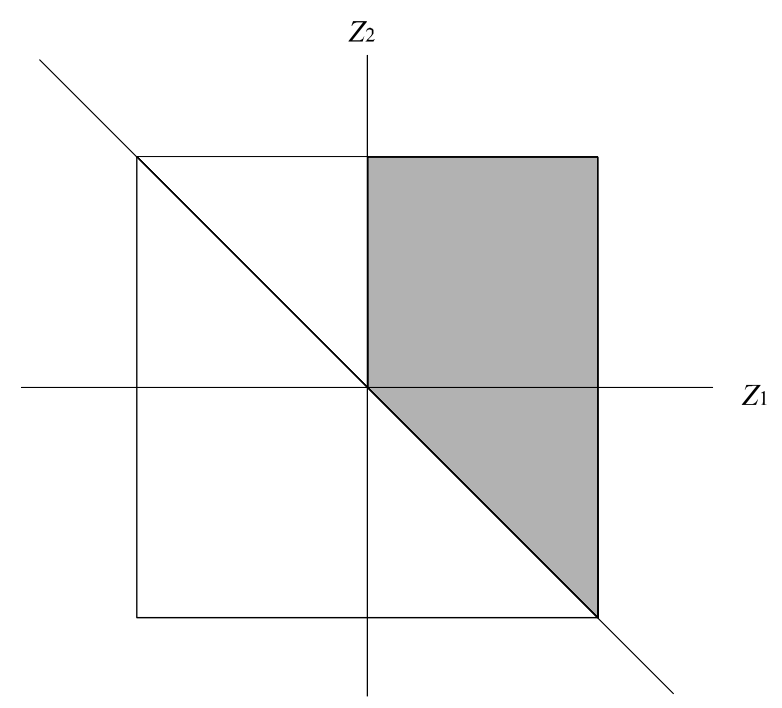

Rechts der Ordinate ist die Bedingung $z_{1}>0$ und oberhalb der Winkelhalbierenden die Bedingung $z_{2}>-z_{1} \Leftrightarrow z_{1}+z_{2}>0$ erfüllt. Im Bereich der schraffierten Schnittfläche treffen beide Bedingungen zu, ihre Größe von $1 / 4+1 / 8=3 / 8$ entspricht der oben 198.7 abgeleiteten Überlebenswahrscheinlichkeit für zwei Perioden.

Für Runde 3 und nachfolgende kann bisher noch keine analytische Lösung erzielt werden, die zu einem handhabbaren Ergebnis führt, wie die nachfolgenden Absätze zeigen. ${ }^{161}$ Daher muß die Hypothese, daß Varianz und Verteilung keinen Einfluß auf die Überlebenswahrscheinlichkeit ausüben, wenn die Zuwächse stetig und symmetrisch um Null verteilt sind, experimentell geprüft werden (s. Unterabschnitt 5.3.1.6.

Überlebenswahrscheinlichkeit in der 3. Runde:

$$
\begin{array}{ll}
K_{3} & =z_{3}+K_{2}=z_{1}+z_{2}+z_{3} \\
\mathrm{p}_{\mathrm{uil}}\left(K_{3}>0\right) & \equiv \mathrm{p}\left[\left(K_{3}>0\right) \wedge\left(K_{2}>0\right) \wedge\left(K_{1}>0\right)\right]
\end{array}
$$

161 Vgl. ZWICKER (1981, S. 75). 


$$
=\mathrm{p}\left(K_{3}>0 \mid K_{2}>0 \wedge K_{1}>0\right) \cdot \mathrm{p}_{\mathrm{ül}}(2),
$$

ohne Berücksichtigung der Ergebnisse in den Vorrunden:

$$
\begin{aligned}
\mathrm{p}\left(K_{3} \leq 0\right) & =\mathrm{p}\left(z_{1}+z_{2}+z_{3} \leq 0\right)=\mathrm{p}\left[z_{3} \leq-\left(z_{1}+z_{2}\right)\right] \\
& =\int_{z_{1}+z_{2}} \mathrm{p}\left[z_{3} \leq-\left(z_{1}+z_{2}\right) \mid\left(z_{1}+z_{2}\right)\right] \cdot \mathrm{f}\left(z_{1}+z_{2}\right) \cdot \mathrm{d}\left(z_{1}+z_{2}\right) \\
& =\int_{z_{1}+z_{2}} \mathrm{~F}_{z_{3}}\left[-\left(z_{1}+z_{2}\right)\right] \cdot \mathrm{f}\left(z_{1}+z_{2}\right) \cdot \mathrm{d}\left(z_{1}+z_{2}\right) \\
\mathrm{p}\left(K_{3}>0\right) \quad & =\mathrm{p}\left(z_{1}+z_{2}+z_{3}>0\right)=\mathrm{p}\left[z_{3}>-\left(z_{1}+z_{2}\right)\right] \\
& =\int_{z_{1}+z_{2}} \mathrm{p}\left(z_{3}>-\left(z_{1}+z_{2}\right) \mid z_{1}+z_{2}\right) \cdot \mathrm{f}\left(z_{1}+z_{2}\right) \cdot \mathrm{d}\left(z_{1}+z_{2}\right) \\
& =\int_{z_{1}+z_{2}}\left[1-\mathrm{F}_{z_{3}}\left[-\left(z_{1}+z_{2}\right)\right]\right] \cdot \mathrm{f}\left(z_{1}+z_{2}\right) \cdot \mathrm{d}\left(z_{1}+z_{2}\right)
\end{aligned}
$$

mit Berücksichtigung der Ergebnisse in den Vorrunden:

$$
\begin{aligned}
\mathrm{p}_{\mathrm{ül}}\left(K_{3}>0\right) & =\int_{z_{1}>0, z_{1}+z_{2}>0}\left[1-\mathrm{F}_{z 3}\left[-\left(z_{1}+z_{2}\right)\right]\right] \cdot \mathrm{f}\left(z_{1}+z_{2}\right) \cdot \mathrm{d}\left(z_{1}+z_{2}\right) \\
& =\int_{z_{1}>0, z_{1}+z_{2}>0} \mathrm{f}\left(z_{1}+z_{2}\right) \cdot \mathrm{d}\left(z_{1}+z_{2}\right)-\int_{z_{1}>0, z_{1}+z_{2}>0} \mathrm{~F}_{z 3}\left[-\left(z_{1}+z_{2}\right)\right] \cdot \mathrm{f}\left(z_{1}+z_{2}\right) \cdot \mathrm{d}\left(z_{1}+z_{2}\right) .
\end{aligned}
$$

Dieser Ausdruck ist wegen seiner Integrationsgrenzen nicht handhabbar. Die nachfolgenden Umformungen vermögen die Integrationsgrenzen nicht zu verändern und sind daher nur von akademischem Interesse.

Da die Einzahlungen $z_{i}$ symmetrisch um Null verteilt sind, folgt:

$$
\begin{aligned}
& \mathrm{p}_{\mathrm{uil}}\left(K_{3}>0\right) \\
& =\int_{z_{1}>0, z_{1}+z_{2}>0} \mathrm{f}\left(z_{1}+z_{2}\right) \cdot \mathrm{d}\left(z_{1}+z_{2}\right)-\int_{z_{1}>0, z_{1}+z_{2}>0}\left[1-\mathrm{F}_{z_{3}}\left(z_{1}+z_{2}\right)\right] \cdot \mathrm{f}\left(z_{1}+z_{2}\right) \cdot \mathrm{d}\left(z_{1}+z_{2}\right) \\
& =\int_{z_{1}>0, z_{1}+z_{2}>0} \mathrm{f}\left(z_{1}+z_{2}\right) \cdot \mathrm{d}\left(z_{1}+z_{2}\right)-\int_{z_{1}>0, z_{1}+z_{2}>0} \mathrm{f}\left(z_{1}+z_{2}\right) \cdot \mathrm{d}\left(z_{1}+z_{2}\right)+\int_{z_{1}>0, z_{1}+z_{2}>0} \mathrm{~F}_{z_{3}}\left(z_{1}+z_{2}\right) \cdot \mathrm{f}\left(z_{1}+z_{2}\right) \cdot \mathrm{d}\left(z_{1}+z_{2}\right) \\
& =\int_{z_{1}>0, z_{1}+z_{2}>0} \mathrm{~F}_{z_{1}}\left(z_{1}+z_{2}\right) \cdot \mathrm{f}\left(z_{1}+z_{2}\right) \cdot \mathrm{d}\left(z_{1}+z_{2}\right)
\end{aligned}
$$

Überlebenswahrscheinlichkeit in der t. Runde:

$$
K_{t} \quad=z_{t}+K_{t-1}=\sum_{i=1}^{t} z_{i}
$$




$$
\begin{aligned}
\mathrm{p}_{\mathrm{ül}}\left(K_{t}>0\right) & =\mathrm{p}\left(K_{t}>0 \wedge K_{t-1}>0 \wedge \ldots \wedge K_{1}>0\right) . \\
& =\mathrm{p}\left(K_{t}>0 \mid K_{t-1}>0 \wedge K_{t-2}>0 \wedge \ldots \wedge K_{1}>0\right) \cdot \mathrm{p}_{\text {ül }}(t-1) .
\end{aligned}
$$

Für die Überlebenswahrscheinlichkeit gilt analog zur dritten Runde:

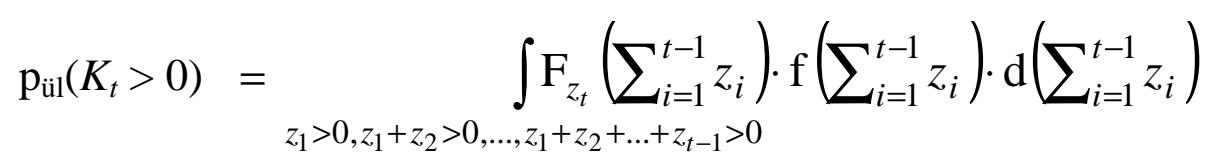

Für großes $t$ konvergieren $\mathrm{F}(\cdot)$ und $\mathrm{f}(\cdot)$ gegen eine Normalverteilung. Dennoch kann wie erwähnt für die Überlebenswahrscheinlichkeit kein Grenzwert berechnet werden, weil die Integrationsgrenzen nicht handhabbar sind.

Auch wenn die Überlebenswahrscheinlichkeiten für Runde drei und später nicht abgeleitet werden können, so ist es dennoch relativ einfach möglich zu zeigen, daß die Varianz die Überlebenswahrscheinlichkeit in jeder beliebigen Runde nicht beeinflußt, wenn die Einzahlungen stetig um Null verteilt sind. Das Kapital in der $t$. Runde beträgt nach (200D) $\sum_{i=1}^{t} z_{i}$. Eine höhere Varianz der Einzahlungen läßt sich durch Multiplikation mit einer Konstanten $a$ ausdrücken, so daß ein alternativer Kapitalstrom mit höherer Varianz der Einzahlungen als

$$
K_{t}^{\prime} \quad=\sum_{i=1}^{t} a \cdot z_{i}=a \cdot \sum_{i=1}^{t} z_{i}
$$

formuliert werden kann. ${ }^{162}$ Eine Erhöhung der Varianz führt also in diesem Modell zu einer affinen Transformation des ursprünglichen Kapitalstroms. $K_{t}^{\prime}$ ist das $a$-fache von $K_{t}$. Da die Konstante $a$ eine positive Zahl ist, besitzen $K_{t}$ und $K_{t}^{\prime}$ stets das gleiche Vorzeichen, wie Schaubild 202.1 beispielhaft veranschaulicht. Die Überlebenswahrscheinlichkeit wird daher nicht von der Varianz beeinflußt.

Dieses Ergebnis gilt für jeden beliebigen stetigen Verteilungstyp der Einzahlungen, deren Erwartungswert Null ist. Für diskrete Verteilungen hängt die Überlebenswahrscheinlichkeit, wie (1951] zeigt, schon in der ersten Runde von der Varianz ab. Einen Einfluß besitzt die Varianz ebenfalls, wenn die Einzahlungen einen nichtneutralen Erwartungswert aufweisen, wie man sich leicht qualitativ klar machen kann: Der Erwartungswert der Einzahlung der ersten Runde betrage Eins. Ist die Spanne der Verteilung der Einzahlungen kleiner als Eins, besteht keine Insolvenzgefahr, diese entsteht erst bei einer größeren Spanne der Verteilung. Wie sehr die Varianz der Einzahlung die Überlebenswahrscheinlichkeit beeinflußt, läßt sich in der ersten Runde mit Hilfe der Verteilungsfunktion der Einzahlung und in der zweiten Periode unter

162 Die Varianz von $z$ erhöht sich durch die Multiplikation mit der Konstanten $a$ um das $a^{2}$-fache, vgl. Übersicht 166.1. 
Umständen durch (1970) algebraisch berechnen. Für höhere Perioden wird dies im übernächsten Abschnitu anhand von Monte-Carlo-Simulationen untersucht.

Schaubild 202.1: Kapitalverlauf bei verschiedener Varianz der Einzahlung

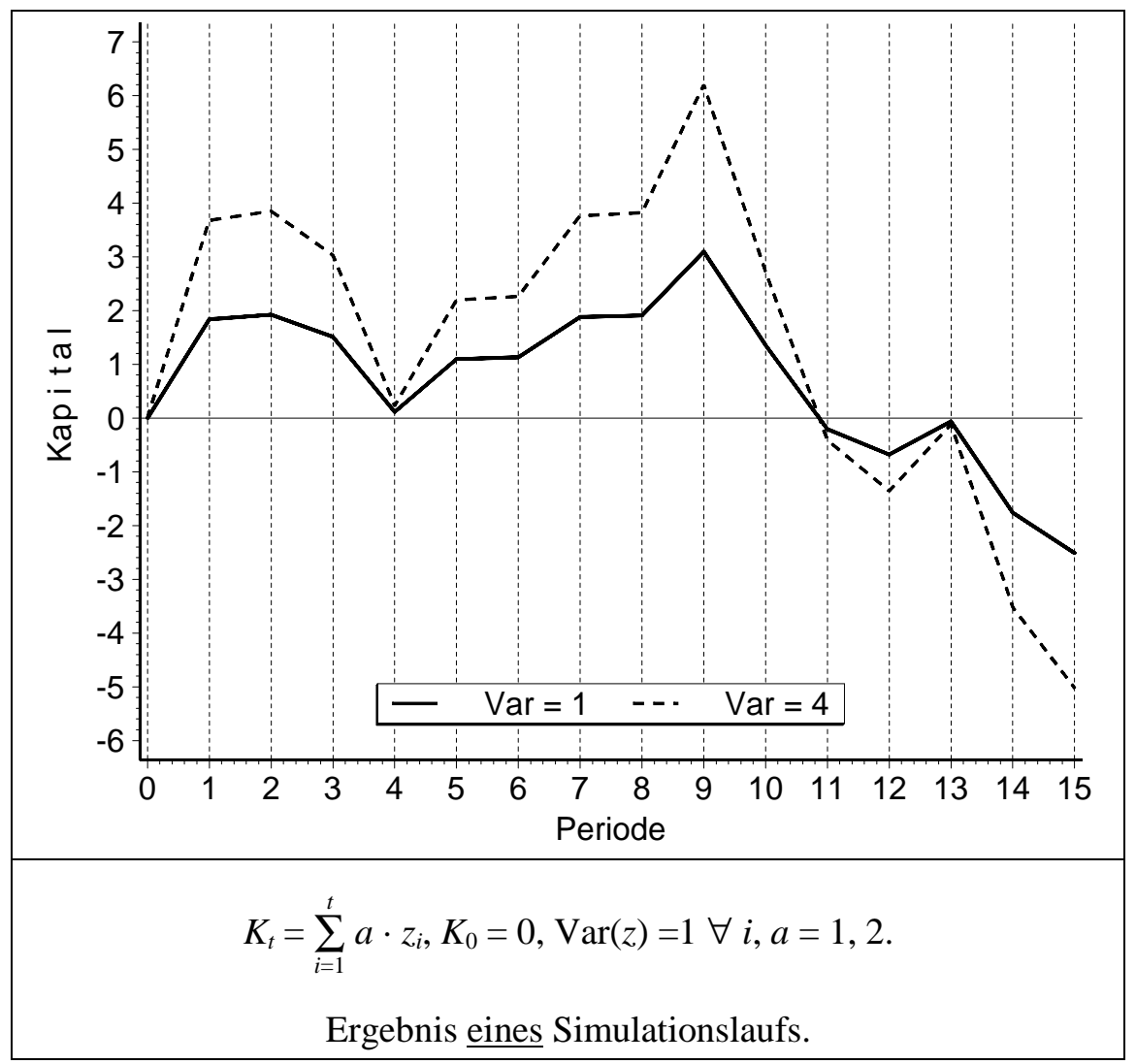

Wir heben nun die bisherige Annahme, das Startkapital sei Null, auf, $\boldsymbol{K}_{\mathbf{0}}$ ist also beliebig. Dies führt in der ersten Runde zu folgenden Modifikationen:

$$
\begin{array}{ll}
K_{1} & =K_{0}+z_{1} \\
\mathrm{p}\left(K_{1} \leq 0\right) & =\mathrm{p}\left(K_{0}+z_{1} \leq 0\right)=\mathrm{p}\left(z_{1} \leq-K_{0}\right)=F_{z 1}\left(-K_{0}\right) \\
\mathrm{p}\left(K_{1}>0\right) & =\mathrm{p}\left(z_{1}>-K_{0}\right)=1-F_{z 1}\left(-K_{0}\right) .
\end{array}
$$

Sind die Einzahlungen symmetrisch verteilt, vereinfacht sich die Überlebenswahrscheinlichkeit wegen 198.5 zu:

$$
\mathrm{p}\left(K_{1}>0\right) \quad=F_{z 1}\left(K_{0}\right) \text {. }
$$

Es ist also nicht mehr der Wert der Verteilungsfunktion der Einzahlung an der Stelle Null, sondern für $K_{0}$ bzw. $-K_{0}$ zu bestimmen. Für ein Startkapital von 1 und gleichverteilte, ganzzahlige Einzahlungen zwischen -1 und 1 beträgt die Überlebenswahrscheinlichkeit in der ersten Runde :

$$
\begin{aligned}
\mathrm{p}_{\mathrm{ül}}(1) & =\mathrm{p}_{\mathrm{ül}}\left(K_{1}>0\right)=\mathrm{p}\left(K_{0}+z_{1}>0\right) \\
& =\mathrm{p}\left(1+z_{1}>0\right)=\mathrm{p}\left(z_{1}>-1\right)
\end{aligned}
$$




$$
=2 / 3
$$

Wenn die Spanne der möglichen Einzahlungen größer ist, z.B. $z_{1} \sim \operatorname{uiid}\{-2,-1,0,1$, 2\}, ergibt sich

$$
\mathrm{p}_{\mathrm{ül}}(1) \quad=\mathrm{p}\left(z_{1}>-1\right)=3 / 5
$$

und für $z_{1} \sim \operatorname{uid}\{-3,-2,-1,0,1,2,3\}$

$$
\mathrm{p}_{\text {ül }}(1) \quad=\mathrm{p}\left(z_{1}>-1\right)=4 / 7 \text {. }
$$

Die Überlebenswahrscheinlichkeit ist abhängig von der Anzahl der Einzahlungsmöglichkeiten. Bezeichnen wir die Anzahl der positiven Realisationsmöglichkeiten von $z_{1}$ wie in 195,1 mit $k$, so gilt:

$$
\mathrm{p}_{\mathrm{ül}, k}(1) \quad=\mathrm{p}\left(z_{1}>-1\right)=\frac{k+1}{2 k+1}
$$

und für beliebiges Startkapital:

$$
\mathrm{p}_{\ddot{\mathrm{uil}}, k}(1) \quad=\mathrm{p}\left(z_{1}>-K_{0}\right)=\frac{k+K_{0}}{2 k+1} .
$$

Beide Ausdrücke konvergieren für eine zunehmende Spanne der Verteilung der Einzahlung gegen 1/2. Bei stark steigendem Startkapital nähert sich die Überlebenswahrscheinlichkeit Eins.

Gehen wir nun zur zweiten Runde über. Unabhängig von der Verteilung der Einzahlung gilt analog zu $K_{0}=0$ :

$$
\begin{aligned}
& K_{2}=K_{1}+z_{2}=K_{0}+z_{1}+z_{2} \\
& \mathrm{p}_{\text {ül }}\left(K_{2}>0\right) \equiv \mathrm{p}\left[\left(K_{1}>0\right) \wedge\left(K_{2}>0\right)\right] .
\end{aligned}
$$

Beschränken wir uns wiederum zunächst auf die Wahrscheinlichkeiten in der zweiten Runde, also ohne Berücksichtigung des Ausgangs der ersten Runde:

$$
\begin{aligned}
\mathrm{p}\left(K_{2} \leq 0\right) & =\mathrm{p}\left(K_{0}+z_{1}+z_{2} \leq 0\right)=\mathrm{p}\left(z_{2} \leq-z_{1}-K_{0}\right) \\
& =\int_{z_{1}} \mathrm{p}\left(z_{2} \leq-z_{1}-K_{0} \mid z_{1}\right) \cdot \mathrm{f}\left(z_{1}\right) \cdot \mathrm{d} z_{1} \\
& =\int_{z_{1}} \mathrm{~F}_{z 2}\left(-z_{1}-K_{0}\right) \cdot \mathrm{f}\left(z_{1}\right) \cdot \mathrm{d} z_{1} \\
\mathrm{p}\left(K_{2}>0\right) \quad & =\mathrm{p}\left(K_{0}+z_{1}+z_{2}>0\right)=\mathrm{p}\left(z_{2}>-z_{1}-K_{0}\right) \\
& =\int_{z_{1}} \mathrm{p}\left(z_{2}>-z_{1}-K_{0} \mid z_{1}\right) \cdot \mathrm{f}\left(z_{1}\right) \cdot \mathrm{d} z_{1} \\
& =\int_{z_{1}}\left[1-\mathrm{F}_{z_{2}}\left(-z_{1}-K_{0}\right)\right] \cdot \mathrm{f}\left(z_{1}\right) \cdot \mathrm{d} z_{1}
\end{aligned}
$$


Für die Überlebenswahrscheinlichkeit bis zur zweiten Runde dürfen wiederum nur solche Ausprägungen von $z_{1}$ aufsummiert werden, die zum Überleben in der ersten Runde geführt haben, für die also gilt: $z_{1}>-K_{0}$ :

$$
\begin{aligned}
& \mathrm{p}_{\mathrm{uil}}\left(K_{2}>0\right)=\int_{z_{1}>-K_{0}}\left[1-\mathrm{F}_{z_{2}}\left(-z_{1}-K_{0}\right)\right] \cdot \mathrm{f}\left(z_{1}\right) \cdot \mathrm{d} z_{1} \\
&=\int_{z_{1}}>-K_{0} \\
& \mathrm{f}\left(z_{1}\right) \cdot \mathrm{d} z_{1}-\int_{z_{1}}>-K_{0} \\
& \mathrm{~F}_{z_{2}}\left[-\left(z_{1}+K_{0}\right)\right] \cdot \mathrm{f}\left(z_{1}\right) \cdot \mathrm{d} z_{1} .
\end{aligned}
$$

Der Minuend läßt sich als Verteilungsfunktion ausdrücken, wie die nebenstehende Graphik verdeutlicht:

$$
\int_{z 1>-K 0} \mathrm{f}\left(z_{1}\right) \cdot \mathrm{d} z_{1}=1-\mathrm{F}_{z 1}\left(-K_{0}\right)
$$

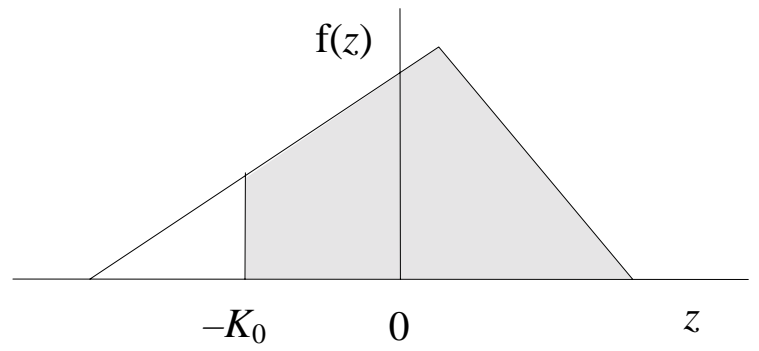

$1-\mathrm{F}_{z 1}\left(-K_{0}\right)$ gibt somit die Obergrenze der Überlebenswahrscheinlichkeit an, da der zweite Summand in 2042 negativ ist. Sie beträgt 1/2, wenn kein Startkapital vorhanden und die Einzahlungen symmetrisch, stetig und neutral verteilte Zufallsvariablen sind, und nimmt mit steigendem Startkapital zu.

Für symmetrisch neutrale Verteilungen kann die Beziehung 198,5 genutzt werden, so daß die Überlebenswahrscheinlichkeit sich unter dieser Bedingung vereinfacht:

$$
\begin{aligned}
\mathrm{p}_{\mathrm{uil}}\left(K_{2}>0\right) & =\int_{z_{1}>-K_{0}}\left[1-\mathrm{F}_{z_{2}}\left(-z_{1}-K_{0}\right)\right] \cdot \mathrm{f}\left(z_{1}\right) \cdot \mathrm{d} z_{1} \\
& =\int_{z_{1}}>-K_{0} \\
& =\int_{z_{1}}>-K_{0}
\end{aligned}
$$

Für $K_{0}=0$ ergibt dieser Term, wie aus 1987 hervorgeht, 3/8. Es läßt sich leicht zeigen, daß für wachsendes $K_{0}$ der Term und damit die Überlebenswahrscheinlichkeit größer wird et vice versa. ${ }^{163}$ Der genaue Wert der Verteilungsfunktion $\mathrm{F}_{z}$ an der Stelle $K_{0}$ ist abhängig von der Verteilung der Einzahlung $z$. Liegt also ein positives Startkapital vor, ist die Überlebenswahrscheinlichkeit nicht mehr unabhängig von Verteilungstyp und Varianz.

163 Für wachsendes $K_{0}$ sinkt die untere Integrationsgrenze, der Bereich, über den das Integral gebildet wird, vergrößert sich also. Zum zweiten wächst auch der Integrand mit zunehmenden $K_{0}$, da definitionsgemäß die Verteilungsfunktion $\mathrm{F}(\cdot)$ eine monoton wachsenden Funktion in den ersten beiden Quadranten ist und $\mathrm{f}(z)$ ebenfalls nur positive Werte annehmen kann. 
Schaubild 205,1 zeigt dies deutlich. Es veranschaulicht das gleiche Modell wie Schaubild 202.1, mit dem einzigen Unterschied, daß ein Startkapital in Höhe von 1 vorhanden ist. Diese Veränderung bewirkt, daß $K_{t}^{\prime}=K_{0}+\sum_{i=1}^{t} a \cdot z_{i}$ nicht mehr ein konstantes Vielfaches von $K_{t}=K_{0}+\sum_{i=1}^{t} z_{i}$ ist. ${ }^{164}$ Die höhere Varianz der Einzahlungen führt bei den vorliegenden Zufallszahlen zu einer Insolvenz in Periode 12, während bei einer kleinen Varianz das Kapital in dieser Runde positiv ist.

Schaubild 205.1: Kapitalverlauf bei verschiedener Varianz der Einzahlung und positivem Startkapital

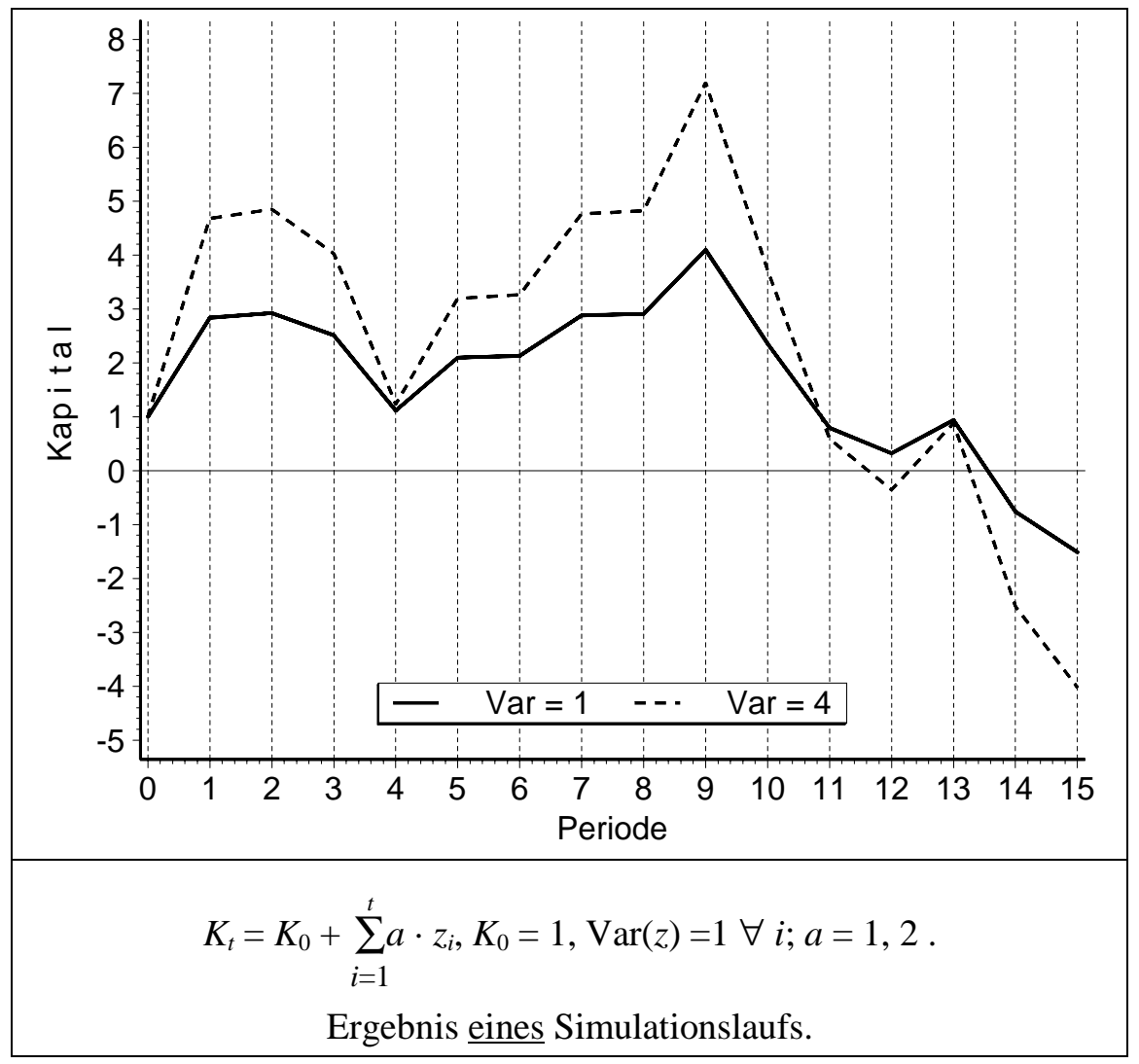

Auch für das Modell mit positivem Startkapital gilt, daß ab Runde 3 bisher noch keine brauchbare analytische Lösung erzielt werden kann. Daher werden hierfür ebenfalls im übernächsten Abschnitt Ergebnisse von Monte-Carlo-Simulationen vorgestellt. Die Bestimmungsgleichungen der Überlebenswahrscheinlichkeit seien dennoch der Vollständigkeit halber aufgeführt.

164 Vielmehr ist nun $K_{t}^{\prime}-1$ eine affine Transformation von $K_{t}-1$. Die „Affinitätslinie“ hat sich um eine Einheit nach oben verschoben; allgemein verschiebt sie sich um $K_{0}$. 
Überlebenswahrscheinlichkeit in der 3. Runde:

$$
\begin{aligned}
K_{3} & =K_{2}+z_{3}=K_{0}+z_{1}+z_{2}+z_{3} \\
\mathrm{p}\left(K_{3} \leq 0\right) & =\mathrm{p}\left(K_{0}+z_{1}+z_{2}+z_{3} \leq 0\right)=\mathrm{p}\left[z_{3} \leq-\left(K_{0}+z_{1}+z_{2}\right)\right] \\
& =\int_{z 1+z 2} \mathrm{p}\left[z_{3} \leq-\left(z_{1}+z_{2}+K_{0}\right) \mid\left(z_{1}+z_{2}\right)\right] \cdot \mathrm{f}\left(z_{1}+z_{2}\right) \cdot \mathrm{d}\left(z_{1}+z_{2}\right) \\
& =\int_{z 1+z 2} \mathrm{~F}_{z 3}\left(-\left(z_{1}+z_{2}+K_{0}\right) \cdot \mathrm{f}\left(z_{1}+z_{2}\right) \cdot \mathrm{d}\left(z_{1}+z_{2}\right)\right. \\
\mathrm{p}\left(K_{3}>0\right) & =\int_{z 1+z 2} \mathrm{p}\left[z_{3}>-\left(z_{1}+z_{2}+K_{0}\right) \mid\left(z_{1}+z_{2}\right)\right] \cdot \mathrm{f}\left(z_{1}+z_{2}\right) \cdot \mathrm{d}\left(z_{1}+z_{2}\right) \\
& =\int_{z 1+z 2}\left[1-\mathrm{F}_{z 3}\left[-\left(z_{1}+z_{2}+K_{0}\right)\right]\right] \cdot \mathrm{f}\left(z_{1}+z_{2}\right) \cdot \mathrm{d}\left(z_{1}+z_{2}\right) \\
\mathrm{p}_{\mathrm{ül}}\left(K_{3}>0\right) & =\int_{z_{1}>0, z_{1}+z_{2}>0}\left[1-\mathrm{F}_{z 3}\left[-\left(z_{1}+z_{2}+K_{0}\right)\right]\right] \cdot \mathrm{f}\left(z_{1}+z_{2}\right) \cdot \mathrm{d}\left(z_{1}+z_{2}\right) \\
& =\mathrm{p}\left[K_{3}>0 \mid\left(K_{2}>0 \wedge K_{1}>0\right)\right] \cdot \mathrm{p} \text { ül }(2) \cdot
\end{aligned}
$$

Überlebenswahrscheinlichkeit in der t. Runde:

Wir definieren:

$$
Z_{t} \quad \equiv \sum_{j=1}^{t} z_{j}
$$

Dies gestattet es, den Kapitalbestand vereinfacht darzustellen:

$$
\begin{aligned}
K_{t} & =K_{t-1}+z_{t}=K_{0}+\sum_{j=1}^{t} z_{j}=K_{0}+Z_{t} \\
\mathrm{p}_{\mathrm{ül}}\left(K_{t}>0\right) & =\underset{z_{1}>0, z_{1}+z_{2}>0, \ldots, z_{1}+z_{2}+\ldots+z_{t-1}>0}{ } \\
& =\mathrm{p}\left[K_{t}>0 \mid\left(K_{t-1}>0 \wedge K_{t-1}>0\right) \wedge \ldots \wedge K_{1}>0\right] \cdot \mathrm{p}_{\ddot{u ̈ l}}(t-1) .
\end{aligned}
$$

Zum Abschluß der Betrachtung additiver Systeme seien nun Modelle mit fester Auszahlung in jeder Periode untersucht. Diese Verallgemeinerung führt in der ersten Runde zu folgenden Modifikationen:

$$
\begin{array}{ll}
K_{1} & =K_{0}+z_{1}-a \\
\mathrm{p}\left(K_{1}>0\right) & =\mathrm{p}\left(z_{1}>-K_{0}+a\right)=1-F_{z 1}\left(-K_{0}+a\right) .
\end{array}
$$

Sind die Einzahlungen symmetrisch-neutral verteilt, vereinfacht sich die Überlebenswahrscheinlichkeit wegen (198.5] zu:

$$
\mathrm{p}\left(K_{1}>0\right) \quad=F_{z 1}\left(K_{0}-a\right) .
$$


Definieren wir den Saldo aus Startkapital und Auszahlung in der ersten Runde als Nettostartkapital: $K_{0}^{n} \equiv K_{0}-a$, erlaubt dies, die oben erzielten Ergebnisse für Modelle ohne Auszahlung Modelle mit Auszahlung übertragen.

Für die zweite Runde läßt sich Entsprechendes zeigen. Für das Kapital gilt:

$$
K_{2}=K_{1}+z_{2}-2 \cdot a=K_{0}-2 a+z_{1}+z_{2} \text {. }
$$

$K_{0}$ und $a$ sind zeitinvariante Konstanten. Definieren wir analog zur ersten Runde $K_{0,2}^{n} \equiv$ $K_{0}-2 a$, kann daher das Kapital in der zweiten Runde mit und ohne Auszahlung formal in der gleichen Struktur ausgedrückt werden:

$$
K_{2}=K_{0}-2 a+z_{1}+z_{2}=K_{0,2}^{n}+z_{1}+z_{2} .
$$

Die Wahrscheinlichkeit eines positiven Kapitals in der zweiten Runde ohne Auszahlung beträgt nach (203.2)'

$$
\mathrm{p}\left(K_{2}>0\right) \quad=\int_{z_{1}}\left[1-\mathrm{F}_{z_{2}}\left(-z_{1}-K_{0}\right)\right] \cdot \mathrm{f}\left(z_{1}\right) \cdot \mathrm{d} z_{1}
$$

Ersetzen wir in diesem Ausdruck $K_{0}$ durch $K_{0,2}^{n}$, erhalten wir die verallgemeinerte Wahrscheinlichkeit eines positiven Kapitals in der zweiten Runde für ein Modell mit Auszahlung:

$$
\mathrm{p}\left(K_{2}>0\right) \quad=\int_{z_{1}}\left[1-\mathrm{F}_{z_{2}}\left(-z_{1}-K_{0,2}^{n}\right)\right] \cdot \mathrm{f}\left(z_{1}\right) \cdot \mathrm{d} z_{1} .
$$

Für die Überlebenswahrscheinlichkeit bis zur zweiten Runde dürfen wiederum nur Ausprägungen von $z_{1}$ aufsummiert werden, die die Überlebensbedingung der ersten Runde erfüllen, für die also gilt: $z_{1}>-K_{0}^{n}=-K_{0}+a$ :

$$
\mathrm{p}_{\mathrm{ül}}\left(K_{2}>0\right)=\int_{z_{1}>-K_{0}+a}\left[1-\mathrm{F}_{z_{2}}\left(-z_{1}-K_{0,2}^{n}\right)\right] \cdot \mathrm{f}\left(z_{1}\right) \cdot \mathrm{d} z_{1} .
$$

Auflösen der Abkürzung $K_{0,2}^{n}$ führt schließlich zu:

$$
\mathrm{p}_{\mathrm{ül}}\left(K_{2}>0\right)=\int_{z_{1}>-K_{0}+a}\left[1-\mathrm{F}_{z_{2}}\left(-z_{1}-K_{0}+2 a\right)\right] \cdot \mathrm{f}\left(z_{1}\right) \cdot \mathrm{d} z_{1} .
$$

Für symmetrische Verteilungen vereinfacht sich die Formel analog zu (204.3):

$$
\mathrm{p}_{\mathrm{uul}}\left(K_{2}>0\right)=\int_{z_{1}>-K_{0^{+}}+a} \mathrm{~F}\left(z_{1}+K_{0}-2 a\right) \mathrm{f}\left(z_{1}\right) \cdot \mathrm{d} z_{1} .
$$

Der Unterschied zu den Formeln ohne Einzahlung, 2041) und 204,3, besteht darin, daß $K_{0}$ in der Integrationsgrenze durch $K_{0}-a$ und als Argument der Verteilungsfunktion durch $K_{0}-2 a$ zu ersetzen ist. Eine detaillierte Herleitung befindet sich im Anhang, Abschnitt 9.4. 
Als letztes einfaches Modell mit genau einer stochastischen Einzahlung betrachten wir nun Einzahlungen, die vom Kapital in der Vorperiode abhängen. Ökonomisch können sie als stochastische Verzinsung interpretiert werden.

Das Kapital sei definiert als

$$
K_{t} \quad=K_{t-1}+K_{t-1} \cdot z_{t}=K_{t-1} \cdot\left(1+z_{t}\right) .
$$

Der stochastische Zinsfuß $z_{t}$ sei weiterhin eine iid-Variable und das Startkapital $K_{0}$ größer als Null. Diese Annahme wird gewählt, da anderenfalls keine positive Kapitalentwicklung möglich wäre. Die Überlebenswahrscheinlichkeit in der ersten Runde ist nicht von der Höhe des (annahmegemäß positiven) Startkapitals abhängig, sondern nur vom Vorzeichen des Aufzinsungsfaktors $q_{t} \equiv 1+z_{t}$. Ist er negativ, falliert das System in der ersten Runde.

Die Überlebenswahrscheinlichkeit für die erste Runde lautet:

$$
\begin{aligned}
K_{1} & =K_{0} \cdot q_{1}=K_{0} \cdot\left(1+z_{1}\right) \\
\mathrm{p}_{\text {ül }}\left(K_{1}>0\right) & =\mathrm{p}\left(1+z_{1}>0\right)=\mathrm{p}\left(z_{1}>-1\right)=1-\mathrm{F}_{z_{1}}(-1) \\
& =\mathrm{p}\left(q_{1}>0\right)=1-\mathrm{F}_{q_{1}}(0) .
\end{aligned}
$$

Für das Kapital der folgenden Runden gilt:

$$
\begin{array}{ll}
K_{2} & =K_{1} \cdot\left(1+z_{2}\right)=K_{0} \cdot\left(1+z_{1}\right) \cdot\left(1+z_{2}\right)=K_{0} \cdot q_{1} \cdot q_{2} \\
K_{t} & =K_{0} \cdot \prod_{i=1}^{t} q_{t} .
\end{array}
$$

Der Erwartungswerts des Aufzinsungsfaktors ist per definitionem

$$
\mathrm{E}(q) \quad=\mathrm{E}(z+1)=\mathrm{E}(z)+1 .
$$

Die stochastischen Kenngrößen der Aufzinsungsfaktoren sind aufgrund ihrer iidEigenschaft unabhängig vom Index:

$$
\begin{aligned}
& \mathrm{E}\left(q_{i}\right)=\mathrm{E}\left(q_{j}\right) \equiv \mathrm{E}(q), \operatorname{Var}\left(q_{i}\right)=\operatorname{Var}\left(q_{j}\right) \equiv \operatorname{Var}(q), \\
& \mathrm{F}\left(q_{i}\right)=\mathrm{F}\left(q_{j}\right) \equiv \mathrm{F}(q), \mathrm{f}\left(q_{i}\right)=\mathrm{f}\left(q_{j}\right) \equiv \mathrm{f}(q), \forall i, j .165
\end{aligned}
$$

Für den Erwartungswert des Kapitals gilt somit (ohne Berücksichtigung der Überlebenswahrscheinlichkeit):

$$
\begin{array}{ll}
\mathrm{E}\left(K_{1}\right) & =K_{0} \cdot[\mathrm{E}(z)+1] \\
\mathrm{E}\left(K_{2}\right) & =K_{0} \cdot[\mathrm{E}(z)+1]^{2} \\
\mathrm{E}\left(K_{t}\right) & =K_{0} \cdot[\mathrm{E}(z)+1]^{t}=K_{0} \cdot[\mathrm{E}(q)]^{t} .
\end{array}
$$

165 Daraus darf selbstverständlich nicht der Schluß gezogen werden, $\mathrm{E}\left(q_{i}\right) \cdot \mathrm{E}\left(q_{j}\right)$ sei äquivalent zu $\mathrm{E}\left(q_{i}^{2}\right)$. Seien z.B. $q_{i}$ und $q_{j}$ standardnormalverteilte Zufallsvariablen, ergibt der erste Ausdruck Null und der zweite Eins. ( $q_{i}^{2}$ ist $\chi^{2}$-verteilt mit einem Freiheitsgrad, Näheres siehe Abschnitt 4.1.) 
Die Überlebenswahrscheinlichkeit in der zweiten und allen weiteren Runden hängt wie in der ersten Runde nicht vom Betrag des (positiven) Startkapitals ab, sondern nur von den Vorzeichen der Aufzinsungsfaktoren $q_{i}$ :

$$
\begin{aligned}
& \mathrm{p}\left(K_{2}>0\right) \quad=\mathrm{p}\left(K_{0} \cdot q_{1} \cdot q_{2}>0\right)=\mathrm{p}\left(q_{1} \cdot q_{2}>0\right) \\
& \mathrm{p}\left(K_{t}>0\right) \quad=\mathrm{p}\left(K_{0} \cdot \prod_{i=1}^{t} q_{t}>0\right)=\mathrm{p}\left(\prod_{i=1}^{t} q_{t}>0\right) .
\end{aligned}
$$

Trotz der iid-Eigenschaft kann diese Wahrscheinlichkeit nicht in ein Produkt von Wahrscheinlichkeiten zerlegt werden, wie in Abschnitt 4.4 auf Seite 178 ausgeführt wurde. Die beiden nachfolgenden Beispiele zeigen auf, wie groß der Fehler sein kann, der entsteht, wenn die Zerlegung als Näherung für die Wahrscheinlichkeit des Produktes verwendet wird. Untersucht wird eine diskrete (a) und eine stetige (b) Gleichverteilung. Anschließend wird die Überlegung zur Bestimmung der Überlebenswahrscheinlichkeit für zwei und mehr Runden fortgeführt.

(a) Für die iid-Zufallsvariablen $z_{1}$ und $z_{2}$ gelte, daß sie ganzzahlig gleichverteilt zwischen -1 und 1 seien. Die Wahrscheinlichkeit für eine positive Realisation beträgt daher für jede Zufallsvariable $z_{i} 1 / 3$, so daß das Produkt der Wahrscheinlichkeiten 1/9 ergibt:

$$
\mathrm{p}\left(z_{1}>0\right) \cdot \mathrm{p}\left(z_{2}>0\right)=1 / 3 \cdot 1 / 3=1 / 9 .
$$

Wie groß ist die Wahrscheinlichkeit, daß das Produkt aus $z_{1}$ und $z_{2}$ größer als Null ist?

Die nebenstehende Matrix zeigt die Ausprägungsmöglichkeiten des Produktes von $z_{1}$ und $z_{2}$. Zwei von neun Möglichkeiten sind größer als Null. Die Wahrscheinlichkeit für $\left(z_{1} \cdot z_{2}>0\right)$ ist daher mit 2/9 genau doppelt so groß wie das Produkt der Einzelwahrscheinlichkeiten: $\mathrm{p}\left(z_{1}>0\right) \cdot \mathrm{p}\left(z_{2}>\right.$ $0)$.

(b) $z_{1}$ und $z_{2}$ seien wieder iid-Variablen, die nun stetig gleichverteilt

\begin{tabular}{|c||ccc|}
\hline & -1 & 0 & 1 \\
\hline \hline-1 & 1 & 0 & -1 \\
0 & 0 & 0 & 1 \\
1 & -1 & 0 & 1 \\
\hline
\end{tabular}
seien zwischen -1 und 1 . Die Wahrscheinlichkeit eine positiven Realisation beträgt daher für beide Variablen $1 / 2$ und das Produkt der Wahrscheinlichkeiten 1/4:

$$
\mathrm{p}\left(z_{1}>0\right) \cdot \mathrm{p}\left(z_{2}>0\right)=1 / 2 \cdot 1 / 2=1 / 4 .
$$

Für das Ereignis $z_{1} \cdot z_{2}>0$ gibt es zwei Möglichkeiten: beide Zufallsvariable realisieren sich positiv oder beide realisieren sich negativ. Die Wahrscheinlichkeit für jede der beiden Kombinationen beträgt $1 / 4$, ihre Summe also 1/2:

$$
\begin{aligned}
\mathrm{p}\left(z_{1} \cdot z_{2}>0\right) & =\mathrm{p}\left(\left[\left\{z_{1}>0\right\} \cap\left\{z_{2}>0\right\}\right] \cup\left[\left\{z_{1}<0\right\} \cap\left\{z_{2}<0\right\}\right]\right. \\
& =1 / 4+1 / 4=1 / 2 .
\end{aligned}
$$

Wie im diskreten Beispiel ist die Wahrscheinlichkeit des Produktes doppelt so groß wie das Produkt der Wahrscheinlichkeiten. 
Die Wahrscheinlichkeitsverteilung eines Produktes kann für bestimmte Verteilungen, u.a. die Normalverteilung, nach (1741) angegeben werden, allerdings nur für Verteilungen, die sich ausschließlich positiv realisieren. In SPRINGER (1979) und FISZ (1965, S. 57) wird zwar auch die Formel der Dichtefunktion eines allgemeinen Produktes von Zufallsvariablen, die die Positivität nicht voraussetzt, angegeben; diese Formel entzieht sich aber aufgrund ihrer Komplexität im allgemeinen einer algebraischen oder numerischen Bestimmung.

Daher habe ich einen anderen Ansatz entwickelt, der es erlaubt, mit wenig mathematischem Aufwand die Überlebenswahrscheinlichkeit abzuleiten, wenn die iidAnnahme erfüllt ist. Die Herleitung benötigt nicht die Überlebenswahrscheinlichkeit in der $t$. Runde, die für (151,2 und (151,3 von zentraler Bedeutung ist:

$$
\mathrm{p}_{\text {ül }}(t) \quad=\mathrm{p}\left[K_{t}>0 \mid \ddot{\mathrm{ul}}(t-1)\right] \cdot \mathrm{p}_{\text {ül }}(t-1)
$$

Der Gedankengang beginnt bei der gemeinsamen Wahrscheinlichkeitsfunktion von $K_{1}$ und $K_{2}$ :

$$
\mathrm{p}_{\text {ül }}(2) \quad=\mathrm{p}\left(K_{1}>0 \wedge K_{2}>0\right) .
$$

Einsetzen von $K_{1}=q_{1} \cdot K_{0}$ und $K_{2}=q_{2} \cdot K_{1}=q_{2} \cdot q_{1} \cdot K_{0}$ führt zu:

$$
\mathrm{p}_{\mathrm{ül}}(2) \quad=\mathrm{p}\left(q_{1} \cdot K_{0}>0 \wedge q_{2} \cdot q_{1} \cdot K_{0}>0\right) \text {. }
$$

Da das Startkapital $K_{0}$ definitionsgemäß positiv ist, wird die Wahrscheinlichkeit ausschließlich durch die Aufzinsungsfaktoren $q_{i}$ determiniert. Die Bestimmungsgleichung der Überlebenswahrscheinlichkeit vereinfacht sich daher zu:

$$
\mathrm{p}_{\mathrm{üi}}(2) \quad=\mathrm{p}\left(q_{1}>0 \wedge q_{2} \cdot q_{1}>0\right) .
$$

Die zweite Bedingung in dieser Gleichung kann nur eintreten, wenn beide Aufzinsungsfaktoren positiv oder beide negativ sind. Ein negativer Aufzinsungsfaktor $q_{1}$ ist durch die erste Bedingung ausgeschlossen, daher muß in der zweiten Bedingung $q_{2}$ ebenfalls positiv sein, damit diese Bedingung wahr wird. Die Überlebenswahrscheinlichkeit für zwei Runden vereinfacht sich daher weiter zu:

$$
\mathrm{p}_{\mathrm{uil}}(2) \quad=\mathrm{p}\left(q_{1}>0 \wedge q_{2}>0\right) \text {. }
$$

$q_{1}$ und $q_{2}$ sind im Gegensatz zu $K_{1}$ und $K_{2}$ stochastisch unabhängige Zufallsveränderliche. Daher kann die gemeinsame Wahrscheinlichkeit in ein Produkt von Einzelwahrscheinlichkeiten zerlegt werden. Da die Aufzinsungsfaktoren identisch verteilt sind, stimmen die Einzelwahrscheinlichkeiten überein. Somit gilt für die Überlebenswahrscheinlichkeit:

$$
\begin{aligned}
\mathrm{p}_{\mathrm{ül}}(2) & =\mathrm{p}\left(q_{1}>0\right) \cdot \mathrm{p}\left(q_{2}>0\right)=\left[\mathrm{p}\left(q_{1}>0\right)\right]^{2} \\
& =[\mathrm{p}(q>0)]^{2} .
\end{aligned}
$$

Für die Überlebenswahrscheinlichkeit bis zur $t$. Runde gilt entsprechend:

$$
\mathrm{p}_{\mathrm{uul}}(t) \quad=[\mathrm{p}(q>0)]^{t} .
$$


Da $\mathrm{p}(q>0)$ die Überlebenswahrscheinlichkeit in der ersten Runde repräsentiert, ist die Überlebenswahrscheinlichkeit für $t$ Runden eine unmittelbare Funktion der Überlebenswahrscheinlichkeit der ersten Periode:

$$
\mathrm{p}_{\mathrm{ül}}(t)=\left[\mathrm{p}_{\mathrm{ül}}(1)\right]^{t} .
$$

Die Überlebenswahrscheinlichkeit fällt daher streng monoton mit der Zeit in Form einer Exponentialfunktion oder ist konstant Eins.

Auch in dieses Modell wird abschließend eine Auszahlung eingeführt. Das Kapital sei nun definiert als

$$
\begin{aligned}
K_{t} & =K_{t-1}+K_{t-1} \cdot z_{t}-a=K_{t-1} \cdot\left(1+z_{t}\right)-a . \\
& =K_{t-1} \cdot q_{t}-a .
\end{aligned}
$$

Wie im Modell ohne Einzahlung ist ein positives Startkapital zum Überleben Voraussetzung. Während ohne Einzahlungen die Höhe des Startkapitals bedeutungslos für die Überlebenswahrscheinlichkeit ist, ist sie nun neben den Einzahlungen entscheidend. Es liegt auf der Hand, daß bei symmetrisch neutralen Einzahlungen ein Startkapital in Höhe der Auszahlung zu einer Ruinwahrscheinlichkeit von 50\% in der ersten Runde führt.

Die Überlebenswahrscheinlichkeit der ersten Periode lautet:

$$
\begin{aligned}
\mathrm{p}_{\text {ïl }}\left(K_{1}>0\right) & =\mathrm{p}\left[K_{0} \cdot\left(1+z_{1}\right)>a\right] \\
& =\mathrm{p}\left(K_{0} \cdot q_{1}>a\right) \\
& =\mathrm{p}\left(q_{1}>\frac{a}{K_{0}}\right) \\
& =1-\mathrm{F}_{q_{1}}\left(\frac{a}{K_{0}}\right) .
\end{aligned}
$$

Ist der Aufzinsungsfaktor symmetrisch um Eins verteilt, beträgt daher die Überlebenswahrscheinlichkeit für $a>K_{0}$ weniger als $1 / 2$ et vice versa.

Für das Kapital der folgenden Runden gilt:

$$
\begin{aligned}
K_{2} & =K_{1} \cdot\left(1+z_{2}\right)-a=\left[K_{0} \cdot\left(1+z_{1}\right)-a\right] \cdot\left(1+z_{2}\right)-a \\
& =\left[K_{0} \cdot q_{1}-a\right] \cdot q_{2}-a \\
K_{3} & =\left[\left[K_{0} \cdot q_{1}-a\right] \cdot q_{2}-a\right] \cdot q_{3}-a .
\end{aligned}
$$

Die geschlossene Form dieser Differenzengleichung gibt Maple, ein für viele Plattformen verfügbares Programm zur Computeralgebra, an mit: 


$$
K_{t} \quad=-\left(\prod_{n 1=0}^{t-1} \mathrm{q}\left(\_n 1+1\right)\left(a\left(\sum_{-n 2=0}^{t-1} \frac{1}{\prod_{-n 1=0}^{n 2} \mathrm{q}\left(\_n 1+1\right)}\right)-\mathrm{K}(0)\right)\right.
$$

Die Summations- oder Multiplikationsvariable wird in Maple durch einen führenden Unterstrich gekennzeichnet. Kleine Vereinfachungen der Gleichung führen zu:

$$
K_{t} \quad=\prod_{i=1}^{t} q_{i} \cdot\left(K_{0}-a \cdot \sum_{j=1}^{t} \frac{1}{\prod_{i=1}^{j} q_{i}}\right) .
$$

Die Überlebenswahrscheinlichkeit kann bereits für die zweite Runde kaum noch angegeben werden, da die Gleichung zeigt, daß die Verteilungsfunktion eines Produktes von zwei Zufallsvariablen $\left(q_{1} \cdot q_{2}\right)$ zu bestimmen ist. Für $t$ Runden wären entsprechend Verteilungsfunktionen der Produkte von $t$ Zufallsvariablen zu berechnen.

Die Formeln vereinfachen sich entscheidend, wenn die Aufzinsungsfaktoren in jeder Periode gleich sind. Unter dieser Voraussetzung gilt für das Kapital:

$$
\begin{aligned}
K_{t} & =K_{0} \cdot q^{t}-\frac{a \cdot q^{t}-a}{q-1} \\
& =K_{0} \cdot q^{t}-\frac{a \cdot\left(q^{t}-1\right)}{q-1} .
\end{aligned}
$$

Die Voraussetzung gleicher Aufzinsungsfaktoren ist in einem stochastischen Kontext sehr viel beschränkender, als es zunächst erscheinen mag. Sie bedeutet nicht nur, daß die Aufzinsungsfaktoren identisch verteilt sind, sondern auch, daß sie stochastisch zu 100\% abhängig sind. Nur dann ist sichergestellt, daß die Aufzinsungsfaktoren ab der zweiten Periode exakt gleich ausfallen, wie der erste Aufzinsungsfaktor. ${ }^{166}$ Da diese Bedingungen für ein Überlebensmodell zu restriktiv sind, wird dieser Ansatz nicht weiter verfolgt und die Überlebenswahrscheinlichkeiten in Simulationen untersucht.

In der Tabelle 213.1 sind die wichtigsten Ergebnisse, die zur Überlebenswahrscheinlichkeit einfacher dynamischer Systeme analytisch bisher abgeleitet werden konnten, zusammengestellt. Im nächsten Abschnitt wird das Konzept der Markovketten erläutert. Diese Technik gestattet es, auf relativ einfache Weise die Überlebenswahrscheinlichkeit in dynamischen Systeme, die durch Differenzengleichung erster Ordnung gekennzeichnet sind, numerisch für beliebige Perioden zu berechnen. Voraussetzung sind diskret verteilte Einzahlungen.

166 In Abschnitt 4.4 wird der Unterschied zwischen Quadraten von Zufallsveränderlichen - hier sind die Ausprägungen der Zufallsveränderliche exakt gleich - und Produkten von Zufallsveränderliche demonstriert. 
Tabelle 213.1: Zusammenfassung: analytische Ergebnisse zur Überlebenswahrscheinlichkeit

\begin{tabular}{|c|c|c|c|c|c|c|c|c|}
\hline $\begin{array}{l}\text { Ver- } \\
\text { knüp- } \\
\text { fung }\end{array}$ & $\begin{array}{c}\text { Pe- } \\
\text { ri- } \\
\text { ode }\end{array}$ & Modell & $\begin{array}{c}\text { Ver- } \\
\text { tei- } \\
\text { lung* }\end{array}$ & $K_{0}$ & $\begin{array}{c}\mathrm{E}(z) \\
\mathrm{E}(q) \\
* *\end{array}$ & $\begin{array}{c}\text { Überlebens- } \\
\text { wahrscheinlichkeit }\end{array}$ & $i i d^{* * *}$ & $\begin{array}{c}\text { Formel } \\
\text { bzw. } \\
\text { Seite }\end{array}$ \\
\hline $\begin{array}{l}\text { ohne } o \text {. } \\
\text { additiv }\end{array}$ & 1 & $K_{1}=z_{1}-a$ & bel. & 0 & bel. & $1-F_{z_{1}}(a)$ & - & $(192.1)$ \\
\hline " & " & $K_{1}=z_{1}$ & $\begin{array}{l}\text { dis- } \\
\text { kret }\end{array}$ & " & 0 & $\frac{k}{2 k+1}$ & - & (195.1) \\
\hline " & " & " & $\begin{array}{c}\text { sym- } \\
\text { stet }\end{array}$ & " & " & $1 / 2$ & - & S. 195 \\
\hline ohne & $t$ & $K_{t}=z_{t}-a$ & bel. & - & bel. & $\prod_{i=1}^{i}\left[1-F_{z_{i}}(a)\right]$ & {$\left[1-F_{z}(a)\right]^{t}$} & $(193.1)$ \\
\hline additiv & 2 & $K_{2}=z_{1}+z_{2}$ & " & 0 & " & $\int_{\mathbf{z}_{1}>0}\left[1-\mathrm{F}_{z 2}\left(-z_{1}\right)\right] \cdot \mathrm{f}\left(z_{1}\right) \cdot \mathrm{d} z_{1}$ & $\int_{z>0}\left[1-\mathrm{F}_{z}(-z)\right] \cdot \mathrm{f}(z) \cdot \mathrm{d} z$ & $\frac{1972}{(197,3)}$ \\
\hline " & " & " & $\begin{array}{l}\text { sym- } \\
\text { stet }\end{array}$ & " & 0 & $\int_{\mathbf{z}_{1}>0} \mathrm{~F}_{z 2}\left(z_{1}\right) \cdot \mathrm{f}\left(z_{1}\right) \cdot \mathrm{d} z_{1}$ & $3 / 8$ & (198.7) \\
\hline " & 1 & $K_{1}=K_{0}+z_{1}-a$ & bel. & bel. & bel. & $1-F_{z 1}\left(-K_{0}+a\right)$ & - & $(202,1)$ \\
\hline " & 1 & " & $\begin{array}{l}\text { sym- } \\
\text { stet }\end{array}$ & " & 0 & $F_{z 1}\left(K_{0}+a\right)$ & - & $(202,2)$ \\
\hline " & 1 & " & $\begin{array}{l}\text { dis- } \\
\text { kret }\end{array}$ & " & 0 & $\frac{k+K_{0}}{2 k+1}$ & - & $(203,1)$ \\
\hline " & 2 & $\begin{aligned} K_{2}= & K_{0}+z_{1} \\
& +z_{2}\end{aligned}$ & bel. & $"$ & bel. & $\int_{z 1>-K_{0}}\left[1-\mathrm{F}_{z_{2}}\left(-z_{1}-K_{0}\right)\right] \mathrm{f}\left(z_{1}\right) \mathrm{d} z_{1}$ & $\int_{z>-K_{0}}\left[1-\mathrm{F}\left(-z-K_{0}\right)\right] \mathrm{f}(z) \mathrm{d} z$ & $(204,1)$ \\
\hline " & " & " & $\begin{array}{l}\text { sym- } \\
\text { stet }\end{array}$ & " & 0 & $\int_{z_{1}} \mathrm{~F}_{-K_{0}} \mathrm{~F}_{z_{2}}\left(z_{1}+K_{0}\right) \mathrm{f}\left(z_{1}\right) \mathrm{d} z_{1}$ & $\int_{z>-K_{0}} \mathrm{~F}\left(z+K_{0}\right) \mathrm{f}(z) \mathrm{d} z$ & $(204,3$ \\
\hline " & " & $\begin{array}{l}K_{2}=K_{0}+z_{1} \\
+z_{2}-2 \cdot a\end{array}$ & bel. & $"$ & bel. & $\begin{array}{l}\int_{z 1>-K_{0^{+}} a}\left[1-\mathrm{F}_{z_{2}}\left(-z_{1}-K_{0}+\right.\right. \\
\quad 2 a)] \cdot \mathrm{f}\left(z_{1}\right) \mathrm{d} z_{1}\end{array}$ & $\begin{array}{l}\int_{z>-K_{0}+a}\left[1-\mathrm{F}\left(-z-K_{0}+\right.\right. \\
2 a)] \cdot \mathrm{f}(z) \mathrm{d} z\end{array}$ & $(204.2$ \\
\hline " & " & " & $\begin{array}{l}\text { sym- } \\
\text { stet }\end{array}$ & " & 0 & $\int_{z 1>-K_{0^{+a}}} \mathrm{~F}\left(z_{1}+K_{0^{-}}-2 a\right) \mathrm{f}\left(z_{1}\right) \mathrm{d} z_{1}$ & $\int_{z>-K_{0}+a} \mathrm{~F}\left(z+K_{0}-2 a\right) \mathrm{f}(z) \mathrm{d} z$ & $(207,3$ \\
\hline $\begin{array}{l}\text { multi- } \\
\text { plikativ }\end{array}$ & 1 & $K_{1}=K_{0} \cdot q_{1}$ & bel. & $>0$ & bel. & $1-\mathrm{F}_{q_{1}}\left(\frac{a}{K_{0}}\right)$ & - & $(208,1)$ \\
\hline " & $t$ & $K_{t}=K_{0} \cdot \prod_{i=1}^{t} q_{t}$ & " & " & " & - & {$\left[1-\mathrm{F}_{q}(0)\right]^{t}$} & $(F 11,1)$ \\
\hline \multicolumn{9}{|c|}{$\begin{array}{l}\text { * } \quad \text { sym-stet = symmetrisch stetige Verteilung } \\
* * * \quad \text { Überlebenswahrscheinlichkeit, wenn die Einzahlungen identisch verteilt sind. } \\
\text { Alle Einzahlungen sind stochastisch unabhängig. }\end{array}$} \\
\hline
\end{tabular}




\subsection{Markovketten als Instrument zur Berechnung von Überlebens- wahrscheinlichkeiten bei diskreten Einzahlungen}

Bei den bisher verwendeten random walk Modellen des Typs $K_{t}=K_{t-1}+z_{t}$ hängt der Zustand der endogenen Variablen $K$ in der Stufe $t$ von einer Zufallsveränderlichen $z$ und dem Zustand in der Vorperiode ab. Weiter zurückliegende Stufen beeinflussen $K_{t}$ nicht unmittelbar. Daher erfüllt ein solches Modell die Voraussetzung, die an einfache eindimensionale Markovprozesse gestellt werden.

Markovprozesse bezeichnen ein mathematisches Modell für stochastische Prozesse, die nicht in die mittelbare Zukunft fortwirken. ${ }^{167}$ Dies hat zwei Implikationen, die im Grunde äquivalent sind:

a) Die Zufallsgröße $K_{t}$ wird außer vom Zufallsterm ausschließlich von ihrer Ausprägung in der unmittelbaren Vorstufe bestimmt. Daher ist die Position von $K_{t}$ auf der Zeitachse unerheblich:

$$
K_{r}=K_{s} \text {, wenn } k_{r-1}=k_{s-1} \forall r, s \leq T \text {. }
$$

Der Großbuchstabe $K$ bezeichnen die Zufallsveränderliche $K$ und die Gesamtheit ihrer Eigenschaften in den Perioden $r$ bzw. $s$, der Kleinbuchstabe $k$ die Realisation der Zufallsveränderlichen. Die Zufallsvariable $K_{r}$ und $K_{s}$ haben daher die gleichen stochastischen Eigenschaften, wenn ihre Realisationen in der Vorperiode identisch waren. Die Realisationen in der aktuellen Periode hingegen stimmen in der Regel nicht überein.

b) Der Pfad, auf dem $k_{t-1}$ erreicht wurde, hat keine Bedeutung. $k_{t-1}$ führt immer, unabhängig von seiner geschichtlichen Entwicklung, zur Zufallsgröße $K_{t}$. Insbesondere führt jedes beliebige $k_{t-2}$, das zu $k_{t-1}$ geführt hat, auch zu der Zufallsvariable $K_{t}$ :

$$
\left(K_{t} \mid k_{t-1}, k_{t-2, i}\right)=\left(K_{t} \mid k_{t-1}, k_{t-2, j}\right) \forall i, j
$$

oder allgemeiner:

$$
\left(K_{t} \mid k_{t-1}, k_{t-r, i}\right)=\left(K_{t} \mid k_{t-1}, k_{t-r, j}\right) \quad \forall i, j, r>1 .
$$

Markovketten sind eine Teilmenge von Markovprozessen. Bei Markovketten werden nur diskrete Zeitpunkte betrachtet. Daher können die bisher betrachteten Überlebenswahrscheinlichkeiten mit Hilfe von Markovketten dargestellt werden. Dies bietet den großen Vorteil, die Überlebenswahrscheinlichkeit in diskreten Modellen auch für höhere Runden relativ bequem berechnen zu können. ${ }^{168}$ Betrachten wir dazu wieder das Modell, das wir schon zuvor verwendet haben:

$$
\begin{array}{ll}
K_{t} & =K_{t-1}+z_{t}, K_{0}=0, z_{t} \sim \operatorname{uiid}\{-2,-1,0,1,2\} \\
\mathrm{p}_{\text {ül }}(t) & \equiv \mathrm{p}\left(K_{t}>0 \wedge K_{t-1}>0 \wedge \ldots \wedge K_{1}>0\right) .
\end{array}
$$

\footnotetext{
167 FERSCHL (1977) nennt sie „Prozesse ohne Nachwirkungen“.

168 Voraussetzung ist ein Rechner, der Matrizen quadrieren kann.
} 
Nach der ersten Runde sind fünf Kapitalbestände möglich: -2, -1, 0, 1 und 2. Diese Kapitalbestände werden auch Zustände genannt. Von diesen fünf Zuständen sind die unteren drei nichtpositiv und führen zum Ausscheiden. Zur Vereinfachung sei angenommen, daß nichtpositive Kapitalbestände in den nächsten Runden unverändert bleiben. Somit beträgt die Wahrscheinlichkeit, daß ein nichtpositiver Kapitalbestand in der Folgerunde in einen anderen Kapitalbestand übergeht, Null und daß er seinen Zustand beibehält, Eins. Die Wahrscheinlichkeit des Übergangs einer Variablen in einen anderen Zustand beim Wechsel der Periode wird als Übergangswahrscheinlichkeit bezeichnet. Die Summe der Übergangswahrscheinlichkeiten für alle in der nächsten Runde möglichen Zustände beträgt Eins. Für nichtpositive Zustände von $K$ beträgt die Übergangswahrscheinlichkeit für jegliche Veränderung Null, da sie sich annahmegemäß nicht mehr verändern, und für keine Veränderung Eins. Dies ist Tabelle 215.1 für die Übergänge von Periode 1 nach 2 dargestellt. Die Insolvenzkapitalbestände der ersten Runde $(-2,-1,0)$ sind schraffiert. In diesem Bereich lauten die Elemente der Hauptdiagonale - sie geben die Wahrscheinlichkeit einer Nicht-Veränderung an Eins, alle anderen Elemente sind Null.

Beträgt die erste Einzahlung $z_{1}$ Eins, können in der zweiten Runde die Kapitalbestände $-1,0,1,2,3$ mit gleicher Wahrscheinlichkeit erreicht werden, bei $z_{1}=2$ verschieben sich die möglichen Kapitalstände um Eins nach rechts. Die Wahrscheinlichkeiten der Übergänge von positiven Kapitalbeständen in Runde $1 \mathrm{zu}$ den in Runde 2 sind ebenfalls Tabelle 215.1 dargestellt.

\section{Tabelle 215.1: Übergangswahrscheinlichkeiten in einem einfachen diskreten Überlebensmodell}

\begin{tabular}{|c|c|c|c|c|c|c|c|c|c|c|c|}
\hline & \multicolumn{7}{|c|}{$K_{2}$} & \multirow{2}{*}{$\frac{1}{\mathrm{p}\left(K_{2}>0 \mid K_{1}>0\right)}$} & \multirow{2}{*}{$\begin{array}{c}\text { (2) } \\
\mathrm{p}\left(K_{1}\right)\end{array}$} & \multirow[b]{2}{*}{ (1) - (2) } \\
\hline & & -2 & -1 & 0 & 1 & 2 & 3 & 4 & & & \\
\hline \multirow{5}{*}{$K_{1}$} & -2 & 1 & 0 & 0 & 0 & 0 & & & 0 & .2 & 0 \\
\hline & -1 & 0 & 1 & 0 & 0 & 0 & & & 0 & .2 & 0 \\
\hline & 0 & 0 & 0 & 1 & 0 & 0 & & & 0 & .2 & 0 \\
\hline & 1 & 0 & .2 & .2 & .2 & .2 & .2 & & .6 & .2 & .12 \\
\hline & 2 & 0 & 0 & .2 & .2 & .2 & .2 & .2 & .8 & .2 & .16 \\
\hline \multicolumn{11}{|c|}{ Summe: } & .28 \\
\hline \multicolumn{12}{|c|}{$K_{t}=K_{t-1}+z_{t}, K_{0}=0, z_{t} \sim \operatorname{uiid}\{-2 \ldots 2\}$} \\
\hline
\end{tabular}

Rechts der gestrichelten Linie in der Matrix beträgt das Kapital in der zweiten Runde mehr als Null. Gleiches gilt für das Kapital in der ersten Runde unterhalb des schraffierten Bereichs. Der „Überlebensteil“ der Matrix befindet sich daher im südöstlichen Teil der Matrix. Die Übergangswahrscheinlichkeiten sind hier kursiv dargestellt. 
Wird mit $K_{1}=1$ in die zweite Runde gestartet, lautet die Überlebenswahrscheinlichkeit für diese Runde 0.6, bei einem Startwert von zwei lautet sie 0.8 (Spalte (1)). Werden diese bedingten Wahrscheinlichkeiten mit der Wahrscheinlichkeit des zugehörigen Startkapitals gewichtet (Spalte (2) und aufsummiert, ergibt sich für die Überlebenswahrscheinlichkeit bis zum Ende der zweiten Runde der Wert 0.28 (rechts unten).

Die Überlebenswahrscheinlichkeiten von Periode 1 zu Periode 3 ergeben sich durch Quadrierung der Übergangsmatrix von Periode 1 nach 2. Die Übergangsmatrix der Tabelle 215.1 enthält nur Kapitalbestände bis Vier, nach Periode drei aber kann das Kapital maximal Sechs betragen. Daher wird die Übergangsmatrix um zwei Spalten ergänzt. Die neue Matrix ist (wie die ursprüngliche) nicht quadratisch (dies ist bekanntlich eine Voraussetzung zur Quadrierung). Folglich sind der Matrix vier Zeilen hinzuzufügen. Durch diese Erweiterung enthält die Matrix viele nichtmögliche Übergänge. Sie erhalten die Wahrscheinlichkeit Null.

Die erweiterte Matrix kann nun quadriert werden. Das Ergebnis ist in Tabelle 216.1 dargestellt. Die Tabelle beschreibt alle Kapitalbestände, die nach der dritten Runde auftreten können (in der zweiten, invers dargestellten Zeile), und ihre Wahrscheinlichkeit in Abhängigkeit von der Einzahlung in der ersten Runde. Weniger interessant sind die Zeilen 1 bis 3, da definitionsgemäß für $K_{1} \leq 0$ das System falliert, und die unteren vier Zeilen, da Kapitalbestände größer als zwei in der ersten Runde nicht möglich sind. Sie wurden, wie erläutert, nur eingefügt, um die Quadrierung zu ermöglichen, inhaltlich sind sie ohne Bedeutung.

Tabelle 216.1: Übergangswahrscheinlichkeiten von Periode 1 nach 3

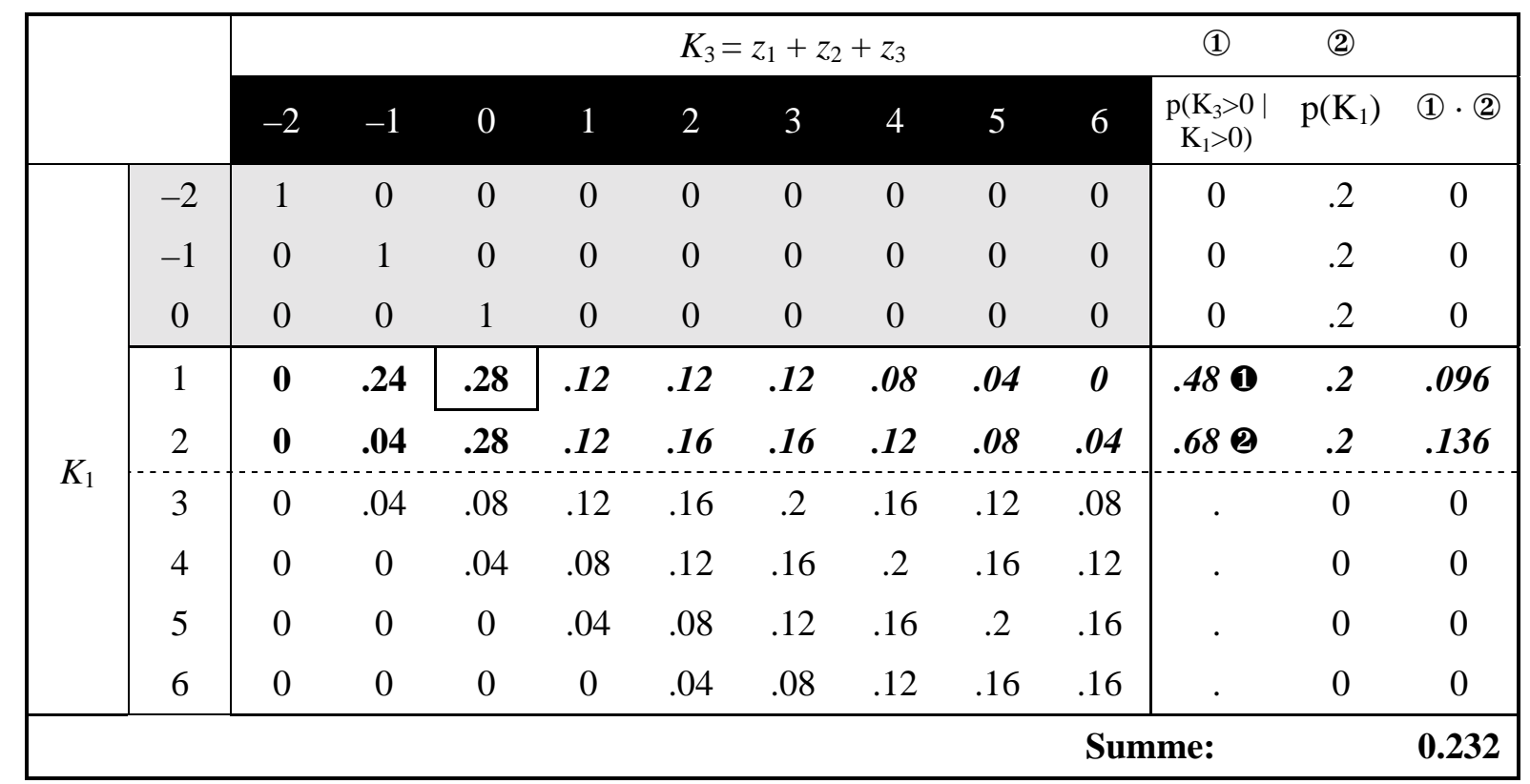

Die Überlebenswahrscheinlichkeit bis zum Ende der dritten Runde ist in den fett gedruckten Zeilen 4 und 5 unterhalb der schraffierten Fläche ausgewiesen. In den ersten drei Spalten dieser beiden Zeilen ist das Kapital in der dritten Runde kleiner 
oder gleich Null, so daß hier das System ebenfalls falliert. Das Modell überlebt in dieser Darstellung, wenn $K_{3}$ größer als Null und $K_{1}$ Element der Menge $\{1,2\}$ ist.

Zur Erläuterung der Übergangsmatrix sei eine Zelle exemplarisch hergeleitet: Die Übergangswahrscheinlichkeit eines Kapitalbestandes von 1 in der ersten Runde hin zu einem Kapitalbestand von Null in der dritten Runde, in Tabelle 216.1 durch den umrahmten Wert gekennzeichnet. Gegeben ist also ein Kapitalbestand von Eins nach der ersten Runde. In der zweiten Runde sind (s.o.) Einzahlungen von -2, -1, 0, 1 oder 2 möglich, so daß in dieser Runde gleichwahrscheinliche Kapitalbestände von $-1,0,1$, 2 oder 3 auftreten können. Die in Periode 3 möglichen Kapitalbestände sind in Tabelle 217.1 dargestellt, dabei ist die Regel beibehalten, daß nicht-positive Kapitalbestände in einer Runde in den Folgeperioden nicht verändert werden. Dadurch ist sichergestellt, daß positive Kapitalbestände in $t=3$ nicht auf unzulässige Weise erreicht werden, z.B. würde der Einzahlungspfad $K_{3}=z_{1}+z_{2}+z_{3}=1+(-1)+1=1$ die Überlebensbedingung nicht erfüllen. Ist ein Kapital (nicht Kapitalzuwachs) in einer Periode nichtpositiv, kann kein positiver Kapitalstand mehr erreicht werden.

Tabelle 217.1: Bedingte Kapitalbestände in der dritten Runde, Bedingung: $K_{1}=1$

\begin{tabular}{|c|c|ccccc|}
\hline \multicolumn{2}{|c|}{$z_{2} \rightarrow$} & -2 & -1 & 0 & 1 & 2 \\
\cline { 2 - 6 } & $K_{2} \rightarrow$ & -1 & 0 & 1 & 2 & 3 \\
\hline \hline \multirow{3}{*}{$z_{3}$} & -2 & -1 & 0 & -1 & 0 & 1 \\
& -1 & -1 & 0 & 0 & 1 & 2 \\
& 0 & -1 & 0 & 1 & 2 & 3 \\
& 1 & -1 & 0 & 2 & 3 & 4 \\
& 2 & -1 & 0 & 3 & 4 & 5 \\
\hline \multicolumn{7}{|c|}{ kursive Zahlen: Kapitalbestände in Periode 3, } \\
& \multicolumn{5}{c}{ Schraffur: $K_{3}=0}$. \\
\hline
\end{tabular}

Das Ereignis $K_{3}=0$ tritt 7 mal auf. Unter der Bedingung $K_{1}=1$ kann $K_{3}$ als $z_{3}+z_{2}+$ 1 geschrieben werden. Insgesamt sind für $\left\{K_{3} \mid K_{1}=1\right\} 25$ Möglichkeiten vorhanden. Folglich resultiert für das Ereignis $K_{3}=0$ die Wahrscheinlichkeit $7 / 25=0.28$. Auf ähnliche Weise können die anderen Übergangswahrscheinlichkeiten der Tabelle 216.1 bestimmt werden.

Aus den Übergangswahrscheinlichkeiten ergibt sich die bedingte Überlebenswahrscheinlichkeit (Spalte (1)). Gegeben $K_{1}$, beträgt sie für die dritte Runde (s. (1) in Tabelle 216.1]:

$$
\mathrm{p}\left(K_{3}>0 \wedge K_{2}>0 \mid K_{1}=1\right)=0.12+0.12+0.12+0.08+0.04+0=0.48
$$

und gegeben $K_{1}=2$ (s. (2) in Tabelle 216.1):

$$
\mathrm{p}\left(K_{3}>0 \wedge K_{2}>0 \mid K_{1}=2\right)=0.12+0.16+0.16+0.12+0.08+0.04=0.68 \text {. }
$$


Werden diese beiden bedingten Überlebenswahrscheinlichkeiten mit den (gleichen) Wahrscheinlichkeiten für $K_{1}=1$ bzw. 2 gewichtet und anschließend aufsummiert (Spalte (2), ergibt sich als Überlebenswahrscheinlichkeit bis zur dritten Runde 0.232.

Auf entsprechende Weise kann die Überlebenswahrscheinlichkeit für vier und mehr Runden bestimmt werden. In Schaubild 2181 ist dies dargestellt. Es zeigt sich, daß die Überlebenswahrscheinlichkeit mit zunehmendem $t$ rasch gegen Null konvergiert.

\section{Schaubild 218.1: Übergangswahrscheinlichkeiten in einem einfachen diskreten Überlebensmodell in Abhängigkeit von der Zeit}

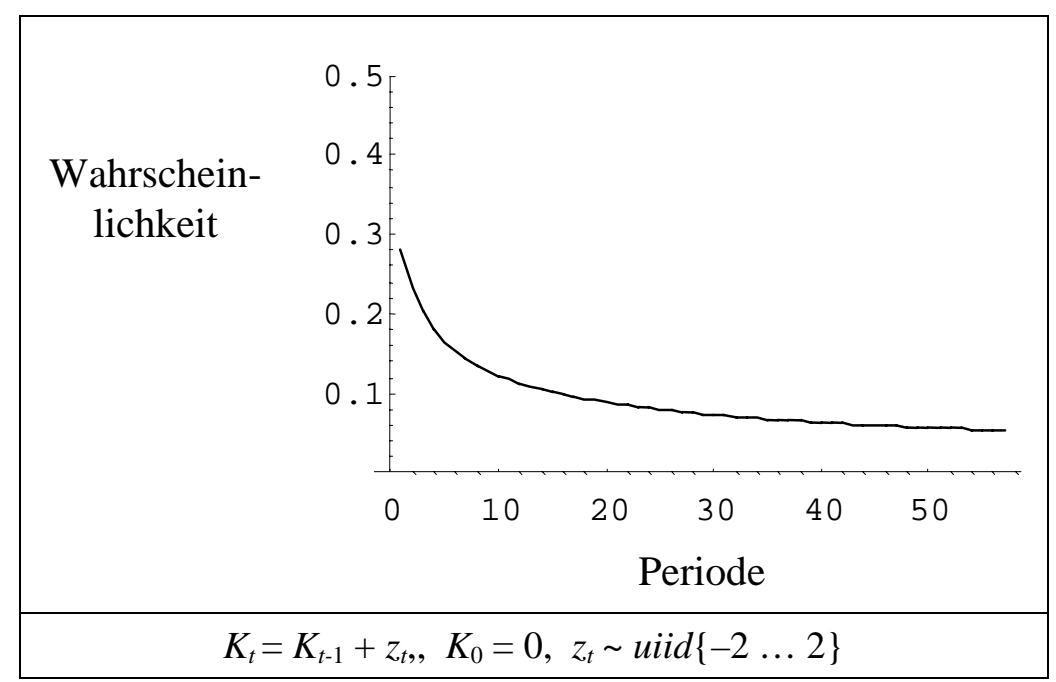

Wichtig ist zum Abschluß festzuhalten, daß diese Überlebenswahrscheinlichkeiten analytisch ermittelt sind. Sie sind daher, von Rundungsfehlern abgesehen, exakt. Dies ist ein wesentlicher Unterschied zu den Ergebnissen der Monte-Carlo-Simulationen, die in den nächsten beiden Abschnitten vorgestellt werden und eine, zum Großteil unbekannte Varianz aufweisen. Im nächsten Abschnitt werden die in Abschnitt 5.1 abgeleiteten Grundsätze auf konkrete Differenzengleichungen übertragen. Die Abhandlung erfolgt getrennt für additive und multiplikative Einzahlungen.

\subsection{Konkrete Beispiele mit einer stochastischen Einzahlung}

Im vorvorigen Abschnitt wurden drei Typen von einfachen Überlebensmodellen vorgestellt:

(a) Modelle ohne dynamische Verknüpfung

$$
K_{t} \quad=z_{t}-a
$$

(b) Modelle mit additiver Verknüpfung

$$
K_{t} \quad=K_{t-1}+z_{t}-a
$$

(c) Modelle mit multiplikativer Verknüpfung

$K_{t} \quad=K_{t-1} \cdot\left(1+z_{t}\right)-a$. 
Bei allen Modellen erfolgt eine Differenzierung zwischen den Varianten $K_{0}=0$ und $K_{0}>0$ sowie $a=0$ und $a>0$. Modell (a) ist bereits an einem Beispiel illustriert worden (vgl. Schaubild 1941. Die Modelle (b) und (c) werden nun auf Beispiele angewendet, wobei zwischen stetigen und diskreten Verteilungen unterschieden wird. Auszahlungen sind nur in Simulationsbeispielen für die Modelle (b) und (c) berücksichtigt.

\subsubsection{Additive Verknüpfung zwischen Kapital und Einzahlung}

Wir betrachten in diesem Unterabschnitt ausschließlich Modelle, in denen der Erwartungswert der Einzahlung nicht vom aktuellen Kapitalbestand abhängt (Modell b). Begonnen wird mit diskreten Einzahlungen, zunächst ein Beispiel mit größerer, dann eines mit kleiner Spanne der Einzahlungen, schließlich mit beliebiger Varianz. Den Abschluß bildet ein Beispiel mit stetigen Einzahlungen.

Die Überlebenswahrscheinlichkeit wird für diese Beispiele analytisch abgeleitet oder enumerativ ermittelt. Dies bedeutet, daß, von einer Ausnahme abgesehen, die Überlebenswahrscheinlichkeit nur für die ersten beiden Perioden bestimmt werden kann. Daher wird der Unterabschnitt durch die Vorstellung von Monte-Carlo-Simulationsergebnissen für höhere Perioden abgerundet.

\subsubsection{Ganzzahlige Einzahlungen zwischen -2 und +2}

Für die ersten beiden Runden gelte für das Kapital:

$\begin{array}{ll}K_{1} & =z_{1} \\ K_{2} & =K_{1}+z_{2}=z_{1}+z_{2} .\end{array}$

In Tabelle 220.1 sind die Kapitalbestände in der zweiten Runde für alle Kombinationen von $z_{1}$ und $z_{2}$ aufgeführt.

Für dieses Beispiel ist die Wahrscheinlichkeit eines positiven Kapitalbestandes in der ersten Runde gleich der Wahrscheinlichkeit einer positiven Einzahlung: $\mathrm{p}\left(K_{1}>0\right)$ $=\mathrm{p}\left(z_{1}>0\right)$. Da die Einzahlungen identisch und unabhängig verteilt sind, ist die Wahrscheinlichkeit für $z_{1}>0$ gleich der Wahrscheinlichkeit für $z_{2}>0$. Sie beträgt $2 / 5$. (Die bedingte Wahrscheinlichkeit für $z_{2}>0$, gegeben $z_{1}$, lautet wegen der stochastischen Unabhängigkeit zwischen $z_{1}$ und $z_{2}$ ebenfalls $2 / 5$.)

Überleben bedeutet das gemeinsame Eintreten der beiden Ereignisse $\left\{K_{1}>0\right\}$ und $\left\{K_{2}>0\right\}$. Die Gemeinsamkeit des Eintretens kann durch das Mengensymbol „へ“ ausgedrückt werden. Die Wahrscheinlichkeit des zweiten Ereignisses wird durch den Ausgang des ersten Ereignisses beeinflußt, daher sind beide Ereignisse nicht stochastisch unabhängig.

Für das zweite Ereignis, ein positiver Kapitalbestand in der zweiten Runde, gibt es zwei Möglichkeiten:

(a) beide Einzahlungen sind größer als Null: $\left\{z_{1}>0\right\} \cap\left\{z_{2}>0\right\}$,

(b) es sind zwar nicht beide Einzahlungen positiv, aber ihre Summe ist größer als Null: $\left\{z_{1}+z_{2}>0\right\}$. 
Tabelle 220.1: Kapital in der zweiten Runde in Abhängigkeit von den Einzahlungen und Auszählen der Überlebenswahrscheinlichkeit

\begin{tabular}{|c|c|c|c|c|c|c|}
\hline$i$ & $z_{1} z_{2}$ & -2 & -1 & $\mathbf{0}$ & 1 & 2 \\
\hline 1 & -2 & -4 & -3 & -2 & -1 & 0 \\
\hline 2 & -1 & -3 & -2 & -1 & 0 & 1 \\
\hline 3 & 0 & -2 & -1 & 0 & 1 & 2 \\
\hline 4 & 1 & -1 & 0 & 1 & 2 & 3 \\
\hline 5 & 2 & 0 & 1 & 2 & 3 & 4 \\
\hline
\end{tabular}

Legende:

relative Häufigkeit

\begin{tabular}{|c|c|c|}
\hline helle Schraffur & Pleite in der ersten Runde $\left(K_{1} \leq 0\right)$ & $15 / 25$ \\
\hline dunkle Schraffur & Pleite in der zweiten Runde & $3 / 15$ \\
\hline mit (irgendeiner) Schraffur & Pleite-Wahrscheinlichkeit insgesamt & $18 / 25$ \\
\hline unterhalb des fetten Striches & $K_{2}>0$ & $10 / 25$ \\
\hline ohne Schraffur & Überlebensfälle für zwei Runden & $7 / 25$ \\
\hline
\end{tabular}

Für das Berechnen des gemeinsamen Eintretens von $\left\{K_{1}>0\right\}$ und $\left\{K_{2}>0\right\}$, also $\left\{K_{1}>0\right\} \cap\left\{K_{2}>0\right\}$, können ebenfalls die Bedingungen (a) und (b) benutzt werden. Allerdings mit einer Einschränkung: von (b) können nur die Fälle berücksichtigt werden, in denen $z_{1}>0$ ist, da das Gegenereignis $z_{1} \leq 0$ und die Bedingung (a) sich gegenseitig ausschließen. Die Bedingung (b) wird damit zu:

$$
\left\{z_{1}+z_{2}>0\right\} \backslash\left\{z_{1} \leq 0\right\}=\left\{z_{1}+z_{2}>0\right\} \cap\left\{z_{1}>0\right\} .
$$

In dieser Formulierung ist die Schnittmenge von (a) und ( $b^{\prime}$ ) nicht leer. Um dies zu erreichen, sind aus ( $b^{\prime}$ ) alle Fälle zu entfernen, die auch der Bedingung (a) genügen:

$\left(b^{\prime \prime}\right) \quad\left\{z_{1}+z_{2}>0\right\} \cap\left\{z_{1}>0\right\} \backslash\left(\left\{z_{1}>0\right\} \cap\left\{z_{2}>0\right\}\right)$.

$\left(\mathrm{b}^{\prime \prime}\right)$ sind letztlich alle Kombination von $z_{1}$ und $z_{2}$, wo die Summe und der erste Summand positiv sind, der zweite hingegen nicht.

$\left.\left(b^{\prime \prime \prime}\right) \quad\left\{z_{1}+z_{2}>0\right\} \cap\left\{z_{1}>0\right\} \cap\left\{z_{2} \leq 0\right\}\right)$.

Der Vorteil einer leeren Schnittmenge zwischen (a) und ( $\left.b^{\prime \prime \prime}\right)$ besteht darin, daß nun einfaches Addieren der beiden Möglichkeiten für das gemeinsame Eintreten von $\left\{K_{1}\right\rangle$ $0\}$ und $\left\{K_{2}>0\right\}$, (a) und ( $\left.b^{\prime \prime \prime}\right)$, die Überlebenswahrscheinlichkeit für zwei Runden ergibt: 


$$
\begin{aligned}
\mathrm{p}_{\text {ül }}(2) \quad & =\mathrm{p}\left(\left\{K_{1}>0\right\} \cap\left\{K_{2}>0\right\}\right. \\
& =\mathrm{p}\left[(\mathrm{a}) \vee\left(\mathrm{b}^{\prime \prime \prime}\right)\right] \\
& =\mathrm{p}\left[\left(\left\{z_{1}>0\right\} \cap\left\{z_{2}>0\right\}\right) \cup\left(\left\{z_{1}+z_{2}>0\right\} \cap\left\{z_{1}>0\right\} \cap\left\{z_{2} \leq 0\right\}\right)\right] \\
& =\mathrm{p}\left(\left\{z_{1}>0\right\} \cap\left\{z_{2}>0\right\}\right)+\mathrm{p}\left(\left\{z_{1}+z_{2}>0\right\} \cap\left\{z_{1}>0\right\} \cap\left\{z_{2} \leq 0\right\}\right) .
\end{aligned}
$$

Wie aus Tabelle 2201 leicht durch Abzählen zu ermitteln ist, beträgt die erste Wahrscheinlichkeit 4/25. Die Bedingung $\left\{z_{1}+z_{2}>0\right\}$ erfüllen 10 Kombinationen, davon sind, um die zweite Wahrscheinlichkeit zu ermitteln, vier Fälle in der südöstlichen Ecke der Tabelle abzuziehen, die bereits durch Bedingung (a) abgedeckt sind, und weitere drei, bei denen $z_{1}$ nicht größer als Null ist. Es verbleiben somit drei Kombinationen. Die zweite Wahrscheinlichkeit ist also 3/25 und die gesamte Wahrscheinlichkeit 7/25. Diese Überlebenswahrscheinlichkeit wird in der Tabelle durch den Bereich ohne Schraffur angezeigt.

Die Überlebenswahrscheinlichkeit kann nach 1521, 151.5 und 151.2 auch geschrieben werden als

$$
\begin{aligned}
\mathrm{p}_{\text {ül }}\left(K_{2}>0\right) & \equiv \mathrm{p}\left(K_{2}>0 \wedge K_{1}>0\right) \\
& =\frac{\mathrm{p}\left[\left\{K_{1}>0\right\} \cap\left\{K_{2}>0\right\}\right]}{\mathrm{p}\left(K_{1}>0\right)} \cdot \mathrm{p}\left(K_{1}>0\right) \\
& =\mathrm{p}\left(K_{2}>0 \mid K_{1}>0\right) \cdot \mathrm{p}\left(K_{1}>0\right) .
\end{aligned}
$$

Da in diesem Beispiel $K_{1}$ und $z_{1}$ übereinstimmen, kann auch geschrieben werden:

$$
\mathrm{p}_{\text {ül }}\left(K_{2}>0\right)=\mathrm{p}\left(K_{2}>0 \mid z_{1}>0\right) \cdot \mathrm{p}\left(z_{1}>0\right) \text {. }
$$

$K_{2}$ ist eine Funktion von $K_{1}$ und daher stochastisch abhängig von $K_{1}$. Folglich ist der Ausdruck $\mathrm{p}\left(K_{2}>0 \wedge K_{1}>0\right)$ nicht äquivalent zu p $\left(K_{2}>0\right) \cdot \mathrm{p}\left(K_{1}>0\right) . z_{1}$ und $z_{2}$ hingegen sind stochastisch unabhängig.

Die bedingte Wahrscheinlichkeit in der letzten Formel kann leicht in Tabelle 220.1 abgezählt werden: sie beträgt 7/10. Mit $\mathrm{p}\left(z_{1}>0\right)=2 / 5$ folgt für die Überlebenswahrscheinlichkeit bis zur zweiten Runde:

$$
\mathrm{p}_{\mathrm{uul}}\left(K_{2}>0\right)=\frac{7}{10} \cdot \frac{2}{5}=\frac{7}{25} \text {. }
$$

Ein anderer Weg, die Überlebenswahrscheinlichkeit zu bestimmen, verläuft über den Bayes-Ansatz, der auf den Seite 152 und 196f beschrieben ist. Einfache arithmetische Umformungen führen $\mathrm{zu}$ :

$$
\begin{aligned}
\mathrm{p}_{\mathrm{uil}}\left(K_{2}>0\right) & \equiv \mathrm{p}\left(K_{2}>0 \wedge K_{1}>0\right) \\
& =\mathrm{p}\left(z_{1}+z_{2}>0 \wedge z_{1}>0\right) \\
& =\mathrm{p}\left(z_{2}>-z_{1} \wedge z_{1}>0\right) .
\end{aligned}
$$


Tabelle 222.1: Berechnung der Überlebenswahrscheinlichkeit für die zweite Runde nach dem Bayes-Ansatz, ohne Startkapital

\begin{tabular}{|c|c|c|c|c|c|}
\hline$i$ & $z_{1 i}$ & $\mathrm{p}\left(z_{1 i}\right)$ & $-z_{1 i}$ & $\mathrm{p}\left(z_{2}>-z_{1 i}\right)$ & $\mathrm{p}\left(z_{2}>-z_{1 i}\right) \cdot \mathrm{p}\left(z_{1 i}\right)$ \\
\hline 1 & -2 & $1 / 5$ & 2 & 0 & 0 \\
\hline 2 & -1 & $1 / 5$ & 1 & $1 / 5$ & $1 / 25$ \\
\hline 3 & 0 & $1 / 5$ & 0 & $2 / 5$ & $2 / 25$ \\
\hline 4 & 1 & $1 / 5$ & -1 & $3 / 5$ & $3 / 25$ \\
\hline 5 & 2 & $1 / 5$ & -2 & $4 / 5$ & $4 / 25$ \\
\hline Summen: & $\begin{array}{l}\sum_{z_{1 i}} \\
\sum_{z_{1 i}>0}\end{array}$ & \multicolumn{3}{|c|}{ (nicht schraffierter Bereich) } & $\begin{array}{l}10 / 25 \\
7 / 25\end{array}$ \\
\hline \multicolumn{6}{|c|}{$K_{1}=z_{1}, K_{2}=z_{1}+z_{2}, z_{i} \sim \operatorname{uiid}\{-2,-1,0,1,2\}$} \\
\hline
\end{tabular}

Daraus folgt nach (19T.3] in Verbindung mit [131.5] für diskrete Verteilungen:

$$
\mathrm{p}_{\text {ül }}\left(K_{2}>0\right)=\sum_{z_{i}>0} \mathrm{p}\left(z_{2}>-z_{1 i} \mid z_{1}\right) \cdot \mathrm{p}\left(z_{1 i}\right)
$$

Die Summationsbedingung $z_{i}>0$ ist, wie aus Tabelle 222.1 leicht abzulesen ist, nur für $i=4$ und für $i=5$ erfüllt. Daher folgt:

$$
\begin{aligned}
\mathrm{p}_{\text {ül }}\left(K_{2}>0\right) & =\sum_{i=4}^{5} \mathrm{p}\left(z_{2}>-z_{1 i}\right) \cdot \mathrm{p}\left(z_{1 i}\right) \\
& =3 / 5 \cdot 1 / 5+4 / 5 \cdot 1 / 5 \\
& =7 / 25 .
\end{aligned}
$$

Die Überlebenswahrscheinlichkeit bis zur zweiten Runde beträgt also für ein Modell mit fünf ganzzahligen, gleichwahrscheinlichen, neutralen Einzahlung ohne Startkapital 0.28. Es stellt sich die Frage, wie stark ein positives Startkapital die Überlebensfähigkeit verbessert.

Das Modell ist nun gegeben durch:

$$
\begin{array}{ll}
K_{1} & =K_{0}+z_{1} \\
K_{2} & =K_{1}+z_{2}=K_{0}+z_{1}+z_{2} \\
z_{i} & \sim \operatorname{uiid}\{-2,-1,0,1,2\} .
\end{array}
$$

Die Überlebensbedingungen lauten, vgl. (204,1) und (131,3):

$$
\begin{aligned}
& \mathrm{p}_{\text {ül }}\left(K_{1}>0\right)=\mathrm{p}\left(z_{1}>-K_{0}\right) \\
& \mathrm{p}_{\text {ül }}\left(K_{2}>0\right)=\mathrm{p}\left[\left(z_{1}>-K_{0}\right) \wedge\left(z_{2}>-z_{1}-K_{0}\right)\right]
\end{aligned}
$$




$$
=\sum_{z_{1}}>-K_{0} \mathrm{p}\left(z_{2}>-z_{1}-K_{0}\right) \cdot \mathrm{f}\left(z_{1}\right)
$$

Die Auswirkungen des Startkapitals auf die Überlebenswahrscheinlichkeit der ersten Runde sind im vorvorigen Abschnitt durch die Formel

$$
\mathrm{p}_{\ddot{\mathrm{uil}}, k}(1) \quad=\mathrm{p}\left(z_{1}>-K_{0}\right)=\frac{k+K_{0}}{2 k+1}
$$

quantifiziert worden. Um die Überlebenswahrscheinlichkeit für die zweite Runde zu bestimmen, ist in Tabelle 223,1 das Analogon zu Tabelle 222,1. mit der Modifikation $K_{0}=1$ dargestellt.

Tabelle 223.1: Berechnung der Überlebenswahrscheinlichkeit für die zweite Runde nach dem Bayes-Ansatz, mit Startkapital

\begin{tabular}{|c|ccccc|}
\hline$i$ & $z_{1 i}$ & $\mathrm{p}\left(z_{1 i}\right)$ & $-\left(z_{1 i}+K_{0}\right)$ & $\mathrm{p}\left(z_{2}>-z_{1 i}-K_{0}\right)$ & $\begin{array}{c}\mathrm{p}\left(z_{2}>-z_{1 i}-\right. \\
\left.K_{0}\right) \cdot \mathrm{p}\left(z_{1 i}\right)\end{array}$ \\
\hline 1 & -2 & $1 / 5$ & 1 & $1 / 5$ & $1 / 25$ \\
2 & -1 & $1 / 5$ & 0 & $2 / 5$ & $2 / 25$ \\
3 & 0 & $1 / 5$ & -1 & $3 / 5$ & $3 / 25$ \\
4 & 1 & $1 / 5$ & -2 & $4 / 5$ & $4 / 25$ \\
5 & 2 & $1 / 5$ & -3 & 1 & $5 / 25$ \\
\hline Summen: & $\sum$ & & & $15 / 25$ \\
& $z_{1 i}$ & & & \\
& $z_{1 i}>-K_{0}$ & & \\
& $K_{1}=K_{0}+z_{1}, K_{2}=K_{0}+z_{1}+z_{2}, z_{i} \sim$ uiid $\{-2,-1,0,1,2\}, K_{0}=1$ \\
\hline
\end{tabular}

Die Summationsbedingung $z_{i}>0$ ist, wie aus der Tabelle leicht abzulesen ist, nur für $i=3,4$ und 5 erfüllt:

$$
\begin{aligned}
\mathrm{p}_{\text {ül }}\left(K_{2}>0\right) & =\sum_{i=3}^{5} \mathrm{p}\left(z_{2}>-z_{1}-K_{0}\right) \cdot \mathrm{f}\left(z_{1}\right) \\
& =3 / 5 \cdot 1 / 5+4 / 5 \cdot 1 / 5+1 \cdot 1 / 5 \\
& =12 / 25 .
\end{aligned}
$$

Die Überlebenswahrscheinlichkeit bis zur zweiten Runde beträgt also für ein Modell mit einem Startkapital von 1 und fünf ganzzahligen, gleichwahrscheinlichen, neutralen Einzahlungen $0.48 \mathrm{im}$ Vergleich zu 0.28 ohne Startkapital. Für weitere 
Startkapitale ergeben sich durch analoge Berechnungen folgende Überlebenswahrscheinlichkeiten:

Tabelle 224.1: Konkrete Berechnung der Überlebenswahrscheinlichkeit für die zweite Runde nach dem Bayes-Ansatz für variierte Startkapitale

\begin{tabular}{|c|c|c|c|c|c|c|c|c|}
\hline Startkapital & -2 & -1 & 0 & 1 & 2 & 3 & 4 & 5 \\
\hline Überlebenswahrscheinlichkeit & 0 & $3 / 25$ & $7 / 25$ & $12 / 25$ & $17 / 25$ & $22 / 25$ & $24 / 25$ & 1 \\
\hline
\end{tabular}

Die Berechnungen hierzu sind im Anhang, Abschnitt 9.3 wiedergegeben.

Steht zu Beginn ein Startkapital von fünf oder mehr zur Verfügung, kann es in zwei Perioden nicht aufgezehrt werden; die Überlebenswahrscheinlichkeit beträgt daher 1 . Wird auf der anderen Seite mit einem Kapital von -2 oder weniger gestartet, falliert das System bereits mit Sicherheit in der ersten Runde. Schaubild 224 1 visualisiert diesen Zusammenhang, der einen leicht sigmoiden Verlauf zeigt.

Schaubild 224.1: Überlebenswahrscheinlichkeit für zwei Runden in Abhängigkeit vom Startkapital, diskretes Modell

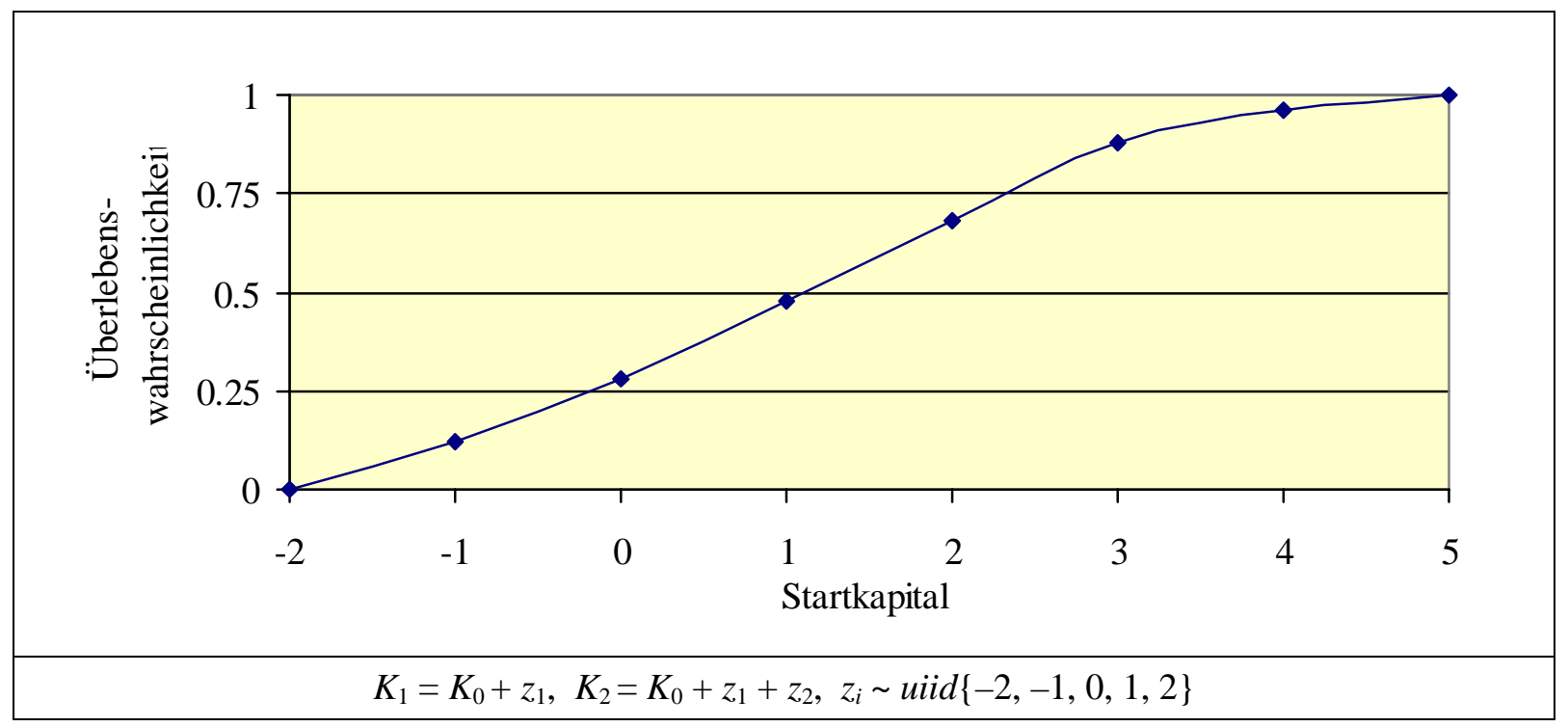

\subsubsection{Ganzzahlige gleichverteilte Einzahlungen zwischen -1 und +1}

Diese Einzahlungen unterscheiden sich vom vorigen Beispiel durch eine geringere Varianz. Wirkt sich diese Varianzreduktion auf die Überlebenswahrscheinlichkeit aus? Bei stetigen Verteilungen ist dies, wie oben gezeigt wurde, nicht der Fall. Die Berechnungen erfolgen ganz ähnlich wie bei dem vorherigen Beispiel anhand der Tabelle 225.1 und sind daher nur gerafft wiedergegeben. 
Tabelle 225.1: Kapital in der zweiten Runde in Abhängigkeit von den Einzahlungen und Auszählen der Überlebenswahrscheinlichkeit, kleinere Varianz

\begin{tabular}{|c|c||ccc|}
\hline$i$ & $\boldsymbol{z}_{\mathbf{1}} \boldsymbol{z}_{\mathbf{2}}$ & $\mathbf{- 1}$ & $\mathbf{0}$ & $\mathbf{1}$ \\
\hline \hline 1 & $-\mathbf{1}$ & -2 & -1 & 0 \\
2 & $\mathbf{0}$ & -1 & 0 & 1 \\
3 & $\mathbf{1}$ & 0 & 1 & 2 \\
\hline \multicolumn{5}{|c}{$K_{1}=z_{1}, K_{2}=z_{1}+z_{2}, z_{i} \sim \operatorname{uiid}\{-1,0,1\}$} \\
\hline
\end{tabular}

\begin{tabular}{|c|c|c|}
\hline \multicolumn{2}{|l|}{ Legende: } & relative \\
\hline & Pleite in der ersten Runde $\left(K_{1} \leq 0\right)$ & $6 / 9$ \\
\hline & Pleite in der zweiten Runde & $1 / 9$ \\
\hline mit (irgendeiner) Schraffur & Pleite-Fälle insgesamt & $7 / 9$ \\
\hline unterhalb des fetten Striches & $K_{2}>0$ & $3 / 9$ \\
\hline ohne Schraffur & Überlebensfälle für zwei Runden & $2 / 9$ \\
\hline
\end{tabular}

$$
\begin{array}{ll}
\mathrm{p}\left(z_{i}>0\right) & =1 / 3 \\
\mathrm{p}_{\text {ül }}(2) & =\mathrm{p}\left(\left\{K_{1}>0\right\} \cap\left\{K_{2}>0\right\}\right. \\
& =\mathrm{p}\left[\left(\left\{z_{1}>0\right\} \cap\left\{z_{2}>0\right\}\right) \cup\left(\left\{z_{1}+z_{2}>0\right\} \cap\left\{z_{1}>0\right\} \cap\left\{z_{2} \leq 0\right\}\right)\right] \\
& =\mathrm{p}\left(\left\{z_{1}>0\right\} \cap\left\{z_{2}>0\right\}\right)+\mathrm{p}\left(\left\{z_{1}+z_{2}>0\right\} \cap\left\{z_{1}>0\right\} \cap\left\{z_{2} \leq 0\right\}\right) .
\end{array}
$$

Wie aus Tabelle 2251 leicht durch Abzählen zu ermitteln ist, beträgt die erste Wahrscheinlichkeit 1/9. Die Bedingung $\left\{z_{1}+z_{2}>0\right\}$ erfüllen 3 Kombinationen. Um die zweite Wahrscheinlichkeit zu ermitteln, sind davon der Fall in der südöstlichsten Ecke der Tabelle abzuziehen, der bereits durch Bedingung (a) abgedeckt ist, und ein weiterer nördlich davon, wo $z_{1}$ nicht größer als Null ist. Somit verbleibt eine Kombination. Die zweite Wahrscheinlichkeit ist also 1/9 und die gesamte Wahrscheinlichkeit 2/9. Diese Überlebenswahrscheinlichkeit wird in der Tabelle durch den Bereich ohne Schraffur angezeigt.

Abzählen der Überlebenswahrscheinlichkeit nach (151.2 führt zu:

$$
\mathrm{p}_{\text {ül }}\left(K_{2}>0\right)=\mathrm{p}\left(K_{2}>0 \mid z_{1}>0\right) \cdot \mathrm{p}\left(z_{1}>0\right) \text {. }
$$

Für die bedingte Wahrscheinlichkeit ist ausschließlich die rechte Spalte der Tabelle $225.1 \mathrm{zu}$ betrachten, da nur hier $z_{1}$ positiv ist. In zwei von drei Fällen nimmt das Kapital unter dieser Bedingung positive Werte an. Also gilt:

$$
\mathrm{p}_{\text {ül }}\left(K_{2}>0\right)=2 / 3 \cdot 1 / 3=2 / 9 \text {. }
$$


Bei $z_{i} \in\{-1,0.1\}$ beträgt die Überlebenswahrscheinlichkeit für zwei Runden also $2 / 9 \approx 0.22$, bei $z_{i} \in\{-2,-1,0,1,2\}$ hingegen $7 / 25=0.28$ (s.o.). Die Überlebenswahrscheinlichkeit nimmt also bei diskreten Verteilungen mit der Varianz der Verteilung $\mathrm{zu}$.

Die Ursache liegt darin, daß bei diskreten, ganzzahligen, um Null symmetrischen Verteilungen dennoch die Überlebenswahrscheinlichkeit in der ersten Runde nicht $1 / 2$ beträgt, da $z_{1}=0$ für das Überleben per definitionem nicht ausreicht. Je größer die Spanne der Verteilung, desto mehr nähert sich die Überlebenswahrscheinlichkeit in der ersten Runde $1 / 2$ an:

$$
\lim _{k \rightarrow \infty}\left(\frac{k+1}{2 k+1}\right)=1 / 2
$$

und in der zweiten Runde $3 / 8$ [vgl. (198.7)] .

Die zeitliche Entwicklung der Überlebenswahrscheinlichkeit läßt sich für diskrete Einzahlungen auch über die zweite Periode hinaus leicht mit Hilfe des Konzepts der zellulären Automaten ${ }^{169}$ darstellen, wenn die Einzahlungen gleichwahrscheinlich sind. Dies sei an dem gerade behandelten Beispiel mit drei Einzahlungsmöglichkeiten demonstriert.

Ein bestimmter Kapitalbestand $K_{t}$ führt zu drei potentiellen Kapitalbeständen in der nächsten Runde. Dies bedeutet umgekehrt, daß die Wahrscheinlichkeit - oder bei vielen Wiederholungen die Häufigkeit - eines Kapitalbestandes in einer Runde von den Häufigkeiten benachbarter Kapitalbestände in der Vorperiode abhängt. Diese Art der Abhängigkeit kann einfach in Tabellenkalkulationsprogramme übertragen werden.

Tabelle 227.1 zeigt, wie mit Hilfe eines zellulären Automaten die Häufigkeiten von Kapitalbeständen in bestimmten Runden bestimmt werden können. Dargestellt sind nur Kapitalbestände, deren Entstehungspfad die Überlebensbedingung erfüllt. Die Zeilen für $t=2$ oder 3 seien stellvertretend erklärt.

In Runde 2 treten die Kapitalbestände „Eins“ und „Zwei“ jeweils einmal auf. In der nächsten Runde beträgt das Kapital 1, wenn der Vorperiodenkapitalbestand „Eins“ unverändert geblieben ist oder wenn der Vorperiodenkapitalbestand „Zwei“ und die folgende Einzahlung -1 lautete. (Ein Vorperiodenkapitalbestand von „Null“ braucht nicht berücksichtigt zu werden, da er die Überlebensbedingung nicht erfüllt.) Es gibt also zwei Möglichkeiten, die auf Überlebenspfaden liegen, für einen Kapitalbestand von „Eins“ in der dritten Runde. Entsprechend berechnen sich die Häufigkeiten für $K_{3}=2$ und $K_{3}=3$ und die anderen Perioden.

169 Vgl. GAYLORD \& NiSHIDATE (1996), die zelluläre Automaten mit Hilfe des ComputeralgebraProgramms Mathematica implementieren, sowie BALMANN (1994) Kap. 4.3. Zur Verwendung von Automaten zur Messung der Komplexität in nichtkooperativen Spielen vgl. KALAI (1997). 
Tabelle 227.1: Zeitliche Entwicklung der Verteilung von Kapitalbeständen

\begin{tabular}{|c|c|c|c|c|c|c|c|c|c|c|c|c|c|}
\hline \multirow{3}{*}{$t$} & \multirow{3}{*}{$\begin{array}{l}\text { Anz. } \\
\text { Ges. } \\
\text { KBest. }\end{array}$} & \multicolumn{10}{|c|}{ Kapitalbestand } & \multirow{3}{*}{$\begin{array}{c}\text { Anz. } \\
\text { Übl.K } \\
\text { Best. }\end{array}$} & \multirow{3}{*}{$\begin{array}{l}\text { Ül. } \\
\text { Wa. }\end{array}$} \\
\hline & & 1 & 2 & 3 & 4 & 5 & 6 & 7 & 8 & 9 & 10 & & \\
\hline & & \multicolumn{10}{|c|}{ Häufigkeit } & & \\
\hline 1 & 3 & 1 & & & & & & & & & & 1 & 0.333 \\
\hline 2 & 9 & 1 & 1 & & & & & & & & & 2 & 0.222 \\
\hline 3 & 27 & 2 & 2 & 1 & & & & & & & & 5 & 0.185 \\
\hline 4 & 81 & 4 & 5 & 3 & 1 & & & & & & & 13 & 0.160 \\
\hline 5 & 243 & 9 & 12 & 9 & 4 & 1 & & & & & & 35 & 0.144 \\
\hline 6 & 729 & 21 & 30 & 25 & 14 & 5 & 1 & & & & & 96 & 0.132 \\
\hline 7 & 2187 & 51 & 76 & 69 & 44 & 20 & 6 & 1 & & & & 267 & 0.122 \\
\hline 8 & 6561 & 127 & 196 & 189 & 133 & 70 & 27 & 7 & 1 & & & 750 & 0.114 \\
\hline 9 & 19683 & 323 & 512 & 518 & 392 & 230 & 104 & 35 & 8 & 1 & & 2122 & 0.108 \\
\hline 10 & 59049 & 835 & 1353 & 1422 & 1140 & 726 & 369 & 147 & 44 & 9 & 1 & 6036 & 0.102 \\
\hline
\end{tabular}

Anz.Ges.K.Best.: Anzahl aller möglichen Kapitalentwicklungspfade, berechnet als $3^{t}$ (Kombinationen mit Berücksichtigung der Anordnung). Jeder der Pfade hat die gleiche Wahrscheinlichkeit. $3^{t}$ ist gleichzeitig die Anzahl der Wiederholungen; auf diese Wiederholungsanzahl beziehen sich die Häufigkeiten im rechten Teil der Tabelle.

Anz.ÜblK.Best.: Anzahl der Kapitalentwicklungspfade, die die Überlebensbedingung erfüllen. Ül.Wa.: Überlebenswahrscheinlichkeit bis zur Runde $t$.

Interessant ist an den Ergebnissen, daß bei wenigen Perioden der häufigste Kapitalbestand zwei ist und sich dieser Modalwert mit zunehmender Rundenzahl langsam erhöht. Schaubild 227.1 macht dies für 6 und 10 Perioden deutlich. $N$ ist die Anzahl der Wiederholungen. Sie ist so gewählt, daß auf das jeweils maximale Kapital eine Häufigkeit von 1 fällt.

Schaubild 227.1: Häufigkeit von Kapitalbeständen nach sechs bzw. zehn Runden

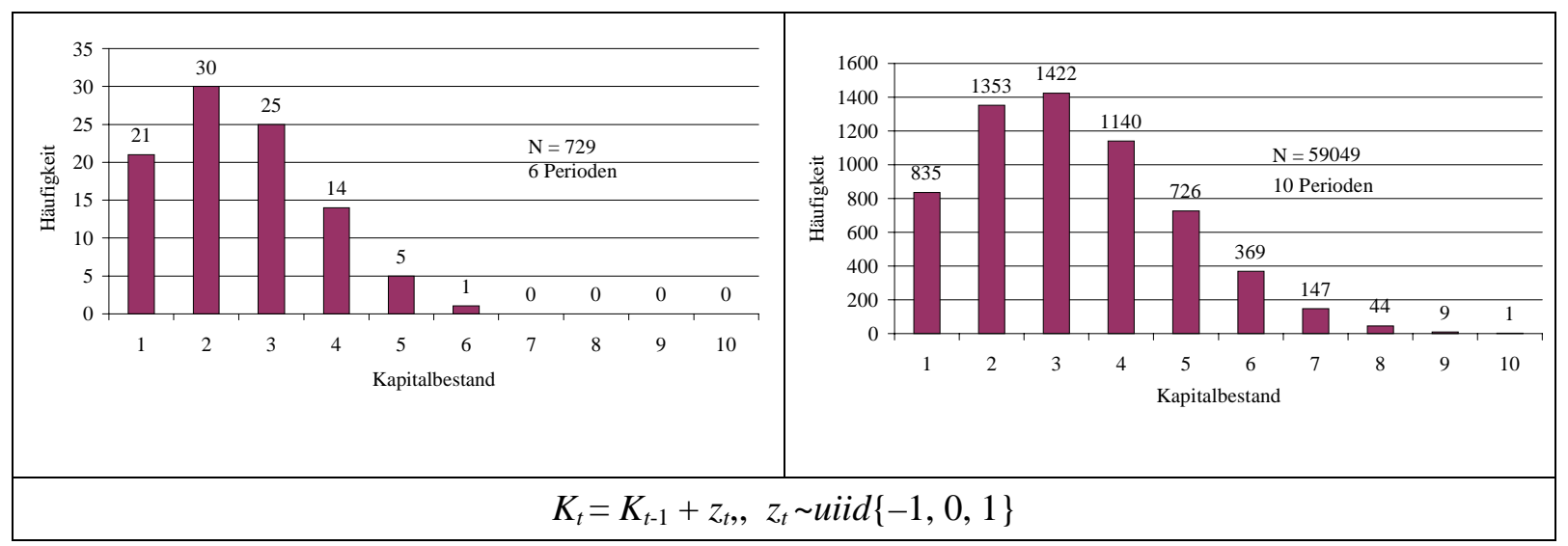




\subsubsection{Diskrete Gleichverteilung allgemein}

Wir hatten gesehen, daß bei einer gleichmäßigen Verteilung mit $z_{i} \in Z=\{-1,0,1\}$ die Überlebenswahrscheinlichkeit für zwei Runden $2 / 9$ und bei $Z=\{-2,-1,0,1,2\} 7 / 25$ beträgt. Tabelle 228.1 zeigt die Realisationen für $Z=\{-3,-2,-1,0,1,2,3\}$ und, im schraffierten Bereich, für $Z=\{-4,-3,-2,-1,0,1,2,3,4\}$.

Tabelle 228.1: Realisationen für die zweite Periode eines Systems mit diskreten gleichverteilten Zufallsveränderlichen

\begin{tabular}{|c|c||c|ccccccc|c|}
\hline$i$ & $z_{\mathbf{1}} \boldsymbol{z}_{\mathbf{2}}$ & $\mathbf{- 4}$ & $\mathbf{- 3}$ & $\mathbf{- 2}$ & $\mathbf{- 1}$ & $\mathbf{0}$ & $\mathbf{1}$ & $\mathbf{2}$ & $\mathbf{3}$ & $\mathbf{4}$ \\
\hline \hline 1 & $-\mathbf{4}$ & & & & & & & & & \\
$\vdots$ & $\vdots$ & & & & & & & & & \\
5 & $\mathbf{0}$ & & & & & & & & & \\
6 & $\mathbf{1}$ & & -2 & -1 & 0 & 1 & 2 & 3 & 4 & 5 \\
7 & $\mathbf{2}$ & & -1 & 0 & 1 & 2 & 3 & 4 & 5 & 6 \\
8 & $\mathbf{3}$ & & 0 & 1 & 2 & 3 & 4 & 5 & 6 & 7 \\
9 & $\mathbf{4}$ & 0 & 1 & 2 & 3 & 4 & 5 & 6 & 7 & 8 \\
\hline
\end{tabular}

Aus ihr können folgende Schlußfolgerungen gezogen werden:

- Bezeichnen wir die Anzahl der positiven Elemente in Z wie in 203 1 mit $k$, so beträgt die Überlebenswahrscheinlichkeit in der ersten Runde nach dieser Formel:

$$
\mathrm{p}_{\mathrm{ül}, k}(1)=\frac{k}{2 \cdot k+1}
$$

da das Startkapital als Null angenommen ist.

- Die Anzahl der Kapitalbestände in der zweiten Runde unter Berücksichtigung ihrer zeitlichen Entstehung wächst quadratisch. Sie beträgt $(2 k+1)^{2}$.

- Die Zahl der Überlebensfälle $\left(a_{i \ddot{ }}\right)$ in der zweiten Runde erhöht sich mit zunehmendem $k$ um $3 k-1$ :

- $a_{u ̈ l, k}=a_{u ̈ l, k-1}+3 k-1$.

- Überführen dieser Differenzengleichung in eine geschlossene Form, ergibt

$$
a_{i i l, k}=1 / 2 k \cdot(1+3 k) \text {. }
$$

- Die Überlebenswahrscheinlichkeit für zwei Runden ist der Quotient aus der Zahl der Überlebensfälle und der Gesamtzahl der Kapitalbestände:

$$
p_{\ddot{u l}, k}(2)=\frac{a_{\ddot{u l l}, k}}{(1+2 k)^{2}}=1 / 2 \frac{k+3 k^{2}}{(1+2 k)^{2}} \text {. }
$$


Für $k=3$ errechnen sich $a_{\ddot{u ̈ l}, 3}=1 / 2 \cdot 3 \cdot(1+3 \cdot 3)=15$ Überlebensfälle. Sie befinden sich in obiger Tabelle unterhalb der durchgezogenen, fetten Linie im nicht-schraffierten Bereich. Die Zahl der Überlebensfälle wächst für $k=4$ auf 26. Die Überlebenswahrscheinlichkeiten betragen $\frac{15}{(2 \cdot 3+1)^{2}}=\frac{15}{49}$ für $k=3$ und $\frac{26}{(2 \cdot 4+1)^{2}}=\frac{26}{81}$ für $k=4$.

Schaubild 229.1 illustriert die Überlebenswahrscheinlichkeit in den ersten beiden Perioden in Abhängigkeit von $k$ und damit von der Varianz der Verteilung. Die Graphik legt die Vermutung nahe, daß die Kurven jeweils konvergieren.

\section{Schaubild 229.1: Überlebenswahrscheinlichkeiten für diskrete Verteilungen unterschiedlicher Varianz}

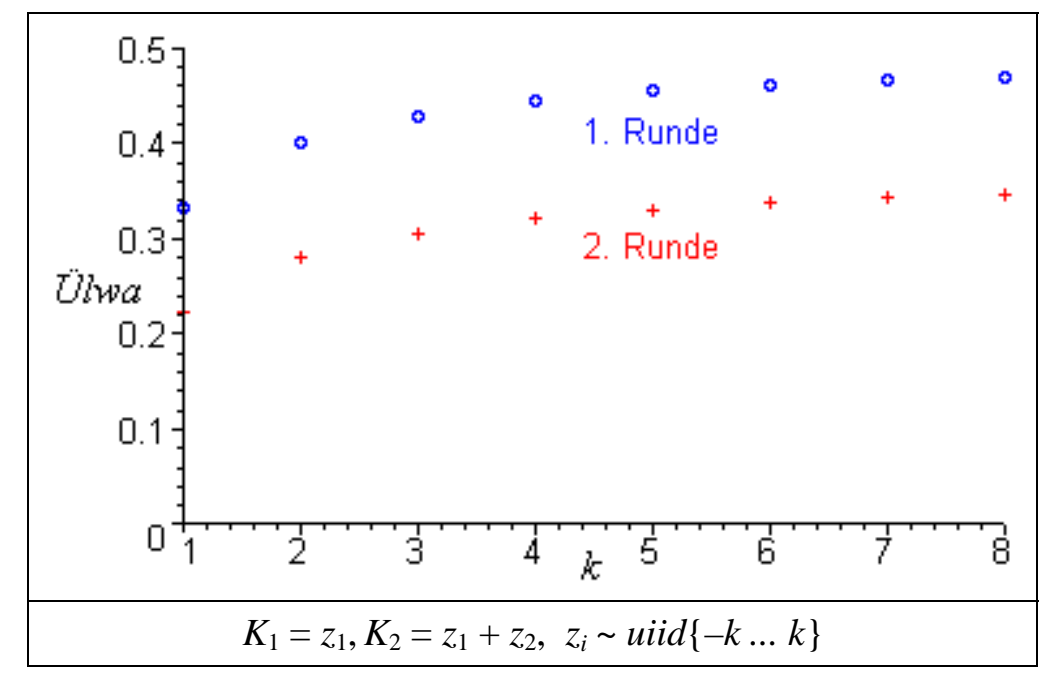

(228.1) konvergiert offensichtlich gegen $1 / 2$, der Überlebenswahrscheinlichkeit der ersten Runde für stetig um Null verteilte Einzahlungen. Eine Grenzwertbetrachtung von 228, führt über die Regel von BERNOULLI-L'HOSPITIAL:

$$
\lim _{x \rightarrow a} \frac{\mathrm{f}(x)}{\mathrm{g}(x)}=\frac{\lim _{x \rightarrow a} \mathrm{f}^{\prime}(x)}{\lim _{x \rightarrow a} \mathrm{~g}^{\prime}(x)}=\frac{\lim _{x \rightarrow a} \mathrm{f}^{\prime \prime}(x)}{\lim _{x \rightarrow a} \mathrm{~g}^{\prime \prime}(x)}
$$

$\mathrm{zu}:$

$$
\begin{aligned}
\lim _{k \rightarrow \infty} \mathrm{p}_{\mathrm{ül}, k} & =\lim _{k \rightarrow \infty} \frac{k+3 k^{2}}{2 \cdot(1+2 k)^{2}} \\
& =\frac{\lim _{x \rightarrow \infty}(1+6 k)}{\lim _{x \rightarrow \infty} 2 \cdot 2 \cdot(1+2 k) \cdot 2}
\end{aligned}
$$




$$
\begin{aligned}
& =\frac{\lim _{x \rightarrow \infty} 6}{\lim _{x \rightarrow \infty} 8 \cdot 2} \\
& =3 / 8 .
\end{aligned}
$$

Die Überlebenswahrscheinlichkeit bis zur zweiten Runde konvergiert für eine diskrete Verteilung also bei wachsendem $k$ ebenfalls gegen die entsprechende Wahrscheinlichkeit einer stetigen Verteilung [s. (1987]]. Im folgenden wird ein Beispiel mit stetig verteilten Einzahlungen vorgestellt.

\subsubsection{Stetige Verteilungen}

Die Einzahlungen $z$ seien gleichverteilt im Intervall $[a, b]$. Dichte- und Verteilungsfunktion der Gleichverteilung lauten unter Verwendung der Heaviside-Funktion:

$$
\begin{array}{ll}
\mathrm{f}(z) & =\frac{1}{b-a} \cdot \mathrm{H}(z-a) \cdot \mathrm{H}(b-z) \\
\mathrm{F}(z) & =\frac{z-a}{b-a} \cdot \mathrm{H}(z-a) \cdot \mathrm{H}(b-z)+\mathrm{H}(z-b) .
\end{array}
$$

Für die Kapitalbestände gilt wie üblich:

$$
\begin{aligned}
& K_{1}=z_{1}+K_{0} \\
& K_{2}=K_{0}+K_{1}+z_{2}=K_{0}+z_{1}+z_{2},
\end{aligned}
$$

so daß die Überlebenswahrscheinlichkeit der ersten Runde nach (202.1)

$$
\begin{aligned}
\mathrm{p}_{\mathrm{ül}}(1) & =1-F_{z 1}\left(-K_{0}\right) \\
& =1-\frac{-K_{0}-a}{b-a} \cdot \mathrm{H}\left(-K_{0}-a\right) \cdot \mathrm{H}\left(b+K_{0}\right)-\mathrm{H}\left(-K_{0}-b\right)
\end{aligned}
$$

lautet. Ohne Startkapital vereinfacht sich die Formel zu:

$$
\mathrm{p}_{\mathrm{ül}}\left(1 \mid K_{0}=0\right)=1-\frac{-a}{b-a} \cdot \mathrm{H}(-a) \cdot \mathrm{H}(b)-\mathrm{H}(-b) .
$$

Ist das Minimum der Einzahlung größer als Null, ist in der ersten Runde keine Insolvenz möglich; umgekehrt führen ausschließlich negative Einzahlungen mit einer Wahrscheinlichkeit von Eins zum Ruin. Der Faktor $\mathrm{H}(-a) \cdot \mathrm{H}(b)$ stellt sicher, daß die Überlebenswahrscheinlichkeit nur dann zwischen 0 und 1 liegt, wenn die Einzahlungen sowohl negativ als auch positiv sein können, andernfalls ergibt der Faktor Null. Ist der Faktor Null, beträgt die Überlebenswahrscheinlichkeit Eins, es sei denn, der Subtrahend $\mathrm{H}(-b)$ reduziert die Überlebenswahrscheinlichkeit auf Null, wenn $b$ negativ ist.

Für zwei Runden läßt sich die Überlebenswahrscheinlichkeit ohne Startkapital nach (197.2) ausdrücken als: 


$$
\begin{aligned}
\mathrm{p}_{\mathrm{ül}}\left(K_{2}>0\right) & =\int_{z_{1} \geq 0}\left[1-\mathrm{F}_{z_{2}}\left(-z_{1}\right)\right] \cdot \mathrm{f}\left(z_{1}\right) \cdot \mathrm{d} z_{1} \\
& =\int_{0}^{b} \frac{\left(1-\frac{\mathrm{H}(-z-a) \mathrm{H}(b+z)(-z-a)}{b-a}-\mathrm{H}(-z-b)\right) \mathrm{H}(z-a) \mathrm{H}(b-z)}{b-a} d z
\end{aligned}
$$

Diesen Ausdruck kann ich nicht weiter vereinfachen. Die Differenz $1-\mathrm{H}(-z-b)$ aus dem ersten Faktor ergibt zwar entweder Eins oder Null; dennoch kann der Term nicht zu $\mathrm{H}(b+z)$ zusammengefaßt werden, weil für $b=z=0$ die Funktion $\mathrm{H}(b+z)$ Null ergibt, die Funktion 1- $\mathrm{H}(-z-b)$ hingegen Eins.

Der letzte Faktor des Zählers, $\mathrm{H}(b-z)$, bewirkt, daß der Integrand Null wird, sobald $z$ größer als $b$ ist. Diese Vorschrift ist nicht redundant: $b$ bildet zwar die Obergrenze der Integration, aber nur, wenn für $b$ eine positive Zahl gewählt wird. Ist $b$ negativ, wird von Null absteigend bis $b$ integriert. Dieses Integral wäre negativ, wenn nicht der genannte Faktor für $z>b<0$ den Integranden auf Null setzte.

Wie in der ersten Runde besteht für Gleichverteilungen mit $b \leq 0$ auch in der zweiten Runde keine Überlebensmöglichkeit. Umgekehrt gibt es für Verteilungen mit a $>0$ keine Ruinwahrscheinlichkeit. Interessant sind daher wiederum nur Parameterkombinationen mit $a<0<b$. Unter dieser Annahme vereinfacht sich die Überlebenswahrscheinlichkeit zu:

$$
\begin{aligned}
& \mathrm{p}_{\mathrm{uil}}\left(K_{2}>0 \mid a \leq 0<b\right)= \\
& -\frac{a^{2} \operatorname{signum}(a+b)+2 a b \operatorname{signum}(a+b)+b^{2} \operatorname{signum}(a+b)+a^{2}+2 a b-5 b^{2}}{4 \cdot(b-a)^{2}}
\end{aligned}
$$

Die Signum-Funktion liefert den Wert 1, wenn ihr Argument positiv ist, 0, wenn es Null, und -1 , wenn es negativ ist. Es ist offensichtlich, daß die Überlebenswahrscheinlichkeit entscheidend von der Relation zwischen $|a|$ und $b$ abhängt. Daher wird eine Fallunterscheidung getroffen: (1) $b$ ist größer als der Betrag von $a$ und (2) $b$ ist kleiner als der Betrag von $a .{ }^{170}$ Diese Unterscheidung führt zu folgenden Ausdrücken für die Überlebenswahrscheinlichkeit:

$$
\begin{aligned}
& \mathrm{p}_{\text {ül }}\left(K_{2}>0 \mid a<0<b \wedge b>-a\right)=-\frac{1 / 2 a^{2}+a b-b^{2}}{(b-a)^{2}} \\
& \mathrm{p}_{\text {ül }}\left(K_{2}>0 \mid a<0<b \wedge b<-a\right)=\frac{3 b^{2}}{2 \cdot(b-a)^{2}} .
\end{aligned}
$$

170 Falls $|a|=b$, liegt ein Modell mit einer symmetrischen Verteilung vor, deren Eigenschaften in Abschnitt 5.1 für beliebige symmetrisch um Null verteilte Zufallsvariablen abgeleitet sind, vgl. insbesondere (198.7). 
Um eine Vorstellung zu gewinnen, wie die Überlebenswahrscheinlichkeit von den Parametern der Gleichverteilung abhängt, ist jene in Schaubild 232,1 für variierenden Parameter $b$ und festen Parameter $a$ dargestellt. Die Überlebenswahrscheinlichkeit zeigt mit wachsender Spanne der Verteilung einen sigmoiden Verlauf, obwohl die obigen Bestimmungsgleichungen für $p_{\text {ül }}(2)$ nur zweiten Grades sind.

\section{Schaubild 232.1: Überlebenswahrscheinlichkeit für zwei Runden bei gleich- verteilten stetigen Einzahlungen, untere Grenze $a$ konstant, obere Grenze $b$ zunehmend}

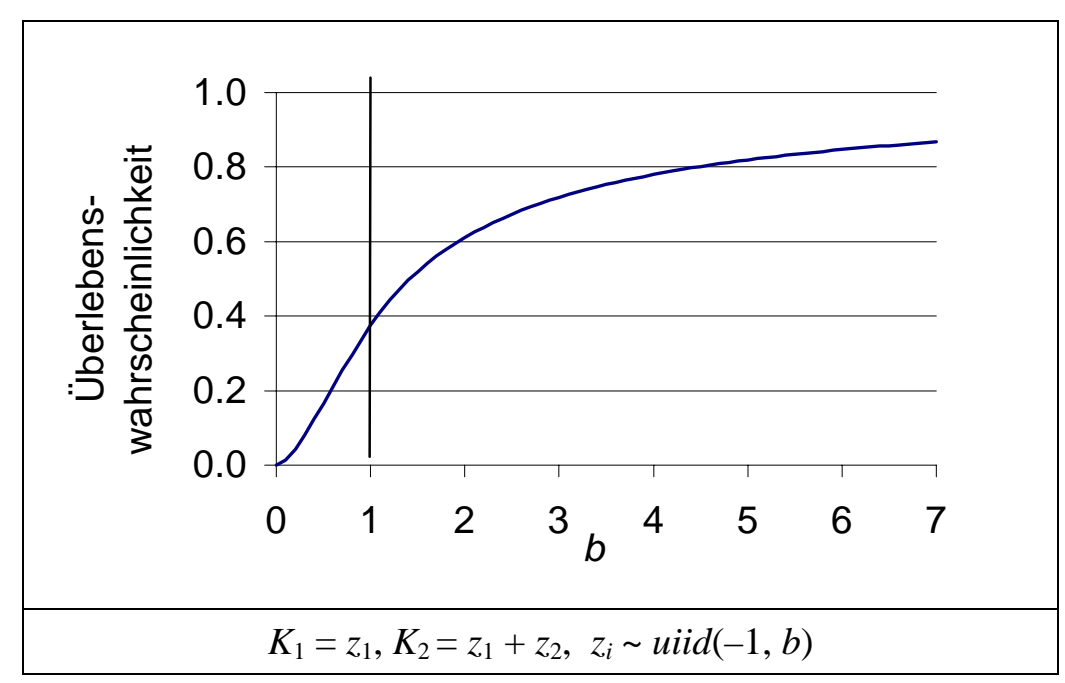

Die Ursache des sigmoiden Verlaufs liegt in den Fallunterscheidungen der Bestimmungsgleichungen begründet: für $b<1$ trifft die obere und für $b>1$ die untere Gleichung zu. Schaubild 232,2 zeigt die Bestandteile der zusammengesetzten Kurve: für sie wird links von $b=1=-a$ der gepunktete und rechts davon der durchgezogene Graph verwendet.

Schaubild 232.2: Bestandteile des sigmoiden Kurvenverlaufs aus Schaubild 232.1

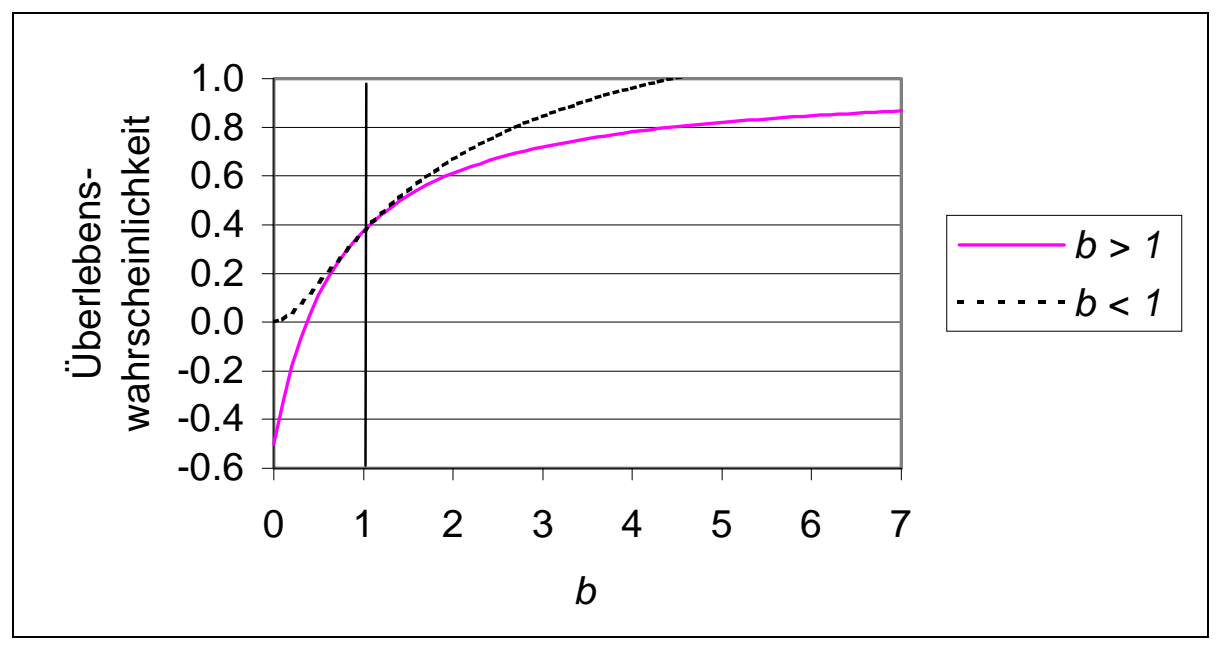


Für $b=1$ ist die Verteilung symmetrisch, und die Überlebenswahrscheinlichkeit beträgt $3 / 8$, wie es in Abschnitt 5.1 für allgemeine symmetrische Verteilungen um Null abgeleitet wurde, vgl. (198.7) und Fußnote 170.

In diesem Beispiel wird gleichzeitig Varianz und Erwartungswert der Verteilung variiert. Wie bereits in Abschnitt 5.1 gezeigt wurde, beeinflußt die Varianz bei symmetrisch um Null verteilten stetigen Zufallsvariablen die Überlebenswahrscheinlichkeit für zwei Perioden nicht. Wie wirkt sich hingegen eine Verschiebung des Erwartungswertes bei Konstanthalten der Varianz aus?

In Schaubild 2331 sind Überlebenswahrscheinlichkeiten für eine Gleichverteilungen mit einer konstanten Spanne von 1 dargestellt, d.h. $b=a+1$. Die Graphik beginnt mit $(a=-1, b=0)$. Die Überlebenswahrscheinlichkeit ist hier Null und steigt mit zunehmendem $a$ an, bis sie für $a=0$ Eins erreicht. Die Ergebnisse für $b=0$ und $a$ $=0$ liegen auf der Hand, weniger offensichtlich ist das Steigungsverhalten des Graphen. Er zeigt wieder einen leicht s-förmigen Verlauf.

\section{Schaubild 233.1: Überlebenswahrscheinlichkeit für zwei Runden bei gleich- verteilten stetigen Einzahlungen, Varianz konstant, Erwartungswert zunehmend}

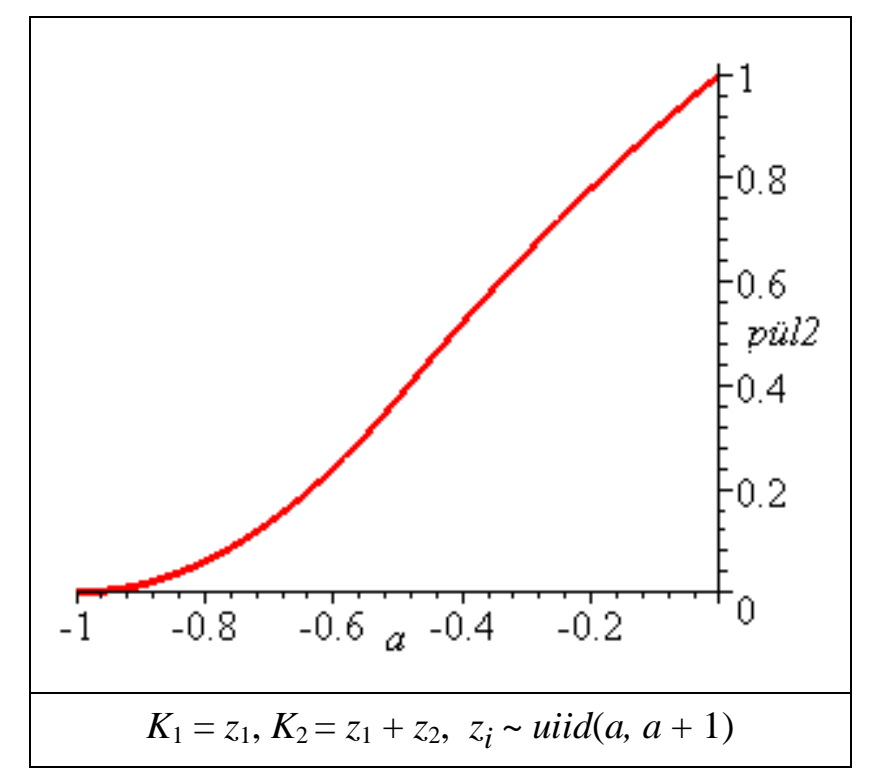

Dies erklärt sich ebenfalls aus dem quadratischen Term in der Ableitung der Überlebenswahrscheinlichkeit nach dem Parameter $b$ im Zusammenhang mit den Fallunterscheidungen bei $a=-1 / 2:^{171}$

$$
\begin{aligned}
\frac{\partial\left[\mathrm{p}_{\mathrm{uil}}(2) \mid b=a+1\right]}{\partial a}= & -\operatorname{sign}(1, a+1 / 2) \cdot a^{2}-2 \operatorname{sign}(a+1 / 2) \cdot a-\operatorname{sign}(1, a+1 / 2) \cdot a \\
& -2 \operatorname{sign}(a+1 / 2)-1 / 4 \operatorname{sign}(1, a+1 / 2)+a+2 .
\end{aligned}
$$

171 Um die nachfolgende Formel abzuleiten, wird in (231.1) zunächst $b$ durch $a+1$ ersetzt und dieser Ausdruck anschließend nach $a$ differenziert. 
Ist Startkapital vorhanden, beträgt die Überlebenswahrscheinlichkeit für zwei Runden

$$
\mathrm{p}_{\mathrm{ül}}(2)=\int_{-K O}^{\infty} \frac{\left(1-\frac{\mathrm{H}(-z-K 0-a) \mathrm{H}(b+z+K 0)(-z-K 0-a)}{b-a}-\mathrm{H}(-z-K 0-b)\right) \mathrm{H}(z-a) \mathrm{H}(b-z)}{b-a} d z .
$$

Schaubild 234.1 visualisiert diese Gleichung für $a=-1=-b$. Ist das Startkapital kleiner als -1, kann das System keinesfalls die erste Runde überleben. Bei größerem Startkapital steigt die Überlebenswahrscheinlichkeit für zwei Perioden nahezu linear an. Auffällig ist der Knick in der Kurve bei $K_{0}=1$. Links davon erhöht ein vergrößertes Startkapital die Überlebenswahrscheinlichkeit in beiden Perioden. Ab einem Startkapital von Eins kann in der ersten Periode keine Insolvenz eintreten, eine weitere Steigerung des Startkapitals verbessert nur noch die Überlebenschancen in der zweiten Runde. Die Überlebenswahrscheinlichkeit steigt daher ab $K_{0}=1$ deutlich langsamer und nähert sich Eins. Übertreffen die finanziellen Mittel zu Anfang Zwei, ist auch in der zweiten Periode keine Insolvenz möglich.

\section{Schaubild 234.1: Überlebenswahrscheinlichkeit für zwei Runden bei gleichver- teilten stetigen Einzahlungen in Abhängigkeit vom Startkapital}

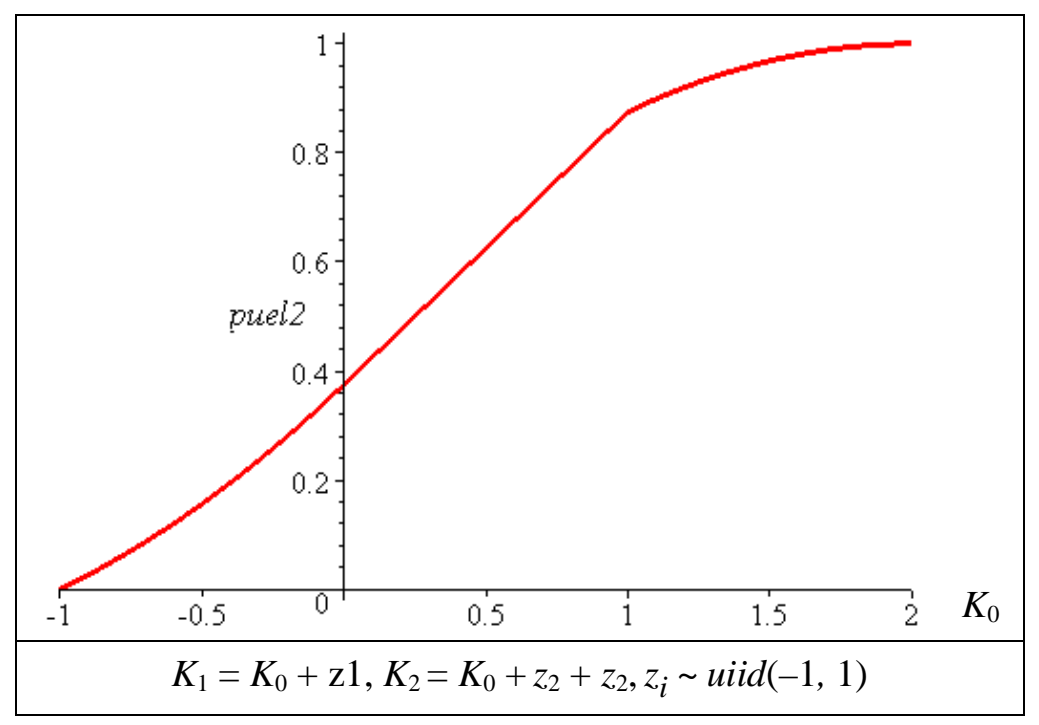

Als letztes analytisches Beispiel werden die Formeln der Überlebenswahrscheinlichkeit kurz auf die Normalverteilung angewandt. Die Überlebenswahrscheinlichkeiten bei vorhandenem Startkapital betragen:

$$
\begin{array}{ll}
\mathrm{p}_{\text {ül }}(1) & =1-F_{z 1}\left(-K_{0}\right) \\
\mathrm{p}_{\mathrm{ül}}(2) & =\int_{z_{1}}>-K_{0}
\end{array}
$$$$
\text { (202.11) }
$$ 
Einsetzen der allgemeinen Dichte- und Verteilungsfunktion der Normalverteilung führt zu nicht handhabbaren Ausdrücken ${ }^{172}$. Werden die Parameter der Normalverteilung vorgegeben, vereinfacht sich die Formel für die erste Runde stark, während der Ausdruck für die zweite Runde auch für eine Standardnormalverteilung sehr komplex bleibt:

$$
\begin{aligned}
& \mathrm{p}_{\text {ül }}[1 \mid z \sim \mathrm{N}(0,1)]=1 / 2+1 / 2 \cdot \operatorname{erf}\left(1 / 2 \cdot \sqrt{2} \cdot K_{0}\right) \\
& \mathrm{p}_{\text {ül }}[2 \mid z \sim \mathrm{N}(0,1)]=\int_{-K O}^{\infty} \frac{1}{4} \frac{\left(1+\operatorname{erf}\left(\frac{1}{2} \sqrt{2} z+\frac{1}{2} \sqrt{2} K 0\right)\right) \mathbf{e}^{\left(-\frac{1}{2} z^{2}\right)} \sqrt{2}}{\sqrt{\pi}} d z .
\end{aligned}
$$

Bei einem Startkapital in Höhe der Varianz von Eins beträgt die Überlebenswahrscheinlichkeit für eine Runde $84 \%$, da die Werte einer Standardnormalverteilung sich zu $68 \%$ zwischen $-\sigma^{2}$ und $\sigma^{2}$ befinden. ${ }^{173}$ Sie geht für zwei Runden auf $71 \%$ zurück Zum Vergleich: Ohne Startkapital betragen die Wahrscheinlichkeiten $1 / 2$ und $3 / 8$, vgl. (198.7).

\section{Schaubild 235.1: Überlebenswahrscheinlichkeit für zwei Runden bei standard- normalverteilten Einzahlungen in Abhängigkeit vom Startkapital}

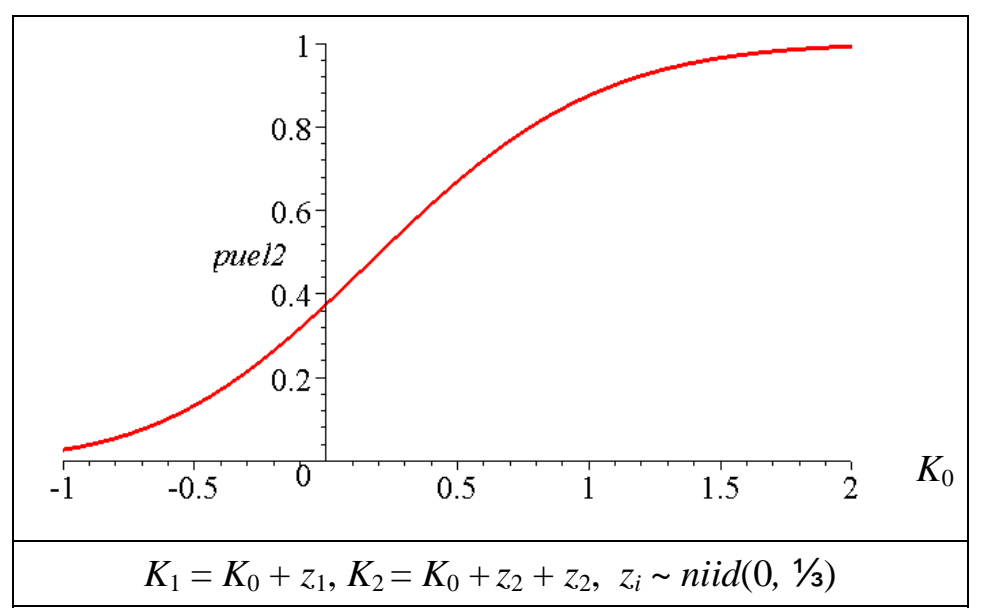

172 Zum Beispiel in der ersten Runde:

173 Damit sind $68+(100-68) / 2=84 \%$ der Werte kleiner als $\sigma^{2}$.

$$
\operatorname{pül}(1)=1-\left(\lim _{x \rightarrow(-\infty)}-\frac{1}{2} \frac{\sqrt{\pi} \sigma \operatorname{erf}\left(\frac{1}{2} \frac{\sqrt{2} x}{\sigma}-\frac{1}{2} \frac{\mu \sqrt{2}}{\sigma}\right)}{\sqrt{\pi \sigma^{2}}}-\frac{1}{2} \frac{\operatorname{erf}\left(\frac{1}{2} \frac{\sqrt{2}(K 0+\mu)}{\sigma}\right) \sqrt{\pi} \sigma}{\sqrt{\pi \sigma^{2}}}\right) .
$$


In Schaubild 2351 sind Überlebenswahrscheinlichkeiten für zwei Runden in Abhängigkeit vom Startkapital abgetragen. Die Varianz der Einzahlungen ist auf $1 / 3$ und der Erwartungswert auf Null festgesetzt. Damit stimmen die Einzahlungen in den Momenten mit den gleichverteilten Einzahlungen aus dem Beispiel in Schaubild 234,1 überein.

Der Unterschied in der Überlebenswahrscheinlichkeit zwischen den beiden Typen von Einzahlungen ist relativ gering. Zwei Unterschiede sind erkennbar. Zum einen nähert sich der Graph asymptotisch Null bzw. Eins, da die normalverteilten Einzahlungen nicht beschränkt sind. Daher entfallen zum anderen auch die Gründe für einen Knick des Graphen an der Stelle $K_{0}=1$, der die rechteckverteilten Einzahlungen kennzeichnet.

Als nächstes werden für die genannten Beispiele Simulationen durchgeführt, um die theoretischen Ergebnisse zu überprüfen und auf mehr als zwei Perioden auszudehnen.

\subsubsection{Monte-Carlo-Simulationsergebnisse für die Überlebenswahr- scheinlichkeit bis zur zweiten Runde}

Bei diskreten, gleichverteilten Einzahlungen ist nach 2282 für zwei Runden eine Überlebenswahrscheinlichkeit, abhängig von der Anzahl der positiven Einzahlungsmöglichkeiten $k$, von

$$
p_{i \ddot{l}, k}(2) \quad=1 / 2 \frac{k+3 k^{2}}{(1+2 k)^{2}}
$$

zu erwarten. Die nachfolgende Aufstellung zeigt Ergebnisse einer Monte-CarloSimulation (MCS) für dieses Szenario mit $-2 \leq z \leq 2$.

\begin{tabular}{|c|c|c|c|c|c|c|}
\hline \multicolumn{2}{|c|}{ Variable } & \multirow{2}{*}{$\frac{\mathrm{N}}{100000}$} & \multirow{2}{*}{$\frac{\text { Mean }}{0.0042}$} & \multirow{2}{*}{$\frac{\text { Std Dev }}{1.4135}$} & \multirow{2}{*}{$\frac{\text { Min }}{-2}$} & \multirow{2}{*}{$\frac{\text { Maximum }}{2}$} \\
\hline $\mathrm{Z1}$ & 1. Einz & & & & & \\
\hline $\mathrm{Z} 2$ & 2. $\operatorname{Einz}$ & 100000 & 0.0061 & 1.4131 & -2 & 2 \\
\hline H1 & p_ül (1) & 100000 & 19999 & 11529 & 1 & 39947 \\
\hline $\mathrm{H} 2$ & $p(K 2>0)$ & 100000 & 20084 & 11544 & 1 & 40082 \\
\hline H3 & p_ül (2) & 100000 & 14054 & 8087 & 1 & 28048 \\
\hline
\end{tabular}

In 39947 Fällen (vgl. (1) von 100000 Wiederholungen wird die erste Runde überlebt und in 25048 Fällen (2) auch die zweite Runde. Die theoretisch zu erwartende Häufigkeit lautet für die erste Runde 40000 und für zwei Runden

$$
\mathrm{p}_{\ddot{\mathrm{ui}}, 2}(2) \quad=1 / 2 \frac{2+3 \cdot 2^{2}}{(1+2 \cdot 2)^{2}}=7 / 25=28000 / 100000 \text {. }
$$

Der Fehler durch die Simulation beträgt $-0.13 \%$ bzw. $+0.17 \%$. 
Für eine Verteilung mit kleinerer Spanne, $z \in\{-1,0,1\}$, erreicht die Simulation ein etwas schlechtere Übereinstimmung mit den theoretischen Werten.

diskrete, gleichverteilte Einzahlungen mit kleinerer Varianz: $z \in\{-1,0,1\}$

\begin{tabular}{lllrrrr}
\multicolumn{2}{c}{ Variable } & N & Mean & Std Dev & Min & Maximum \\
\hline Z1 & 1. Einz & 100000 & 0.0031 & 0.8159 & -1 & 1 \\
Z2 & 2. Einz & 100000 & 0.0030 & 0.8167 & -1 & 1 \\
H1 & P_ül(1) & 100000 & 16759 & 9639 & 1 & $\mathbf{3 3 4 5 0}$ \\
H2 & P(K2>0) & 100000 & 16793 & 9669 & 1 & $\mathbf{3 3 4 8 5}$ \\
H3 & P_ül(2) & 100000 & 11194 & 6446 & 1 & $\mathbf{2 2 3 2 5}$
\end{tabular}

Der theoretischen Wahrscheinlichkeit von $1 / 3$ steht ein Simulationsergebnis von $33.45 \%$ gegenüber, ein Fehler von $0.39 \%$. Für zwei Runden wird der theoretische Wert von

$$
\mathrm{p}_{\mathrm{u} i, 1}(2) \quad=1 / 2 \frac{1+3 \cdot 1^{2}}{(1+2 \cdot 1)^{2}}=2 / 9=22222 / 100000
$$

mit 22325 und einem Fehler von $0.46 \%$ noch etwas stärker verfehlt. Offensichtlich ist bei dieser sehr engen diskreten Verteilung der Zufallsfehler größer als bei einer Verteilung mit mehr Realisationsmöglichkeiten.

Wie werden die theoretischen Wahrscheinlichkeiten bei Simulationen mit stetigen Verteilungen getroffen? Bei symmetrisch um Null verteilten Einzahlungen ist für die erste Runde eine Überlebenshäufigkeit von $50 \%$ zu erwarten. Für zwei Runden verringert sie sich auf $3 / 8=37500 / 100$ 000, s. (198.7). Die Fehler betragen, wie aus den nachfolgenden Aufstellungen leicht nachzurechnen ist, in der ersten Runde $0.22 \%$ und in der zweiten $0.59 \%$. Wie theoretisch abgeleitet, ist auch in der Simulation, die mit gleichen Folge von Pseudozufallszahlen durchgeführt wurde, die Überlebenswahrscheinlichkeit unabhängig von der Varianz.

stetige, gleichverteilte Einzahlungen mit kleinerer Varianz: $-2 \leq \mathrm{z} \leq 2$

\begin{tabular}{lllrrrr}
\multicolumn{2}{c}{ Variable } & N & Mean & Std Dev & Min & Maximum \\
\hline Z1 & 1. Einz & 100000 & 0.0041 & 1.1546 & -2 & 1.9999 \\
Z2 & 2. Einz & 100000 & 0.0049 & 1.1536 & -2 & 1.9999 \\
H1 & p_ül (1) & 100000 & 25098 & 14451 & 1 & $\mathbf{5 0 1 1 2}$ \\
H2 & p(K2>=0) & 100000 & 25122 & 14445 & 1 & $\mathbf{5 0 1 8 1}$ \\
H3 pül (2) & 100000 & 18911 & 10859 & 1 & $\mathbf{3 7 7 2 3}$
\end{tabular}

stetige, gleichverteilte Einzahlungen mit größerer Varianz: $-3 \leq \mathrm{z} \leq 3$

\begin{tabular}{llrrrrr}
\multicolumn{2}{l}{ Variable } & $\mathrm{N}$ & Mean & Std Dev & Min & Maximum \\
Z1 & 1. Einz & 100000 & 0.0062 & 1.7319 & -3 & 2.9999 \\
Z2 & 2. Einz & 100000 & 0.0074 & 1.7304 & -3 & 2.9998 \\
H1 & p_ül (1) & 100000 & 25098 & 14451 & 1 & $\mathbf{5 0 1 1 2}$ \\
H2 & p(K2>=0) & 100000 & 25122 & 14445 & 1 & $\mathbf{5 0 1 8 1}$ \\
H3 pül (2) & 100000 & 18911 & 10859 & 1 & $\mathbf{3 7 7 2 3}$
\end{tabular}


Die Simulationsergebnisse bestätigen die theoretischen Erwartungen:

- Bei stetigen Verteilungen beträgt die Überlebenswahrscheinlichkeit für zwei Runden unabhängig von der Varianz der Einzahlungen $3 / 8$.

- Bei diskreten Verteilungen hingegen ist die Überlebenswahrscheinlichkeit varianzabhängig.

Tabelle 238.1: Überlebenswahrscheinlichkeit für zwei Perioden

\begin{tabular}{|c|c|c|c|c|c|c|c|c|c|}
\hline OBS & \multicolumn{2}{|c|}{ Verteilung } & \multicolumn{2}{|c|}{$\begin{array}{l}\text { Schranke } \\
\text { untere obere }\end{array}$} & \multirow{2}{*}{$\frac{\text { Varianz }}{1.15}$} & \multirow{2}{*}{$\begin{array}{l}p_{\text {ül }}(1) \\
49.97\end{array}$} & \multirow{2}{*}{$\frac{\mathrm{p}\left(K_{2}>0\right)}{50.00}$} & \multirow{2}{*}{$\frac{\mathrm{p}_{\text {uil }}(2)}{37.44}$} & \multirow{2}{*}{$\begin{array}{c}\mathrm{p}\left(K_{2}>0\right. \\
\left.\mid K_{1}>0\right) \\
74.93\end{array}$} \\
\hline 1 & stetig & gleich & -2 & 2 & & & & & \\
\hline 2 & stetig & gleich & -3 & 3 & 1.73 & 49.97 & 50.00 & 37.44 & 74.93 \\
\hline 3 & stetig & gleich & -5 & 5 & 2.89 & 49.97 & 50.00 & 37.44 & 74.93 \\
\hline 4 & stetig & gleich & -10 & 10 & 5.77 & 49.97 & 50.00 & 37.44 & 74.93 \\
\hline 5 & diskret & gleich & -2 & 2 & 2.0 & 40.02 & 40.00 & 28.06 & 70.12 \\
\hline 6 & diskret & gleich & -3 & 3 & 4.0 & 42.90 & 42.80 & 30.64 & 71.41 \\
\hline 7 & diskret & gleich & -5 & 5 & 10.0 & 45.53 & 45.45 & 33.09 & 72.67 \\
\hline 8 & diskret & gleich & -10 & 10 & 36.7 & 47.70 & 47.60 & 35.13 & 73.65 \\
\hline 9 & diskret & gleich & -50 & 50 & 850.0 & 49.50 & 49.55 & 37.00 & 74.74 \\
\hline 10 & diskret & gleich & -100 & 100 & 3367 & 49.75 & 49.76 & 37.22 & 74.81 \\
\hline 11 & diskret & gleich & -200 & 200 & 13400 & 49.87 & 49.88 & 37.33 & 74.86 \\
\hline 12 & diskret & gleich & -500 & 500 & 83500 & 49.93 & 49.95 & 37.40 & 74.90 \\
\hline 13 & diskret & gleich & -5000 & 5000 & $8.3 \mathrm{E}+06$ & 49.97 & 49.99 & 37.44 & 74.93 \\
\hline 14 & diskret & gleich & -100000 & 100000 & $3.3 \mathrm{E}+09$ & 49.97 & 50.00 & 37.44 & 74.93 \\
\hline 15 & stetig & normal & & & 1.15 & 50.05 & 49.94 & 37.53 & 75.00 \\
\hline 16 & stetig & normal & & & 1.73 & 50.05 & 49.94 & 37.53 & 75.00 \\
\hline 17 & stetig & normal & & & 2.89 & 50.05 & 49.94 & 37.53 & 75.00 \\
\hline 18 & stetig & normal & & & 5.77 & 50.05 & 49.94 & 37.53 & 75.00 \\
\hline 19 & stetig & normal & & & 5.77 & 49.92 & 50.01 & 37.45 & 75.02 \\
\hline 20 & stetig & gleich & -10 & 10 & 5.77 & 49.99 & 49.98 & 37.48 & 74.97 \\
\hline \multicolumn{10}{|c|}{$K_{1}=z_{1}, K_{2}=z_{1}+z_{2}, \mathrm{E}\left(z_{i}\right)=0$} \\
\hline \multicolumn{2}{|c|}{$\begin{array}{l}\mathrm{p}_{\mathrm{ull}}(1), \mathrm{p}_{\mathrm{ull}}(2) \\
\mathrm{p}\left(K_{2}>0 \mid K_{1}>0\right)\end{array}$} & $\begin{array}{l}\text { Überleb } \\
\text { Überleb } \\
\text { Runde }\end{array}$ & $\begin{array}{l}\text { nswahrs } \\
\text { nswahrs } \\
\text { urde erre } \\
\end{array}$ & $\begin{array}{l}\text { heinlichk } \\
\text { heinlichk } \\
\text { cht. }\end{array}$ & $\begin{array}{l}\text { it in der er } \\
\text { it in der }\end{array}$ & $\begin{array}{l}\text { ten bzy } \\
\text { weiten }\end{array}$ & zweite & unde & e zweite \\
\hline
\end{tabular}

Umfangreichere Simulationsergebnisse sind in Tabelle 238.1 dargestellt. Sie zeigen, daß bei stetig verteilten Einzahlungen die Überlebenswahrscheinlichkeit in keiner Weise von der Varianz der Einzahlungen tangiert wird, während bei diskreten Verteilungen die Überlebenswahrscheinlichkeit sich mit zunehmender Streuung der bei stetigen Verteilungen annähert. Sie zeigen auch, daß bei stetig-symmetrisch neutralen 
Verteilungen der Verteilungstyp sich nicht auf die Überlebenswahrscheinlichkeit auswirkt.

\subsubsection{6 Überlebenswahrscheinlichkeit für mehr als zwei Perioden}

Mit analytischen Verfahren kann die Überlebenswahrscheinlichkeit im Falle stetig verteilter Einzahlungen nur bis zur zweiten Periode berechnet werden. Daher werden für höhere Runden in diesem Unterabschnitt Monte-Carlo-Simulationen durchgeführt. Die Einzahlungen sind die einzigen Zufallsvariablen. Sie sind, wenn nichts anderes vermerkt ist, identisch, stetig, symmetrisch, neutral, stochastisch unabhängig und gleichverteilt. Angaben zur Varianz in einem Szenario, beispielsweise in einer Schaubildüberschrift, beziehen sich stets auf die Varianz der Einzahlungen.

Für ein einfaches additives Modell $K_{t}=K_{t-1}+z_{t}$ mit stetig gleichverteilten Einzahlungen im Intervall $[-2,2]$ ergibt sich der in Schaubild 239.1 dargestellte Verlauf der Überlebensquote für die ersten 50 Perioden. Der Begriff „Überlebensquote“ hebt hervor, daß MC-Studien numerische Ergebnisse liefern und keine Überlebenswahrscheinlichkeit im analytischen Sinne. Die Überlebensquote stellt das Verhältnis dar, das sich in der Simulation zwischen der Anzahl der Überlebensfälle und der Anzahl der Wiederholungen ergeben hat. Sie ist also eine relative Häufigkeit und keine Wahrscheinlichkeit im strengen Sinne. Von dieser Unterscheidung abgesehen, werden die Begriffe synonym verwendet.

\section{Schaubild 239.1: Überlebensquoten für stetig und diskret gleichverteilte Einzahlungen, additives Modell}

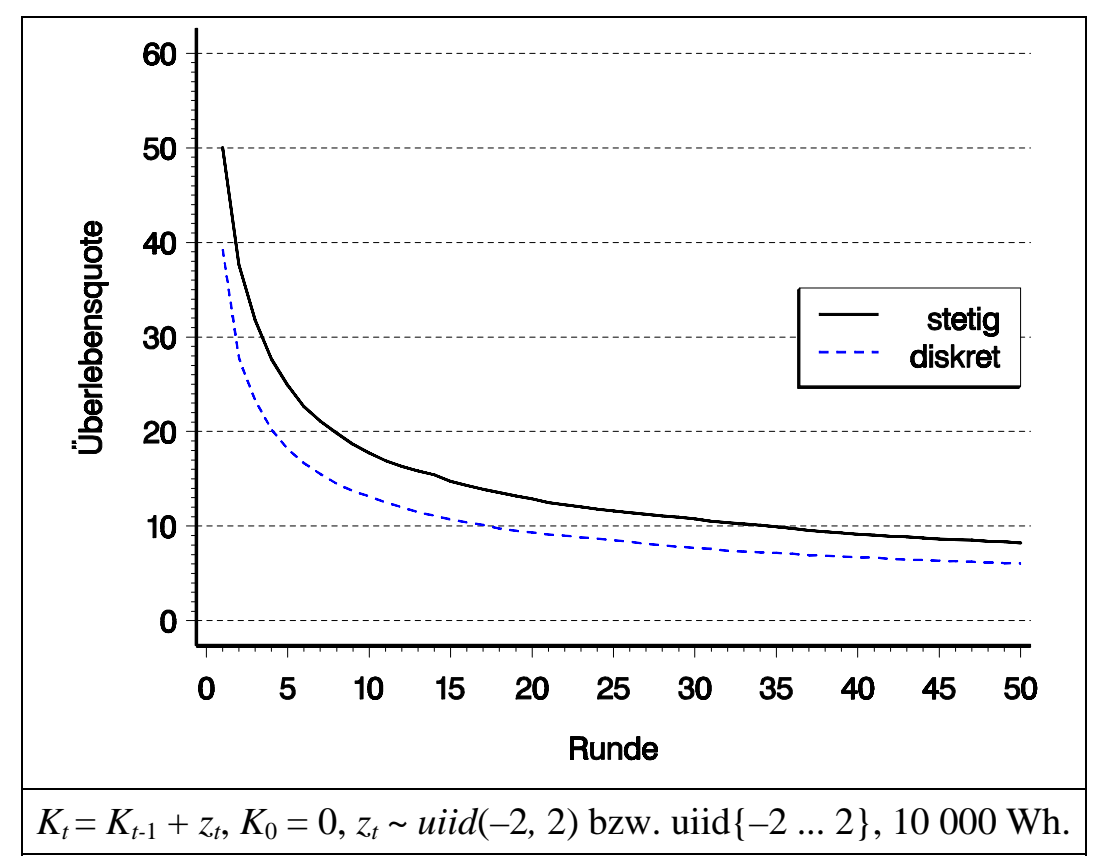

Die Überlebenswahrscheinlichkeit fällt sowohl für diskrete als auch für stetig verteilte Einzahlungen hyperbelartig ab. Bei einer stetigen Verteilung hat sich die Überlebenswahrscheinlichkeit nach fünf Runden bereits halbiert, während sie die 10\%-Marke 
erst in Runde 35 erreicht. Der analytisch abgeleitete Vorsprung einer stetigen Verteilungen in bezug auf die Überlebenswahrscheinlichkeit in den ersten beiden Perioden gegenüber einer diskreten Verteilung verringert sich mit der Periodenzahl ein wenig, bleibt aber auch in der 50. Periode noch deutlich erkennbar.

Trotz gleicher Spanne der stetigen und der diskreten Verteilung unterscheiden sich die beiden Verteilungen in der Varianz. Die Varianz der stetigen Gleichverteilung ist bekanntlich

$$
\operatorname{Var}(X) \quad=\frac{(o-u)^{2}}{12} \text {. }
$$

Die Varianz einer gleichmäßigen Verteilung erhalte ich aus dem bekannten Ansatz für diskrete Verteilungen:

$$
\operatorname{Var}(X) \equiv \sum_{i=1}^{n} \frac{\left(x_{i}-\bar{x}\right)^{2}}{n}
$$

nach einigen Umformungen als:

$$
\operatorname{Var}(X) \quad=\frac{(o-u+1)^{2}-1}{12} \cdot 174
$$

Daher beträgt die Varianz einer stetigen Verteilung im Intervall [-2, 2] 4/3, während die Varianz der diskreten Verteilung im gleichen Intervall mit 2 deutlich größer ist. Damit stetige und diskrete Gleichverteilung die gleiche Varianz aufweisen, sind die Grenzen der stetigen Verteilung im Vergleich zur diskreten um $1 / 2$ nach außen zu verschieben:

$$
\operatorname{Var}\{u \ldots o\}=\operatorname{Var}(u-1 / 2, o+1 / 2) .
$$

Es nicht möglich, durch eine Erhöhung der Varianz der stetigen Verteilung die beiden Kurven in Schaubild 239.1 in Deckung $\mathrm{zu}$ bringen. In Abschritt 5.1 ist gezeigt worden, daß die Überlebenswahrscheinlichkeit bei um Null verteilten Zufallsvariablen und Startkapital 0 nicht von der Varianz abhängt. Tabelle 24C.1 demonstriert, daß MC-Simulationen dieses Ergebnis exakt widerspiegeln.

\section{Tabelle 240.1: Überlebenswahr- scheinlichkeit bei unterschiedlicher Varianz im additiven Modell}

\begin{tabular}{|c|ccc|}
\hline \multirow{2}{*}{ Runde } & \multicolumn{3}{|c|}{$\begin{array}{c}\text { Überlebenswahrscheinlich- } \\
\text { keit bei einer Halbspanne } \\
\text { der Einzahlungen von } \ldots\end{array}$} \\
\cline { 2 - 4 } & 1 & 2 & 3 \\
\hline 1 & 49.78 & 49.78 & 49.78 \\
2 & 37.26 & 37.26 & 37.26 \\
3 & 31.35 & 31.35 & 31.35 \\
4 & 27.15 & 27.15 & 27.15 \\
5 & 24.48 & 24.48 & 24.48 \\
6 & 22.45 & 22.45 & 22.45 \\
7 & 20.90 & 20.90 & 20.90 \\
8 & 19.46 & 19.46 & 19.46 \\
9 & 18.28 & 18.28 & 18.28 \\
10 & 17.46 & 17.46 & 17.46 \\
\hline$K_{t}=K_{t-1}+z_{t}, K_{0}=0, z_{t} \sim$ uiid $(-b, b)$, \\
10 & 000 Wiederholungen \\
\hline
\end{tabular}

174 Für natürliche Zahlen stimmt diese Formel mit der Varianz überein, die BosCH (1992, S. 168f) für eine gleichmäßige Verteilung im geschlossenen Intervall $[1, m]$ mit $\left(m^{2}-1\right) / 12$ angibt. 
Diskrete und stetige Einzahlungen nähern sich in bezug auf ihre Überlebenswahrscheinlichkeit an, wenn die Varianz der diskreten Verteilung erhöht wird. In Unterabschnitt 5.3.1.3 ist für die ersten beiden Runden beschrieben worden, wie die Überlebenswahrscheinlichkeiten diskreter Verteilungen mit zunehmender Varianz rasch gegen die einer entsprechenden stetigen Verteilung konvergieren, vgl. Schaubild 241,1. Simulationsergebnisse bestätigen diese Eigenschaft: Auch für höhere Perioden nähern sich die Überlebenswahrscheinlichkeiten mit zunehmender Varianz sehr schnell der stetigen Verteilung an. Bei einer Halbspanne von Zehn, das heißt, bei Einzahlungen, die gleichmäßig zwischen -10 und 10 verteilt sind, ist kaum noch ein Unterschied zwischen diskreter und stetiger Verteilung zu erkennen. Gleichmäßige Verteilungen mit 20 oder mehr Ausprägungen sind daher gut durch stetige Verteilung zu approximieren bzw. umgekehrt.

\section{Schaubild 241.1: Überlebensquote bei unterschiedlicher Varianz einer diskreten Verteilung im additiven Modell}

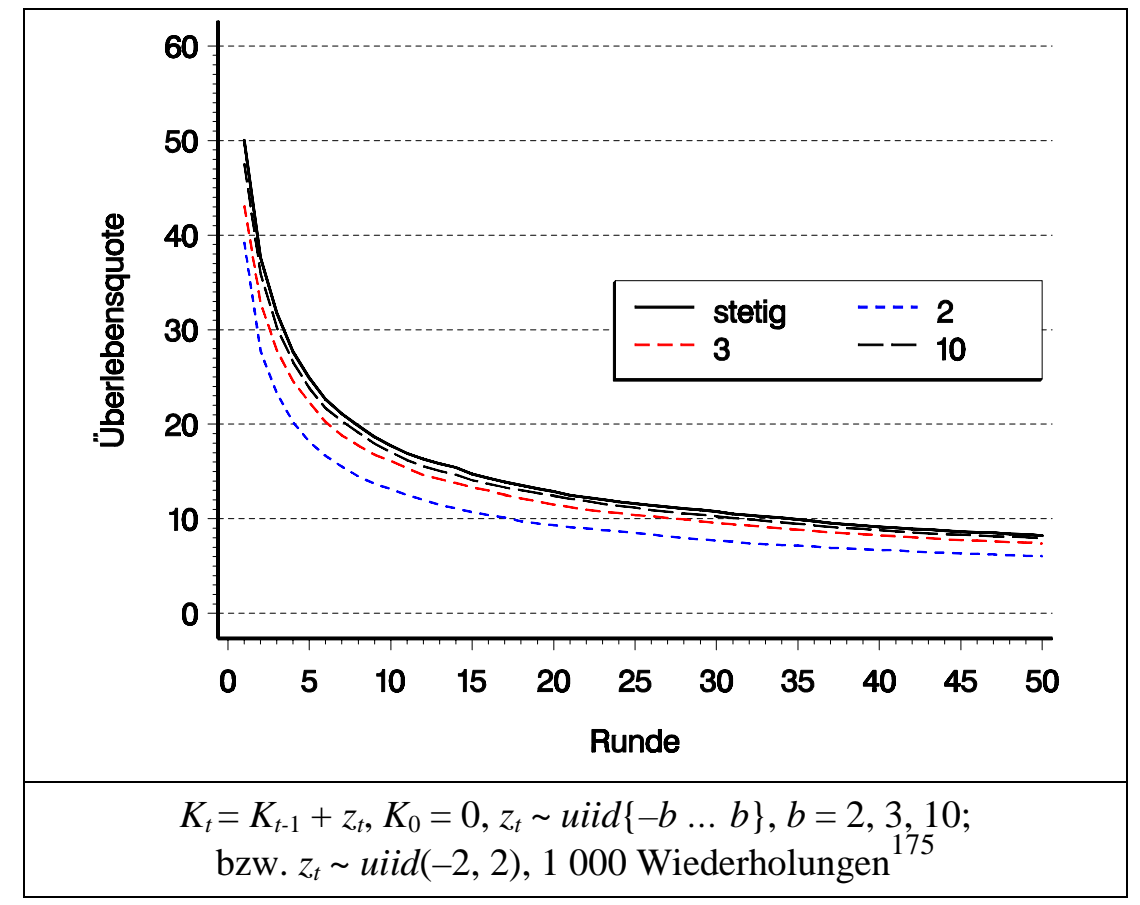

Bei den bisher vorgestellten Simulationsstudien betrug das Startkapital jeweils Null und die Einzahlungen waren gleichverteilt. Im folgenden werden diese beiden Annahmen variiert.

Steht ein positives Startkapital zur Verfügung, übt die Varianz, wie (204.1) und (204.3] für symmetrische Verteilungen zeigen, auch für stetige Verteilungen einen Einfluß auf die Überlebenswahrscheinlichkeit aus. Der Einfluß der Varianz nimmt mit

175 Die Notation $z_{t} \sim \operatorname{uiid}(-b \ldots b)$ bedeutet, daß die Zufallsvariable $z_{t}$ gleichmäßig im abgeschlossenen Intervall $\left[\begin{array}{lll}-b & \ldots & b\end{array}\right]$ verteilt ist, während durch die Schreibweise $\operatorname{uiid}(-b, b)$ eine stetige Gleichverteilung symbolisiert wird. Die Symmetrie der Intervalle um Null impliziert einen Erwartungswert von Null. 
dem Startkapital zu. Schaubild 245i zeigt, daß eine Zunahme der Varianz trotz gleichbleibenden Erwartungswertes đie Überlebenswahrscheinlichkeit erheblich senkt.

Der Einfluß der Varianz auf die Überlebenswahrscheinlichkeit verringert sich mit zunehmender Periodenzahl. Die Differenz der Überlebensquoten geht kontinuierlich zurück, wie Tabelle 242.1 zum einen für Einzahlungen zwischen -2 und 2 und zum anderen für Einzahlungen zwischen -80 und 80 darstellt. Währenddessen bleibt der relative Unterschied in der Überlebenswahrscheinlichkeit zwischen den Verteilungen nahezu konstant.

Tabelle 242.1: Vergleich von Überlebensquoten bei unterschiedlicher Varianz und positivem Startkapital im additiven Modell

\begin{tabular}{|c|c|c|c|c|}
\hline \multirow{2}{*}{ Runde } & \multicolumn{2}{|c|}{ Überlebensquoten } & \multirow{2}{*}{$\begin{array}{l}\text { Differenz der } \\
\text { Überlebens- } \\
\text { quoten }\end{array}$} & \multirow{2}{*}{$\begin{array}{l}\text { Quotient der } \\
\text { Überlebens- } \\
\text { quoten }\end{array}$} \\
\hline & Halbspanne $=2$ & Halbspanne $=80$ & & \\
\hline 1 & 74.8 & 24.3 & 50.6 & 1.5 \\
\hline 2 & 62.1 & 23.8 & 38.3 & 1.6 \\
\hline 4 & 48.6 & 20.5 & 28.0 & 1.7 \\
\hline 6 & 41.0 & 18.1 & 22.9 & 1.8 \\
\hline 8 & 36.6 & 16.4 & 20.2 & 1.8 \\
\hline 10 & 33.0 & 15.0 & 18.0 & 1.8 \\
\hline 12 & 30.1 & 13.6 & 16.6 & 1.8 \\
\hline 14 & 28.1 & 12.4 & 15.7 & 1.8 \\
\hline 16 & 26.5 & 11.9 & 14.6 & 1.8 \\
\hline 18 & 25.2 & 11.4 & 13.8 & 1.8 \\
\hline 20 & 23.9 & 10.8 & 13.2 & 1.8 \\
\hline 22 & 23.0 & 10.5 & 12.5 & 1.8 \\
\hline 24 & 22.1 & 10.0 & 12.1 & 1.8 \\
\hline 26 & 21.2 & 9.6 & 11.6 & 1.8 \\
\hline 28 & 20.6 & 9.3 & 11.3 & 1.8 \\
\hline 30 & 20.1 & 9.1 & 11.0 & 1.8 \\
\hline 32 & 19.4 & 8.9 & 10.5 & 1.8 \\
\hline 34 & 18.8 & 8.5 & 10.3 & 1.8 \\
\hline 36 & 18.3 & 8.3 & 9.9 & 1.8 \\
\hline 38 & 17.8 & 8.2 & 9.6 & 1.9 \\
\hline 40 & 17.4 & 8.1 & 9.3 & 1.9 \\
\hline 42 & 16.9 & 7.8 & 9.1 & 1.9 \\
\hline 44 & 16.5 & 7.6 & 8.9 & 1.8 \\
\hline 46 & 16.2 & 7.4 & 8.7 & 1.9 \\
\hline 48 & 15.8 & 7.2 & 8.6 & 1.8 \\
\hline 50 & 15.6 & 7.1 & 8.4 & 1.8 \\
\hline$K_{t}$ & $K_{0}=1, z_{t} \sim u i i$ & $b, b), b \stackrel{\wedge}{=}$ Halbsp & e, $10000 \mathrm{Wic}$ & ungen \\
\hline
\end{tabular}


Schaubild 243.1: Überlebensquote bei unterschiedlicher Varianz und positivem Startkapital im additiven Modell

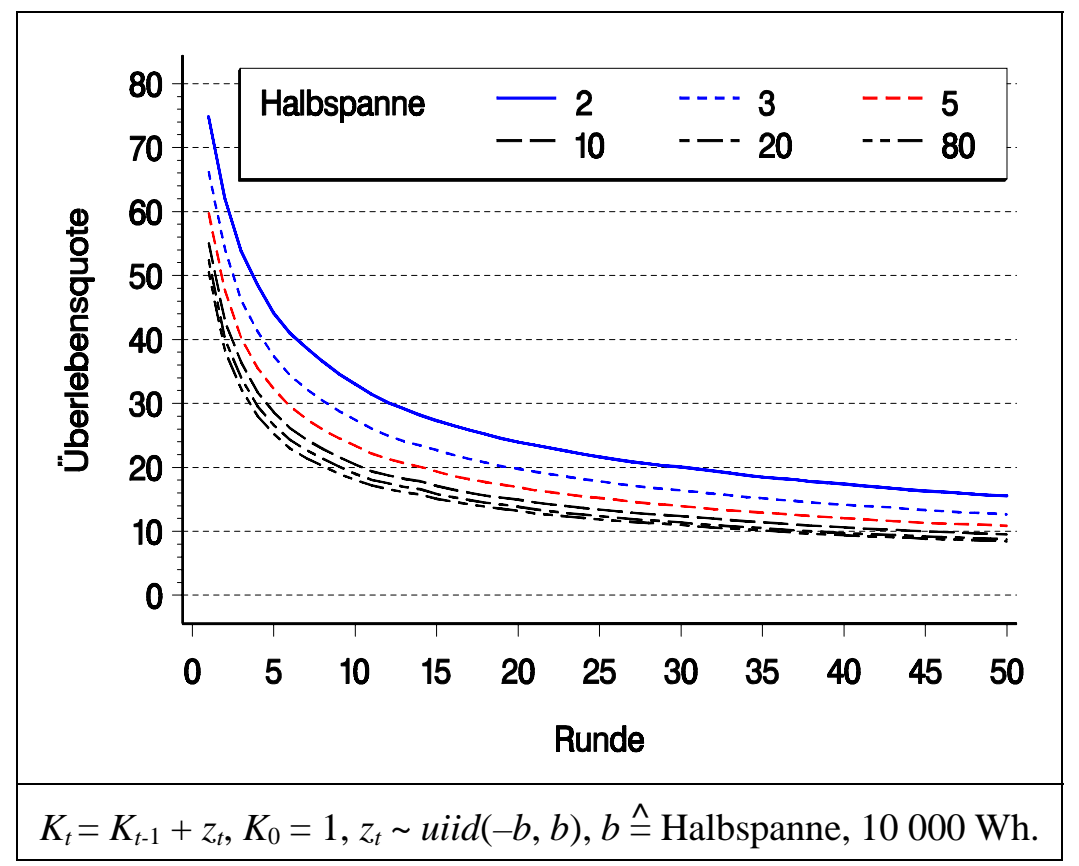

Als nächstes wird der Einfluß des Startkapitals auf die Überlebenswahrscheinlichkeit untersucht. Die unterste Kurve in Schaubild 2432 stellt die Referenzsituation dar mit stetig gleichverteilten Einzahlungen zwischen -2 und 2 und einem Startkapital von Null. Diese Kurve findet sich auch in den Schaubildern 2411] und 239.1. Sie unterscheidet sich darin, daß die Anzahl der Perioden aus Gründen der Rechenzeit und einer übersichtlicheren Darstellung auf 20 reduziert ist

\section{Schaubild 243.2: Überlebensquote bei unterschiedlichem Startkapital im} additiven Modell

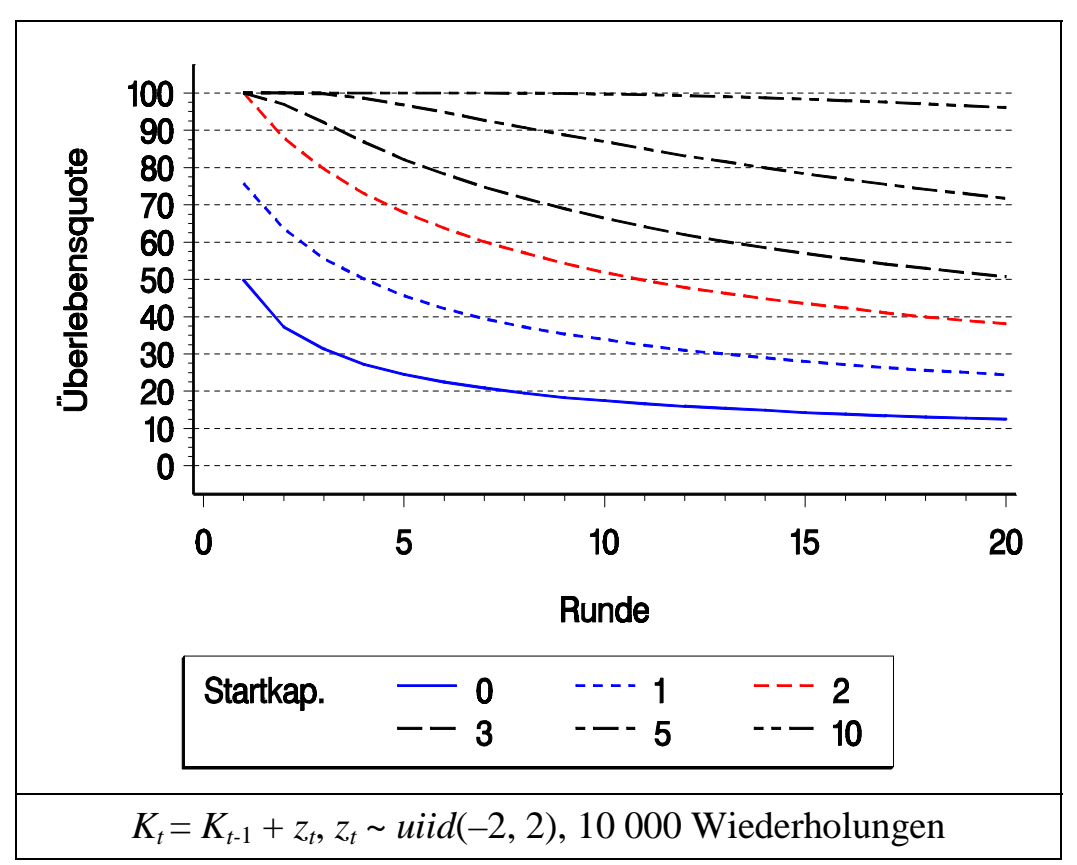


Steht Startkapital zur Verfügung, vermindert sich die Insolvenzgefahr deutlich. Bei $K_{0}=10 \mathrm{kann}$ rechnerisch frühestens ab der 5. Periode eine Insolvenz eintreten, die Wahrscheinlichkeit ist aber zunächst vernachlässigbar. Das per Simulation ermittelte Insolvenzrisiko liegt beispielsweise in der achten Runde bei $0.03 \%$ und bleibt bis zur 20. Periode unter $5 \%$.

Ist kein Startkapital vorhanden, ist der Verteilungstyp der Einzahlungen in der ersten Runde für die Überlebenswahrscheinlichkeit ohne Belang, solange die Verteilungen stetig, symmetrisch und neutral sind. Denn die Wahrscheinlichkeit einer zu Insolvenz führenden Einzahlung, das heißt einer Einzahlung kleiner als Null und damit kleiner als der Median, hängt unter diesen Einschränkungen nicht vom Verteilungstyp ab. Beginnt die Simulation mit Startkapital, müssen sich beschränkte von unbeschränkten Verteilungen unterscheiden. Beträgt beispielsweise $K_{0}$ Zwei, kann das System praktisch in der ersten Runde nicht fallieren, wenn die Einzahlungen stetig zwischen -2 und 2 gleichverteilt sind. ${ }^{176}$ Bei einer Normalverteilung mit gleichen Momenten hingegen existiert selbstverständlich eine positive Wahrscheinlichkeit für eine erste Einzahlung kleiner als -2 und damit eine positive Ruinwahrscheinlichkeit für die erste Runde, die sich algebraisch berechnen läßt. Sie beträgt 4\%. Die Normalverteilung ist also einer Gleichverteilung unterlegen, und zwar im Sinne einer höheren Insolvenzwahrscheinlichkeit.

Für zwei Runden kann die Überlebenswahrscheinlichkeit nach der in Abschnitt 5.1 abgeleiteten Beziehung 204.B

$$
\mathrm{p}_{\text {ül }}\left(K_{2}>0\right)=\int_{z_{1}>-K_{0}} \mathrm{~F}\left(z_{1}+K_{0}\right) \mathrm{f}\left(z_{1}\right) \mathrm{d} z_{1}
$$

berechnet werden. ${ }^{177}$ Werden Verteilungs- und Dichtefunktionen entsprechend eingesetzt, ergibt sich für normalverteilte Einzahlungen mit $\mu=0, \sigma=4 / 3$ und $K_{0}=2$ eine Wahrscheinlichkeit von $87.5 \%$. Für gleichverteilte Einzahlungen ergibt sich zufälligerweise die gleiche Wahrscheinlichkeit. Die Entwicklung der Überlebenswahrscheinlichkeit für höhere Runden sollen nun Simulationen zeigen, deren Ergebnisse in Schaubild 245.1 dargestellt sind. Die Ordinate beginnt abweichend von den anderen Abbildungen nicht bei Null, um die Unterschiede zwischen Normal- und Gleichverteilung zu verdeutlichen. Wie die analytischen Ergebnisse für die ersten beiden Runden vermuten lassen, verliert die Gleichverteilung in höheren Runden ihre Überlegenheit. Allerdings ist die Differenz nur sehr gering. Deutlich höhere Überlebenswahrscheinlichkeiten weisen ab der zweiten Periode dreieckverteilte Einzahlungen auf. In der ersten Runde ist bei dreieckverteilten wie bei normalverteilten Einzahlungen eine Insolvenz möglich, da die minimale Einzahlung symmetrisch-neutral dreieckverteilter Einzahlungen mit einer Varianz von $4 / 3$ das Startkapital in einer Höhe von $-4 / 3 \cdot \sqrt{3} \approx$ -2.3 übersteigt.

\footnotetext{
176 Die Varianz einer stetigen Gleichverteilung zwischen -2 und 2 beträgt 4/3.

177 Diese Formel gilt für symmetrisch neutrale Einzahlungen.
} 


\section{Schaubild 245.1: Überlebensquote bei normal- , gleich- oder symmetrisch drei- eckverteilten Einzahlungen mit gleicher Varianz, additives Modell}

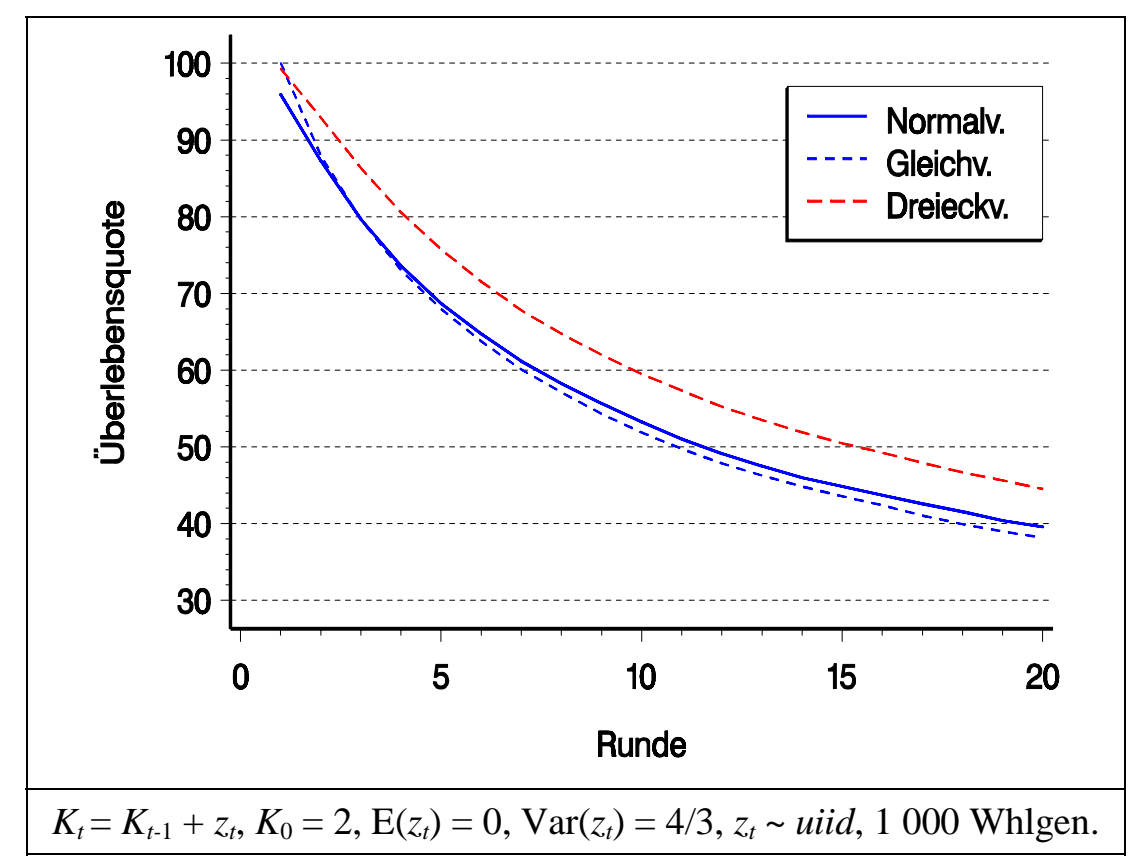

Als letzte Variation von Systemen mit additiver Verknüpfung wird den Modellen eine konstante Auszahlung hinzugefügt. Als Referenz dient das bisherige Modell, das um ein Startkapital von Zehn erweitert wird. Dieses Referenzmodell entspricht der obersten Linie in Schaubild 243.2.

Sind im Referenzmodell die Einzahlungen deterministisch (also wegen ihrer Neutralitätseigenschaft gleich Null), ergeben periodische Auszahlungen in Höhe von Eins in der zehnten Runde einen Kapitalbestand, der Null beträgt. Definitionsgemäß erfüllt dieser Kapitalbestand nicht die Überlebensbedingung (s. Abschnitt 4.2, so daß die Lebenserwartung neun Perioden beträgt. Eine infinitesimale Verringerung des Startkapitals bewirkt keine Veränderung der Lebensdauer, während eine infinitesimale Vergrößerung zu einem positiven Kapitalbestand in der zehnten Runde führt und damit die Lebensdauer auf zehn Perioden erhöht.

Welche Lebensdauer ist bei stochastischen, symmetrisch neutralen Einzahlungen zu erwarten? Nehmen wir dazu vereinfachend an, es gäbe nur in der zehnten Periode eine stochastische Einzahlung mit einem Median von Null. Sie wird sich hälftig größer und hälftig kleiner als Null realisieren, mit der Konsequenz, daß auch das Kapital in der zehnten Periode zu 50\% positiv und zu 50\% negativ ausfällt. Das System wird folglich mit gleicher Wahrscheinlichkeit die zehnte Runde überleben wie fallieren. Die Überlebenswahrscheinlichkeit für zehn Runden beträgt somit 1/2 und die mittlere Lebensdauer 9.5 Perioden. 
Kann dieser Gedankengang auf alle Perioden übertragen werden, so daß bei stochastisch unabhängigen, neutral symmetrischen Einzahlungen der Erwartungswert des Alters ebenfalls 9.5 beträgt?

Stochastische Einzahlungen in allen Perioden führen zu einem Kapital in der neunten Periode, dessen Erwartungswert $K_{0}-9 a=10-9 \cdot 1=1$ beträgt. Das Addieren einer symmetrisch neutralen Zufallsvariablen $\mathrm{zu} K_{9}$ und anschließende Subtraktion von Eins, der Auszahlung in der zehnten Runde, führen zu einer Zufallsvariable $K_{10}$, deren Median Null ist. Daher beträgt auch bei stochastischen Einzahlungen in allen Runden die Überlebenswahrscheinlichkeit für 10 Runden 1/2. Dieser Schluß wird durch Simulationen, deren Ergebnis in Schaubild 246.1 abgebildet ist, bestätigt. Die Überlebenswahrscheinlichkeit für eine Auszahlung von Eins ist eine umgekehrt logistische Funktion mit einem Wendepunkt bei zehn Runden und 50\% Überlebensquote.

\section{Schaubild 246.1: Überlebensquote für variierte Auszahlungen im additiven Modell}

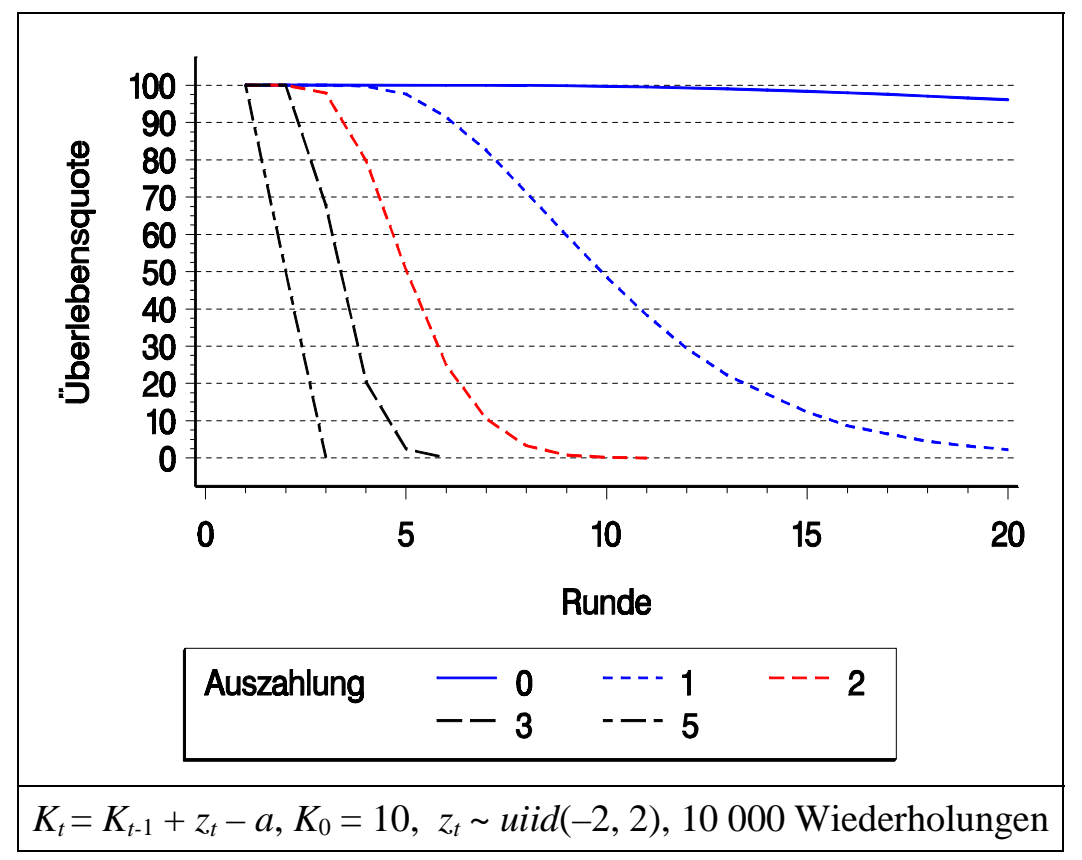

Für die Überlebenswahrscheinlichkeit spielt es daher keine Rolle, ob nur die Einzahlung in der letzten Runde stochastisch ist oder ob dies für alle Einzahlung zutrifft. Gleiches gilt nicht für die Lebensdauer, wie später gezeigt wird.

Als nächstes wird der Frage nachgegangen, ob der Verlauf der Überlebensquote von der Varianz der neutralen Einzahlung abhängt. Zu diesem Zweck werden aus technischen Gründen Startkapital und Auszahlung jeweils verzehnfacht.

Schaubild 247.1 zeigt das naheliegende Ergebnis: Je größer die Varianz der Einzahlungen, desto früher ist ein Ruin möglich. Gleichzeitig erlauben erst größere Varianzen eine längere Lebensdauer. Alle Kurven schneiden sich in einem Punkt, bei zehn Runden und einer Überlebensquote von $1 / 2$. Offensichtlich fallieren die Systeme in der 
Hälfte der Wiederholungen in Runde zehn oder früher. Daher können zehn Perioden als „Medianalter“ aufgefaßt werden. Es ist unabhängig von der Varianz der Einzahlungen.

\section{Schaubild 247.1: Überlebensquote für variierte Varianzen und feste Auszahlung im additiven Modell}

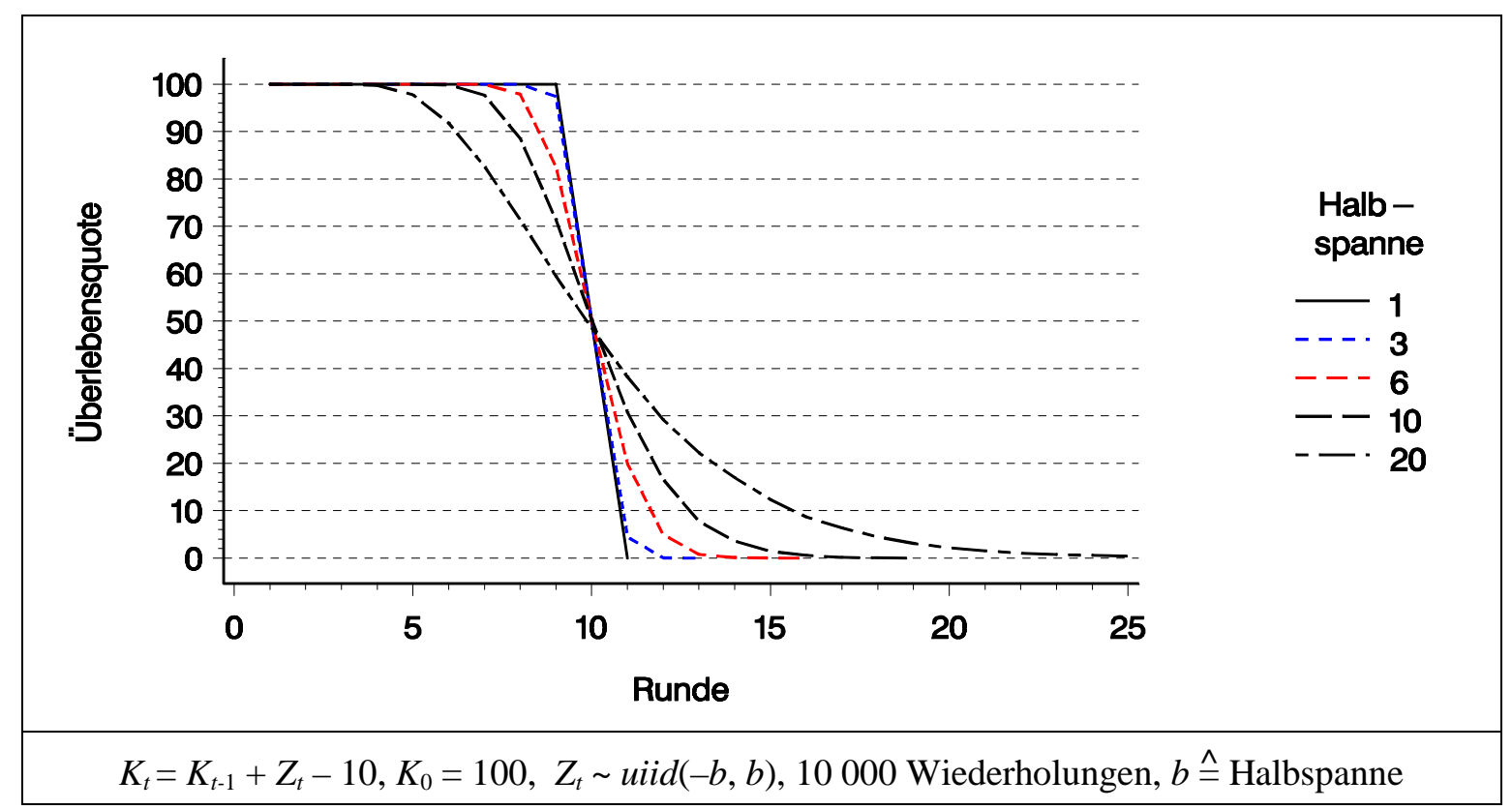

Anders sieht es mit dem mittleren Alter im Unterschied zum Medianalter aus. Sehr hohe Varianzen führen in Einzelfällen zum Ausscheiden in der ersten Runde. Ein früheres Insolvenzalter ist selbstverständlich nicht möglich. Umgekehrt ermöglichen große Varianzen, daß Perioden erreicht werden, die mehr als eine doppelt so groß sind wie das Medianalter. Die Altersverteilung wird dadurch unsymmetrisch und ihr Erwartungswert vergrößert sich mit zunehmender Varianz. Dies ist in Schaubild 248,1 dargestellt.

Das Schaubild zeigt ebenfalls, daß trotz steigender Lebenserwartung der Vermögensendwert sinkt. Dieser Effekt darf nicht überbewertet werden. Das Simulationsmodell, das dieser Graphik zugrunde liegt, enthält keine wirksame Altersbegrenzung. ${ }^{178}$ Daher geht jedes Unternehmen bei den gewählten Parametern irgendwann Pleite. Bei hoher Varianz ist der (negative) Kapitalbestand, der zum Ausscheiden führt, natürlich größer als bei kleiner Varianz. Deswegen kann der Zusammenhang zwischen erreichtem Kapital und Lebensdauer nur sinnvoll für ein fest vorgegebenes Zieljahr untersucht werden. Schaubild 2482 zeigt das Ergebnis für zehn Perioden.

178 Aus technischen Gründen ist eine Altersgrenze erforderlich. Sie ist so hoch angesetzt, daß sie in keiner der Wiederholungen erreicht wird. Um dieses sicherzustellen, wird für jedes Simulationsexperiment die maximal erreichte Periode mit der gesetzten Altersgrenze verglichen, so daß diese im Bedarfsfall erhöht werden kann. 
Schaubild 248.1: Durchschnittlich erreichtes Alter und mittleres Kapital für variierte Varianzen und feste Auszahlung im additiven Modell

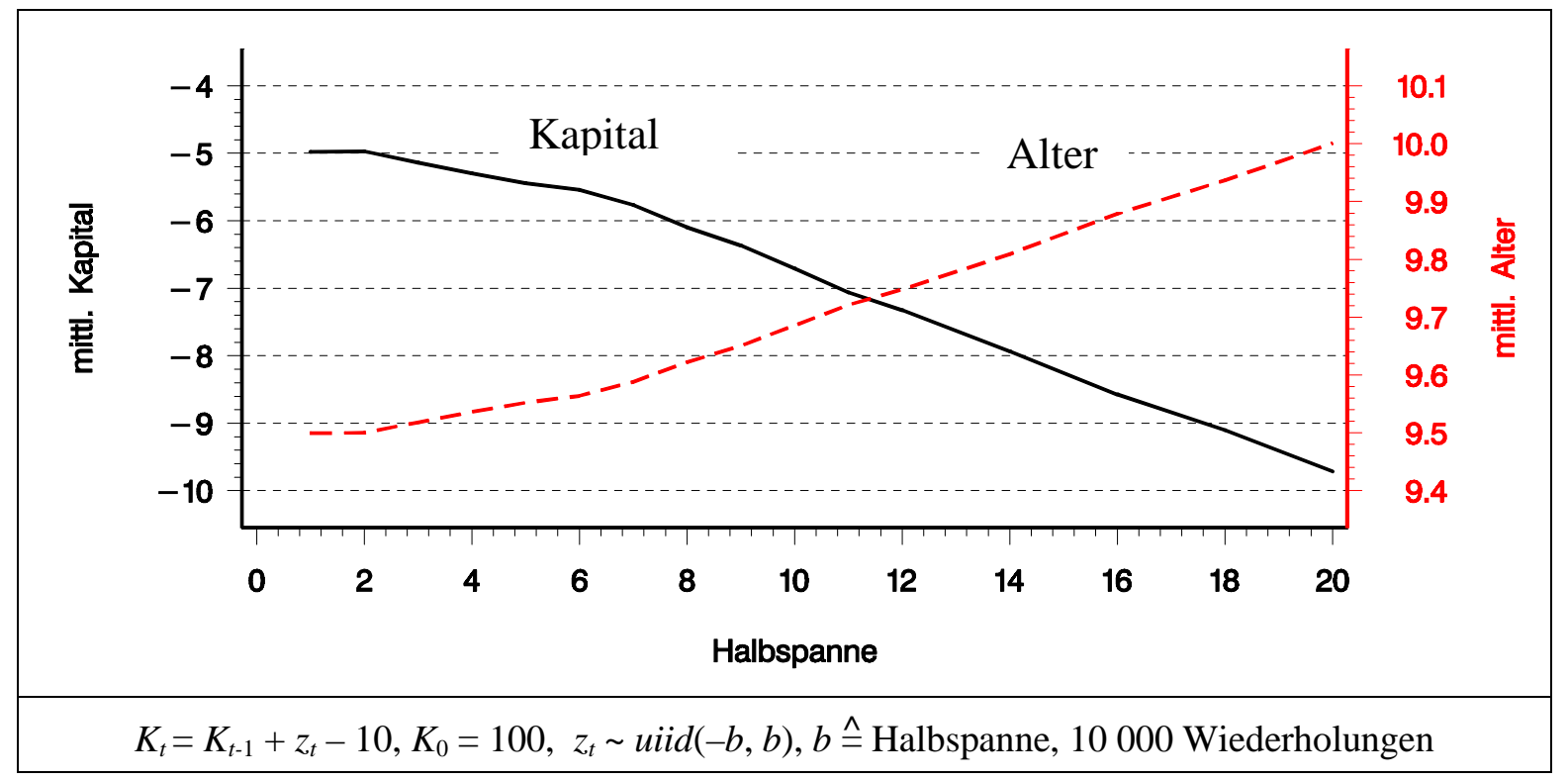

Schaubild 248.2: Lebensdauer und mittleres Kapital für variierte Varianzen, feste Auszahlung und Planungshorizont von zehn Perioden, additives Modell

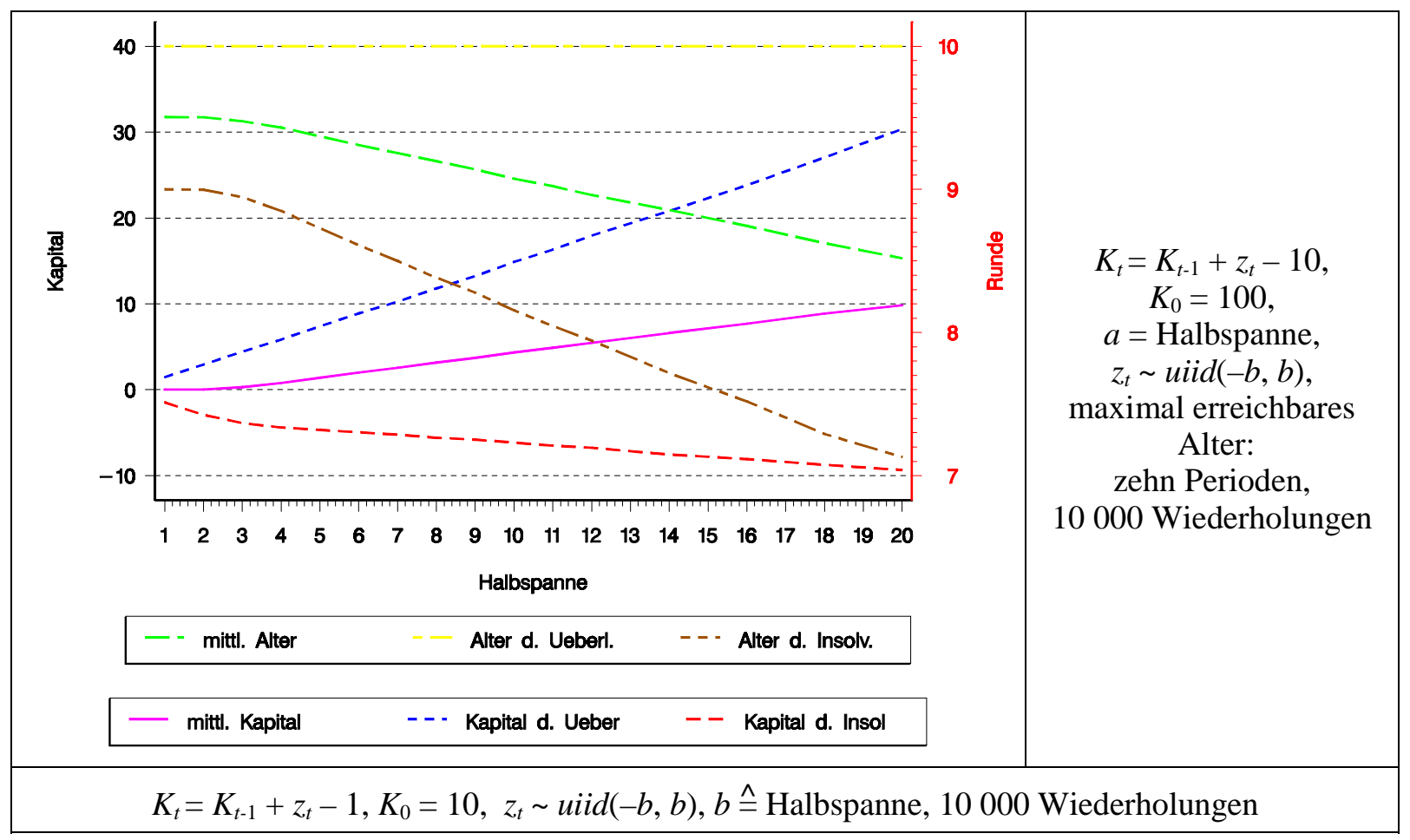

Die drei Kurven für das Alter in Schaubild 248.2 sind weniger interessant, weil ihr Verlauf deduzierbar ist: (a) Die Überlebenden erreichen das vorgegebene maximale Alter (oberste Kurve). (b) Bei höherer Varianz wird Insolvenz früher möglich, die 
Insolvenzfälle treten daher in früheren Perioden auf (untere Alterskurve). (c) Die mittlere Lebenserwartung, als Mittelwert aus einer konstanten und einer fallenden Kurve, nimmt zusammen mit der Varianz ab. Zum (negativen) Kapitalbestand in der Insolvenzperiode ist bereits ausgeführt worden, daß er mit der Varianz abnehmen muß (unterste Kurve). Im Umkehrschluß steigt das Kapital der Überlebenden mit zunehmender Varianz an (obere Kapitalkurve). Nicht a priori vorhersehbar ist, welcher Effekt überwiegt. Bei den gewählten Daten nimmt das Kapital über alle Unternehmen zu. Eine Steigerung der Einzahlungsvotalität führt also zu einer Erhöhung des Erwartungswertes des Kapitals in der zehnten Periode.

Abschließende Simulationen bestätigen die naheliegende Vermutung, daß eine Auszahlung durch einen entsprechenden Erwartungswert der Einzahlungen kompensiert werden kann. Veränderungen von Auszahlung und Erwartungswert der Einzahlung, die die Differenz $\mathrm{E}(z)-a$ konstant lassen, beeinflussen die Überlebenswahrscheinlichkeit nicht. Daher führt z.B. eine Verringerung des Erwartungswertes der Einzahlungen auf $-1,-2,-3$ oder -5 zu den gleichen Überlebenswahrscheinlichkeiten wie eine Erhöhung der Auszahlungen um1, 2, 3 oder 5.

\subsubsection{Multiplikative Verknüpfung zwischen Kapital und Einzahlung}

Für die Modellfamilie $K_{t}=K_{t-1} \cdot q_{t}-a$, also für eine multiplikative Verknüpfung von Kapital und Einzahlung, werden im wesentlichen die gleichen Simulationen wie für die additiven Modelle vorgestellt. Auch hier sind die Einzahlungen die einzige stochastische Komponente des Modells, so daß sich Angaben zur Varianz stets auf die Einzahlungen beziehen. Für ihre Verteilung gilt weiterhin, daß sie, wenn nichts anderes vermerkt ist, stetig, symmetrisch und neutral sowie daß die Einzahlungen in allen Perioden die gleiche Verteilung aufweisen und stochastisch unabhängig sind.

Eine kleine Umformulierung des Modells erleichtert aus technischen Gründen die Simulation: Der Aufzinsungsfaktor $q$ wird in $1+$ Zinssatz zerlegt und der Zins nicht dezimal, sondern in Prozent $(i)$ angegeben: ${ }^{179}$

$$
\begin{aligned}
K_{t} & =K_{t-1} \cdot q_{t}-a \\
& =K_{t-1} \cdot\left(1+z_{t}\right)-a \\
& =K_{t-1} \cdot\left(100+i_{t}\right) / 100-a .
\end{aligned}
$$

Diese Schreibweise offenbart, daß für $a=0$ Insolvenz nur möglich ist, wenn der Zinssatz -100 unterschreitet. Daher gibt es für neutrale Einzahlungen mit einer Halbspanne von 100 praktisch in keiner Runde eine Insolvenzwahrscheinlichkeit. Für nur geringfügig größere Spannbreiten geht die Überlebenswahrscheinlichkeit bereits stark zurück, wie Schaubild 250,1 zeigt.

179 Zinssätze in Prozent erlaubt es, für ihre Erwartungswerte weitgehend ganze Zahlen zu verwenden. Ganze Zahlen steigern die Recheneffizienz des verwendeten Simulationsprogramms und erleichtern die Programmierung. 
Schaubild 250.1: Überlebensquote bei unterschiedlicher Varianz, multiplikatives Modell mit kleinem Startkapital

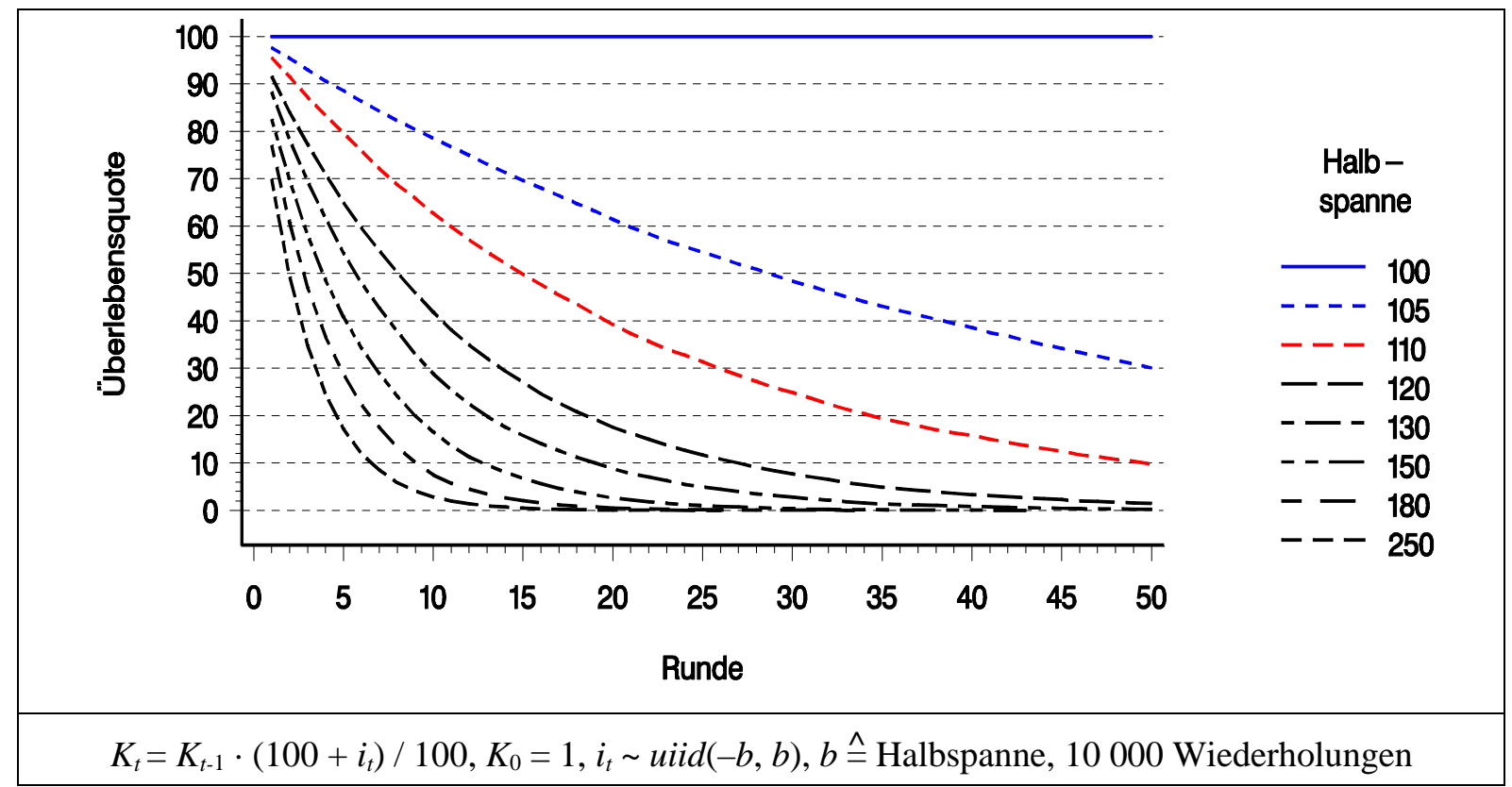

Der Einfluß, den die Varianz der Verzinsung auf die Überlebenswahrscheinlichkeit in einer bestimmten Runde ausübt, ist offensichtlich nicht linear. In Schaubild 250,2 ist die Halbspanne des Zinssatzes gegen die Überlebenswahrscheinlichkeit in der Runde 10 bzw. 5 abgetragen.

Schaubild 250.2: Überlebensquote bei unterschiedlicher Varianz für die Perioden fünf und zehn, multiplikatives Modell

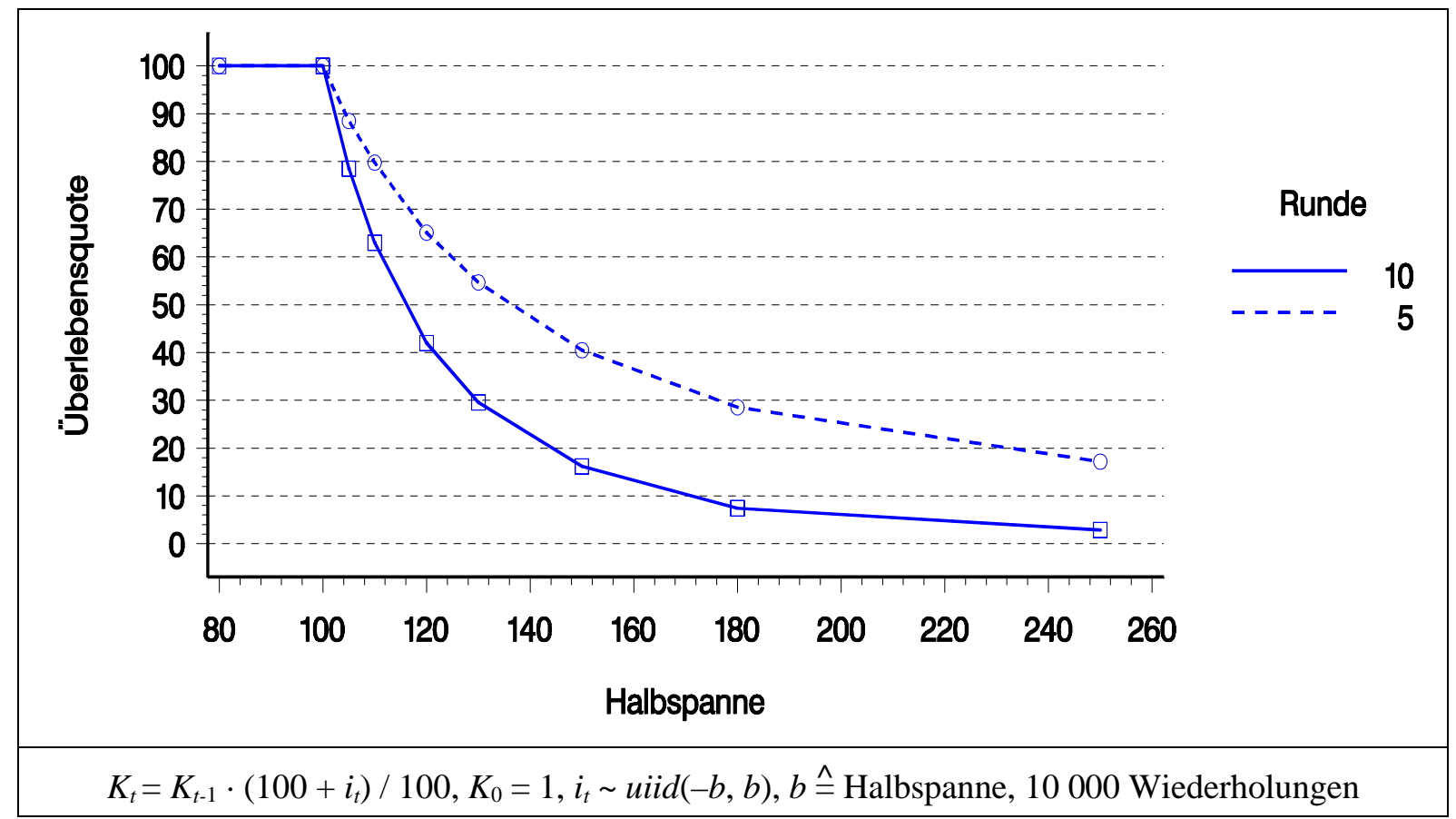


Das Schaubild zeigt, daß Varianzen, die die Halbspanne nicht auf über 100 erhöhen, unschädlich sind. Geringfügig größere Variabilität führt zu einem steilen Abfall der Überlebenswahrscheinlichkeit, die sich bei größerer Volatilität in Form einer Hyperbel Null nähert. Der hyperbolische Charakter, d.h. die Krümmung der Kurve, nimmt mit der betrachteten Rundenzahl zu.

Die verwendeten stochastischen Zinssätze sind arithmetisch um ein Vielfaches größer als die Einzahlungen im vorigen Unterabschnitt. Daher ist der Unterschied zwischen diskreten und stetig verteilten Zufallszahlen vernachlässigbar, wie Schaubild 251.1 an einem Beispiel demonstriert.

\section{Schaubild 251.1: Überlebensquote für stetig und diskret gleichverteilte Einzahlungen, multiplikatives Modell}

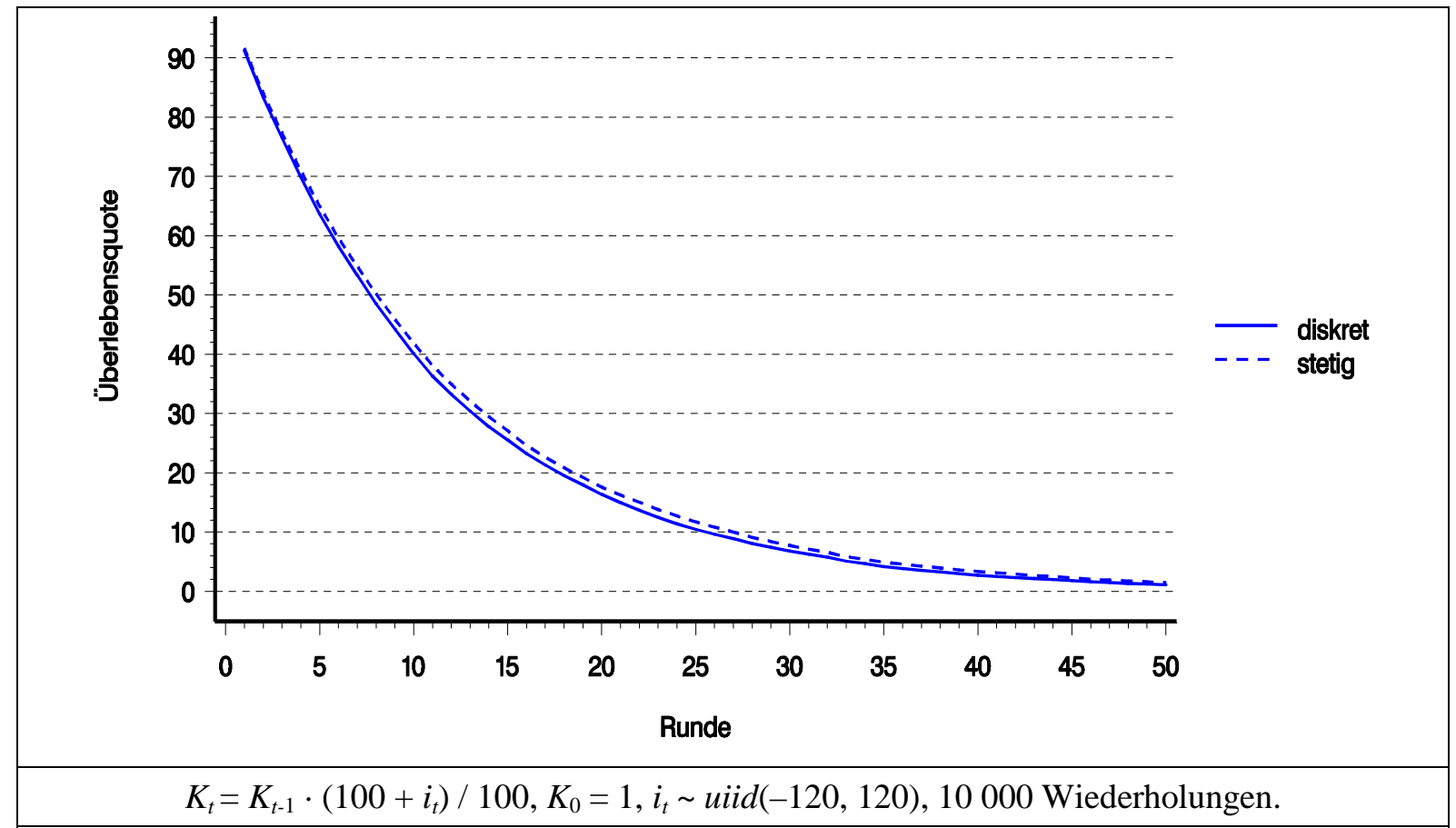

Das Startkapital wirkt sich in Modellen ohne Auszahlung, wie in Abschnitt 5.1 auf Seite 208 nachgewiesen, nicht auf die Insolvenzgefahr aus. Bevor das Startkapital in Modellen mit Auszahlung variiert wird, zeigt Schaubild 252.1 die Effekte von verschiedenen Auszahlungshöhen bei einem Startkapital von Zehn und neutralen Einzahlungen.

Auf den ersten Blick birgt die Graphik keine Überraschungen. Mit zunehmender Auszahlung fällt die Überlebenswahrscheinlichkeit rascher ab. Betrachten wir dennoch das Modell genauer. 


\section{Schaubild 252.1: Überlebensquote bei variierten Auszahlungen, multiplikatives Modell}

\begin{tabular}{|c|c|ccc|}
\hline & 90 & & \\
8
\end{tabular}

Ohne Auszahlung ist keine Insolvenz möglich, da der Aufzinsungsfaktor wegen der vorgenommenen Spezifikation seiner Verteilung $1 / 2$ (entsprechend $i=-50$ ) nicht unterschreiten kann. Ohne Einzahlung, also in einem deterministischen Modell, würde das System, wenn die Auszahlung Eins und das Startkapital Zehn beträgt, wie bei additiven Einzahlungen (s. S. 245 genau neun Perioden überleben. In der zehnten wäre das Kapital bereits Null und damit definitionsgemäß Zahlungsunfähigkeit eingetreten. Bei einer stochastischen Einzahlung in der zehnten Periode, die neutral und symmetrisch ist, wird das System mit gleicher Wahrscheinlichkeit die zehnte Runde überleben wie fallieren, die mittlere Lebensdauer beträgt somit 9.5 Perioden. Es bestehen also keine Unterschiede zwischen additivem und multiplikativem Modell (vgl. Schaubilder 246,1 und 252,1].

Anders sieht es aus, wenn es in jeder Periode eine stochastische Einzahlung gibt. Schaubild 253,1 zeigt die Altersverteilung für dieses Szenario. Sie verläuft sehr unsymmetrisch. Da das Minimum des Aufzinsungsfaktors auf $1 / 2$ festgesetzt ist, kann eine Insolvenz frühestens in der dritten Periode eintreten, wie die nachfolgende Aufstellung zeigt:

\begin{tabular}{|c|c|c|c|c|}
\hline Periode & $\begin{array}{c}\text { Kapital am } \\
\text { Anfang }\end{array}$ & $\begin{array}{c}\text { minimale } \\
\text { Einzahlung }\end{array}$ & Auszahlung & $\begin{array}{c}\text { minimales } \\
\text { Kapital am Ende }\end{array}$ \\
\hline \hline 1 & 10 & -5 & 1 & 4 \\
\hline 2 & 4 & -2 & 1 & 1 \\
\hline 3 & 1 & $1 / 2$ & 1 & $-1 / 2$ \\
\hline \multicolumn{5}{|c|}{$K_{t}=K_{t-1} \cdot q_{t}-1, K_{0}=10, q_{t}=1 / 2$} \\
\hline
\end{tabular}


Nach oben sind dem erreichbaren Alter keine Grenzen gesetzt. Aus Schaubild 253.1 ist ersichtlich, daß das häufigste Alter Sechs und der Median Sieben beträgt. Eine Berechnung des Mittelwertes kann aus den bisherigen Daten nicht erfolgen, da das maximale Alter aus rechentechnischen Gründen auf 50 begrenzt ist. Die Wahrscheinlichkeit, ein höheres Alter zu erreichen, bewegt sich zwar im Promillebereich, dennoch könnte sie das mittlere Alter signifikant verändern, da große und sehr große Lebensdauern theoretisch vorkommen können.

\section{Schaubild 253.1: Beispiel einer Altersverteilung in einem multiplikativen Modell}

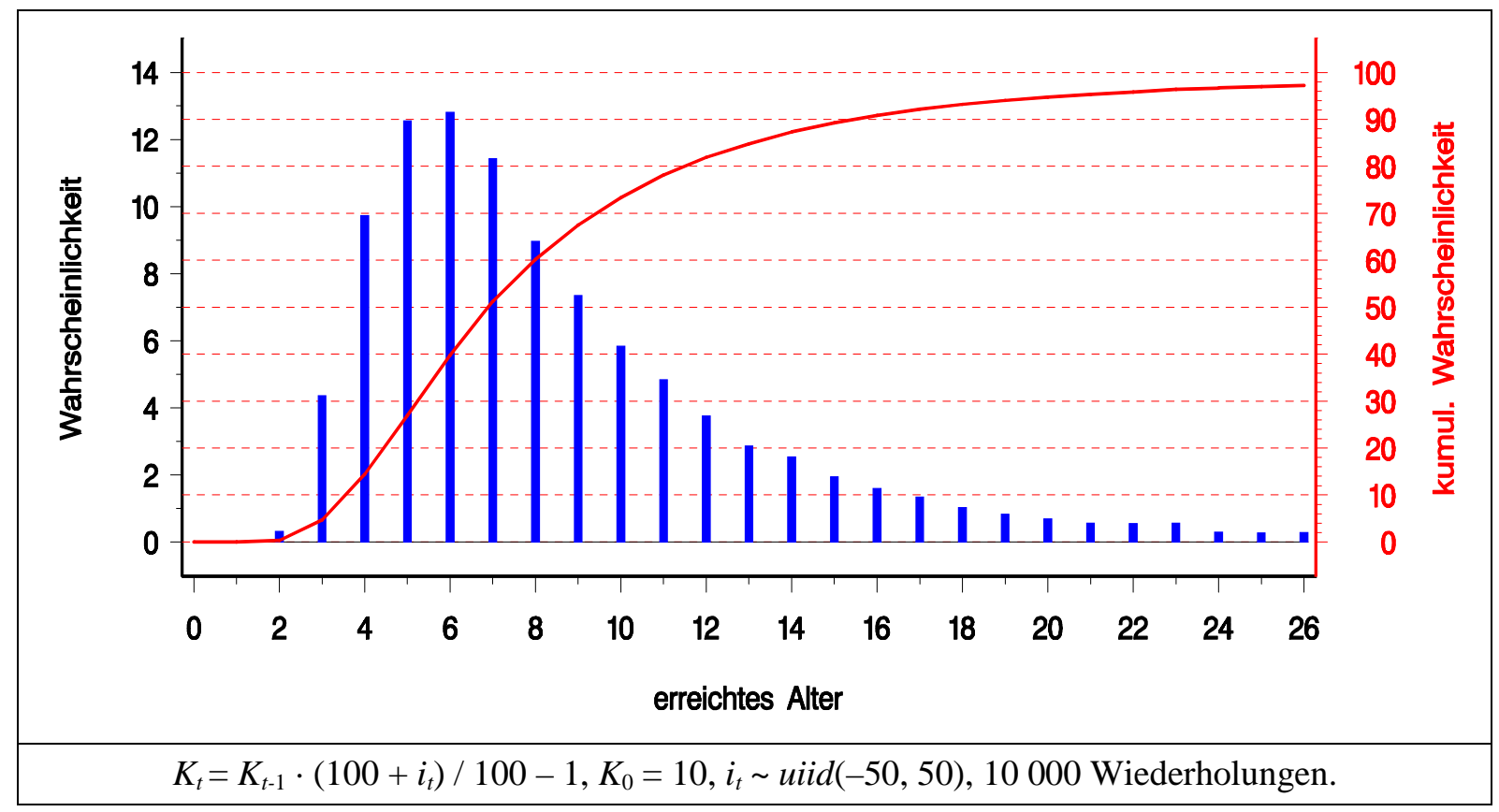

Daher wird die Simulation mit entsprechenden Änderungen wiederholt. Die höchsten erreichten Perioden sind 112, 115, 134 und 150 Perioden. Sie treten jeweils genau ein Mal bei 10000 Wiederholungen auf. Dies verdeutlicht, daß das maximal erreichte Alter in hohem Maße ein stochastischer Wert ist. Und in der Tat führt eine Wiederholung der Simulation ${ }^{180}$ zu Lebensdauern in der Spitze von 121, 176, 233 und 304.

180 Die Simulationen werden im allgemeinen mit einem sog. Seed von 65 durchgeführt. Für diese Wiederholungssimulation wurde ein Seed von 5 verwendet.

Wie in Unterabschnitt 2.1.4.2 ausgeführt, werden für Monte-Carlo-Simulationen Pseudozufallszahlen verwendet. Dies sind streng deterministische Abfolgen von Ziffern. Daher ist jede Reihe durch ihre Startsequenz eindeutig charakterisiert. Diese Sequenz bezeichnet man in der Computerterminologie als Seed. Wird für die Simulationen ein anderer Seed als 65 verwendet, wird dies ausdrücklich vermerkt, um die Ergebnisse reproduzieren zu können.

Die Auswahl des Seeds geschah nach folgendem Kriterium: Der Anteil der Zufallszahlen, die negativ vom Erwartungswert abweichen, soll 1/2 möglichst nahe kommen, damit die Anzahl positiver und die Anzahl negativer Abweichungen möglichst übereinstimmen. Formal formuliert: die Differenz zwischen dem Median der gezogenen Zufallszahlen und ihrem (theoretischen) Erwartungswert (nicht arithmetischem Mittelwert) wird minimiert. 
Die Unterschiede zwischen den beiden Simulationsdurchläufen zeigen deutlich, daß das Maximum einer simulierten Verteilung in weiten Bereichen schwanken kann. Diese Varianz des Maximums kann nicht durch eine Erhöhung des Stichprobenumfangs reduziert werden. Mehr Wiederholungen verschieben, bildlich gesprochen, das Maximum nach außen und verringern damit nicht seine Variabilität. Beispielsweise führen eine Million Wiederholungen anstelle von $10000 \mathrm{zu}$ Altersmaxima von 235, 347, 407 und 487, jeweils mit absoluter Häufigkeit 1.

Die Streuung des Maximums beeinflußt die mittlere Lebensdauer abgeschwächt, da die Extremwerte nur sehr geringe Wahrscheinlichkeiten besitzen. Bei einem Seed von 5 beträgt das mittlere Alter 9.309 und bei dem üblichen Seed von 65 verändert es sich auf 9.346. Diese Differenz von 0.037 beruht ausschließlich auf der Wahl anderer Zufallszahlen. Schwankungen in dieser Größenordnung, hier ein halbes Prozent, sind daher nicht systematisch und können nicht interpretiert werden.

Die Abweichungen des Simulationsergebnisses von 9.3 zu 9.5, dem vermuteten Erwartungswert der Lebensdauer, ist um ein Vielfaches größer. Die Einführung einer stochastischen Einzahlung, deren Erwartungswert Null ist, führt also offensichtlich zu einer Lebenserwartung und damit zu einer Überlebenswahrscheinlichkeit, die beide deutlich geringer sind, als aufgrund von theoretischen Überlegungen vermutet.

Eine Ursache dieser Diskrepanz könnte in Schwächen der Simulationstechnik begründet liegen. Sehr große Werte besitzen in diesem Modell nur eine sehr kleine Wahrscheinlichkeit. In einem Simulationsexperiment können sie auch in sehr großen Stichproben praktisch nicht auftreten, wenn Einzelwahrscheinlichkeiten und Stichprobenumfang zusammen zu theoretischen absoluten Häufigkeiten kleiner als Eins führen, wie im Exkurs über das Petersburger Paradoxon ausführlich beschrieben (Abschnitt 4.5. Dadurch wird der Mittelwert nach unten verzerrt. Eine Vergrößerung des Stichprobenumfangs erhöht das zu erwartende Maximum der Altersverteilung. Vermutungen, mit dem größeren Maximum wachse auch der Mittelwert der Lebenserwartung, bestätigen sich dennoch nicht. Wie in Schaubild 255.1] dargestellt, nimmt das durchschnittliche Alter bei einer Verzehnfachung der Wiederholungen deutlich ab und steigt bei der nächsten Zehnerpotenz geringfügig wieder an. Ein Experiment mit $10^{7}$ Wiederholungen kann wegen begrenzter Ressourcen nicht durchgeführt werden.

Der Rückgang der Lebenserwartung zwischen $10^{4}$ und $10^{5}$ Wiederholungen ist deutlich größer als der Unterschied zwischen den beiden Folgen von Zufallszahlen und daher wohl nicht zufallsbedingt.

Untersucht wurden alle Seeds des Programmpakets $S A S^{\circledR}$ zwischen 1 und 200. Der Seed von 65 erfüllt die gestellte Anforderung am weitesten: Bei einem Stichprobenumfang von 100000 Wiederholungen realisieren sich 50004 Zufallszahlen kleiner als der Erwartungswert, der als Null festgesetzt ist. Das arithmetische Mittel beträgt 0.00048 .

Die Differenz zwischen Erwartungswert und arithmetischen Mittelwert minimiert ein Seed von 118 mit $\bar{x}=2 \cdot 10^{5}$. Hierbei liegt die Anzahl negativer Realisationen mit 49859 um 141 unter der optimalen Anzahl von 50000. 


\section{Schaubild 255.1: Durchschnittliches Alter in einem multiplikativen Modell}

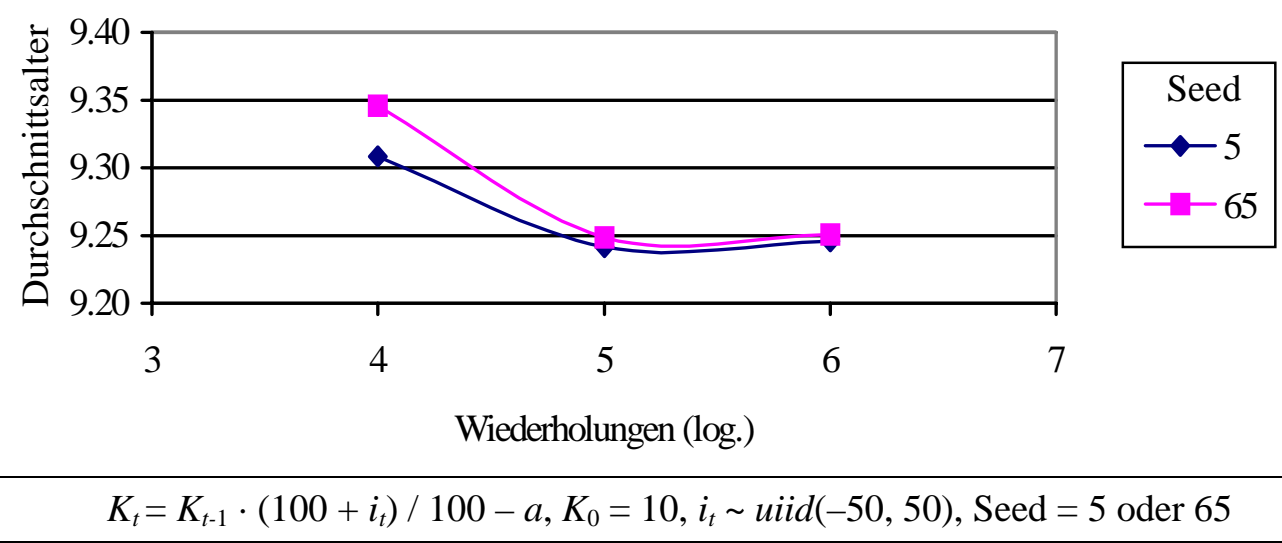

Die Experimente mit gesteigerter Wiederholungszahl liefern also keine Anhaltspunkte für eine Erklärung, warum das mittlere Alter deutlich unter 9.5 Perioden bleibt. Die Frage muß daher zunächst unbeantwortet bleiben. Untersucht werden kann hingegen, welchen Einfluß die Varianz der Einzahlungen auf die Lebensdauer ausübt. Schaubild 255.2 gibt hierauf eine eindeutige Antwort.

\section{Schaubild 255.2: Lebenserwartung bei variierter Varianz in einem multiplikativen Modell}

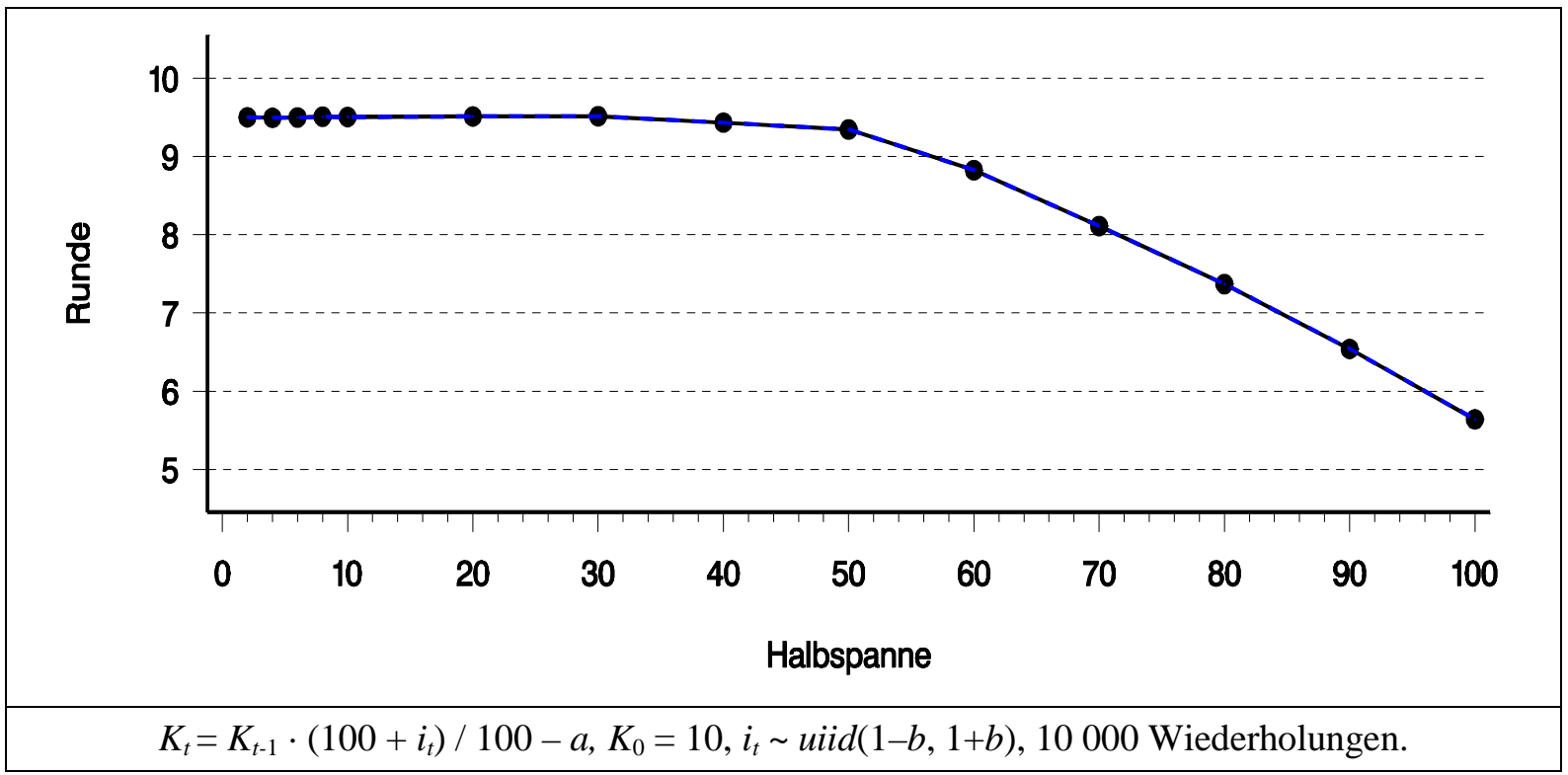

Für kleine Varianzen wird die theoretische Ableitung bestätigt, die mittlere Lebenserwartung beträgt 9.5 Perioden. Wächst die Varianz, fällt die Lebenserwartung drastisch ab. Daher ist es in diesem Beispiel auch für eine risikoneutrale Entscheiderin sinnvoll, eine wenig volatile Anlageform zu bevorzugen. Ursache dieses Kurvenverlaufs ist die Nicht-Umkehrbarkeit von Kapitalpfaden, die die Nullinie unterschritten haben: Auf der einen Seite werden überdurchschnittlich hohe Zuwächse in den ersten 
Perioden im statistischen Mittel in späteren Runden wieder ausgeglichen. Diese Möglichkeit besteht auf der anderen Seite bei außergewöhnlich geringen Zuwächsen nicht mehr, wenn Insolvenz eingetreten ist. Diese Asymmetrie verstärkt sich bei größerer Varianz der Einzahlungen und erklärt die abfallende Steigung des Graphen.

Die nachfolgende Graphik zeigt die Wahrscheinlichkeit, eine vorgegebene Runde in Abhängigkeit von der Streuung des Zinssatzes zu erreichen. In der obersten Kurve ist diese Wahrscheinlichkeit für einen Planungshorizont von fünf Perioden abgetragen. Bei kleiner Spanne des Zinssatzes besteht kein Risiko, dieses Alter nicht zu erreichen. Erst durch eine größere Streuung des Zinssatzes wird Insolvenz möglich, wie in Abschnitt 4.3 in Zusammenhang mit Schaubild 156.1 erläutert. Daher fällt die Überlebenswahrscheinlichkeit mit Zunahme der Varianz.

\section{Schaubild 256.1: Überlebensquote für eine vorgegebene Periode in Abhängigkeit von der Varianz der Verzinsung, multiplikatives Modell mit} Auszahlung

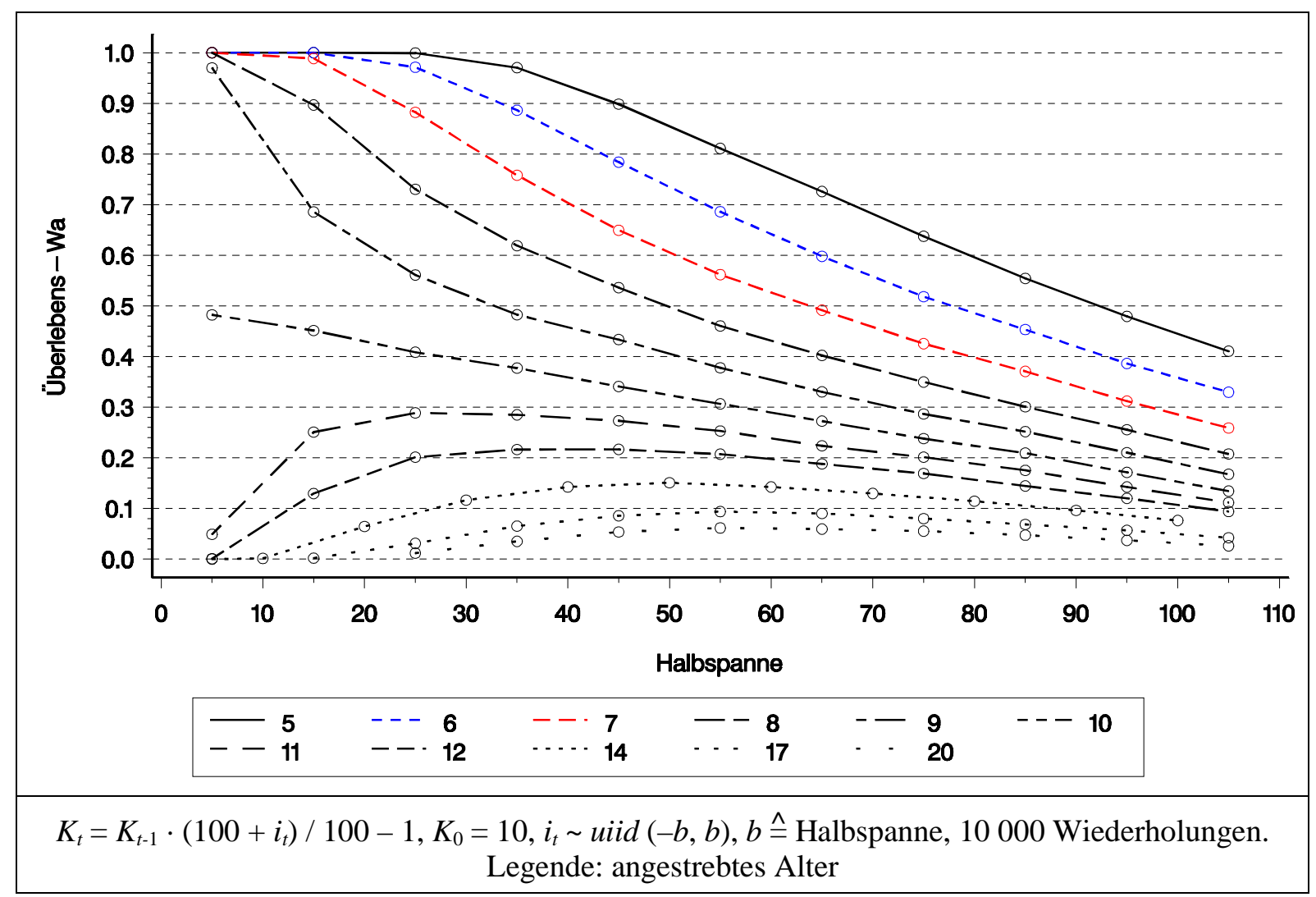

Wird eine höhere Lebensdauer angestrebt, können im Vergleich zu fünf Perioden schon geringere Varianzen dieses Überlebensziel gefährden. Ein Alter von neun Perioden wird bereits bei der kleinsten untersuchten Schwankungsbreite des Zinssatzes von $\pm 5 \%$ nicht mit Sicherheit erreicht.

Auffällig sind die Sprünge zur nächsten und übernächsten Altersstufe. Zehn Perioden werden auch bei kleinster Varianz nur in knapp der Hälfte der Fälle überlebt. Mit 
größerer Streuung fällt die Kurve ab. Die Ursache ist die gleiche, die auch zu einer Abnahme der durchschnittlichen Lebenserwartung (Schaubild 255.2) führt: Eine infinitesimale Varianz ist Voraussetzung, um überhaupt das bei deterministischen Einzahlungen unmögliche Alter von zehn Perioden zu erreichen. Eine minimale Varianz führt zu einer Überlebenswahrscheinlichkeit für neun Perioden von $1 / 2$ und für zehn Perioden von ebenfalls 1/2. Jede Steigerung der Varianz kann diese Überlebenswahrscheinlichkeit nicht erhöhen, im Gegenteil: Nachdem eine kritische Varianzgrenze überschritten worden ist, wird ein Ausscheiden vor der neunten Periode möglich und daher die Überlebenswahrscheinlichkeit für neun Runden gesenkt. Die kritische Zinsvolatilität liegt offensichtlich unterhalb von $\pm 5 \%$.

Die Kurven für einen längeren Planungshorizont zeigen einen deutlich anderen Verlauf. Hier ist eine größere Mindestvarianz Voraussetzung, um das gewünschte Alter zu erreichen. Beispielsweise kann ein Alter von 17 oder 20 Perioden bei einer Streuung des Zinssatzes von $\pm 5 \%$ nicht erzielt werden. Eine Lebensdauer von elf Runden besitzt die größte Wahrscheinlichkeit, wenn der Zinssatz um $\pm 25 \%$ schwankt. Größere Votalitäten verringern die Überlebenswahrscheinlichkeit wieder.

Überlebenswahrscheinlichkeiten für mehr als zehn Perioden zeigen also deutliche Maxima. Zur Erklärung können die bereits genannten Gründe herangezogen werden. Eine gewisse Streuung der Einzahlungen ist Voraussetzung, um ein Alter zu erreichen, das bei deterministischen Einzahlungen unmöglich ist.

Die nachfolgende Graphik 2571 demonstriert, daß zwischen hoher Lebenserwartung und großer Überlebenswahrscheinlichkeit ein Zielkonflikt bestehen kann.

\section{Schaubild 257.1: Überlebensquote bei variierter Varianz in einem multiplikativen Modell mit höherem Startkapital}

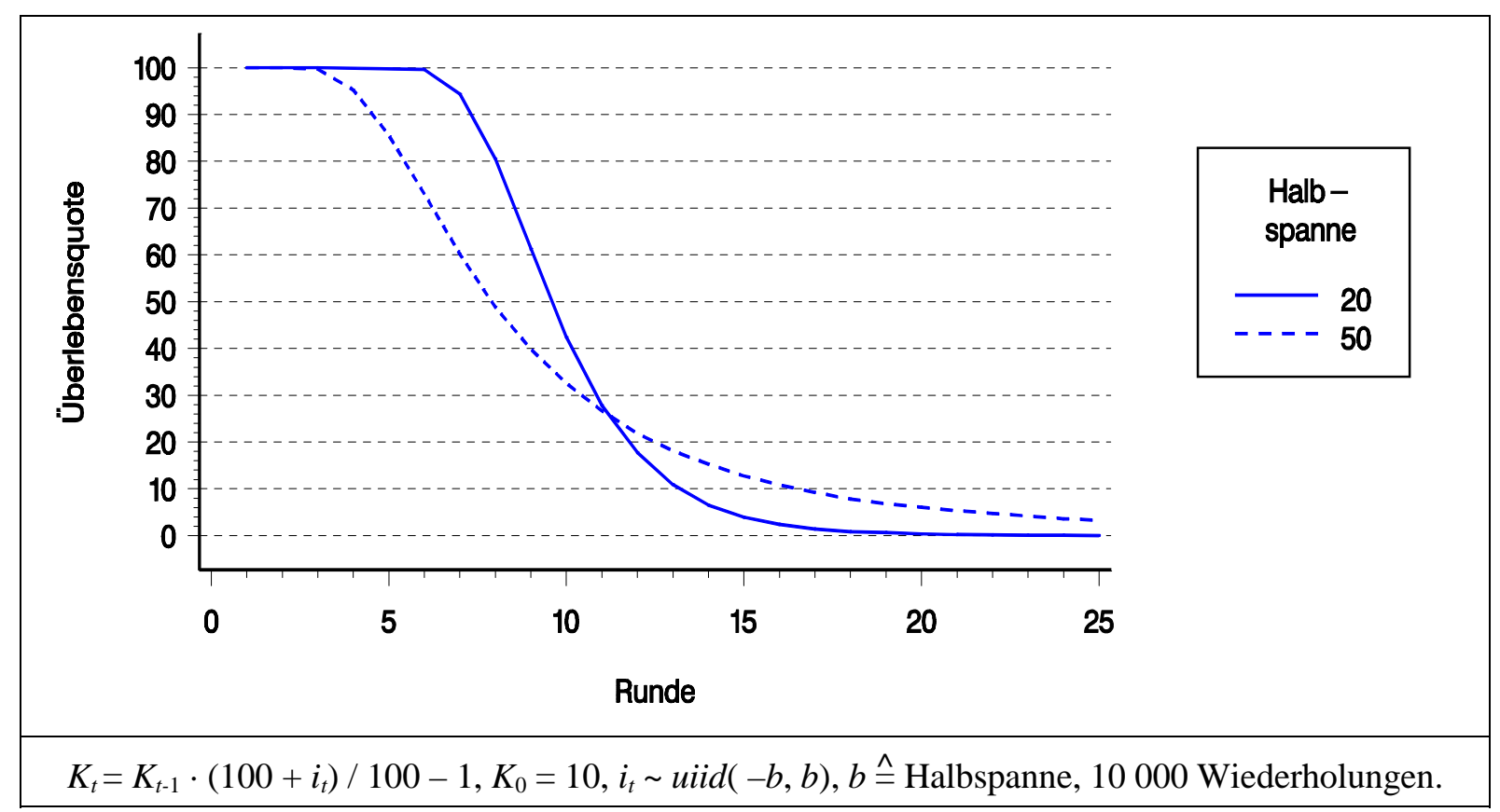


Die Überlebenswahrscheinlichkeit kann, wie bereits erwähnt, nur für eine vorbestimmte Runde angegeben werden. Steht die Maximierung der Lebensdauer im Vordergrund, ist es vorteilhaft, eine kleine Varianz der Einzahlung zu wählen, wie im vorherigen Schaubild gezeigt. Zinssätze mit einer Schwankung von $\pm 20 \%$ lassen ein Alter von 9.5 Perioden erwarten, Zinsen mit einer Schwankung von $\pm 50 \%$ nur von 9.3 Perioden. Weniger streuende Zinsen führen ebenso zu einer größeren Überlebenswahrscheinlichkeit, wenn der Planungshorizont zehn Perioden nicht überschreitet. Hat die Entscheiderin hingegen die Runde 15 im Blick, so wird bei Wahl der volatileren Einzahlung diese Runde mit größerer Wahrscheinlichkeit erreicht, als bei der Einzahlungsalternative mit der geringeren Varianz. Volatilere Zinsen senken also die Lebenserwartung und erhöhen gleichzeitig die Wahrscheinlichkeit, zwölf und mehr Perioden zu überleben.

\subsection{Modelle mit stochastischer und fester Einzahlung (Portfolio-Modelle)}

In diesem Abschnitt wird ein Investor betrachtet, der in jeder Periode konstante Auszahlungen zu bestreiten hat. Ihm stehen zwei Anlagemöglichkeiten (ein Portfolio) zur Verfügung:

- eine spekulative mit im Durchschnitt hoher Rendite $\tilde{q}$, z.B. Aktien mit stochastischer Dividende (abgekürzt mit ,Aktien“), und

- eine sichere mit geringerer Verzinsung $\bar{q}$, z.B. Finanzanlagen mit festem Zins (abgekürzt mit ,FinAnl“).

$\tilde{q}$ und $\bar{q}$ werden als Aufzinsungsfaktoren interpretiert. Der Kapitalbestand zu Ende einer Periode kann daher geschrieben werden als:

$$
K_{t}^{e} \quad=\text { FinAnl }_{t} \cdot \bar{q} \quad+\text { Aktien }_{t} \cdot \tilde{q} \quad-a .
$$

$\mathrm{Zu}$ Anfang jeder Periode hat sich der Investor zu entscheiden, wie er sein Portfolio auf die beiden Anlageformen aufteilen will:

$$
\begin{aligned}
K_{t}^{a} & =\text { FinAnl }_{t} & & + \text { Aktien }_{t} \\
& =\beta \cdot K_{t}^{a} & & +(1-\beta) \cdot K_{t}^{a} .
\end{aligned}
$$

Somit ergibt sich für das Kapital am Periodenende:

$$
\begin{aligned}
& K_{t}^{e} \quad=\beta \cdot K_{t}^{a} \cdot \bar{q} \quad+(1-\beta) \cdot K_{t}^{a} \cdot \tilde{q}-a \\
& =K_{t}^{a} \cdot(\beta \cdot \bar{q}+(1-\beta) \cdot \tilde{q})-a .
\end{aligned}
$$

Da das Kapital zu Beginn einer Periode definitorisch gleich dem Kapitalbestand am Ende der Vorperiode ist:

$$
K_{t}^{a} \quad=K_{t-1}^{e},
$$

kann das Kapital am Ende von Periode $t$ auch geschrieben werden als: 


$$
K_{t}^{e} \quad=K_{t-1}^{e} \cdot(\beta \cdot \bar{q}+(1-\beta) \cdot \tilde{q}) \quad-a .
$$

Für das Modell gelten folgende zusätzliche Annahmen:

(a) Wenn das Kapital am Ende einer Periode nicht positiv ist, scheidet das Unternehmen aus.

(b) Die Portfolioaufteilung $\beta$ wird zu Beginn des Planungszeitraumes festgelegt und kann in den weiteren Runden nicht geändert werden.

(c) Der Erwartungswert der Rendite der Aktien ist höher als der Festzins: $\mathrm{E}(\tilde{q})>\bar{q}>1$.

(d) Die realisierte Rendite der Aktien kann den Festzins unterschreiten: $\operatorname{Min}(\tilde{q})<\bar{q}$.

(e) Das Startkapital ist deutlich größer als die feste Auszahlung: $K_{0} \gg>a$.

(f) Die Finanzanlage reicht nicht aus, die feste Auszahlung zu decken: $K_{0} \cdot \bar{q}<a$.

Ohne die Annahmen b) und c) wäre die Fragestellung trivial: Überträfe die Rendite der Aktien auch im schlechtesten Fall den Festzins, gäbe es kein Entscheidungsproblem. Ohne die Annahme f) wäre ein Überleben sicher möglich. Die Aufnahme eines Startkapitals in das Modell ist notwendig, da anderenfalls keine Einnahmen erzielt werden könnten.

SZPIRO (1997) benutzt ein ähnliches Modell zur Untersuchung von Risikoaversion mit Hilfe genetischer Algorithmen. Er untersucht eine Population aus Agenten mit zufällig ausgewählten Verhaltensweisen, die einem Selektionsdruck unterworfen sind. Die Verhaltensweisen erfahren im Generationenwechsel nicht zielgerichtete Veränderungen (Mutationen). Es stellt sich heraus, daß sich langfristig Verhaltensweisen durchsetzten, die statt reiner Gewinnmaximierung einen Portfoliomix bevorzugen. Die hierin zum Ausdruck kommende Risikoaversion entspricht in ihrer Größenordnung Aversionskoeffizienten, die auch in empirischen Erhebungen gefunden werden.

Im weiteren soll in einer Monte-Carlo-Simulation folgender Frage nachgegangen werden:

\section{Welcher Wert für $\beta$ maximiert die Überlebenswahrscheinlichkeit?}

Das Oberziel „maximale Überlebenswahrscheinlichkeit“ kann operational in zwei Subziele aufgegliedert werden, die nicht immer harmonieren werden (vgl. hierzu die Ausführungen im vorherigen Abschnitt). Maximale Überlebenswahrscheinlichkeit kann einerseits bedeuten, die Wahrscheinlichkeit, eine vorgegebene endliche Runde zu erreichen, zu maximieren, oder anderseits, ohne konkreten Planungshorizont eine maximale Lebensdauer anzustreben. Letzteres Ziel ist nicht sehr hilfreich. Zum einen ist es nicht sehr plausibel, daß eine durchschnittliche Lebensdauer von 200 einer von 180 Jahren lexikographisch vorgezogen wird. Zum anderen ist es für Simulationsexperimente aus rechentechnischen Gründen vorteilhaft, die maximale Lebensdauer zu begrenzen.

Für die in Tabelle 260.1 aufgelisteten Parameter zeigt Schaubild 260.1 den Verlauf der Überlebenswahrscheinlichkeit (gestrichelte Linie) bei einer sukzessiven Erhöhung 
des Anteils der stochastischen Anlage. Bei ausschließlich fester Anlage ist es annahmegemäß nicht möglich, zehn Perioden zu überleben. Mit Zunahme der stochastischen Geldanlage steigt die Überlebenswahrscheinlichkeit steil an und erreicht bei $40 \%$ ihren höchsten Wert.

Tabelle 260.1: Parameter eines Portfoliomodells

\begin{tabular}{|c|c|c||c|c|c|}
\hline Variable & Kürzel & Wert & Variable & Kürzel & Wert \\
\hline \hline Startkapital & $K_{0}$ & 500 & maximal erreichbares Alter & & 10 \\
\hline fester Zins & $\bar{q}$ & 1.10 & stochastischer Zins & $\tilde{q}$ & 1.13 \\
\hline Auszahlung & $a$ & 100 & Anzahl Wiederholungen & & 10000 \\
\hline
\end{tabular}

\section{Schaubild 260.1: Portfoliowahl und Überlebensquote für 10 Perioden}

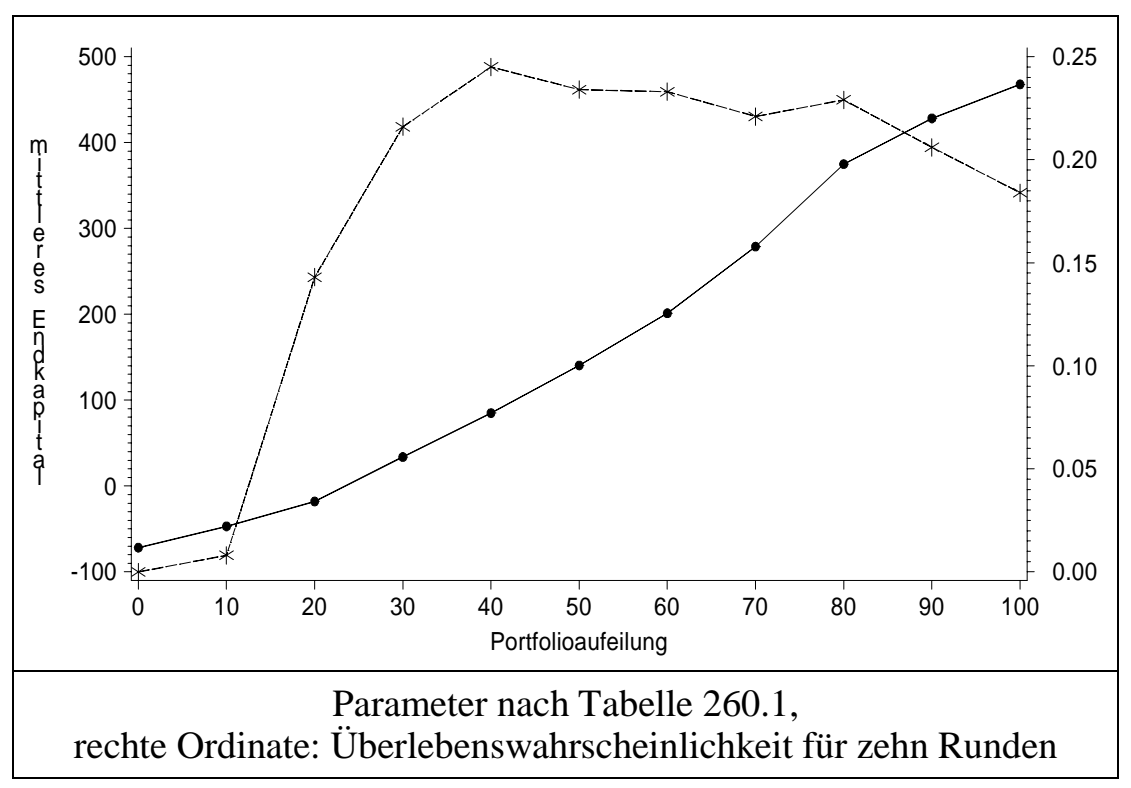

Die zweite Kurve (durchgezogene Linie) beschreibt die Entwicklung des durchschnittlichen Kapitals. Überlebende Unternehmen gehen in diese Rechnung mit ihrem Endvermögen in der zehnten Runde ein, ausscheidende Unternehmen mit dem (negativen) Kapitalstand, das sie in der Runde ihrer Insolvenz aufwiesen.

Das so berechnete durchschnittliche Endkapital steigt monoton. Auch nachdem die Überlebenswahrscheinlichkeit ihr Maximum überschritten hat, nimmt das durchschnittliche Kapital weiter zu. Offensichtlich vergrößert die Erhöhung des stochastischen Anlageanteils das Endvermögen der verbleibenden Unternehmen so sehr, daß die Abnahme der Anzahl der überlebenden Unternehmen überkompensiert werden kann.

Das nächste Schaubild 2611 zeigt neben dem Kapitalverlauf das durchschnittlich erreichte Alter. Bereits bei einem stochastischen Anlageanteil von 20\% wird scheinbar der Erwartungswert der Lebensdauer maximiert. Diese Graphik ist aus den bereits 
genannten Gründen jedoch irreführend: da das maximal erreichbare Alter auf zehn begrenzt ist, ist das durchschnittlich erreichte Alter nach unten verzerrt.

\section{Schaubild 261.1: Portfoliowahl und Lebensdauer}

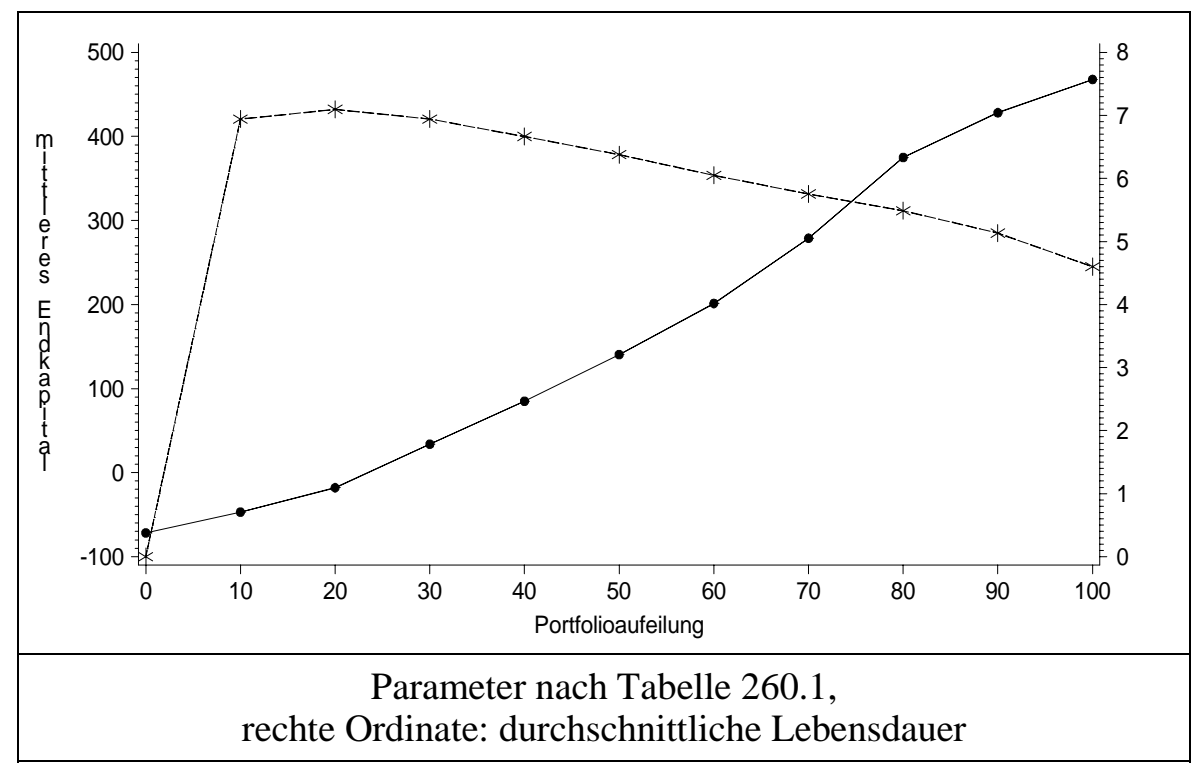

\subsection{Beispiele eines einfachen stochastischen Betriebsentwicklungs- modells}

Nachdem in den vorigen Abschnitten die „Überlebensfähigkeit“ einfacher Systeme untersucht wurde, stehen in diesem und dem folgenden Abschnitt stärker anwendungsorientierte Systeme im Vordergrund, ein einfaches Betriebsentwicklungsmodell und ein Planspiel.

Mit Betriebsentwicklungsmodellen wird versucht, die Entwicklung von Betrieben oder Unternehmen in Abhängigkeit von relevanten Einflußfaktoren zu analysieren. Bei der Konstruktion eines solchen Modells ist $\mathrm{zu}$ entscheiden, welche Einflußfaktoren aufgenommen und welche Ergebnisgrößen in ihrem Verlauf untersucht werden sollen. Deckt das Modell einen längeren Zeitraum ab, kann es nützlich sein, einen endogenen Entscheidungsprozeß abzubilden. Betriebsentwicklungsmodelle werden damit gleichzeitig zu Entscheidungsmodellen.

In der Praxis hängt die Entscheidungsfindung von mannigfaltigen Einflüssen ab und ist zum Teil mit rationalen Kriterien nicht nachvollziehbar. Dies wird z.B. an den Schwierigkeiten deutlich, das Verhalten von Menschen, auch von Wirtschaftssubjekten, die sich in einem marktwirtschaftlichen Wettbewerb befinden, vorherzusagen. Zielfunktionen von Wirtschaftssubjekten sind in der Regel mehrdimensional, wobei die Erreichung eines Ziels die Erfüllung eines anderen oft erschwert oder gar ausschließt (hoher Grad von Zielkonkurrenz). Die Beiträge einer Entscheidungsalternative zur Erfüllung der Subziele sind häufig nur vage bekannt, und eine genauere Evaluierung ist in der zeitlichen Kürze der Entscheidungssituation nicht möglich. 
Ein Modell muß von dieser Komplexität menschlicher Verhaltensweisen abstrahieren. Jemand, der/die ein Modell baut, wird sich daher immer sehr stark beschränken (vgl. Unterabschnitt 2.1.2). Dennoch müssen in einem Entscheidungsmodell alle Faktoren, die die Wahlhandlungen beeinflussen sollen, vollständig aufgenommen werden. ${ }^{181}$ Die Verarbeitung der Einflüsse zu einer Entscheidung kann in einem Simulationsmodell nicht durch eine black box erfolgen, sondern ist umfassend abzubilden. Die Art der Informationsverarbeitung läßt sich in einem Modell nur mit Hilfe rationaler Methoden ${ }^{182}$ nachzeichnen. Die Anzahl entscheidungsrelevanter Einflüsse sollte so gering und ihre Verknüpfung so einfach wie möglich sein, damit das Modell leicht verständlich und weniger anfällig gegen Fehlspezifikation ist. Andererseits muß das Modell auch ausreichend komplex sein, um Antworten auf die interessierenden Fragen geben zu können.

Wie zu Ende von Unterabschnitt 2.1.4.3 angerissen, lassen sich bei Betriebsentwicklungsmodellen zwei Typen, die quasi Pole eines Kontinuums bilden, unterscheiden. Beim ersten Typ werden Einproduktunternehmen mit wenigen Produktionsfaktoren (i.d.R. zwei) oder Zweiproduktunternehmen, die nur einen Faktor verwenden, untersucht. ${ }^{183}$ Dieses einfache Produktionsprogramm erlaubt es, auch nichtlineare Produktionsfunktionen zu verwenden oder Interdependenzen der Marktteilnehmer, die in einem Oligopol auftreten, zu studieren. Die Entscheidungen der Unternehmerinnen zielen auf Gewinnmaximierung o.ä., die Methode stützt sich auf Ableiten einer Gewinnfunktion.

Hier sei ein einfaches Beispiel dieses Typs entwickelt. Untersuchungsgegenstand ist die Frage, ob das Verfolgen des Ziels Gewinnmaximierung gleichzeitig auch eine Maximierung der Überlebenswahrscheinlichkeit bedeutet. ${ }^{184}$ Dazu sei ein Unternehmen mit folgenden Eigenschaften definiert:

- Es besitze zu Beginn ein Eigenkapital in Höhe von $E K_{0}$.

- Es nehme im zu Beginn festgelegten, jährlich gleichen Umfang Fremdkapital ( $F K$ ) auf, der Fremdkapitalzins sei deterministisch und betrage $i$.

- Das Gesamtkapital weise eine stochastische Gesamtkapitalrentabilität $r_{t}$ auf.

- In jeder Periode fallen konstante Auszahlungen $a$ an.

- Die Eigenkapitalfortschreibung erfolge nach der Differenzengleichung erster Ordnung:

$$
E K_{t+1} \quad=E K_{t}+\left(E K_{t}+F K\right) \cdot r_{t}-F K \cdot i-a .
$$

181 Dies schließt gegebenenfalls die Aufnahme stochastischer, entscheidungsbeeinflussender Faktoren ein.

Rationale Methoden sind nicht auf Gewinnmaximierung beschränkt. Entscheidend ist, daß der Entscheidungsprozeß in geschlossener Form modelliert werden kann. Intuitive Entscheidungen entziehen sich einer Abbildung in einem Modell.

184

Beispiel hierfür sind FISCHER (1987), BRANDES $(1989,1990)$.

Diese These wird u.a. von FRIEDMAN (1953) vertreten (s. Abschnitt 4.3). 
- Ein Unternehmen scheide aus, sobald sein Eigenkapital am Ende einer Periode kleiner als Null oder gleich Null ist.

Die Parameter (siehe Tabelle 263.1] sind so gewählt, daß erstens ohne Fremdkapitalaufnahme ein nachhaltiges Überleben nicht möglich ist, und zweitens der Fremdkapitalzins unter dem Erwartungswert der Gesamtkapitalrendite liegt.

Bei Kalkulation mit Erwartungswerten - also ohne Berücksichtigung des Risikos - sind bei diesen Parametern mit hohen Fremdkapitalaufnahmen hohe Gewinne verbunden. Doch wie verhält es sich mit der Überlebenswahrscheinlichkeit?

Tabelle 263.1:Parameter des Überlebensmodells

\begin{tabular}{|c|c|c|c|}
\hline$a$ & $E K$ & $r$ & $i$ \\
\hline 5 & 10 & $\sim \operatorname{uiid}(0,0.6)$ & 0.2 \\
\hline
\end{tabular}

Die Antwort auf diese Frage wird entscheidend vom Planungshorizont bestimmt. Auf lange Sicht sind die Überlebenschancen eines Unternehmens ohne Fremdkapital sehr gering, da die festen Auszahlungen die durchschnittlichen Erträge des Eigenkapitals übersteigen. Auf kurze Sicht (bis zu zwei Perioden) hingegen können schuldenfreie Unternehmen nicht ausscheiden, weil auch bei minimalen Renditen das Eigenkapital ausreicht, über zwei Jahre die festen Auszahlungen zu bestreiten.

Realisierten sich die Renditen in jeder Periode in Höhe ihres Mittelwertes, so würde das Eigenkapital der Unternehmen ohne Fremdkapitalaufnahme am Ende der vierten Periode negativ. Der Erwartungswert der Lebensdauer ist also zwischen drei und vier Perioden zu vermuten. Simulationen mit 1000 Unternehmen ohne Fremdkapitalaufnahme bestätigen diese Überlegung (s. Tabelle 263.2). Nach zwei Perioden sind noch alle, nach drei mehr als die Hälfte und nach vier weniger als die Hälfte der Unternehmen im Rennen. ${ }^{185}$

Tabelle 263.2: Anzahl überlebender Unternehmen (keine Fremdkapitalaufnahme)

\begin{tabular}{|c|c|c|c|c|c|c|c|c|c|c|c|c|c|c|c|c|}
\hline Perioden & 2 & 3 & 4 & 5 & 6 & 7 & 8 & 9 & 10 & 11 & 12 & 13 & 14 & 15 & 16 & 17 \\
\hline Anzahl & 1000 & 654 & 273 & 100 & 42 & 25 & 10 & 7 & 4 & 1 & 1 & 1 & 1 & 1 & 1 & 0 \\
\hline \multicolumn{11}{|c|}{ Es starten 1000 Unternehmen } \\
\hline
\end{tabular}

Schaubild 264.1 demonstriert Ergebnisse für vier Perioden und eine jährliche konstante Fremdkapitalaufnahme. ${ }^{186}$ Die obere Kurve gibt die Anzahl der Unternehmen an (von 1000), die ein positives Eigenkapital aufweisen, die untere das durchschnittliche Eigenkapital aller 1000 Unternehmen $^{187}$, jeweils nach vier Perioden und in Abhängigkeit von der Fremdkapitalaufnahme. Es zeigt sich, daß das Eigenkapital im Mittel aller

\footnotetext{
185 Durch lineare Interpolation ergibt sich eine durchschnittliche Lebenserwartung von $3+(654-500) /(654-273)=3.4$ Perioden.

Das heißt, die Fremdkapitalaufnahme der ersten Periode wird in den folgenden Perioden beibehalten.

einschließlich des negativen Eigenkapitals der ausgeschiedenen Unternehmen
} 
Unternehmen mit der Kreditaufnahme wächst (und damit erst recht im Durchschnitt der überlebenden Unternehmen). Im Gegensatz dazu wirkt sich eine sehr hohe Fremdkapitalaufnahme auf die Überlebenswahrscheinlichkeit leicht ungünstig aus. Gewinnmaximierung durch verstärkten Einsatz von Krediten vermindert also in diesem Beispiel geringfügig die Überlebenswahrscheinlichkeit.

\section{Schaubild 264.1: Anzahl überlebender Betriebe nach vier Perioden und mittleres Eigenkapital}

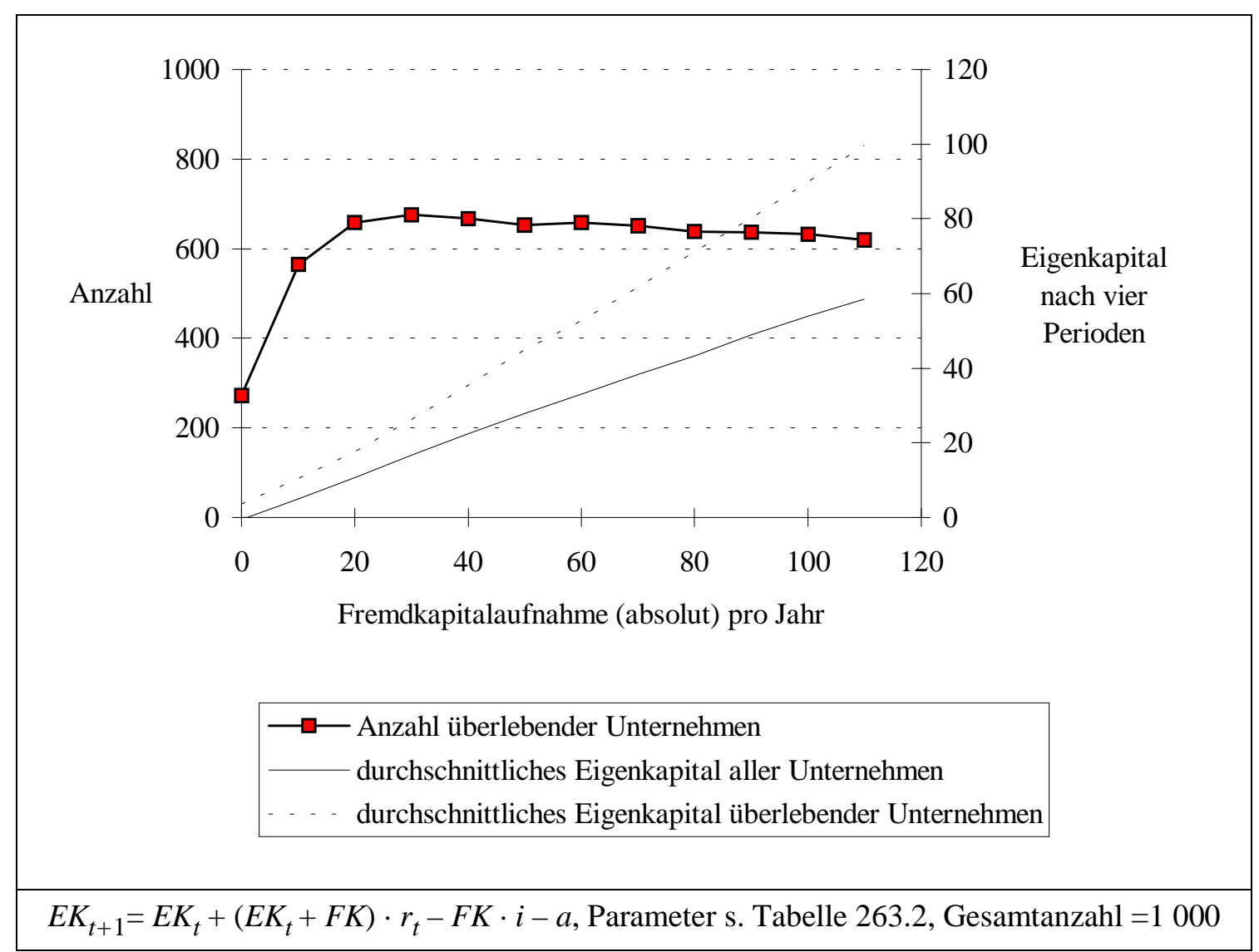

BRANDES (1989, S. 12) zeigt für ein ähnliches, etwas komplexeres Beispiel ebenfalls, daß die Wahl der gewinnmaximierenden Strategien, also maximaler Kreditaufnahme, nicht auch die Ruinwahrscheinlichkeit minimiert. ${ }^{188}$ Kleine Modelle dieser Art sind daher bereits geeignet, der FRIEDMANschen Gewinnmaximierungshypothese zu widersprechen.

In diese Modelle lassen sich sehr leicht Modifikationen einbauen:

- "situationsabhängige" statt fester Kreditaufnahme,

- variable statt fester Entnahmen

- Änderung des Planungshorizonts (ein Beispiel befindet sich im Anhang),

188 Im Gegensatz zu dem hier vorgestellten Modell bestimmt BRANDES nicht explizit den die Überlebenswahrscheinlichkeit maximierenden Fremdkapitalanteil. 
- Auswahl zwischen Produktionsverfahren, die sich in Erwartungswert und Streuung unterscheiden (wenn das Modell um diese Möglichkeiten ergänzt wird).

Die genannten Erweiterungen werden an dieser Stelle nicht weiter verfolgt. Statt dessen werden im nächsten Kapitel Betriebsentwicklungsmodelle des zweiten Typs näher beschrieben, in denen Unternehmen mit differenzierteren Produktions- und Faktoreinsatzmöglichkeiten betrachtet werden. Den Abschluß dieses Kapitels über einfache Überlebensmodelle bildet eine empirische Analyse eines seit vielen Jahren eingesetzten Unternehmensplanspiels unter Überlebensgesichtspunkten.

\section{6 Überlebensaspekte im Planspiel „Puten und Perlhühner““}

Seit zehn Jahren wird am Lehrstuhl von Prof. Brandes, Institut für Agrarökonomie der Universität Göttingen, das Unternehmensplanspiel "Puten und Perlhühner" vorlesungsbegleitend von Studierenden im Hauptdiplom gespielt (BRANDES et al. 1990 ${ }^{189}$ ). Kurz gesagt, haben die Beteiligten in diesem mehrperiodischen Spiel die Möglichkeit, als Unternehmer bzw. Unternehmerinnen zwischen zwei relativ ähnlichen Produktionsverfahren und einer stochastischen Geldanlage zu wählen. Die Produktion setzt Anlageinvestitionen voraus. Die zur Auswahl stehenden Anlagen unterscheiden sich in ihren variablen und festen Kosten. Entscheidend ist, daß die Preisbildung endogen erfolgt, also vom Verhalten der Mitbewerber abhängig und daher für die einzelne Unternehmensleitung schwer abzuschätzen ist. Die Parameter des Spiels sind so festgelegt, daß mit ausschließlicher Geldanlage die laufenden Kosten im Mittel nicht gedeckt sind, so daß die Unternehmen, wollen sie überleben, gezwungen sind zu investieren.

Auswertungen von Spielverläufen ergeben, daß die Resultate von Strategien kaum vorhersagbar sind und konträre Strategien zu gleichen Ergebnissen führen können. Die Graphiken in Schaubild 2661 zeigen auf der Abszisse die Summe der Produktion in den ersten drei Perioden als Maß für den Expansionsdrang eines Unternehmens und auf der Ordinate das erzielte Eigenkapital am Ende des Spiels (nach 7 bzw. 8 Perioden). Die eingezeichneten Graphen stellen lineare bzw. kubische Regressionen dar. Es zeigt sich, daß expansive Strategien erfolgversprechender sind. Die Korrelationen zwischen Produktionsmenge und Eigenkapital am Ende sind deutlich positiv (0.43 in 1994/95 und $0.64 \mathrm{im}$ Jahr davor, Irrtumswahrscheinlichkeit kleiner als $0.1 \%$ ). Für risikoaverse Entscheider ist aber nicht nur der Erwartungswert von Interesse, sondern auch das mit einer Entscheidung verbundene Risiko. Daher werden in einem zweiten Schritt die Beträge der KQ-Residuen mit der Summe der Produktion korreliert. Die Beziehung erweist sich als gesichert positiv $(\rho=0.48, \alpha \leq 0.05 \%)$. Es besteht also ein trade off zwischen Streuung und Erwartungswert, so daß eine Entscheidung für eine bestimmte Produktionsmenge von der individuellen

189 Die jeweils aktuellste Spielbeschreibung findet sich im Internet unter http://www.gwdg.de/ uaao/brandes/pup/spiel.htm. 
Risikoeinstellung des Entscheiders abhängt. Allerdings kann es auch Fälle geben, wo trotz gegebenen trade offs alle Entscheider, gleich welcher Risikoeinstellung, den höheren Erwartungswert wählen werden, wie in Abschnitt \$.r ausgeführt worden ist.

\section{Schaubild 266.1: Vergleich expansiver und vorsichtiger Strategien beim Unternehmensplanspiel „Puten und Perlhühner““}

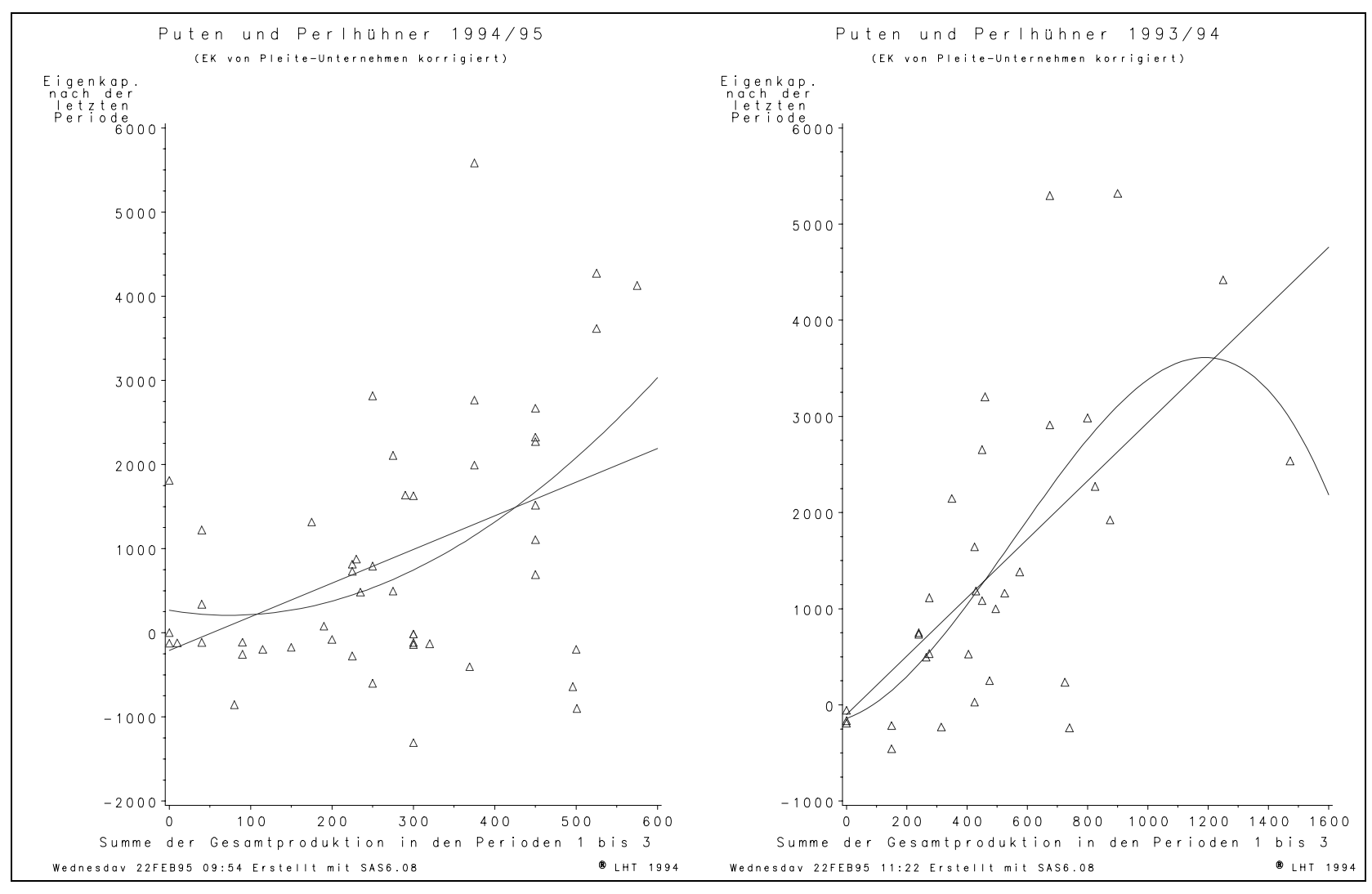

Die Methode zur Einschränkung des Unbestimmtheitsbereichs in Abschnitt 3.1 basiert auf der Überlegung, daß Alternativen, die neben einem höheren Erwartungswert auch ein größeres Minimum aufweisen, präferiert werden. Angewandt auf die Ergebnisse des Investitionsproblems im Planspiels „Puten und Perlhühner“ bedeutet dieser Ansatz, daß es wichtig ist, worst-case-Erwartungen zu bilden, um Erkenntnisse über das bei einer bestimmten Produktionsmenge kleinstmögliche Eigenkapital zu gewinnen. Die in den obigen Abbildungen dargestellten Wertepaare aus Produktion und Eigenkapital können nur als Realisationen von Zufallsstichproben interpretiert werden. Daher stellen die empirisch gefundenen, also die in der Vergangenheit realisierten Minima nur grobe Fingerzeige für die möglichen Extremwerte dar. Die eingezeichnete Regressionsgerade entspricht im Grunde einer Ausgleichsgeraden mit Optimalitätseigenschaften nur für die konkrete Stichprobe. Wegen des zufälligen Charakters der Stichprobe ist die Regressionsgerade selbst ebenfalls stochastisch; die dargestellte Gerade besitzt aufgrund der Werte der konkreten Stichprobe lediglich die größte Wahrscheinlichkeit, andere Verläufe der Regressionsgeraden lassen sich aber nicht ausschließen. Die Punkte der Regressionsgeraden sind Punktprognosen für das Eigen- 
kapital in Abhängigkeit vom Produktionsumfang. Andere Regressionsgeraden empirischer Daten führten zu anderen Punktprognosen. Um den Bereich von Punktprognosen, die eine bestimmte minimale Wahrscheinlichkeit besitzen, zu illustrieren, ist die Regressionsgerade in Schaubild 201 von einem 99\%-Konfidenzintervall für den Erwartungswert des vorhergesagten wertes umgeben.

\section{Schaubild 267.1: Expansion und zu erwartendes minimales Eigenkapital}

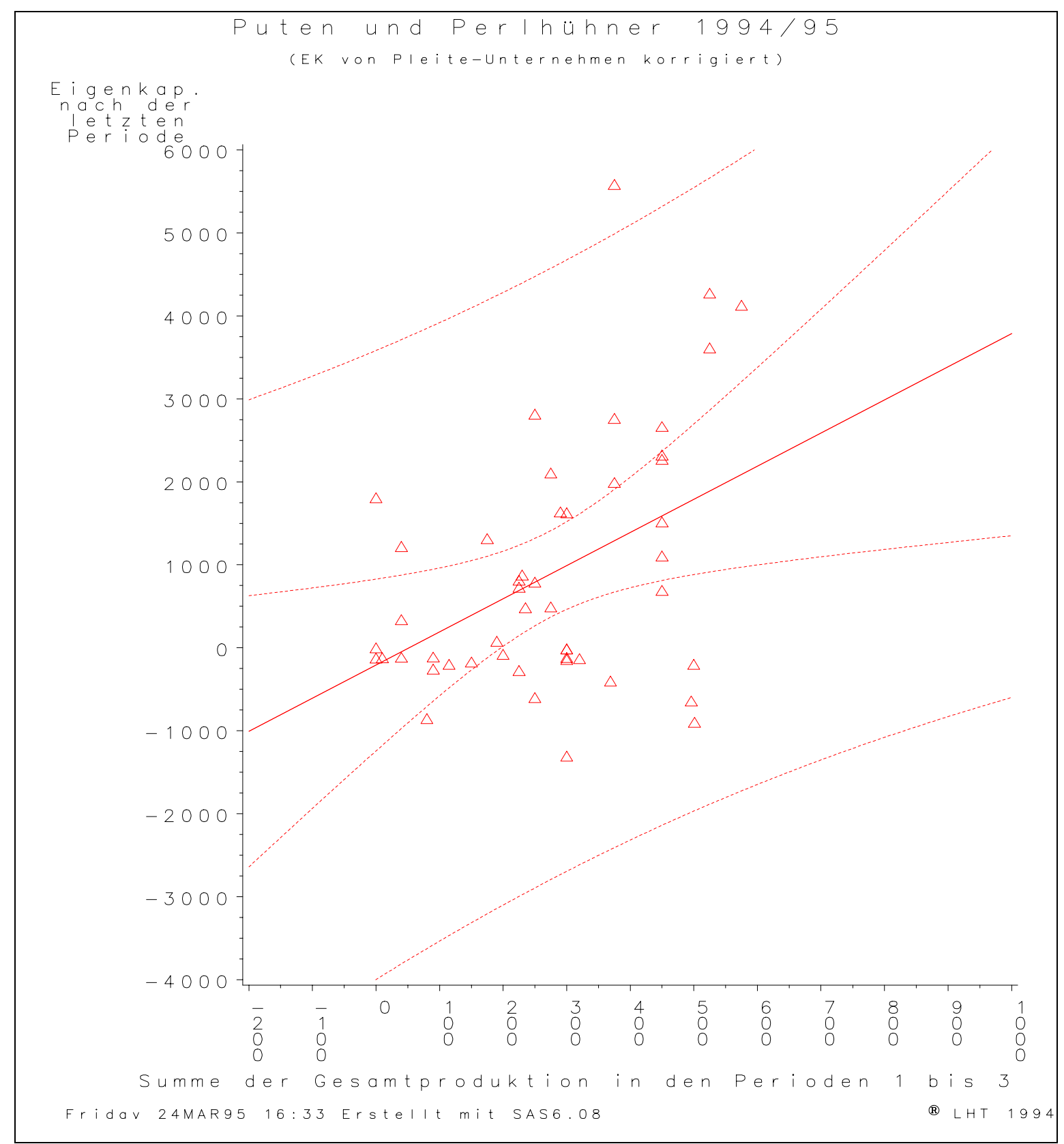

Dieses 99\%-Konfidenzintervall ist durch das innere Paar von gestrichelten Linien dargestellt. Es berücksichtigt nur den Fehler, der aus einer Fehlschätzung der Regressionsgerade hervorgehen kann. Die abhängige Variable wird zusätzlich noch durch den allgemeinen Störterm beeinflußt. Ein Vertrauensbereich für die Vorhersage eines ein- 
zelnen Wertes, und nicht seines Erwartungswertes, ist daher stets größer. Seine Darstellung erfolgt durch das äußere Paar von gestrichelten Linien. Dies bedeutet, daß unter ausschließlicher Zugrundelegung der Stichprobeninformation die Wahrscheinlichkeit von Werten außerhalb der äußeren gestrichelten Linien $1 \%$ beträgt. Insbesondere beläuft sich die Wahrscheinlichkeit, bei einem bestimmten Investitionsvolumen am Ende ein Eigenkapital geringer als die unterste gestrichelte Linie zu erzielen, auf ein halbes Prozent.

Im Gegensatz zu der Argumentation im Exkurs in Abschnitt 3.1 ist die Verteilung hier nicht beschränkt. Akzeptiert die Entscheiderin statt der Minima der Verteilung das untere $1 \%$-Konfidenzintervall als Untergrenzenargument für ihre Nutzenfunktion, dann impliziert dies für sie, daß die „Minima“ ansteigen. Es wäre dann für eine solche Entscheiderin sinnvoll, das größte Investitionsvolumen zu wählen.

In diesem Kapital wurde in einfache Überlebensmodelle eingeführt, die sich als Differenzengleichungen erster Ordnung darstellen lassen. Bis zur zweiten Runde ließen sich zum Großteil analytische und damit allgemeingültige Lösungen ableiten. Darüber hinaus konnten Erkenntnisse über die Überlebenswahrscheinlichkeit nur mit Hilfe von Monte-Carlo-Simulationen gewonnen werden. Die in den beiden letzten Abschnitten vorgestellten Beispiele von Betriebsentwicklungsmodellen leiten über zum nächsten Kapitel, das die Analyse von Überlebenswahrscheinlichkeiten in einem komplexeren Betriebsentwicklungsmodell zum Gegenstand hat. 


\section{Ein stochastisches Betriebsentwicklungs- und Überlebensmodell}

\begin{tabular}{|c|c|}
\hline 6.1 & Strategien und Umweltszenarien in Betriebsentwicklungsmodellen ..........269 \\
\hline & Investitionsverhalten und Erwartungsbildung ..... \\
\hline & Ausgangslage, Umweltentwicklung und Entnahmen .. \\
\hline 6.2 & Modellbeschreibung. \\
\hline 6.3 & Ergebnisse ausgewählter Strategien im deterministischen Modell .............288 \\
\hline 6.4 & 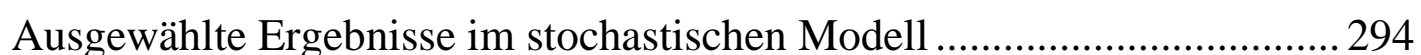 \\
\hline & 5.4.1 Vergleich der Strategien im stochastischen Modell ...................... 294 \\
\hline & anzen im stochastischen Modell \\
\hline
\end{tabular}

Nach der Diskussion einfacher Überlebensmodelle im vorigen Kapitel wird nun ein stochastisches Betriebsentwicklungsmodell vorgestellt, das einen stark vereinfachten landwirtschaftlichen Betrieb auf einem polypolistischen Markt repräsentiert. Das Kapitel beginnt mit der Beschreibung von Strategien zur Erhöhung der Überlebensfähigkeit von Unternehmen und Auswirkungen von Umweltvariablen auf die Ruinwahrscheinlichkeit. Anschließend werden das verwendete Betriebsentwicklungsmodell erläutert sowie seine Ergebnisse dargestellt und interpretiert.

\subsection{Strategien und Umweltszenarien in Betriebsentwicklungsmodellen}

Unternehmen verfolgen im allgemeinen das übergeordnete Ziel der Existenzsicherung ihres Betriebes. Da dieses Ziel wegen der Komplexität realer Entscheidungssituationen kaum operational ist, müssen Subziele oder Mittel definiert werden, deren Zusammenhänge mit dem Oberziel nur mit (subjektiven) Wahrscheinlichkeitsaussagen beschrieben werden können. Implikationen dieser Mittel zeigen sich insbesondere im Investitions- und Konsumverhalten. Die hierbei verfolgte Strategie beeinflußt ganz wesentlich die Überlebenswahrscheinlichkeit.

Werden solche Strategien modelliert, so ist ihre relative Vorzüglichkeit abhängig von der Art und Weise, wie die Stochastizität der Umweltentwicklung in das Modell eingeht. Das heißt, Strategieerfolg und Umweltmodellierung stehen in einem engen Zusammenhang. Darüber hinaus hängen die Aussichten einer Strategie auch von der Ausgangslage des Unternehmens ab. Diese genannten Einflußfaktoren werden im folgenden beschrieben. 


\subsubsection{Investitionsverhalten und Erwartungsbildung}

Investitionsentscheidungen in der Landwirtschaft sind durch folgende Besonderheiten gekennzeichnet:

- Es besteht große Unsicherheit über zukünftige Entwicklungen.

Landwirte sind teilweise von üblichen marktabhängigen Preisschwankungen betroffen; teilweise sind die Preise administriert und die Landwirte daher einem Politikänderungsrisiko unterworfen. Das Politikänderungsrisiko betrifft nicht nur Preissprünge, sondern auch Änderungen der rechtlichen Rahmenbedingungen. Als Stichwörter seien genannt: Dungeinheiten, Milchquoten, Flächenstillegung, Umwelt- und Naturschutzauflagen, Bundesimmissionsschutzgesetz.

- Falsche Großinvestitionsentscheidungen lassen sich innerhalb einer Generation kaum ausgleichen, da in der Regel nur eine oder höchstens zwei dieser Entscheidungen im Laufe einer Unternehmerlaufbahn getroffen werden. Investitionsentscheidungen sind daher besonders riskant.

- Investitionen sind häufig sehr langfristiger Natur und in hohem Maße irreversibel. Anderweitige Verwendungsmöglichkeiten durchgeführter Investitionen sind beschränkt, die Opportunitätskosten insbesondere von Gebäuden sind bezogen auf den Anschaffungspreis sehr gering. Entscheidend für das Risiko langfristiger Investitionen ist daher, wie sehr sie den Betrieb festlegen bzw. zu welchen Kosten sie es dem Unternehmen ermöglichen, Entscheidungen zu revidieren, wenn Zukunftserwartungen nachträglich geändert werden müssen.

Dem Treffen guter Investitionsentscheidungen kommt folglich für die Überlebensfähigkeit zentrale Bedeutung zu. Drei Aspekte seien im folgenden näher erläutert: die Fremdkapitalaufnahme, die Wachstumsgeschwindigkeit und die Erwartungsbildung.

Zwischen den beiden erstgenannten Punkten besteht ein enger Zusammenhang, denn Wachstum ist in größerem Umfange ohne Fremdkapital kaum möglich. Wird andererseits ein behutsames Wachstumsziel vorgegeben, so braucht eventuell der Fremdkapitalaufnahmespielraum nicht ausgenutzt zu werden.

Eine Mindestbedingung zur Durchführung von Investitionen in einem Modell ist, daß diese wirtschaftlich sind, d.h., daß sich der erwartete kalkulatorische Gewinn durch die Investition erhöht. Eine sich aus einer reinen Gewinnmaximierung ergebende Investitionssumme eignet sich gut als Referenzinvestitionsstrategie. Stärker expansive Strategien sind in einem Modell mit einer polypolistischen Marktstruktur sinnlos, wenn Mengenanpasserverhalten unterstellt wird, also ein einzelnes Unternehmen das Verhalten der Konkurrenz und damit letztlich den Preis nicht beeinflussen kann. Die Referenzstrategie ist daher in einem Polypolmodell gleichzeitig die Obergrenze für ein rentables Investitionsvolumen.

In vielen Überlebensmodellen (z.B. FISCHER 1988, BRANDES 1989, 1990, BURMESTER 1996) werden Strategien untersucht, die in einer Polypolsituation nicht anwendbar sind. Denn bei einer polypolistischen Marktstruktur können Alternativen zur Gewinnmaximierung letztlich nur zum Inhalt haben, durch vorsichtigeres Wachs- 
tum auf im Mittel mögliche Gewinne zu verzichten, um dafür die Überlebenswahrscheinlichkeit zu erhöhen. Folgende Vorgaben zur Höhe des Investitionsvolumens sind einzeln oder in Kombination als Bestandteile von Investitionsstrategien denkbar (vgl. WITT 1986; PORTER 1995, S. 62; für den landwirtschaftlichen Bereich besonders: HAAS 1997, BURMESTER 1996, Kap. 4; KÜHL 1992 S. 104ff, 110f; WALKER \& HELMERS 1984):

- Die Investitionen pro Jahr werden auf eine feste Summe beschränkt. Dieses Limit kann konstant sein oder jährlich wachsen.

- Die Investitionsvolumina orientieren sich an der bisherigen Kapazität.

- Für die Finanzierung von Investitionen wird ein Mindest-Eigenkapitalanteil gefordert.

- Die Höhe der Investitionen wird an die finanzielle Situation des Unternehmens geknüpft:

$\diamond$ Es wird nur dann investiert, wenn bestimmte Kennzahlen der Verteilung des erwarteten Gewinns vorgegebene Grenzen übersteigen. Diese Grenzen können den durchschnittlichen Gewinn, den Mindestgewinn oder ein Quantil des Gewinns betreffen. Ähnliche Verknüpfungen sind mit dem aktuellen Eigenkapital oder dem Eigenkapitalzuwachs möglich, der durch die Investition erwartetet wird.

$\diamond$ Das Unternehmen investiert jedes Jahr einen bestimmten Anteil des Gewinns oder des Eigenkapitals.

$\diamond$ Investitionen sind in der Regel mit Kreditaufnahme verbunden. Die Verschuldung oder der Kapitaldienst darf vorbestimmte Anteile des Eigenkapitals oder Gewinns nicht übersteigen.

$\diamond$ Im Zuge der Ausführung der Investition darf eine Mindestliquidität (im Sinne des verfügbaren kurzfristigen Kreditspielraums) nicht unterschritten werden.

- Nach Investitionen erfolgt eine Konsolidierungspause.

- Investitionen bedingen Erwartungen an eine positive Preisveränderung.

- Investitionstätigkeit setzt nur ein, wenn die Gewinnentwicklung unbefriedigend verläuft (satisfizierendes Verhalten).

- Die Planung erfolgt mit geringeren als den erwarteten Preisen. Die Unternehmerin nimmt sogenannte Risikoabschläge vor.

- Statt eines gewinnmaximierenden Produktmixes werden für die Produktionsverfahren Mindest- und/oder Höchstanteile oder für die absoluten Produktionsmengen Bandbreiten festgesetzt (Diversifikation).

Unternehmensstrategien können sich neben Investitionen im engeren Sinne u.a. auch auf generelle Finanzrestriktionen und die Erwartungsbildung erstrecken. Eine der bekanntesten Empfehlungen ist die Goldene Bilanzregel. Sie besagt, daß die kurzfristigen Kredite das Umlaufvermögen nicht überschreiten dürfen. Andere Leitlinien beziehen sich auf das gesamte Fremdkapital und fordern, daß es bestimmte Prozent- 
sätze des Eigenkapitals nicht überschreiten dürfe. Das bekannte Konzept der kurz- und langfristigen Kapitaldienstgrenzen ist eher ein Indikator für Unternehmenskrisen als ein Instrument zur Investitionsevaluierung. Denn eine gewinnbringende Investition erhöht die Kapitaldienstfähigkeit, auch wenn die Kapitaldienstgrenze überschritten sein mag.

Die ex-ante-Rentabilität von Investitionen hängt entscheidend von den getroffenen Zukunftsannahmen ab. Daher kommt der Erwartungsbildung in Investitionsentscheidungen große Bedeutung zu. ${ }^{190}$ Sie wird häufig in die Kategorien der autoregressiven und der rationalen Erwartungsbildung unterteilt. Autoregressive Erwartungen bezüglich einer Variablen basieren ausschließlich auf der Zeitreihe dieser Variablen. Es lassen sich naive (auch klassische genannt), extrapolative und adaptive Formen unterscheiden.

Bei naiven (Preis-)Erwartungen gehen die Wirtschaftssubjekte davon aus, daß sich der aktuelle Preis nicht ändert: ${ }^{191}$

$$
\hat{p}_{t} \quad=p_{t-1} \text {. }
$$

Diese Form der Preiserwartung wird z.B. für das Entstehen des Schweinezyklus verantwortlich gemacht. ${ }^{192}$ Weniger naive Erwartungen werden als Funktion gewichteter Mittel vergangener Preise, z.B. der letzten drei Jahre, dargestellt:

$$
\hat{p}_{t} \quad=1 / 2 \cdot p_{t-1}+1 / 3 \cdot p_{t-2}+1 / 6 \cdot p_{t-3} \text {. }
$$

Extrapolative Preiserwartungen fußen auf der Vermutung, daß nicht die Preise fortbestehen, sondern der in der Vergangenheit beobachtete Preistrend. Zum Preis des Vorjahres wird ein Vielfaches der Preisänderung des letzten Jahres addiert. Diese Summe bildet die Preiserwartung für dieses Jahr (GoODWIN 1947):

$$
\hat{p}_{t} \quad=p_{t-1}+\alpha \cdot\left(p_{t-1}-p_{t-2}\right) .
$$

$\alpha$ bewegt sich in der Regel um 1 .

Bei adaptiven Preiserwartungen wird Lernen unterstellt (NERLOVE 1958). Ausgangspunkt ist nicht, wie bei den extrapolativen Erwartungen, der im Vorjahr realisierte, sondern der für das Vorjahr erwartete Preis. Er wird um die im Vorjahr erlebte Überraschung (also die Differenz zwischen eingetretenem und erwartetem Preis) korrigiert:

$$
\begin{aligned}
\hat{p}_{t} & =\hat{p}_{t-1}+\alpha \cdot\left(p_{t-1}-\hat{p}_{t-1}\right) \\
& =\alpha \cdot p_{t-1}+(1-\alpha) \cdot \hat{p}_{t-1}
\end{aligned}
$$

190 Zu einer empirischen Studie der Erwartungsbildung von Landwirten vgl. SULEWSKI et al. (1994).

191 Vgl. EZEKIEL (1938), zit. n. MUTH (1961, S. 331).

192 Erstmals von HANAU (1930) beschrieben und später ausführlich von ihm im Handwörterbuch der Sozialwissenschaften (1956) dargestellt. 
Durch fortgesetzte Substitution lassen sich die Erwartungsgrößen der rechten Gleichungsseite durch reale Vorperiodenpreise ersetzen:

$$
\hat{p}_{t} \quad=\alpha \cdot \sum_{s=1}^{\infty}(1-\alpha)^{s} \cdot p_{t-s} .
$$

Die Gewichte der Preise zurückliegender Jahre, $(1-\alpha)^{s}$, bilden eine abnehmende geometrische Reihe und konvergieren für $0<\alpha<1$ gegen Null. Daher kann die Summation nach wenigen Perioden abgebrochen werden. Letztlich unterscheiden sich somit adaptive Preiserwartungen nicht sehr von den "weniger naiv" genannten, die auch einer abnehmenden, aber meist keiner geometrischen Reihe folgen.

Adaptive und extrapolative Erwartungen sind sehr geeignet, wenn davon ausgegangen werden kann, daß die zu antizipierende Variable, oder ihre Veränderungsrate, einem Trend folgt; sie liefern hingegen keine Handhabe bei der Prognose von Wendepunkten oder gar Extrema. ${ }^{193}$ Gibt es außerhalb der Geschichte der Variablen Hinweise auf einen bevorstehenden Bruch, so wäre es daher wenig rational, diese Information zu mißachten.

Rationale Erwartungen ${ }^{194}$ sind dadurch gekennzeichnet, daß die Wirtschaftssubjekte nicht (nur) die vergangene Zeitreihe einer Variablen betrachten, wie bei den autoregressiven Erwartungen, sondern alle verfügbaren Informationen richtig ausnutzen. In der strengen Form besitzen dabei alle Wirtschaftssubjekte - im Sinne des Modells vollständige und korrekte Kenntnis des Verursachungsprozesses dieser Variablen. ${ }^{195}$ Daher kommen alle zu ex ante richtigen Erwartungen. ${ }^{196}$ Dies bedeutet jedoch nicht, daß Irrtümer ausgeschlossen sind (BENDER 1982), sondern nur, daß die Irrtümer stochastisch und im Mittel gleich Null sind. Das heißt, der Erwartungswert des Erwartungsirrtums ist Null.

Wird z.B. eine Größe, die Gegenstand der Erwartungsbildung ist, von einem stochastischen Term beeinflußt, so ist nach den Regeln rationaler Erwartungen den Wirtschaftssubjekten das Bildungsgesetz des stochastischen Terms bekannt, nicht aber seine konkrete Realisation. Benutzen nun die Wirtschaftssubjekte zur Einbeziehung

193 Darauf weist z.B. NEUMANN (1979, S. 375) hin.

Eine umfassende Zusammenstellung nichtrationaler Erwartungen, die über die hier beschriebenen hinausgeht, liefert der schon genannte Aufsatz von SULEWSKI et al. (1994, S. 304f).

194 Der Begriff geht auf einen Artikel von MUTH in der Zeitschrift ECONOMETRICA aus dem Jahre 1961 zurück. Seitdem sind sie sehr populär in den Wirtschaftswissenschaften (z.B. WITTMANN 1985, GABISCH \& LORENZ 1987, SCHNABL 1988, SARGENT 1987). THIJSSEN (1996) z.B. untersucht das Investitionsverhalten von Landwirten im Zusammenhang mit ihrer Erwartungsbildung, RIBHEGGE (1987) die Grenzen ihrer Anwendbarkeit und GÜTH (1998), ob rationale Kostenerwartungen evolutionär stabil sind. Der Monetarismus basiert auf rationalen Erwartungen, der in den 80er Jahren die vorherrschende Doktrin in der westlichen Wirtschaftspolitik (Thatcherism, Reaganomics) war (BARTMANN \& JOHN 1985, SHEFFRIN 1983). NERLOVE $(1979,1983)$ entwikkelte seine adaptiven Erwartungen weiter zu sogenannten quasi-rationalen Erwartungen und sieht sie als Alternative zu vollständig rationalen Erwartungen (1993).

$196 \mathrm{Zu}$ einer Kritik dieses Konzeptes vgl. BARTMANN \& JOHN (1985, S. 221ff). 
des stochastischen Terms in ihre Entscheidungsfindung statt des Erwartungswertes einen entsprechenden Zufallszahlengenerator, so kommen sie zwar zu unterschiedlichen, aber dennoch alle zu korrekten Erwartungen.

Eine Form weniger strenger rationaler Erwartungen sei am Beispiel eines sehr einfachen Marktmodells (vgl. Schaubild 274 1] erläutert.

Schaubild 274.1: Einfaches Beispiel eines Modells mit rationalen Erwartungen

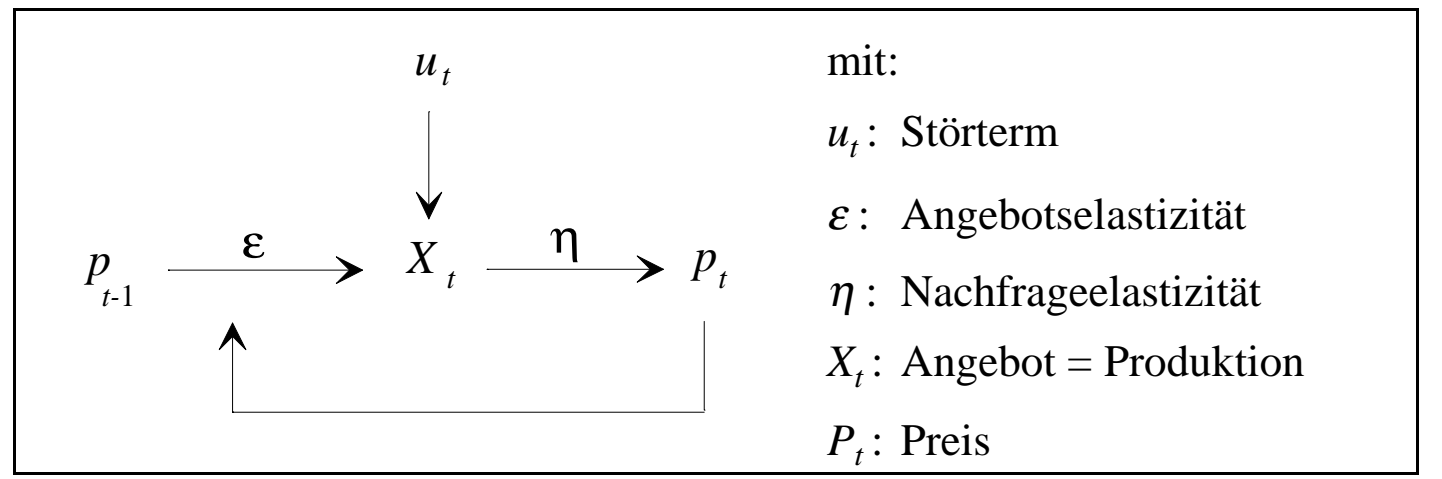

Die Preise der Vorperiode bestimmen über eine konstante Angebotselastizität $\varepsilon$ zuzüglich des Störterms $u_{t}$ die Angebotsmenge $X_{t},{ }^{197}$ die ihrerseits, bedingt durch die ebenfalls konstante Nachfrageelastizität $\eta$ nur zu einem Preis $p_{t}$ vollständig abgesetzt werden kann (keine Lagerhaltung). Die Unternehmen besitzen Kenntnis der Modellstruktur, nicht aber der Elastizitäten. Sie schätzen die Angebotselastizität, in Schaubild $274 b_{\text {mit }} \hat{e}$ bezeichnet, und berechnen mit ihrer Hilfe das vermutete Angebot $\hat{X}_{t}$. Mittels der geschätzten Nachfrageelastizität erwarten sie den Marktpreis $\hat{p}_{t}$, der dann ihr individuelles Angebot $x_{t}$ bestimmt. $^{198}$

\section{Schaubild 274.2: Bestimmung der Produktionsmenge eines Unternehmens mit rationalen Erwartungen}

$p_{t-1} \stackrel{\hat{\varepsilon}_{t}}{\longrightarrow} \hat{X}_{t} \stackrel{\hat{\eta}_{t}}{\longrightarrow} \hat{p}_{t} \longrightarrow x_{t} \wedge$ : erwartete Größen

Die Schätzung der unbekannten Elastizitäten kann entweder durch Bildung autoregressiver Erwartungen, z.B.

197 Genauer gesagt, bestimmen relative Preisänderungen über die Elastizität relative Mengenänderungen. Da aber zu einem bestimmten Zeitpunkt alter Preis und alte Menge vorliegen, determiniert letztlich, vom Störterm einmal abgesehen, nur der neue Preis über die Elastizität die neue Menge.

198

Dieses Modell ist nur dann schlüssig, wenn unterstellt werden kann, daß ein einzelnes Unternehmen wegen seiner Größe keinen Einfluß auf den Marktpreis ausübt. Anderenfalls muß ein simultanes Preis-Mengen-Gleichgewicht bestimmt werden. Hierfür zeigen HINNERS-TOBRÄGEL \& BRANDES (1997, S. 311) eine iterative Vorgehensweise für einen Oligopolmarkt. 


$$
\hat{e}_{t} \quad=\alpha \cdot \sum_{s=1}^{5}(1-\alpha)^{s} \cdot \varepsilon_{t-s},
$$

oder über ein Regressionsmodell erfolgen:

$$
\frac{X_{t+1}-X_{t}}{X_{t}}=\varepsilon \cdot \frac{p_{t}-p_{t-1}}{p_{t-1}}+u_{t} .
$$

Rationale Erwartungen werden in der deutschen Wirtschaft offensichtlich wenig eingesetzt. Eine Umfrage im Jahr 1994 bei den 500 größten westdeutschen Industrieunternehmen ergab, daß zur Prognose insbesondere vier Instrumente eingesetzt werden: intern erstellte Szenarien oder Studien, Mitarbeitergespräche, Trendextrapolation und extern erstellte Szenarien oder Studien (WELGE \& Al-LAHAM 1997, 797f). Autoregressive Erwartungsbildung ist daher, obgleich sie sich zur strategischen Unternehmensplanung weniger eignet, in der Praxis sehr viel relevanter als rationale Erwartungsbildung, die vermutlich teilweise in den zugekauften Studien verwendet wird.

Solide Erwartungen und angepaßte Investitionsstrategien können allein nicht das Überleben eines Unternehmens garantieren. Entscheidend ist auch die betriebliche Situation zum Zeitpunkt der Planung, die Entwicklung der Zukunft sowie das Entnahmeverhalten.

\subsubsection{Ausgangslage, Umweltentwicklung und Entnahmen}

Auch gut geplante Investitionen können sich als verlustbringend herausstellen, weil sich die Umwelt anders als angenommen entwickelt hat. In einem Unsicherheit integrierenden Betriebsentwicklungsmodell kann daher nicht nur mit Erwartungswerten zufallsbehafteter Parameter gerechnet werden. Aussagekräftiger ist es, die unsicheren Größen sind mit ihren Realisationen im Zeitablauf aufzunehmen. Besondere ökonomische Bedeutung kommt dabei den Preisen von Produkten und Faktoren (einschl. Zinsen) und den Erträgen (insbesondere in der Landwirtschaft) zu. Aber auch die Verfügbarkeit von Krediten, Pachtflächen und Arbeitskräften oder auch Lieferrechten sowie die rechtlichen Rahmenbedingungen können sich ändern und die Überlebensfähigkeit entscheidend beeinflussen.

In einem Modell kann die Veränderung von Parametern deterministisch (Szenarioanalyse) oder stochastisch erfolgen. Stochastische Parameter können um einen festen Wert schwanken oder einen Trend oder/und Zyklus überlagern. Die Realisation einer Zufallsveränderlichen im Zeitablauf braucht nicht unabhängig von den bereits erfolgten Realisationen in der Vergangenheit zu sein (Stichwort Autokorrelation). Außerdem ist es möglich, daß einige Zufallsveränderliche sich in jeder Periode gegenseitig beeinflussen, also eine Kovarianz besteht. 
Serielle Korrelationen und Kovarianzen werden in Betriebsentwicklungsmodellen im allgemeinen ausgeklammert, weil sie schwer zu implementieren sind. ${ }^{199}$ Wenn die Realisationen einer Zufallsveränderlichen abhängig sind von ihrer/ihren Realisation(en) in der/den Vorperiode(n), spricht man von Autokorrelation. Gilt dabei, daß auf die Realisation der Zufallsvariablen $\xi$ in Periode $t+1$ nur ihre Realisation in $t$ einwirkt und dies in der Form

$$
\begin{array}{lll}
x_{t+1}=\rho \cdot x_{t}+u & \text { mit } u: & \text { Störterm, } \rho \text { : Korrelationskonstante, } \\
& x_{t+1}, x_{t}: & \text { Realisationen der Zufallsvariable } \xi,
\end{array}
$$

geschrieben werden kann, so liegt Autokorrelation erster Ordnung vor.

Sind die Realisationen der Zufallsveränderlichen abhängig von Realisationen anderer Zufallsveränderlicher zum gleichen Zeitpunkt, spricht man von Kovarianz bzw. Korrelation. Sie soll kurz veranschaulicht werden.

Die Zufallsveränderliche $\xi$ sei normalverteilt mit Erwartungswert $\mu_{1}$ und Varianz $\sigma_{1}^{2}$ und kann daher bekanntlich als lineare Transformation der standardnormalverteilten Zufallsveränderlichen $\zeta_{S N 1}$ ausgedrückt werden: $\xi=\mu_{1}+\sigma_{1} \cdot \xi_{S N 1}{ }^{200}$ Eine Zufallsvariable $\eta$, die definiert sei als:

$\eta$

$$
\begin{aligned}
& =\sqrt{1-r^{2}} \cdot \sigma_{2} \cdot \xi_{S N 2}+r \cdot \sigma_{2} \cdot \xi_{S N 1}+\mu_{2}, \xi_{S N 2} \sim \mathrm{N}(0,1) \\
& =\sqrt{1-r^{2}} \cdot \sigma_{2} \cdot \xi_{S N 2}-r \cdot \frac{\sigma_{2}}{\sigma_{1}} \cdot \mu_{1}+\mu_{2}, \xi \sim \mathrm{N}\left(\mu_{1}, \sigma_{1}\right),
\end{aligned}
$$

besitzt dann die Momente $\mu_{2}$ und $\sigma_{2}^{2}$ und eine Korrelation von $r$ zu $\xi^{201}$ Die Korrelation ist dabei offensichtlich nicht abhängig von den Momenten von $\xi$. Wichtig ist beim Erzeugen von Zufallszahlen, die eine vorgegebene Korrelation aufweisen sollen, vor allem, daß $\eta$ mit Hilfe der gleichen Zufallsvariablen wie $\xi$, hier $\zeta_{S N 1}$, und eines eigenständigen stochastischen Terms, hier $\zeta_{S N 2}$, generiert wird.

Neben der Streuung eines Parameters ist sein Trend von erheblicher Bedeutung. Bei einem geknickten Preistrend beispielsweise ist von Interesse, ob anfänglich steigende und später fallende Preise andere Auswirkungen auf die Überlebenswahrscheinlichkeit haben als ein umgekehrter Trendverlauf, unter den Voraussetzungen, daß die langfristigen Erwartungswerte der Preise übereinstimmen und feste Preise in Höhe der Erwartungswerte das Überleben sichern würden.

Besteht große Unsicherheit über die zukünftige Entwicklung, können gravierende negative Überraschungen nicht ausbleiben. Hier wirkt eine gute Ausstattung des

199 In neuerer Software zur Risikoanalyse sind hierfür Routinen enthalten, z.B. in dem schon erwähnten Programm RISK ®.

200 Vgl. Fußnote 144.

201 Vgl. BRANDES \& BUdDE (1980, S. 52 mit Vorzeichenfehler) und KREYSZIG (1975, Abschnitt 107). BRANDES \& BUDDE zeigen auch einen Ansatz, korrelierte dreieckverteilte Zufallszahlen zu erzeugen. 
Unternehmens mit Fläche, Eigenkapital und Arbeitskräften als Puffer und erhöht die Stabilität, da im Eigentum des Unternehmens befindliche Faktoren die Liquidität entlasten und das Risiko einer Zahlungsunfähigkeit vermindern. Langfristig kann eine bessere Ausgangslage Insolvenzgefahren durch Unwirtschaftlichkeit, die zu einer Überschuldung führt, nicht abwenden, sondern den Konkurs nur hinausschieben.

Auch die Höhe der Entnahmen und der Einlagen bzw. der im Notfall möglichen Einlagen beeinflußt die Überlebenswahrscheinlichkeit. Modellimplementationen von Entnahmen bewegen sich zwischen zwei Polen, von denen jeder für sich unangemessen ist, nämlich einer rein exogenen oder einer rein endogenen Festlegung der Entnahmen. Exogene Entnahmen können u.U. den Gewinn (abzgl. notwendiger Tilgungen) übertreffen und endogen bestimmte Entnahmen das Existenzminimum unterschreiten. Für das Management eines Unternehmens ist es wichtig zu wissen, in welcher Höhe durch feste, nicht von Unternehmenserfolg abhängige Entnahmen „Kosten“ entstehen. Szenario-Analysen vermögen die Nachteile eines solchen Auszahlungsschemas im Vergleich zu flexibleren Regelungen, wie sie im Familienbetrieb üblich sind, abzuschätzen.

Neben der Höhe ist auch die zeitliche Verteilung der Entnahmen von Bedeutung. Als eine Zielsetzung könnte die Maximierung der auf Dauer erreichbaren mittleren Breite des Entnahmenstroms unterstellt werden. Dies läßt jedoch Zeitpräferenzen außer acht (ADAM et al. 1994, S. 549). Je geringer die Gegenwartspräferenz ist, desto größer kann im allgemeinen die nicht diskontierte Summe der Entnahmen sein. Jede heute entnommene Mark reduziert das Einkommenspotential in der Zukunft. Das Ausmaß der Verringerung ist sowohl zeitabhängig, z.B. von der erwarteten Zukunft, als auch niveauabhängig: Bei höheren Entnahmen ist der Grenzverlust tendenziell größer. Dabei sind Bereiche stabilen Grenzverlustes mit anschließenden Sprüngen wahrscheinlich. Die Formulierung eines Entnahmeziels muß daher neben der Höhe Fristigkeit und Verteilung enthalten.

Nach diesen grundsätzlichen Ausführungen zu den Implikationen von Strategien und Umweltmodellierung auf Probleme der Ruinwahrscheinlichkeit setzt sich der verbleibende Teil des Kapitels mit einem konkreten Betriebsentwicklungsmodell auseinander, das geeignet ist, Überlebensfragen realitätsnäher als die Modelle in Kapitel 5 abzubilden. Ergebnisse für ausgewählte Strategien und Umwelt- und Betriebsszenarien werden vorgestellt.

\subsection{Modellbeschreibung}

Modelle der betrieblichen Entwicklung stellen immer ein Kompromiß zwischen Realitätsnähe auf der einen und Rechenbarkeit und Überschaubarkeit auf der anderen Seite dar. In Überlebensmodellen steht naturgemäß der Verlauf endogener Variablen in der Zeit im Vordergrund des Interesses. Daher sollten Überlebensmodelle dynamisch sein. Um die Auswirkungen von Unsicherheit und Risiko analysieren zu können, ist es darüber hinaus sinnvoll, dem Modell einige stochastische Variablen hinzuzufügen. Sto- 
chastisch dynamische Modelle entziehen sich, wenn nicht sehr rigide Annahmen getroffen werden, einer analytischen Lösung (vgl. Unterabschnitt 2.1.4.1). Daher wird in dieser Arbeit ein Monte-Carlo-Simulationsansatz gewählt.

Überlebensfragen sind in einem Unternehmen kurz- und auch langfristig von Bedeutung. Daher muß ein Modell einen ausreichenden Zeithorizont besitzen. Um für diese Fristigkeit ausreichend Entwicklungsvariabilität anbieten zu können, sollte das Modell Investitions- und Fremdfinanzierungsmöglichkeiten enthalten. Besteht zwischen den Investitionsalternativen oder in anderen Bereichen Wahlfreiheit, ist das Modell um ein Entscheidungsmodul zu ergänzen.

Kurz zusammengefaßt empfiehlt es sich daher, ein Betriebsentwicklungsmodell zur Analyse von Überlebenswahrscheinlichkeiten so zu gestalten, daß es dynamisch und stochastisch ist, Investitionsmöglichkeiten enthält und gleichzeitig ein Entscheidungsmodell darstellt.

Im folgenden wird ein Modell vorgestellt, das diesen Anforderungen genügt. Als Simulationsmodell kann es filigraner gestaltet sein als ein analytisch zu lösendes Modell. Dennoch wird es möglichst einfach gehalten, um Übersichtlichkeit zu gewährleisten und Rechenzeit zu sparen. Ein schlichtes Modell hat zudem den Vorteil, weniger fehleranfällig zu sein. Beobachtete Effekte lassen sich leichter isolieren und auf ihre Ursachen zurückführen. Der Aufbau des Modells wird in den folgenden Absätzen zunächst grob skizziert. Daran anknüpfend erfolgt eine nähere Erläuterung einiger wichtiger Details. Den Abschluß dieses Abschnitts bildet die Beschreibung der dynamischen Eigenschaften des Modells in seiner deterministischen Variante unter der Verhaltensannahme einfacher Gewinnmaximierung.

\section{Überblick:}

Modelliert wird ein stark stilisierter landwirtschaftlicher Betrieb in einem polypolistischen Markt. Ihm stehen zwei vereinfachte Produktionsverfahren ("Pflanzenproduktion" und "Tierproduktion") und eine Geldanlagemöglichkeit zur Verfügung. Die Produktion setzt Investitionen voraus. Die Möglichkeit, gegen Entgelt zusätzliche Produktionsfaktoren (Boden, Arbeit, Kapital) einzusetzen, erlaubt betriebliches Wachstum. Die Entwicklung des Betriebes ist ungewiß, da einige Umweltvariablen als Zufallsveränderliche formuliert sind. Sie wird mit Hilfe der Technik einer Monte-Carlo-Studie unter besonderer Beachtung der Überlebenswahrscheinlichkeit simuliert.

Für die Existenzsicherung sind Fragen der alternativen Verwendung von Faktoren und der Finanzierung von besonderer Bedeutung. Deswegen steht im Modell ein differenziertes Angebot von kurz- und langfristigen Krediten mit unterschiedlichen Zinssätzen und Höchstgrenzen und eine Anlagemöglichkeit für Eigenkapital zur Verfügung. Es wird angenommen, daß für die unternehmenseigenen Faktoren keine Verwendungsmöglichkeiten außerhalb des Unternehmens vorhanden sind, daß sie also keine Opportunitätskosten verursachen (von Finanzanlagen abgesehen), und daß dem 
Unternehmen kein unentgeltliches Kapital von außen zufließt, z.B. keine Privateinlagen.

Die "Modellandwirtin" bildet Erwartungen über die Zukunft und plant auf Grundlage dieser Erwartungen und einer vorgegebenen Strategie ihren Betrieb. Diese Planung wird im Modell mit Hilfe der einperiodischen linearen Programmierung abgebildet. Die Landwirtin kann folglich unmittelbar immer nur Entscheidungen für eine Periode treffen.

Nach Ende der ersten Periode befindet sich ihr Betrieb in der Regel nicht in der Situation, die sie in der Planung angenommen hatte, da sich die stochastischen Größen anders realisiert haben als erwartet. Auf Grundlage der eingetreten Größen plant die Landwirtin für die nächste Periode. Dieser Prozeß der "rollenden Planung” - Erwartungsbildung, Planung, Realisation, Überraschung, Neuplanung - setzt sich bis zum Ende des Planungshorizontes fort. So entsteht ein Entwicklungspfad des Betriebes.

Um zu zuverlässigen Ergebnissen zu kommen, werden für die gleiche Strategie viele Entwicklungspfade erzeugt, jeweils mit anderen Zufallszahlen. So ergibt sich eine Verteilung für den Entwicklungspfad einer Strategie. Diese EntwicklungspfadVerteilung kann abschließend mit Verteilungen anderer Strategien verglichen werden, um z.B. Aussagen über Unterschiede in der Überlebenswahrscheinlichkeit zwischen zwei Strategien treffen zu können.

Die Technik der rollenden Planung, d.h. in jeder Periode eine neue Planung unter Berücksichtigung der veränderten betrieblichen Ausgangslage, erfordert, daß im Simulationsmodell alle $\mathrm{Zu}$ - und Abgänge an Kapazitäten des Betriebes festgehalten werden. Dazu gehören z.B. Maschinen- und Gebäudebestand, Eigenkapital, Verbindlichkeiten und Kreditspielräume. Zu diesem Zweck ist ein kleines Buchführungsmodul erstellt worden, das die notwendigen Variablen fortschreibt. Die mathematischen Grundlagen des Programms sind im Anhang, Unterabschnitt 9.6.3 dokumentiert.

Besondere Beachtung gilt in der vorliegenden Simulationsstudie dem Insolvenzrisiko. In der Realität gibt es sicherlich eine Vielzahl von Gründen, die eine Unternehmensleitung veranlassen, die Weiterführung ihres Unternehmens einzustellen. Dazu gehören Erwartungen an die Zukunft, die "erlittene" Vergangenheit und Vorstellungen über alternative Verwendungsmöglichkeiten der eigenen Faktoren außerhalb des Unternehmens. Für ein Modell ist es notwendig, eine eindeutige Schwelle für den Übergang zur Insolvenz festzulegen.

Es wird festgelegt, daß ein Unternehmen dann und nur dann ausscheidet, wenn es sich überschuldet hat. Dies wird am Ende jeder Periode in der Bilanz an der Position des Eigenkapitals überprüft. Ist es negativ, scheidet das Unternehmen unabwendbar aus. Illiquidität als zweite mögliche Insolvenzursache kann im Modell nicht auftreten, da ein Strafkredit bereitsteht, der zu einem sehr hohen Zins in unbegrenzter Höhe aufgenommen werden kann. Zu beachten ist, daß der Boden nicht bilanziert wird, um die Bewertungsproblematik zu vermeiden. Ökonomisch kann die Ausscheideschwelle 
so interpretiert werden, daß ein Unternehmen geschlossen wird, wenn das Grundvermögen angegriffen werden müßte.

Insolvenz kann im vorliegenden Betriebsentwicklungsmodell ebenfalls nicht durch das Verhalten anderer Unternehmen ausgelöst werden. Denn das Modell ist so gestaltet, daß jeweils nur ein Unternehmen untersucht wird. Auf diese Weise treffen alle Strategien auf exakt die gleiche Umwelt, so daß ungestörte Vergleiche der Strategien möglich sind.

Überlegungen, Strategien in einem Marktmodell einem direktem Vergleich untereinander $\mathrm{zu}$ unterziehen, wurden fallengelassen. Ergebnisse von FISCHER $(1988)^{202}$, AXELROD (1991) ${ }^{203}$, SigMund (1995, S. 188f) und BURMESTER ((1996, Abschnitte 4.3 und 4.4) bestätigen den naheliegenden Gedankengang, daß die relative Vorzüglichkeit einer Strategie entscheidend davon abhängt, welche anderen Strategien auf dem Markt vertreten sind. Träten mehrere Unternehmen gleichzeitig an, wie es auch in anderen Simulationsstudien gehandhabt wird (z.B. GERYBADZE 1982, BRANDES 1989), wären Kombinationseffekte und Überlagerungen nicht auszuschließen.

Die „optimalen“ Entscheidungen eines Akteurs wären dann nicht nur von den eigenen in der Vergangenheit getroffenen Entscheidungen, sondern auch von denen der Kollegen abhängig. Da die verfolgten Strategien dem jeweils anderen in der Regel höchstens annähernd bekannt sind, kann es streng genommen unter diesen Voraussetzungen keine rationale Strategie geben (ADAM et al. 1994, S. 549, SIGMUND 1995).

Ein Beispiel für Kombinationseffekte liefert BURMESTER (1996). In seinem Simulationsmodell fällt eine Strategie vom ersten Platz weit nach hinten, wenn eine bestimmte andere Strategie weggelassen wird. Rangfolgen lassen sich daher nur genau für die spezifizierte Menge von Strategien aufstellen, die Rangfolgen sind für Teilmengen der Strategien bereits nicht mehr gültig, genauso wenig für erweiterte Strategiemengen.

Für AXELRODs Strategienvergleich beim Gefangenen-Dilemma-Spiel gilt diese Kritik ebenfalls, obwohl in seinem Wettbewerb jeweils zwei Strategien unmittelbar gegeneinander antreten (AXELROD 1991). Auch die relative Vorzüglichkeit zweier Strategien wird durch eine Veränderung von anderen Strategien (Modifikation, Wegfall oder Hinzufügen einer Strategie) beeinflußt: Zum Beispiel sei A besser als B und nicht besser als C und D, B hingegen sei besser als C und D; "besser" werde nur ordinal gemessen. Dann ist die Strategie B häufiger vorteilhafter als A. Fallen die Strategien $\mathrm{C}$ und $\mathrm{D}$ weg, dreht sich die Reihenfolge um.

Aus den genannten Gründen konkurrieren in dieser Arbeit die Strategien nicht gegeneinander, sondern alle Verhaltensweisen treten gegen die gleiche Umwelt an. Die resultierenden Reihenfolgen der Strategien sind dann zwar umweltabhängig, aber

\footnotetext{
202 Vgl. insbesondere S. 88.

203 2. Auflage; die erste erschien 1984.
} 
unbeeinflußt von anderen (zufällig) auf dem Markt vertretenen Typen von Konkurrenten.

\section{Im einzelnen:}

Gegenstand des Betriebsentwicklungsmodells ist ein 2-Produkt-Unternehmen. Jedes Produkt wird über ein spezifisches Produktionsverfahren mit linearen Produktionsfunktionen erzeugt. Die Daten der zwei Produktionsverfahren Pflanzenproduktion und Tierproduktion und die übrigen Koeffizienten sind den Kennziffern des KTBLTaschenbuchs Landwirtschaft 1996/97 und den Richtwert-Deckungsbeiträgen der Landwirtschaftskammer Hannover 1995 für die Verfahren Wintergerste und Schweinemast entnommen. Zur Produktion sind Investitionen, Umlaufkapital, Fläche, Arbeit und in der Tierproduktion zusätzlich freie Dungeinheiten erforderlich.

Der Bereich der Investitionsplanung weist im Vergleich zu anderen Betriebsentwicklungsmodell einige Besonderheiten auf, die die Finanzierung und die Zusammensetzung des Investitionsbündels betreffen. Die Finanzierungsart einer Investition kann frei gewählt werden. Als Ergebnis der Optimierung ist für jedes Investitionsobjekt ein Fremdkapitalanteil von 0 bis $100 \%$ möglich. ${ }^{204}$ Um das optimale Investitionsprogramm zu bestimmen, benutzen BALMANN (1995) und BURMESTER (1996) Heuristiken. Das Modell von BALMANN bringt alle durchführbaren Investitionsobjekte in eine Rangfolge und wählt das rentabelste aus. Danach werden erneut unter Berücksichtigung der ersten Investition alle Investitionsalternativen evaluiert, und es erfolgt eine zweite Wahl. Dieser iterative Prozeß wird fortgeführt, bis die für eine Investition erforderlichen betrieblichen Kapazitäten erschöpft sind. BURMESTER benutzt eine ähnliche Approximation. Zunächst werden die für eine Mindestproduktion erforderlichen Investitionen beschafft und anschließend iterativ der jeweils profitabelste Produktionszweig erweitert (BURMESTER 1996, Unterabschnitt 3.2.2).

Diese Heuristiken führen nicht mit Sicherheit zum gewinnmaximierenden Produktionsprogramm, wie ein kleines Beispiel zeigt: Es mögen drei Investitionsobjekte zur Auswahl stehen, $A, B$ und $C$. $A$ sei profitabler als $B$ oder $C$. Die Kapazitäten des Betriebes erlauben jede der Investitionen durchzuführen und darüber hinaus auch $B$ und $C$ gemeinsam, aber nicht $A$ zusammen mit einer anderen. Die genannten Heuristiken werden in diesem Beispiel das Investitionsobjekt $A$ auch dann erwerben, wenn die Objekte $B$ und $C$ zusammen einen höheren Gewinn erbrächten.

Diese möglichen Nachteile werden vermieden, wenn die Zusammensetzung des Investitionsprogramms selber unmittelbarer Gegenstand einer simultanen Optimierung ist. Die Implementierung dieser Forderung in das Betriebsentwicklungsmodell bedient sich im Grundsatz des in Abschnitt 3.4 entwickelten Formulierungsvorschlages für ein einperiodisches LP.

204 Andere Modelle schreiben oft feste Fremdkapitalanteile zur Finanzierung einer Investition vor (z.B. BALMANN 1995). Dies führt zu überhöhten Kosten, wenn ausreichend Eigenmittel zur Verfügung stehen. 
Die betriebseigene Arbeitskapazität beträgt eine Arbeitskrafteinheit (AK). Sie kann durch Fremd-AK aufgestockt werden. Das Modell bietet neben der stundenweisen Beschäftigung von Aushilfen auch die Möglichkeit, feste AK einzustellen. Eine ganzjährige AK ist pro Stunde billiger als eine Aushilfe; daher muß die Aktivität "ganzjährige AK" im Tableau ganzzahlig formuliert werden. Anderenfalls würde sie in Bruchteilen realisiert, und die Aktivität "Aushilfen" wäre überflüssig. Die Aufnahme von ganzzahligen Aktivitäten führt zu Verzerrungen der Dualwerte des LPTableaus; daher wird auf ihre Interpretation verzichtet.

Die Betriebsfläche kann durch Zupacht in unbegrenztem Umfang zu konstanten Preisen aufgestockt werden. Die Pachtdauer beträgt stets eine Periode. Die Produktionskosten sind auf eigenen und gepachteten Flächen gleich. Insbesondere werden keine höheren Transportkosten berücksichtigt, wie dies im Modell von BALMANN (1995) geschieht. ${ }^{205}$

Die langfristige Finanzierung einer Investition erfolgt stets fristenkongruent über Annuitätendarlehen (Zinssatz 6 oder 9\%). Beide Darlehen sind in ihrer Höhe begrenzt. Für den kurzfristigen Finanzbedarf stehen ein Dispo- und ein Überziehungskredit zur Verfügung mit Zinssätzen von 12 und 16\%, beide ebenfalls begrenzt. Ein dritter kurzfristiger Kredit ist sehr teuer (20\%) und unbegrenzt, er fungiert als Strafkredit. Die kurzfristigen Kredite haben eine Laufzeit von einem Jahr. Sie können auch zur Finanzierung von Investitionen herangezogen werden. Die differenzierte Abbildung des Kapitalmarktes ist ein Unterschied zu anderen Modellen, in denen nur ein Fremdkapitalzins berücksichtigt wird (FISCHER 1988, BALMANN 1995, BURMESTER 1996). Sie erfordert ein entsprechendes LP-Tableau, das in Abschnitt 3.4 aus bekannten Ansätzen, die aber nicht gleichzeitig mehr als einen Fremdkapitalzinssatz und einen Habenzins integrieren können, entwickelt worden ist.

Statt des hier verwendeten einperiodisch formulierten LPs käme grundsätzlich auch ein mehrperiodisches in Frage. Dieses böte u.a. den Vorteil, auch den Zeitpunkt von Investitionen optimieren zu können. ${ }^{206}$ Eine rollende Planung ist mit mehrperiodischen wie mit einperiodischen LPs möglich. Die Nachteile mehrperiodischer LP-Tableaus liegen vor allen in ihrer Unübersichtlichkeit und der erforderlichen Rechenzeit. Eine Monte-Carlo-Simulation mit mehrperiodischen LPs überstiege die verfügbare Rechenkapazität. Die Einwände bezüglich mangelnder Praxisrelevanz der linearen Programmierung gelten in der mehrperiodischen Variante verstärkt. Daher erfolgt in dieser Arbeit eine einperiodische Formulierung des LP-Tableaus. Es wird nach jeder Periode fortgeschrieben und in der nächsten Periode neu berechnet.

205 Im BALMANN-Modell sind steigende Transportkosten modelliert, um ein Gleichgewicht auf dem Bodenmarkt zu gewährleisten. Da in meinem Modell Marktgleichgewichte keine Rolle spielen, 206 erscheinen konstante Produktionskosten ausreichend.

206 Zu damit verbundenen Problemen vgl. NiPPEL (1994, S. 594 ff). 
Das LP-Tableau ist trotz stochastischer Umwelt deterministisch. ${ }^{207}$ Die Modellandwirtin nutzt es als Planungsinstrument. Sie weiß, welche Variablen stochastisch sind, und ist in der Lage, für diese Zufallsveränderlichen rationale Erwartungen zu bilden. Das heißt, sie kennt das Bildungsgesetz der stochastischen Variablen, nicht aber ihre konkreten Ausprägungen, und kalkuliert daher in jeder Periode mit ihren Erwartungswerten. Mit diesen erwarteten Werten kann das LP-Tableau berechnet werden, als wäre das Modell deterministisch.

Die Ergebnisse der Optimierung bestimmen die Produktions- und Investitionsentscheidungen für die aktuelle Periode. Nach der Planung realisieren sich die Zufallsveränderlichen anhand vorgegebener Verteilungen. Sie werden im Gegensatz zu vielen anderen Simulationsmodellen (z.B. KÜHL 1992, GROSSKOPF 1973) nicht als diskrete, sondern als stetige Zufallsveränderliche eingeführt. Die Realisierungen der Zufallsveränderlichen bestimmen das tatsächliche Ergebnis, das in der Regel vom erwarteten Ergebnis abweichen wird. Anhand des eingetretenen Ergebnisses werden die Bilanz erstellt und der Gewinn ermittelt. Die Bilanz dokumentiert neben der Entwicklung des Eigenkapitals sowie des Sachanlage- und Umlaufvermögens insbesondere Veränderungen der Faktorausstattung. Dazu gehören die Produktionskapazität und nicht ausgeschöpfte Kreditspielräume. Die aktualisierten Bestände an Faktoren ${ }^{208}$ gehen als Eingangsvariablen in das LP-Tableau der nächsten Periode ein. Die Planung für die Folgeperiode erfolgt unter Kenntnis der Ergebnisse der abgelaufenen Perioden, so daß sie im Lichte der bis dahin Realität gewordenen Zukunft erfolgen kann.

In Schaubild 2851 ist das Simulationsprogramm als Flußdiagramm dargestellt. Das Programm liest zunächst das LP-Tableau ein und prüft es auf Konsistenz. AnschlieBend kann das Tableau online modifiziert werden, um die Spezifika des gewünschten Szenarios einzugeben. Veränderbare Koeffizienten betreffen das Unternehmen (Ausstattung, verfolgte Strategie), seine Umwelt (Preise, Erträge, Zinsfüße; eingegeben entweder als Konstanten oder in Form von Verteilungsfunktionen) sowie Steuerungsdaten der Simulation (Anzahl Perioden, Anzahl Wiederholungen). Aus den Umweltdaten werden automatisch rationale Erwartungen gebildet und dem LP-Tableau als feste Werte übergeben. Nach einer erneuten Konsistenzprüfung wird das Tableau als Basistableau, auf das zu Beginn jeder neuen Wiederholung zurückgegriffen wird, abgespeichert und für die erste Runde optimiert. Im gleichen Programmabschnitt,

207 Das Basistableau ist im Anhang, Unterabschnitt 9.6.2, dokumentiert und im Detail erläutert.

208 Die Faktorfortschreibung erwies sich als diffiziles Problem. Vergleichsweise übersichtlich ist noch die Fortschreibung der Produktionskapazität: Die Nutzungsdauer der Maschinenausstattung für die Pflanzenproduktion betrage zwölf Perioden, und die Stallbauinvestitionen werden nach achtzehn Perioden abgeschrieben. Dann setzt sich der aktuelle Bestand an Produktion aus den Beschaffungen der letzten elf bzw. siebzehn Perioden zusammen.

Aufwendiger ist die Bestimmung der aktuellen Fremdkapitalbelastung. Bei zwei Zinssätzen und zwei Laufzeiten können pro Periode vier verschiedene Kredite in Anspruch genommen werden, die über zwölf bzw. achtzehn Perioden bedient werden müssen. Insgesamt ist eine Verwaltung für 60 Kredite mit je drei Kennzahlen (Zins, Tilgung, Restschuld) vorzuhalten. Im Anhang (Unterabschnitt 9.6.3) ist die Fortschreibung im Einzelnen erläutert. 
„erster Lauf“ genannt, werden alle Zufallszahlen, die für den gesamten Simulationsdurchlauf benötigt werden, generiert und für alle Zinssätze und Nutzungsdauern ein Kreditplan erstellt, der in den späteren Runden nur mit der aktuellen Höhe der Kreditaufnahme gefüllt werden muß.

Nachdem das Tableau optimiert ist, werden mit Hilfe der gezogenen Zufallszahlen das tatsächliche Ergebnis bestimmt und eine Gewinn-und-Verlust-Rechnung (GuV) sowie eine Bilanz erstellt. Damit ist die erste Runde beendet.

$\mathrm{GuV}$ und Bilanz dienen zum einen dazu, den wirtschaftlichen Erfolg des Unternehmens zu beschreiben, und zum anderen, Produktionskapazitäten, freie Kreditspielräume und die Liquidität fortzuschreiben. Die aktualisierten Bilanzpositionen finden in der nächsten Runde als Kapazitätsvektor (RHS) des fortgeschriebenen LP-Tableaus Verwendung. Dieser innere Kreislauf setzt sich fort, bis die gewünschte Anzahl Perioden erreicht ist $(t=2$ bis aPer). Danach ist das Unternehmen einmal über den gesamten Planungshorizont durchgerechnet. Um zu Wahrscheinlichkeitsaussagen zu gelangen, wird dieser Modelldurchlauf vollständig wiederholt (von $w=2$ bis $a W h$ ). Die Anzahl der Wiederholungen ist so zu bemessen, daß die Ergebnisse stabil sind. In der Literatur werden meist 50 bis 100 Wiederholungen durchgeführt. ${ }^{209}$

Die Ergebnisse der Simulation werden im nächsten Abschnitt dargestellt. Zum Abschluß der Modellbeschreibung sei zuvor noch erläutert, wie sich der Betrieb im Modell unter der Verhaltensannahme einfacher Gewinnmaximierung entwickelt, wenn alle Parameter deterministisch und bekannt sind. Dieses Szenario wird als Grundmodell bezeichnet.

Die Darstellung des Grundmodells geschieht an dieser Stellte aus folgendem Grund: Die Erstellung eines Modells erfolgt in einem rekursiven Prozeß, wie in Unterabschnitt 2.1.3 ausgeführt ist. Abwechselnd werden Modellparameter variiert und das dynamische Verhalten des Systems betrachtet, bis die Anpassung des Modells an das abzubildende System zufriedenstellt. Die dynamischen Eigenschaften eines Modells, die zu seiner Kalibrierung benutzen worden sind, werden daher wie die Modellparameter als Bestandteile einer vollständigen Modellbeschreibung aufgefaßt.

209 Vgl. z.B. LeAtham et al. (1986), BURMeSTER (1996). Protil (1997, S. 41ff) beschreibt unter Hinweis auf LAW \& KELTON (1992) eine Vorgehensweise, mit der die erforderliche Anzahl Wiederholungen endogen bestimmt werden. Generell ist die Wiederholungszahl abhängig von der interessierenden Statistik. Mittelwerte bedürfen beispielsweise weniger Repetitionen als Dezile, weil die Verteilung an den Rändern „dünner“ ist als in der Mitte. Daher sind die meisten Wiederholungen für die Schätzung von Extrema erforderlich (vgl. die Ausführungen dazu in Abschnitt 3.2 und zum Petersburger Paradox in Abschnitt 4.5). 
Schaubild 285.1: Ablaufschema des Simulationsprogramms

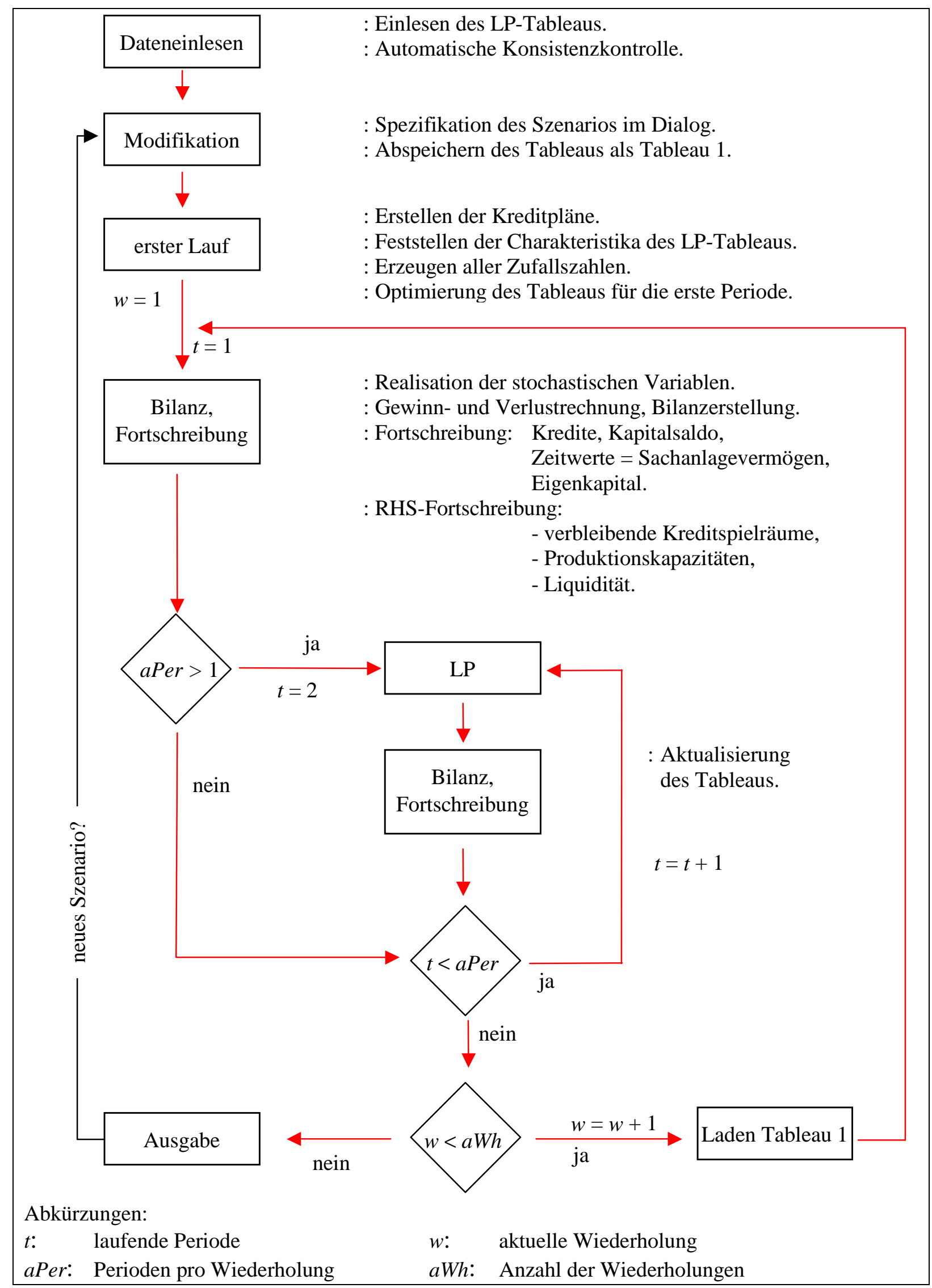




\section{Eigenschaften des deterministischen Grundmodells}

Die wichtigsten Parameter des Grundmodells sind in Tabelle 286,1 dargestellt, die übrigen sind im Anhang (Abschnitt 9.6.1 dokumentiert.

Gewinnmaximierung bei vollkommener Voraussicht und festen Parametern ergibt eine Betriebsorganisation, wie sie in Schaubild 2871 im Zeitablauf abgetragen ist. Beide Produktionsrichtungen werden verfolgt. Dafür ist eine begrenzte Zupacht von Flächen erforderlich. Da dem Unternehmen 100 ha Eigentumsfläche zur Verfügung stehen, ist die Zupacht eine lineare Funktion des Umfangs der Pflanzenproduktion.

Tabelle 286.1: Parameter des Betriebsentwicklungsmodells (Auszug)

\begin{tabular}{|l|c|}
\hline \multicolumn{1}{|c|}{ Variable } & 2500 \\
\hline \hline $\begin{array}{l}\text { Maschinenneuwert pro ha in der } \\
\text { Pflanzenproduktion }\end{array}$ & 12 \\
\hline Nutzungsdauer Maschinen & 24 \\
\hline $\begin{array}{l}\text { Produktpreis in der Pflanzen- } \\
\text { produktion }\end{array}$ & 70 \\
\hline $\begin{array}{l}\text { Ertrag pro ha in der } \\
\text { Pflanzenproduktion }\end{array}$ & 1000 \\
\hline $\begin{array}{l}\text { variable Kosten der } \\
\text { Pflanzenproduktion pro ha }\end{array}$ & 680 \\
\hline Deckungsbeitrag Pflanzenproduktion & 0 \\
\hline $\begin{array}{l}\text { Produktionskapazität zu Beginn der er- } \\
\text { sten Periode in der Pflanzenproduktion }\end{array}$ & und $20 \%$ \\
\hline $\begin{array}{l}\text { Liquidität (Eigenkapital } \\
\text { der ersten Periode }\end{array}$ & zu Beginn $5 \cdot 10^{5}$ \\
\hline $\begin{array}{l}\text { kurzfristige Zinssätze } \\
\text { langfristige Kreditobergrenzen }\end{array}$ & 4000 \\
\hline Zins für Finanzanlagen & 40 \\
\hline Lohnkosten einer Arbeitskraftstunde & 27 \\
\hline Anzahl Perioden & 200 \\
\hline
\end{tabular}

\begin{tabular}{|l|c|}
\hline \multicolumn{1}{|c|}{ Variable } & 1100 \\
\hline \hline $\begin{array}{l}\text { Baukosten eines Stallplatzes in der } \\
\text { Tierproduktion }\end{array}$ & 18 \\
\hline Nutzungsdauer Stallbauten & 3 \\
\hline Produktpreis in der Tierproduktion & 210 \\
\hline $\begin{array}{l}\text { Ertrag in der Tierproduktion (kg } \\
\text { Schlachtgewicht pro Jahr und Platz) }\end{array}$ & 500 \\
\hline $\begin{array}{l}\text { variable Kosten der Tierproduktion } \\
\text { pro Stallplatz }\end{array}$ & 130 \\
\hline Deckungsbeitrag Tierproduktion & 0 \\
\hline $\begin{array}{l}\text { Produktionskapazität zu Beginn der } \\
\text { ersten Periode in der Tierproduktion }\end{array}$ & 30000 \\
\hline Entnahmen pro Periode & 6 und $9 \%$ \\
\hline $\begin{array}{l}\text { Auf Angabe von Einheiten wird verzichtet, wenn } \\
\text { sie ohne besonderen Informationswert erscheinen. }\end{array}$ \\
\hline langfristige Zinssätze $10^{4}$ \\
\hline kurzfristige Kreditobergrenzen & 250 \\
\hline Pacht & je \\
\hline Jahreskosten einer Arbeitskraft & \\
\hline
\end{tabular}

Die Verkaufserlöse aus pflanzlicher und tierischer Produktion stimmen bis zur zehnten Periode in etwa überein. Die Pflanzenproduktion und mit ihr die Zupacht erreichen in dieser Runde ein Hochplateau. Ab der 15. Periode fallen sie langsam zugunsten der Tierproduktion ab, die ihr Maximum nach 30 Perioden erreicht. Ursache sind die knapp werdenden Dungeinheiten, die eine weitere Ausdehnung verhindern.

210 Eigenkapital wird ohne Wertansatz für Boden ausgewiesen, vgl. S. 279. 
Fremdarbeitskräfte werden zu keiner Zeit weder in Form von Aushilfen noch von ständig Beschäftigten eingesetzt.

Schaubild 287.1: Entwicklung von Tier- und Pflanzenproduktion, Zupacht und AKh-Zukauf bei Gewinnmaximierung unter Sicherheit (in Mastplätzen, ha bzw. Stunden)

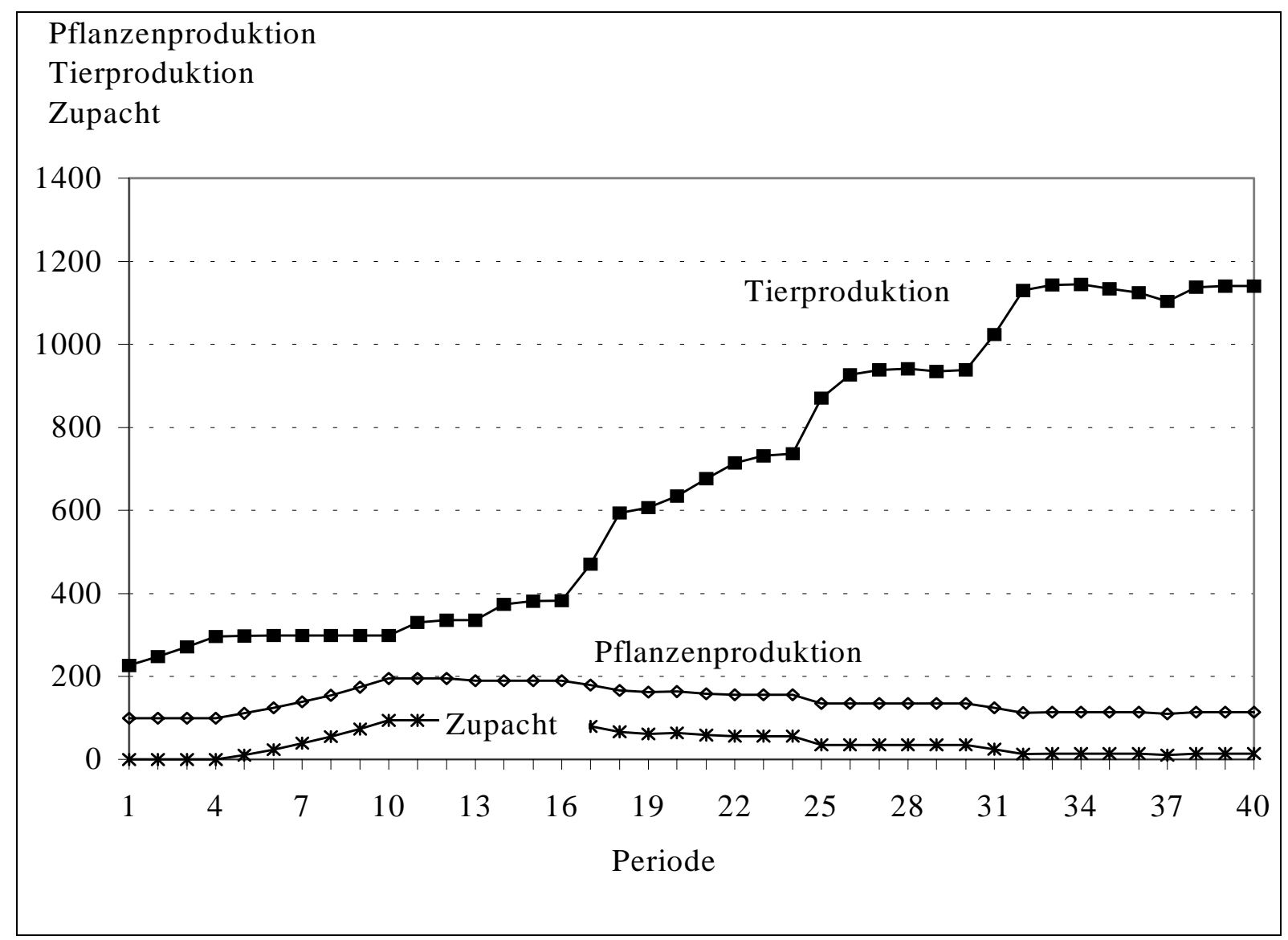

Die Entwicklung der Betriebsorganisation mag ein wenig überraschen, insbesondere der Rückgang der Zupacht ab der 15. Periode; sie dient dennoch der Gewinnmaximierung. Denn nicht nur der Gewinn, auch die übrigen Finanzkennzahlen zeigen eine positive Entwicklung (s. Schaubild 2881. Dabei zeigt der Gewinn die für den LOHMANN-RUCHTI-Effekt (vgl. Abschnitt 2.4 typischen Einbrüche. Auch der Kapitalsaldo (Geldvermögen abzgl. Fremdkapital) weist einige lokale Minima auf. Beide Kurvenverläufe sind verursacht durch hohe Ersatzinvestitionen für Maschinen (Nutzungsdauer 12 Jahre) und Stallbauten (Nutzungsdauer 18 Jahre). 


\section{Schaubild 288.1: Zeitliche Entwicklung der Finanzkennzahlen bei Gewinnmaximierung unter Sicherheit}

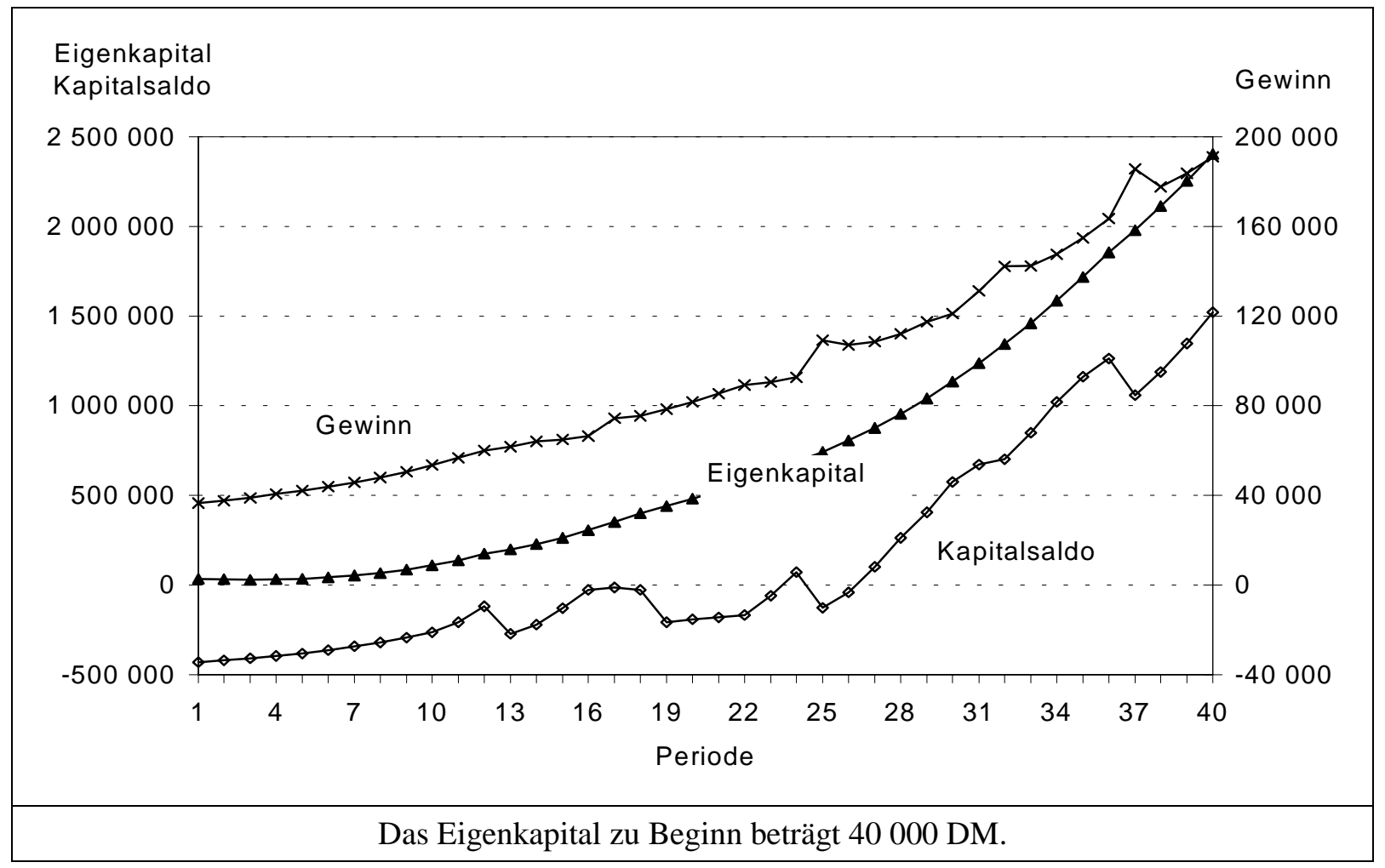

\subsection{Ergebnisse ausgewählter Strategien im deterministischen Modell}

In diesem Abschnitt werden die Ergebnisse einiger Strategien im Vergleich zur Referenzstrategie, der unbedingten Gewinnmaximierung (s. S. 270 und den vorangegangenen Unterabschnitt), für das deterministische Modell vorgestellt. Zum einen wird eine Diversifikationsstrategie untersucht. Sie beinhaltet einen Zwang zu einer Angleichung der Investitionsvolumina. Eine Kombination verschiedener Produktionsverfahren gilt allgemein als eine Möglichkeit, das Risiko zu senken. Zum anderen plant die Entscheiderin nicht mit den von ihr erwarteten Preisen, sondern nimmt Abschläge für den Getreide- und den Schweinepreis vor. Die Abschläge sind gestaffelt. Diese Strategiengruppe wird im folgenden als Risikoabschlagsstrategie bezeichnet.

Für jede Verhaltensannahme werden die Auswirkungen auf das Produktionsprogramm und die Wirtschaftlichkeit unter der Voraussetzung vollständiger Voraussicht untersucht. Die relative Rentabilität dieser Strategien im Vergleich zu einer Gewinnmaximierung kann ohne Vorhandensein von Unsicherheit nur negativ sein. Die Untersuchung dient der Vorbereitung der Analyse der Strategien im stochastischen Modell, wo neben der Wirtschaftlichkeit der Verhaltensweisen ihre Implikationen in bezug auf Fragen der Überlebenswahrscheinlichkeit im Vordergrund stehen. 


\section{Diversifikation}

Die Diversifikationsstrategie ist auf Grundlage der Referenzstrategie spezifiziert. Sie unterscheidet sich von der reinen Gewinnmaximierung durch eine zusätzliche Bedingung: Das Verhältnis der Produktionsumfänge von Pflanzen- und Tierproduktion beträgt $25: 11{ }^{211}$ Dieser Quotient wird gewählt, damit das Investitionsvolumen der beiden Produktionsverfahren in den ersten zwölf Runden (die Nutzungsdauer der Maschinen beträgt 12 Perioden) gleich hoch ist. ${ }^{212}$ Danach stimmen wegen der Unterschiedlichkeit der Nutzungsdauern die Investitionsvolumina nicht mehr überein.

\section{Schaubild 289.1: Produktionsverfahren bei Diversifikation im deterministischen Modell}

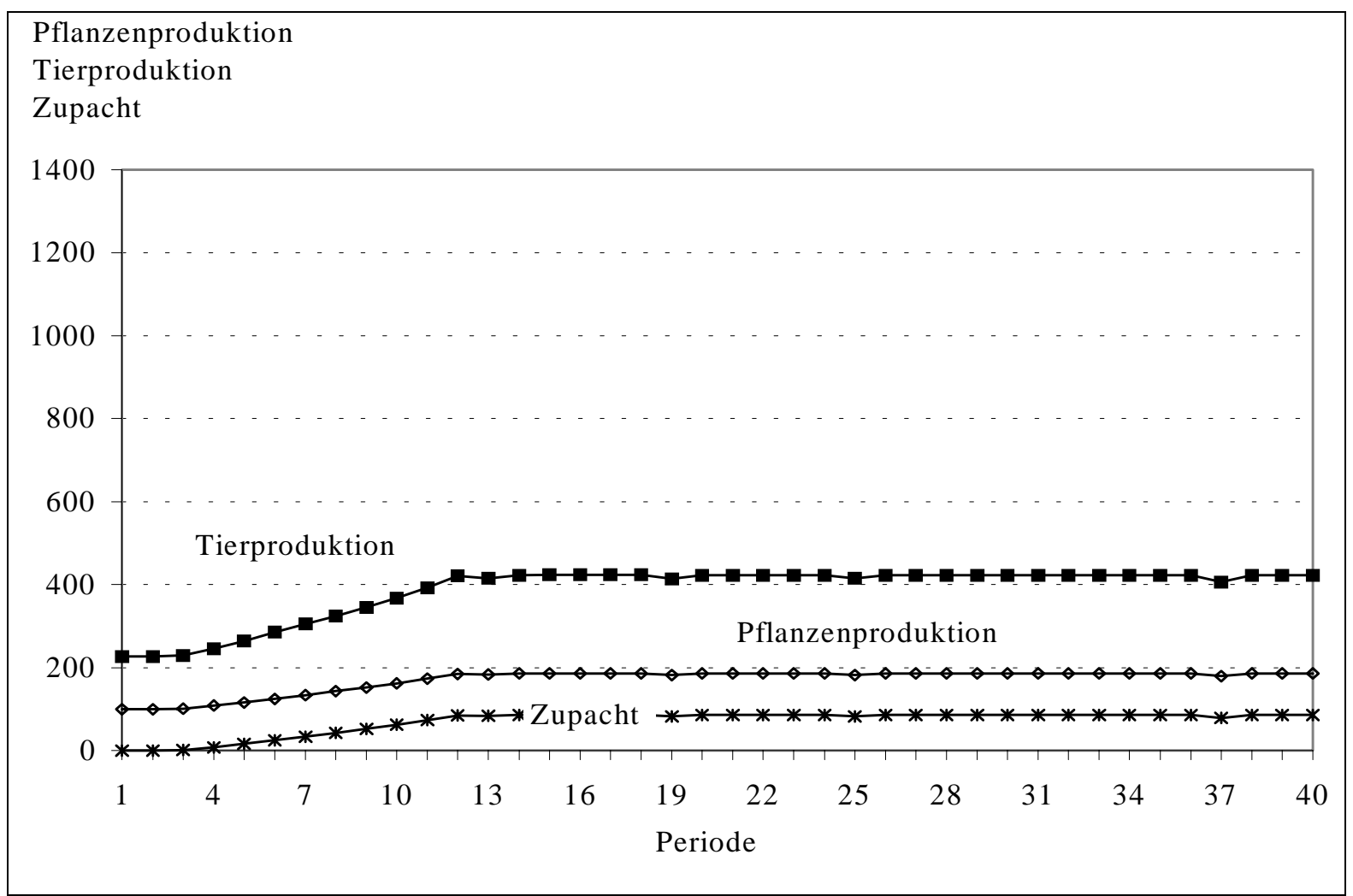

Die Diversifikationsauflage fördert, wie Schaubild 2891 $\mathrm{im}$ Vergleich zu Schaubild 287.1 und Tabelle 2961 ${ }^{13}$ zeigen, pflanzliche Produktion und Zupacht nur leicht, so daß die Tierproduktion, die bei der Referenzstrategie im zeitlichen Durchschnitt den fünffachen Umfang im Vergleich zur Pflanzenproduktion aufweist, zurückgefahren

211 Auf die Modellierung eines Korridors wird verzichtet, da sich die Tierproduktion aufgrund ihrer größeren Wettbewerbskraft stets am oberen Rand des Korridors bewegen würde.

212 Die Anschaffungspreise der Investitionen der beiden Produktionsrichtungen stehen genau in diesem Verhältnis zueinander, vgl. Tabelle 286.1.

213 Die Tabelle enthält auch die Ergebnisse anderer Strategien im deterministischen wie auch im stochastischen Modell. Ihre Beschreibung erfolgt später. 
werden muß. Das durchschnittliche Niveau in der Tierproduktion liegt bei knapp 400 Einheiten, im Vergleich zu 675 bei Gewinnmaximierung.

Die finanziellen Auswirkungen sind weit geringer, als es die starke Einschränkung der Tierhaltung vermuten läßt. In Tabelle 294.1 sind wichtige Finanzkennzahlen der Strategien zusammengefaßt. Das Eigenkapital liegt in der letzten Runde $12 \%$ niedriger als bei unbeschränkter Gewinnmaximierung. Der Gewinn beträgt in der letzten Periode $84 \%$ des Referenzgewinns, während der durchschnittliche Gewinn nur um 9\% zurückgeht. Diversifikation wirkt also vor allem in höheren Perioden gewinnschmälernd. Dies zeigt auch der Verlauf der Gewinnkurve in Schaubild 2901 im Vergleich zum Referenzgewinnverlauf in Schaubild 2881. Sehr gut sind auch in diesen Schaubildern die durch den LOHMANN-RUCHTI-Effekt bedingten Gewinnrückgänge in einzelnen Perioden erkennbar.

\section{Schaubild 290.1: Finanzkennzahlen bei Diversifikation im deterministischen Modell}

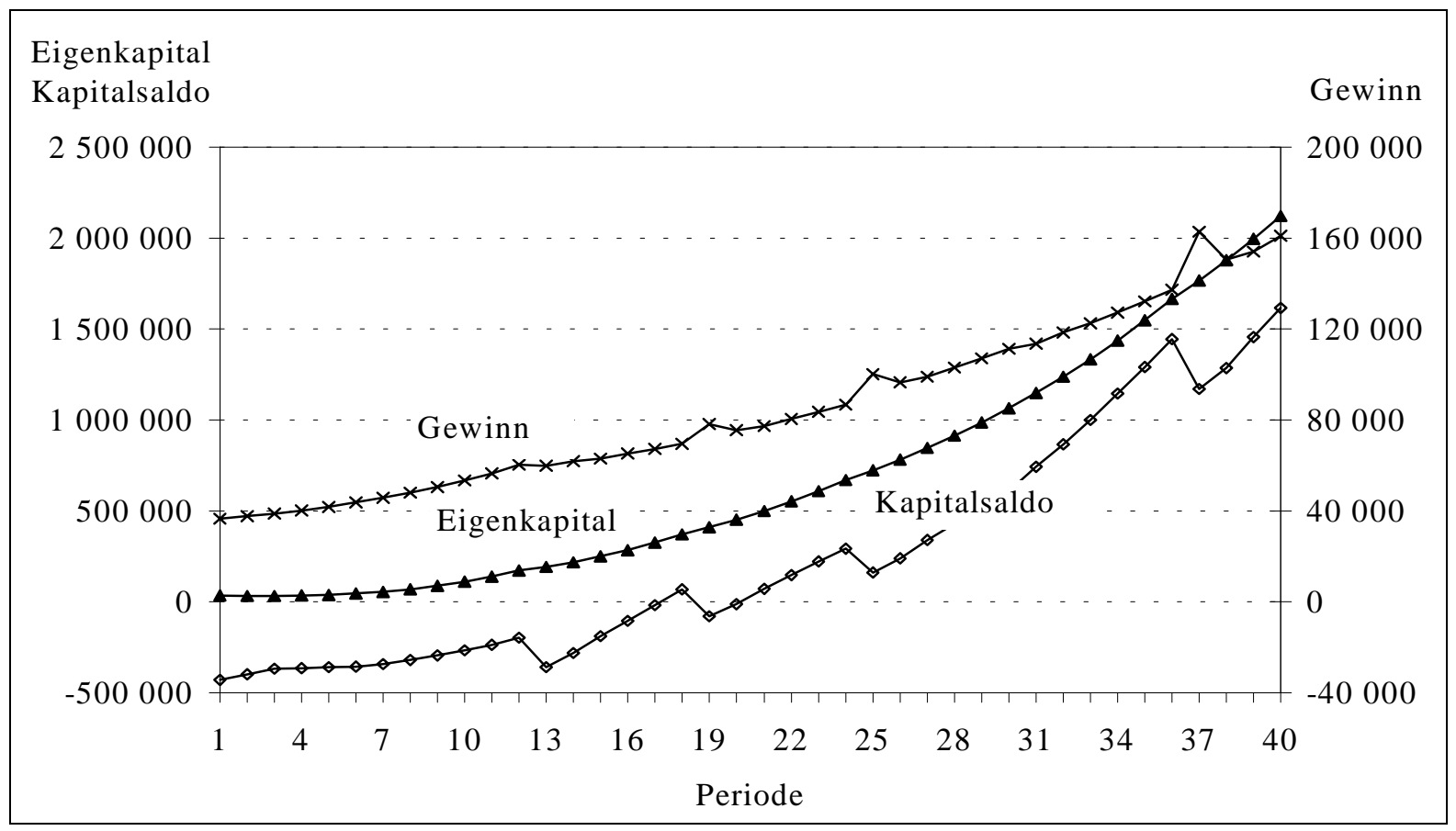

\section{Risikoabschläge}

Bei dieser Strategie trifft die Unternehmerin ihre Investitionsentscheidungen nicht auf Grundlage der erwarteten Preise, sondern sie verwendet reduzierte Ansätze. Beispielhaft werden im folgenden die Auswirkungen von zwei Abschlagsstrategien im deterministischen Modell analysiert: einem 10\%igem Preisabschlag bei Getreide und einem 2.5\%igem bei Schweinen. Die realisierten Preise und alle anderen Koeffizienten bleiben unverändert. Die sich aus diesen Preisannahmen ergebenden Deckungsbeiträge sind im Anhang in den Tabellen 3371 und Tabelle 3381 berechnet. Der Gewinnbeitrag der Pflanzenproduktion bleibt positiv, auch wenn die Maschinen mit dem teueren 
Annuitätendarlehen zu 9\% finanziert werden. Hingegen kann die Tierproduktion nur dann zum Gewinn des Unternehmens beitragen, wenn die Investitionen nicht mit dem teueren langfristigen Kredit finanziert werden müssen.

Schaubild 291.1: Produktionsverfahren mit Risikoabschlägen im deterministischen Modell

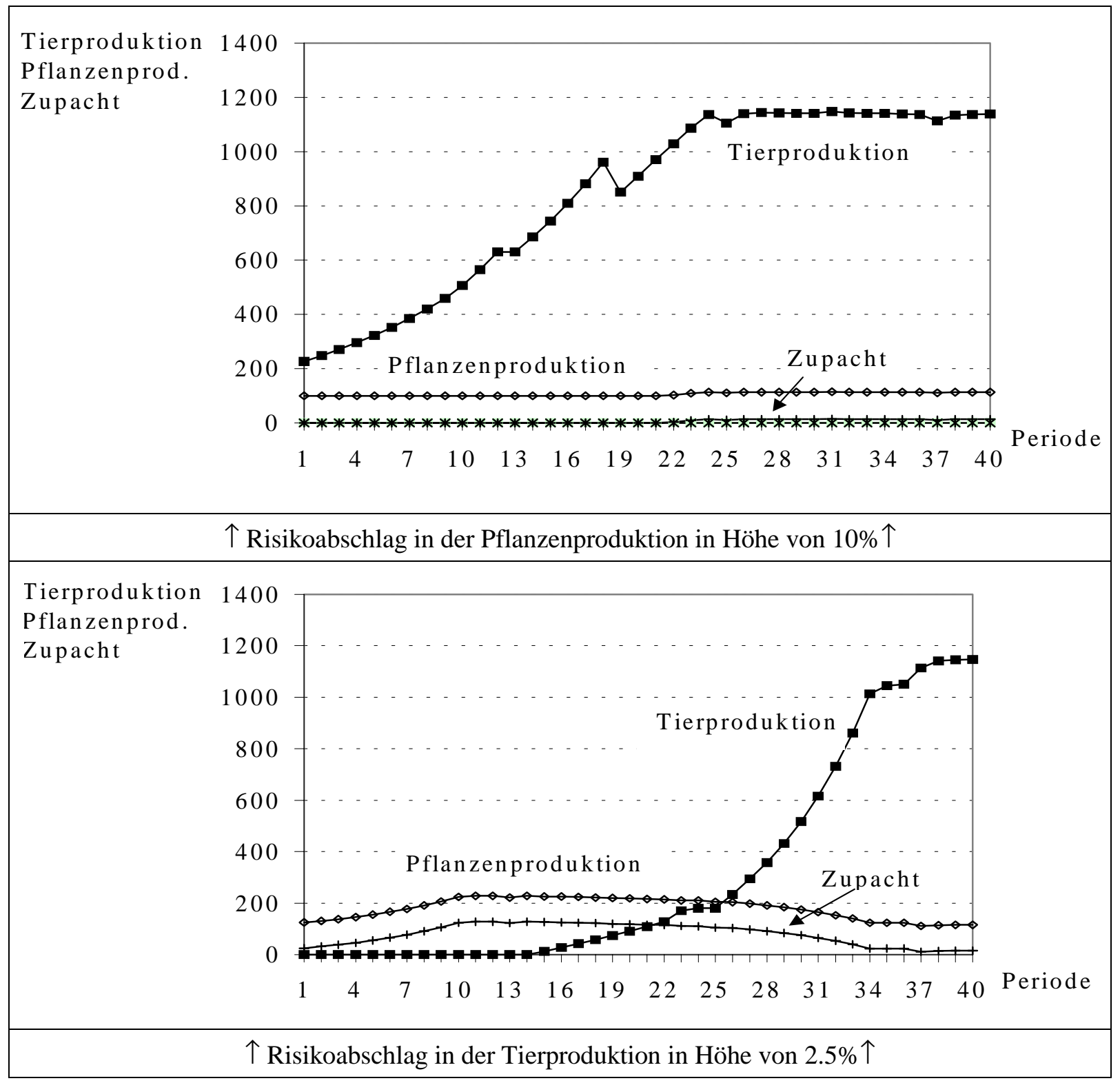

Bei einer Kalkulation mit um 10\% reduzierten Getreidepreisen wird im Vergleich zur Referenzstrategie wie erwartet deutlich weniger in Pflanzenproduktion und mehr in Tierproduktion investiert. Pachtflächen setzt das Unternehmen nahezu nicht mehr ein (siehe Schaubild 2911). Reduzierte Schweinepreisansetzungen erlauben in den ersten Perioden keine Tierproduktion, da diese zu dem teuren Kredit nicht rentabel ist und der billige besser durch die Pflanzenproduktion verwertet wird. Erst wenn ausreichend Eigenmittel erwirtschaftet worden sind, kann die Tierhaltung aufgenommen 
werden. Sie erreicht gegen Ende des Planungshorizontes trotz des Preisabschlages das gleiche Level wie im linken Schaubild. Preisabschläge in der untersuchten Größenordnung können also langfristig nicht die relative Wettbewerbskraft der beiden Produktionsverfahren verändern. Im Mittel jedoch lassen Risikoabschläge in der Pflanzenproduktion den mittleren Umfang der Tierproduktion stark ansteigen, während vorsichtigere Schweinepreiserwartungen zu einem spiegelbildlichen Ergebnis führen. In Tabelle gestelit.

Die finanziellen Auswirkungen von Risikoabschlägen sind in Schaubild 293,1 abgebildet und in Tabelle 2941 in aggregierter Form aufgeführt. Die Graphik bei Getreidepreisabschlägen ähnelt der der Diversifikationsstrategie aus Schaubild 290.1. Preisabschläge in der Tierproduktion drücken den finanziellen Erfolg zu Anfang weniger und später deutlich stärker als Abschläge im konkurrierenden Produktionsverfahren Pflanzenproduktion.

Die Durchschnittszahlen der Tabelle 294.11 bringen diese Entwicklung nicht zum Ausdruck. Im Mittel über 40 Perioden führen Preisabschläge in der Tierproduktion zu einem höheren Gewinn als Getreidepreisabschläge. In der letzten Periode liegt der Gewinn bei Getreidepreisabschlägen hingegen um 10 Prozentpunkte höher. Dieser positive langfristige Trend für die Getreidepreisabschläge wird bei Betrachtung des Eigenkapitals noch deutlicher. Der Verlust dieser Strategie im Vergleich zu reiner Gewinnmaximierung beträgt in der 40 . Periode nur $2 \%$, während Schweinepreisabschläge das Eigenkapital um 18\% vermindern.

Zum Vergleich der Strategien erscheinen über die Zeit gebildete Durchschnitte wenig sinnvoll. Zur Einschätzung der Ertragskraft eines Unternehmens werden im allgemeinen nur die Gewinne der letzten Jahre berücksichtigt. ${ }^{215}$ Hohe Gewinne in der Vergangenheit führen in dem vorliegenden Modell nicht zu höheren Entnahmen, da diese für alle Strategien in gleicher Höhe fixiert sind und alle Eigenkapitalzuwächse sofort und vollständig investiert oder angelegt werden. Eine Gegenüberstellung der Strategien über den Gewinn muß sich folglich auf die letzten Perioden beziehen. Wie Tabelle 294.1 zu entnehmen ist, stimmen die Rangfolgen der Strategien in bezug auf den durchschnittlichen Gewinn und den Gewinn in der letzten Periode nicht überein. Dies liegt in Ungenauigkeiten bei der Berechnung des Eigenkapitals bzw. des Gewinn begründet. Wie im Anhang im Unterabschnitt 9.6.3 dargestellt, basiert die Berechnung des Eigenkapitals auf linearen Abschreibungen. Im Gegensatz dazu erfolgt die Zielwertoptimierung mit Hilfe von Annuitäten (vgl. Abschnitte 3.4 und 9.6.2. Ein Annuitätendarlehen ist durch progressive Tilgungen gekennzeichnet. Die implizite progressive Abschreibung in der Bestimmung des Zielwertes, aus der der Gewinn abgeleitet

214 Die Tabelle enthält auch Ergebnisse von Varianten der Risikoabschlagsstrategie für das deterministische Modell, die hier nicht besprochen werden.

215 In der Rechtsprechung beispielsweise werden zur Bestimmung der Ertragskraft eines Unternehmens die Gewinne der letzten drei bis sechs Jahre herangezogen (mündliche Auskunft von E. BAHRS, Institut für Agrarökonomie, 08.08.1998). 
wird (vgl. Unterabschnitt 9.0.3.8), und die lineare Abschreibung, die für die Fortschreibung des Eigenkapitaiverwendet wird, führt zu der genannten Differenz.

\section{Schaubild 293.1: Finanzkennzahlen bei Risikoabschlägen im deterministischen Modell}

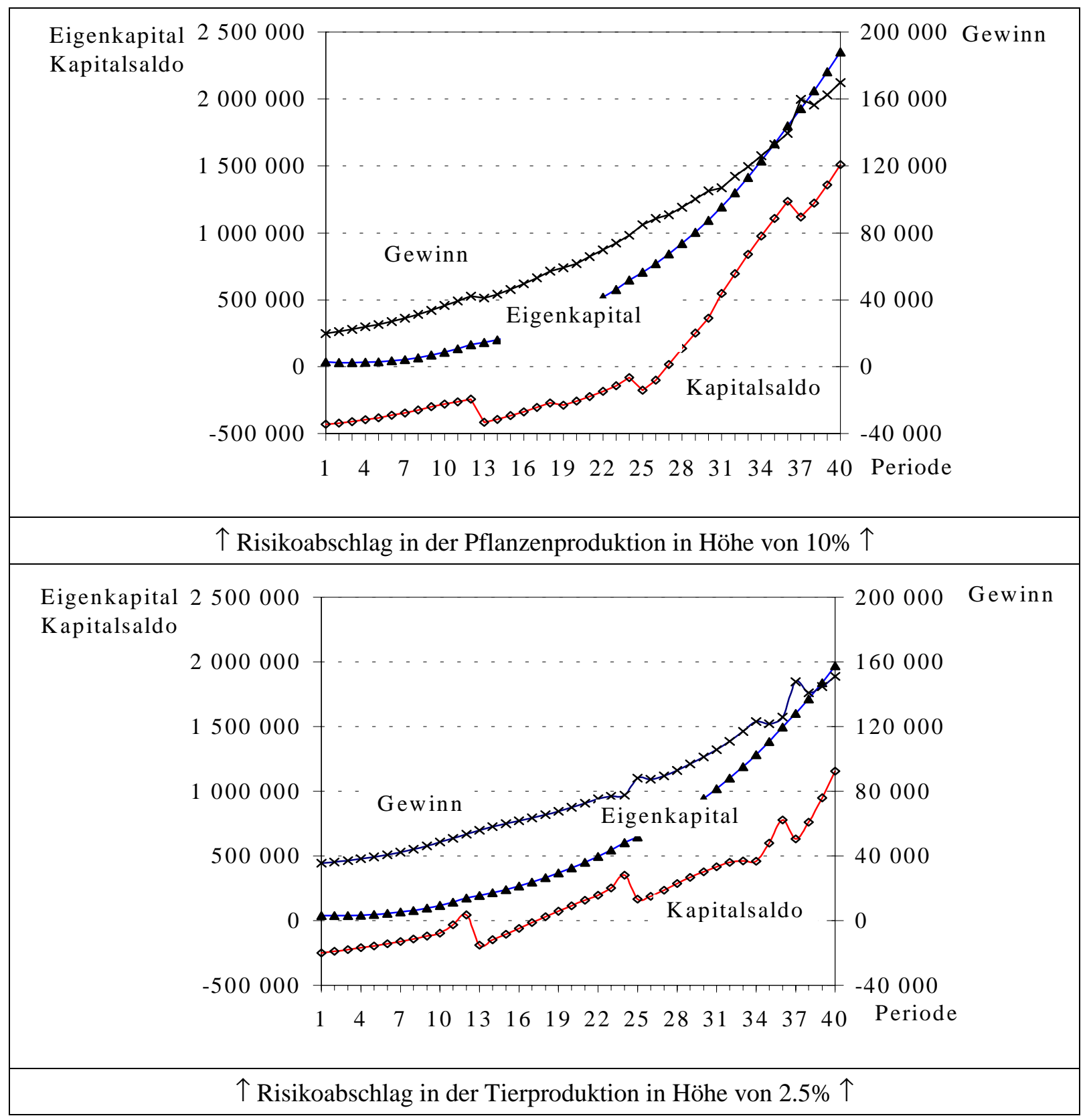

Vergleiche der Strategien scheinen über das Eigenkapital einfacher möglich als über den Gewinn. Der Verkaufswert eines Unternehmens wird nicht vom mittleren Eigenkapital der vergangenen 40 Jahre bestimmt, sondern richtet sich eher nach dem Eigenkapitalbestand der aktuellen Periode, dem Vermögensendwert. Dieser scheint daher ein geeigneter Maßstab zu sein, Strategien in ihren wirtschaftlichen Auswirkungen zu beurteilen. Das Kriterium Eigenkapital besitzt zudem den Vorteil, daß die Rangfolgen 
der Strategien zwischen durchschnittlichem Eigenkapital und dem Vermögensendwert nicht divergieren.

Tabelle 294.1: Wichtige Finanzkennziffern von vier Strategien im deterministischen Modell

\begin{tabular}{||c||c|c|c|c|c|c|c|c|}
\hline \multicolumn{1}{|c||}{ Strategie } & \multicolumn{5}{c|}{ Gewinn } & \multicolumn{4}{c|}{ Eigenkapital } \\
\cline { 2 - 10 } & $\varnothing$ über alle Perioden & \multicolumn{2}{c|}{ letzte Periode } & $\varnothing$ über alle Perioden & \multicolumn{2}{|c|}{ letzte Periode } \\
\cline { 2 - 9 } & absolut & relativ & absolut & relativ & absolut & relativ & absolut & relativ \\
\hline \hline Referenz & 94355 & 100 & 190907 & 100 & 735591 & 100 & 2403711 & 100 \\
Diversifikation & 85447 & 91 & 161154 & 84 & 678114 & 92 & 2122990 & 88 \\
Risikoabschlag & 75055 & 80 & 169895 & 89 & 702705 & 96 & 2353470 & 98 \\
$\begin{array}{c}\text { PP 10\% } \\
\text { Risikoabschlag } \\
\text { TP 2.5 \% }\end{array}$ & 79057 & 84 & 151029 & 79 & 616735 & 84 & 1969351 & 82 \\
\hline \multicolumn{3}{|c|}{40 Perioden, PP = Pflanzenproduktion, TP = Tierproduktion } \\
\hline
\end{tabular}

\subsection{Ausgewählte Ergebnisse im stochastischen Modell}

Als Referenz dient das oben vorgestellte deterministische Modell. Für das stochastische Modell werden die Produktpreise in den Produktionsverfahren Pflanzen- und Tierproduktion als Zufallsvariablen modelliert: Die Produktpreise schwanken gemäß einer symmetrischen Dreiecksverteilung unkorreliert und stochastisch unabhängig um $\pm 10 \%$ um ihren Mittelwert. Eine Dreieckverteilung wird gewählt, weil sie zum einen im Gegensatz zu einer Normalverteilung beschränkt ist und zum anderen zu ihren Rändern hin im Unterschied zu einer Gleichverteilung abnehmende Wahrscheinlichkeiten aufweist. Alle anderen Variablen bleiben deterministisch. Zwei Modelle werden unterschieden: In Modell I ist nur der Getreidepreis stochastisch, in Modell II ist darïber hinaus auch der Schweinepreis eine Zufallsvariable.

\subsubsection{Vergleich der Strategien im stochastischen Modell}

Die Simulation wird bis zu 600 Mal wiederholt. Jede Wiederholung erstreckt sich über 40 Perioden. Folglich beträgt das maximale Alter, das ein Unternehmen erreichen kann, ebenfalls 40 Perioden. In die Analysen aufgenommen werden außer den schon beschrieben Strategien auch Getreidepreisabschläge in anderer Höhe und Kombinationen mit Preisabschlägen in der Tierproduktion. Eine Variation der Schweinepreisabschläge erfolgt nicht, da bei deutlich geringeren Preisen keine wirtschaftliche Schweinemast möglich ist (vgl. Tabelle $3381 \mathrm{im}$ Anhang). Die genaue Staffelung der Preisabschläge kann Tabelle 296.1 entnommen werden. 
Wie unterscheiden sich stochastisches und deterministisches Modell? Da die Modellandwirtin rationale Erwartungen bildet, ${ }^{216}$ plant sie mit dem Erwartungswert der Preise, bei den Risikoabschlagsstrategien vermindert um einen Abschlag. Ihre Produktion ist somit in der ersten Periode unabhängig von der Unsicherheit der Preise. Die wirtschaftliche Situation hingegen wird durch die realisierten Preise, die im allgemeinen anders ausfallen als erwartet, verbessert oder verschlechtert. Damit ist die Ausgangslage für die zweite Runde eine andere. Dies bewirkt trotz gleicher Preiserwartungen eine veränderte Produktion in der zweiten und den folgenden Perioden.

Im Mittel wird sich der erwartete Preis realisieren. Dennoch stimmt das über 600 Wiederholungen gemittelte Produktionsprogramm in den beiden stochastischen Modellen und dem deterministischen Modell nicht vollständig überein, wie Tabelle 296.1 zu entnehmen ist. Die Unterschiede zwischen den beiden stochastischen Modellen sind für gleiche Strategien sehr gering. Offensichtlich verändert die Aufnahme eines zweiten stochastischen Preises das mittlere Produktionsprogramm nicht. Im Vergleich von stochastischen Preisen zu festen Preisen sind ebenfalls keine nennenswerten Differenzen zu verzeichnen, mit einer Ausnahme: bei Gewinnmaximierung wird bei einem oder zwei stochastischen Preisen deutlich mehr Tier- und etwas weniger Pflanzenproduktion betrieben als bei festen Preisen.

Die deutlichen Unterschiede des Produktionsprogramm zwischen den Strategien im deterministischen Modell bleiben also im stochastischen Modell erhalten. Wie wirken sich die Strategien auf die zentrale Frage, die Überlebenswahrscheinlichkeit, aus? Dies wird im folgenden für Modell I ausführlich erörtert. Für Modell II schließt sich eine geraffte Darstellung an.

Für die Überlebenswahrscheinlichkeit ergeben sich ebenfalls deutliche Unterschiede zwischen den Strategien im Modell I. Sie sind in Tabelle 297.1 zusammengefaßt:

- Bei Verwendung der Referenzstrategie fallieren 30 der 600 Unternehmen innerhalb der ersten 40 Perioden. Die Insolvenzen sind zeitlich sehr stark konzentriert, alle ereignen sich in den Runden 2 bis 8. In den Perioden zuvor reicht das Startkapital aus, negative Preisausschläge abzufangen, danach haben die erfolgreichen Unternehmen genügend Eigenkapital gebildet, um Preiseinbrüche zu überstehen. Die durchschnittliche Überlebensdauer beträgt hier 38.2 Perioden ${ }^{217}$.

- Diversifizieren die Unternehmen ihre Produktion, können sie dadurch die Insolvenzwahrscheinlichkeit um 20\% auf 24 fallierende Unternehmen senken. Die durchschnittliche Überlebensdauer steigt auf 38.6 Perioden.

216 Auch bei der Risikoabschlagsstrategie bildet die Unternehmerin rationale Erwartungen. Sie weiß um die Unsicherheit ihrer Erwartungen und basiert daher ihre Planung auf reduzierten Ansätzen.

217 Dieser Wert ist au den Gründen, auf die in Unterabschnitt 5.3.2, S. 253 hingewiesen wurde, nach unten verzerrt, da das maximale Alter auf 40 Perioden begrenzt ist. 
- Risikoabschläge in der Pflanzenproduktion wirken sich nicht auf die Überlebensfähigkeit aus. Wie bei der Gewinnmaximierung fallieren 30 von 600 Unternehmen. Auch die durchschnittlichen Lebensdauern stimmen exakt überein (38.3 Perioden).

Tabelle 296.1: Wichtige Produktionskennziffern der Strategien

\begin{tabular}{|c|c|c|c|c|c|c|}
\hline \multirow[b]{2}{*}{ Strategie } & \multicolumn{6}{|c|}{ Mittelwerte über alle Perioden und Wiederholungen des Umfangs der ... } \\
\hline & \multicolumn{2}{|c|}{\begin{tabular}{c}
\multicolumn{2}{c}{ Tierproduktion } \\
determin. stochast.
\end{tabular}} & \multicolumn{2}{|c|}{ Pflanzenproduktion } & \multicolumn{2}{|c|}{ Pacht } \\
\hline \multicolumn{7}{|c|}{ Modell I: Getreidepreise $\pm 10 \%$} \\
\hline Referenz & 673 & 693 & 143 & 138 & 43 & 38 \\
\hline Diversifikation & 386 & 383 & 170 & 168 & 70 & 68 \\
\hline Risikoabschlag (PP 10\%) & 839 & 835 & 106 & 106 & 6 & 6 \\
\hline \multicolumn{7}{|c|}{ Modell II: Getreidepreise $\pm 10 \%$ und Schweinepreise $\pm 10 \%$} \\
\hline Referenz & 673 & 694 & 143 & 137 & 43 & 37 \\
\hline $\begin{array}{c}\text { Diversifikation } \\
\text { Risikoabschläge: }\end{array}$ & 386 & 383 & 170 & 168 & 70 & 68 \\
\hline PP5\% & 839 & 830 & 106 & 106 & 6 & 6 \\
\hline PP10\% & 839 & 828 & 106 & 106 & 6 & 6 \\
\hline $\mathrm{PP} 15 \%$ & 839 & 825 & 106 & 106 & 6 & 6 \\
\hline PP $20 \%$ & 462 & 461 & 0 & 0 & 0 & 0 \\
\hline TP $2.5 \%$ & 319 & 275 & 180 & 182 & 80 & 82 \\
\hline PP 5\%, TP $2.5 \%$ & 727 & 699 & 106 & 105 & 6 & 5 \\
\hline PP $10 \%$, TP $2.5 \%$ & 708 & 687 & 104 & 104 & 4 & 4 \\
\hline PP15\%, TP $2.5 \%$ & 670 & 645 & 100 & 100 & 0 & 0 \\
\hline PP $20 \%$, ТP $2.5 \%$ & 252 & 252 & 0 & 0 & 0 & 0 \\
\hline
\end{tabular}

Ein Test auf Signifikanz mit den in Abschnitt 3.3 beschriebenen Prüfverfahren ergibt, daß Anteilswerte von 24/600 und 30/600 sich nicht signifikant unterscheiden; die Irrtumswahrscheinlichkeit $\alpha$ beträgt 0.4. Numerische Simulationen führen zu dem Ergebnis, daß die gefundenen Häufigkeitsunterschiede erst beim Sechsfachen der Wiederholungszahl, also bei 3600 Wiederholungen, mit $\alpha=5 \%$ signifikant unterschiedlich sind. Eine Irrtumswahrscheinlichkeit von unter $1 \%$ wird ab 6000 Iterationen erreicht. Der Test liefert also keine Bestätigung für die Hypothese, daß sich Referenzund Diversifikationsstrategie signifikant in den Insolvenzzahlen unterscheiden. Dies bedeutet nicht, daß kein systematischer Unterschied bestehen kann, sondern nur, daß er, falls er bestünde, von diesem Test nicht erkannt wird. Daher wäre es sinnvoll, andere parametrische Tests zu entwickeln oder simulative Testverfahren, wie das Boot- 
strapping, zu erproben. Dafür war in dieser Arbeit kein Raum mehr. Somit sind die nachfolgenden Ergebnisse mit entsprechender Vorsicht zu interpretieren.

Die finanziellen Ergebnisse der Strategien faßt Tabelle 2971 zusammen. Einige Kennziffern sind in Schaubild 2991 grafisch abgetragen sowie im Anhang als Poster präsentiert. Im folgenden werden zunächst die Hauptresultate vorgestellt und abschließend einige Auffälligkeiten diskutiert.

Tabelle 297.1: Vermögensendwerte und Überlebenswahrscheinlichkeiten der Strategien im stochastischen Modell

\begin{tabular}{|c|c|c|c|c|c|c|}
\hline \multirow[b]{2}{*}{ Strategie } & \multirow{2}{*}{$\begin{array}{l}\text { Ausscheide- } \\
\text { rate } \\
\text { (2) }\end{array}$} & \multicolumn{4}{|c|}{ Eigenkapital in der letzten Runde (Vermögensendwert) } & \multirow{2}{*}{$\begin{array}{l}\varnothing \text { "Lebens- } \\
\text { dauer" } \\
\quad 7\end{array}$} \\
\hline & & $\operatorname{determin}_{(3)}^{* *}$ & $\begin{array}{l}\text { alle } \\
\text { (4) }\end{array}$ & $\begin{array}{l}\text { Überlebende } \\
\text { (5) }\end{array}$ & $\begin{array}{c}\text { Ausge- } \\
\text { schiedene } * * *\end{array}$ & \\
\hline \multicolumn{7}{|c|}{ Modell I: Getreidepreis $\pm 10 \%$} \\
\hline Referenz & $5 \%$ 2 & 2403711 & 2270758 & 2390538 & -5048 & 38.2 \\
\hline Diversifikation & $4 \%$ (1) & 2122990 & 2029077 & 2113785 3 & -3900 & 38.6 \\
\hline $\begin{array}{l}\text { Risikoabschlag } \\
\text { PP } 10 \%\end{array}$ & $5 \%$ 2 & 2353470 & 2236626 & 2354609 (2) & -5048 & 38.2 \\
\hline \multicolumn{7}{|c|}{ Modell II: Getreide- und Schweinepreis $\pm 10 \%$} \\
\hline Referenz & $19 \% 0$ & 2411674 & 2015688 & 2484710 & -5679 & 33.3 \\
\hline $\begin{array}{c}\text { Diversifikation } \\
\text { Risikoabschläge: }\end{array}$ & $12 \% \boldsymbol{5}$ & 2122990 & 1903261 & $2159300 \boldsymbol{6}$ & -4410 & 35.7 \\
\hline PP 5\%* & $19 \% \nabla$ & 2353795 & 1959733 & 2430698 (2) & -5327 & 33.1 \\
\hline$P P 10 \%$ & $18 \% \quad \boldsymbol{6}$ & 2353470 & 1959650 & $2412173 \boldsymbol{3}$ & -5477 & 33.3 \\
\hline PP $15 \%$ & $19 \% \nabla$ & 2353470 & 1944879 & $2407373 \mathbb{4}$ & -5639 & 33.2 \\
\hline PP 20\%* & $100 \% \boldsymbol{\theta}$ & -27083 & -20092 & - & -20092 & 1.8 \\
\hline $\mathrm{TP} 2.5 \%$ & $6 \% \quad 4$ & 1969351 & 1830769 & 19513348 & -3771 & 37.9 \\
\hline PP 5\%, TP $2.5 \%$ & $0.3 \% \quad \boldsymbol{1}$ & 1993440 & 1960636 & $1967202 \boldsymbol{0}$ & -2577 & 39.9 \\
\hline PP $10 \%$, ТP $2.5 \%$ & $0.3 \% \quad \mathbf{1}$ & 1997322 & 1946365 & $1952884 \boldsymbol{7}$ & -2909 & 39.9 \\
\hline PP $15 \%$, ТР $2.5 \%$ & $0.3 \%$ & 1935894 & 1875737 & $1882020 \boldsymbol{9}$ & -2909 & 39.9 \\
\hline PP $20 \%, \mathrm{TP} 2.5 \% *$ & $100 \% \quad \boldsymbol{9}$ & -20746 & -19998 & - & -19998 & 2.0 \\
\hline \multicolumn{7}{|c|}{$\begin{array}{c}\text { 40 Perioden, } 600 \text { Wiederholungen, } \mathrm{PP}=\text { Pflanzenproduktion, } \mathrm{TP}=\text { Tierproduktion } \\
\text { (123456089: Rangziffern } \\
\text { Kursiv dargestellte Zeilen kennzeichnen die Ergebnisse nicht dominierter Strategien. }\end{array}$} \\
\hline
\end{tabular}

Als Prüfstein des finanziellen Erfolgs einer Strategie wird der Vermögensendwert der überlebenden Unternehmen herangezogen. Risikoneutrale Akteure würden sich am Erwartungswert des Eigenkapitals orientieren; dies entspricht dem Vermögensendwert aller Unternehmen. Demgegenüber beachten risikoscheue Entscheider den trade off zwischen Überlebenswahrscheinlichkeit und Eigenkapital im Überlebensfall. Eigenka- 
pitaleinbußen einer Strategie im Vergleich zur Referenzstrategie werden daher als Kosten dieser Strategien aufgefaßt. Die Eigenkapitalsituation der Überlebenden unterscheidet sich nicht stark von der im deterministischen Modell, wie aus Tabelle 2971 im Vergleich von Spalte (3) zu Spalte (5) ersichtlich ist. Daher bleiben auch die Unterschiede zwischen den Strategien weitgehend erhalten. Die Risikoabschlagsstrategie, die in puncto Überlebenswahrscheinlichkeit keine Vorteile bringt, führt zu den geringsten Kosten. Das Eigenkapital der Überlebenden in der letzten Runde geht kaum zurück (1.5\%). Demgegenüber erfordert die risikomindernde Strategie Diversifikation erhebliche finanzielle Einbußen. Das Eigenkapital nimmt um 12\% ab.

Modell II beinhaltet stochastischen Getreide- und Schweinepreise mit einer Spanne von $\pm 10 \%$. In diesem Modell erfolgt eine stärkere Aufgliederung der Risikoabschlagsstrategien. Vier Stufen des Abschlags auf den Getreidepreis werden untersucht (5, 10, 15 und 20\%), jeweils allein und in Kombination mit einem Abschlag in der Tierproduktion (2.5\%). Unter diesen Strategien führt Gewinnmaximierung wie in Modell I zum höchsten Eigenkapital. Strategien, die Preisabschläge bei Getreide zwischen 5 und $15 \%$ vornehmen, verbessern ebenfalls wie in Modell I kaum die Überlebensaussichten und verursachen nur geringe Gewinneinbußen. Kleinere Ruinwahrscheinlichkeiten können durch Diversifikation oder verstärkt durch Risikoabschläge in der Tierproduktion erzielt werden. Bereits die Insolvenzhäufigkeiten der Diversifikationsstrategie unterscheiden sich signifikant von der Referenzstrategie $(\alpha<0.1 \%)$. Das gleiche Signifikanzniveau weisen die Differenzen zwischen Diversifikation und Risikoabschlägen in der Tierproduktion auf. Eine Kombination von Abschlägen in der Tier- und Pflanzenproduktion bietet nahezu eine Überlebensgarantie (zwei Insolvenzen auf 600 Wiederholungen), während alleinige moderate Abschläge in der Pflanzenproduktion fast wirkungslos bleiben. Sehr hohe Abschläge in der Pflanzenproduktion von 20\% führen hingegen mit Sicherheit zum Ruin des Unternehmens. Die Ursache liegt darin, daß bei um ein Fünftel reduzierten Preisen die Pflanzenproduktion nicht mehr wirtschaftlich betrieben werden kann (s. Tabelle 296.1] und ausschließlich mit Tierproduktion der Betrieb auch bei festen Preisen nicht überlebensfähig ist, erkennbar am negativen Eigenkapital im deterministischen Modell (s. Spalte (3) in Tabelle 297.1]. Bei der Entscheidung über die Höhe von Preisabschlägen sind daher die Stabilitätsbereiche der Produktionsverfahren zu berücksichtigen.

Die in schwarze Kreise eingeschlossenen Ziffern in Tabelle 297.1 bringen die relative Vorzüglichkeit einer Strategie in Bezug auf ein Kriterium zum Ausdruck. Als Rangkriterien sind die Ausscheiderate (s. Spalte (2) in der Tabelle) und das Endvermögen der überlebenden Unternehmen (5) ausgewählt worden. Die Ausscheiderate gibt den Prozentsatz der Unternehmen (oder äquivalent dazu der Wiederholungen) an, die das Zieljahr, die 40. Periode, nicht erreicht haben. Die sich aus diesem Beurteilungskriterium ergebende Reihenfolge der Strategien stimmt mit der Plazierung nach durchschnittlicher Lebensdauer (7) überein. 


\section{Schaubild 299.1: Vermögensendwerte und Überlebenswahrscheinlichkeiten der Strategien im stochastischen Modell II}

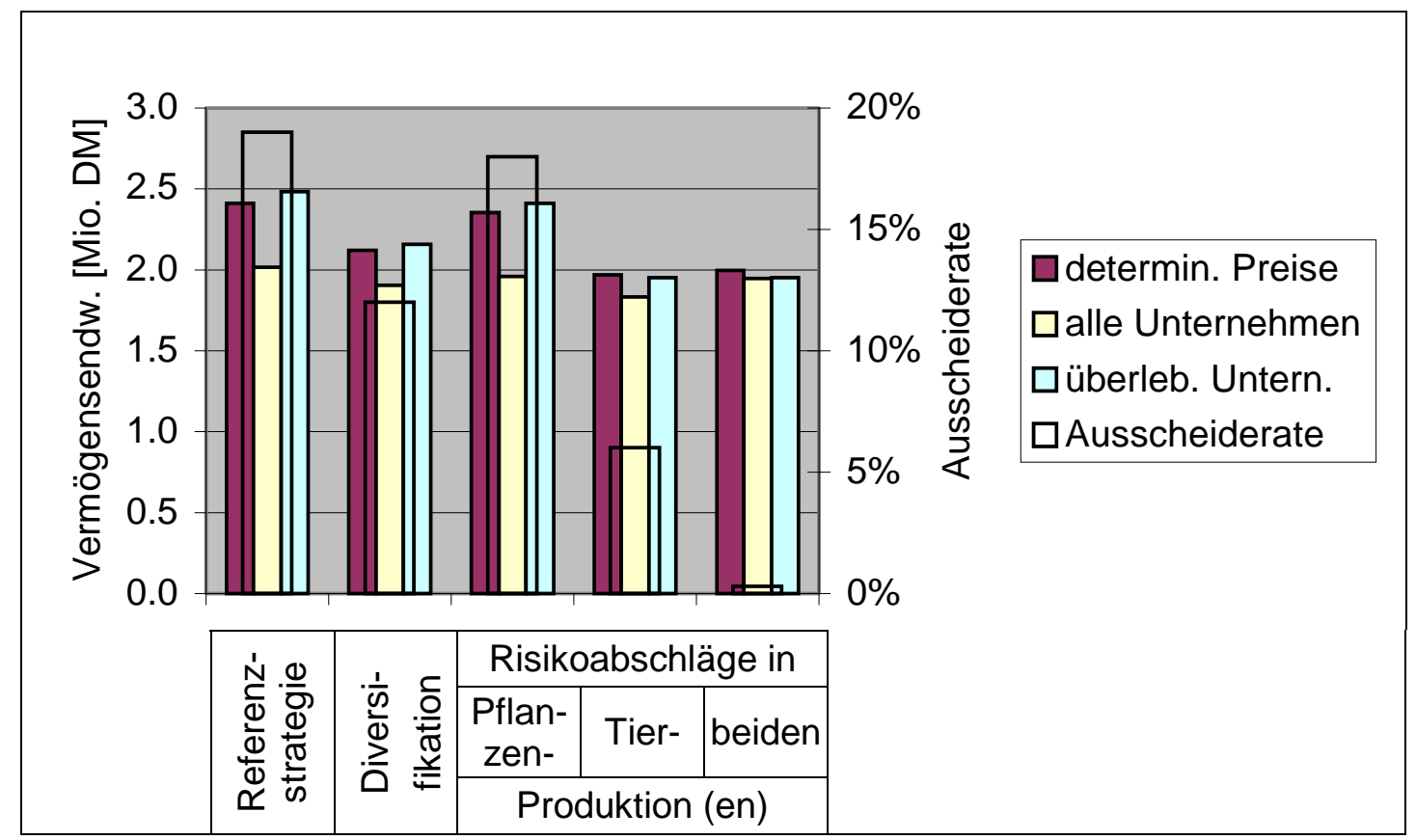

Einige der simulierten Strategien kommen für niemanden in Frage, der risikoavers ist, da sie (schwach) dominiert werden. Dies sind zunächst die Strategien mit 20\%igem Risikoabschlag in der Pflanzenproduktion, da sie als einzige mit Sicherheit zum Ruin führen. Weiter werden dominiert:

- 5\%iger und 15\%iger Getreidepreisabschlag von der Referenzstrategie

- nur Preisabschlag in der Tierproduktion von zusätzlichem Getreidepreisabschlag in Höhe von 5 oder $10 \%$

- Preisabschlag in der Tierproduktion und zusätzlich ein Getreidepreisabschlag von mehr als 5\% von Getreidepreisabschlag von nur 5\% (PP 5\% ^ TP 2.5\%).

Die nicht dominierten Strategien sind kursiv dargestellt. Sie bilden die Auswahlmenge für einen risikoaversen Entscheider: \{Referenz, Diversifikation, PP 10\%, PP 5\% ^ TP $2.5 \%$ \}. Zwischen ihnen muß die Unternehmerin eine Entscheidung treffen.

Das Eigenkapital der Ausgeschiedenen in Tabelle 297.1 soll nicht ökonomisch interpretiert werden; es bestätigt jedoch eine wahrscheinlichkeitstheoretische Aussage aus Abschnitt5.3 Je größer die Stichprobe, desto kleiner ist c.p. ihr Minimum, oder der Anteil der Verteilung, der einen bestimmten Wert unterschreitet. Die Tabelle zeigt, daß eine höhere Insolvenzrate (entsprechend einer größeren Stichprobe) auch zu niedrigerem durchschnittlichen Eigenkapital der ausscheidenden Unternehmen führt.

Abschließend seien noch einige Auffälligkeiten des Finanzergebnisses erläutert. Zunächst zum Eigenkapital in der letzten Runde. Betragsmäßig ist das Eigenkapital aller Unternehmen (Spalte (4)) im stochastischen Modell stets geringer ist als unter Sicherheit. Die Ursache hierfür liegt darin, daß ausscheidende Unternehmen nicht mehr zur 
Eigenkapitalakkumulation beitragen können und damit den Mittelwert senken, während im deterministischen Modell alle Unternehmen die letzte Runde erreichen.

Folglich sollte der mittlere Vermögensendwert ausschließlich der überlebenden Unternehmen größer sein als im deterministischen Modell, da ein Ausklammern des unteren Teils einer Verteilung den Erwartungswert des verbleibenden Teils der Verteilung steigen läßt. Der in diesem Sinne bedingte Erwartungswert übertrifft den Erwartungswert der gesamten Verteilung, wie in Abschnitt 4.2 ausgeführt wurde. In den Simulationsergebnissen ist dieser Effekt nur bei hohen Ausscheideraten festzustellen. Bei geringen Insolvenzraten wird der Vermögensendwert des deterministischen Modells knapp unterschritten. Auf die Ursachen dieses Ergebnisses kann hier nicht weiter eingegangen werden.

Eine weitere Besonderheit betrifft die Szenarien mit einem Risikoabschlag in der Tierproduktion von $2.5 \%$ und in der Pflanzenproduktion von 5 bzw. $10 \%$. Das Szenario mit der schärferen Verhaltensrestriktion (Preisabschlag in der Pflanzenproduktion von $10 \mathrm{im} \mathrm{Vergleich} \mathrm{zu} \mathrm{5 \% )} \mathrm{darf} \mathrm{keinen} \mathrm{höheren} \mathrm{Gewinn} \mathrm{aufweisen.} \mathrm{Daß} \mathrm{das} \mathrm{ausge-}$ wiesene Eigenkapital in der letzten Periode dennoch bei der mehr einschränkenden Strategie höher ausfällt, liegt in den oben (s. S. 292] beschriebenen Ungenauigkeiten bei der Berechnung des Eigenkapitals begründet. Im Anhang sind die Ergebnisse beider Strategien dokumentiert. Tabelle 388.1 zeigt, daß die weniger einschränkende Strategie in jeder Periode zu einem höheren Zielwert führt, während die Werte des Eigenkapitals zunächst gleich sind und ab der 23. Periode sich ein Vorteil für den $10 \%$-Abschlag ergibt.

Nach der Diskussion der Auswirkungen von Strategien auf die Überlebenswahrscheinlichkeit wird nun der Datenkranz modifiziert. Dabei beschränkt sich die Betrachtung auf die Referenzstrategie. In Tabelle 301.1 sind die Ergebnisse für Betriebsszenarien zusammengestellt, die sich im verfügbaren Startkapital unterscheiden. Eine Erhöhung des Startkapitals um 10 TDM auf 50 TDM reduziert die Insolvenzrate auf zwei Drittel. Dieser Anstieg ist signifikant. Die Irrtumswahrscheinlichkeit liegt unter $0.1 \%$. Eine weitere Steigerung um 10 TDM führt wiederum zu einer Verringerung der Insolvenzrate um ein Drittel auf nunmehr 2\%. Auch dieser Unterschied erweist sich als signifikant mit einer Irrtumswahrscheinlichkeit von 1.2\%. Absolut gemessen ist die zweite Erhöhung des Startkapitals um 10 TDM weniger effektiv als die erste. Eine Veränderung des Startkapitals von 40 auf 50 TDM reduziert die Ruinwahrscheinlichkeit um 13 Prozentpunkte, eine weitere Erhöhung um 10 TDM nur noch um 4 Prozentpunkte. Umgekehrt sind die Auswirkungen höheren Startkapitals auf den Vermögensendwert der Überlebenden. Eine Erhöhung des Startkapitals um 10 TDM läßt den Vermögensendwert nach 12 Perioden dieser Gruppe um 26 TDM ansteigen; stehen weitere 10 TDM an Startkapital zur Verfügung, führen diese zu einer Vergrößerung des Vermögensendwertes um 33 TDM. Bezogen auf den Erwartungswert des Eigenkapitals in der 12. Periode ist eine lineare Beziehung zwischen Startkapital und Eigenkapital festzustellen. Auffällig ist der starke Abfall des Endvermögens 
insolventer Unternehmen: Je größer das Startkapital, desto mehr wird im Modell investiert; daher führen bei größerem mengenmäßigen Umsatz Insolvenzen zu höheren Kapitalunterdeckungen.

Tabelle 301.1: Vermögensendwerte und Überlebenswahrscheinlichkeiten der Strategien im stochastischen Modell: Variation des Startkapitals

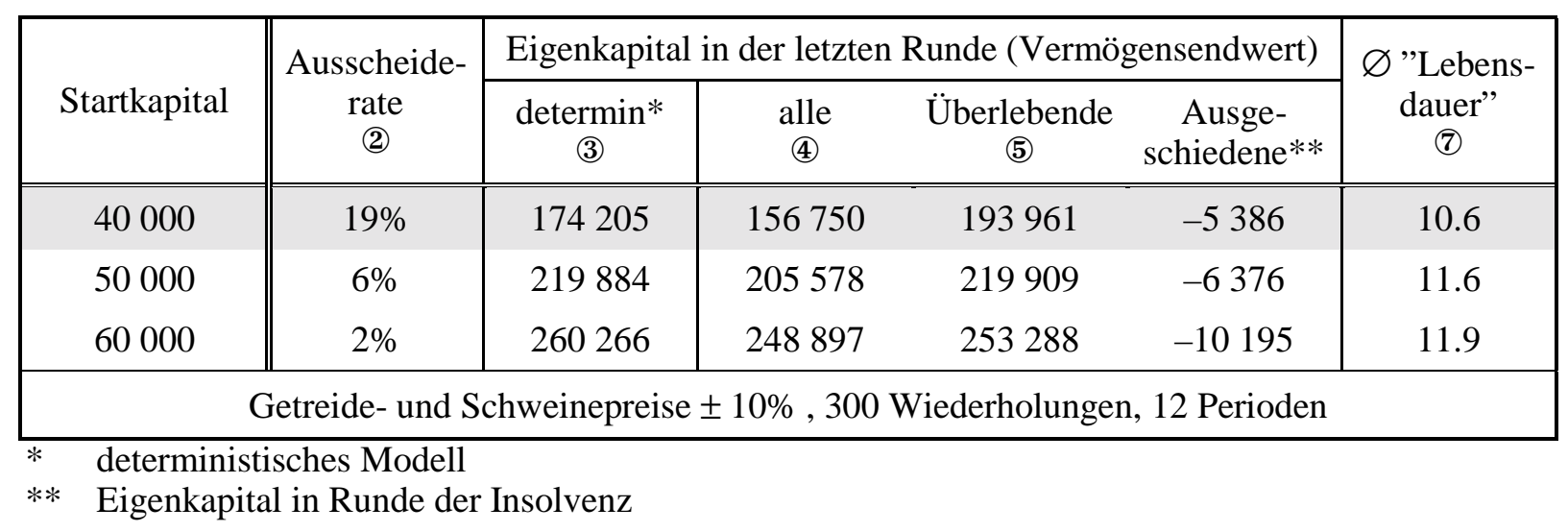

Die Abnahme der Ausscheiderate mit zunehmender Unternehmensgröße, hier gemessen am Startkapital, deckt sich mit einer These der Organisationstheorie zur Erklärung der Überlebenswahrscheinlichkeit von Unternehmen (vgl. Unterabschnitt 2.3.1.1. Die liability of smallness besagt, daß kleine Unternehmen unter ungünstigeren terms of trade wirtschaften und daher einem größeren Insolvenzrisiko unterworfen sind. Die Ergebnisse der Simulation können dennoch nicht zur Unterstützung dieser These herangezogen werden. Denn in der Simulation sind die terms of trade konstant und hängen nicht von der Betriebsgröße ab. Daß trotz konstanter terms of trade die Insolvenzrate mit der Betriebsgröße korreliert ist, kann die Theorie der liability of smallness nicht erklären.

Als letzte Szenariogruppe wird die übrige Faktorausstattung variiert. Da unter der Referenzstrategie in keiner der 600 Wiederholungen eine Insolvenz nach der 8. Runde beobachtet wurde, wird die Periodenzahl auf 12 verringert. Bei einer Verdoppelung der Eigentumsfläche treten keine Insolvenzen mehr auf, siehe Tabelle 302.1. Das Eigenkapital nach der 12. Periode nimmt auf das 3.5-fache zu. Eine Verdoppelung der betrieblichen Arbeitskapazität (bei 100 ha Fläche) wirkt sich kaum positiv aus, ${ }^{218}$ weder in bezug auf die Überlebenswahrscheinlichkeit noch auf den finanziellen Erfolg. Arbeit ist offensichtlich nicht knapp. Dies wird bereits aus dem Verzicht auf die Beschäftigung von Aushilfen in den bisherigen Szenarien deutlich. Auch im deterministischen Modell wird die betriebliche Arbeitskapazität erst ab der 30. Periode ausgeschöpft. ${ }^{219}$ Eine Faktorausstattung mit 200 ha und 2 Arbeitskräften führt ebenfalls nur zu geringen finanziellen Verbesserungen im Vergleich zu einer Arbeitskraft.

\footnotetext{
218 Die Entnahmen sind konstant gehalten, um eine ceteris-paribus-Analyse zu ermöglichen. Eine 219 gleichzeitige Verdoppelung der Entnahmen ließe die Ruinwahrscheinlichkeit stark ansteigen.

Eine der Ursachen dafür ist, daß im Modell keine Zeitspannen enthalten sind.
} 
Wird als alleinige Veränderung der Faktorausstattung die Arbeitskapazität verdoppelt, ergibt sich eine geringfügige Verringerung des Vermögensendwertes. Dieses unplausible Ergebnis beruht auf den beschriebenen unterschiedlichen Abschreibungsmethoden für die Gewinnermittlung und die Eigenkapitalfortschreibung (s. S. 292). Die Gewinne liegen bei zwei Arbeitskräften in jeder Periode höher als bei nur einer Arbeitskraft (nicht ausgewiesen).

Als nächstes wird die Dungeinheitengrenze pro ha halbiert. Ein größerer Betrieb mit zwei Arbeitskräften und 200 ha wird durch diese verschärfte Auflage nicht tangiert, weder in seiner Überlebenswahrscheinlichkeit noch in seinem wirtschaftlichen Erfolg. Auch bei dem Ausgangsbetrieb (100 ha, 1 AK) führt eine Reduzierung der Dungeinheiten nicht $\mathrm{zu}$ einer Erhöhung der Insolvenzrate. Die finanziellen Auswirkungen dieser Restriktion sind sowohl im deterministischen wie im stochastischen Modell bei beiden Betriebsgrößen sehr gering.

Diese Ergebnisse bestätigen ebenfalls, daß größere Betriebe, hier gemessen an der Flächenausstattung, auch bei konstanten terms of trade einem geringeren „Insolvenzrisiko ausgesetzt sind.

\section{Tabelle 302.1: Vermögensendwerte und Überlebenswahrscheinlichkeiten der Strategien im stochastischen Modell: Variation der Faktor- ausstattung}

\begin{tabular}{|c|c|c|c|c|c|c|}
\hline \multirow{2}{*}{$\begin{array}{l}\text { Faktoraus- } \\
\text { stattung }\end{array}$} & \multirow{2}{*}{$\begin{array}{l}\text { Ausscheide- } \\
\text { rate } \\
\text { (2) }\end{array}$} & \multicolumn{4}{|c|}{ Eigenkapital in der letzten Runde (Vermögensendwert) } & \multirow{2}{*}{$\begin{array}{c}\varnothing \text { „Lebens- } \\
\text { dauer” } \\
7\end{array}$} \\
\hline & & $\operatorname{determin}^{*} * *$ & alle & $\begin{array}{l}\text { Überlebende } \\
\text { (5) }\end{array}$ & \begin{tabular}{c|} 
Ausge- \\
schiedene $* * *$
\end{tabular} & \\
\hline 100 ha, 12 Per. & $19 \%$ & $\begin{array}{r}174205 \\
46204^{+}\end{array}$ & 156750 & 193961 & -5386 & 10.6 \\
\hline 200 ha & $0 \%$ & 704499 & 702956 & 702956 & - & 12 \\
\hline $2 \mathrm{AK}$ & $18 \%$ & $\begin{array}{rl}172 & 670 \\
46 & 286^{+}\end{array}$ & 155152 & 190507 & -5909 & 10.6 \\
\hline 2 AK, 200ha & $0 \%$ & 735730 & 732194 & 732194 & - & 12 \\
\hline $\begin{array}{l}100 \mathrm{DE}, 2 \mathrm{AK}, \\
200 \mathrm{ha}\end{array}$ & $0 \%$ & 735730 & 745241 & 745241 & - & 12 \\
\hline $100 \mathrm{DE} *$ & $19 \%$ & 2370289 & 1982932 & 2459488 & -5453 & 33.1 \\
\hline 100 ha $*$ & $19 \%$ & 2411674 & 2015688 & 2484710 & -5679 & 33.3 \\
\hline \multicolumn{7}{|c|}{$\begin{array}{l}\text { Getreide- und Schweinepreise } \pm 10 \%, 300 \text { Wiederholungen, } 12 \text { Perioden } \\
\text { Es ist nur die veränderte Faktorausstattung im Vergleich zur Referenzsituation angegeben. }\end{array}$} \\
\hline $\begin{array}{ll}* & 40 \text { Periode } \\
* * & \text { determinist } \\
* * * & \text { Eigenkapit } \\
+ & \text { durchschni }\end{array}$ & $\begin{array}{l}\text { isches Modell } \\
\text { l in Runde der }\end{array}$ & nsolvenz & & & & \\
\hline
\end{tabular}

Zum Abschluß dieses Kapitels werden die Auswirkungen von Experimenten mit veränderter Preisvarianz untersucht. 


\subsubsection{Vergleich von Preisvarianzen im stochastischen Modell}

In Abschnitt 4.3 wurde abgeleitet, daß eine zunehmende Varianz der Einzahlungen die Überlebenswahrscheinlichkeit senkt, wenn ohne Varianz keine Insolvenzgefahr besteht. Dieser theoretische Forderung entspricht das Simulationsmodell, wie Tabelle 303.1 zu entnehmen ist. Bei einer Halbspanne des Getreidepreises von $\pm 1 \%$ falliert kein Unternehmen. Eine Preishalbspanne von $20 \%$ vervierfacht die Insolvenzrate im Vergleich zum Referenzmodell mit zehnprozentiger Spanne. Eine weitere Vergrößerung der Spanne läßt die Ausscheidewahrscheinlichkeit langsamer ansteigen. Dies stützt die theoretische Ableitung aus Abschnitt 4.3 und Kapitel 5. daß bei sehr hohen Varianzen die Überlebenswahrscheinlichkeit wieder ansteigt: Bei kleinen Preisvarianzen ist eine Insolvenz ausgeschlossen. Sie wird erst durch größere Einnahmeschwankungen möglich. Daher steigt die Insolvenzrate mit der Varianz an. Bei extrem großer Varianz kann das Eigenkapital bereits in der ersten Runde aufgebraucht werden und eine Insolvenz nachsichziehen. Eine weitere Varianzerhöhung kann die Ruinwahrscheinlichkeit nicht mehr erhöhen, aber sie verbessert auf der anderen Seite den wirtschaftlichen Erfolg der Überlebenden (der bedingte Erwartungswert steigt), der deren Überlebensaussichten in der Folgezeit verbessert und die Ruinwahrscheinlichkeit absenkt.

Tabelle 303.1: Vermögensendwerte und Überlebenswahrscheinlichkeiten bei Gewinnmaximierung und Variation der Getreidepreisstreuung

\begin{tabular}{|c||c|ccc|c|}
\hline \multirow{2}{*}{ Strategie } & Ausgeschiedene & \multicolumn{3}{|c|}{ Eigenkapital } & durchschnittliche \\
\cline { 3 - 5 } & $(\%)$ & Alle & Überlebende & Ausgeschiedene & "Lebensdauer" \\
\hline \hline Referenz $( \pm 10 \%)$ & 8 & 2242468 & 2437764 & -3436 & 37.1 \\
$\pm 01 \%$ & 0 & 2403732 & 2403732 & - & 40.0 \\
$\pm 20 \%$ & 28 & 1839590 & 2559474 & -11541 & 29.8 \\
$\pm 40 \%$ & 57 & 1295676 & 3039219 & -19629 & 19.1 \\
$\pm 60 \%$ & 72 & 991972 & 3621052 & -30448 & 13.8 \\
$\pm 90 \%$ & 80 & 869061 & 4510849 & -41385 & 10.2 \\
$\pm 120 \%$ & 79 & 1155322 & 5744747 & -64652 & 10.5 \\
\hline \multicolumn{7}{|c|}{ Jedes Szenario mit 100 Wiederholungen über 40 Perioden. } \\
\hline
\end{tabular}





\section{Zusammenfassung}

Dynamische Systeme unterliegen Einflußfaktoren, die sich auf deren Überlebenswahrscheinlichkeit auswirken können. Ein Beispiel für ein solches System sind landwirtschaftliche Großbetriebe in Ostdeutschland. Ein beträchtlicher Teil dieser Betriebe mußte in den vergangenen Jahren schließen. Da die Datenbasis nicht ausreicht, um quantitative empirische Untersuchungen zu den Bestimmungsfaktoren des Erfolgs oder Scheiterns solcher Unternehmen durchführen zu können, müssen hierfür andere Methoden herangezogen werden. In der vorliegenden Arbeit werden derartige Faktoren anhand von Unternehmensmodellen und einfacheren dynamischen Systemen grundsätzlich untersucht.

Zunächst werden in Kapitel 2 die bekanntesten Theorien zur Analyse der Überlebensfähigkeit von Unternehmen beschrieben und mögliche methodische Herangehensweisen diskutiert. Ein besonderer Schwerpunkt wird dabei auf Modellanalysen gelegt. Hierbei stehen Simulationsmodelle im Zentrum des Interesses, deren Grundprinzipien, Vor- und Nachteile sowie Anwendungsgebiete eingehend erläutert werden. Als Beispiel für eine Modellanalyse wird ein einfaches Betriebsentwicklungsmodell konstruiert, um den sogenannten LOHMANN-RUCHTI-Effekt zu illustrieren und den Anwendungsbereich dieses Theorems auszuweiten. Es wird gezeigt, daß trotz deterministischer und konstanter Umwelt ein positiver Gewinntrend in einzelnen Perioden Einbrüche aufweisen kann. Ursache des Effektes sind vorübergehende Kapazitätserweiterungen, die aus den Abschreibungsansätzen finanziert werden.

Kapitel 3 setzt sich zunächst mit methodischen Problemen von Betriebsentwicklungsmodellen und der Entscheidungsfindung in unsicheren Situationen auseinander. Es wird für Unsicherheit, die durch beschränkte Verteilungen charakterisiert ist, ein Algorithmus entwickelt, der den Unbestimmtheitsbereich erheblich einschränken kann. Der nächste Abschnitt dieses Kapitels weist auf die Schwierigkeiten hin, Signifikanztests für Häufigkeitsunterschiede in Monte-Carlo-Simulationen durchzuführen. Dieses Problem ist für die vorliegende Arbeit von besonderer Bedeutung, da in MonteCarlo-Simulationen Insolvenzen Ereignisse darstellen, deren Häufigkeit gezählt wird. Ruinhäufigkeiten beispielsweise besitzen aufgrund ihrer Entstehungsart in MonteCarlo-Überlebensmodellen keine Varianz und können im Gegensatz zu anderen Kennziffern, wie Gewinn, Umsatz, Produktionsmenge, nicht mit Hilfe von t-Tests auf Signifikanz geprüft werden. Das in der Arbeit entwickelte Verfahren basiert auf Tests, die zur Beurteilung von unterschiedlichen Anteilswerten Verwendung finden. Es wird auf die besondere Fragestellung in Monte-Carlo-Simulationen erweitert. Als Ergebnis steht eine Tabelle zur Verfügung, aus der für beliebige Monte-Carlo-Studien die Signifikanz von Häufigkeitsunterschieden abgelesen werden kann. Die Tabelle gilt für 100 Wiederholungen; sie kann leicht für andere Stichprobengrößen angepaßt werden.

In Betriebsentwicklungsmodellen ist die lineare Programmierung eine häufig benutzte Methode. Die Literatur zur einperiodischen Variante der linearen Program- 
mierung behandelt Investitionen nur am Rande. Da Investitionen jedoch ein wesentliches Element der betrieblichen Entwicklung bilden, setzt sich der letzte Teil dieses methodischen Kapitels mit den Problemen auseinander, Investitionen in die linearen Programmierung zu integrieren. Es wird ein Ansatz entwickelt, der eine simultane Optimierung von Investitionen und Finanzierung ermöglicht. Dabei ist die Anzahl verschiedener Zinssätze auf der Haben- und Sollseite prinzipiell unbeschränkt.

Kapitel 4 nähert sich der Problematik der Überlebenswahrscheinlichkeit aus der wahrscheinlichkeitstheoretischen Perspektive an. Summen und Produkte von Zufallsveränderlichen werden als Beispiel benutzt, um die analytischen Schwierigkeiten aufzuzeigen, die bei der Ableitung der Wahrscheinlichkeitsverteilung von Funktionen von Zufallsvariablen entstehen. Produkte von Zufallsveränderlichen, wie zum Beispiel Erlöse als Produkt aus stochastischen Preisen und stochastischen Erträgen, spielen in der Ökonomik eine bedeutende Rolle. Es wird demonstriert, daß weder ein Quadrat noch ein Produkt von Normalverteilungen wieder eine Normalverteilung ergeben und auch nicht durch eine Normalverteilung approximiert werden können. Das Kapitel schließt mit einem Exkurs über mathematische Probleme in Monte-Carlo-Simulationen. Dazu wird das bekannte Petersburger Paradoxon mit Hilfe einer solchen MonteCarlo-Simulation nachgebildet. Es stellt sich heraus, daß der bekanntlich unendliche Erwartungswert dieses Spieles auch bei sehr hoher Wiederholungszahl nicht annähernd erreicht wird. Die Ursachen dieser schlechten Abbildung werden aufgezeigt und problematisiert.

In Kapitel 5 erfolgt die Vorstellung einfacher Überlebensmodelle. Während für eine Analyse von Erwartungswerten und Streuungen in dynamischen Systemen die Kenntnis der Momente dieser Verteilungen und ihrer zeitlichen Entwicklungen ausreicht, muß für eine Quantifizierung von Überlebenswahrscheinlichkeiten die Wahrscheinlichkeitsverteilung als Ganzes in jeder Periode bekannt sein. Dies führt bei stetigen Verteilungen in der Regel ab der dritten Periode zu unüberwindbaren methodischen Problemen. Daher kann die Überlebenswahrscheinlichkeit von dynamisch stetigen Systemen für mehrere Perioden nur mit Hilfe von Monte-Carlo-Simulationen beleuchtet werden. Dieser Aufgabe ist Kapitel 5 im besonderen gewidmet. Das Kapitel gliedert sich in die Betrachtung von Modellen mit nur einer stochastischen Einzahlung und Modellen, in denen zwischen einer stochastischen und einer deterministischen Einzahlung zu wählen ist. Für die erstgenannten Modelle wird zwischen Einzahlungen, die additiv, und Einzahlungen, die multiplikativ mit dem Kapital der Vorperiode verbunden sind, unterschieden. Für diskret additive Einzahlungen wird das Konzept der Markov-Ketten benutzt, um die Überlebenswahrscheinlichkeit auch für beliebige Runden analytisch berechnen zu können. Den Abschluß des Kapitels bildet ein Abschnitt, der sich mit Überlebensgesichtspunkten des dynamischen Planspiels „Puten \& Perlhühner" auseinandersetzt. Die in Kapitel 3 abgeleitete Technik zur Reduzierung des Unsicherheitsbereichs bei Entscheidungsproblemen wird auf dieses Spiel angewandt, um ineffiziente Investitionsstrategien aus der Auswahlmenge ausschließen zu können. 
Im abschließenden Kapitel 6 wird ein Betriebsentwicklungsmodell entworfen. Mit seiner Hilfe werden Einflußfaktoren auf die Überlebenswahrscheinlichkeit landwirtschaftlicher Betriebe untersucht. Das Modell bildet einen stilisierten landwirtschaftlichen Betrieb $a b$, der sich zur Betriebsplanung der sogenannten rollenden Planung bedient. Das enthaltene LP ist einperiodisch formuliert und optimiert simultan den Produktions-, Investions- und Finanzbereich des Unternehmens. Der Einfluß ,,vorsichtiger" Wachstumsstrategien und verschiedener Faktorausstattungen wird untersucht. Entgegen der herrschenden Theorie konnte in den Simulationen beobachtet werden, daß bereits einfache Diversifikationen oder Risikoabschläge die Insolvenzhäufigkeit in bestimmten Situationen deutlich senken. Große Unternehmen erweisen sich auch bei expansiver Investitionspolitik als weniger gefährdet als kleine. Dieses Ergebnis wird unter konstanten terms of trade erzielt; schlechtere Austauschbeziehungen sind also keine Voraussetzung für eine empirisch häufig zu beobachtende größere Ruinrate kleiner Unternehmen. 



\section{Literaturverzeichnis}

Abramowitz, Milton \& Irene A. Stegun (1970): Handbook of mathematical functions with formulas, graphs, and mathematical tables. 9. Aufl., Washington: United States Department of Commerce.

Adam, Dietrich, Gerhard Schünemann \& Rainer Sibbel (1994): Deterministisches Chaos im Verhalten dynamischer Investitions- und Finanzierungsmodelle. WiSt 23, Heft 11, S. 546-551.

Alchian, Armen A. (1950): Uncertainty, evolution, and economic theory. Journal of Political Economy 58, S. 211-221.

Amburgey, T. L., D. Kelly \& W. P. Barnett (1993): Resetting the clock: The dynamics of organizational change and failure. Administrative Science Quarterly 38, S. 51-73, zit. nach Woywode (1998, S. 40).

Amman, Hans M., David A. Kenndrick \& John Rust (Hg.) (1996): Handbook of computational economics. Amsterdam: Elsevier.

Anderson, Jock R., John L. Dillon \& J. Brian Hardacker (1977): Agricultural decision analysis. Ames, Iowa: University Press .

Aumann, Robert J. (1997): The St. Petersburger Paradox: A discussion of some recent coments. Journal of Economic Theory 14, S. 443- 445.

Axelrod, Robert (1991): Die Evolution der Kooperation. 2. Aufl., München: Oldenbourg.

Axelrod, Robert (1997): Advancing the art of simulation in the sozial sciences. Complexity 3, Nr. 2, S. 16-22.

Axelrod, Robert (1997): The complexity of cooperation. Princeton: University Press.

Bailey, DeeVon \& James W. Richardson (1985): Analysis of selected marketing Strategies: A whole-farm simulation approach. American Journal of Agriculture Economics S. 813-820.

Balmann, Alfons (1994): Ansätze zur Erklärung einer Dominanz und Persistenz "suboptimaler" Betriebsgrößenstrukturen in der Landwirtschaft. Agrarwirtschaft 43, S. 227-236.

Balmann, Alfons (1995): Pfadabhängigkeiten in Agrarstrukturentwicklungen. Berlin: Duncker \& Humblot.

Balmann, Alfons (1997): Farm Based Modelling of Regional Structural Change. European Review of Agricultural Economics 24, (1), S. 85-108. 
Balmann, Alfons, C. Laskowski und Martin Odening (1998):

Kreditwürdigkeitsanalyse landwirtschaftlicher Unternehmen mit Hilfe multivariater statistischer Verfahren. In: Spilke, J. \& R. Doluschitz (Hg.): Referate der 19. GIL-Jahrestagung.

Balmann, Alfons, Hermann Lotze \& Steffen Noleppa (1998):

Agrarsektormodellierung auf der Basis 'typischer Betriebe'. Agrarwirtschaft 47, Heft 5, S. 222-230 und Heft 6, S. 251-258.

Bamberg, Günter \& Adolf Gerhard Coenenberg (1989): Betriebswirtschaftliche Entscheidungslehre. München: Vahlen.

Bamberg, Günter \& Franz Baur (1987): Statistik. 5. Aufl., München Wien: R. Oldenbourg.

Banse, Martin \& Wolfgang Muench (1998): Die Einführung der Gemeinsamen Agrarpolitik in den Beitrittsländern Mitteleuropas und die Agenda 2000 - landwirtschaftliche und volkswirtschaftliche Effekte. Agrarwirtschaft 47, Heft 3-4, S. 180-189.

Barlett, Peggy F. (1993): American dreams, rural realities: familiy farms in crisis. Chapel Hill, London: University of North Carolina Press.

Bartmann, Hermann \& Klaus-Dieter John (1985): Entscheidungen, Erwartungen und Kontrakte bei Unsicherheit. Jahrbuch für Nationalökonomik und Statistik 200/3, Stuttgart. G. Fischer, S. 217-228.

Bäuerle, P. (1987): Finanzielle Planung mit Hilfe heuristischer Kalküle. Frankfurt: Lang.

Bellman, Richard (1967): Dynamische Programmierung und selbstanpassende Regelprozesse. München: Oldenbourg.

Bender, Dieter (1982): Finanzmarkttheorie des Wechselkurses. $H d W W$ 9, Stuttgart: Fischer, Mohr: Tübingen, Göttingen: Vandenhoeck \& Ruprecht, S. 748764.

Benjamin, Jaxk R. \& C. Allin Cornell (1970): Probability, Statistics and Decision for Civil Engineers. New York: McGraw-Hill.

Berg, Ernst \& Friedrich Kuhlmann (1993): Systemanalyse und Simulation. Stuttgart: Ulmer.

Berger, Thomas \& Wilhelm Brandes (1998): Evolutionäre Ansätze in der Agrarökonomik. Agrarwirtschaft 47, Heft 7, S. 275-282.

Berger, Thomas (1996): Fuzzy-KW. Ein Programm zur Berechnung von FuzzyKapitalwerten. Working Paper 26, Wirtschafts- und Sozialwissenschaften und der Landwirtschaftlich-Gärtnerischen Fakultät, Humboldt-Universität. Berlin. 
Berger, Thomas (1999): Diffusion von Innovationen in pfadabhängigen Systemen Ein bioökonomisches Simulationsmodell für den regionalen Agrarstrukturwandel in Chile. Dissertation, Institut für Agrarökonomie der Universität Göttingen.

Bernoulli, Daniel (1738/1954): Exposition of a new theory of measurment of risk. Econometrica 22, S. 23-36, Übersetzung eines Aufsatzes von 1738 von Louise Sommer.

Bleymüller, Josef, Günther Gehlert \& Herbert Gülicher (1998): Statistik für Wirtschaftswissenschaftler. 11. überarbeitete Aufl., München: Vahlen.

Blohm, Hans \& Klaus Lüder (1995): Investition. 8. Aufl., München: Vahlen.

Bode, Oliver (in Vorbereitung): Quantitative Analyse dynamischer nichtlinearer Panelmodelle. Dissertation, Institut für Agrarökonomie der Universität Göttingen.

Bokelmann, Wolfgang (1993): Früherkennung von Unternehmenskrisen. Unveröffentlichte Habilitationsschrift, Hannover.

Bølviken, Erik \& Eva Skovlund (1996): Confidence Intervalls from Monte Carlo Tests. Journal of American Statistical Association 91, Nr. 435, Theory and Methods, S. 1071-1078.

Borz, Jürgen (1993): Statistik für Sozialwissenschaftler. 4. Aufl., Berlin: Springer.

Bosch, Karl (1992): Statistik-Taschenbuch. München: Wien.

Bouzaher, A, R. Cabe, S.R. Johnson, A. Manale \& J.F. Shogren (1995): CEEPES: An evolving system for agroenvironmetal policy. In: Milon, J.W. \& J.F. Shogren (Hg.): Intergrating Economic and Eological Indicators: Practical Methods for Environmetal Policy Analysis. Praeger, S. 67-89. Zit. nach Oriade \& Dillon (1997) S. 51f.

Brandes, Wilhelm \& Emil Woermann (1969): Landwirtschaftliche Betriebslehre. Band 1: Allgemeiner Teil. Theorie und Planung des landwirtschaftlichen Betriebes. 2. Aufl., Hamburg, Berlin: Parey.

Brandes, Wilhelm \& Hans-Joachim Budde (1980): Compri- eine computergestützte Planung risikobehafteter Investitionen. Göttinger Schriften zur Agrarökonomie 47.

Brandes, Wilhelm \& Martin Odening (1992): Investition, Finanzierung und Wachstum in der Landwirtschaft. Stuttgart: Ulmer.

Brandes, Wilhelm (1985): Über die Grenzen der Schreibtischökonomie. Tübingen: Mohr.

Brandes, Wilhelm (1989): Überlegungen und Modellrechnungen zum natürlichen Selektionsargument. Diskussionsbeitrag 8905, Institut für Agrarökonomie der Universität Göttingen. 
Brandes, Wilhelm (1990): Überlegungen und Modellrechnungen zur Fruchtbarkeit von Maximierungsprinzip und Gleichgewichtskonzept.

Diskussionsbeitrag 9001, Institut für Agrarökonomie der Universität Göttingen.

Brandes, Wilhelm, Guido Recke \& Thomas Berger (1997): Produktions- und Umweltökonomik. Band 1, Stuttgart: Ulmer.

Brandes, Wilhelm, Hans-.Joachm Budde \& Eberhard Sperling (1980): A computerized planning method for risky investments. European Review of Agricultural Economics 7, (2), S. 147-157.

Brandes, Wilhelm, Helmut Müller \& Hans-Peter Weikard (1990): Puten und Perlhühner. Jahrbuch für Nationalökonomie und Statistik 207, Heft 3, S. 139-205.

Bratley, P., B.L. Fox \& L.E Schrage (1987): A guide to simulation. 2. Aufl., New York.

Bronstein, I.N. \& K.A. Semendjajew (1991): Taschenbuch der Mathematik. 25. Aufla., Moskau: Nauka.

Brüderl, Josef, Peter Preisendoerfer \& Rolf Ziegler (1996): Der Erfolg neugegründeter Betriebe : eine empirische Studie zu den Chancen und Risiken von Unternehmensgründungen. Berlin: Duncker \& Humblot.

Buchner, R \& J. Weinreich (1971): Der Einfluß des Abschreibungsverfahrens auf die Kapazitätsveränderung im Zeitablauf beim Marx-Engels-Effekt. Zeitschrift für betriebswirtschaftliche Forschung 23, S. 454-466.

Budde, Hans-Joachim (1974): Optimale Anpassung der Schweineproduktion an zyklische und saisonale Preisbewegungen. Dissertation, Institut für Agrarökonomie der Universität Göttingen.

Burmester, Christian (1996): Existenzsicherung von Unternehmen bei unsicheren Erwartungen. Berlin: Duncker \& Humblot.

Buscher, Udo \& Folker Roland (1992): Fuzzy-Set-Modelle in simultanen Investitions- und Produktionsplanungen. Institut für Betriebswirtschaftliche Produktions- und Investitionsforschung der Universität Göttingen.

Carrol, G. R. (1983): A stochastic model of organizational mortality: Review and reanalysis. Social science research 12, S. 303-329.

Casti, John L. (1997): Can you trust it? On the reliability of computer simulation and the validity of models. Comolexity 2, S. 8-11.

Chatfield, Chris (1996): The analysis of time series. 5. Aufl., London: Chapman \& Hall. 
Chien, Ying I. \& Granett L. Bradford (1976): A sequential model of the farm firm growth process. American Journal of Agricultural Economics 58, S. 456465.

Colson, François, Alain Blogowski, Bernard Dechambre, Eduardo Chia, Didier Désarménien \& Bruno Dorin (1993): Prévenier les défaillances financières en agriculture. Cahiers d'économie et sociologie rurales 29 , S. 21-44.

Cox, D.R. \& D.V. Hinkley (1974): Theoretical Statistics. London: Chapman \& Hall.

Csáki, Csaba (1985): Simulation and Systems Analysis in Agriculture. Amsterdam: Elsevier.

Day, R.H. (1963): Recursive Programming and Production Response. Amsterdam.

Day, R.H. (1965): Probability distributions of field crop yields. Journal of Farm Economics 47, S. 713-741.

Day, R.H. (1982): The complex dynamics offarm growth. MRG Working Paper, Nr. 8205, University of Southern California, Los Angeles.

Day, R.H. (1987a): The evolving economy. European Journal of Operational Research 30, S. 251-257.

Day, R.H. (1987b): Economic development in the very long run. MRG Working Paper, Nr. 8732, University of Southern California, Los Angeles.

Day, R.H. (1989): Dynamic systems, adaption and economic evolution. MRG Working Paper, Nr. 8908, University of Southern California, Los Angeles.

Dillon, C.R., J.W. Mjelde \& B.A. McCarl (1989): Biopysical simulation in support of crop production decisions: A case study in the blackland region of Texas. South journal of agricultural economics 21, S. 73-86.

Dobson, Annette J. (1983): Introduction to statistical modelling. London: Chapman \& Hall.

Domschke, Wolfgang \& Andreas Drexl (1991): Einführung in Operations Research. 2. Aufl., Berlin: Springer.

Efron, Bradley \& Robert J. Tibshirani (1993): An introduction to the bootstrap. New York: Chapman \& Hall.

Efron, Bradley (1979): Bootstrap methods: another look on the jackknife. Annals of Statistics 7, S. 1-26.

Efron, Bradley (1982): The jackknife, the bootstrap, and other resampling plans. Philadelphia: Society of Industrial and Applied Mathematics.

Eiselt, Horst A. \& Helmut von Frajer (1977): Operations research handbook. Berlin: de Gruyter. 
Eisenführ, Franz \& Martin Weber (1993): Rationales Entscheiden. Berlin, Heidelberg: Springer.

Epps, Thomas W. (1978): Financial Risk and the Petersburger Paradox: Comment. Journal of Economic of Finance 33, Nr. 5, S. 1455-1456.

Ericscon, R. \& A. Pakes (1995): Markov-perfect industry dynamics. Review of economic studies 62, Nr. 10, S. 53-82.

Eurostat (1997): Auswirkungen einer weltweiten Liberalisierung der Märkte für Getreide, Ölsaaten und Hülsenfrüchte auf die Landwirtschaft der Europäischen Union. Ergebnisse wechselseitig verknüpfter Modelle auf verschiedenen Untersuchungsebenen.

Everding, Stefan (1996): Früherkennung von Kreditbetrug mit Hilfe bankmäßiger Kreditwürdigkeitsprüfungen. Hamburg: Kovac.

Ezekiel, M. (1938): The cobweb theorem. Quarterly journal of economics 52, S. 255280.

Feichtinger, Gustav \& Michael Kopel (1994): Nichtlineare dynamische Systeme und Chaos: Neue Impulse für die Betriebswirtschaftslehre? Zeitschrift für Betriebswirtschaft 64, Heft 1, S. 7-34.

Ferschl, Franz (1997): Stochastische Prozesse in der Ökonomie. $H d W W 7$, Stuttgart: Fischer.

Firor, John (1993): Herausforderung Weltklima: Ozonloch, globale Erwärmung und saurer Regen. Heidelberg: Spektrum.

Fischer, Klaus (1988): Oligopolistische Marktprozesse: Einsatz verschiedener PreisMengen-Strategien unter Berücksichtigung von Nachfrageträgheit. Heidelberg. Physica.

Fisz, Marek (1965): Wahrscheinlichkeitstheorie und mathematische Statistik. 1965,3. Aufl., Leipzig: VEB Deutscher Verlag der Wissenschaften.

Flusser, Vilém (1992): Mit dem Zufall gegen den Zufall spielen. Universitas Heft 12, S. 1164-1169.

Förschle, Gerhard \& Manfred Kropp (1995): Unternehensfinanzierung. 2. Aufl., Bonn: Economica.

Förstner, K., G. Bamberg \& R. Henn (1973): Einführung in die Wahrscheinlichkeitsrechnung. Meisenheim am Glan: Anton Hain.

Foster, John (1998): Competition, competitiv selection and economic evolution. Working Paper, Max-Planck-Institut zur Erforschung von Wirtschaftssystemen, Jena.

Friedman, Milton (1953): Essays in positive economics. Chicago: University Press. 
Fuchs, Clemens. (1988): Regionalvergleich, Preisprognosen und Strategiemodelle zur Wirtschaftlichkeit der Schweineproduktion in der Bundesrepublik Deutschland. Dissertation am Institut für Landwirtschaftliche Betriebslehre der Universität Hohenheim. Frankfurt am Main: Strothe.

Gabisch, Günter \& Hans-Walter Lorenz (1987): Business Cycle Theory - a Survey of Methods and Concepts. Berlin: Springer.

Gabler (1988): Wirtschafts-Lexikon. 12. Aufl., Wiesbaden: Gabler.

Gajanana, T. M. (1994): Farm planning under risk - motad approach. Indian journal of economics 75, S. 93-110.

Gal, Tomas (Hg.) (1991): Grundlagen des Operations Research. 3. Aufl., Band 1, Berlin: Springer.

Gaylord, Richard J. \& Kazume Nishidate (1996): Modelling nature: cellular automata simulations with Mathematica. New York: Springer.

Gerybadze, Alexander (1982): Inovation, Wettbewerb und Evolution. Tübingen: Mohr.

Gibbons, Robert (1992): Game theory for appied economists. Princeton: University Press.

Goodwin, R.M. (1947): Dynamical coupling with especial reference to markets having production lags. Econometrica 15, S. 181-204.

Green, William H. (1993): Econometric Analysis. Englewood Cliffs: Prentice-Hall.

Grochla, E. \& N. Szyperski (1973): Modell- und computergestützte Unternehmensplanung. Wiesbaden.

Groos, Stefan (1994): Entwicklung eines Entscheidungs-Unterstützungs-Systems für den Investitions- und Liquiditätsbereich landwirtschaftlicher Unternehmen. JLU-Gießen, Forschungsberichte der Agrarinformatik Nr. 3.

Großkopf, Werner (1973): Die Bewertung des landwirtschaftlichen Betriebes mit Hilfe der Simulation. Berichte über Landwirtschaft 51, Heft 1, Hamburg \& Berlin: Parey, S. 114-123.

Günther, Hans-Otto \& Manfred Gronalt (1994): Computergestützte Entscheidungsanalyse eines Kapazitätserweiterungsprojektes. WiSt 23, Heft 11, S. 643-478.

Güth, Werner (1998): Are rational cost expectation evolutionary stable? ifo Studien 44, S. 1-14.

Haas, Gisela (1997): Mit Finanzstrategien die Zukunft als Landwirt sichern. Ernährungsdienst, 12.3.97, S. 4. 
Haegert, Lutz (1970): Die Aussagefähigkeit der Dualvariablen und wirtschaftliche Deutung der Optimalitätsbedingungen beim Chance-Constraint Programming. In: Hax, Herbert (Hg.): Entscheidungen bei unsicheren Erwartungen S. 101-128. Köln/Opladen.

Haen, Hartwig de (1971): Dynamisches Regionalmodell der Produktion und Investition in der Landwirtschaft. Agrarwirtschaft, Sonderheft 43. Hannover: Strothe.

Haen, Hartwig de (1973): Landwirtschaftliche Strukturprojektionen mit Hilfe von Simulationsmodellen. Agrarwirtschaft 22, S. 157-173.

Hanau, Arthur (1930): Die Prognose der Schweinepreise. Vierteljahreshefte zur Konjunkturforschung, Sonderheft 18.

Hanau, Arthur (1956): Schweinezyklus. HdSW 9, Stuttgart: 171-173.

Hanf (1986): Entscheidungslehre. München: R. Oldenbourg.

Hanf, C.-H. \& G. Schiefer (1982): Introduction to planing and decision models. Arbeitsbericht 82/2, Institut für landwirtschaftliche Betriebs- und Arbeitslehre, Kiel.

Hanf, Claus Henning (1996): Beziehungen zwischen Haushalt und Betrieb in der Landwirtschaft. In: Hagedorn, Konrad (Hg.): Institutioneller Wandel und Politische Ökonomie von Landwirtschaft und Agrarpolitik S. 47-66.

Hardach, F.W. \& Karl Hax (1958): Der Geltungsbereich des Kapazitätserweiterungs-Effektes. Zeitschrift für handelswissenschaftliche Forschung, Neue Folge 10, S. 530-545.

Harpain, H.H. (1989): Zur Abschätzung von Unternehmensentwicklungen mittels Systemsimulation. In: Schriften der Gesellschaft für Wirtschafts- und Sozilwissenschaften des Landbaues e.V (Hg.): Neuere Forschungskonzepte und-methoden in den Wirtschafts- und Sozialwissenschaften des Landbaues 25, Landwirtschaftsverlag Münster Hiltrup, S. 371-375.

Harsany, John C. (1995): Games with incomplete information. American Economic Review 85, Nr. 3, S. 291- 303.

Hauser, Martin (1989): Früherkennung von Krisen industrieller Unternehmungen durch Portfolio-Analyse. Freiburg (Breisgau): Hochschulverlag.

Hax, Herbert (1970): Investitionsentscheidungen bei unsicheren Erwartungen. In: Hax, H. (Hg.): Entscheidungen bei unsicheren Erwartungen, S. 129-140. Köln: Westdeutscher Verlag. 
Hax, Karl (1958): Die Bedeutung der betrieblichen Abschreibungs- und Investitionspolitik für das wirtschaftliche Wachstum der modernen Industriestaaten. Zeitschrift für handelswissenschaftliche Forschung, Neue Folge 10, S. 247-257.

Hazel, P. B. R. \& R. D. Norton (1986): Mathematical Programming for economic analysis in agriculture. New York: MacMillan Publishing Company.

Hazel, Peter B. R. (1971): A linear alternative to quadratic and semivariance programming for farm planning under uncertainty. American Journal of Agricultural Economics 53, S. 53-62.

Heithues, T. (1966): A recursive programming model of farm growth in Northern Germany. Journal of Farm Economics 48, S. 668-684.

Hensche, Hans-Ulrich, Martin Spielhoff \& Harald Vogt (1998):

Schweinefleischproduktion im Jahr 2000 und 2010 - Szenarien und zukunftsrobuste Strategien. Forschungsberichte des Fachbereichs Soest, Universität-Gesamthochschule Paderborn.

Hillier, Frederik S. \& Gerald. J. Liebermann (1988): Operations research. 4. Auflage, München: Oldenbourg.

Hillmann, Axel (1998): PC-Simulation einer offenen Volkswirtschaft. WiSt 27, Heft 3 , S. 137-144.

Hinners-Tobrägel, Ludger \& Wilhelm Brandes (1997): Das Planspiel "Wachsen oder Weichen". Zeitschrift für Agrarinformatik 5 S. 88-96.

Hinners-Tobrägel, Ludger \& Wilhelm Brandes (1997): Einübung in Produktionsund Spieltheorie: Das Planspiel "Wachsen oder Weichen". Agrarwirtschaft 46, S. 309-313.

Hinners-Tobrägel, Ludger (1993): Einige Simulationen mit dem l1-Schätzer für die lineare Einfachregression. Diplomarbeit, Institut für Agrarökonomie der Universität Göttingen.

Hirschauer, Norbert \& Albert Gierend (1998): Risiken im Plan berücksichtigen. Neue Landwirtschaft, Heft 7, S. 14-19.

Holzapfel, Ralph (1997): Standortplanung mit einem Fuzzy Entscheidungsansatz. Dissertation, Institut für Agrarökonomie, Universität Göttingen.

Hubig, Christoph (1994): Das Risiko des Risikos. Universitas 49, Heft 4, S. 310-318.

Hüls, Dagmar (1995): Früherkennung insolvenzgefährdender Unternehmen. Düsseldorf: IDW.

Illing, Gerhard (1995): Spieltheorie in den Wirtschaftswissenschaften. WiSt 24, Heft 10, S. 509-515.

Intrilligator, Michael D. (1971): Mathematical Optimazation and Economic Theory. Englewood Cliffs, N.J.: Prentice-Hall. 
Isermeyer, F., Fasterding, F., Heitmann, D., Hemme, T., Hinrichs, P., Kleinhanß, W., Manegold, D. \& Schefski, A. (1996): Software Use in the FAL ,Model Family“. Arbeitsbericht der Gruppe "Daten und Modelle" der Bundesforschungsanstalt für Landwirtschaft Braunschweig-Völkenrode (FAL).

Iwai, Katsuhito (1984): Schumpetrian dynamics. An evolutionary model of innovation and imitiation. Journal of Economic Behavior and Organization 5, North-Holland, S. 159-190.

Jacobs, A. (1998): Paralleler Einsatz von Betriebs- und Sektormodellen. Dissertation in Vorbereitung, Braunschweig (FAL).

Jaspersen, Thomas (1997): Investition: computergestützte Verfahren und Controlling im Investitionsproze $\beta$. München: Oldenbourg.

Javanovic, Boyan (1982): Selection and the evolution of industries. Econometrica 50, S. 649-670.

Jerger, Jürgen (1992): Das St. Petersburger Paradoxon. WiSt 21, Heft 8, S. 407-410.

Johnson, S.L., R.M. Adams \& G.M. Perry (1991): The on-fram costs of reducing groundwater pollution. American Journal of Agricultural Economics 73, S. 1063-1073.

Johnson, S.R. \& Gordon C. Rausser (1977): System Analysis and simulation: a survey of applications in agricultural and ressource economics. In: Martin, Lee R. (Hg.), A survey of agricultural economics literature, Bd. 2, Mineapolis.

Judge, G.G., W.E. Griffiths, R.C. Hill, H. Lütkepohl \& T.-C. Lee (1985): The theory and practise of econometrics. 2. Aufl., New York: Wiley.

Judge, G.G., W.E. Griffiths, R.C. Hill, H. Lütkepohl \& T.-C. Lee (1988):

Introduction to the theory and practise of econometrics. 2. Aufl., New York: Wiley.

Kalai, Ehud (1996): Games, Computers, and O.R. Proceedings of the Seventh Annual ACM-SIAM Symposium on Discrete Algorithms, S. 468-473. Über Internet verfügbar über http://www.kellogg.nwu.edu/research/math/Down-papers.htm (16.4.98).

Kellog, R.L., J.E. Jr. Easley \& T. Johnson (1988): Optimal timing of harvest for the North Carolina bay scallop fishery. American Journal of Agricultural Economics 70, S. 50-62.

Kendall, Maurice George \& Alan Stuart (1977): The advanced theory of statistics, Band 1. 4. Aufl., London: Charles Griffin \& company limited.

Kienle, Richard von (o.J.): Fremdwörterlexikon. München: Keyser. 
King, R.P., D.W. Lybecker, A. Regmi \& S.M. Swinton (1993): Bioeconomic models of crop production systems: design, development and use. Review of economic studies 15, S. 389-401.

Kleinhanß, W. (1998): Modellverbund in der FAL. Schriftenreihe der Bundesforschungsanstalt für Landwirtschaft Braunschweig-Völkenrode.

Kofler, Eduard (1989): Prognosen und Stabilität bei unvollständiger Information. Frankfurt a.M.: Campus.

Köhne, Manfred (1966): Theorie der Investition in der Landwirtschaft. Berichte über Landwirtschaft, 182. Sonderheft. München, Berlin: Parey.

Köhne, Manfred (1968): Die Verwendung der linearen Programmierung zur Betriebsentwicklungsplanung in der Landwirtschaft. Agrarwirtschaft, Sonderheft 25. Hannover: Strothe.

Kopel, Michael \& Gustav Feichtinger (1995): Rationalität und komplexes Verhalten. Zeitschrift für betriebswirtschafltiche Forschung 47, Heft 6, S. 545-557.

Kopel, Michael (1996): Kontrolliertes Chaos: Ein Ausweg aus der Unternehmenskrise. Zeitschrift für Betriebswirtschaft 66, Heft 4, S. 487503.

Krekó, Béla (1974): Optimierung, nichlineare Modelle. Berlin: VEB Deutscher Verlag der Wissenschaften.

Kreyszig, Erwin (1975): Statistischen Methoden und ihre Anwendung. 2. Aufl., Göttingen: Vandenhoeck \& Ruprecht.

Kromphardt, J. (1982): Wirtschaftswissenschaft - II. Methoden und Theorienbildung in der Volkswirtschaftslehre. HdWW Bd. 9, S. 904-936.

Kruschwitz, Lutz (1995): Finanzierung und Investition. Berlin; New York: de Gruyter.

Kruschwitz, Lutz (1995a): Investitionsrechnung. 6. erw. Aufl. - Berlin; New York: de Gruyter.

Kühl, Jens (1992): Analyse langfristiger Entwicklungspfade und Entwicklungsstrategien landwirtschaftlicher Betriebe. Dissertation an der agrarwissenschaftlichen Fakultät der Christian-Albrechts-Universität Kiel.

Kühnberger, Manfred, Peter Eckstein \& Martina Woithe (1996): Die Diskriminanzanalyse als ein Instrument zur Früherkennung negativer Unternehmensentwicklungen. Zeitschrift für Betriebswirtschaft 66, Heft 12, S. 1449-1464. 
Küpper, Hans-Ulrich (1995): Unternehmhensplanung und -steuerung mit pagatorischen oder kalkulatorischen Erfolgsrechnungen? Zeitschrift für betriegbswirtschaftliche Forschung Sonderheft 34, S. 19-50.

Kuratorium für Technik und Bauwesen in der Landwirtschaft e.V. (Hg.) (1996): KTBL-Taschenbuch Landwirtschaft. 18. Aufl. Münster-Hiltrup: Landwirtschaftsverlag.

Kurz, Heiko (1991): Vergleichende Beurteilung von Ansätzen zur Berücksichtigung des Risikos in linearen Betriebsplanungsmodellen. Diplomarbeit am Institut für Agrarökonomie der Universität Göttingen.

Landwirtschaftskammer Hannover (Hg.) (1995): Richtwert-Deckungsbeiträge.

Lange, C. \&W. Lorenz (1993): Computergestützte Lehre am Beispiel des IS-LM-ZZDiagramms. Jahrbücher für Nationalökonomie und Statistik 212/1-2, S. 88-104.

Lange, C. \&W. Lorenz (1995): IS-LM-Analyse auf dem PC. WiSt 24, Heft 11, S. 593-596.

Larson, Harold J. (1969): Introduction to probability theory and statistical inference. Monterey: Wiley.

Law, A.M. \& W.D. Kelton (1992): Simulation modelling and analysis. 2. Aufl., New York: McGraw-Hill.

Leatham, David J., Greogry M. Perry, M. Edward Rister \& James W.

Richardson (1986): Farm survival and performance under alternative financial conditions and credit policies. Agribusiness 2, Nr. 3, S. 321337.

Lentz, Wolfgang (1993): Numerische Optimierungsverfahren und ihre Verwendung in dynamischen Modellen. In: Berg, Ernst \& Friedrich Kuhlmann (Hg.): Systemanalyse und Simulation, S. 269-338. Stuttgart: Ulmer.

Linsmeier, Thomas J. \& Neil D. Pearson (1996): Risk management: an introduction to Value at Risk. Working paper, Univerity of Illinois at UrbanaChampaign.

Litwin, Ulrike (1986): Zum optimalen Informationsaufwand in der Landwirtschaft. Dissertation, Institut für Agarökonomie der Universität Göttingen.

Lohmann, Karl \& Peter Rühmann (1989): Marktverzinsung und Erhaltungskonzeptionen bei abnutzbaren Anlagegegenständen. ZfB 59, Heft 12.

Lohmann, Karl (1991): Kapazitäts- und Finanzierungswirkungen von Investitionen in abnutzbare Anlagegegenstände. In: Lücke, Wolfgang \& Klaus Schulz (Hg.): Standort Deutschland: Personal, Investition, Internationalität, Wiesbaden: Gabler. 
Lohmann, M. (1949): Abschreibungen, was sie sind und was sie nicht sind. Der Wirtschaftsprüfer 2, S. 253-257.

Maccarl, Bruce (1989): Linear approximation using motad and separable programming: should it be done? American Journal of Agricultural Economics 71, S. 158.

Maleka, P. (1993): An application of Target MOTAD Model to crop production in Zambia. Agricultural economics 9, S. 15-36.

Markowitz, H.M. (1959): Portfolio Selection. New York: Wiley.

Markowitz, H.M. (1977): An algorithm for finding undominated portfolios. In: Levy, Haim \& Marshall Sarnat (Hg.): Financial decision making under uncertainty, New York: Academic Press.

Meadows, Dennis; Donella Meadows, Erich Zahn \& Peter Milling (1973): Die Grenzen des Wachstums: Bericht des Club of Rome zur Lage der Menschheit. Reinbeck: Rowohlt.

Meadows, Donella; Dennis Meadows \& JØrgen Randers (1993): Die neuen Grenzen des Wachstums. 7. Aufl., Stuttgart: DVA.

Menger, Karl (1934): Das Unsicherheitsmoment in der Wertlehre. Zeitschrift für Nationalökonomie 51, S. 459-485.

Menger, Karl (1967): The role of Uncertainty in Economics. In: Shubik, Martin (Hg.): Essays in mathematical economics, S. 211-231.

Miranda, Mario J. and Joseph W. Glauber (1993): Intraseason demand for fall potatoes under rational expectations. Journal of Agricultural Economics 75, S. 104-112.

Montmort, Pierre Rémond de (1708): Essai d'analyse sur les jeux de hazard. Paris.

Muchow, Russel C. \& Jennifer A. Bellamy (Hg.) (1991): Climatic Risk in Crop Production: Models and Management for the Semiarid Tropics and Subtropics. Wallingford: CAP.

Müller, R. (1976): Zur Analyse der Agrarstrukturentwicklung einer Kleinregion mit einem Simulationsmodell. Agrarwirtschaft, Sonderheft 68. Hannover: Strothe.

Müller, R.A.E. (1977): Regionale Flächen-, Ertrags- und Produktionsvorausschätzung für die Bundesrepublik Deutschland. Dissertation, Kiel.

Müller, Ralf (1992): Finanzwirtschaftlilche Beurteilung landwirtschaftlicher Unternehmen bei der Kreditvergabe. Dissertation am Fachbereich Agrarwissenschaften der Universität Gießen.

Muth, John F. (1996): Rational expectations and the theory of price movements. Econometrica 29, Heft 3, S. 315-335. 
Naylor, T.H.; J.L. Balintfy; D.S. Burdick \& Kong Chu (1966): Computer Simulation Techniques. New York.

Nelson, Richard R. \& Sydney G. Winter (1982): An evolutionary theory of economic change. Cambridge: Belknap Press of Harvard University Press.

Nerlove, Marc (1958): Adaptaive expectations and cobweb phenomena. Quarterly Journal of Economics 73, Mai, S. 227-240.

Nerlove, Marc (1979): The dynamics of supply: retrospect and prospect. American Journal of Agricultural Economics 61, S. 874-888.

Nerlove, Marc (1983): Expectations, plans, and realizations in theory and practise. Econometrica 51, Heft 5, S. 1251-1279.

Nerlove, Marc (1993): Quasi-rational expectations, an alternative to fully rational expectations to U.S. beef cattle supply. Unveröffentlichtes Manuskript, ausgegeben bei einem Vortrag im Institut für Agrarökonomie der Universität Göttingen im Januar 1993.

Neubert, H. (1951): Die Anlagenfinanzierung aus Abschreibungen. Zeitschrift für handelswissenschaftliche Forschung, Neue Folge 3, S. 367-383 u. S. 415-423.

Neumair, Urs (1998): A general model of corporate failure and survival - A complexity theory approach. Dissertation, Bamberg.

Neumann, Klaus \& Morlock, Martin (1993): Operations Research. München: Hanser.

Neumann, Klaus (1975): Operations Research Verfahren. Band 1, München: Hanser.

Neumann, Manfred, J.M. (1979): Rationale Erwartungen in Makromodellen. Ein kritischer Überblick. Zeitschrift für Wirtschafts- und Sozialwissenschaften 99, Heft 4, Berlin: Duncker \& Humblot.

Nielsen, Lars Tyge (1992): The utility of infinite menus. Economics Letters 39, S. 4347.

Nippel, Peter (1994): Der optimale Investitionszeitpunkt unter Berücksichtigung zukünftigen technischen Fortschritts. WiSt 23, Heft 11, S. 594-596.

Noell, Christian (1996): Mehr Sicherheit oder Gewinn. DLG-Mitteilungen, Heft 12, S. 30-32.

o.V. (1998): Szenarien und Strategien für die Schweinefleischerzeugung. Agra-Europe 46, Nr. 13, Sonderbeilage.

Odening, Martin (1991): Zur Bestimmung des optimalen Verschuldungsgrades. Agrarwirtschaft 40, Heft 5, S. 148-153. 
Odening, Martin (1994): Früherkennung in landwirtschaftlichen Unternehmen. Vortrag im agrarökonomischen Kolloquium des Instituts für Agrarökonomie, Göttingen, im Sommersemester 1994.

Odening, Martin (1994): Komplexreduktion in Entscheidungsmodellen. Frankfurt a.M.: Lang.

Odening, Martin (1996): Estimating viability and competitivness of farms in transformation countries. Quarterly Journal of International Agriculture 35, Nr. 1.

Olfert, Klaus (1992): Investition. 5. Aufl., Ludwigshafen (Rhein): Kiehl.

Orcutt, Guy H. (1960): Simulation of economic systems. American Economic Review 50, Nr. 5, S. 893-907.

Oriade, Caleb A. \& Carl R. Dillon (1997): Developments in biopysical and bioeconomic simulation of agricultural systems: a review. Americal Journal of Agricultural Economics 73, S. 45-58.

Paetow, Holger (1997): Simulation dynamischer Marktprozesse mit Spreadsheets. WiSt 26, Heft 11, S. 587-591.

Pagenkopf, Jürgen (1981): Simulation. Handwörterbuch der Wirtschaftswissenschaft Bd. 6, S. 536-549.

Perridon, Louis \& Manfred Steiner (1995): Finanzwirtschaft der Unternehmung. 8. Aufl., München: Vahlen.

Peter, Gregor (1994): Eine Ermittlung der langfristigen Durchschnittskostenkurve von Marktfruchtbetrieben anhand des "economic engineering" Ansatzes. Dissertation, Institut für Agrarökonomie der Universität Göttingen.

Pinkwart, Andreas (1993): Katastrophentheorie und Unternehmenskrise - Der Beitrag der Katastrophentheorie zur Erklärung von Unternehmenskrise und -insolvenz. Zeitschrift für Betriebswirtschaft 63, Heft 9, S. 873-895.

Protil, Roberto Max (1997): Effizienz- und Risikoanalyse der Materiallogistik in landwirtschaftlichen Genossenschaften anhand eines Simulationsmodells. Göttingen: Cuvillier.

Quenouille, M. (1949): Approximate tests of correlation in time series. Journal of Royal Statistics Society B 11, S. 18-44.

Rasch, Dieter (1976): Einführung in die mathematische Statistik. Berlin: VEB Deutscher Verlag der Wissenschaften.

Reichling, Peter (1996): Performance: Glück oder Können? WiST 25, Heft 6, S. 286291.

Ribhegge, Hermann (1987): Grenzen der Theorie rationaler Erwartungen. Tübingen: Mohr. 
Richardson, J.W. \& C.J. Nixon (1986): Description of FLIPSIM V: a general firm level simulation model. Texas Agric. Exp. Station 49, S. 49ff. Zit. nach Oriade \& Dillon (1997), S. 49.

Rinne, Horst (1997): Taschenbuch der Statistik. 2. Aufl., Thun: Deutsch.

Rohatgi, Vijay K. (1984): Statistical Inference. New York: Wiley.

Ruchti, H. (1942): Die Bedeutung der Abschreibung für den Betrieb. Berlin.

Sachs, L. (1984): Angewandte Statistik. 6. Aufl., Berlin: Springer.

Salassi, M.E.; B.R. Deeleman \& J.G. Hamill (1987): Economic survivability of Mississippi rice farms: a deterministic simulation approach. South journal of agricultural economics 19, Heft 2, S. 163-173. Zit. nach Oriade \& Dillon (1997) S. 53.

Samuelsen, Paul A. (1977): St. Petersburg paradoxes: defanged, dissected, and historically described. Journal of Economic Literature 15, Nr. 1, S. 2455.

Sargent, Thomas J. (1987): Rational expectations. In: Eatwell, John, Murray Milgate und Peter Newman (Hg.): The New Palgrave. A dictionary of economics 4, London: MacMillan, New York: Stockton Press, Tokyo: Maruzen Company, S. 76-79.

SAS Institute (1990): SAS/Graph. Bd. 1.

Schanz, Günther (1988): Modell. In: Gabler Wirtschafts-Lexikon. 12. Aufl., S. 433f, Wiesbaden: Gabler.

Scheid, Harald (1992): Über den Zufall. Universitas 47, Heft 12, S. 1135-1144.

Schellberg, Bernhard (1994): Insolvenzprognosemodelle. Stuttgart: M \& P.

Schmitt, Günter (1988): Wie optimal ist eigentlich die ,optimale“ Betriebsgröße in der Landwirtschaft? Agrarwirtschaft 37, S. 234-245.

Schmitt, Günter (1992): Der Zusammenhang zwischen Organisationsform und Betriebsgröße in der Landwirtschaft: Eine institutionenökonomische Erklärung. Berichte über Landwirtschaft 70, S. 505-525.

Schmitt, Günter (1993): Haushalts- und Betriebsgröße in der Landwirtschaft: Zum Problem der optimalen Betriebsgröße und des optimalen Betriebgrößenwachstums. Berichte über Landwirtschaft 71, S. 377-398.

Schnabl, Herrmann (1988): Theoreme der Erwartungsbildung. WiSt 17, Heft 12, 620-624.

Schneeberger, Hans (1998): The bootstrap-method: Discussion and proposal for improvment. Jahrbücher für Nationalökonomie und Statistik 217, /1, S. 108-115. 
Schoppe, Siegfried (1995): Moderne Theorie der Unternehmung. München; Wien: Oldenbourg.

Schumpeter, Joseph A. (1965): Geschichte der ökonomischen Analyse. Erster Teilband, Göttingen: Vandenhook \& Ruprecht.

Scupin, Jörg \& Thomas Kühne (1998): Lohnt sich die Investition? Bauernzeitung 39, Heft 32, S. 12-14.

Selten, Reinhard (1990): Bounded rationality. Zeitschrift für die gesamte Staatswissenschaft (JITE) 146, S. 649-658.

Sennetti, John T. (1976): On Bernoulli, shape, financial risk and the St. Petersburger Paradox. Journal of Economic of Finance 31, Nr. 3, S. 960-962.

Shapley, Lloyd S. (1977): The St. Petersburger Paradox: A con game? Journal of Economic Theory 14, S. 439-442.

Shapley, Lloyd S. (1977a): Lotteries and menus: A comment on unbounded utilities. Journal of Economic Theory 14, S. 446-453.

Sheffrin, Steven M. (1983): Rational expectations. Cambridge: Cambridge university press.

Sigmund, Karl (1995): Games of life. London: Penguin.

Sinn, Hanns-Werner (1980): Ökonomische Entscheidungen bei Ungewißheit. Tübingen: Mohr.

Sommer, Louise (1954): Exposition of a new theory of measurment of risk. Econometrica 22, S. 23-36, Übersetzung eines Aufsatzes von Daniel Bernoulli (1738).

Sondergeld, Lutz \& Stefan Voß (1997): Optimierung mit dem Ameisensystem. WiSt 26, Heft 11, S. 568-573.

Springer, M.D. (1979): The Algebra of Random Variables. New York: Wiley.

Steinhauser, Hugo, Cay Langbehn \& Uwe Peters (1989): Einführung in die landwirtschaftliche Betriebslehre. Band 1: Allgemeiner Teil. 4. Aufl. Stuttgart: Ulmer.

Stigler, George J. (1958): The economies of scale. Journal of law and economics 1, S. 54-71.

Stinchcombe, A.L. (1965): Organizations and social structure. In: March, J.G. (Hg.): Handbook of organisation, S. 153-193, Chicago: Rand McNeally.

Sulewski, Travis, John Spriggs \& R.A. Schoney (1994): Agricultural producer price expectation. Canadian Journal of Agricultural Economics 42, S. 301310.

Szpiro, George G. (1997): The emergence of risk aversion. Complexity 2, Heft 2, S. 31-39. 
Thijssen, Geert (1996): Farmers' investment behavior: an empirical assessment of two specifications of expectations. American Journal of Agricultural Economics 78, Februar.

Tries, Bruno (1998): Kalibrierung ökonomischer Planungsmodelle mittels Fuzzy-LP. Landbauforschung Völkenrode, Heft 1 S. 35-37.

Tukey, J.W. (1958): Bias and confidence in not quite large samples. Annals of Mathematical Statistics 29, (Abstract) S. 614.

Tukey, J.W. (1972): Data analysis, computation and mathematics. Quarterly Journal of Applied Mathematics 33, S. 51-65.

Vester, Frederic (1991): Unsere Welt-ein vernetztes System. 7. Aufl., München: DTV.

Wagner, Joachim (1995): PC-Simulation eines Zwei-SektorenGleichgewichtsmodells. WiSt 24, Heft 2, S. 89-95.

Waldman, Michael (1994): Systematic errors and the theory of natural selection. American Economic Review 84, Heft 6.

Walker, Paul (1996): A chronology of game theory. Internet http://www.canterbury.ac.nz/econ/hist.htm (6.8.97, 16h44).

Weiber, Rolf \& Jost Adler (1995): Der Einsatz von Unsicherheitreduktionsstrategien im Kaufprozeß: Eine informationsökonomische Analyse. In: Kaas, Klaus P. (Hg.), Kontrakte, Geschäftsbeziehungen, Netzwerke - Marketing und Neue Institutionenökonomik, S. 61-78, Düsseldorf.

Weinschenck, G. (1977): Zur Anwendung der Systemforschung und der Simulation in der landwirtschaftlichen Sektoranalyse. Bericht 5/77 - Vorträge zum 3. Forschungskolloquium des Lehrstuhl für Wirtschaftslehre des Landbaus Kiel, S. 37-69.

Weiß, Christoph R. (1996): Exits from a declining sector: econometric evidence from a panel of upper-Austrian farms 1980-1990. Arbeitspapier Nr. 9601, Institut für Volkswirtschaftslehre, Universität Linz, Österreich.

Welge, M.K. \& A. Al-Laham (1997): Stand der strategischen Planungspraxis in der deutschen Industrie. Zeitschrift für betriebswirtschaftliche Forschung 49, Heft 9, S. 790-806.

Wholey, D. R., J. B. Christianson \& S. M. Sanchez (1992): Organisational size and failure among health maintenance organisations. American Sociological Review 57, S. 829-842, zit. nach Woywode (1998, S. 40) .

Wiesemann, Hans-Olaf (1994): Dynamische Optimierung. WiSt 23, Heft 7, S. 364368.

Winter, Sidney G. Jr. (1964): Economic "Natural Selection" and the theory of the firm. Yale Economic Essays 4, 1, 
Winter, Sydney G. (1987): Competition and selection. The new Palgrave Bd. 1, S. 545-548.

Winter, Sydney G. (1987a): Natural selection and evolution. The new Palgrave Bd. 3, S. 614-617.

Witt, Ulrich (1986): Firms' market behavior under imperfect information and economic natural selection. Journal of Economic Behavior and Organisation 7, North-Holland, S. 265-290.

Witt, Ulrich (1979): Marktprozesse: neoklassische versus evolutorische Theorie der Preis- und Mengendynamik. Athenaeum Oekonomie 3, Wirtschaftswissenschaftliche Dissertation, Göttingen.

Witt, Ulrich (1985): Individualistische Grundlagen der evolutorischen Ökonomik. Mannheim: Univ., Habil.-Schriften.

Witt, Ulrich (1994): Wirtschaft und Evolution. Einige neuere theoretische Entwicklungen. WiSt 23, Heft 10, 503-512.

Witte, Thomas, Jörg Frieder Deppe \& Axel Born (1975): Lineare Programmierung. Wiesbaden: Gabler.

Wittmann, Uli (1985): Das Konzept rationaler Preiserwartungen. Berlin: Springer.

Wöhe, Günter (1996): Einführung in die Allgemeine Betriebswirtschaftslehre. 19. Aufl., München: Vahlen.

Wossink, Ada (1990): Analysis of future agricultural change: a farm economics approach applied to Dutch arable farming. Proefschrift Wageningen.

Woywode, Michael (1998): Determinanten der Überlebenswahrscheinlichkeit von Unternehmen : eine empirische Überpruefung organisationstheoretischer und industrieökonomischer Erklärungsansaetze. Baden-Baden: Nomos.

Zwicker, Eckart (1981): Simulation und Analyse dynamischer Systeme in den Wirtschafts- und Sozialwissenschaften. Berlin: de Gryter. 



\section{Anhang}

9.1 Programm zum Petersburger Paradoxon ................................................... 330

9.2 Ableitung der Überlebenswahrscheinlichkeit für zwei Perioden für ein

Modell mit additiver stochastischer Einzahlung, Startkapital und fester

Auszahlung ........................................................................................... 331

9.3 Berechnung der Überlebenswahrscheinlichkeit für die zweite Runde

hach dem Bayes-Ansatz für unterschiedliche Startkapitale.........................331

9.4 Uberlebenswahrscheinlichkeit und Planungshorizont ................................. 333

9.5 Simulationsprogramm zur Bestimmung der

Uberlebenswahrscheinlichkeit für ein und zwei Runden .........................334

$9.6 \quad$ Einzelheiten des Betriebsentwicklungsmodells aus Kapitel 6....................335

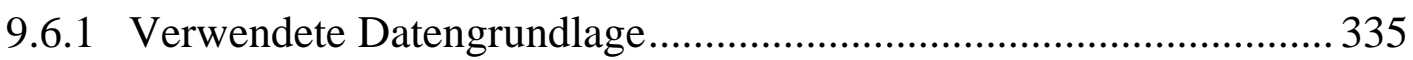

9.6.2 Beschreibung des LP-Tableaus................................................ 338

9.6.2.1 Komponenten der Zielfunktion .................................. 341

9.6.2.2 Liquiditätsrestriktionen (Liqui) ....................................... 345

p.6.2.3 Weitere Restriktionen und Kapazitäten .............................346

9.6.3 Fortschreibung des LP-Tableaus auf die nächste Periode ................ 349

9.6.3.1 Maschinenkapazität und Stallplätze zu Beginn einer

Periode ...................................................................... 349

\subsection{Berechnungsmethode A (verwendete}

Methode)..................................................... 350

9.6.3.1.2 Berechnungsmethode B .................................. 351

9.6.3.2 Fortschreibung des Maschinenzeitwertes:

Maschinenzeitwert zu Beginn der Periode $t$..................... 351

9.6.3.3 Vervollständigen des Saldos der Ein- und

Auszahlungen und Fortschreibung der Liquidität ..............352

9.6.3.4 Fortschreibung des Fremdkapitals und des totalen

Kapitalsaldos.......................................................... 353

0.6.3.5 $\quad$ Bestimmung des gesamten Zinssaldos ...............................356 
9.6.3.6 $\quad$ Fortschreibung der Kreditobergrenzen.............................356

9.6.3.7 Eigenkapital ........................................................ 357

9.6.3.8 $\quad$ Bestimmung des Gewinns ............................................ 357

\subsubsection{Zusammenfassung von Kennziffern zur}

Betriebsbeschreibung....................................................... 358

9.6 .4 Das Simulationsprogramm.......................................................... 361

9.6 .5 Der Ergebnisausdruck ............................................................. 380

9.6.6 Posterdarstellungen der Ergebnisse des

Betriebsentwicklungsmodells aus Kapitel 6 .................................. 388

\subsection{Programm zum Petersburger Paradoxon}

Das nachfolgende Listing enthält den Code eines Programms, geschrieben in der Sprache REXX, mit dem der Spielverlauf des Petersburger Paradoxons, das in Abschnitt 4.5 beschrieben ist, simuliert werden kann.

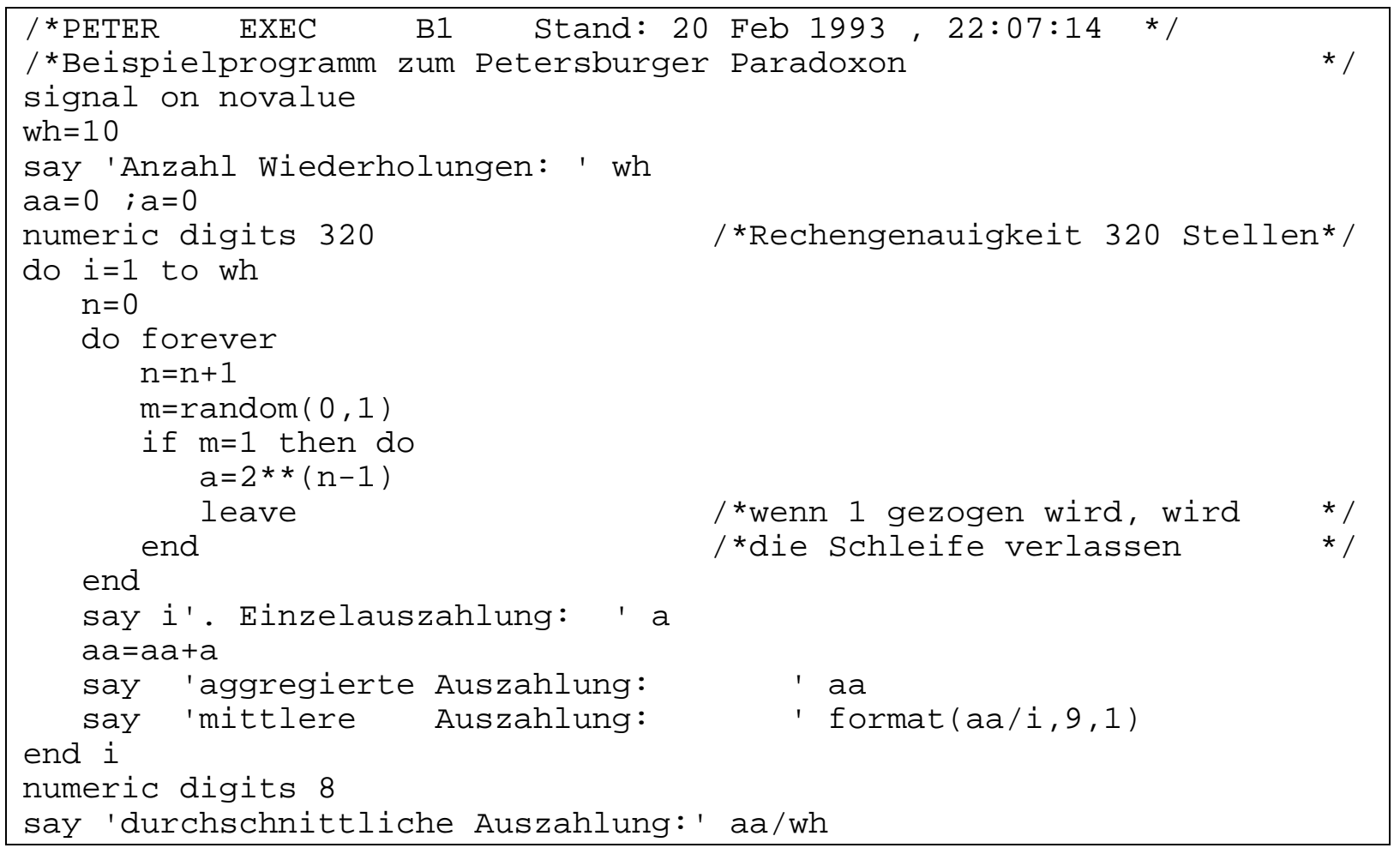




\subsection{Ableitung der Überlebenswahrscheinlichkeit für zwei Perioden für ein Modell mit additiver stochastischer Einzahlung, Startkapital und fester Auszahlung}

In diesem Abschnitt wird die Formel 2042] aus Abschnitt5.1 hergeleitet. Sie gibt die Wahrscheinlichkeit an, daß die endogene Variable $K$ in dem dynamischen System $K_{t}=$ $K_{t-1}+z_{t}-a$ sich in den Perioden eins und zwei größer als Null realisiert. $z_{t}$ wird als stochastische Einzahlung, $a$ als konstante Auszahlung und $K_{t}$ als Kapitalbestand aufgefaßt.

$$
\begin{array}{ll}
K_{1} & =K_{0}+z_{1}-a \\
\mathrm{p}\left(K_{1}>0\right) & =\mathrm{p}\left(z_{1}>-K_{0}+a\right)=1-F_{z 1}\left(-K_{0}+a\right) \\
K_{2} & =K_{1}+z_{2}-a=K_{0}-2 a+z_{1}+z_{2} \\
\mathrm{p}_{\mathrm{uil}}\left(K_{2}>0\right) & =\mathrm{p}\left[\left(K_{1}>0\right) \wedge\left(K_{2}>0\right)\right] \\
\mathrm{p}\left(K_{2}>0\right) & =\mathrm{p}\left(K_{0}-2 a+z_{1}+z_{2}>0\right)=\mathrm{p}\left(z_{2}>-z_{1}-K_{0}+2 a\right) \\
& =\int_{z_{1}} \mathrm{p}\left(z_{2}>-z_{1}-K_{0}+2 a \mid z_{1}\right) \cdot \mathrm{f}\left(z_{1}\right) \cdot \mathrm{d} z_{1} \\
& =\int_{z_{1}}\left[1-\mathrm{F}_{z_{2}}\left(-z_{1}-K_{0}+2 a\right)\right] \cdot \mathrm{f}\left(z_{1}\right) \cdot \mathrm{d} z_{1} \\
\mathrm{p}_{\mathrm{ül}}\left(K_{2}>0\right) & =\int_{z_{1}>-K_{0}+a}\left[1-\mathrm{F}_{z_{2}}\left(-z_{1}-K_{0}+2 a\right)\right] \cdot \mathrm{f}\left(z_{1}\right) \cdot \mathrm{d} z_{1}
\end{array}
$$

Für symmetrische Verteilungen vereinfacht sich die Formel analog zu (204.3):

$$
\mathrm{p}_{\mathrm{uil}}\left(K_{2}>0\right)=\int_{z_{1}>-K_{0}+a} \mathrm{~F}\left(z_{1}+K_{0}-2 a\right) \mathrm{f}\left(z_{1}\right) \cdot \mathrm{d} z_{1}
$$

\subsection{Berechnung der Überlebenswahrscheinlichkeit für die zweite Runde nach dem Bayes-Ansatz für unterschiedliche Startkapitale}

Dieser Abschnitt dient der Einzelberechnung der Überlebenswahrscheinlichkeit für zwei Perioden. Die Ergebnisse sind in Tabelle 224.1 bereits zusammengefaßt dargestellt. Die Berechnung erfolgt nach 223,1. 
Tabelle 332.1: Berechnung der Überlebenswahrscheinlichkeit für die zweite Runde nach dem Bayes-Ansatz, $K_{0}=-1$

\begin{tabular}{|c|c|c|c|c|c|}
\hline$i$ & $z_{1 i}$ & $\mathrm{p}\left(z_{1 i}\right)$ & $-\left(z_{1 i}+K_{0}\right)$ & $\mathrm{p}\left(z_{2}>-\mathrm{Z}_{1}-K_{0}\right)$ & $\mathrm{p}\left(z_{2}>-z_{1 i}-K_{0}\right) \cdot \mathrm{p}\left(\mathrm{z}_{1 i}\right)$ \\
\hline 1 & -2 & $1 / 5$ & 3 & 0 & 0 \\
\hline 2 & -1 & $1 / 5$ & 2 & 0 & 0 \\
\hline 3 & 0 & $1 / 5$ & 1 & $1 / 5$ & $1 / 25$ \\
\hline 4 & 1 & $1 / 5$ & 0 & $2 / 5$ & $2 / 25$ \\
\hline 5 & 2 & $1 / 5$ & -1 & $3 / 5$ & $3 / 25$ \\
\hline Summen: & $\begin{array}{c}\sum_{z_{1 i}} \\
\sum_{z_{1 i}>-K_{0}}\end{array}$ & \multicolumn{3}{|c|}{ (nicht schraffierter Bereich) } & $6 / 25$ \\
\hline \multicolumn{6}{|c|}{$K_{1}=K_{0}+z_{1}, K_{2}=K_{0}+z_{1}+z_{2}, z_{i} \sim \operatorname{uiid}\{-2,-1,0,1,2\}$} \\
\hline
\end{tabular}

Tabelle 332.2: Berechnung der Überlebenswahrscheinlichkeit für die zweite Runde nach dem Bayes-Ansatz, $K_{0}=2$

\begin{tabular}{|c|c|c|c|c|c|}
\hline$i$ & $z_{1 i}$ & $\mathrm{p}\left(z_{1 i}\right)$ & $-\left(z_{1 i}+K_{0}\right)$ & $\mathrm{p}\left(z_{2}>-\mathrm{z}_{1 i}-K_{0}\right)$ & $\mathrm{p}\left(z_{2}>-z_{1 i}-K_{0}\right) \cdot \mathrm{p}\left(\mathrm{z}_{1 i}\right)$ \\
\hline 1 & -2 & $1 / 5$ & 0 & $2 / 5$ & $2 / 25$ \\
\hline 2 & -1 & $1 / 5$ & -1 & $3 / 5$ & $3 / 25$ \\
\hline 3 & 0 & $1 / 5$ & -2 & $4 / 5$ & $4 / 25$ \\
\hline 4 & 1 & $1 / 5$ & -3 & 1 & $5 / 25$ \\
\hline 5 & 2 & $1 / 5$ & -4 & 1 & $5 / 25$ \\
\hline Summen: & $\sum_{z_{1 i}}$ & \multirow{2}{*}{\multicolumn{3}{|c|}{ (nicht schraffierter Bereich) }} & $19 / 25$ \\
\hline & $\sum_{z_{1 i}>-K_{0}}$ & & & & $17 / 25$ \\
\hline \multicolumn{6}{|c|}{$\mathrm{K}_{1}=\mathrm{K}_{0}+\mathrm{z}_{1}, \mathrm{~K}_{2}=\mathrm{K}_{0}+\mathrm{z}_{1}+\mathrm{z}_{2}, \mathrm{z}_{\mathrm{i}} \sim \operatorname{uiid}\{-2,-1,0,1,2\}$} \\
\hline
\end{tabular}


Tabelle 333.1: Berechnung der Überlebenswahrscheinlichkeit für die zweite Runde nach dem Bayes-Ansatz, $K_{0}=3$

\begin{tabular}{|c|c|c|c|c|c|}
\hline$i$ & $z_{1 i}$ & $\mathrm{p}\left(z_{1 i}\right)$ & $-\left(z_{1 i}+K_{0}\right)$ & $\mathrm{p}\left(z_{2}>-\mathrm{z}_{1}-K_{0}\right)$ & $\mathrm{p}\left(z_{2}>-z_{1 i}-K_{0}\right) \cdot \mathrm{p}\left(\mathrm{z}_{1 i}\right)$ \\
\hline 1 & -2 & $1 / 5$ & -1 & $3 / 5$ & $3 / 25$ \\
\hline 2 & -1 & $1 / 5$ & -2 & $4 / 5$ & $4 / 25$ \\
\hline 3 & 0 & $1 / 5$ & -3 & 1 & $5 / 25$ \\
\hline 4 & 1 & $1 / 5$ & -4 & 1 & $5 / 25$ \\
\hline 5 & 2 & $1 / 5$ & -5 & 1 & $5 / 25$ \\
\hline \multirow[t]{2}{*}{ Summen: } & $\sum_{z_{1 i}}$ & & & & $22 / 25$ \\
\hline & $\sum_{z_{1 i}>-K_{0}}$ & & $\sum_{z_{1 i}}$ & & $22 / 25$ \\
\hline \multicolumn{6}{|c|}{$\mathrm{K}_{1}=\mathrm{K}_{0}+\mathrm{z}_{1}, \mathrm{~K}_{2}=\mathrm{K}_{0}+\mathrm{z}_{1}+\mathrm{z}_{2}, \mathrm{z}_{\mathrm{i}} \sim \operatorname{uiid}\{-2,-1,0,1,2\}$} \\
\hline
\end{tabular}

Tabelle 333.2: Berechnung der Überlebenswahrscheinlichkeit für die zweite Runde nach dem Bayes-Ansatz, $K_{0}=4$

\begin{tabular}{|c|c|c|c|c|c|}
\hline $\mathrm{i}$ & $\mathrm{z}_{1 \mathrm{i}}$ & $\mathrm{p}\left(\mathrm{z}_{1 \mathrm{i}}\right)$ & $-\left(\mathrm{z}_{1 \mathrm{i}}+\mathrm{K}_{0}\right)$ & $\mathrm{p}\left(\mathrm{z}_{2}>-\mathrm{z}_{1 \mathrm{i}}-\mathrm{K}_{0}\right)$ & $\mathrm{p}\left(\mathrm{z}_{2}>-\mathrm{Z}_{1 \mathrm{i}}-\mathrm{K}_{0}\right) \cdot \mathrm{p}\left(\mathrm{z}_{1 \mathrm{i}}\right)$ \\
\hline 1 & -2 & $1 / 5$ & -2 & $4 / 5$ & $4 / 25$ \\
\hline 2 & -1 & $1 / 5$ & -3 & 1 & $5 / 25$ \\
\hline 3 & 0 & $1 / 5$ & -4 & 1 & $5 / 25$ \\
\hline 4 & 1 & $1 / 5$ & -5 & 1 & $5 / 25$ \\
\hline 5 & 2 & $1 / 5$ & -6 & 1 & $5 / 25$ \\
\hline \multirow[t]{2}{*}{ Summen: } & $\sum_{z_{1 i}}$ & & & & $24 / 25$ \\
\hline & $\sum_{z_{1 i}>-K_{0}}$ & & $\sum_{z_{1 i}}$ & & $24 / 25$ \\
\hline \multicolumn{6}{|c|}{$\mathrm{K}_{1}=\mathrm{K}_{0}+\mathrm{z}_{1}, \mathrm{~K}_{2}=\mathrm{K}_{0}+\mathrm{z}_{1}+\mathrm{z}_{2}, \mathrm{z}_{\mathrm{i}} \sim \operatorname{uiid}\{-2,-1,0,1,2\}$} \\
\hline
\end{tabular}

\section{4 Überlebenswahrscheinlichkeit und Planungshorizont}

Dieser Abschnitt ergänzt das einfache stochastische Betriebsentwicklungsmodell aus Abschnitt 5.5 um ein Szenario mit längerer Planungsdauer. Das nachfolgende Schaubild zeigt, daß die optimale Fremdkapitalaufnahme vom Planungshorizont abhängig ist. War bei vier Perioden (vgl. Schaubild 204 lichkeit mit einer Fremdkapitalaufnahme von 30 verbunden, so ist bei einem Planungshorizont von sechs Perioden eine verdoppelte Fremdkapitalaufnahme optimal. 
Schaubild 334.1: Anzahl überlebender Betriebe nach sechs Perioden und mittleres Eigenkapital

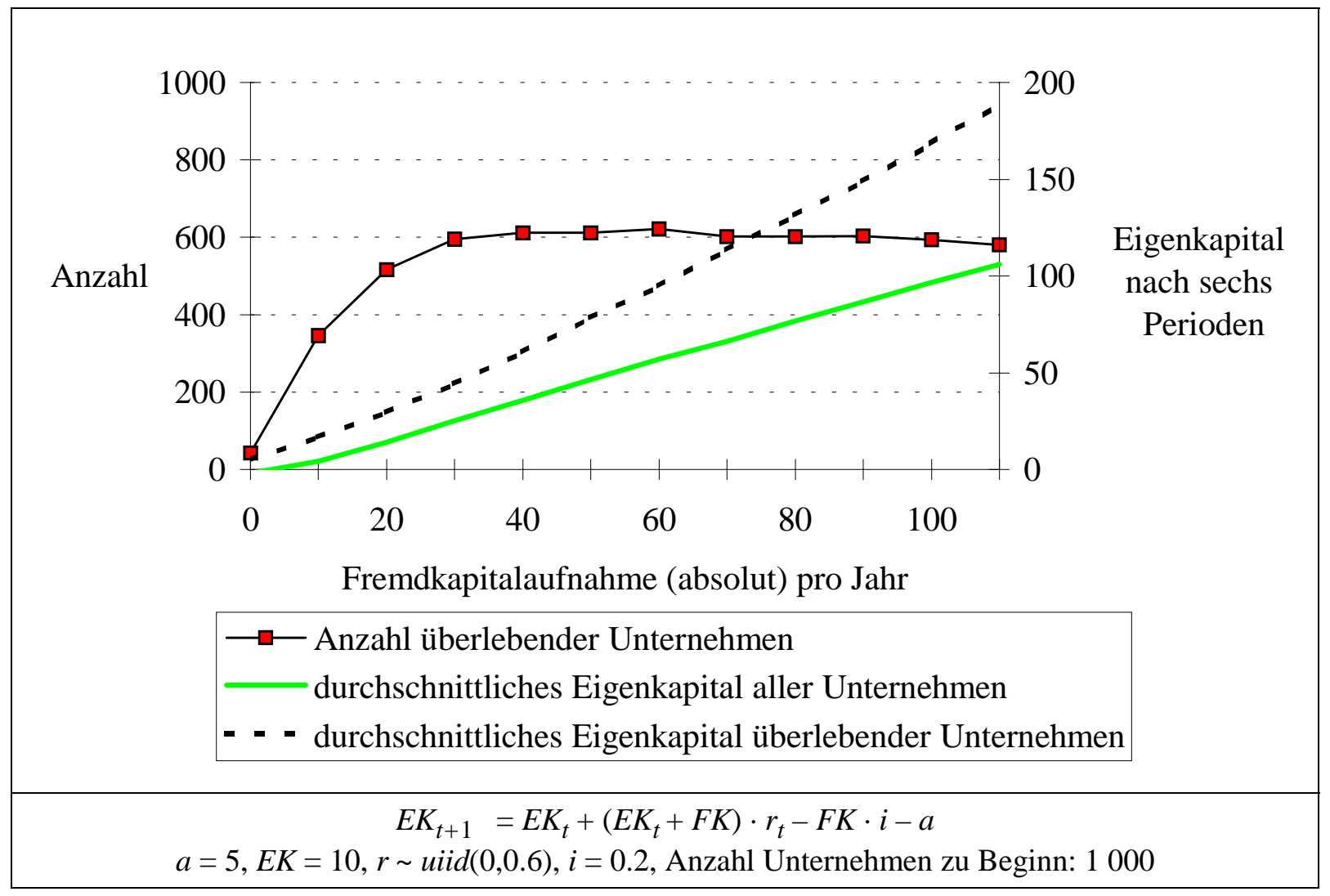

\subsection{Simulationsprogramm zur Bestimmung der Überlebens- wahrscheinlichkeit für ein und zwei Runden}

Vgl. Unterabschnitt 5.3.1.5

Die Simulationen wurden mit folgendem $S{ }^{\circledR}{ }^{\circledR}$-Programm durchgeführt:

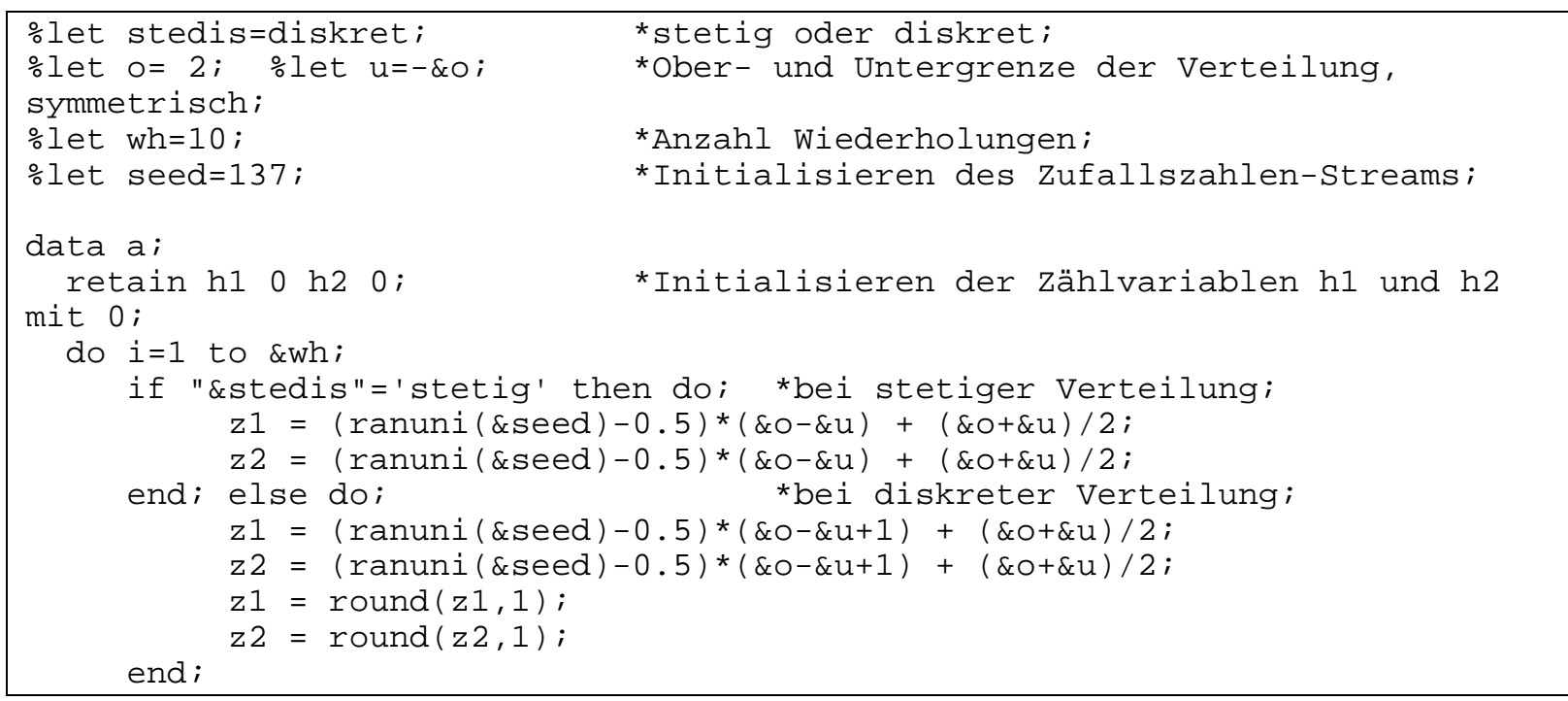




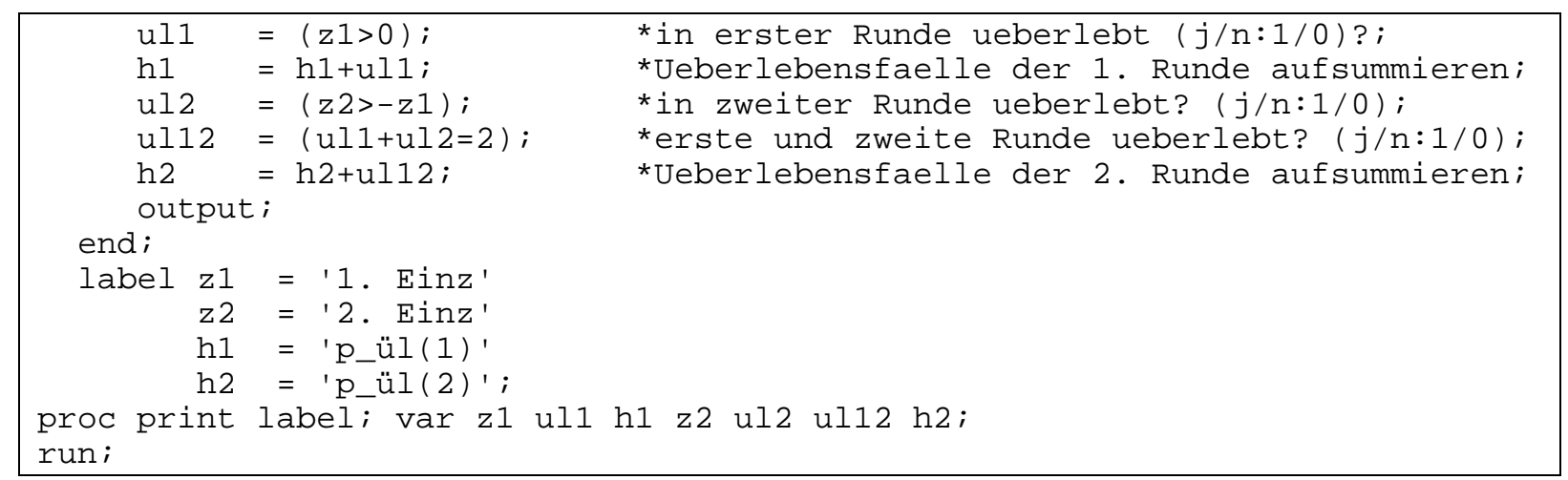

Dieses Programm liefert folgendes Ergebnis:

\begin{tabular}{|c|c|c|c|c|c|c|c|}
\hline \multicolumn{2}{|c|}{ stedis=diskret, } & \multicolumn{3}{|c|}{$u=-2, \quad o=2, \quad w h=10$} & \multirow[b]{2}{*}{ UL2 } & \multirow[b]{2}{*}{ UL12 } & \multirow[b]{2}{*}{ p_ül (2) } \\
\hline OBS & 1. Einz & UL1 & p_ül (1) & Einz & & & \\
\hline 1 & -1 & 0 & 0 & 2 & 1 & 0 & 0 \\
\hline 2 & 1 & 1 & 1 & 0 & 1 & 1 & 1 \\
\hline 3 & -1 & 0 & 1 & 1 & 0 & 0 & 1 \\
\hline 4 & -1 & 0 & 1 & 2 & 1 & 0 & 1 \\
\hline 5 & 2 & 1 & 2 & -2 & 0 & 0 & 1 \\
\hline 6 & -1 & 0 & 2 & 1 & 0 & 0 & 1 \\
\hline 7 & 2 & 1 & 3 & -1 & 1 & 1 & 2 \\
\hline 8 & -2 & 0 & 3 & 1 & 0 & 0 & 2 \\
\hline 9 & -1 & 0 & 3 & 0 & 0 & 0 & 2 \\
\hline 10 & 2 & 1 & 4 & -2 & 0 & 0 & 2 \\
\hline
\end{tabular}

In diesem Beispiel mit zehn Wiederholungen überleben $40 \%$ der Unternehmen die erste Runde (letzte Zeile, 4. Spalte). Ebenfalls in vier von zehn Fällen ist die zweite Einzahlung positiv (5. Spalte). Dennoch überleben nur $20 \%$ beide Runden (letzte Spalte).

\subsection{Einzelheiten des Betriebsentwicklungsmodells aus Kapitel 6}

Das Betriebsentwicklungsmodell ist in Kapitel 5 nur in groben Zügen skizziert worden. Seine ökonomischen und technischen Einzelheiten sind in diesem Abschnitt näher beschrieben: die Datengrundlage der verwendeten Produktionsverfahren, das LPTableau und die Fortschreibung des einperiodischen LPs auf die nächste Periode.

\subsubsection{Verwendete Datengrundlage}

Das Betriebsentwicklungsmodell enthält die Produktionsverfahren Pflanzenproduktion und Tierproduktion. Ihre Koeffizienten sind im wesentlichen den Richtwert-Dekkungsbeiträgen der Landwirtschaftskammer Hannover, abgekürzt mit RW-DB, und dem KTBL-Taschenbuch Landwirtschaft, Abkürzung KTBL, entnommen. In den ausgewiesenen Deckungs- und Gewinnbeiträgen sind keine Arbeitskosten enthalten. Kursive Zeilen kennzeichnen die Veränderungen gegenüber der Ausgangssituation. 
Tabelle 336.1: Arbeitswirtschaft

\begin{tabular}{|c|c|c|c|}
\hline Variable & Quelle & Wert & Kommentar \\
\hline Bruttoarbeitstage & & 261 & $365-52 \cdot 2$ \\
\hline $\begin{array}{l}\text { bezahlte Feiertage und } \\
\text { Urlaub }\end{array}$ & $\begin{array}{l}\text { KTBL, } \\
\text { S. } 55\end{array}$ & 31 & $12 \%$ der Bruttoarbeitstage \\
\hline Nettoarbeitstage & & 230 & eigene Berechnungen \\
\hline Nettoarbeitsstunden/AK/Jahr & & 1840 & 8 Stunden/Tag \\
\hline $\begin{array}{l}\text { Bruttolohn einschl. Lohn- } \\
\text { nebenkosten für Aushilfen }\end{array}$ & $\begin{array}{l}\text { KTBL, } \\
\text { S. } 55\end{array}$ & $27 \mathrm{DM} / \mathrm{h}$ & $\begin{array}{l}16.10 \mathrm{DM} / \mathrm{h}+43 \% \mathrm{Nebenkosten} \\
+16 \% \text { für Anleitung, schlechtere Arbeits- } \\
\text { qualität im Vergleich zu Fest-AK und unsi- } \\
\text { chere Verfügbarkeit }\end{array}$ \\
\hline Jahreskosten einer Voll-AK & & 48000 & $\begin{array}{l}261 \text { Arbeitstage } \cdot 8 \mathrm{~h} \cdot 16.10 \mathrm{DM} / \mathrm{h}+43 \% \\
\text { Lohnnebenkosten }\end{array}$ \\
\hline
\end{tabular}

Tabelle 336.2: Produktionsverfahren Pflanzenproduktion

\begin{tabular}{|c|c|c|c|}
\hline Variable & Quelle & Wert & Kommentar \\
\hline Ertrag & $\begin{array}{l}\text { RW-DB, } \\
\text { S. } 32\end{array}$ & $70 \mathrm{dt}$ & Wintergetreide, mittlere Intensität \\
\hline Preis & " & $24 \mathrm{DM} / \mathrm{dt}$ & \\
\hline variable Kosten & $" \prime$ & $1000 \mathrm{DM} / \mathrm{ha}$ & einschließlich Lohndrusch \\
\hline Arbeitszeitanspruch & " & $8 \mathrm{~h}$ & \\
\hline Maschinenneuwert & & $2500 \mathrm{DM} / \mathrm{ha}$ & $\begin{array}{l}\text { ohne Mähdrescher, eigene Berechnungen } \\
\text { nach KTBL }\end{array}$ \\
\hline Nutzungsdauer & & 12 Jahre & eigene Berechnungen nach KTBL \\
\hline$\varnothing$ Umlaufkapital & " & 667 & Dauer: 8 Monate, Betrag: variable Kosten \\
\hline => DB ohne Zinsanspruch & & $680 \mathrm{DM} / \mathrm{ha}$ & $70 \mathrm{dt} \cdot 24 \mathrm{DM} / \mathrm{dt}-1000 \mathrm{DM}$ \\
\hline $\begin{aligned}=> & \text { Kapitalkosten der } \\
& \text { Maschinen }\end{aligned}$ & & $282,298,349$ & bei $5 ; 6$ bzw. $9 \%$ Zins \\
\hline $\begin{array}{l}\text { => Gewinnbeitrag ohne } \\
\text { / mit Zinsanspruch des } \\
\text { Umlaufkapitals }\end{array}$ & & $\begin{array}{l}398,382, \\
365,341 \\
362,271\end{array}$ & $\begin{array}{l}\text { DB - Kapitalkosten (für 5, } 6 \text { und } 9 \% \\
\text { Zinsen) }\end{array}$ \\
\hline
\end{tabular}


Tabelle 337.1: Produktionsverfahren Pflanzenproduktion bei 10\%iger Preisreduzierung

\begin{tabular}{|c|c|c|c|}
\hline Variable & Quelle & Wert & Kommentar \\
\hline Ertrag & $\begin{array}{l}\text { RW-DB, } \\
\text { S. } 32\end{array}$ & $70 \mathrm{dt}$ & Wintergerste, mittlere Intensität \\
\hline Preis & $"$ & $24 \cdot 0.9 \mathrm{DM} / \mathrm{dt}$ & \\
\hline variable Kosten & " & $1000 \mathrm{DM} / \mathrm{ha}$ & einschließlich Lohndrusch \\
\hline Arbeitszeitanspruch & $"$ & $8 \mathrm{~h}$ & \\
\hline Maschinenneuwert & & $2500 \mathrm{DM} / \mathrm{ha}$ & $\begin{array}{l}\text { ohne Mähdrescher, eigene Berechnungen } \\
\text { nach KTBL }\end{array}$ \\
\hline Nutzungsdauer & & 12 Jahre & eigene Berechnungen nach KTBL \\
\hline$\varnothing$ Umlaufkapital & $"$ & 667 & Dauer: 8 Monate, Betrag: variable Kosten \\
\hline$=>$ DB ohne Zinsanspruch & & $512 \mathrm{DM} / \mathrm{ha}$ & $70 \mathrm{dt} \cdot 24 \cdot 0.9 \mathrm{DM} / \mathrm{dt}-1000 \mathrm{DM}$ \\
\hline $\begin{aligned}=> & \text { Kapitalkosten der } \\
& \text { Maschinen }\end{aligned}$ & & $282,298,349$ & bei 5; 6 bzw. $9 \%$ Zins \\
\hline $\begin{aligned}=> & \text { Gewinnbeitrag ohne } \\
& / \text { mit Zinsanspruch des } \\
& \text { Umlaufkapitals }\end{aligned}$ & & $\begin{array}{lll}230, & 214, & 163 \\
197, & 174, & 103\end{array}$ & $\begin{array}{l}\text { DB - Kapitalkosten (für 5, } 6 \text { und } 9 \% \text { Zinsen) } \\
\text { DB - Kapitalkosten - Verzinsung des } \\
\text { Umlaufkapitals }\end{array}$ \\
\hline
\end{tabular}

Tabelle 337.2: Produktionsverfahren Tierproduktion

\begin{tabular}{|c|c|c|c|}
\hline Variable & Quelle & Wert & Kommentar \\
\hline Fleischertrag & $\begin{array}{l}\text { RW-DB, } \\
\text { S. } 90\end{array}$ & $\begin{array}{c}210 \mathrm{~kg} / \\
\text { Mastplatz/Jahr }\end{array}$ & $\begin{array}{l}92 \mathrm{~kg} \text { Schlachtgewicht pro Mastschwein, } \\
\text { 2.3 Durchgänge }\end{array}$ \\
\hline Preis & $"$ & $3 \mathrm{DM} / \mathrm{kg} \mathrm{SG}$ & einschl. MWSt \\
\hline variable Kosten & " & 500 DM/Platz & 216 DM/Tier, Futterverwertung 1:3 \\
\hline $\mathrm{AKh}$ & " & 0.8 & $0.3 \mathrm{~h}$ pro Mastschwein, etwas erhöht \\
\hline Stallplatz-Neuwert & & $1100 \mathrm{DM}$ & eigene Berechnungen nach KTBL \\
\hline Nutzungsdauer & " & 18 Jahre & " \\
\hline$\varnothing$ Umlaufkapital & & $158.70 \mathrm{DM}$ & $\begin{array}{l}100 \text { DM Ferkelkosten }+ \text { Hälfte der sonstigen } \\
\text { variablen Kosten pro Tier }\end{array}$ \\
\hline$=>$ DB ohne Zinsanspruch & & 130 DM/Platz & $\begin{array}{l}210 \mathrm{~kg} \cdot 3 \mathrm{DM} / \mathrm{kg}-500 \mathrm{DM} \\
=57 \mathrm{DM} / \text { Tier }\end{array}$ \\
\hline $\begin{aligned}=> & \text { Kapitalkosten eines } \\
& \text { Stallplatzes }\end{aligned}$ & & $94,101,125$ & bei $5 ; 6$ bzw. $9 \%$ Zins \\
\hline $\begin{array}{l}\text { => Gewinnbeitrag ohne } \\
\text { / mit Zinsanspruch des } \\
\text { Umlaufkapitals }\end{array}$ & & $\begin{array}{llr}36, & 29, & 5 \\
28, & 19, & -10\end{array}$ & DB - Kapitalkosten (für 5, 6 und $9 \%$ Zinsen) \\
\hline
\end{tabular}


Tabelle 338.1: Produktionsverfahren Tierproduktion bei 2.5\% iger Preisreduzierung

\begin{tabular}{|c|c|c|c|}
\hline Variable & Quelle & Wert & Kommentar \\
\hline Fleischertrag & $\begin{array}{l}\text { RW-DB, } \\
\text { S. } 90\end{array}$ & $\begin{array}{c}210 \mathrm{~kg} / \\
\text { Mastplatz/Jahr }\end{array}$ & $\begin{array}{l}92 \mathrm{~kg} \text { Schlachtgewicht pro Mastschwein, } \\
2.3 \text { Durchgänge }\end{array}$ \\
\hline Preis & $"$ & $\begin{array}{l}3 \cdot 0.975 \\
D M / k g S G\end{array}$ & einschl. MWSt \\
\hline variable Kosten & $"$ & 500 DM/Platz & 216 DM/Tier, Futterverwertung 1:3 \\
\hline $\mathrm{AKh}$ & $"$ & 0.8 & $0.3 \mathrm{~h}$ pro Mastschwein, etwas erhöht \\
\hline Stallplatz-Neuwert & & $1100 \mathrm{DM}$ & eigene Berechnungen nach KTBL \\
\hline Nutzungsdauer & & 18 Jahre & $"$ \\
\hline$\varnothing$ Umlaufkapital & & $158.70 \mathrm{DM}$ & $\begin{array}{l}100 \text { DM Ferkelkosten }+ \text { Hälfte der sonstigen } \\
\text { variablen Kosten pro Tier }\end{array}$ \\
\hline$=>D B$ ohne Zinsanspruch & & 114 DM/Platz & $210 \mathrm{~kg} \cdot 3 \mathrm{DM} / \mathrm{kg}-500 \mathrm{DM} \stackrel{\wedge}{=} 57 \mathrm{DM} /$ Tier \\
\hline $\begin{aligned}=> & \text { Kapitalkosten eines } \\
& \text { Stallplatzes }\end{aligned}$ & & $94,102,125$ & bei $5 ; 6$ bzw. $9 \%$ Zins \\
\hline $\begin{aligned}= & \text { Gewinnbeitrag ohne } \\
& \text { / mit Zinsanspruch des } \\
& \text { Umlaufkapitals }\end{aligned}$ & & $\begin{array}{rr}21, & 13,-11 \\
13, & 4,-25\end{array}$ & $\begin{array}{l}\text { DB - Kapitalkosten (für 5, } 6 \text { und } 9 \% \\
\text { Zinsen) }\end{array}$ \\
\hline
\end{tabular}

\subsubsection{Beschreibung des LP-Tableaus}

In diesem Unterabschnitt wird das verwendete LP-Tableau vorgestellt und vor allem formal erläutert. Eine inhaltliche Beschreibung und Begründung erfolgte im Textteil. Das Tableau ist in Tabelle 340.1 wiedergegeben. In Tabelle 339.1 werden die im Tableau verwendeten Abkürzungen erläutert. 
Tabelle 339.1: Zusammenfassung der im Tableau verwendeten Abkürzungen

\begin{tabular}{|c|l|l|}
\hline \multicolumn{2}{|c|}{ ID_-Spalte (Restriktionen) } \\
\hline 1 & ZieI & Zielfunktion \\
\hline 2 & Flaeche & Ackerfläche \\
\hline 3 & MaKapaz & Maschinenkapazität \\
\hline 4 & StaIIpI & Stallplätze \\
\hline 5 & Liqui & Liquidität, verfügbare Mittel \\
\hline 6 & EKKorr & $\begin{array}{l}\text { Korrektur für Investitionen mit } \\
\text { Eigenmitteln }\end{array}$ \\
\hline 7 & OgIKr1 & $\begin{array}{l}\text { Obergrenze für langfristige } \\
\text { Kreditaufnahme 1 (nur für Inv.) }\end{array}$ \\
\hline 8 & OgIKr2 & dito für Kreditaufnahme 2 \\
\hline 9 & OgkFK1 & $\begin{array}{l}\text { Obergrenze für kurzfristige } \\
\text { Kreditaufnahme 1 }\end{array}$ \\
\hline 10 & OgkFK2 & dito für Kreditaufnahme 2 \\
\hline 11 & AKStd & Arbeitskraftstunden \\
\hline 12 & DE & Dungeinheiten \\
\hline 13 & OgMasCh & $\begin{array}{l}\text { Obergrenze für Maschinen- } \\
\text { investitionen }\end{array}$ \\
\hline 14 & OgStI Ib & Obergrenze für Stallbau \\
\hline 15 & PPgTP & Diversifikation \\
\hline 16 & upper & $\begin{array}{l}\text { Obergrenze für bestimmte } \\
\text { Aktivitäten }\end{array}$ \\
\hline 17 & intAK & Integerrestriktion für Fremd-AK \\
\hline 18 & int & $\begin{array}{l}\text { Integerrestriktion für } \\
\text { bestimmte Aktivitäten }\end{array}$ \\
\hline 19 & kKapSaI & kurzfristiger Kapitalsaldo \\
\hline 20 & kZiSa Id & kurzfristiger Zinssaldo \\
\hline 21 & MaInv & $\begin{array}{l}\text { Summe Maschinen- } \\
\text { investitionen }\end{array}$ \\
\hline 23 & EinAusS & $\begin{array}{l}\text { Saldo der Ein- und } \\
\text { Auszahlungen }\end{array}$ \\
\hline
\end{tabular}

\begin{tabular}{|c|c|}
\hline \multicolumn{2}{|r|}{ Variablen (Aktivitäten) } \\
\hline _ID_ & Name der Restriktion \\
\hline${ }^{R} \mathrm{RHS} \_$ & Right Hand Side, Wert der Restriktion \\
\hline _TYPE_ & Type der Restriktion \\
\hline PfIPrn & Pflanzenproduktion \\
\hline MaInvEK & Maschineninvestition mit Eigenkapital \\
\hline MaIIKrI-2 & $\begin{array}{l}\text { Maschineninvestition mit langfristigem } \\
\text { Kredit } 1 \text { bis } 2\end{array}$ \\
\hline MaIkKrI-3 & $\begin{array}{l}\text { Maschineninvestition mit kurzfristigem } \\
\text { Kredit } 1 \text { bis } 3\end{array}$ \\
\hline Tierprn & Tierproduktion (Schweinemast) \\
\hline StInVEK & Stallbau mit Eigenkapital \\
\hline StIIKrI-2 & Stallbau mit langfristigem Kredit 1 bis 2 \\
\hline StIkKrI-3 & Stallbau mit kurzfristigem Kredit 1 bis 3 \\
\hline$A K$ & feste Fremdarbeitskräfte \\
\hline$A K h$ & $\begin{array}{l}\text { variable Fremdarbeitskraft } \\
\text { (stundenweise) }\end{array}$ \\
\hline Pacht & Zupacht von Ackerflächen \\
\hline FinAnI & Finanzanlagen (Laufzeit 1 Jahr) \\
\hline EntgZi & $\begin{array}{l}\text { entgangene Zinsen durch Investitionen } \\
\text { mit Eigenkapital }\end{array}$ \\
\hline$k F K 1$ & billiger kurzfr. Kredit (Laufzeit 1 Jahr) \\
\hline$k F K 2$ & $\begin{array}{l}\text { mittelteurer kurzfr. Kredit (Laufzeit } 1 \\
\text { Jahr) }\end{array}$ \\
\hline$k F K 2$ & teurer kurzfr. Kredit (Laufzeit 1 Jahr) \\
\hline
\end{tabular}




\section{Tabelle 340.1: LP-Tableau des Grundmodells, erste Periode}

\begin{tabular}{|c|c|c|c|c|c|c|c|c|c|c|c|c|}
\hline NR & ${ }_{-} \mid D_{-}$ & _ TYPE_ & RHS & PFLPRN & MAI NVEK & MAI LKR I & MAI LKR2 & MAI KKR I & MAI KKR 2 & MAI KKR 3 & TI ERPRN & STI NVEK \\
\hline 1 & Zi el & $\max$ & & 680.0 & 282.1 & 298.2 & -349.1 & 403.6 & -481.0 & 563.1 & 130.0 & 94.1 \\
\hline 2 & Flaeche & Le & 100 & 1 & & & . & . & & . & . & \\
\hline 3 & MaKapaz & Le & & 1 & -1 & -1 & -1 & -1 & -1 & -1 & . & \\
\hline 4 & Stal|p| & Le & & & & . & . & . & . & . & 1 & -1 \\
\hline 5 & Liqui & Le & 40000 & 666.7 & 2500 & . & . & . & . & . & 158.7 & 1100 \\
\hline 6 & EKKorr & Le & & & -2500 & . & . & . & & . & . & -1100 \\
\hline 7 & $0 \mathrm{~g} \mid \mathrm{FK} 1$ & Le & 500000 & & & 2500 & . & . & & . & . & \\
\hline 8 & $0 \mathrm{~g} / \mathrm{FK} 2$ & Le & 500000 & . & & . & 2500 & . & & . & . & \\
\hline 9 & OgkFK 1 & Le & 50000 & . & & . & . & 2500 & . & . & . & \\
\hline 10 & $0 \mathrm{gkFK} 2$ & Le & 50000 & . & & . & . & . & 2500 & . & . & . \\
\hline 11 & AKSt d & Le & 1840 & 8.0 & 0.4 & 0.4 & 0.4 & 0.4 & 0.4 & 0.5 & 0.8 & 0.2 \\
\hline 12 & $D E$ & Le & 200 & . & & . & . & . & . & . & 0.2 & \\
\hline 13 & OgMasch & I e & 2500 & . & 1 & 1 & 1 & 1 & 1 & 1 & . & \\
\hline 14 & $0 g S t|| b$ & Ie & 1000 & . & & . & . & . & . & . & . & 1 \\
\hline 15 & PPgTP & eq & & -2500 & & . & . & . & . & . & 1100 & \\
\hline 16 & upper & upperbd & & . & 1000 & 1000 & 1000 & 1000 & 1000 & 1000 & . & 2000 \\
\hline 17 & int AK & integer & & . & & 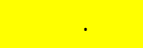 & . & . & - & . & . & \\
\hline 18 & $\mathrm{int}$ & free & & . & 1 & 1 & 1 & 1 & 1 & 1 & . & 1 \\
\hline 19 & kKapSal & free & & . & & . & . & 2500 & 2500 & 2500 & . & \\
\hline 20 & kZi Sald & free & & . & & . & . & 300 & 400 & 500 & . & \\
\hline 21 & MaI n v & free & & . & 1 & -1 & -1 & -1 & -1 & -1 & . & \\
\hline 22 & $S t I \| n v$ & free & & . & & . & . & . & . & . & . & -1 \\
\hline 23 & EinAus $S$ & free & & & 2500 & & . & 2800 & 2900 & 3000 & . & 1100 \\
\hline
\end{tabular}

Schraffierter Bereich: Koeffizientenmatrix $A$, um die Zielfunktion (erste Zeile) und den Kapazitätenvektor $b$ (RHS), links angeordnet, ergänzt. Zahlen mit mehr als drei Stellen links vom Dezimalpunkt sind aus Platzgründen nicht dezimal, sondern rechtsbündig ausgerichtet. 
Tabelle 340,1] Fortsetzung

\begin{tabular}{|c|c|c|c|c|c|c|c|c|c|c|c|c|c|}
\hline [I LKR I & STI LKR2 & STI KKRI & STI KKR2 & STI KKR3 & $A K$ & AKH & PACHT & ENTGZI & FI NANL & KF K 1 & KFK2 K & KF K 3 & $N R$ \\
\hline 101.6 & .125 .6 & -151.7 & -189.1 & -228.6 & 48000 & -27 & 250.0 & 0.05 & 0.05 & .0 .12 & -0.16 & .0 .2 & 1 \\
\hline . & . & . & . & . & & . & 1 & & . & . & . & & 2 \\
\hline . & . & . & . & . & & . & & & . & . & . & & 3 \\
\hline-1 & -1 & -1 & -1 & -1 & & . & & & . & . & . & & 4 \\
\hline . & . & . & . & . & 48000 & 27 & 250.0 & & 1 & $\cdot 1$ & -1 & -1 & 5 \\
\hline . & . & . & . & . & & . & & 1 & . & . & . & & 6 \\
\hline 1100 & . & . & . & . & & . & & . & . & . & . & & 7 \\
\hline . & 1100 & . & . & . & & . & & & . & . & . & & 8 \\
\hline . & . & 1100 & & . & & . & & & . & 1 & & & 9 \\
\hline . & . & . & 1100 & . & & . & & & . & . & 1 & & 10 \\
\hline 0.2 & 0.2 & 0.2 & 0.2 & 0.3 & -1840 & -1 & 0.1 & & . & . & . & & 11 \\
\hline . & . & . & . & . & & . & -2.0 & & . & . & 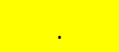 & & 12 \\
\hline . & & . & & & & . & & & . & . & & & 13 \\
\hline 1 & 1 & 1 & 1 & 1 & & . & & & . & . & . & & 14 \\
\hline . & . & . & . & . & & . & & & . & . & . & & 15 \\
\hline 2000 & 2000 & 2000 & 2000 & 2000 & 100 & 1380 & & . & . & . & . & & 16 \\
\hline . & . & . & . & . & 1 & . & & & . & . & . & & 17 \\
\hline 1 & 1 & 1 & 1 & 1 & 1 & . & & . & . & . & . & & 18 \\
\hline . & . & 1100 & 1100 & 1100 & . & . & & & -1 & 1 & 1 & 1. & 19 \\
\hline . & . & 132.0 & 176.0 & 220.0 & & . & & & .0 .05 & 0.12 & 0.16 & 0.2 & 20 \\
\hline . & & . & . & . & & . & & & . & . & . & ' & 21 \\
\hline$\cdot 1$ & -1 & $\cdot 1$ & $\cdot 1$ & -1 & & & & & . & . & & & 22 \\
\hline & & 1232 & 1276 & 1320 & 48000 & 27 & 250.0 & & .0 .05 & 0.12 & 0.16 & 0.2 & 23 \\
\hline
\end{tabular}

Die folgenden Erläuterungen beschreiben das Tableau zeilenweise, zunächst die Zielfunktion und die Aktivitäten, dann die Restriktionen, die Besonderheiten enthalten. Sie beginnt jeweils mit dem Kürzel für die Aktivität bzw. Restriktion, dann folgt die Position in der erweiterten Koeffizientenmatrix $A$ (s. Tabelle 340.1] und anschließend der Klartextname. Die nächste Zeile enthält in kursiver Schrift eine Kurzbeschreibung und der nächste Absatz eine ausführlichere Erläuterung, soweit dies notwendig erscheint.

\subsubsection{Komponenten der Zielfunktion}

In diesem Unterabschnitt werden neben den Koeffizienten der Zielfunktion auch die Aktivitäten des Tableaus beschrieben. Daher gliedert sich die Kurzbeschreibung in zwei Teile, die Charakteristika der Aktivität, gekennzeichnet durch ein Quadrat $\square$, und ihren Zielbeitrag, eingeleitet durch ein Dreieck $\Delta$.

\section{Ziel $\quad A(1 ;$.$) \quad Zielfunktion$}

Maximierung eines Proxies für den erwarteten kalkulatorischen Gewinn einer Periode

Der Zielwert im LP-Tableau stimmt nicht vollständig mit dem Periodengewinn des Unternehmens überein. Siehe hierzu Unterabschnitt 9.6.3.8 


\section{PflPrn $\quad A(1 ; 2) \quad$ Pflanzenproduktion}

\section{Pflanzenproduktion (Wintergetreide)}

\section{$\Delta$ Deckungsbeitrag ohne Zinskosten}

In dieser Zelle ist keine Berücksichtigung der Festkosten der zur Pflanzenproduktion notwendigen Maschineninvestitionen möglich, da diese Kosten auch getragen werden müssen, wenn in einzelnen Jahren nicht oder nicht voll produziert werden sollte. Die Eckdaten des Produktionsverfahrens sind in Tabelle 336.2 erläutert.

\section{MaInvEK-MaInvkKr3 A(1;3-8) Maschineninvestitionen}

Anschaffung eines Maschinenaggregats, das die Bewirtschaftung eines Hektars Ackerfläche ermöglicht, mit unterschiedlicher Finanzierung

$\Delta$ Jahreskosten

Investitionen können entweder mit Eigenkapital oder mit Kredit finanziert werden. Es stehen fünf Kreditarten zur Verfügung: drei kurzfristige, die später unter „kurzfristige Kreditaktivitäten" auf Seite 344 näher beschrieben sind, und zwei langfristige, die sich jeweils im Zinssatz unterscheiden. Die langfristigen Kredite sind zinsgünstiger als die kurzfristigen und können ausschließlich zur fristenkongruenten Finanzierung von Investitionen genutzt werden.

Da die jährlichen Kosten einer Investition u.a. vom Zinssatz abhängig sind, ist für jede Finanzierungsform einer Investition eine eigene Investitionsaktivität zu formulieren. In die Zielfunktion gehen jeweils die Jahreskosten ein. Diese betragen, weil angenommen wird, daß keine Reparaturen anfallen: $A \cdot$ Wiedergewinnungsfaktor $(N, i)$, mit $A=$ Anschaffungspreis, $N=$ Nutzungsdauer der Investition, $i=$ Zinssatz. $A$ . Wiedergewinnungsfaktor entsprechen den in Investitionskalkülen üblichen Jahreskosten:

Maschinenkosten $=$ Abschreibung $+A \cdot f \cdot i$.

Denn die Konstante $f$ wird gerade so bestimmt, daß die Identität $A$ - Wiedergewinnungsfaktor $=$ Abschreibung $+A \cdot f \cdot i$ gegeben ist (BRANDES \& WOERMANN 1969, S. 104). $f$ ist daher eine Funktion von $N$ und $i$ und stets kleiner als 1.

Durch diesen Ansatz werden in der Zielfunktion Zinskosten, die sich nicht direkt am Anschaffungspreis, sondern am durchschnittlich zu verzinsenden Anlagewert $A \cdot f$ orientieren, verwendet. Zinsansprüche von $A \cdot i$ wären einfacher zu implementieren, führten jedoch zu zu hohen Kosten und damit zu suboptimalen, da zu geringen Investitionsvolumina.

Da in der Zielfunktion mit der Annuität die Kapitalkosten einer Investition bereits vollständig erfaßt sind, dürfen Investitionsaktivitäten nicht an anderen Stellen im Tableau zu weiteren Kapitalkosten führen (vgl. ,entgangene Zinsen|' auf S. 343 und in Unterabschnitt. 9.6.2.2 Liquiditätsrestriktionen, Maschineninvestitionen mit Kredit' auf S. 345, etwa durch die Inanspruchnahme von Liquidität. 


\section{$\begin{array}{lll}\text { Tierprn } & A(1 ; 9) & \text { Tierproduktion }\end{array}$}

Tierproduktion

$\Delta$ Deckungsbeitrag ohne Zinskosten

Hier ist wie in der Pflanzenproduktion keine Berücksichtigung der Festkosten des Stallbaus möglich, da diese Festkosten auch getragen werden müssen, wenn nicht oder nicht voll produziert wird. Die Eckdaten des Produktionsverfahrens sind in Tabelle 337.2 Rerläutert.

\section{StInvEK-StInvKr3 $\quad A(1 ; 10-15) \quad$ Stallbauinvestitionen}

Erstellung eines Stallplatzes

$\Delta$ Jahreskosten einschließlich aller Nebenkosten, mit unterschiedlicher Finanzierung

Für Stallbauinvestitionen gilt das gleiche wie für Maschineninvestitionen.

$A K$

$$
\text { A(1;16) }
$$

Arbeitskräfte

ganzjährig beschäftigte Arbeitskraft

$\Delta$ Jahreslohnsumme für eine feste Arbeitskraft, einschließlich Lohnnebenkosten

Arbeitskräfte können nur ganzjährig eingestellt werden. Daher ist die Aktiviät AK als Integervariable vereinbart.
AKh
$A(1 ; 17)$
Arbeitskraftstunden

\section{Aushilfen}

\section{$\Delta$ Stundenlohn für nicht-ständige Arbeitskräfte}

Die Lohnkosten für Aushilfen sind so gewählt, daß das Jahresarbeitsvolumen einer AK (s. Restriktion Arbeitskapazität) günstiger von einer ständigen AK abgedeckt wird als über Aushilfen.

\section{Pacht}

$A(1 ; 18)$

\section{Zupacht}

zugepachtete Ackerfläche in ha

$\Delta$ Preis für zugepachtete Flächen pro ha

Die Pachtverträge laufen jeweils über ein Jahr. Eine Verpachtung ist nicht möglich.

EntgZi entgangene Zinsen

Hilfsvariable. Sie dient dazu, ein korrektes Investitionsvolumen zu erhalten.

$\Delta$ Eigenkapitalzinssatz, Zins für Finanzanlagen $\left(i_{E K}\right)$

Investitionen, die mit Eigenkapital durchgeführt werden, beanspruchen Liquidität und verursachen daher auf diesem Wege Opportunitätskosten. Denn die für Investitionen aufgewendeten Finanzmittel stehen nicht mehr für andere Verwendungen zur Verfü- 
gung. Sie reduzieren insbesondere den Umfang der Finanzanlagen. Die Opportunitätskosten betragen daher $A \cdot i$.

Gleichzeitig ist eine Investition mit Eigenkapital in der Zielfunktion mit ihrer Annuität belastet (s. ,Maschineninvestitionen'; S. 342). Es kommt daher zu einer Doppelzählung [genauer: dem $(1+f)$ fachen] der Zinskosten. Aus diesem Grund ist die Aktivität „entgangene Zinsen“ eingefügt. Sie erhöht im Umfang der mit Eigenkapital finanzierten Investitionen den Zielwert um $A \cdot i$, also um die entgangenen Zinsen der wegen der Investition im Tableau nicht möglichen Finanzanlagen.

\section{FinAnl A(1;19) Finanzanlagen}

Finanzanlagen mit einjähriger Laufzeit

$\Delta$ Zinssatz $i_{E K}$

Es können nur Wertpapiere mit einjähriger Laufzeit erworben werden. Da dies die einzige Anlagemöglichkeit für freie Mittel im Tableau bietet, entspricht der Zins der Finanzanlagen den Opportunitätskosten des Eigenkapitals.

\section{kFK1-3 A(1;16-18) kurzfristige Kreditaktivitäten}

Kurzfristiges Fremdkapital mit unterschiedlichem Zinsfuß, Laufzeit ein Jahr

$\Delta$ Zinsfiuße $i_{F K_{l}}, i_{F K_{2}}$ und $i_{F_{3}}$

Finanzierungsfragen sind für den Erfolg von Investitionen von besonderer Bedeutung. Daher wird den Unternehmen ein differenziertes Kreditangebot bereitgestellt. $k F K l$, $k F K 2$ und $k F K 3$ sind kurzfristige Kredite mit einer Laufzeit von einem Jahr und unterschiedlichen Zinssätzen. Die beiden zinsgünstigeren Kredite können nur bis zu bestimmten Obergrenzen aufgenommen werden, während der teuere Kredit unbeschränkt zur Verfügung steht. Er fungiert quasi als „Strafkredit“ (vgl. in Unterabschnitt 9.6.2.3 den Punkt, Kreditrestriktionen', S. 346.

Neben den kurzfristigen werden auch langfristige Kredite angeboten. Diese sind ausschließlich für Investitionen zugelassen und unter, Maschineninvestitionen auf Seite 342 beschrieben. Eine Investition kann auch mit kurzfristigem Fremdkapital finanziert werden. Geschieht dies, dürfen dennoch dadurch nicht die Umfänge der Kreditaktivitäten $k F K 1$ bis $k F K 3$ erhöht werden, weil sonst die Investition mit vollen Zinskosten belastet würde. Wie dies erreicht wird, ist in Unterabschnitt 9.6.2.2 unter dem Punkt, Maschineninvestitionen mit Kreditł auf Seite 345 beschrieben.

Die Aktivitäten $k F K 1$ bis $k F K 3$ dienen also ausschließlich der Finanzierung des Umlaufkapitals. Zur sprachlichen Unterscheidung von den in den Investitionsaktivitäten implizit enthaltenen Kreditaufnahmen werden diese expliziten Kredite $k F K 1$ bis $k F K 3$ Kreditaktivitäten genannt und mit $F K$ anstelle von $K r$ für die Investitionskredite abgekürzt. 
Nach der Beschreibung der Aktivitäten und der Zielfunktion werden nun die wichtigsten Restriktionen erläutert. Von besonderem Interesse sind in einem Modell, das Insolvenzgefahren untersucht, die Liquiditätsrestriktionen.

\subsubsection{Liquiditätsrestriktionen (Liqui)}

Alle Auszahlungen außer den Zinsen entstehen zu Beginn einer Periode, während die Einzahlungen (Deckungsbeiträge und Zinseinnahmen) am Ende einer Periode fällig werden.

Liquidität, die in Anspruch genommen wird, verursacht Kosten. Entweder können weniger Finanzanlagen erworben werden oder die Auszahlungen müssen über Kreditaufnahme refinanziert werden.

\section{RHS \\ $\boldsymbol{A}(5 ; 1)$ \\ Right Hand Side}

Ausstattung des Betriebes am Anfang der Periode mit Liquidität

PflPrn A(5;2) Pflanzenproduktion

Bedarf der Pflanzenproduktion an Umlaufkapital, vgl. Tabelle 336.2.

MaInvEK A(5;3) Maschineninvestitionen mit EK

Anschaffungswert eines Maschinenaggregates, finanziert mit Eigenkapital

Investitionen aus Eigenkapital reduzieren im Gegensatz zu fremdfinanzierten Investitionen direkt die Liquidität. Die Liquiditätswirksamkeit ist erforderlich, weil Mittel, die für Investitionen verwendet worden sind, selbstverständlich nicht noch ein zweites Mal ausgegeben werden können. Andererseits verursachen Investitionen mit Eigenkapital dadurch Opportunitätskosten von $A \cdot i$, weil in Höhe der eigenfinanzierten Investitionen Finanzanlagen nicht mehr möglich sind. Diese „Doppelzählung“ der Zinskosten wird durch die Aktivität „EntgZi““ (s. S. 343] ausgeglichen.

\section{MaIlKr1-MaIkKr3 A(5;3) Maschineninvestitionen mit Kredit}

Investitionen, die mit lang- oder kurzfristigem Kredit finanziert sind, enthalten bereits in der Zielfunktion die Kapitalkosten und dürfen daher direkt keine Liquidität beanspruchen, weil es sonst zu einer Doppelzählung der Zinsen käme. Sie verringern aber den Kreditspielraum (näheres unter „Kreditrestriktionen“ auf Seite 346.

\section{Tierprn $\quad A(5 ; 9) \quad$ Tierproduktion}

Bedarf der Tierproduktion an Umlaufkapital, vgl. Tabelle 337.2. 


\section{StInvEK-StInvFK3 $\quad A(5 ; 10-15) \quad$ Stallbauinvestitionen}

Für die Liquiditätswirkungen von Stallbauinvestitionen gilt das gleiche wie für Maschineninvestitionen.

\section{AK, AKh, Pacht und Kredite}

Die Liquiditätswirkungen der restlichen Aktivitäten AK, AKh, Pacht und Kredite sind ohne Besonderheiten (Kredite erhöhen die Liquidität, daher ist ihr Koeffizient -1). Die Koeffizienten von $A K, A K h$ und Pacht entsprechen mit umgekehrten Vorzeichen den Zielbeiträgen dieser Aktivitäten.

\subsubsection{Weitere Restriktionen und Kapazitäten}

\section{Eigenkapitalkorrektur (EKKorr)}

Diese Restriktion stellt sicher, daß entgangene Zinsen (s.o.) nur in dem Umfang gutgeschrieben werden, wie die Finanzierung der Investitionen mit Eigenkapital erfolgt.

\section{Kreditrestriktionen (OglFK1, OglFK2, OgkFK1, OgkFK2)}

Der Umfang der langfristigen Kredite ist beschränkt (Zeilen 7 und 8: OglFK1 und OglFK2). Sie können nur für Investitionen in Anspruch genommen werden und besitzen keine Verbindungen zur Liquiditätsrestriktion im Tableau.

Die beiden billigeren der kurzfristigen Kredite (Zeile 9 und 10: OgkFK1 und OglkK2) sind ebenfalls beschränkt, der teuerste steht unbegrenzt zur Verfügung und ist daher nicht mit einer eigenen Restriktion im Tableau vertreten. Die kurzfristigen Kredite liefern im Gegensatz zu den langfristigen im Tableau unmittelbar Liquidität.

Fremdfinanzierte Investitionen beanspruchen aus den unter ,Maschineninvestitionen“ auf Seite 342 und ,Liquiditätsrestriktionen" auf Seite 345 genannten Gründen direkt keine Liquidität. Sie reduzieren jedoch den Kreditspielraum für die beiden billigeren kurzfristigen Kreditaktivitäten und verringern damit indirekt die Liquidität (s. Tabelle 336.1].

\section{Arbeitskapazität (AKStd)}

Dem Unternehmen steht eine nicht entlohnte Arbeitskraft zur Verfügung. Sie leistet ebenso wie Fremdarbeitskräfte 1840 Stunden im Jahr (s. Tabelle 336.1).

\section{Dungeinheiten (DE)}

Die tierische Produktion ist über diese Restriktion mit der bewirtschafteten Fläche verknüpft. Pro Hektar können bis zu zehn Schweine gemästet werden. Die Kapazität kann durch Flächenzupacht aufgestockt werden. 


\section{Obergrenzen der Investitionen (OgMasch, OgStllb)}

Die Restriktionen 13 und 14 begrenzen den Umfang der Investitionen pro Jahr. Sie saldieren jeweils die nach Finanzierung aufgespaltenen Investitionsaktivitäten und verhindern ein überschäumendes Wachstum, das sonst unter extrem günstigen, zufallsbedingten Konstellationen möglich wäre. Die Obergrenzen sind für den Simulationszeitraum konstant.

\section{Diversifikation (PPgTP)}

Diese Restriktion garantiert in der dargestellten Form, daß der Umfang der Pflanzenproduktion und der Umfang der Tierproduktion exakt übereinstimmen. Durch Ändern des Typs der Restriktion von „eq“ zu ,free“ kann sie für Simulationsexperimente ausgeschaltet werden.

\section{Integer-Restriktion}

Stallplätze, Maschinen und Arbeitskräfte können nur in ganzen Einheiten beschafft bzw. angestellt werden. Dies wird sichergestellt, indem den Variablen der Typ „,integer" zugewiesen ist. Durch Ändern des Typs der Restriktion zu ,free" können auch diese Restriktionen für Simulationsexperimente ausgeschaltet werden. Dies gestattet rasche Vergleiche zwischen ganzzahligen und in dieser Hinsicht unbeschränkten Lösungen. In Zeile 18 z.B. ist die Ganzzahligkeitsbedingung, die für Investitionen und Vollarbeitskräfte gilt, deaktiviert. Für die Arbeitskräfte bleibt sie dennoch durch Zeile 17 weiterhin wirksam.

Ganzzahlige Variablen müssen aus programmtechnischen Gründen in ihrem Umfang nach oben begrenzt werden (nach unten sind sie es durch die implizite Nichtnegativitätsbedingung für alle Variablen automatisch). In SAS kann durch eine Restriktion des Typs ,,upperbd“ für beliebig viele Aktivitäten in einer Zeile eine Obergrenze vereinbart werden (Zeile 16). Die Restriktionen OgMasch und OgStllb sind inhaltlich begründet, für die Ganzzahligkeitsbedingungen sind sie nicht erforderlich.

\section{„freie Restriktionen“}

Das Tableau enthält ferner einige Restriktionen vom Typ ,free“. Dies sind keine Restriktionen im bekannten Sinne. Sie können dazu genutzt werden, die gewichteten Umfänge ausgewählter Aktivitäten zu aggregieren. Diese Summe wird im LP-Ergebnis als ungenutzte Kapazität (Rest) ausgewiesen. Folgende Aggregate werden gebildet:

- kurzfristiger Kapitalsaldo (kKapSal)

- kurzfristiger Zinssaldo (kZiSald)

- Umfang der Maschinen- und Stallbauinvestitionen (MaInv und StllInv)

- Saldo der Ein- und Auszahlungen (EinAusS).

Der kurzfristige Kapitalsaldo im Tableau setzt sich aus kurzfristigen Krediten und Finanzanlagen zusammen. Die kurzfristigen Kredite ihrerseits bestehen aus den Kreditaktivitäten 1 bis 3 und mit kurzfristigem Kredit 1 bis 3 finanzierten Investitionen 
(Maschinen oder Stallbau). Dieser Saldo weist im Soll ein negatives Vorzeichen auf. Er gibt Aufschluß über die Belastung des Betriebes mit kurzfristigen Verbindlichkeiten bzw. über sein Finanzvermögen. Ein totaler Kapitalsaldo kann im Tableau nicht bestimmt werden, da die in Vorperioden aufgenommenen Kredite im Tableau nicht erfaßt werden.

Die Restschuld der langfristigen älteren Kredite kann im verwendeten Tableau nicht ermittelt werden. Daher wird für das Betriebsentwicklungsmodell die Restschuld der langfristigen Kredite tableauextern $\mathrm{zu}$ dem vom LP ermittelten kurzfristigen Kapitalsaldo addiert und diese Summe als (gesamter) Kapitalsaldo ausgewiesen (s. Unterabschnitt 9.6.3.4 Fortschreibung des Fremdkapitals und des totalen Kapitalsaldos, beginnend auf Seite 353). Ist der Saldo im Soll, geht ihm ein negatives Vorzeichen voran.

Der kurzfristige Zinssaldo besteht aus Zinsen für die vorgenannten kurzfristigen Kredite (explizite und einperiodische Investitionskredite) und den Erträgen der Finanzanlagen (positives Vorzeichen). Es geht hier also im Unterschied zur Zielfunktion um real gezahlte oder erhaltene Zinsen und nicht um kalkulatorische Zinskosten. Der kurzfristige Zinssaldo weist wie der Kapitalsaldo im Soll ein negatives Vorzeichen auf. Ein totaler Zinssaldo kann aus den gleichen Gründen wie beim Kapitalsaldo nicht ausgewiesen werden.

Die Zeile MaInv summiert alle Aktivitäten, die zu einer Ausdehnung der Maschinenkapazität führen (unabhängig von der Finanzierung). Analoges gilt für die Zeile StllInv für Stallplätze.

In der Zeile EinAusS werden alle Ein- und Auszahlungen in der laufenden Periode erfaßt, soweit dies im LP-Tableau möglich bzw. sinnvoll ist. Sie dient als Hilfsgröße zur Bestimmung des kurzfristigen Kreditbedarfs in der Folgeperiode. Daher werden auch die Anschaffungswerte der mit langfristigen Krediten finanzierten Investitionen nicht berücksichtigt. Die Deckungsbeiträge gehen nicht ein, da sie als stochastische Größen nicht genau bekannt sind. Die realisierten Deckungsbeiträge werden in der Tableaufortschreibung außerhalb des Tableaus dem Saldo hinzugefügt.

\begin{tabular}{|l|l|l|}
\hline EinAusS $=$ & - & Auszahlungen für Arbeitskräfte und Pacht \\
& - & $\begin{array}{l}\text { Anschaffungswerte der mit kurzfristigen Krediten oder Eigenkapital finanzierten } \\
\text { Investitionen } \\
\text { Zinsen dieser kurzfristigen Investitionskredite } 1\end{array}$ \\
\hline- & Saldo der Zinszahlungen für Finanzanlagen und kurzfristige Kreditaktivitäten 2 \\
\hline & $\begin{array}{l}\text { Nicht: Anschaffungswerte der mit langfristigem Kredit finanzierten Investitionen } \\
\text { Realisierte Deckungsbeiträge ( } \\
\text { verfahren) Saldo der Ein- und Auszahlungen der Produktions- }\end{array}$ \\
\hline
\end{tabular}

Die letzten beiden Positionen, 1 und 2, bilden zusammen den kurzfristigen Zinssaldo (s.o). 
Um die Zinsen für die kurzfristigen Investitionskredite zu erfassen, werden im Tableau in der Zeile EinAusS unter den Investitionsaktivitäten MaIkKrl-2 und StIkKr1-2 nicht der Anschaffungswert (A), sondern das $(1+i)$ fache davon eingetragen.

Dem Unternehmen entstehen weitere Auszahlungen, die im Tableau zweckmäßigerweise nicht erfaßt werden: Kapitaldienst für langfristige Kredite und Entnahmen. Die Werte für die in der aktuellen Periode neu aufgenommenen Kredite könnten im Tableau problemlos berechnet werden. Ein Berücksichtigen der Altschulden hingegen würde das Tableau sehr stark vergrößern. In jeder Periode können vier verschiedene Kredite aufgenommen werden: zwei Laufzeiten mal zwei Zinssätze. Bei einer mittleren Kreditlaufzeit von 15 Jahren ergeben sich daraus $(15-1) \cdot 4=56$ mögliche Altkredite, die als Aktivitäten in das Tableau einzuführen wären. Daher werden Zinsen und Tilgungen aller langfristigen Kredite tableauextern berechnet (vgl. Unterabschnitt 9.6.3.4. beginnend auf S. 353.

\subsubsection{Fortschreibung des LP-Tableaus auf die nächste Periode}

Das Ergebnis der Vorperiode beeinflußt die Ausgangsausstattung für die aktuelle Periode. Verändern können sich beispielsweise der Kreditspielraum bzw. das freie Kapital und die Produktionskapazität. Das LP-Tableau ist so formuliert, daß Vorperiodenergebnisse sich nur auf die Kapazitäten des Betriebes auswirken. Daher ist nur der RHS-Vektor des Tableaus in jeder Periode zu aktualisieren. Dieser Unterabschnitt beschreibt die Fortschreibung der RHS-Variablen.

Darüber hinaus werden einige Kennzahlen fortgeschrieben, die Einblick in die Situation des Betriebes geben, z.B. Eigenkapital und Sachanlagevermögen. Dies geschieht außerhalb des Tableaus, weil anderenfalls das Tableau sehr unübersichtlich würde. Auch die Aktualisierung des Fremdkapitalkontos erscheint außerhalb des Tableaus einfacher als innerhalb, wie in Unterabschnitt 9.6.2.3 auf Seite 349 erläutert ist. Der Unterabschnitt schließ mit der Berechnung des Gewinns aus dem Zielwert des LP-Tableaus.

In diesem Unterabschnitt werden folgende Kürzel verwendet:

$$
\begin{array}{ll}
\text { MaKapaz }^{a} & =\text { Maschinenkapazität zu Periodenbeginn } \\
\text { MaSchInv } & =\text { Maschineninvestitionen (Anzahl) } \\
\text { Stallpla } & =\text { Kapazität an Stallplätzen } \\
\text { StalIInV } & =\text { Bau von Stallplätzen (Anzahl) } \\
\text { MaZw } & =\text { Maschinenzeitwert } \\
a & =\text { am Anfang der Periode } \\
e & =\text { am Ende der Periode }
\end{array}
$$

\subsubsection{Maschinenkapazität und Stallplätze zu Beginn einer Periode}

Beide Größen sind Kapazitäten des Betriebes und werden daher im LP-Tableau als Werte für die RHS-Spalte verwendet. 
In die Maschinenkapazität zu Beginn der Periode $t$ gehen alle Maschinen ein, die zu diesem Zeitpunkt noch nicht abgeschrieben sind, also einen Zeitwert größer als Null haben. Die Investitionen des Jahres $t$ gehen nicht in die Kapazitätsberechnung für dieses Jahr $t$ ein, da dieser Wert, wie gesagt, als RHS-Wert benutzt wird.

\subsection{Berechnungsmethode A (verwendete Methode)}

Kerngedanke: Bestimmung der noch nutzbaren Investitionen der Vorperioden

Beispiel: $\quad$ Maschineninvestition, $N=4$

Eine im Jahr 0 angeschaffte Maschine kann bei vierjähriger Nutzungsdauer in den Jahren 0, 1, 2 und 3 benutzt werden. Die Maschinenkapazität zu Beginn des Jahres 4 (also der RHS-Wert im Tableau) wird daher bestimmt durch Investitionen, die in den Jahren 3, 2 und 1 getätigt wurden, jedoch nicht mehr durch Investitionen des Jahres 0 und ebenfalls nicht durch die in 4 getätigt werdenden Investitionen (s. folgende Übersicht).

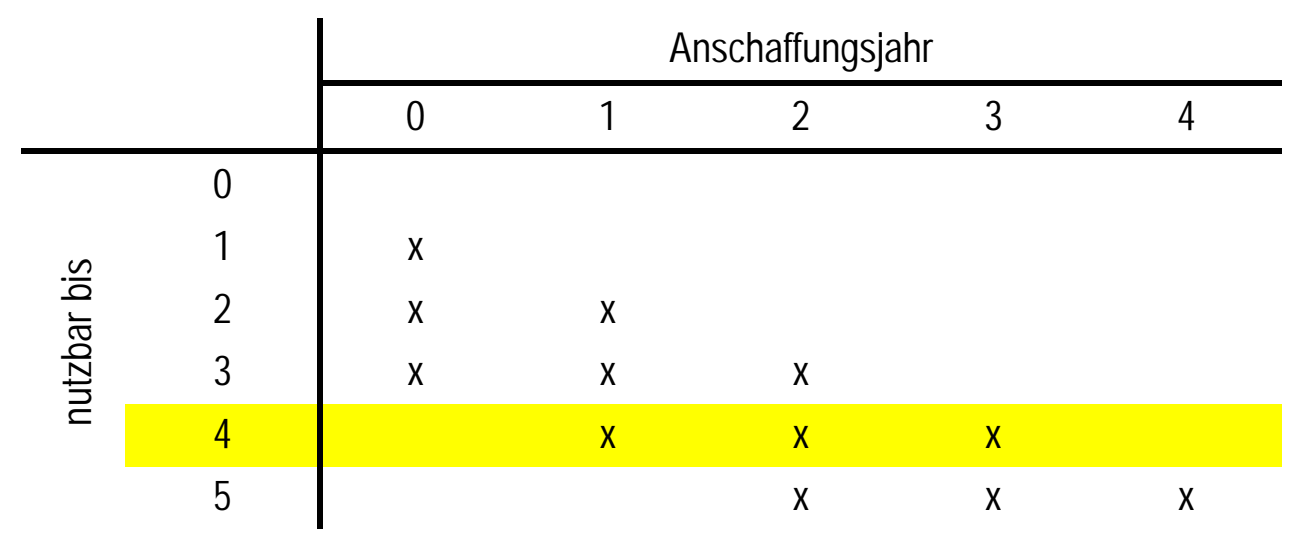

Also gilt für die Kapazität im Tableau:

$$
\operatorname{MaKapaz}(4)=\operatorname{MaschInv}(3)+\operatorname{MaschInv}(2)+\operatorname{MaschInv}(1)
$$

oder allgemein für ein beliebiges Jahr:

$$
\begin{aligned}
\operatorname{MaKapaz}^{a}(t) & =\operatorname{MaschInv}(t-1)+\operatorname{MaschInv}(t-2)+\operatorname{MaschInv}(t-3) \\
& =\operatorname{MaschInv}(t-1)+\operatorname{MaschInv}(t-2)+\operatorname{MaschInv}(t-(N-1)) \\
& =\sum_{i=1}^{N-1} \operatorname{MaschInv}(t-i)
\end{aligned}
$$

Analog:

$$
\operatorname{Stallpla}(t)=\sum_{i=1}^{N-1} \operatorname{StalIInv}(t-i) \text {. }
$$




\subsection{Berechnungsmethode B}

Kerngedanke: Ausgehend von der Kapazität zu Beginn der Vorperiode wird mittels der $\mathrm{Zu}$ - und Abgänge in den Vorperioden die Kapazität zu Beginn der aktuellen Periode bestimmt.

Beispiel: $\quad$ Maschineninvestitionen, $N=4$

Eine im Jahr 0 angeschaffte Maschine kann in den Jahren 0, 1, 2 und 3 benutzt werden, Ende 3 wird sie unbrauchbar. Die Maschinenkapazität zu Beginn des Jahres 4 (also der RHS-Wert im Tableau) wird bestimmt durch:

- Kapazität zu Beginn der Vorperiode, also des Jahres 3,

- zzgl. Investitionen in der Vorperiode,

- abzgl. in der Vorperiode unbrauchbar gewordenen Anlagen, d.h., in 0 angeschafften Maschinen:

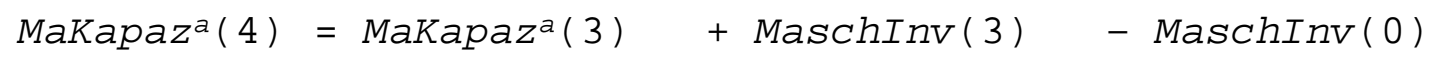

oder allgemein für ein beliebiges Jahr:

$$
\begin{aligned}
\operatorname{MaKapaz}^{a}(t) & =\operatorname{MaKapaz}^{a}(t-1)+\operatorname{MaschInv}(t-1)-\operatorname{MaschInv}(t-4) \\
& =\operatorname{MaKapaz}^{a}(t-1)+\operatorname{MaschInv}(t-1)-\operatorname{MaschInv}(t-\mathrm{N}) .
\end{aligned}
$$

Analog:

$$
\text { Stallplaa }(t)=\operatorname{Stallpla}(t-1)+\operatorname{StalIInv}(t-1)-\operatorname{StalIInv}(t-N) \text {. }
$$

Diese Vorgehensweise empfiehlt sich, wenn die Lösung numerisch bestimmt wird. Denn bei diesem Verfahren werden unabhängig von der Nutzungsdauer stets nur drei Variablen (Bestand in $t-1$, Investitionen in $t-1$, Investitionen in $t-N$ ) verrechnet, während bei Methode A $N-1$ Investitionen saldiert werden. Wird eine analytische Lösung der Differenzengleichung angestrebt, ist Verfahren B aufwendiger, da die Ordnung der Differenzengleichung um Eins höher ist als bei Methode A. Diese höherer Ordnung wird in vielen Fällen eine analytische Lösung unmöglich machen (vgl. hierzu die Ausführungen zu Differenzengleichungen im Abschnitt 2.4 über den LOHMANN-RUCHTIEffekt). Im Simulationsprogramm wird trotz geringen Mehraufwand an Rechenzeit Methode A verwendet, damit numerische und analytische Lösungsansätze die gleiche Struktur aufweisen.

\subsubsection{Fortschreibung des Maschinenzeitwertes: Maschinenzeitwert zu Beginn der Periode t}

In den Maschinenzeitwert zu Ende einer Periode $t\left[\mathrm{MaZw}^{e}(t)\right]$ gehen alle Maschinen ein, die zu diesem Zeitpunkt noch brauchbar sind, also einen Zeitwert größer als Null haben. Die Abschreibung der aktuellen Periode ist abgezogen. Wegen des unterschiedlichen Termins (Anfang bzw. Ende einer Periode) beziehen sich Kapazitäts- und Zeitwertberechnungen nicht auf die gleichen Maschinen. 
Beispiel: $\quad N=4$

$$
\begin{aligned}
& \operatorname{MaZw}^{e}(4)=\operatorname{MaschInv}(4) \cdot A \cdot 3 / 4+\operatorname{MaschInV}(3) \cdot A \cdot 1 / 2+\operatorname{MaschInV}(2) \cdot A \cdot 1 / 4 \\
& \operatorname{MaZw}_{W}{ }^{e}(t)=\operatorname{MaschInV}(t) \cdot A \cdot 3 / 4+\operatorname{MaschInV}(t-1) \cdot A \cdot 1 / 2+\operatorname{MaschInV}(t-2) \cdot A \cdot 1 / 4 \\
& =\operatorname{MaschInv}(t) \cdot A \cdot 3 / N+\operatorname{MaschInv}(t-1) \cdot A \cdot 2 / N+\operatorname{MaschInV}(t-2) \cdot A \cdot 1 / N \\
& =\operatorname{MaschInv}(t) \cdot A \cdot \frac{N-1}{N}+\operatorname{MaschInv}(t-1) \cdot A \cdot \frac{N-2}{N}+\operatorname{MaschInv}(t-2) \cdot A \cdot \frac{N-3}{N} \\
& =\sum_{i=1}^{N-1} \operatorname{MaschInV}(t-i+1) \cdot A \cdot \frac{N-i}{N} \text {. }
\end{aligned}
$$

\section{Analog:}

$\operatorname{StallZw}(t)=\sum_{i=1}^{N-1} \operatorname{StallInv}(t-i+1) \cdot A \cdot \frac{N-i}{N}$.

$M a Z w^{e}$ und $S t a l I Z W^{e}$ ergeben zusammen das Sachanlagevermögen.

Die dargestellte Berechnung für den Maschinenzeitwert bezieht sich jeweils auf das Ende einer Periode. Das im Mittel in einer Periode vorhandene Maschinenkapital wird dadurch systematisch unterschätzt. Dies führt im Extrem dazu, daß ein Unternehmen, das nur Gebrauchtmaschinen mit einer Restnutzungsdauer von einem Jahr kauft, bei dieser Berechnungsmethode einen Maschinenzeitwert von Null aufweist. Dies ist bei der Interpretation der Ergebnisse zu beachten.

\subsubsection{Vervollständigen des Saldos der Ein- und Auszahlungen und Fortschreibung der Liquidität}

Im Tableau werden nicht alle Auszahlungen, die einem Unternehmen entstehen, erfaßt, weil es ansonsten unverhältnismäßig aufgebläht würde. Der im Tableau ermittelte Einnahmen/Ausgaben-Saldo (EinAusS, vgl. S. 353] umfaßt Investitionen, die mit Eigenkapital oder kurzfristigen Krediten finanziert sind, Finanzanlagen und die übrigen einperiodischen Kredite. Zur Vervollständigung werden außerhalb des Tableaus folgende Größen in Ansatz gebracht:

- realisierte Deckungsbeiträge der Produktionsverfahren,

- Tilgungen der langfristigen Kredite, aufgenommen in dieser und allen Vorperioden,

- Zinsen der langfristigen Kredite, aufgenommen in dieser und allen Vorperioden,

- Entnahmen.

Der neue Saldo nach der Fortschreibung wird mit EASav bezeichnet. Ist diese Größe negativ, vermindert sich die zu Periodenbeginn vorhandene Liquidität um diesen Betrag bzw. zu Beginn der Folgeperiode ist eine entsprechend höhere Summe an kurzfristigem Fremdkapital aufzunehmen. 


\begin{tabular}{|l|l|l|l|}
\hline EASav & $=$ & & ausgeführt in Unterprogramm: $^{220}$ \\
\hline & + & EinAusS & Tableau \\
& + & realisierte DB der Produktionsverfahren & Fortschreibung \\
& - & Tilgungen der langfristigen Kredite & Fortschreibung \\
& - & Zinsen der langfristigen Kredite & Fortschreibung \\
& - & Entnahmen & Fortschreibung \\
\hline
\end{tabular}

Dieser Stand des kurzfristigen Kapitalkontos wird mit Liquidität (Liqui) bezeichnet, gleichgültig, ob es sich im Soll oder im Haben befindet. Mit der Liquidität wird der Kapitaldienst der Altschulden in die Kalkulation des aktuellen Tableaus eingefügt. Für die Fortschreibung der Liquidität gilt zusammengefaßt:

\begin{tabular}{|c|c|c|c|}
\hline \multirow[t]{2}{*}{ Liqui $_{t+1}$} & \multirow{2}{*}{$\begin{array}{l}= \\
+ \\
+\end{array}$} & \multirow[b]{2}{*}{$\begin{array}{l}\text { Liqui }_{t} \\
\text { vervollständigter Saldo der Ein- und Auszahlungen } \\
\left(\text { EASav }_{t}\right)\end{array}$} & ausgeführt in Unterprogramm: \\
\hline & & & $\begin{array}{l}\text { Tableau } \\
\text { Fortschreibung (353.1) }\end{array}$ \\
\hline
\end{tabular}

Ist die Liquidität zu Anfang der Periode negativ, so ist sie unverzüglich durch Aufnahme von kurzfristigem Fremdkapital auszugleichen.

\subsubsection{Fortschreibung des Fremdkapitals und des totalen Kapitalsaldos}

Im vorigen Kapitel wurde die Liquidität zu Beginn der nächsten Periode abgeleitet. Diese Liquidität ist einer von zwei Bausteinen für den gesamten Kapitalsaldo. Der fehlende Baustein sind die Restschulden der langfristigen Kredite. Diese werden jeweils am Ende der Periode vor der in der aktuellen Periode fälligen Tilgung festgestellt (zur Begründung s. Unterabschnitt 9.6.4.

Der Fremdkapitalbestand in einer Periode ergibt sich nach der Bilanzgleichung als Fremdkapital in der Vorperiode zzgl. Zugänge abzgl. Abgänge. Die Umsetzung dieser einfachen Vorschrift ist ähnlich der Bestimmung der Maschinenkapazität in einem Betriebsentwicklungsmodell mit mehreren Zinssätzen und Laufzeiten aufwendig. Sie wird für den Fremdkapitalbestand detailliert dargestellt und für die aggregierten Zinsen und Tilgungen nur angegeben.

Zur Bestimmung der Restschulden sei zunächst unterstellt, daß der Betrieb nur im Jahre 3 einen Kredit aufgenommen habe. Da Kredite grundsätzlich nur am Periodenanfang aufgenommen werden, beträgt das Alter dieses Kredites am Ende des Jahres 5 $(t=5)$ drei Jahre $(J=3)$. Seine Restschulde $(t)$ beläuft sich Ende 5 vor der Tilgung auf:

Restschulde(5) = Kreditsumme $\quad-$ Tilgung(4) - Tilgung(3)

220 Diese Spalte verweist auf Unterprogramme des Simulationsprogramms, das in Unterabschnitt 9.6.4 dokumentiert ist. 
oder allgemein:

$$
\text { Restschulde }(t, J) \quad=\text { Kreditsumme } \quad-\sum_{j=1}^{J-1} \operatorname{Tilgung}(t-j) .
$$

Da es sich bei den langfristigen Krediten ausschließlich um Annuitätendarlehen handelt, ist der Tilgungsanteil nicht konstant, sondern ergibt sich aus der Differenz von (über die Jahre konstanter) Annuität und Zinsanteil:

Tilgung $(t)=$ Annuität $-\operatorname{Zinsen}(t)$.

Damit gilt für die bisher geleisteten (zwei) Tilgungen $(J=3$, s.o.):

$$
\sum_{j=1}^{J-1} \operatorname{Tilgung}(t-j)=(J-1) \cdot \text { Annuität }-\sum_{j=1}^{J-1} \operatorname{Zinsen}(t-j)
$$

und für die Restschulde(t):

$$
\begin{aligned}
& \text { Restschulde }(t, J)=\text { Kreditsumme }-\left[(J-1) \cdot \text { Annuität }-\sum_{j=1}^{J-1} \operatorname{Zinsen}(t-j)\right] \\
& \text { Restschulde }(t, J)=\text { Kreditsumme }-(J-1) \cdot \text { Annuität } \quad+\sum_{j=1}^{J-1} \operatorname{Zinsen}(t-j) .
\end{aligned}
$$

Das Unternehmen kann in allen Jahren und nicht nur vor $J$ Jahren, wie bisher untersucht, einen Kredit in wechselnder Höhe aufnehmen. Aus Vereinfachungsgründen ist von einer bestimmten Kreditart, definiert durch Laufzeit $N$ und Zinssatz $i$, pro Periode jeweils nur die Aufnahme eines Kredits möglich. Für vorgegebene Laufzeit und vorgegebenen Zinssatz kann daher jeder Kredit unverwechselbar durch sein Alter bestimmt und eindeutig mit $J$ indiziert werden ( $N, i$ gegeben). Ebenso können Zinsen, Tilgung und Restschulden einer Kreditart eindeutig mit $J$ indiziert werden, sofern $J$ in Periode $t+1$ ebenfalls um Eins erhöht wird.

Nehmen wir nun als Beispiel einen Kredit, der (am Anfang des Jahres) 0 aufgenommen wurde und eine Laufzeit von $N=4$ hat. Dieser Kredit ist somit in den Jahren 0, 1, 2 und 3 zurückzuzahlen. Positive Valuta vor der Tilgung hat dieser Kredit in den gleichen Jahren. Für die gesamten Restschulden (mit Krediten mit 4jähriger Laufzeit) am Ende des Jahres 3 sind daher nur die Restschulden der Kredite zu berücksichtigen, die 0 [Restschulde $\left.(0,4)^{221}\right]$ und später aufgenommen wurden, also nicht älter als 4 Jahre sind.

In den folgenden Formeln bezeichnet das vorletzte Argument (oder Index) der Funktion Restschuld in der ersten Gleichung das Kreditaufnahmejahr und in den folgenden das Alter, das letzte Argument in allen Gleichungen die Laufzeit. Die gesamten Restschulden von Krediten mit 4jähriger Laufzeit am Ende des Jahres 3, genannt Restschuldene $(3,4)$, betragen also:

Restschulden $^{e}(3,4)=$ Restschulde $^{e}(3,4)+$ Restschulde $^{e}(2,4)+\operatorname{Restschuld}^{e}(1,4)+$ Restschulde $(0,4)$

221 Der zweite Index bezeichnet hier die Laufzeit des Kredites und nicht sein Alter wie in Rest$\operatorname{schuld}^{e}(t, J)$. 
$\operatorname{Restschulden}^{e}(t, 4)=\operatorname{Restschuld}^{e}(1,4)+\operatorname{Restschuld}^{e}(2,4)+\operatorname{Restschuld}^{e}(3,4)+\operatorname{Restschuld}^{e}(4,4)$

$\operatorname{Restschulden}^{e}(t, N)=\sum_{J=1}^{N} \operatorname{Restschuld}^{e}(J, N)$

$$
=\sum_{J=1}^{N}\left[\operatorname{Kreditsumme}(J, N)-(J-1) \cdot \operatorname{Annuität}(J, N)+\sum_{j=1}^{J-1} \operatorname{Zinsen}(t-j, J, N)\right] .
$$

Die Unternehmen können in zwei Objekte mit jeweils unterschiedlicher Nutzungsdauer $(N)$ investieren. Da bei langfristigen Krediten stets fristenkongruent finanziert wird, sind zwei $(a N)$ Kreditlaufzeiten ${ }^{222}$ zu berücksichtigen. Die Kredite werden zu zwei (ai) Zinssätzen ${ }^{223}$ (i) angeboten. Dadurch sind insgesamt vier Kreditarten zu berücksichtigen. Die Summe aller RESTSCHULDENe der langfristigen Kredite im Jahre $t$ ist daher:

$$
\begin{gathered}
\operatorname{RESTSCHULDENe}(t)=\sum_{N=1}^{a N} \quad \sum_{i=1}^{a i} \operatorname{Restschulden} e(t, i, N) \\
=\sum_{N=1}^{a N} \sum_{i=1}^{a i} \sum_{J=1}^{N} \operatorname{Restschulde}_{(t, J, i, N)} \\
=\sum_{N=1}^{a N} \sum_{i=1}^{a i} \quad \sum_{J=1}^{N}[\operatorname{Kreditsumme}(J, i, N)-(J-1) \cdot \operatorname{Annuität}(J, i, N) \\
\left.+\sum_{j=1}^{J-1} \operatorname{Zinsen}(t-j, J, i, N)\right] .
\end{gathered}
$$

Es wird also differenziert zwischen (jeweils im Jahr $t$, daher ist dieser Index weggelassen):

- der Restschuld eines konkreten Kredits, spezifiziert durch Alter, Laufzeit und Zinssatz:

Restschulde $(J, i, N)$,

- der Restschuld einer Kreditart beliebigen Alters, gekennzeichnet durch Laufzeit und Zinssatz:

Restschuldene $(i, N)$,

- und der Summe aller langfristigen Verbindlichkeiten, den RESTSCHULDENe.

Neben der Verschuldung des Betriebes ist von Interesse, welchen Kapitaldienst er zu leisten hat. Für die in einer Periode $t$ zu zahlenden Zinsen für alle langfristigen Kredite gilt mit der für RESTSCHULDEN eingeführten Notation:

$$
\text { Zinsen }(t) \quad=\sum_{N=1}^{a N} \sum_{i=1}^{a i} \sum_{J=1}^{N} \operatorname{Zins}(t, J, i, N) .
$$

Der Zins $(t, J, i, N)$ ist jeweils die in $t$ zu entrichtende Verzinsung eines konkreten Kredits, spezifiziert durch Alter, Laufzeit und Zinssatz: Zins $(t, J, i, N)$. Entsprechend wird

\footnotetext{
$222 a N=$ Anzahl Nutzungsdauern

$223 a i=$ Anzahl Zinsfüße
} 
unter Tilgung $(t, J, i, N)$ der in $t$ fällige Rückzahlungsbetrag eines konkreten Kredites verstanden, so daß sich für die Tilgungen insgesamt analog ergibt:

$$
\text { Tilgungen }(t) \quad=\sum_{N=1}^{a N} \sum_{i=1}^{a i} \sum_{J=1}^{N} \operatorname{Tilgung}(t, J, i, N) \text {. }
$$

Für die Valuta nach der Tilgung folgt:

${ }^{n T} \operatorname{RESTSCHULDENe}(t)=\operatorname{RESTSCHULDENe}(t)-\operatorname{Tilgungen}(t)$.

Die so bestimmten Restschulden, Zinsen und Tilgungen tragen positive Vorzeichen!

Der langfristige, gesamte oder totale Kapitalsaldo errechnet sich somit, wie zu Beginn des Abschnittes eingeführt, zu:

\begin{tabular}{|l|l|l|l|}
\hline \multirow{2}{*}{ gesamter Kapitalsaldo } & $=$ & & berechnet in Unterprogramm: \\
\cline { 3 - 4 } & + & Fortschreibung \\
neue Liquidität & Fortschreibung \\
RESTSCHULDENe & Fortschreibung \\
& - & Tilgung & Fortschreibung \\
\hline
\end{tabular}

Diese Größe wird im Ergebnisausdruck in Unterabschnitt 9.6.5 unter tKapSaldo ausgewiesen.

\subsubsection{Bestimmung des gesamten Zinssaldos}

Im Tableau wird der kurzfristige Zinssaldo (s. S. 348 bestimmt. Hinzu kommen die Zinsen für die langfristigen Kredite:

\begin{tabular}{|l|l|l|}
\hline gesamter Zinssaldo $=$ & + & $\begin{array}{l}\text { kurzfristiger Zinssaldo } \\
\text { Zinsen für langfristige Kredite }\end{array}$ \\
\hline
\end{tabular}

Diese Größe wird im Ergebnisausdruck unter ZinsSaldo ausgewiesen.

\subsubsection{Fortschreibung der Kreditobergrenzen}

Die im Tableau ausgewiesenen Kreditobergrenzen limitieren die Gesamtverschuldung mit den einzelnen Krediten. Der in einer Folgeperiode noch verfügbare Kreditspielraum bestimmt sich daher aus der im Tableau genannten Obergrenze (Kreditspielraum $\left._{0, i}\right)$ abzüglich der Restschulden dieser Kreditart nach der Tilgung.

\begin{tabular}{|l|l|l|}
\hline Kreditspielraum $_{t+1, i}=$ & + & $\begin{array}{l}\text { Kreditspielraum }_{0, i} \\
\text { Restschuld }_{t, i} \text { nach der Tilgung }\end{array}$ \\
\hline
\end{tabular}

$i$ steht für die zwei limitierten langfristigen Kreditarten (vgl. Unterabschnitt 9.6.2.3. Die einperiodischen Kredite werden nach jeder Runde zurückgezahlt, daher ist der Kreditspielraum bei kurzfristigen Krediten in jeder Periode gleich. Allerdings erfordert 
eine negative Anfangsliquidität die sofortige Aufnahme von kurzfristigen Krediten, so daß der verbleibende nutzbare Kreditspielraum nicht konstant ist.

\subsubsection{Eigenkapital}

Das Eigenkapital setzt sich aus der Differenz von Vermögenswerten und Verbindlichkeiten zusammen. Das Sachvermögen besteht aus dem Zeitwert der Maschinen und Gebäude (lineare Abschreibung, s.o.). Es wird im Ergebnisausdruck als SachAVerm jeweils für das Ende der Periode nach erfolgter Abschreibung ausgewiesen. Etwaige Finanzvermögen geht in die neue Liquidität ein. Daher gilt: Kurzfristige Verbindlichkeiten sind wegen der einjährigen Laufzeit am Ende einer Periode selbstverständlich getilgt, sie gehen als unmittelbarer Kreditbedarf für die nächste Periode negativ in das Eigenkapital ein.

\begin{tabular}{|c|c|c|}
\hline$E K^{e} e_{t}=$ & $\begin{array}{l}+ \\
- \\
+\end{array}$ & $\begin{array}{l}\text { Maschinen- und Gebäudezeitwert (Sachanlagevermögen, SachAVerm) } \\
\text { Restschulden der langfristigen Verbindlichkeiten nach Tilgung }{ }^{n T} R E S T \text { - } \\
\text { SCHULDENe }{ }_{t} \text {, im Soll positives Vorzeichen), Gleichung (356.1) } \\
\text { neue Liquidität (Liquil }{ }_{t+1} \text {, im Soll negatives Vorzeichen), } \\
\text { Gleichung (B53. L), enthält Finanzanlagen bzw. kurzfristigen Kreditbedarf }\end{array}$ \\
\hline
\end{tabular}

\subsubsection{Bestimmung des Gewinns}

Das LP-Tableau dient zur simultanen Optimierung des Produktions-, Investitions- und Finanzierungsprogramms in der aktuellen Periode. Der Zielwert dieser Planungsrechnung weicht daher vom Gewinn als einem Begriff aus der Erfolgsrechnung in der Regel ab. Um vom Zielwert des Tableaus zum Periodengewinn zu gelangen, sind folgende Weiterrechnungen erforderlich:

Der Zielwert berücksichtigt nur die Annuitäten der Investitionen in der aktuellen Periode; die Annuitäten der Altinvestitionen sind daher noch abzuziehen, sofern die Nutzungsdauer der Investitionsgüter noch nicht überschritten ist (Buchstabe a in untenstehender Aufstellung).

Bei Investitionen aus Eigenmitteln sind nur die Abschreibungen gewinnwirksam. In der Zielfunktion haben solche Investitionen jedoch abweichende Auswirkungen. Zum einen ist die Annuität, also Abschreibung und Zinsanspruch, notiert, wie dies für eine Planungsrechnung erforderlich ist. Zum anderen ermöglichen Investitionen mit Eigenkapital im gleichen Umfange die Aktivität „,entgangene Zinsen“. Da Investitionen mit Eigenkapital liquiditätswirksam sein müssen (vgl. ,Maschineninvestitionen mit EK auf Seite 345, ist diese Aktivität erforderlich, um die durch eine Investition mit Eigenkapital verloren gehende Möglichkeit von Finanzanlagen auszugleichen und so eine „Doppelzählung“ der Zinsen zu vermeiden. Daher beeinflussen Investitionen mit Eigenkapital die Zielfunktion dreifach: durch die Annuität, durch nichtmögliche 
Finanzanlagen (Zinsverlust: $A \cdot i$ ) und durch die Aktivität „entgangene Zinsen“ (Zielbeitrag: $A \cdot i$ ). Der Zielbeitrag von Investitionen aus Eigenkapital bestimmt sich also $\mathrm{zu}:$

\begin{tabular}{c|c}
\hline verbal: & in Symbolen: \\
\hline - Annuität & - Annuität \\
- Reduzierung der Finanzanlagen & $-A \cdot i$ \\
+ Aktivität ,entgangene Zinsen“ & $+A \cdot i$
\end{tabular}

Die beiden letzten Effekte gleichen sich aus. Damit reduzieren Investitionen mit Eigenkapital den Zielwert lediglich um die Annuität. Für den Gewinn wird aber nur die Abschreibung benötigt. Daher ist der Zinsanspruch dem Zielwert zur Bestimmung des Gewinns wieder zuzuschlagen (Buchstabe b in untenstehender Aufstellung). ${ }^{224}$

Für Investitionen aus Eigenkapital in Vorperioden sind ebenfalls die zugehörigen Abschreibungen vom Zielwert in Abzug zu bringen, um zum Gewinn zu gelangen (Buchstabe c). Es ergibt sich zusammengefaßt:

\begin{tabular}{|l|l|l|}
\hline Gewinn & $=$ & Zielwert \\
& - & Annuität der Investitionen mit Fremdkapital in den Vorperioden (a) \\
& + & Zinsanspruch von Investitionen mit Eigenkapital in der aktuellen Periode (b) \\
& - & Abschreibung von Investitionen mit Eigenkapital in den Vorperioden (c) \\
\hline
\end{tabular}

\subsubsection{Zusammenfassung von Kennziffern zur Betriebsbeschreibung}

In der nachfolgenden Tabelle werden die in diesem Unterabschnitt 9.6.3 abgeleiteten Variablen zur Beschreibung der betrieblichen Entwicklung zusammengefaßt dargestellt. In der rechten Spalte ist notiert, wofür die Variable benötigt wird bzw. in welchem Unterprogramm des Simulationsprogramms, das in Unterabschnitt 9.6.4 dokumentiert ist, sie berechnet wird.

\footnotetext{
224 Es ist auch möglich, ein Tableau ohne die Aktivität „entgangene Zinsen“ zu konstruieren. Dann ist in der Zielfunktion statt Annuität die Größe $(-$ Annuität $+A \cdot i)$ zu verwenden.

- (Annuität $-A \cdot i)$ läßt sich umformen zu:

$-(A b+A \cdot f \cdot i-A \cdot i)$

$-(A b+(f-1) \cdot A \cdot i)$

und ist daher Abschreibung plus der Verzinsung des durchschnittlichen Komplementärwertes. Da einheitlich Annuitäten in der Zielfunktion stehen sollen, wird dieser Weg allerdings nicht gewählt.
} 
Tabelle 359.1: Kennziffern zur Betriebsbeschreibung

\begin{tabular}{|c|c|c|}
\hline & & enthalten in \\
\hline $\begin{array}{c}\text { kZiSald } \\
\text { (kurzfristiger Zinssaldo) }\end{array}$ & $\begin{array}{l}= \\
-\quad \text { Zinsen für kurzfristige Kreditaktivitäten } \\
-\quad \text { Zinsen für kurzfristige Investitionskredite } \\
+\quad \text { Zinsen für Finanzanlagen }\end{array}$ & $\begin{array}{l}\text { Rest im LP-Ergebnis } \\
\text { Aktivität im Tableau } \\
\text { Aktivität im Tableau }\end{array}$ \\
\hline $\begin{array}{c}\text { EinAusS } \\
\text { (Einnahmen-Ausgaben- } \\
\text { Saldo im Tableau) }\end{array}$ & $\begin{array}{ll}= & \\
+ & \sum \text { Deckungsbeiträge } \\
- & \text { Auszahlungen für Pacht, AK etc. } \\
- & \text { Investitionen mit kurzfristigem Fremd- oder } \\
& \text { Eigenkapital } \\
+ & \text { kurzfristiger Zinssaldo }\end{array}$ & $\begin{array}{l}\text { Rest im LP-Ergebnis } \\
\text { Aktivität im Tableau } \\
\text { Aktivität im Tableau } \\
\text { Aktivität im Tableau } \\
\text { Rest im LP-Ergebnis }\end{array}$ \\
\hline $\begin{array}{c}\text { EASav } \\
\text { (Einnahmen-Ausgaben- } \\
\text { Saldo, vollständig) }\end{array}$ & $\begin{array}{l}= \\
+ \text { EinAusS } \\
- \text { Kapitaldienst für langfristiges Fremdkapital } \\
- \text { Entnahmen }\end{array}$ & $\begin{array}{l}\text { Fortschreibung } \\
\text { Rest im LP-Ergebnis } \\
\text { Fortschreibung } \\
\text { Fortschreibung }\end{array}$ \\
\hline neue Liquidität & $\begin{array}{l}= \\
+ \text { alte Liquidität } \\
+ \text { EASav }\end{array}$ & $\begin{array}{l}\text { für neues Tableau } \\
\text { RHS im altem Tableau } \\
\text { Fortschreibung }\end{array}$ \\
\hline $\begin{array}{c}\text { kKapSal } \\
\text { (kurzfristiger Kapitalsaldo) }\end{array}$ & $\begin{array}{l}= \\
\text { - } \text { kurzfristige Kreditaktivitäten } \\
\text { - } \quad \text { kurzfristige Investitionskredite } \\
+ \text { Finanzanlagen }\end{array}$ & $\begin{array}{l}\text { Rest im LP-Ergebnis } \\
\text { Aktivität im Tableau } \\
\text { Aktivität im Tableau } \\
\text { Aktivität im Tableau }\end{array}$ \\
\hline $\begin{array}{c}\text { Eigenkap } \\
\text { (Eigenkapital) }\end{array}$ & $\begin{array}{l}= \\
+ \text { Sachanlagevermögen } \\
-\quad \text { Restschulden nach Tilgung } \\
+ \text { neue Liquidität }\end{array}$ & $\begin{array}{l}\text { Fortschreibung } \\
\text { Fortschreibung } \\
\text { Fortschreibung } \\
\text { Fortschreibung }\end{array}$ \\
\hline $\begin{array}{c}\text { ZiSaldo } \\
\text { (gesamter Zinssaldo) }\end{array}$ & $\begin{array}{l}= \\
+\quad \text { kurzfristiger Zinssaldo } \\
-\quad \text { Zinsen für langfristige Kredite }\end{array}$ & $\begin{array}{l}\text { Fortschreibung } \\
\text { Rest im LP-Ergebnis } \\
\text { Fortschreibung }\end{array}$ \\
\hline $\begin{array}{c}\text { tKapSaldo } \\
\text { (gesamter Kapitalsaldo) }\end{array}$ & $\begin{array}{l}= \\
+ \text { neue Liquidität } \\
-\quad \text { Restschulden nach Tilgung }\end{array}$ & $\begin{array}{l}\text { Fortschreibung } \\
\text { Fortschreibung } \\
\text { Fortschreibung }\end{array}$ \\
\hline
\end{tabular}




\begin{tabular}{|c|c|c|}
\hline Gewinn & $\begin{aligned}= & \\
+ & \text { Zielwert } \\
- & \text { Annuität der Investitionen mit Fremdkapital } \\
& \text { in den Vorperioden } \\
+ & \text { Zinsanspruch von Investitionen mit Eigen- } \\
& \text { kapital in der aktuellen Periode } \\
- & \text { Abschreibung von Investitionen mit Eigen- } \\
& \text { kapital in den Vorperioden }\end{aligned}$ & $\begin{array}{l}\text { LP-Ergebnis } \\
\text { Fortschreibung } \\
\text { Fortschreibung } \\
\text { Fortschreibung }\end{array}$ \\
\hline \multicolumn{3}{|c|}{ Kontrollrechnungen des gesamten Kapitalsaldos: } \\
\hline tKapSaldo & $\begin{array}{l}= \\
\text { - Restschulden nach Tilgung } \\
+ \text { alte Liquidität } \\
+ \text { Einnahmen-Ausgaben-Saldo, vollständig }\end{array}$ & $\begin{array}{l}\text { Fortschreibung } \\
\text { Fortschreibung } \\
\text { RHS im Tableau } \\
\text { Fortschreibung }\end{array}$ \\
\hline tKapSaldo & $\begin{array}{l}= \\
\text { - Restschulden nach Tilgung } \\
+ \text { alte Liquidität } \\
+ \text { Einnahmen-Ausgaben-Saldo } \\
\text { - Kapitaldienst für langfr. Verbindlichkeiten } \\
\text { - Entnahmen }\end{array}$ & $\begin{array}{l}\text { Fortschreibung } \\
\text { Fortschreibung } \\
\text { RHS im Tableau } \\
\text { Rest im LP-Ergebnis } \\
\text { Fortschreibung } \\
\text { Fortschreibung }\end{array}$ \\
\hline tKapSaldo & 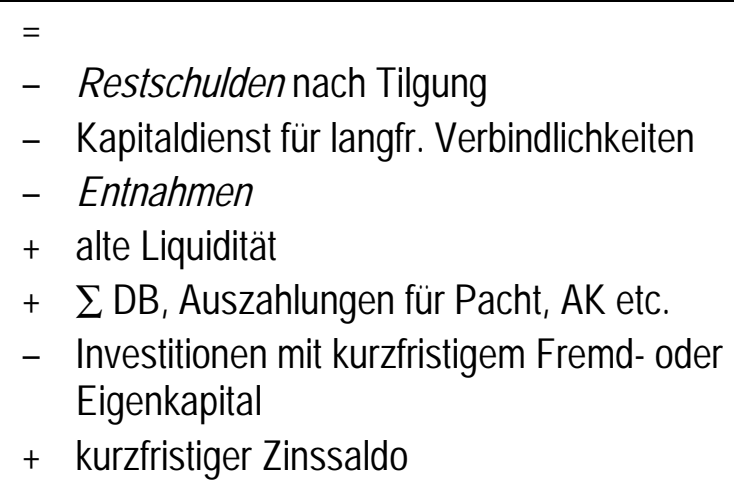 & $\begin{array}{c}\text { Fortschreibung } \\
\text { Fortschreibung } \\
\text { Fortschreibung } \\
\text { Fortschreibung } \\
\text { RHS im Tableau } \\
\text { Aktivitäten i. Tableau } \\
\text { Aktivitäten i. Tableau } \\
\text { Rest im LP-Ergebnis }\end{array}$ \\
\hline tKapSaldo & 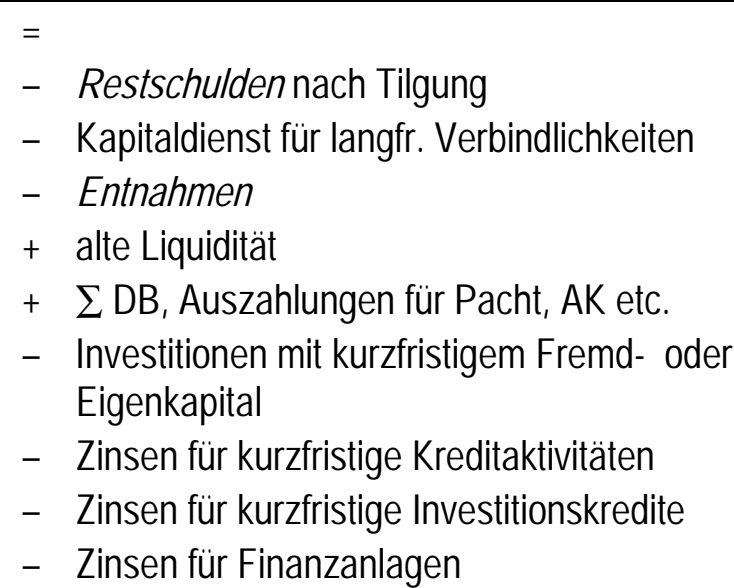 & $\begin{array}{c}\text { Fortschreibung } \\
\text { Fortschreibung } \\
\text { Fortschreibung } \\
\text { Fortschreibung } \\
\text { RHS im Tableau } \\
\text { Aktivitäten i. Tableau } \\
\text { Aktivitäten i. Tableau } \\
\text { Rest im Tableau } \\
\text { Aktivität im Tableau }\end{array}$ \\
\hline
\end{tabular}




\subsubsection{Das Simulationsprogramm}

Mit diesem Programm und einigen weiteren kleineren, die hier nicht wiedergegeben werden, wurden die Ergebnisse des Betriebsentwicklungsmodells in Abschnitt 6.4 erzielt. Alle Programme sind beim Autor (hinners@iamo.uni-halle.de erhältlich.

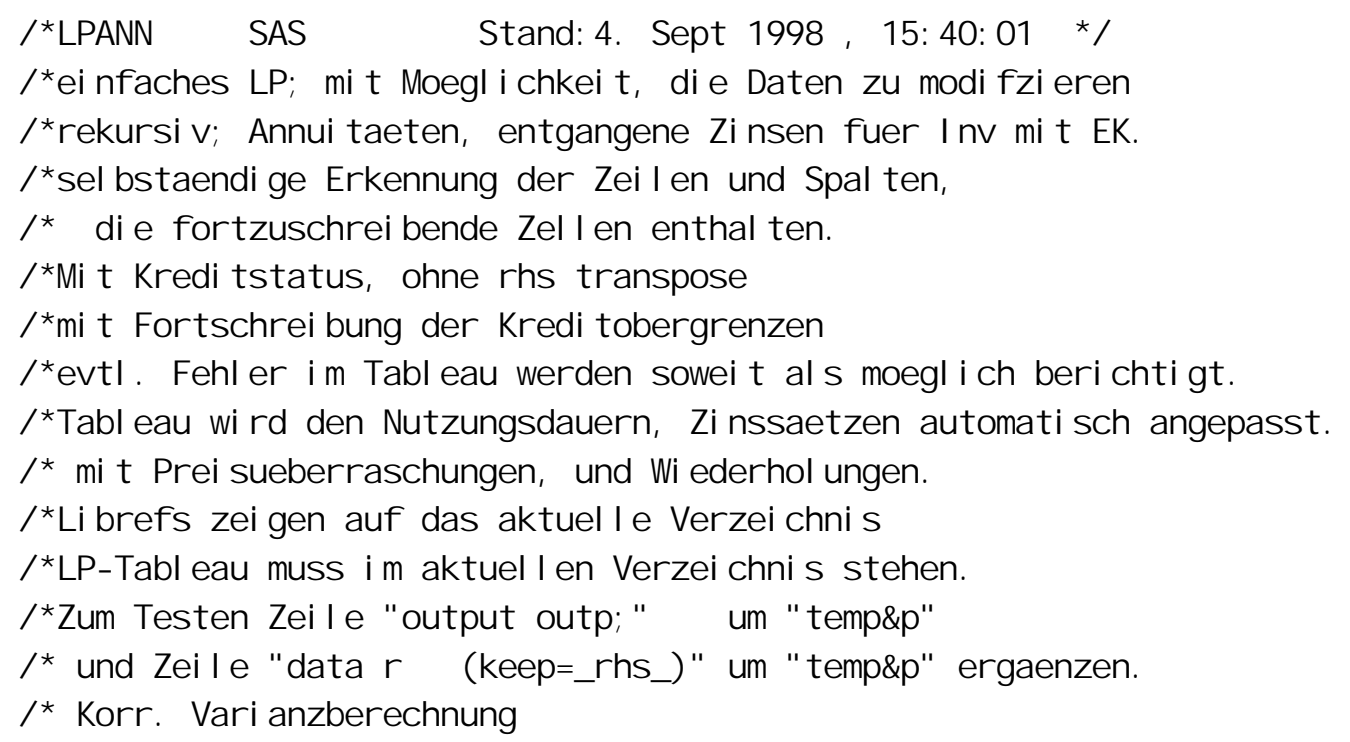

* Die folgenden 3 Variablen werden ggfs. schon beim Aufruf uebergeben.

$\% g l o b a l$ St Wh EndWh aWh;

$\%$ macro init:

$$
\text { \%if \&sysenv=FORE I*im Di alogbetrieb* }
$$

or \&St Wh<0 \%then \%do;

$\%$ put Bed. erf.;

I*Diese Zuweisungen werden nur wirksam, wenn fuer St Wh kein init-Stmt vorliegt*I

$\%$ let St Wh $=1$; Nummer der 1 . Wi ederhol ung

$\%$ let EndWh $=100 ; \quad$ *Nummer der l etzten Wahlg

$\%$ let aWh $=\%$ eval $(\& E n d W h-\& S t W h+1)$;

\%end;

\%mend init; \%init; run;

\%put Wiederholungen von \&St Wh bis \&EndWh, al so \&aWh Wiederhol ungen mit je \&aPer Perioden;

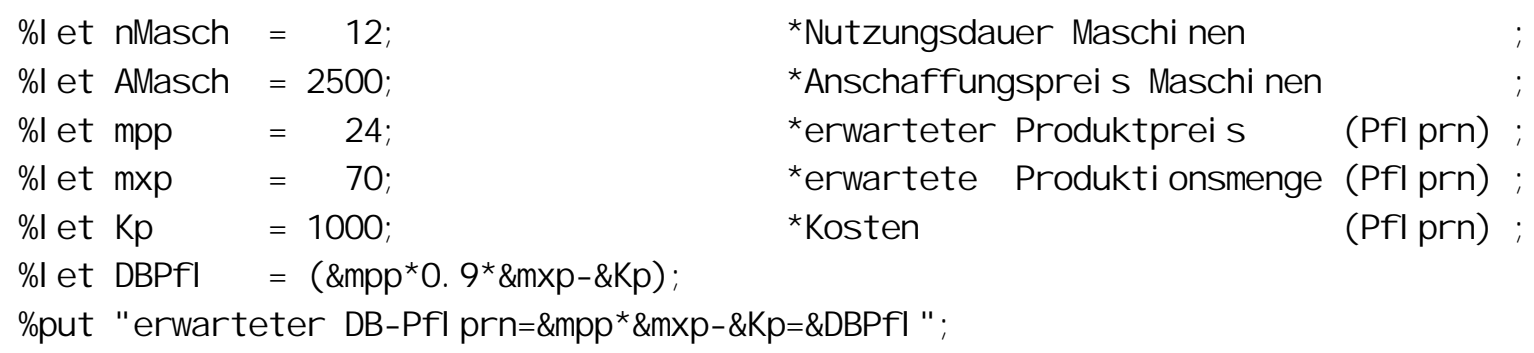




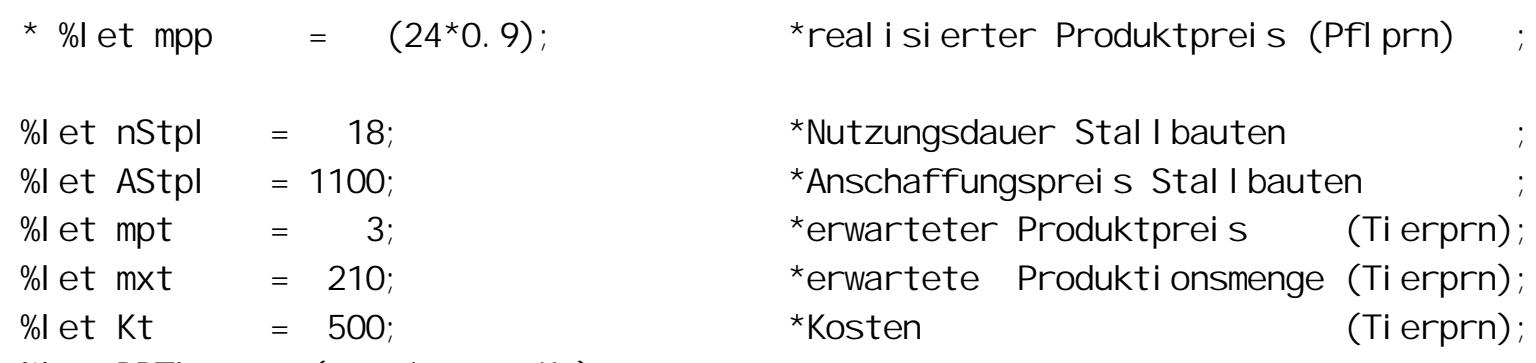

$\%$ et DBTier $=(\& m p t * \& m x t-\& K t)$;

\%put "erwarteter $D B-T i$ erprn=\&mpt*\&mxt $-\& K t=\& D B T i$ er " ;

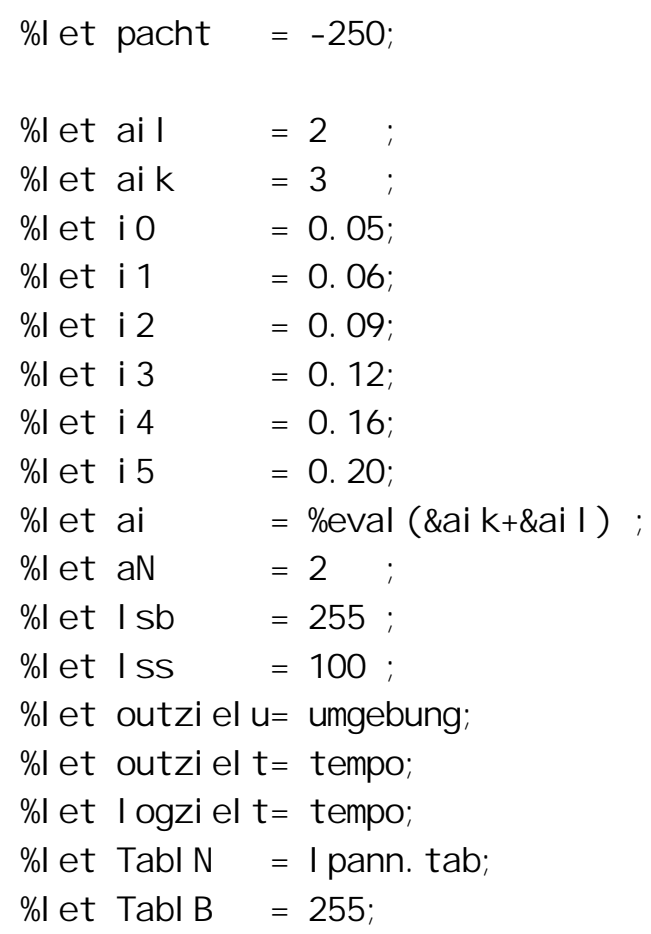

*Pachtpreis

* Anzahl Zi nssaetze fuer Ifr. Kredite;

*Anzahl Zi nssaetze fuer kfr. Kredite;

* Zinssatz fuer Finanzanlagen

*Zinssatz fuer Ifr. Kredit 1

*Zinssatz fuer Ifr. Kredit 2

*Zinssatz fuer kfr. Kredit 1

*Zi nssatz fuer kfr. Kredit 2

*Zinssatz fuer kfr. Kredit 3

*Anzahl Zinssaetze insgesamt

*Anzahl Nutzungsdauern

* Listingbreite fuer Tableaus

* Listingbreite sonst

* Zi el fuer den Umgebungs-Output

* Zi el fuer den zu ueberschr. Output

*Ziel fuer das zu ueberschr. Logfile

* Name des LP-Tableaus

*Breite des LP-Tableaus

* \%let outzielu=print;

*Zi el fuer den zu ueberschr. Output ;

* \% et logzielt $=\log$;

* Zi el fuer das zu ueberschr. Iogfile;

* Parameter der symmetrischen Dreiecksverteil ungen, haeufigster Wert=Erw. wert. ; *u. . = Abweichung nach unten von $m, 0_{\ldots}$. = Abweichung nach oben von $m$.;

* Jeweils in \% vom Mittel wert.;

$\%$ et $u p p=10 ; \%$ et $O p p=10 ; \quad$ *Preisschwankungen in der Planzenproduktion; $\%$ et $u \times p=0 ; \%$ et $0 \times p=0 ; \quad$ *Ertragsschwankungen in der Planzenproduktion; $\%$ let upt $=0 ; \%$ et opt $=0 ; \quad$ *Preisschwankungen in der Tierproduktion $\%$ et $u x t=0 ; \%$ et $0 x t=0 ; \quad$ Eertragschwankungen in der Tierproduktion

$\%$ et dana = w\%eval $($ \&awh/100)p\&upp.t \&upt; *Name des Datensatzes

$*<100: 0, \quad<200: 1,<300: 2,300: 3$;

options noovp ps $=56$ nomprint nomlogic nosource nosymbolgen I $s=\& \mid s s$ nofullstimer;

\%let Aktivita $=$ PfIPrn Malnvek MallKr1 MallKr2 $\quad$ MalkKr1 - MalkKr3

TierPrn StInvEK StIIKr1 StIIKr2 StIkKr1 - StlkKr3

Ak AKh Pacht EntgZi FinAnl kFK1 - kFK3; 
*Aktivitaeten, di e im Ergebni sausdruck aufgef uehrt werden sollen.

(Anfangsbuchstabe $r$ bedeutet, diese "Aktiviaet" ist eine Restriktion). ;

$\%$ et outAkt = rziel Gewinn PfIPrn rMalnv Tierprn rstIIInv

$\begin{array}{llllll}\text { AK AKh Pacht FinAnl IKrl IKr2 } & \end{array}$

$k I K r 1 \quad k l K r 2 \quad k l k r 3 \quad k F K 1 \quad k F K 2 \quad k F K 3$

ITilg rkkapsal KapSaldo Zi Saldo SAVerm Eigenkap;

*Kapazitaeten, die im Ergebnisausdruck aufgefuehrt werden sollen;

$\%$ et outKapaz= MaKapaz Stallpl aLi OglFK1 OglFK2;

*Reste, die im Ergebnisausdruck aufgef uehrt werden sollen

$\%$ et outreste= rFlaeche rMaKapaz rStallpl raKStd rDE

rOgkFK1 rOgkFK2 rOgIFK1 rOgIFK2 rliqui

$\%$ et outsonst $=\mid$ per wh surv pp; *sonstige Variablen im Ergebnisausdruck

\%global p AnzVart AnzZeil t AnzZeile surv

AnLaMaKr AnLaStKr AnLaMaKz AnLaStKz AnzLags

MalnvEK I KrM1 IKrM2 kKrM1 kKrM2 kKrM3 Ml $/$ *Zeilennr. im * *

StInvEK IKrS1 IKrS2 kKrS1 kKrS2 kKrS3 SI /* LP-Ergebnisvektor *

zLi $\quad$ *Zeilennr. im Tableau *I

OglFK1 OglFK2 OgkFK1 OgkFK2 $\quad$ *Kreditobergrenzen *

int v

rhsnamen ergnamen

I*Namen d. Restr. u. Ergebn*I

proc printto new print=\&outziel u; *output in Umgebung. I is, ohne new wuerde angehaengt;

\%macro datenein;

I*aufgerufen aus Haupt programm.

I*Einlesen des Tableaus (liegt als ASClI-File vor).

fil ename tabasc "\&TablN" I recl=260;

data |p.tab;

I*Die obersten 14 Zeilen (Kopfzeilen) werden nicht gelesen

infile tabasc firstobs $=14$ | recl $=\$ T a b \mid B$;

input nr_id_ \$ _type_ \$ _rhs_ EAktivita;

if _id $d_{-}=$int' then $d_{0}$;

I*folgende 2 Zeilen Schalter fuer Ganzzahligkeit

\%l et intvant ; _type $=$ 'FrEE';

I*

_type_ =' int eger';

end;

run; options $|s=\&| s b$;

proc print noobs;

title 'eingelesenes Tableau aus Rohdaten';

run; options $|s=\&| s s$;

I*Kennzahlen des LP-Tableaus bestimmen*/ 
I*Spaltenpositionen der numerischen Variablen im LP-Tableau bestimmen.

*Ablegen der in I p.tab enthal tenen Variablennamen in TabAddre;

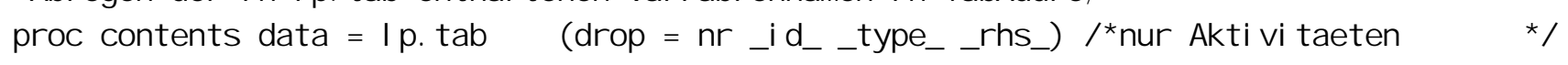
noprint out = Tabaddre (keep = name varnum);

* Sortieren der Variablennamen in TabAddre nach Position in Ip.tab;

proc sort data=TabAddre out =TabAddre (keep=name) ;

by varnum;

data_nul _ ;

set TabÄddre nobs =n ;

call symput('AnzVarT',n); $\quad$ *Anzahl der Aktivitaeten;

select (name);

when('PFLPRN' ) call symput ('sPf|Prn', $n_{-} n_{-}$);

when('MAI NVEK' ) call symput ('sMalnvEK', _ $n_{-}$);

when('MAILKR1' ) cal I symput ('sMall Kr1', $n_{-}$);

when('MAILKR2' ) call symput ('sMall Kr2', , ${ }_{-}$);

when('MAI KKR1' ) call symput ('sMal kKr1', $n_{-}$);

when('MAIKKR2' ) call symput ('sMal kKr2', $\left.{ }_{-} n_{-}\right)$;

when('MAIKKR3' ) call symput ('sMalkKr3', $n_{-}$);

when('TIERPRN' ) call symput ('sTierPrn', ${ }_{-} n_{-}$);

when('STINVEK' ) call symput ('sStI nvEK', , $n_{-}$);

when('STILKR1' ) call symput ('sStIIKr1', _ n_);

when('STILKR2' ) call symput ('sStIIKr2', $n_{-}$);

when('STIKKR1' ) call symput ('sStIKKr1', $\left.{ }_{-} n_{-}\right)$;

when('STIKKR2' ) call symput ('sStIkKr2', $\left.n_{-}\right)$;

when('STIKKR3' ) call symput ('sStIkKr3', $\left.n_{-}\right)$;

when('AK' ) call symput ('sAK', , $n_{-}$);

when('AKH' ) call symput('sAKh', , $n_{-}$);

when('PACHT' ) call symput('sPacht', $\left.n_{-}\right)$;

when('ENTGZI' ) call symput ('sEntgZi', , $n_{-}$);

when('FINANL' ) call symput ('sFinAnl', , $n_{-}$);

when('KFK1' ) call symput ('skKredil', $\left.{ }_{-} n_{-}\right)$;

when('KFK2' ) call symput ('skKredi2', ${ }_{-}{ }_{-}$);

when('KFK3' ) call symput ('skKredi3', ${ }_{-}{ }_{-}$);

otherwi se;

end;

I*Anzahl Zeilen, Zeilennummern ausgewaehlter Restriktionen bestimmen*

I *Kreditobergrenzen auf gleichnamige Makrovariablen Iegen. */

data_null _;

set $\mid p, t a b\left(d r o p=n r_{-}\right.$type_ $) \quad$ nobs $=n$ end =end;

call symput('AnzZeiliT',n); *Anzahl Zeilen;

select (_id $\left.d_{-}\right)$;

when ("Ziel" ) call symput('zZi', , $n_{-}$);

when ("Liqui") call symput ('zLi', , ${ }_{-}$) ;

when ("EKKorr") call symput('zEKK' , ${ }_{-}$) ;

when ("Og|FK1") do;

call symput ('OglFK1', , rhs_ _); 

end;

call symput('zOgIFK1', , $n_{-}$) :

when ("OglFK2" ) do;

call symput ('OglFK2', , rhs _);

end;

call symput (' $z O g \mid F K 2 ', n_{-}$);

when ("OgkFK1") do;

call symput ('OgkFK1', r rhs_);

end;

call symput('zOgkFK1', , $n_{-}$);

when ("OgkFK2") do;

call symput('OgkFK2', , rhs _ $_{-}$;

end;

call symput('zOgkFK2' , $\left.n_{-}\right)_{\text {); }}$

when ("kKapSal") call symput ('zkKapSal', $n_{-}$);

when ("kZiSald") call symput('zkZiSald', ${ }_{-}$- );

when ("EinAusS") call symput('zEA' , ${ }_{-}^{-}$);

otherwi se;

end;

length na $\$ 200$; retain na; *Namen der Restr. auf Macrovariable rhsnamen legen.;

$n a=\operatorname{trim}(n a) \| 1$ ' $\| I_{-} i d_{-}$;

if end then call symput('rhsnamen', na); run;

*konsistentes Anpassen des Tableaus an Vorgaben;

data $t$ t;

set I p.tab(drop=nr_id_ type_ _rhs_);

array az $\left\{\& A n z Z e i I T, \overline{\& A n z}_{\operatorname{Var} T\}}{ }_{-}\right.$temporary_;

array a _numeric_; *alle num. Var. von I p.tab auf array a;

do $i=1$ to \&Anzzeil T;

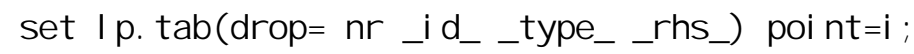

do $j=1$ to \&AnzVarT; $a 2\{i, j\}=a\{j\}$;

end;

end:

*Zi elfunktion (Annuitaeten, $D B$ und Zi nssaetze);

a $2\{\& z Z i \quad, \& S P f \mid \operatorname{Pr} n\}=\& D B P f I$;

a $2\{\& z Z i \quad, \& s \operatorname{TierPr} n\}=\& D B T i$ er ;

a $2\{\& z Z i \quad, \& s$ MalnvEK $\}=-\operatorname{mort}(\& A M a s c h, \ldots, \& i 0, \& n \operatorname{Masch}) ;$

a $2\{\& z Z i \quad, \& s \operatorname{Mal} \mid \operatorname{Kr} 1\}=-\operatorname{mort}(\& A M a s c h, \ldots, \& 1, \& n \operatorname{Masch}) ;$

a $2\{\& z Z i \quad, \& s$ Mal I Kr 2$\}=-\operatorname{mort}(\& A M a s c h, \ldots, \& i 2, \& n M a s c h) ;$

a $2\{\& z Z i \quad, \& s$ Mal kKr 1$\}=-\operatorname{mort}(\& A M a s c h, \ldots, \& 3, \& n M a s c h) ;$

a $2\{\& z Z i \quad, \& s$ Mal kKr 2$\}=-\operatorname{mort}(\& A M a s c h, \ldots, \& i$, \&nMasch);

a $2\{\& z Z i \quad, \& s \operatorname{MalkKr}\}\}=-\operatorname{mort}(\& A M a s c h, \ldots, \& i 5, \& n M a s c h) ;$

a $2\{\& Z Z i \quad, \& S S t \mid n v E K\}=-\operatorname{mort}(\& A S t p \mid, \ldots$, \& $0, \& n S t p \mid) ;$

a $2\{\& Z Z i \quad, \& S S t|| K r 1\}=-\operatorname{mort}(\& A S t p|, \ldots, \& i 1, \& n S t p|)$;

a2 $\{\& z Z i \quad, \& S S t|| K r 2\}=-\operatorname{mort}(\& A S t p \mid, \ldots$, \& 2, \&nStpl );

a $2\{\& z Z i \quad, \& S S t \mid k K r 1\}=-\operatorname{mort}(\& A S t p \mid, \ldots$, \& $3, \& n S t p \mid) ;$

a $2\{\& z Z i \quad, \& S S t \mid k K r 2\}=-\operatorname{mort}(\& A S t p \mid$, , \&i $4, \& n S t p \mid)$; 


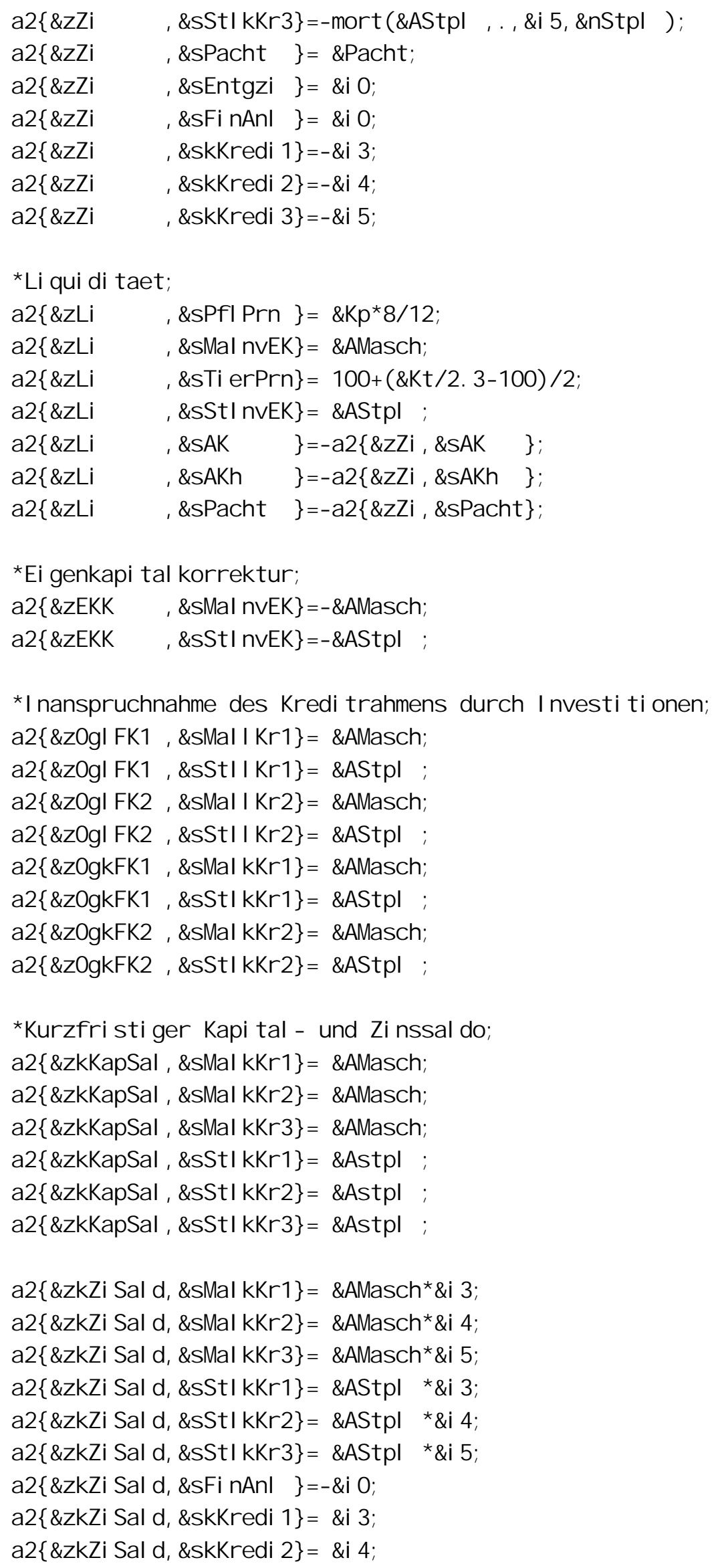


a $2\{\& z k Z i$ Sal d, \&skKredi 3$\}=\& i$;

*Ei nnahmen-Ausgaben-Saldo, idR an ZF angleichen;

a $2\{\& z E A \quad$ \&S MalnvEK $\}=\& A M a s c h$;

a $2\{\& z E A \quad$ \&s Mal kKr 1$\}=\& A M a s c h *(1+\& i 3) ;$

a $2\{\& z E A \quad, \& s \operatorname{Mal} k K r 2\}=\& A \operatorname{Masch} *(1+\& i 4) ;$

a $2\{\& z E A \quad, \& s \operatorname{MalkKr} 3\}=\& A M a s c h *(1+\& i 5)$;

a $2\{\& Z E A \quad, \& S S t \mid n v E K\}=\& A S t p I$;

a $2\{\& z E A \quad, \& S S t \mid k K r 1\}=\& A S t p \mid *(1+\& i 3) ;$

a $2\{\& z E A \quad, \& S S t \mid k K r 2\}=\& A S t p \mid *(1+\& i 4) ;$

a $2\{\& Z E A \quad, \& S S t \mid k K r 3\}=\& A S t p \mid *(1+\& i 5)$;

a $2\{\& Z E A \quad, \& S A K \quad\}=-a 2\{\& Z Z i, \& S A K \quad\} ;$

a2 $\{\& z E A \quad, \& S A K h \quad\}=-a 2\{\& z Z i, \& S A K h \quad\} ;$

a2 $\{\& z E A \quad, \& S P a c h t \quad\}=-a 2\{\& z Z i, \& s P a c h t\} ;$

a $2\{\& z E A \quad, \& s F i n A n l \quad\}=-\& i 0 ;$

a $2\{\& z E A \quad, \& s k \operatorname{kredi} 1\}=\& i 3 ;$

a2 $\{\& z E A \quad, \& s k \operatorname{kredi} 2\}=\& i 4 ;$

a $2\{\& z E A \quad, \& s k \operatorname{kredi} 3\}=\& i 5$;

I*korrekte Inhalte der Zellen des Tableaus rausschreiben. *I

do $i=1$ to \&AnzZeil T;

do $j=1$ to \&AnzVarT;

$a\{j\}=a 2\{i, j\}$;

end;

out put :

end;

st op; run;

I *Form des Ausgangstableaus wiederherstellen.

data Ip.tab;

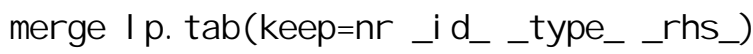

$t t(d r o p=j)$;

run; options $|s=\&| s b$;

proc print noobs:

title 'Tableau der 1. Runde nach automatischer Kontrolle und Anpassung';

\%mend datenein;

$\%$ macro datenman;

I *aufgerufen aus Haupt programm.

$* 1$

I*Modifikationsmoeglichkeit der Daten mit fsedit

I* und anschliessendes Rausschreiben als ASCII-File.

\%put macro datenman wird gestartet!;

fil ename tabasc "\&Tabl N" I recl=260;

proc f sedit data=lp.tab;

I*modifiziertes Tableau wird spaeter in Macro erstlauf gedruckt.

I*Ablegen des modifizierten Tableaus als ASClI-file. 
I*Separates Einlesen der 13 Kopfzeilen aus Rohdatenfile tabasc.

data kopfzeil;

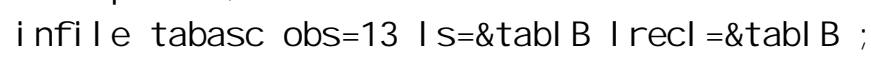

input text 1 \$char200. text 2 \$char\%eval (\&TablB-200-1).;

I*Das SAS-dataset kopfzeil enthaelt in den Variablen text 1 und

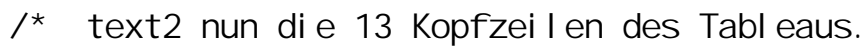

$* 1$

$* 1$

$* 1$

I*Erstellen der Kopfzeile fuer das neue Ascii-Tableau.

data_null _;

set kopfzeil;

file tabasc $\quad|s=\& t a b| B|r e c|=\& t a b \mid B$;

put text 1 \$char200. text 2 \$char\%eval (\&Tabl B-200-1).;

I*Positionsangaben muessen sein, da blanks abgeschn. werden

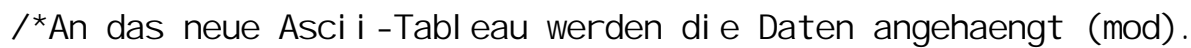

data_null_; *zweiter datastep erforderlich;

set | p.tab;

file tabasc mod $\quad|s=\& t a b| B|r e c|=\& t a b \mid B$;

put nr 2.' ' _ id_\$10. _type_\$10.-r_ rhs 10. (\&Aktivita)(10.2);

run;

\%mend datenman;

$\%$ macro erst Lauf;

$\%$ et $p=1 ; \%$ et $w h=\& S t W h ; * 1$. Periode, St Wh-t e Wi ederhol ung;

* Erzeugen von Tilgungsplaenen fuer alle z.V. stehenden Laufzeiten und Zinssaetze ; dat a Kreditfe $(d r o p=n)$;

$A=1$;

* Kr edi t s umme

$r=0 ; N d=0 ; a n n=0 ; a l=0 ; z i=0 ; t i=0 ;$

$N D=\& n$ Masch ;

do $N=1$ to \&an;

$\%$ do $j=0$ \%t o \&ail;

a $n n=\operatorname{mort}(A, \ldots, \& i \& j, N D)$;

rest schu=A;

$t i=0$;

do al =1 to ND;

$r=\& \& i \& j$;

rest schu=rest schu- $t i$;

$z i=r$ est schu*r;

$\mathrm{ti}=\mathrm{ann}-\mathrm{zi}$;

out put;

end;

\%end;

$N D=\& n S t p l \quad ; \quad$ *maximales Alter der 2. Kreditlaufzeit;

end;

I abel restschu = 'Restschuld am Per. Ende vor Tilgung'

ann $=$ 'Annuitaet'

zi $=$ ' Zinsen'
* nur zur Positionierung der Variablen

* Laufzeit des 1. Kredites

* Schleife ueber die Kreditlaufzeiten

* Schleife ueber die Zinssaetze (lfr. u. kfr.) ;

*Annuit aet

*Vorbelg. d. Restschuld mi t Kreditvol umen

*Vorbelg. d. Zinsen

* Schleife ueber die "Alter" eines Kredites

*aktueller Zi nssatz

* Restschuld am Per. Ende vor der Tilung

* Zi nsen

* Tilgung
$* 1$

$* 1$ 


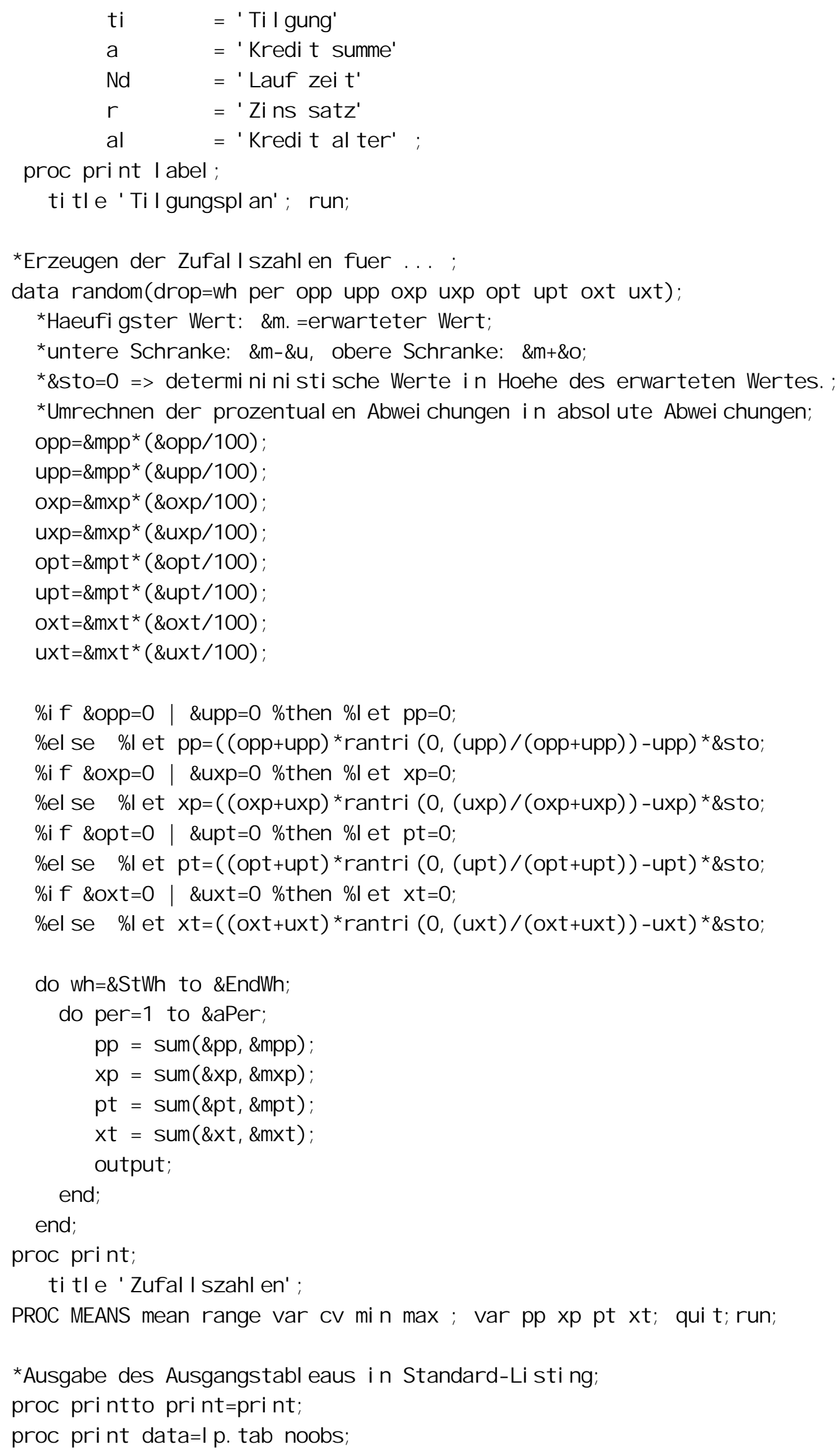


titlel "Tableau der 1. Runde: \&TablN";

title2 '(evtl. durch Macro datenman und Anpassungen modifiziert)';

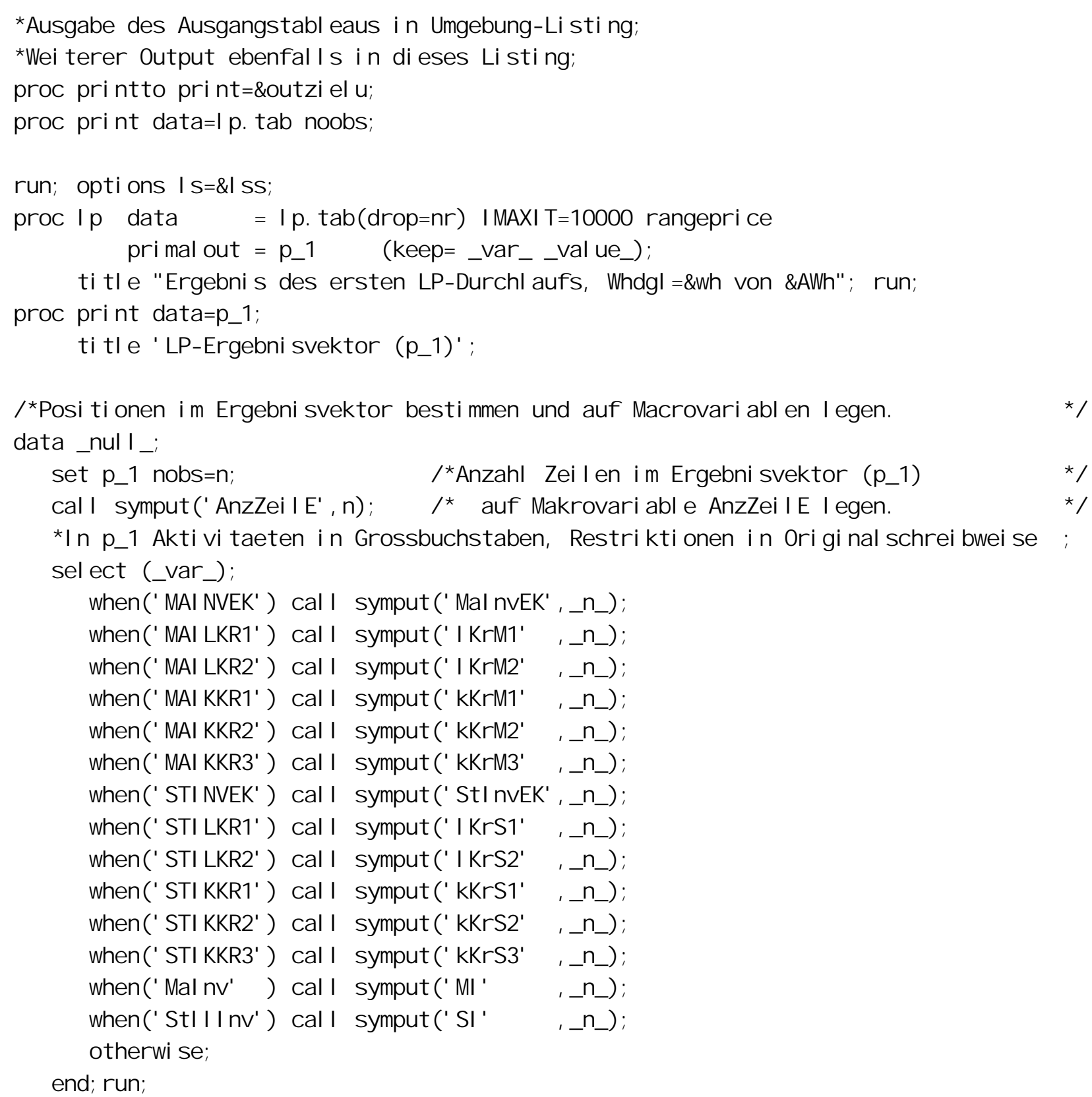

*Erstellen einer Makrovariablen ergnamen. Si e enthaelt die Namen der Variablen der LP-Ergebnisdatei, wobei den urspr. Namen ein "r" vorangestellt wird.

data

length b1 \$162;

$\%$ et $j=1$;

$i=\& A n z \operatorname{Var} T$;

$\%$ do $i=\& A n z \operatorname{Var} T+1 \%$ t o \&AnzZeil E;

$i+1$;

set $p_{-} 1$ point $=$; ;

$b \& j=\operatorname{trim}(b \& j)||^{\prime} r^{\prime}||$ compress(_var_, ' $\left.'{ }_{-}\right)$; 
$\%$ if $\& i=\& A n z \operatorname{Var} T+20 \mid \& i=\& A n z \operatorname{Var} T+40 \%$ then $\%$ do;

call symput ( "namen\&j", tri m(b\&j));

$\%$ et $j=\%$ eval $(\& j+1)$;

length b\&j \$162;

\%end;

\%end;

cal I symput ( "namen\&j", tri $m(b \& j))$;

stop; run;

$\%$ et ergnamen $=$;

$\% d o \quad i=1 \% t o \& j$;

$\%$ et ergnamen $=$ \&ergnamen $\& \&$ namen\&i;

\%end;

$\%$ et ergnamen = \&aktivita \&ergnamen;

* Vorbereitungen fuer die Ergebnisdatei, die in fortschr angelegt wird.

$\%$ if $\& w h=1 \%$ then $\%$ do;

proc datasets library=l p nolist;

delete \&dana; quit;

\%end;

\%mend erstlauf;

\%macro Fortschr;

I*Besti mmung der Anzahl aufzuhebender LP-Ergebnisse.

* Kei ne Macro-Min-Funktion vorhanden, daher Datastep erforderlich.;

data null $_{-}$;

call symput('AnLaMaKr', mi $n(\& p, \quad$ \&nMasch ) ); *max. Alter der Altkredite;

call symput('AnLaStKr', mi $n(\& p$,

\&nstpl ) l; * in 1 . Per. $=1$

cal I symput ('AnLaMaKz', mi $n$ ( $\& p$,

\&nMasch-1 1, ) *max ND der Altinvestition;

call symput ('AnLaStKz', mi $n(\& p$,

\&nStpl-1) 1; *in 1 . Periode $=1$

call symput('AnzLags', mi $n(\& p, \max (\& n$ Masch ,

\&nstpl I) 1; *potentielles Kreditalter:

data $r \quad$ (keep=_rhs_) $/$ *temp\&p*l |*zur Veraend. d. RHS d. Tabl. */ outp(keep=\&outKapaz \&outAkt \&outReste \&outsonst); I*Iiefert 1 Zeile f. Ergebnis. *I

* Di mensionierung von Arrays;

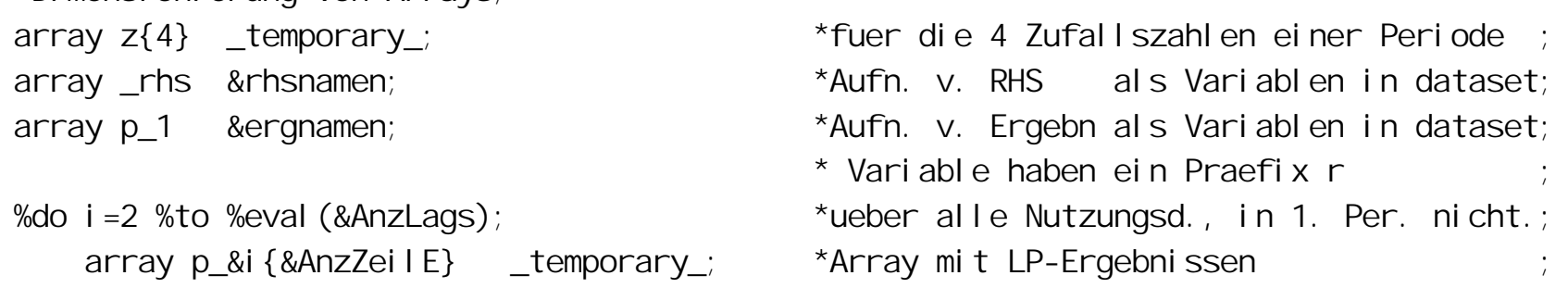
\%end;

* Alter Zinssa, Nutz, dauern

$(a \mathrm{l}, \mathrm{i}, \mathrm{N})$;

array zins $\{\& A n z$ Lags, $0: \& a i l$, \&aN\} _t emporary_; *Zins , Startelement: $(1,0,1)$;

array til g\{\&Anzlags, 0: \&ail, \&aN\} _t emporary_; *Tilung , Startelement: $(1,0,1)$; 
array rsch\{\&Anzlags, $0: \& a i l$, \&aN\} _temporary_; *Restschuld, Startelement: $(1,0,1)$;

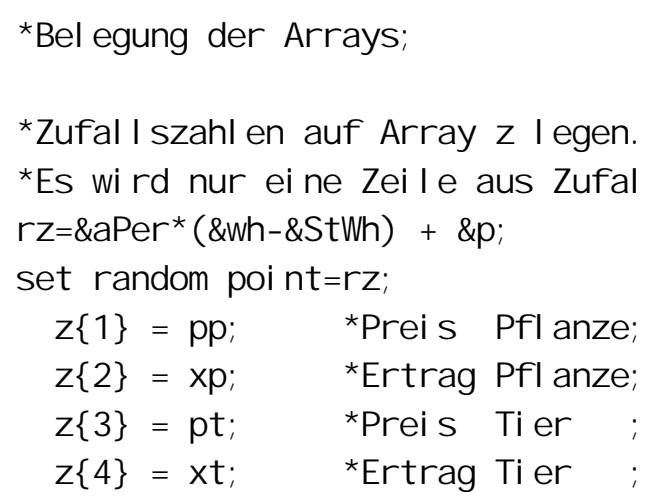

*RHS-Werte auf 1 d-Array _rhs legen. ;

do $i=1$ to \&AnzZeil T;

set aktalkeep = _rhs - $)$ point =i ;

-rhs $\{i\}=$ rhs $_{-}$;

end:

I*LP-Ergebnisse (akutuelles und alte) auf Arrays $p_{-}$? I egen.

I*Aktuell es LP-Ergebnis ist $p_{-} 1$.

$* 1$

$\%$ do $j=1 \%$ o \&AnzLags;

do $i=1$ to \&AnzZeile;

set $p_{-} \& j$ point $=i$;

$p_{-} \& j\{i\}=$ value $;$ end;

\%end;

* Kreditarrays, Einlesen des Tilgungsplanes Kreditfe, erstellt in erstlauf; $j=0 ; b=0$;

$N D=\min (\& p, \& n M a s c h) ; *$ maxi mal es Kreditalter in einer Periode;

$h v=\& n$ Masch;

do $N=1$ to \&aN;

do $i=0$ to \&ail;

do al =1 to ND;

$j+1$;

set kreditfe(keep=zi ti restschu) point =j;

zins $\{a l, i, N\}=z i$;

ti $\mid g\{a \mid, i, N\}=t i$;

$\operatorname{rsch}\{a \mid, i, N\}=r$ est schu;

end;

$j=h v *(i+1)+b$;

end;

$N D=m i n(\& p, \& n S t p l) ;$

$h v=\& n s t p l$;

$b=j$;

end; drop hv b zi ti; 
I*Fortschreibung der Ifr. Kredite: Restschuld am Ende der Periode VOR der Tilgung.*I

1 *Fortschreibung von Zinsen und Tilgung

I zi $n=0 ; \quad$ ti | gl=0; I ti | g2=0; | Kr $1=0 ; \quad \mid K r 2=0 ; \quad A b E K=0 ;$

* $|\mathrm{t}| \mathrm{g} 1=\mid \mathrm{fr}$. bil|liger Kredit, $|\mathrm{ti}| \mathrm{g} 2=\mid \mathrm{fr}$, teuerer Kredit.

* Kapitaldienst der Alt-Kredite und Ab der Inv. aus EK in Vorperioden.

* Bei $\mathrm{N}=4$ heisst dies Kapitaldienste von 3 Krediten mit Alter 2 bis 4.

$\%$ do $i=2 \%$ to \&AnLaMaKr; *Umfang *Anschngsw * (al, i, N) ;

$A b E K=A b E K+p_{-} \& i\{\&$ MaInvEK $\} * \& A M a s c h * t i I g\{\& i, 0,1\} ;$

Izin $=\mid z i n+p_{-} \& i\{\& \mid \operatorname{KrM} 1\} * \& A M a s c h * z i n s\{\& i, 1,1\}$

$+p_{-} \& i\{\& \mid K r M 2\} * \& A M a s c h *$ zins $\{\& i, 2,1\} ;$

$|\mathrm{Ti}| \mathrm{gl}=|\mathrm{Ti}| \mathrm{g} 1+\mathrm{p}_{-} \& i\{\& \mid \mathrm{KrM} 1\} * \& A M a s c h * \operatorname{ti} \mid \mathrm{g}\{\& i, 1,1\} ;$

$|\mathrm{Ti}| g 2=|\mathrm{Ti}| g 2+p_{-} \& i\{\& \mid \mathrm{KrM} 2\} * \& A M a s c h * \operatorname{ti} \mid g\{\& i, 2,1\} ;$

$|\mathrm{Kr} 1=| \mathrm{Kr} 1+p_{-} \&\{\{\& \mid \mathrm{KrM} 1\} * \& \operatorname{MMasch} * \operatorname{rsch}\{\& i, 1,1\} ; * \operatorname{*rsch}<1 ;$

$|\operatorname{Kr} 2=| \operatorname{Kr} 2+p_{-} \& \mid\{\& \mid K r M 2\} * \& A M a s c h * \operatorname{rsch}\{\& i, 2,1\} ; *$ fuer $\& i=3$ ist $r s c h=0$; \%end:

$\%$ do $i=2 \%$ t o \&AnLaSt Kr;

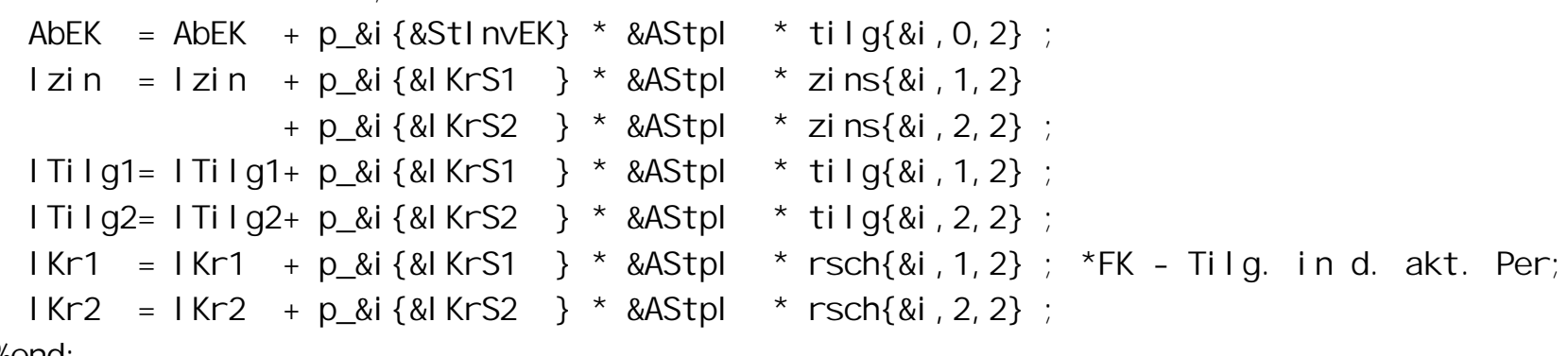

\%end:

*Auswi rkungen der Ueberraschungen auf den Zi el wert.;

rziel $=r z i e l+p f \mid p r n *\left(z\{1\} * z\{2\}-\& m p p^{*} \& m x p\right)$

+ tierprn* $(z\{3\} * z\{4\}-\& m p t \& m x t)$;

* Der Ziel wert des LP-Tableaus beruecksichtigt nur den Kapitialdienst der aktuell aufgenommenen Kredite und nicht die Kapitaldienste (=Annuitaeten) der Altkredite. Di ese sind daher vom Ziel wert abzuziehen, um den Gewinn zu erhalten. (Dank an Jockel!); Gewi nn=rzi el - I zi n- I Ti Ig1- I Ti Igz;

* Der Ziel wert beruecksichtigt nicht die Ab von EK-finanzierten Inv der VoRperioden.; Gewi $n n=G e w i$ nn- AbEK;

* Der Zi el wert beruecksichtigt den ZA auch bei Inv aus EK. Dieser ZA ist aber nicht gewi nnwi rksam. ;

Gewi nn=Gewi nn + (MaInvEK*\&AMasch + StI nvEK*\&AStpl) * \&i 0;

* Hi nzufuegen der Zi nsen und Tilgungen der in der aktuellen Periode auf genommenen Kredite.;
$\%$ et $\mathrm{i}=1 ; \quad \quad \quad{ }^{*} \mathrm{Al}$ t er
al $, i, N^{*} \mid$
$|z i n=| z i n+p_{-} \& i\{\& \mid K r M 1\} * \& A M a s c h *$ zins $\{1,1,1\}$
$+p_{-} \& i\{\& \mid \operatorname{KrM} 2\} * \& A M a s c h * z i n s\{1,2,1\}$;
$|\mathrm{Ti}| g 1=|\mathrm{Ti}| g 1+p_{-} \& i\{\& \mid \mathrm{KrM} 1\} *$ $\& \operatorname{Masch} * \mathrm{ti} \mid g\{1,1,1\}$;
$|\mathrm{Ti}| g 2=|\mathrm{Ti}| g 2+p_{-} \& \mid\{\& \mid \mathrm{KrM} 2\} *$ \&AMasch $* \mathrm{ti} \mid g\{1,2,1\}$; 


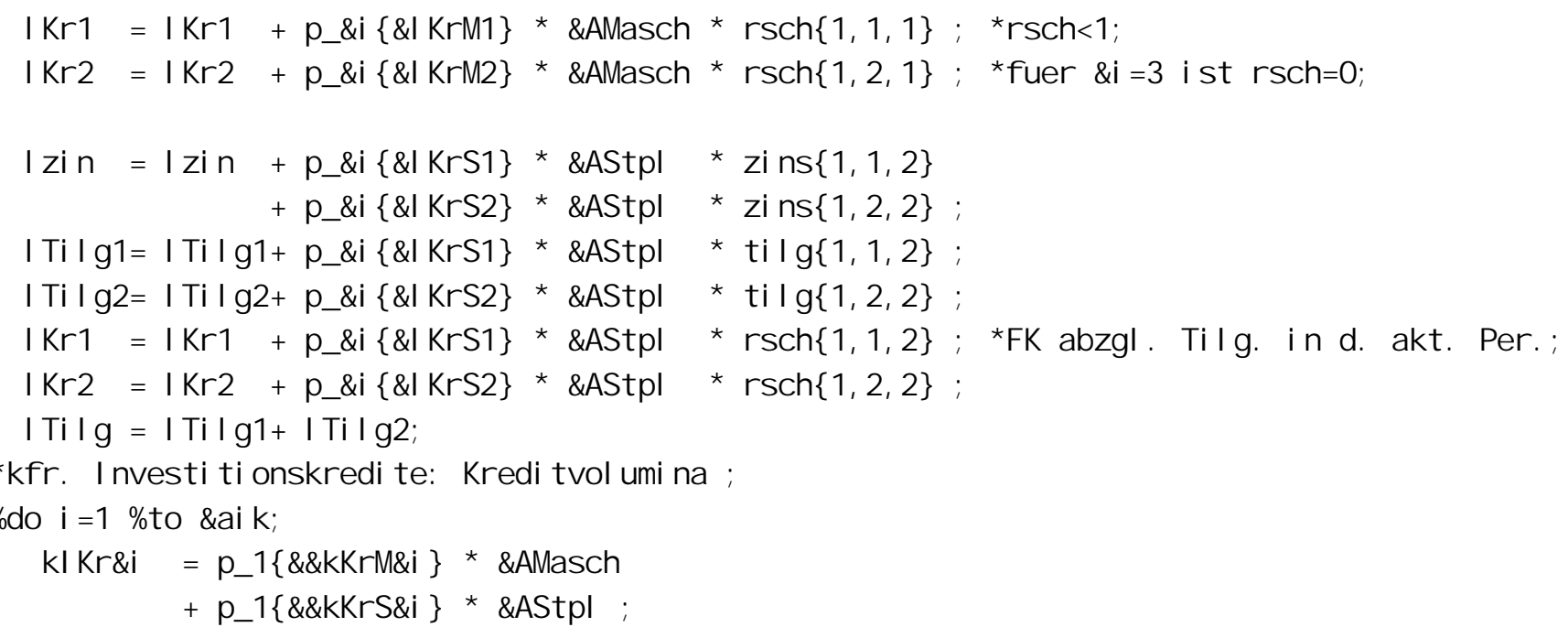

\%end:

*Ei nnahmen/ Ausgaben-Sal do;

* Hi nzufuegen der im Tableau nicht erfassten Positionen. ;

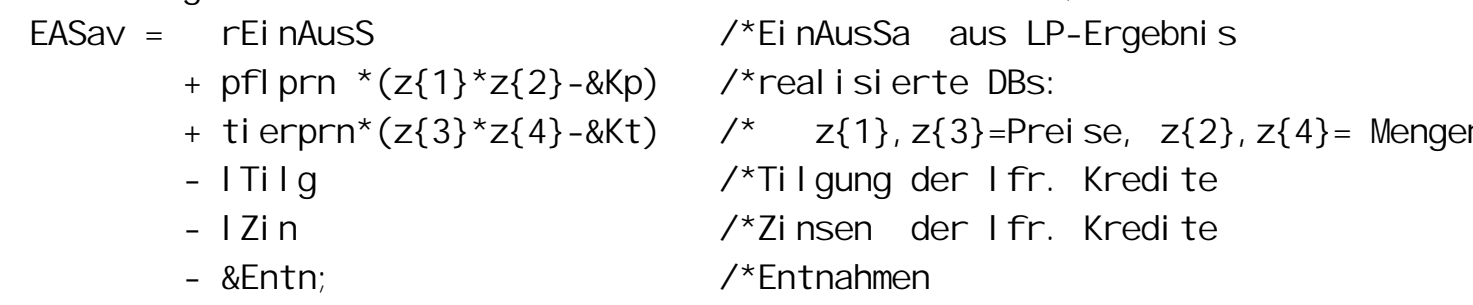

* Kapitaldienst sowohl fuer die in dieser Periode aufgenommenen als auch fuer

Alt kredite.;

* Der Saldo EASav geht in die Berechnung der Liquiditaet der neuen Periode ein.

I*Liquiditaet, Kapitalsaldo

aLi = Liqui; $\quad$ /*aLi: Liquiditaet zu Per, beginn, fuer Output (Kapazitaet).*I

Liqui = /*neue Liquiditaet: notw. Kreditaufn. zu Beginn d. Folgeper*/

I* fuer Fortschreibung des RHS-Vektors. *I

+ aLi $\quad$ *alte Liquiditaet *I

+ EASav; $\quad$ *EinAussa, vollstaendig *I

KapSaldo = $\quad$ I*Saldo aus Finanzanlagen und Fremdkapital nach Tilgung. *I

+ Liqui $\quad$ *neue Liquiditaet *I

- $\mid \mathrm{Kr} 1$ - | Kr 2

+ ITilg; $\quad$ *Ifr. Fremdkapitall nach Tilgung (pos. Vorzeichen) *I

I*Zeitwerte von Maschinen und Staellen (nach $A b)=$ Veraenderungen im Sachanlageverm.*I SAVerm $\quad=0 ; \quad$ I*Sachanlagevermogen am Periodenende nach Abzug d. Abschr.*I $\%$ do i $=1$ to \&AnLaMaKz; $\quad$ *Anzahl * Preis * Zeitwert-Koeffizient *

SAVerm = SAVerm $\quad+p_{-} \& i\{\& M I\} * \& A M a s c h *((\& n M a s c h-\& i) / \& n M a s c h) ;$ \%end;

$\%$ do $\quad i=1 \%$ t $0 \quad \& A n L a S t K z$;

SAVerm = SAVerm $\quad+p_{-} \& i\{\& S I\} * \& A S t p|*(|\& n S t p|-\& i)|$ \&nStpl); \%end; 


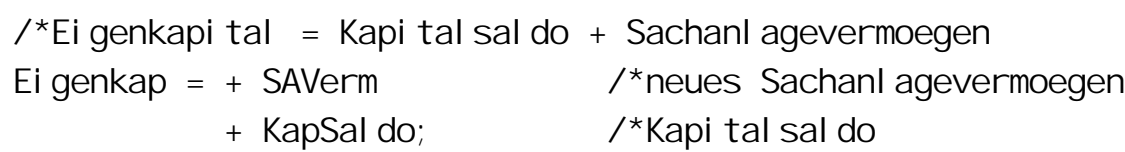

* Di e Variablen IKr? und IZi $n$ weisen Ifr. FK und dessen Zi nsen mit pos. Vorz.n aus. ; ZiSaldo = + rkZiSald $1 *$ Zi nssal do

$\begin{array}{ll}\text { wh } & =\& w h ; \\ \text { l per } & =\& p ;\end{array}$

I Ueberl ebensanal yse* I

surv=0;

if ei genkap<0 then surv=1; | | bei Insolvenz* |

* if KapSaldo-Eigenkap<0 then surv=2;

* if Eigenkap $<0$ \& KapSaldo-Eigenkap $<0$ then surv=12;

call s symput ('surv', surv);

output outp /*temp\&p/; $\quad$ |*Rausschreiben des Ergebnisses fuer akt. Periode *

I*Fortschreibung der RHS fuer naechste Periode.

$I^{*}$ Bisher wurde das Ergeb f. akt. Per. aufbereitet UND rausgeschrieben.

I* Daher koennen jetzt Werte fuer die kommende Periode berechnet werden.

$I^{*}$ Die neue Liquiditaet wurde schon vorher berechnet (Liqui).

*Fortschreibung der Kreditobergrenzen;

OgIFK1 $=\& 0 g|F K 1-| K r 1+|T i| g 1 ; * \& 0 g|F K 1=0 g| F K 1$ aus 1. Periode.;

$O g|F K 2=\& 0 g| F K 2-|K r 2+| T i \mid g 2 ;$

* Kapazitaet an Maschinen und Staellen zu Beginn der Folgeperiode,

d. h., ohne Inv. in der Folgeperiode, da als RHS verwendet.

Bei $N=4$ existieren noch drei nutzbare Maschinenjahrgaenge.

D.h., die schleife geht von 1 (=akt. Peroide) bis 3.;

MaKapaz $=0$;

$\%$ do i $=1 \%$ t o \&AnLaMaKz;

\%end;

MaKapaz = MaKapaz $+p_{-} \& i\{\& M I\} ;$

St al $|p|=0$;

$\%$ do $i=1 \%$ t o \&AnLaSt Kz;

Stal|p|=Stal|p|+p_\&i $\{\& S \mid\} ;$

\%end;

I *veraenderte Daten fuer RHS rausschreiben: OglFKx, MaKapaz, St pl. 


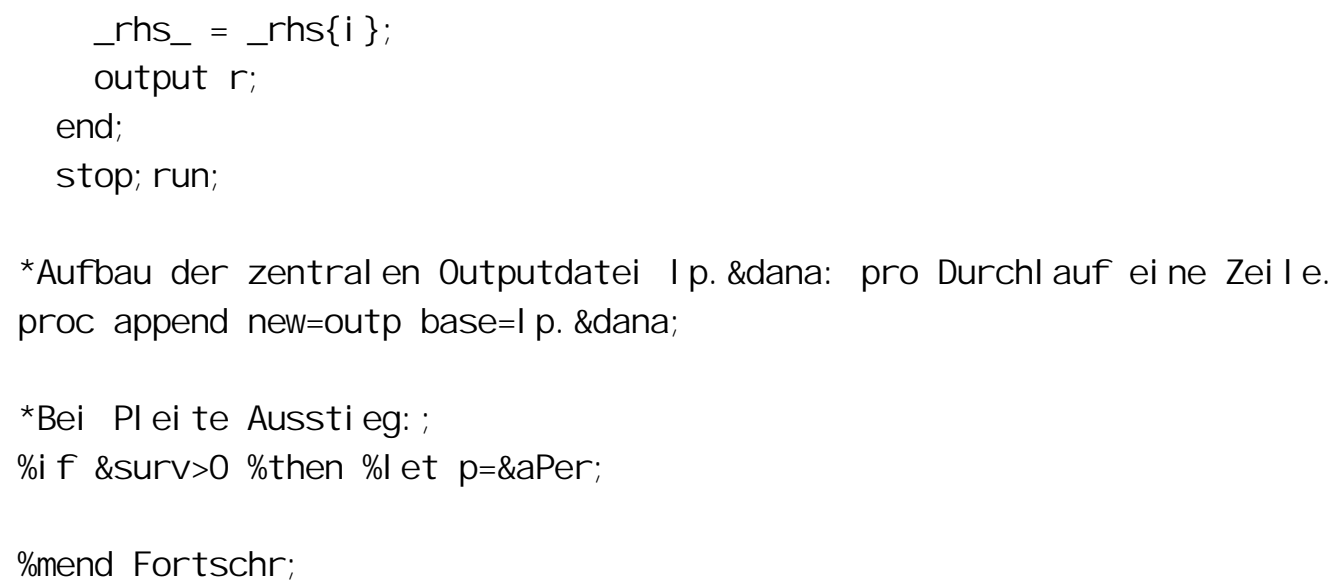

* LP-Ergebnisse des vorigen Laufs vor Start eines neuen LPs neu indizieren. ;

\% et Anzlags=\%eval ( \&Anzlags+1); $\quad$ *Wird erst imfolg. Makro Fortschr. hochgez.*I proc datasets nolist;

delete $p_{-} \%$ val (\&Anzlags ); $\quad$ /*sicherheitshalber, wenn er existiert, $\quad *$ I

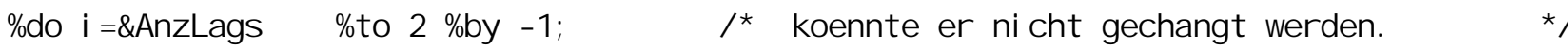
change $p_{-} \%$ eval $(\& i-1)=p_{-} \& i$;

\%end; quit;

proc I p data=akta $(d r o p=n r)$ noprint i maxit $=10000$ noprint

primal out = p_1 ( keep = _var_ _ val ue_);

title "Ergebnis des LP der \&p.. Periode und \&wh.. Wahlg"; run;

\%mend elpee;

\%macr 0 ausgabe;

I*Aufgerufen aus Hauptprogramm.

* Restliche Ausgabe in Standardfiles;

proc printto $\quad \log =\log$;

proc printto print = print; run;

data I p.round (drop=j); *Runden der Ergebnisse fuer Ausgabe

set I p. \&dana;

length _numeric_ 5 ;

array $r\left\{*_{\text {_ }}\right.$ numeric_; *alle numerischen Variablen auf Array $r$ I egen.

do $j=1$ to $\operatorname{dim}(r)$;

$r\{j\}=r$ ound $(r\{j\}, 1)$;

end; 


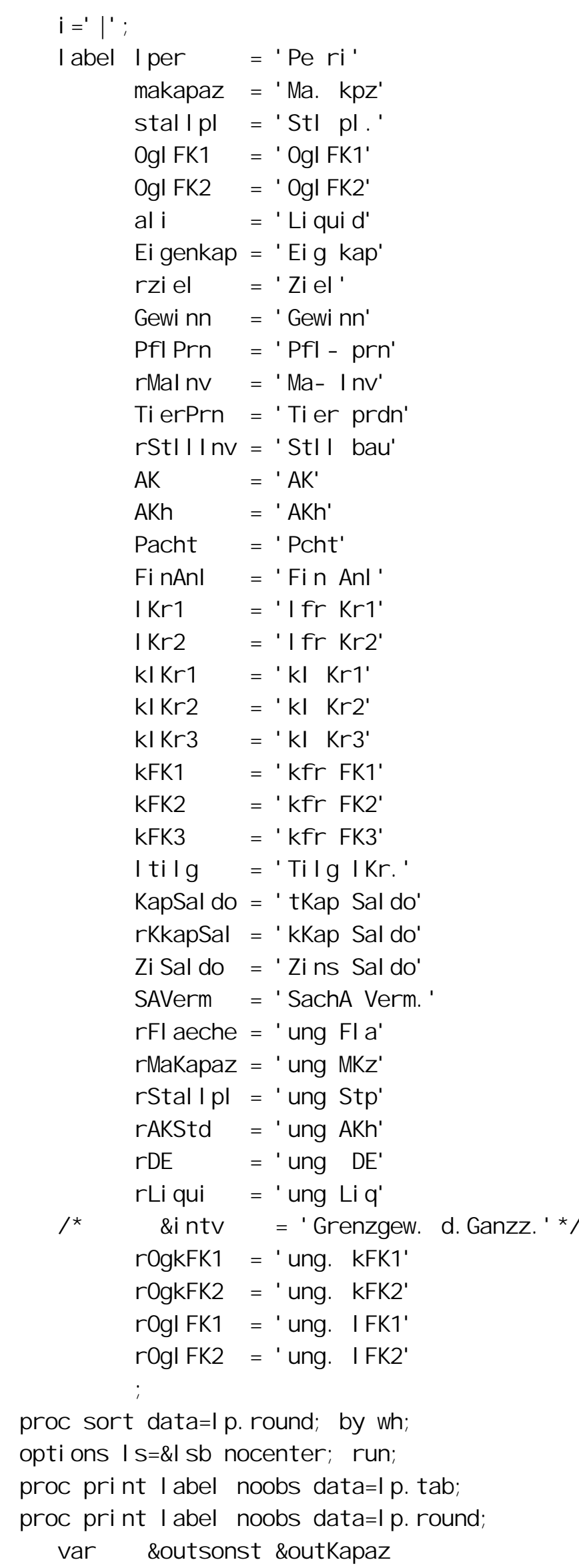


i \&out Akt

i \&outReste ;* \&intv;

by wh;

title1 "Ergebnis mit: Entnahmen = \&Entn, Nutzugungsdauer Maschinen = \&nMasch, Nutzungsdauer Gebaeude = \&nStpl, Wh: \&awh";

title3 'Kapazitaeten zu Periodenbeginn

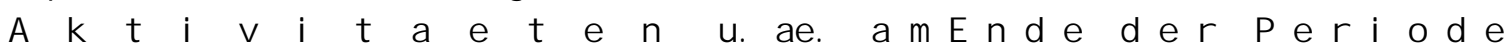

run;

options source mprint; run;

data I p. lastline;

set I p.round;

by wh;

if I ast. wh then output;

proc print noobs;

$\begin{array}{cl}\text { var } & \text { \&outsonst \&outKapaz } \\ \text { i } \quad \text { \&outAkt } \\ \text { i } \quad \text { \&outReste }\end{array}$

title 2 'Mittel werte ueber all e Runden und Wi ederholungen';

proc means data =l p. \&dana; var eigenkap tierprn pflprn akh pacht;

proc sort data=lp.\&dana out=sortsurv; by surv;

proc means ; var eigenkap tierprn pflprnakh pacht;

by surv; run;

title 2 'Mittel werte fuer letzte Zeile fuer jede Wiederholung';

proc means dat $a=1$ p. I astline; var I per surv eigenkap tierprn pflprn akh pacht;

proc sort dat $a=\mid p$.l astline out =sorsurll; by surv;

proc means ; var I per surv eigenkap;

by surv;

run;

\%mend ausgabe;

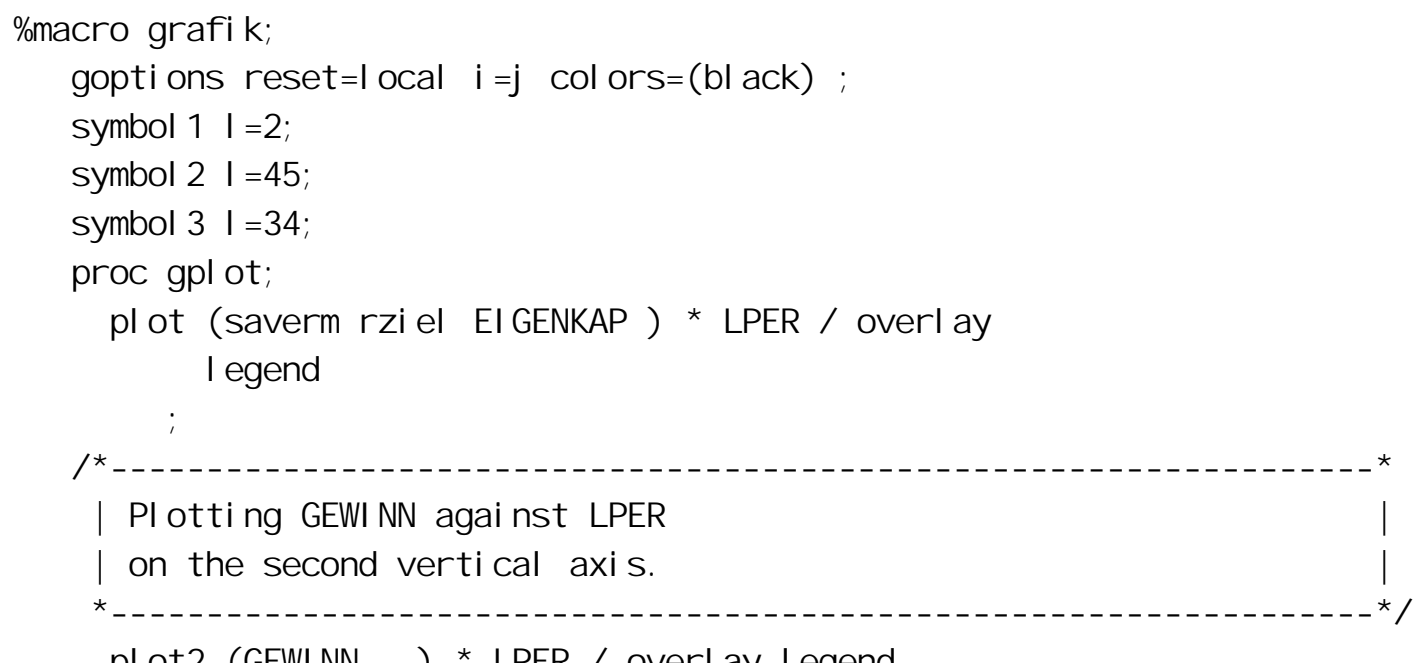

plot2 (GEWINN ) * LPER / overlay l egend 
run;

quit;

\%mend grafik;

$\%$ macro wdhl g;

\%datenein; *Neues Einlesen der Rohdaten. I mmer erforderlich, wenn beim I etzen Mal fuer mehr als eine Periode gerechnet wurde

* \%datenman; *Modifikation der Rohaten mi t fsedit

\%erstlauf; $\quad$ Koordinaten des Tableaus und erste Tableauberechung.

$\%$ d 0 wh $=\& S$ t Wh \%t 0 \&EndWh;

data_null_; title;

file print; put "Wiederholung $\mathrm{Nr}$. \&wh";

$\%$ et $p=1$;

dat a akta;

set I p.tab;

* Fuer wh=1 wird LP in erstlauf (nicht in wh-Schleife enthalten) gerechnet.

$\%$ if $\& w h>1$ \%then $\%$ do;

proc I $p \quad$ data $=a k t a(d r o p=n r) \quad|M A X| T=10000$

primalout $=p_{-} 1$ ( keep = _var_ _value_);

title "Ergebnis des LPS der 1. Runde, Whdgl=\&wh von \&AWh"; run;

\%end;

$\%$ Fortschr;

$\%$ if $\&$ surv $>0$ then $\%$ goto ende;

$\% d o \quad p=2$ \%to \&aPer;

$\%$ el pee;

$\%$ ortschr;

* Bisherige Ausgabe in Umgebung. I st bzw Standard-Saslog.

Nun waehrend der schleife Ausgabe in temporaere Files,

die bei jedem Schleifendurchlauf ueberschrieben werden.;

proc printto new print $=$ \&outzielt; run;

proc printto new log = \&logzielt; run;

\%end;

\%ende: ;

$\%$ end;

\%mend wdhl g;

\%wdhl g;

\%a us gabe;

\%graf i k;

$\%$ ei t daur;

run; 


\subsubsection{Der Ergebnisausdruck}

Ausgewählte Ergebnisse jeder Periode werden protokolliert. Dazu gehören die betrieblichen Kapazitäten auf der Produktions- und der Finanzierungsebene zu Periodenbeginn (z.B. freie Kreditspielräume), ferner Produktion, Investitionen, Finanzierung, Zielwert und Gewinn. Schließlich werden die nicht genutzten betrieblichen Kapazitäten aufgelistet.

Der Ergebnisausdruck entspricht im Kern einem Jahresabschluß, wie er in der Buchführung verwendet wird. Davon abweichend werden die Valuta der Kredite am Periodenende in ihrer Höhe vor der Tilgung dargestellt. Diese Abweichung geschieht aus zwei Gründen:

Die Restschuld vor der Tilgung entspricht der Verschuldung, die der Betrieb während der gesamten Periode trug. Wenn z.B. ein Betrieb einen zweijährigen Kredit über 100 TDM aufnimmt, so ist der Betrieb das ganze erste Jahr über, da eine Kreditaufnahme nur zu Periodenbeginn und eine Tilgung nur zu Periodenende möglich ist, mit eben diesen 100 TDM verschuldet. Die in der Buchführung übliche Kennziffer, Valuta nach Tilgung' hingegen wiese nur 50 TDM aus.

Ein anderer Grund ist aber viel entscheidender. Wenn die Valuta nach der Tilgung ausgewiesen würden, wäre der Umfang der einperiodischen Kredite stets Null. Der Ergebnisausdruck brächte also keine unmittelbare Information über die kurzfristige Verschuldung. Da nicht a priori ausgeschlossen werden kann, daß die kurzfristige Verschuldung (in Verbindung mit anderen Charakteristika) eine wichtige Kennziffer für die finanzielle Stabilität eines Unternehmens ist, werden die Kreditvaluta vor der Tilgung ausgewiesen.

In Tabelle 382.1 ist ein Beispieldurchlauf über 20 Perioden für das Grundmodell (s. Tabelle 340.1] wiedergegeben. Die verwendeten Abkürzungen sind in Tabelle 381.1 erläutert. 
Tabelle 381.1: Erläuterung der im Ergebnisausdruck verwendeten Abkürzungen

\begin{tabular}{|c|c|}
\hline Kürzel* & Bedeutung \\
\hline \multicolumn{2}{|r|}{ Kapazitäten zu Periodenbeginn u. ä. } \\
\hline Peri & laufende Periode \\
\hline WH & aktuelle Wiederholung des Szenarios \\
\hline$S U R V$ & Hat das Unternehmen diese Runde überlebt? $(0=\mathrm{ja}, 1=$ nein $)$ \\
\hline$P P$ & realisierter Preis in der Pflanzenproduktion \\
\hline Ma. kpz & Maschinenkapazität zu Periodenbeginn \\
\hline Stl pl. & Stallplätze zu Periodenbeginn \\
\hline Liquid & Liquidität zu Periodenbeginn \\
\hline OglFK1 & Obergrenze der Aufnahme von langfristigem Fremdkapital mit geringem Zins \\
\hline OglFK2 & Obergrenze der Aufnahme von langfristigem Fremdkapital mit hohem Zins \\
\hline$O g k F K 1$ & Obergrenze der Aufnahme von kurzfristigem Fremdkapital mit geringem Zins \\
\hline$O g k F K 2$ & Obergrenze der Aufnahme von kurzfristigem Fremdkapital mit mittleren Zins \\
\hline \multicolumn{2}{|r|}{ Aktivitäten und andere Betriebscharakteristika am Periodenende } \\
\hline Ziel & Zielwert der LP-Tableau-Optimierung \\
\hline Gewinn & kalkulatorischer Periodengewinn \\
\hline Pfl-prn & Umfang der Pflanzenproduktion (ha) \\
\hline$M a-\operatorname{Inv}$ & Maschineninvestitionen in der aktuellen Periode \\
\hline Tier prdn & Umfang der Tierproduktion (belegte Mastplätze pro Jahr) \\
\hline Stll bau & Stallbauinvestitionen in der aktuellen Periode \\
\hline$A K$ & eingesetzte ganzjährige Fremdarbeitskräfte \\
\hline$A K h$ & zugekaufte Arbeitszeit in Stunden \\
\hline Pcht & zugepachtete Fläche \\
\hline Fin Anl & Finanzanlagen, einjährig \\
\hline lfr Krl & Valuta des langfristigen Kredits mit niedrigem Zins, vor der Tilgung \\
\hline lfr $\mathrm{Kr} 2$ & Valuta des langfristigen Kredits mit hohem Zins, vor der Tilgung \\
\hline$k I K r l$ & Valuta des kurzfristigen Investitionskredits mit niedrigem Zins, vor der Tilgung \\
\hline$k I K r 2$ & Valuta des kurzfristigen Investitionskredits mit mittlerem Zins, vor der Tilgung \\
\hline$k I K r 3$ & Valuta des kurzfristigen Investitionskredits mit hohem Zins, vor der Tilgung \\
\hline Tilg lKr & gesamte Tilgung aller langfristigen Kredite \\
\hline kKap Saldo & kurzfristiger Kapitalsaldo: Finanzanlagen abzgl. kurzfristiges FK (vor d. Tilgung) \\
\hline tKap Saldo & totaler Kapitalsaldo: Finanzanlagen abzgl. gesamtes FK (nach der Tilgung) \\
\hline Zins Saldo & gesamter Zinssaldo \\
\hline SachA Verm. & Sachanlagevermögen \\
\hline Eig kap & Eigenkapital \\
\hline \multicolumn{2}{|r|}{ Reste (ungenutzte betriebliche Kapazitäten) am Periodenende } \\
\hline ung Fla & nicht genutzte Fläche \\
\hline ung $M k z$ & nicht genutzte Maschinenkapazität \\
\hline ung Stp & nicht genutzte Stallplätze \\
\hline ung $D E$ & nicht belegte Dungeinheiten \\
\hline ung $k F K 1$ & ungenutzter Kreditspielraum bei kurzfristigem Kredit mit niedrigem Zins \\
\hline ung $k F K 2$ & ungenutzter Kreditspielraum bei kurzfristigem Kredit mit mittlerem Zins \\
\hline ung lFK1 & ungenutzter Kreditspielraum bei langfristigem Kredit mit niedrigem Zins \\
\hline ung lFK1 & ungenutzter Kreditspielraum bei langfristigem Kredit mit hohem Zins \\
\hline
\end{tabular}


Tabelle 382.1: Ergebnisausdruck für das deterministische Grundmodell (Tabelle 340, 1 ]

\begin{tabular}{|c|c|c|c|c|c|c|c|c|c|c|c|c|c|c|c|c|c|c|c|c|c|c|c|}
\hline & & & Kapa & azita & eten & Peri & odenbe & i $n n$ & & & & A & $\begin{array}{ll}k & t\end{array}$ & & 1 & t & a & $\mathrm{t}$ & e & & u. & & a $m$ \\
\hline $\mathrm{Pe}$ & & & & $\mathrm{Ma}$ & St I & & & & & & $P f I$ & $\mathrm{Ma}$ - & Ti er & $S t \mid 1$ & & & & & & Ifr & If $r$ & kl & kl \\
\hline ri & WH & SURV & PP & $k p z$ & pl. & Li qui d & $O g \mid F K 1$ & $0 g \mid F K 2$ & Zi el & Gewi nn & $\operatorname{prn}$ & I nv & $p r d n$ & bau & AK & AKh & $P c h t$ & Fin & $A \cap I$ & $\mathrm{Kr} 1$ & $\mathrm{Kr} 2$ & $\mathrm{Kr} 1$ & $\mathrm{Kr} 2$ \\
\hline 1 & 1 & 0 & 24 & . & & 4000000 & 500000 & 500000 & 219344 & 286844 & 100 & 100 & 1000 & 1000 & 0 & 0 & 0 & 2424 & 4638 & 0 & 0 & 0 & 0 \\
\hline 2 & 1 & 0 & 24 & 100 & 1000 & 2894151 & 500000 & 500000 & 376519 & 327729 & 113 & 13 & 1130 & 130 & 0 & 0 & 13 & 2461 & 1174 & 0 & 0 & 0 & 0 \\
\hline 3 & 1 & 0 & 24 & 113 & 1130 & 3072645 & 500000 & 500000 & 345936 & 279181 & 115 & 2 & 1147 & 17 & 0 & 0 & 15 & 2787 & 7870 & 0 & 0 & 0 & 0 \\
\hline 4 & 1 & 0 & 24 & 115 & 1147 & 3366783 & 500000 & 500000 & 434053 & 361947 & 115 & 0 & 1149 & 2 & 0 & 0 & 15 & 3101 & 1234 & 0 & 0 & 0 & 0 \\
\hline 5 & 1 & 0 & 24 & 115 & 1149 & 3768008 & 500000 & 500000 & 414519 & 338546 & 115 & 0 & 1149 & 0 & 0 & 0 & 15 & 3504 & 4953 & 0 & 0 & 0 & 0 \\
\hline 6 & 1 & 0 & 24 & 115 & 1149 & 4152160 & 500000 & 500000 & 381730 & 301924 & 115 & 0 & 1149 & 0 & 0 & 0 & 15 & 3889 & 9429 & 0 & 0 & 0 & 0 \\
\hline 7 & 1 & 0 & 24 & 115 & 1149 & 4503842 & 500000 & 500000 & 459096 & 375296 & 115 & 0 & 1149 & 0 & 0 & 0 & 15 & 4241 & 1153 & 0 & 0 & 0 & 0 \\
\hline 8 & 1 & 0 & 24 & 115 & 1149 & 4932933 & 500000 & 500000 & 401868 & 313877 & 115 & 0 & 1149 & 0 & 0 & 0 & 15 & 4670 & 0248 & 0 & 0 & 0 & 0 \\
\hline 9 & 1 & 0 & 24 & 115 & 1149 & 5304799 & 500000 & 500000 & 483674 & 391284 & 115 & 0 & 1149 & 0 & 0 & 0 & 15 & 5042 & 2116 & 0 & 0 & 0 & 0 \\
\hline 10 & 1 & 0 & 24 & 115 & 1149 & 5758474 & 500000 & 500000 & 442457 & 345447 & 115 & 0 & 1149 & 0 & 0 & 0 & 15 & 5495 & 5790 & 0 & 0 & 0 & 0 \\
\hline 11 & 1 & 0 & 24 & 115 & 1149 & 6170931 & 500000 & 500000 & 531192 & 429332 & 115 & 0 & 1149 & 0 & 0 & 0 & 15 & 5908 & 8247 & 0 & 0 & 0 & 0 \\
\hline 12 & 1 & 0 & 24 & 115 & 1149 & 6672123 & 500000 & 500000 & 577493 & 470540 & 115 & 0 & 1149 & 0 & 0 & 0 & 15 & 6409 & 9440 & 0 & 0 & 0 & 0 \\
\hline 13 & 1 & 0 & 24 & 15 & 1149 & 7219616 & 500000 & 500000 & 576657 & 505063 & 115 & 100 & 1149 & 0 & 0 & 40 & 15 & 6705 & 5853 & 0 & 0 & 0 & 0 \\
\hline 14 & 1 & 0 & 24 & 102 & 1149 & 7531980 & 500000 & 500000 & 593708 & 494198 & 115 & 13 & 1149 & 0 & 0 & 5 & 15 & 7236 & 6724 & 0 & 0 & 0 & 0 \\
\hline 15 & 1 & 0 & 24 & 113 & 1149 & 8065293 & 500000 & 500000 & 618962 & 511319 & 115 & 2 & 1149 & 0 & 0 & 1 & 15 & 7798 & 8384 & 0 & 0 & 0 & 0 \\
\hline 16 & 1 & 0 & 24 & 115 & 1149 & 8650311 & 500000 & 500000 & 622264 & 508829 & 115 & 0 & 1149 & 0 & 0 & 0 & 15 & 8387 & 7080 & 0 & 0 & 0 & 0 \\
\hline 17 & 1 & 0 & 24 & 115 & 1149 & 9242063 & 500000 & 500000 & 647795 & 528636 & 115 & 0 & 1149 & 0 & 0 & 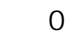 & 15 & 8979 & 9309 & 0 & 0 & 0 & 0 \\
\hline 18 & 1 & 0 & 24 & 115 & 1149 & 9859792 & 500000 & 500000 & 706459 & 581335 & 115 & 0 & 1149 & 0 & 0 & 0 & 15 & 9597 & 7100 & 0 & 0 & 0 & 0 \\
\hline 19 & 1 & 0 & 24 & 115 & 149 & 10536242 & 500000 & 500000 & 666812 & 673532 & 115 & 0 & 949 & 800 & 0 & 0 & 15 & 9425 & 5294 & 0 & 0 & 0 & 0 \\
\hline 20 & 1 & 0 & 24 & 115 & 819 & 10324331 & 500000 & 500000 & 640379 & 595106 & 115 & 0 & 1083 & 264 & 0 & 0 & 15 & 9781 & 1949 & 0 & 0 & 0 & 0 \\
\hline
\end{tabular}


Tabelle 382.1 Fortsetzung

Ende de r p e r i o d e

kl kfr kfr kfr TlgkKap tKap Zins SachA Kr 3 FK1 FK2 FK3 IKr Saldo Saldo Saldo Verm. $\begin{array}{lllllllll}0 & 0 & 0 & 0 & 0 & 2424638 & 2894151 & 121232 & 1268056\end{array}$

$\begin{array}{llllllllll}0 & 0 & 0 & 0 & 0 & 2461174 & 3072645 & 123059 & 1350616\end{array}$

$\begin{array}{llllllllll}0 & 0 & 0 & 0 & 0 & 2787870 & 3366783 & 139393 & 1279382\end{array}$

$\begin{array}{llllllllll}0 & 0 & 0 & 0 & 0 & 3101234 & 3768008 & 155062 & 1188196\end{array}$

$\begin{array}{lllllllllll}0 & 0 & 0 & 0 & 0 & 3504953 & 4152160 & 175248 & 1094422 & 524\end{array}$

$\begin{array}{llllllllll}0 & 0 & 0 & 0 & 0 & 3889429 & 4503842 & 194471 & 1000312 & 5504\end{array}$

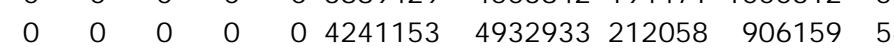

$\begin{array}{lllllllllll}0 & 0 & 0 & 0 & 0 & 4670248 & 5304799 & 233512 & 812000 & 611\end{array}$

$\begin{array}{lllllllll}0 & 0 & 0 & 0 & 0 & 5042116 & 575\end{array}$

$\begin{array}{llllllll}0 & 0 & 0 & 0 & 0 & 5495790 & 617\end{array}$

$\begin{array}{llllllll}0 & 0 & 0 & 0 & 0 & 5908247 & 667\end{array}$

$\begin{array}{llllll}0 & 0 & 0 & 0 & 0 & 6409440\end{array}$

$\begin{array}{llllll}0 & 0 & 0 & 0 & 0 & 6705853\end{array}$

$\begin{array}{llllll}0 & 0 & 0 & 0 & 0 & 7236724\end{array}$

$\begin{array}{llllll}0 & 0 & 0 & 0 & 0 & 7798384\end{array}$

$\begin{array}{lllllll}0 & 0 & 0 & 0 & 0 & 8387080\end{array}$

$\begin{array}{llllll}0 & 0 & 0 & 0 & 0 & 897930\end{array}$

$\begin{array}{lll}0 & 0 & 0\end{array}$

$\begin{array}{llll}0 & 0 & 0 & 0 \\ 0 & 0 & 0 & 0\end{array}$
$0 \quad 9597100 \quad 10536242 \quad 479855$

\begin{tabular}{l|lllll|}
0 & 9425294 & 10324331 & 471265 & 955735 & 11280066
\end{tabular}

$0 \quad 978194910654861 \quad 489097 \quad 1155769 \quad 11810629$
I $R$ e $s \quad t$ e am Periodenende

I ung ung ung ung ung ung. ung. ung. ung. ung $P e$ Eig kap Fla MKz Stp AKh DE KFK1 KFK2 IFK1 IFK2 Liq ri $4162207 \mid \begin{array}{lllllllllll}0 & 0 & 0 & 0 & 0 & 50000 & 50000 & 500000 & 500000 & 0 & 1\end{array}$ $4423261 \mid 00000005000050000500000500000 \quad 0 \quad 2$ $4646165 \mid \begin{array}{lllllllllllll}0 & 0 & 0 & 0 & 0 & 50000 & 50000 & 500000 & 500000 & 0 & 3\end{array}$ $4956204 \mid 0 \quad 0 \quad 0 \quad 0 \quad 05000050000500000500000 \quad 0 \quad 4$ $5246582 \mid 00000005000050000500000500000 \quad 0 \quad 5$

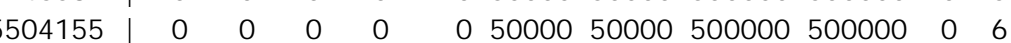
\begin{tabular}{l|lllllllllllll}
5839092 & 0 & 0 & 0 & 0 & 0 & 50000 & 50000 & 500000 & 500000 & 0 & 7
\end{tabular} $6116800 \mid 00000005000050000500000500000 \quad 0 \quad 8$ $6476314 \mid \begin{array}{llllllllllllll}0 & 0 & 0 & 0 & 0 & 50000 & 50000 & 500000 & 500000 & 0 & 9\end{array}$

$05000050000500000500000 \quad 010$ $05000050000500000500000 \quad 011$ 05000050000500000500000012 05000050000500000500000013 $05000050000500000500000 \quad 014$ $05000050000500000500000 \quad 015$ $05000050000500000500000 \quad 016$ $05000050000500000500000 \quad 017$ $05000050000500000500000 \quad 018$ $405000050000500000500000 \quad 019$ $135000050000500000500000 \quad 020$ 
Tabelle 384.1: Ergebnisgrößen der Strategie „Risikoabschlag: 10\% bei Pflanzenproduktion, 2.5\% bei Tierproduktion" im deterministischen Modell

Kapazitaeten zu Periodenbeginn Ma. St I

$\begin{array}{lll}\mathrm{Pi} \text { WH SURV PP kpz pl. } & \\ & \end{array}$

$\begin{array}{llll}1 & 1 & 0 & 24\end{array}$

$\begin{array}{lllll}2 & 1 & 0 & 24 & 100\end{array}$

$\begin{array}{lllll}3 & 1 & 0 & 24 & 100\end{array}$

$\begin{array}{llllll}4 & 1 & 0 & 24 & 100\end{array}$

$\begin{array}{llllll}5 & 1 & 0 & 24 & 100\end{array}$

$\begin{array}{llllll}6 & 1 & 0 & 24 & 100\end{array}$

$\begin{array}{llllll}7 & 1 & 0 & 24 & 100\end{array}$

$\begin{array}{lllll}8 & 1 & 0 & 24 & 100\end{array}$

$\begin{array}{llllll}9 & 1 & 0 & 24 & 100 & 156\end{array}$

$\begin{array}{llllll}10 & 1 & 0 & 24 & 100 & 235\end{array}$

$\begin{array}{llllll}11 & 1 & 0 & 24 & 100 & 329\end{array}$

$\begin{array}{llllll}12 & 1 & 0 & 24 & 100 & 435\end{array}$

$\begin{array}{llllll}13 & 1 & 0 & 24 & 0 & 486\end{array}$

$\begin{array}{llllll}14 & 1 & 0 & 24 & 100 & 486\end{array}$

$\begin{array}{lllllll}15 & 1 & 0 & 24 & 100 & 523\end{array}$

$\begin{array}{llllll}16 & 1 & 0 & 24 & 100 & 563\end{array}$

$\begin{array}{llllll}17 & 1 & 0 & 24 & 100 & 608\end{array}$

$\begin{array}{llllll}18 & 1 & 0 & 24 & 100 & 657\end{array}$

$\begin{array}{llllll}19 & 1 & 0 & 24 & 100 & 711\end{array}$

$\begin{array}{llllll}20 & 1 & 0 & 24 & 100 & 771\end{array}$

$\begin{array}{llllll}21 & 1 & 0 & 24 & 100 & 838\end{array}$

$\begin{array}{llllll}22 & 1 & 0 & 24 & 100 & 911\end{array}$

$\begin{array}{llllll}23 & 1 & 0 & 24 & 100 & 991\end{array}$

$\begin{array}{llllll}24 & 1 & 0 & 24 & 103 & 999\end{array}$

$\begin{array}{llllll}25 & 1 & 0 & 24 & 5 & 992\end{array}$

$\begin{array}{llllll}26 & 1 & 0 & 24 & 100 & 933\end{array}$

$\begin{array}{llllll}27 & 1 & 0 & 24 & 100 & 925\end{array}$

$\begin{array}{llllll}28 & 1 & 0 & 24 & 100 & 911\end{array}$

$\begin{array}{llllll}29 & 1 & 0 & 24 & 100 & 899\end{array}$

$\begin{array}{llllll}30 & 1 & 0 & 24 & 100 & 953\end{array}$

$\begin{array}{llllll}31 & 1 & 0 & 24 & 104 & 1041\end{array}$

$\begin{array}{lllllll}32 & 1 & 0 & 24 & 112 & 1082\end{array}$

$\begin{array}{llllll}33 & 1 & 0 & 24 & 114 & 1101\end{array}$

$\begin{array}{llllll}34 & 1 & 0 & 24 & 114 & 1099\end{array}$

$\begin{array}{lllllll}35 & 1 & 0 & 24 & 111 & 1094\end{array}$

$\begin{array}{llllll}36 & 1 & 0 & 24 & 112 & 1088\end{array}$

$\begin{array}{llllll}37 & 1 & 0 & 24 & 19 & 1082\end{array}$

$\begin{array}{llllll}38 & 1 & 0 & 24 & 112 & 1055\end{array}$

$\begin{array}{lllllll}39 & 1 & 0 & 24 & 114 & 1065\end{array}$

40
Liquid OglFK1 OglFK2 । 40000500000500000 । 44981264819500000 50559280528500000 56807297179500000 63805314829500000 71642333538500000 80713319999500000 $91408281360 \quad 500000$ $104018235318 \quad 500000$ 118885180519500000 136413115364500000 $15623943903500000 \mid 102213$ \begin{tabular}{lll|l}
166979 & 47957500000 & 74558
\end{tabular} \begin{tabular}{llll|l}
166103 & 23858330043 & 108113
\end{tabular} \begin{tabular}{llll|l}
174493 & 26062 & 339721 & 112465
\end{tabular} \begin{tabular}{llll|l}
183774 & 28469 & 350271 & 117268
\end{tabular} \begin{tabular}{ll|l|l|l|l|}
194040 & 31098 & 361770 & 122569
\end{tabular} \begin{tabular}{llll|l}
205395 & $33970 \quad 374304$ & 128418
\end{tabular} \begin{tabular}{ll|l|l|l|}
217954 & 37107 & 387966 & 134874
\end{tabular} \begin{tabular}{llll|l}
231845 & 40534 & 402857 & 141998
\end{tabular} \begin{tabular}{llll|l}
247207 & 44278419089 & 149860
\end{tabular} \begin{tabular}{ll|l|l|l|l|}
264197 & 48367 & 436782 & 158537
\end{tabular} $28298752835456067 \mid 167663$ $298211108839477087 \mid 170076$ $309564165019500000 \mid 141044$ \begin{tabular}{llll|l}
305885 & $60393 \quad 500000$ & 163157
\end{tabular} \begin{tabular}{ll|l|l|l|l|l|l|l|}
314422 & 114647500000 & 162939
\end{tabular} $322668164084500000 \mid 162016$ $331553200344500000 \mid 161312$ \begin{tabular}{ll|l|l|l|}
341188224692 & 500000 & 167187
\end{tabular} $360679258157500000 \mid 177883$ $393770293630500000 \mid 185769$ $471250329028500000 \mid 192013$

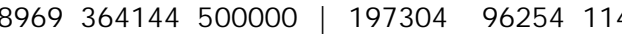
$693624398736500000 \mid 201732102080114$ $804811432533500000 \mid 206973107812114$ $920617465219500000 \mid 185786125163112$ $839485477085500000 \mid 205404122494114$ $948431485919500000 \mid 212410128321114$ \begin{tabular}{llllll|lll}
24 & 114 & 1059 & 1082244 & 491194 & 500000 & 218579 & 135444 & 114
\end{tabular}

\begin{abstract}
A $k$ t $i \quad v \quad$ i $t \quad a$
\end{abstract}
PfI Ma- Tier StI AH

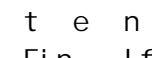
$\begin{array}{ll}\text { Fin } & \text { Ifr } \\ \text { Anl } & \mathrm{Kr} 1\end{array}$ ae. a $m$ $\begin{array}{lll}\mathrm{ffr} & k \mathrm{k} & \mathrm{k}\end{array}$ Ziel Gewinn prn Inv prdn bau AK h Pcht Anl Krl Kr2 Krl Kr2 $\begin{array}{llllllllll}18181 & 18181 & 100 & 100 & 0 & 0 & 0 & 0 & 0 & 0 \\ 250000\end{array}$ $0 \quad 235181$

$0 \quad 219472$

0202821

$0 \quad 185171$

0200948

$0 \quad 242879$

$0 \quad 292774$

$0 \quad 352087$

$0 \quad 422532$

0500000

0500000

$0 \quad 500000178837 \quad 0 \quad 0$

050000016995700

$0500000160279 \quad 0 \quad 0$

050000014972900

$0500000138230 \quad 0 \quad 0$

$0 \quad 500000125696 \quad 0 \quad 0$

$0500000112034 \quad 0 \quad 0$

$0 \quad 500000 \quad 97143 \quad 0 \quad 0$

05000008091100

$0 \quad 500000 \quad 63218 \quad 0 \quad 0$

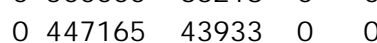

$\begin{array}{lllll}0 & 391161 & 22913 & 0 & 0\end{array}$

$\begin{array}{lllll}0 & 497249 & 0 & 0 & 0\end{array}$

$0439607 \quad 0 \quad 0 \quad 0$

$\begin{array}{lllll}0 & 385353 & 0 & 0 & 0\end{array}$

$\begin{array}{lllll}0 & 342763 & 0 & 0 & 0\end{array}$

$\begin{array}{lllll}0 & 310705 & 0 & 0 & 0\end{array}$

$0275308 \quad 0 \quad 0 \quad 0$

$\begin{array}{lllll}0 & 241843 \quad 0 & 0 & 0\end{array}$

$\begin{array}{llllll}4 & 1041 & 88 & 0 & 0\end{array}$

$\begin{array}{llllll}8 & 1119 & 78 & 0 & 0 & 12\end{array}$

$\begin{array}{llllllll}2 & 1141 & 59 & 0 & 0 & 14 & 62750 & 206370\end{array}$

$\begin{array}{llllllll}0 & 1144 & 43 & 0 & 0 & 14 & 162053 & 170972\end{array}$

$\begin{array}{lllllllll}0 & 1143 & 44 & 0 & 0 & 14 & 268811 & 135856\end{array}$

$\begin{array}{llllllll}3 & 1142 & 48 & 0 & 0 & 14 & 372426 & 101264\end{array}$

$\begin{array}{llllllll}2 & 1142 & 54 & 0 & 0 & 14 & 479917 & 67467\end{array}$

$\begin{array}{llllllll}93 & 1121 & 39 & 0 & 0 & 12 & 389341 & 34781\end{array}$

$\begin{array}{llllllll}2 & 1138 & 83 & 0 & 0 & 14 & 482638 & 22915\end{array}$

$\begin{array}{llllllll}0 & 1140 & 75 & 0 & 0 & 14 & 605556 & 14081\end{array}$

$\begin{array}{lllllllr}0 & 1139 & 79 & 0 & 0 & 14 & 734686 & 8806\end{array}$ 


\section{Tabelle 884.17 Fortsetzung}

End e d e r p e r i o d e

kl kfrkfrer Tilg kKap tKap Zins SachA Kr3 FK1 FK2 FK3 IKr. Saldo Saldo Saldo Verm. Ei

$\begin{array}{llllllllll}0 & 26667 & 0 & 0 & 14819 & -26667 & -190200 & -18200 & 229167\end{array}$

$\begin{array}{lllllllll}0 & 21686 & 0 & 0 & 15708 & -21686 & -168913 & -16713208333\end{array}$

$\begin{array}{lllllllllll}0 & 16107 & 0 & 0 & 16651-16107 & -146014-15101 & 187500\end{array}$

$\begin{array}{llllllllll}0 & 9860 & 0 & 0 & 17650 & -9860 & -121367 & -13352 & 166667\end{array}$

$\begin{array}{lllllllll}0 & 2862 & 0 & 0 & 18709 & -2862 & -94821 & -11454 & 145833\end{array}$

$\begin{array}{lllllllll}0 & 0 & 0 & 0 & 20947 & 0 & -99288 & -12057 & 157570\end{array}$

$\begin{array}{lllllllll}0 & 0 & 0 & 0 & 24239 & 0 & -127232 & -14573 & 194206\end{array}$

$\begin{array}{lllllllll}0 & 0 & 0 & 0 & 28092 & 0 & -160664 & -17566 & 237978\end{array}$

$\begin{array}{llllllll}0 & 0 & 0 & 0 & 32605 & 0 & -200596 & -21125290166\end{array}$

$\begin{array}{lllllllll}0 & 0 & 0 & 0 & 37896 & 0 & -248222 & -25352 & 352275\end{array}$

$\begin{array}{lllllllll}0 & 0 & 0 & 0 & 43903 & 0 & -299858 & -30000 & 421018\end{array}$

$\begin{array}{lllllllll}0 & 0 & 0 & 0 & 47957 & 0 & -285064 & -30000 & 426862\end{array}$

$\begin{array}{lllllllll}0 & 0 & 0 & 0 & 32738 & 0 & -479996 & -46095 & 626336\end{array}$

$\begin{array}{lllllllll}0 & 0 & 0 & 0 & 35740 & 0 & -459724 & -45296 & 613934\end{array}$

$\begin{array}{lllllllll}0 & 0 & 0 & 0 & 39018 & 0 & -437486 & -44425603226\end{array}$

$\begin{array}{llllllll}0 & 0 & 0 & 0 & 42597 & 0 & -413092 & -43476 \\ 0 & 0 & 0 & 0 & 46504388\end{array}$

$\begin{array}{lllllllll}0 & 0 & 0 & 0 & 46504 & 0 & -386331 & -42441587613\end{array}$

$\begin{array}{llllllll}0 & 0 & 0 & 0 & 50769 & 0 & -356973-41313583114\end{array}$

$\begin{array}{lllllllll}0 & 0 & 0 & 0 & 55426 & 0 & -324763-40083 & 581126\end{array}$

$\begin{array}{llllllll}0 & 0 & 0 & 0 & 60510 & 0 & -289425 & -38743581909\end{array}$

$\begin{array}{llllllll}0 & 0 & 0 & 0 & 66060 & 0 & -250653-37282 & 585749\end{array}$

$\begin{array}{lllllllll}0 & 0 & 0 & 0 & 72120 & 0 & -208112 & -35690 & 592961\end{array}$

$\begin{array}{lllllllll}0 & 0 & 0 & 0 & 77025 & 0 & -115863 & -30784 & 558706\end{array}$

$\begin{array}{lllllllll}0 & 0 & 0 & 0 & 79092 & 0 & -25417 & -25532 & 533263\end{array}$

0

0

0

0
0

0

0

0

0

0

0
0

0

0
0 $\begin{array}{llll}0 & 0 & 0 & 57642\end{array}$

$\begin{array}{llll}0 & 0 & 0 & 54254\end{array}$

$\begin{array}{llll}0 & 0 & 0 & 49437\end{array}$

$\begin{array}{llll}0 & 0 & 0 & 43107\end{array}$

$\begin{array}{llll}0 & 0 & 0 & 35396\end{array}$

$\begin{array}{llll}0 & 0 & 0 & 33465\end{array}$

$\begin{array}{llll}0 & 0 & 0 & 35473\end{array}$

$\begin{array}{lllll}0 & 0 & 0 & 35398 & 62750\end{array}$

$\begin{array}{llllll}0 & 0 & 0 & 35115 & 162053\end{array}$

$\begin{array}{llllll}0 & 0 & 0 & 34593 & 268811\end{array}$

$\begin{array}{lllll}0 & 0 & 0 & 33796 & 372426\end{array}$

$\begin{array}{lllll}0 & 0 & 0 & 32687 & 479917\end{array}$

$\begin{array}{llllll}0 & 0 & 0 & 11866 & 389341\end{array}$

$0-133721-29835697788$

$0 \quad-70931-26376 \quad 695040$

$0 \quad-13248-23121700693$

$0 \quad 31897-20566721438$

$0 \quad 65879-18642755270$

$0 \quad 118836-16519775571$

$\begin{array}{lllll}187400-14511789390 & 976790\end{array}$

$300279 \quad-9245 \quad 766180 \quad 1066459$

$443113 \quad-2156 \quad 720328 \quad 1163441$

$\begin{array}{lllll}592360 & 5289 & 675491 & 1267851\end{array}$

737343

$12545642071 \quad 1379415$

885836

$19948 \quad 612503 \quad 1498339$

$\begin{array}{lll}0 & 0 & 0\end{array}$

$8834482638 \quad 934350$

$17380796231 \quad 1612802$

$\begin{array}{lll}0 & 0 & 0\end{array}$

$5275605556 \quad 1073438$

$1124734686 \quad 1214293$
I $R$ e $s$ t $e$ am Periodenende

I ung ung ung ung ung ung. ung. ung. ung. ung pe $38967+0 \quad 0100020023333500002500005000000$ $39420 \mid \begin{array}{llllllllll}0 & 0 & 0 & 1040 & 20028314 & 50000 & 264819 & 500000 & 0 & 2\end{array}$ $41486 \mid \begin{array}{llllllllllll} & 0 & 0 & 0 & 1040 & 200 & 33893 & 50000 & 280528 & 500000 & 0 & 3\end{array}$ $45300 \mid \begin{array}{llllllllllll} & 0 & 0 & 0 & 1040 & 200 & 40140 & 50000 & 297179 & 500000 & 0 & 4\end{array}$

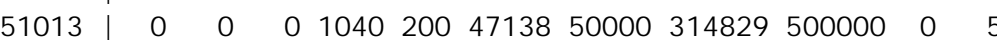
$58282 \mid \begin{array}{lllllllllll}0 & 0 & 0 & 100919450000 & 50000 & 299052500000 & 0 & 6\end{array}$ $66974 \mid \begin{array}{llllllllllll} & 0 & 0 & 0 & 95818250000 & 50000 & 257121500000 & 0 & 7\end{array}$ $77314 \mid \begin{array}{llllllllllll}0 & 0 & 0 & 902 & 169 & 50000 & 50000 & 207226 & 500000 & 0 & 8\end{array}$ $89570 \mid \begin{array}{llllllllll} & 0 & 0 & 83615350000 & 50000 & 147913500000 & 0 & 9\end{array}$ $104052 \mid \begin{array}{lllllllllll} & 0 & 0 & 75813450000 & 50000 & 77468 & 500000 & 0 & 10\end{array}$ $121160 \quad 0 \quad 0 \quad 0 \quad 6711135000050000 \quad 00500000 \quad 0 \quad 11$ \begin{tabular}{l|lllllllll}
41798 & 0 & 0 & 0 & 6411035000050000 & 0 & 500000 & 0 & 12
\end{tabular} \begin{tabular}{l|lllllllllll} 
& 0 & 0 & 0 & 611 & 10350000 & 50000 & 0 & 321163 & 0 & 13
\end{tabular} \begin{tabular}{l|lllllllllll}
54210 & 0 & 0 & 0 & 615 & 95 & 50000 & 50000 & 0 & 330043 & 0 & 14
\end{tabular} \begin{tabular}{l|llllllllll} 
& 0 & 0 & 0 & 581 & 8750000 & 50000 & 0 & 339721 & 0 & 15
\end{tabular}

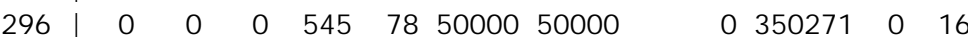

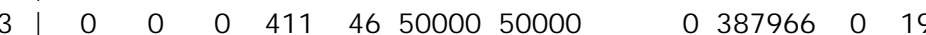
$\begin{array}{lllllllllll}0 & 0 & 0 & 357 & 32 & 50000 & 50000 & 0 & 402857 & 0 & 20\end{array}$ $\begin{array}{llll}0 & 436782 & 0 & 22\end{array}$ 23125000050000 $05000050000 \quad 52835456067 \quad 0 \quad 23$ $0 \quad 50000 \quad 50000 \quad 108839477087 \quad 0 \quad 24$ $0 \quad 5000050000 \quad 2751500000 \quad 0 \quad 25$ $05000050000 \quad 60393500000 \quad 0 \quad 26$ $05000050000 \quad 114647500000 \quad 0 \quad 27$ $05000050000 \quad 157237500000 \quad 0 \quad 28$ $05000050000189295500000 \quad 0 \quad 29$ $05000050000224692500000 \quad 0 \quad 30$ $05000050000258157500000 \quad 0 \quad 31$ $05000050000 \quad 293630500000 \quad 0 \quad 32$ $0 \quad 5000050000 \quad 329028500000 \quad 0 \quad 33$ $05000050000 \quad 364144500000 \quad 0 \quad 34$ $05000050000 \quad 398736500000 \quad 0 \quad 35$ $05000050000 \quad 432533500000 \quad 0 \quad 36$ $0 \quad 50000 \quad 50000 \quad 465219500000 \quad 0 \quad 37$ $05000050000477085500000 \quad 0 \quad 38$ $05000050000485919500000 \quad 0 \quad 39$ $05000050000 \quad 491194500000 \quad 0 \quad 40$ 
Tabelle 386.1: Ergebnisgrößen der Strategie „Risikoabschlag: 5\% bei Pflanzenproduktion, $2.5 \%$ bei Tierproduktion“ im deterministischen Modell

\begin{tabular}{|c|c|c|c|c|c|c|c|c|c|c|c|c|c|c|c|c|c|c|c|c|c|c|}
\hline \multirow{3}{*}{$\mathrm{Pe}$} & \multicolumn{5}{|c|}{ Kapazitaeten } & \multirow[t]{2}{*}{$z u$} & \multirow{2}{*}{\multicolumn{2}{|c|}{ eriodenbeginn }} & \multicolumn{4}{|c|}{ 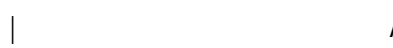 } & A $k$ & $t \quad i$ & \multicolumn{2}{|c|}{ v i } & \multirow{2}{*}{ t } & $a$ & e $n$ & \multicolumn{3}{|c|}{ u. ae. a m } \\
\hline & & & & Ma. & St I & & & & & & $P f I$ & $\mathrm{Ma}$ - & Tier & StII & & AK & & $\begin{array}{l}e \quad t \\
\text { Fin }\end{array}$ & Ifr & Ifr & kl & \\
\hline & WH & SURV & $P P$ & $k p z$ & pl. & Liquid & Og|FK1 & OglFK2 & Zi el & Gewi nn & prn & I nv & $p r d n$ & bau & $A K$ & $\mathrm{~h}$ & Pcht & $A n I$ & $\mathrm{Kr} 1$ & $\mathrm{Kr} 2$ & $\mathrm{Kr} 1$ & $\mathrm{Kr} 2$ \\
\hline 1 & 1 & 0 & 24 & & & 40000 & 500000 & 500000 & & & 100 & 100 & 0 & 0 & 0 & 0 & 0 & 0 & 250000 & 0 & 0 & \\
\hline 2 & 1 & 0 & 24 & 100 & 0 & 44981 & 264819 & 500000 & 6998 & 27178 & 100 & & & 0 & 0 & & & & 235181 & & & \\
\hline 3 & 1 & 0 & 24 & 100 & 0 & 559 & 80528 & 500000 & & & & 0 & 0 & 0 & & & & & & & & \\
\hline 4 & 1 & 0 & 24 & 100 & 0 & 6807 & 97179 & 500000 & & & 100 & 0 & 0 & 0 & 0 & 0 & 0 & 0 & 202821 & 0 & b & \\
\hline 5 & 1 & 0 & 24 & 100 & 0 & 3805 & 314829 & 500000 & 257 & 29437 & 100 & 0 & 0 & 0 & 0 & & & & 185171 & & & \\
\hline 6 & 1 & 0 & 24 & & 0 & & & & & & & 0 & 31 & 31 & & & & & & & 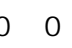 & \\
\hline 7 & 1 & 0 & 24 & 100 & 31 & & 9 & 50 & & & 10 & 0 & 89 & 57 & 0 & 0 & 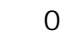 & 0 & & 0 & b & \\
\hline 8 & 1 & 0 & 24 & 100 & 89 & 1408 & 50 & 500 & & & 10 & 0 & 156 & 67 & & & & & & & ) & \\
\hline 9 & 1 & 0 & 24 & 100 & 156 & 104018 & 18 & 500 & & & 10 & 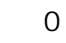 & 23 & 79 & & & & & & & & \\
\hline & 1 & 0 & 24 & 00 & 35 & & & & & & & 0 & & 94 & & & & & & & & \\
\hline & 1 & 0 & 24 & 00 & 329 & & 54 & 500 & & & 10 & 0 & 435 & 106 & & & & & & & D & \\
\hline & 1 & 0 & 24 & 100 & 435 & & & 50 & 110 & & 10 & 0 & 48 & 51 & & & & & 000 & 0 & & \\
\hline & 1 & 0 & 24 & 0 & 86 & & & & & & & 00 & & 0 & & & & & & & & \\
\hline & 1 & 0 & 24 & 100 & 486 & & & & & & 10 & 0 & 5 & 37 & & & & & & & & \\
\hline & 1 & 0 & 24 & & 523 & & & 33 & 12 & & 10 & 0 & 56 & 40 & & & & & 000 & 160279 & & \\
\hline & 1 & 0 & 24 & & 563 & & & & & & & 0 & & 5 & & & & & & & & \\
\hline & 1 & 0 & 24 & 1 & 608 & & & & & & & 0 & & 9 & & & & & & & & \\
\hline & 1 & 0 & 24 & & 657 & & & & & & 10 & ( & & & & & & & & 125696 & & \\
\hline & 1 & 0 & 24 & & 711 & & & & & & 1 & 0 & & 0 & & & & & & & & \\
\hline & 1 & 0 & 24 & 00 & 771 & & & & & & & 0 & & 6 & & & & & & & & \\
\hline & 1 & 0 & 24 & & 838 & & & & & & 10 & ( & & 3 & & & & & & & & \\
\hline & 1 & 0 & 24 & & & & & & & & 1 & 0 & 99 & & & & & & & & & \\
\hline & 1 & 0 & 24 & 100 & 991 & & & & & & & 6 & & 2 & & & & & & & & \\
\hline & 1 & 0 & 24 & 106 & 1032 & & & & & & & 6 & & 87 & & & & & & & & \\
\hline & 1 & 0 & 24 & 1 & 1062 & & & & & & 1 & 94 & & 0 & & & & & & & & \\
\hline & 1 & 0 & 24 & 106 & 994 & & & & & 731 & & 4 & & & & & & 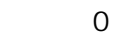 & & & & \\
\hline & 1 & 0 & 24 & 110 & 1021 & & & & & & & 3 & & 113 & & & & 0 & & & & \\
\hline & 1 & 0 & 24 & & 040 & & & & & & & 0 & & 97 & & & & & & & & \\
\hline & 1 & 0 & 24 & & 031 & & & & & & & 0 & & 10 & & & & 0 & & & ? & \\
\hline 3 & 1 & 0 & 24 & 114 & 084 & & & & & & & 0 & & 58 & & & 14 & & & & & \\
\hline 3 & 1 & 0 & 24 & 14 & 142 & & & & & & & 1 & & 6 & & & 15 & & & & 7 & \\
\hline & 1 & 0 & 24 & & & & & & & & & 0 & & & & & 5 & & & & & \\
\hline 33 & 1 & 0 & 24 & 11 & 102 & & & & 3 & & & 0 & & 9 & & & 15 & & & & & \\
\hline 3 & 1 & 0 & 24 & & 1096 & & & & & & & 0 & & 43 & 0 & & 15 & & & & & \\
\hline & 1 & 0 & 24 & & & & & & & & & 0 & & & & & 4 & & & & & \\
\hline 3 & 1 & 0 & 24 & 109 & 1087 & & & & & & 11 & 6 & 1141 & 54 & & & 14 & & 150565 & & ? & \\
\hline 37 & 1 & 0 & 24 & 20 & 1081 & & & & & & & 92 & & 40 & 0 & & 12 & & & 0 & 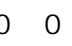 & \\
\hline & 1 & 0 & 24 & 108 & 1055 & & 408416 & & & & 114 & 6 & & 82 & & & 14 & & & & b & \\
\hline & 1 & 0 & 24 & & 1064 & & & & & & & 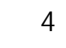 & & & & & 14 & & & 0 & 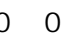 & \\
\hline 40 & 1 & 0 & 24 & 114 & 1058 & 1153090 & 448186 & 500000 & 231519 & 143468 & 114 & 0 & 1139 & 81 & 0 & 0 & 14 & 803552 & 51814 & 0 & D & \\
\hline
\end{tabular}


Fortsetzung Tabelle 386.1

$E$
$k$
$k$

n d e d e r p e r i o d e

kfr kfr kfr Tilg kKap tKap Zins SachA KK2 FK3 IKr. Saldo Saldo Saldo Verm. $26667 \quad 0 \quad 0 \quad 14819 \cdot 26667 \cdot 190200 \cdot 18200229167$ $21686 \quad 0 \quad 0 \quad 15708 \cdot 21686 \cdot 168913 \cdot 16713208333$ $16107 \quad 0 \quad 0 \quad 16651-16107-146014-15101 \quad 187500$ $\begin{array}{lllllllll}9860 & 0 & 0 & 17650 & -9860 & -121367 & -13352 & 166667\end{array}$ $\begin{array}{lllllllll}2862 & 0 & 0 & 18709 & -2862 & -94821 & -11454 & 145833\end{array}$ $\begin{array}{llllllll}0 & 0 & 0 & 20947 & 0 & -99288 & -12057 & 157570\end{array}$ $\begin{array}{llll}0 & 0 & 0 & 24239\end{array}$ $\begin{array}{llll}0 & 0 & 0 & 28092\end{array}$ $\begin{array}{llll}0 & 0 & 0 & 32605\end{array}$ $\begin{array}{llll}0 & 0 & 0 & 37896\end{array}$ $\begin{array}{llll}0 & 0 & 0 & 43903\end{array}$ $\begin{array}{lllll}0 & 0 & 0 & 47957\end{array}$ $\begin{array}{llll}0 & 0 & 0 & 32738\end{array}$ $\begin{array}{llll}0 & 0 & 0 & 35740\end{array}$ $\begin{array}{llll}0 & 0 & 0 & 39018\end{array}$ $\begin{array}{llll}0 & 0 & 0 & 42597\end{array}$ $\begin{array}{llll}0 & 0 & 0 & 46504\end{array}$ $\begin{array}{llll}0 & 0 & 0 & 50769\end{array}$ $\begin{array}{llll}0 & 0 & 0 & 55426\end{array}$ $\begin{array}{llll}0 & 0 & 0 & 60510\end{array}$ $\begin{array}{llll}0 & 0 & 0 & 66060\end{array}$ $\begin{array}{llll}0 & 0 & 0 & 72120\end{array}$ $\begin{array}{llll}0 & 0 & 0 & 79158\end{array}$ $\begin{array}{llll}0 & 0 & 0 & 83610\end{array}$ $\begin{array}{llll}0 & 0 & 0 & 60771\end{array}$ $\begin{array}{llll}0 & 0 & 0 & 59788\end{array}$ $\begin{array}{llll}0 & 0 & 0 & 57392\end{array}$ $\begin{array}{llll}0 & 0 & 0 & 51840\end{array}$ $\begin{array}{llll}0 & 0 & 0 & 44729\end{array}$

$0 \quad-66830-31225878786$

$\begin{array}{lllllllll}0 & 0 & 0 & 46357 & 162329 & 202765 & -17427 & 764384\end{array}$ $\begin{array}{llllll}0 & 0 & 0 & 47154 & 259127\end{array}$

$\begin{array}{lllllll}0 & 0 & 0 & 47815 & 353141\end{array}$

$\begin{array}{lllllll}0 & 0 & 0 & 48316 & 448530\end{array}$

$\begin{array}{llllll}0 & 0 & 0 & 46751 & 528481\end{array}$

$\begin{array}{llllll}0 & 0 & 0 & 45062 & 614166\end{array}$ $\begin{array}{rrrr}0 & -99288 & -12057 & 157570 \\ 0 & -127232 & -14573 & 194206\end{array}$ $0 \cdot 160664-17566 \quad 237978$ $0 \cdot 200596-21125 \quad 290166$ $0 \cdot 248222-25352 \quad 352275$ $0 \cdot 299858 \cdot 30000 \quad 421018$ $0 \cdot 285064 \cdot 30000 \quad 426862$ $0.479996-46095 \quad 626336$ $\begin{array}{llll}0 & -459724 & -45296 & 613934\end{array}$ $\begin{array}{llll}0 & -437486 & -44425 & 603226\end{array}$ $0-413092-43476 \quad 594388$ $\begin{array}{lllll}0 & -386331 & -42441 & 587613\end{array}$ $0.356973-41313 \quad 583114$ $0-324763-40083 \quad 581126$

$0 \cdot 289425 \cdot 38743 \quad 581909$

$0 \cdot 250653-37282 \quad 585749$

$0 \cdot 208112 \cdot 35690 \quad 592961$

$0 \cdot 157890-33954 \quad 600569$

$0 \cdot 111322-32062 \quad 618811$

$0 \cdot 206274-37904 \quad 767526$

$0 \cdot 185050-37512804000$

$0-163639-36971843891$

$\begin{array}{lrrr}0 & -112820 & -34016 \quad 857587\end{array}$

$\begin{array}{lllll}0 & 0 & 0 & 25172 & 512189\end{array}$

$352082 \quad .9586 \quad 704624 \quad 1056706$

$500463 \quad-1817 \quad 6534351153899$

$\begin{array}{llll}651215 & 6082 & 607692 & 1258907\end{array}$

$\begin{array}{lllll}786355 & 13263 \quad 584735 & 1371090\end{array}$

$92605520662 \quad 564588 \quad 1490644$

$\begin{array}{lllll}858689 & 18604 & 747646 & 1606335\end{array}$

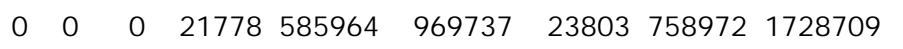

\begin{tabular}{lllllllll|}
0 & 0 & 0 & 17992 & 688654 & 1101276 & 30244 & 756384 & 1857660
\end{tabular}

$\begin{array}{lllllllll}0 & 0 & 0 & 14520 & 803552 & 1241009 & 37069 & 752430 & 1993440\end{array}$
L $\quad s$ t e am periodenende ung ung ung ung ung ung. ung. ung. ung. ung $P e$ I FIa MKz Stp AKh DE KFK1 KFK2 IFK1 IFK2 Liq ri $\begin{array}{llllllllll}0 & 1000 & 200 & 2333350000 & 250000 & 500000 & 0\end{array}$ $41486 \quad 0 \quad 0 \quad 0 \quad 104020033893 \quad 50000280528500000 \quad 0 \quad 3$ $45300 \mid \begin{array}{llllllllllll}0 & 0 & 0 & 1040 & 200 & 40140 & 50000 & 297179 & 500000 & 0 & 4\end{array}$ $51013 \mid 0000010402004713850000314829500000 \quad 0 \quad 5$ \begin{tabular}{l|lllllllllllll}
58282 & 0 & 0 & 0 & 1009 & 194 & 50000 & 50000 & 299052 & 500000 & 0 & 6
\end{tabular} \begin{tabular}{l|llllllllllllll}
66974 & 0 & 0 & 0 & 958 & 182 & 50000 & 50000 & 257121 & 500000 & 0 & 7
\end{tabular} $77314 \mid 000009021695000050000207226500000 \quad 0 \quad 8$ $89570 \mid \begin{array}{lllllllllll}0 & 0 & 0 & 83615350000 & 50000 & 147913 & 500000 & 0 & 9\end{array}$ $104052 \mid \begin{array}{lllllllllllll} & 0 & 0 & 0 & 758 & 134 & 50000 & 50000 & 77468 & 500000 & 0 & 10\end{array}$ \begin{tabular}{l|llllllllllll} 
& 0 & 0 & 0 & 67111350000 & 50000 & 0 & 500000 & 0 & 11
\end{tabular} $6411035000050000 \quad 0500000 \quad 0 \quad 12$ $6111035000050000 \quad 0321163 \quad 0 \quad 13$ $\begin{array}{llllll}615955000050000 & 0 & 330043 & 0 & 14\end{array}$ $\begin{array}{llllllll}5818750000 & 50000 & 0 & 339721 & 0 & 15\end{array}$ $\begin{array}{lllllllll}5457850000 & 50000 & 0 & 350271 & 0 & 16\end{array}$ $\begin{array}{llllllll}505 & 6950000 & 50000 & 0 & 361770 & 0 & 17\end{array}$ $\begin{array}{llllllll}460 & 5850000 \quad 50000 & 0 & 374304 & 0 & 18\end{array}$ $\begin{array}{llllllll}411 & 4650000 & 50000 & 0 & 387966 & 0 & 19\end{array}$ $\begin{array}{llllllll}3573250000 & 50000 & 0 & 402857 & 0 & 20\end{array}$ $\begin{array}{llllllll}297 & 185000050000 & 0 & 419089 & 0 & 21\end{array}$ $\begin{array}{lllllllll}231 & 250000 & 50000 & 0 & 436782 & 0 & 22\end{array}$ $12205000050000 \quad 0456067 \quad 0 \quad 23$ $\begin{array}{llllllll}29 & 0 & 50000 & 50000 & 0 & 477087 & 0 & 24\end{array}$ $\begin{array}{lllllllll}103 & 5000050000 & 0 & 412177 & 0 & 25\end{array}$

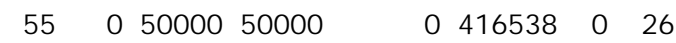

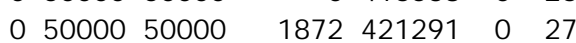
$0 \quad 50000 \quad 50000 \quad 43358 \quad 426471 \quad 0 \quad 28$ $\begin{array}{lllllll}0 & 50000 & 50000 & 81401 & 432118 & 0 & 29\end{array}$ $0 \quad 50000 \quad 50000 \quad 119975 \quad 438273 \quad 0 \quad 30$ $05000050000 \quad 156808 \quad 444983 \quad 0 \quad 31$ $15000050000195852452295 \quad 0 \quad 32$ $25000050000235035460267 \quad 0 \quad 33$ $250000 \quad 50000 \quad 274162 \quad 468955 \quad 0 \quad 34$

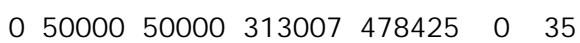
$0 \quad 5000050000 \quad 349435488748 \quad 0 \quad 36$ $0 \quad 50000 \quad 50000 \quad 383244500000 \quad 0 \quad 37$ $0 \quad 5000050000 \quad 408416500000 \quad 0 \quad 38$ $05000050000430194500000 \quad 0 \quad 39$ $05000050000 \quad 448186500000 \quad 0 \quad 40$ 
Tabelle 388.1: Subtraktion der Ergebnisgrößen der Strategie „Risikoabschlag: 10\% bei Pflanzenproduktion, $2.5 \%$ bei Tierproduktion" (Tabelle 384. I) von der Strategie ,Risikoabschlag: $5 \%$ bei Pflanzenproduktion, $2.5 \%$ bei Tierproduktion“" (Tabelle 386. I)

\begin{tabular}{|c|c|c|c|c|c|c|c|c|c|c|c|c|c|c|c|c|c|c|c|c|c|c|}
\hline $\begin{array}{c}\mathrm{Pe} \\
\mathrm{ri}\end{array}$ & WH & SURV & $\begin{array}{c}\text { Preis } \\
\text { Pfl }\end{array}$ & $\begin{array}{l}\text { Ma. } \\
\text { kpz }\end{array}$ & $\begin{array}{l}\text { Stl } \\
\text { pl. }\end{array}$ & $\begin{array}{l}\text { Li- } \\
\text { quid }\end{array}$ & $\begin{array}{c}\text { Og } \\
\text { IFK1 }\end{array}$ & $\begin{array}{c}\mathrm{Og} \\
\text { IFK2 }\end{array}$ & Ziel & $\begin{array}{c}\text { Ge- } \\
\text { winn }\end{array}$ & PP & $\begin{array}{l}\text { Ma } \\
\text { Inv }\end{array}$ & TP & $\begin{array}{l}\text { Stll } \\
\text { bau }\end{array}$ & AK & $\begin{array}{c}\text { AK } \\
h\end{array}$ & Pcht & $\begin{array}{l}\text { Fin } \\
\text { Anl }\end{array}$ & $\begin{array}{l}\text { Ifr } \\
K r 1\end{array}$ & $\begin{array}{l}\text { Ifr } \\
\mathrm{Kr} 2\end{array}$ & $\begin{array}{l}\mathrm{kl} \\
\mathrm{Kr} 1 \mathrm{~K}\end{array}$ & $\begin{array}{c}\mathrm{kl} \\
\mathrm{Kr} 2\end{array}$ \\
\hline 1 & 1 & 0 & 24 & & & 0 & 0 & 0 & 8400 & 8400 & 0 & 0 & 0 & 0 & 0 & 0 & 0 & 0 & 0 & 0 & 0 & 0 \\
\hline 2 & 1 & 0 & 24 & 0 & 0 & 0 & 0 & 0 & 8400 & 8400 & 0 & 0 & 0 & 0 & 0 & 0 & 0 & 0 & 0 & 0 & 0 & 0 \\
\hline 3 & 1 & 0 & 24 & 0 & 0 & 0 & 0 & 0 & 8400 & 8400 & 0 & 0 & 0 & 0 & 0 & 0 & 0 & 0 & 0 & 0 & 0 & 0 \\
\hline 4 & 1 & 0 & 24 & 0 & 0 & 0 & 0 & 0 & 8400 & 8400 & 0 & 0 & 0 & 0 & 0 & 0 & 0 & 0 & 0 & 0 & 0 & 0 \\
\hline 5 & 1 & 0 & 24 & 0 & 0 & 0 & 0 & 0 & 8400 & 8400 & 0 & 0 & 0 & 0 & 0 & 0 & 0 & 0 & 0 & 0 & 0 & 0 \\
\hline 6 & 1 & 0 & 24 & 0 & 0 & 0 & 0 & 0 & 8400 & 8400 & 0 & 0 & 0 & 0 & 0 & 0 & 0 & 0 & 0 & 0 & 0 & 0 \\
\hline 7 & 1 & 0 & 24 & 0 & 0 & 0 & 0 & 0 & 8400 & 8400 & 0 & 0 & 0 & 0 & 0 & 0 & 0 & 0 & 0 & 0 & 0 & 0 \\
\hline 8 & 1 & 0 & 24 & 0 & 0 & 0 & 0 & 0 & 8400 & 8400 & 0 & 0 & 0 & 0 & 0 & 0 & 0 & 0 & 0 & 0 & 0 & 0 \\
\hline 9 & 1 & 0 & 24 & 0 & 0 & 0 & 0 & 0 & 8400 & 8400 & 0 & 0 & 0 & 0 & 0 & 0 & 0 & 0 & 0 & 0 & 0 & 0 \\
\hline 10 & 1 & 0 & 24 & 0 & 0 & 0 & 0 & 0 & 8400 & 8400 & 0 & 0 & 0 & 0 & 0 & 0 & 0 & 0 & 0 & 0 & 0 & 0 \\
\hline 11 & 1 & 0 & 24 & 0 & 0 & 0 & 0 & 0 & 8400 & 8400 & 0 & 0 & 0 & 0 & 0 & 0 & 0 & 0 & 0 & 0 & 0 & 0 \\
\hline 12 & 1 & 0 & 24 & 0 & 0 & 0 & 0 & 0 & 8400 & 8400 & 0 & 0 & 0 & 0 & 0 & 0 & 0 & 0 & 0 & 0 & 0 & 0 \\
\hline 13 & 1 & 0 & 24 & 0 & 0 & 0 & 0 & 0 & 8400 & 8400 & 0 & 0 & 0 & 0 & 0 & 0 & 0 & 0 & 0 & 0 & 0 & 0 \\
\hline 14 & 1 & 0 & 24 & 0 & 0 & 0 & 0 & 0 & 8400 & 8400 & 0 & 0 & 0 & 0 & 0 & 0 & 0 & 0 & 0 & 0 & 0 & 0 \\
\hline 15 & 1 & 0 & 24 & 0 & 0 & 0 & 0 & 0 & 8400 & 8400 & 0 & 0 & 0 & 0 & 0 & 0 & 0 & 0 & 0 & 0 & 0 & 0 \\
\hline 16 & 1 & 0 & 24 & 0 & 0 & 0 & 0 & 0 & 8400 & 8400 & 0 & 0 & 0 & 0 & 0 & 0 & 0 & 0 & 0 & 0 & 0 & 0 \\
\hline 17 & 1 & 0 & 24 & 0 & 0 & 0 & 0 & 0 & 8400 & 8400 & 0 & 0 & 0 & 0 & 0 & 0 & 0 & 0 & 0 & 0 & 0 & 0 \\
\hline 18 & 1 & 0 & 24 & 0 & 0 & 0 & 0 & 0 & 8400 & 8400 & 0 & 0 & 0 & 0 & 0 & 0 & 0 & 0 & 0 & 0 & 0 & 0 \\
\hline 19 & 1 & 0 & 24 & 0 & 0 & 0 & 0 & 0 & 8400 & 8400 & 0 & 0 & 0 & 0 & 0 & 0 & 0 & 0 & 0 & 0 & 0 & 0 \\
\hline 20 & 1 & 0 & 24 & 0 & 0 & 0 & 0 & 0 & 8400 & 8400 & 0 & 0 & 0 & 0 & 0 & 0 & 0 & 0 & 0 & 0 & 0 & 0 \\
\hline 21 & 1 & 0 & 24 & 0 & 0 & 0 & 0 & 0 & 8400 & 8400 & 0 & 0 & 0 & 0 & 0 & 0 & 0 & 0 & 0 & 0 & 0 & 0 \\
\hline 22 & 1 & 0 & 24 & 0 & 0 & 0 & 0 & 0 & 8400 & 8400 & 0 & 0 & 0 & 0 & 0 & 0 & 0 & 0 & 0 & 0 & 0 & 0 \\
\hline 23 & 1 & 0 & 24 & 0 & 0 & 0 & 0 & 0 & 8760 & 8346 & 3 & 3 & 33 & 33 & 0 & 0 & 3 & 0 & 52835 & 0 & 0 & 0 \\
\hline 24 & 1 & 0 & 24 & 3 & 33 & 8674 & -50701 & 0 & 13870 & 8652 & 7 & 4 & 70 & 36 & 0 & 0 & 7 & 0 & 108839 & 0 & 0 & 0 \\
\hline 25 & 1 & 0 & 24 & 7 & 70 & 18416 & $-1 E+05$ & 0 & 16997 & 7111 & 6 & -1 & 62 & -8 & 0 & 0 & 6 & 0 & 2751 & 87823 & 0 & 0 \\
\hline 26 & 1 & 0 & 24 & 6 & 61 & 14893 & -3983 & -83462 & 17689 & 6643 & 10 & 4 & 97 & 35 & 0 & 0 & 10 & 0 & 60393 & 83462 & 0 & 0 \\
\hline 27 & 1 & 0 & 24 & 10 & 96 & 24202 & -59612 & -78709 & 22916 & 6853 & 13 & 3 & 130 & 33 & 0 & 0 & 13 & 0 & 112775 & 78709 & 0 & 0 \\
\hline 28 & 1 & 0 & 24 & 13 & 129 & 33138 & $-1 E+05$ & -73529 & 27801 & 7377 & 14 & 0 & 133 & 3 & 0 & 0 & 14 & 0 & 113879 & 73529 & 0 & 0 \\
\hline 29 & 1 & 0 & 24 & 14 & 132 & 33958 & $-1 E+05$ & -67882 & 28213 & 7537 & 14 & 0 & 131 & -2 & 0 & 0 & 14 & 0 & & 67882 & 0 & 0 \\
\hline 30 & 1 & 0 & 24 & 14 & 131 & 33734 & $-1 E+05$ & -61727 & 27904 & 5433 & 10 & -4 & 101 & -30 & 0 & 0 & 10 & 49382 & 104717 & 61727 & 0 & 0 \\
\hline 31 & 1 & 0 & 24 & 10 & 101 & 72659 & $-1 E+05$ & -55017 & 25784 & 2340 & 3 & -7 & 29 & -72 & 0 & 0 & 3 & 162329 & 101349 & 55017 & 0 & 0 \\
\hline 32 & 1 & 0 & 24 & 3 & 29 & 160848 & -97778 & -47705 & 21213 & 5099 & 1 & -2 & 1 & -28 & 0 & 0 & 1 & 196377 & 97778 & 47705 & 0 & 0 \\
\hline 33 & 1 & 0 & 24 & 1 & 1 & 185530 & -93993 & -39733 & 19150 & 6477 & 1 & 0 & -4 & -4 & 0 & 0 & 1 & 1088 & 93993 & 39733 & 0 & 0 \\
\hline 34 & 1 & 0 & 24 & 1 & -3 & 178378 & -89982 & -31045 & 18298 & 6540 & 1 & 0 & -4 & -1 & 0 & 0 & 1 & 179719 & 89982 & 31045 & 0 & 0 \\
\hline 35 & 1 & 0 & 24 & -2 & -4 & 166159 & -85729 & -21575 & 16682 & 7079 & 0 & 3 & -1 & 3 & 0 & 0 & 0 & 156055 & 85729 & 21575 & 0 & 0 \\
\hline 36 & 1 & 0 & 24 & -3 & -1 & 143361 & -83098 & -11252 & 15583 & 6963 & 0 & 4 & -1 & 0 & 0 & 0 & 0 & 134249 & 83098 & 11252 & 0 & 0 \\
\hline 37 & 1 & 0 & 24 & 1 & -1 & 122194 & -81975 & 0 & 15639 & 7846 & 0 & -1 & 0 & 1 & 0 & 0 & 0 & 122848 & 81975 & 0 & 0 & 0 \\
\hline 38 & 1 & 0 & 24 & -4 & 0 & 110788 & -68669 & 0 & 14111 & 8380 & 0 & 4 & -1 & -1 & 0 & 0 & 0 & 103326 & 68669 & 0 & 0 & 0 \\
\hline 39 & 1 & 0 & 24 & -4 & -1 & 91112 & -55725 & 0 & 13089 & 8396 & 0 & 4 & -1 & 0 & 0 & 0 & 0 & 83098 & 55725 & 0 & 0 & 0 \\
\hline 40 & 1 & 0 & 24 & 0 & -1 & 70846 & -43008 & 0 & 12940 & 8024 & 0 & 0 & 0 & 2 & 0 & 0 & 0 & 68866 & 43008 & 0 & 0 & 0 \\
\hline \multicolumn{4}{|c|}{ Mittelwerte } & 2 & 20 & 36722 & -33636 & -14291 & 13036 & 7747 & 2 & 0 & 19 & 0 & 0 & 0 & 2 & 36183 & 36635 & 16486 & & 0 \\
\hline
\end{tabular}

\subsubsection{Posterdarstellungen der Ergebnisse des Betriebsentwicklungsmodells aus Kapitel [}

Auf den nachfolgenden zwei Seiten sind die wesentlichen Ergebnisse des Betriebsentwicklungsmodells, das in Kapitel 6 diskutiert wurde, als Poster aufbereitet. Das erste Poster legt seinen Schwerpunkt auf eine möglichst umfassende textliche 
Fortsetzung Tabelle 388.1

kl kfr kfr kfr Tilg kKap tKap Zins SachA Eig lung ung ung ung ung ung. ung. ung. ung. ung Pe

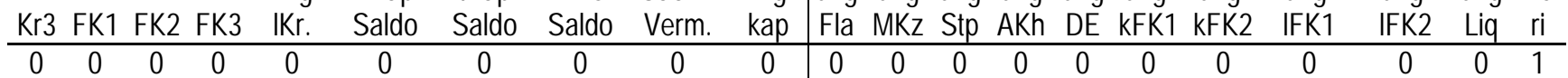

$\begin{array}{llllllllllllllllllllll}0 & 0 & 0 & 0 & 0 & 0 & 0 & 0 & 0 & 0 & 0 & 0 & 0 & 0 & 0 & 0 & 0 & 0 & 0 & 0 & 2\end{array}$

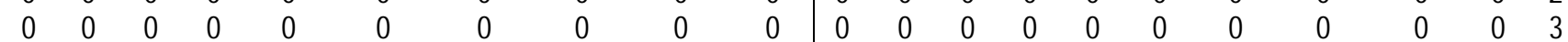

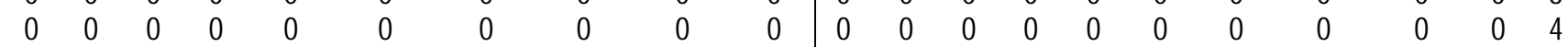

\begin{tabular}{lllllllll|llllllllllll}
0 & 0 & 0 & 0 & 0 & 0 & 0 & 0 & 0 & 0 & 0 & 0 & 0 & 0 & 0 & 0 & 0 & 0 & 0 & 0 & 5
\end{tabular}

\begin{tabular}{lllllllll|llllllllllllll}
0 & 0 & 0 & 0 & 0 & 0 & 0 & 0 & 0 & 0 & 0 & 0 & 0 & 0 & 0 & 0 & 0 & 0 & 0 & 0 & 6
\end{tabular}

$\begin{array}{lllllllllllllllllllll}0 & 0 & 0 & 0 & 0 & 0 & 0 & 0 & 0 & 0 & 0 & 0 & 0 & 0 & 0 & 0 & 0 & 0 & 0 & 0 & 7\end{array}$

\begin{tabular}{lllllllll|llllllllllllll}
0 & 0 & 0 & 0 & 0 & 0 & 0 & 0 & 0 & 0 & 0 & 0 & 0 & 0 & 0 & 0 & 0 & 0 & 0 & 0 & 8
\end{tabular}

$\begin{array}{lllllllllllllllllllllll}0 & 0 & 0 & 0 & 0 & 0 & 0 & 0 & 0 & 0 & 0 & 0 & 0 & 0 & 0 & 0 & 0 & 0 & 0 & 0 & 9\end{array}$

\begin{tabular}{lllllllll|lllllllllllllll}
0 & 0 & 0 & 0 & 0 & 0 & 0 & 0 & 0 & 0 & 0 & 0 & 0 & 0 & 0 & 0 & 0 & 0 & 0 & 0 & 10
\end{tabular}

$\begin{array}{lllllllllllllllllllllll}0 & 0 & 0 & 0 & 0 & 0 & 0 & 0 & 0 & 0 & 0 & 0 & 0 & 0 & 0 & 0 & 0 & 0 & 0 & 0 & 11\end{array}$

\begin{tabular}{lllllllll|llllllllllll}
0 & 0 & 0 & 0 & 0 & 0 & 0 & 0 & 0 & 0 & 0 & 0 & 0 & 0 & 0 & 0 & 0 & 0 & 0 & 0 & 12
\end{tabular}

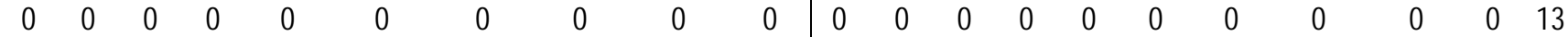

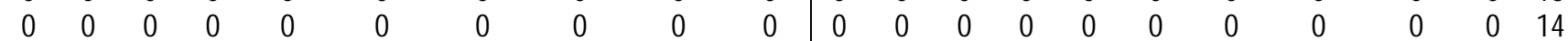

\begin{tabular}{lllllllll|llllllllllll}
0 & 0 & 0 & 0 & 0 & 0 & 0 & 0 & 0 & 0 & 0 & 0 & 0 & 0 & 0 & 0 & 0 & 0 & 0 & 0 & 15
\end{tabular}

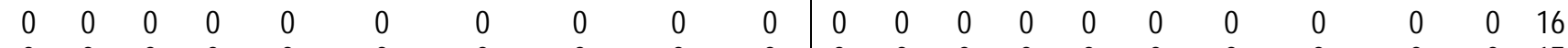

$\begin{array}{llllllllllllllllllllll}0 & 0 & 0 & 0 & 0 & 0 & 0 & 0 & 0 & 0 & 0 & 0 & 0 & 0 & 0 & 0 & 0 & 0 & 0 & 0 & 17\end{array}$

$\begin{array}{lllllllllllllllllllll}0 & 0 & 0 & 0 & 0 & 0 & 0 & 0 & 0 & 0 & 0 & 0 & 0 & 0 & 0 & 0 & 0 & 0 & 0 & 0 & 18\end{array}$

\begin{tabular}{lllllllll|llllllllllll}
0 & 0 & 0 & 0 & 0 & 0 & 0 & 0 & 0 & 0 & 0 & 0 & 0 & 0 & 0 & 0 & 0 & 0 & 0 & 0 & 19
\end{tabular}

$\begin{array}{lllllllllllllllllllllll}0 & 0 & 0 & 0 & 0 & 0 & 0 & 0 & 0 & 0 & 0 & 0 & 0 & 0 & 0 & 0 & 0 & 0 & 0 & 0 & 20\end{array}$

$\begin{array}{lllllllllllllllllllll}0 & 0 & 0 & 0 & 0 & 0 & 0 & 0 & 0 & 0 & 0 & 0 & 0 & 0 & 0 & 0 & 0 & 0 & 0 & 0 & 21\end{array}$

\begin{tabular}{rllllllll|llllllllllll}
0 & 0 & 0 & 0 & 0 & 0 & 0 & 0 & 0 & 0 & 0 & 0 & 0 & 0 & 0 & 0 & 0 & 0 & 0 & 0 & 22
\end{tabular}

$\begin{array}{lllllllllllllllllllll}0 & 0 & 0 & 0 & 2133 & 0 & -42027 & -3170 & 41863 & -164 & 0 & 0 & 0 & -61 & 0 & 0 & 0 & -52835 & 0 & 0 & 23\end{array}$

$\begin{array}{rllllllllllllllllllll}0 & 0 & 0 & 0 & 4518 & 0 & -85905 & -6530 & 85548 & -357 & 0 & 0 & 0 & -121 & 0 & 0 & 0 & -1 \mathrm{E}+05 & 0 & 0 & 24\end{array}$

$\begin{array}{llllllllllllllllllllll}0 & 0 & 0 & 0 & 3129 & 0 & -72553 & -8069 & 69738 & 9252 & 0 & 0 & 0 & -97 & 0 & 0 & 0 & -2751 & -87823 & 0 & 25\end{array}$

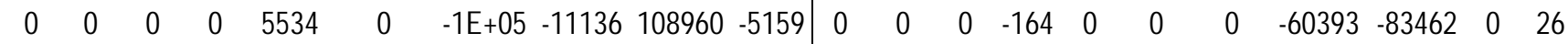

$\begin{array}{lllllllllllllllllllll}0 & 0 & 0 & 0 & 7955 & 0 & -2 E+05 & -13850 & 143198 & -7193 & 0 & 0 & 0 & -217 & 0 & 0 & 0 & -1 E+05 & -78709 & 0 & 27\end{array}$

$\begin{array}{llllllllllllllllllllll}0 & 0 & 0 & 0 & 8733 & 0 & -1 \mathrm{E}+05 & -13450 & 136149 & -8568 & 0 & 0 & 0 & -214 & 0 & 0 & 0 & -1 \mathrm{E}+05 & -73529 & 0 & 28\end{array}$

$\begin{array}{lllllllllllllllllllll}0 & 0 & 0 & 0 & 9333 & 0 & -1 \mathrm{E}+05 & -12583 & 123516 & -9194 & 0 & 0 & 0 & -212 & 0 & 0 & 0 & -1 \mathrm{E}+05 & -67882 & 0 & 29\end{array}$

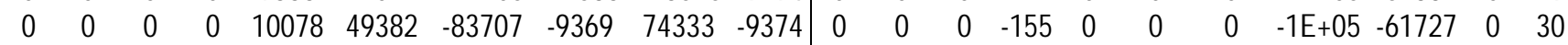

$\begin{array}{lllllllllllllllllllll}0 & 0 & 0 & 0 & 10884 & 162329 & 15365 & -2916 & -25006 & -9641 & 0 & 0 & 0 & -30 & 0 & 0 & 0 & -1 E+05 & -55017 & 0 & 31\end{array}$

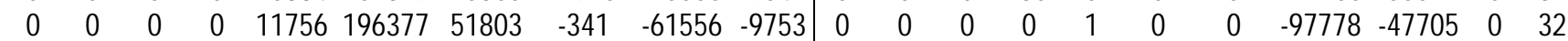

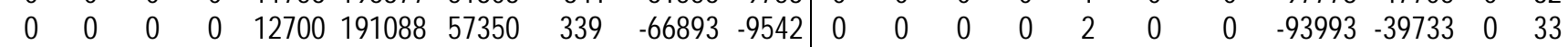

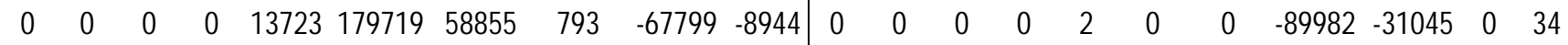

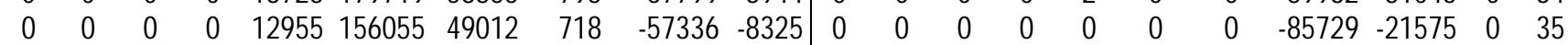

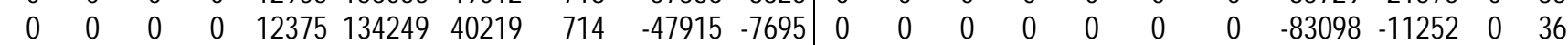

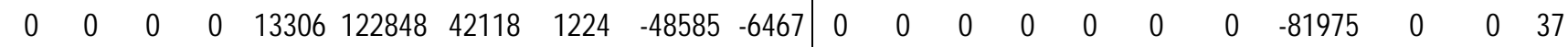

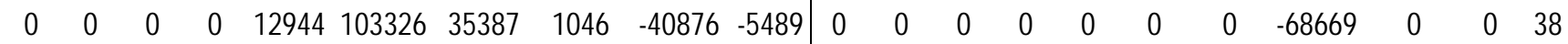

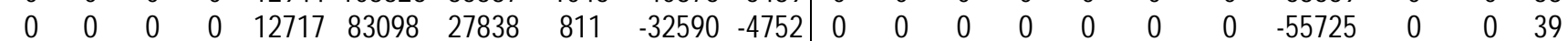

$\begin{array}{rrrrrrrrrrrrrrrrrrrrrrrrrrr}0 & 0 & 0 & 0 & 13396 & 68866 & 26716 & 863 & -30599 & -3882 & 0 & 0 & 0 & 0 & 0 & 0 & 0 & -43008 & 0 & 0 & 40\end{array}$

Wiedergabe des Modells und seiner Ergebnisse. In Gegensatz dazu verzichtet das zweite Poster auf eine sich selbst vollständig erklärende Modellbeschreibung und stellt eine visuelle Darstellung in den Vordergrund. 


\section{What's the best strategy to survive as a farmer? A Monte-Carlo Simulation}

\section{Ludger Hinners-Tobrägel}

\section{Abstract:}

Can other strategies than profit maximisation lead to a higher probability of survival? Results of Monte-Carlo simulations show that in a farm development model behaviour patterns exist, which enlarge the chance of survival compared with profit maximisation. In the special formulation of the presented model simple strategies like diversification or risk discounts on the prices of products reduce the risk of failure significantly.

\section{Key words:}

farm development model; survival analysis; Monte-Carlo simulation

\begin{tabular}{|l|l|}
\hline \multicolumn{1}{|c|}{ The Question } & \multicolumn{1}{c|}{ The Answer } \\
\hline $\begin{array}{l}\text { Is profit maximisation always the best strategy to maintain a farm for } \\
\text { the next generation and to minimise the probability of ruin? }\end{array}$ & $\begin{array}{l}\text { At least within a stochastic dynamic farm model, better strategies } \\
\text { than profit maximisation may exist to minimise ruin probability. }\end{array}$ \\
\hline
\end{tabular}

\section{Model description}

- A very stylised farm is modelled with plant and animal production on a polypolistic market. The farm is a price-taker and can only produce two products with linear technologies in a random environment. The production requires investments. On the market, the farm can borrow loans, hire labour force, and rent land.

- The decision process: linear programming with rational expectations, rolling planning with a planning horizon of one period
- The environment: Prices of products are uncertain. To avoid the success of a strategy depending on the activities of its competitors as in some other studies (Axelrod 1984, Gerybadze 1982, Fischer 1988, Brandes 1989, Sigmund 1995, and Burmester 1996), each strategy is simulated in an identical environment.

- Steps of business planning: building of expectations, planning, realisation, surprise, new planning.

\begin{tabular}{|c|l|l|}
\hline \multicolumn{3}{|c|}{ The Strategies } \\
\hline Reference Strategy & \multicolumn{1}{|c|}{ Diversification Strategy } & \multicolumn{1}{c|}{ Risk Discount Strategy } \\
\hline $\begin{array}{c}\text { Unrestricted profit } \\
\text { maximisation }\end{array}$ & $\begin{array}{l}\text { Investments in plant and animal production are fixed to } \\
\text { be equal although animal production is more profitable. }\end{array}$ & $\begin{array}{l}\text { The farmer calculates the optimal investments } \\
\text { using lower prices than she or he is expecting. }\end{array}$ \\
\hline
\end{tabular}

Table: Final value assets and survivorship probability of the strategies in the stochastic model

\begin{tabular}{|c|c|c|c|c|c|}
\hline \multirow{2}{*}{$\begin{array}{l}\text { strategy } \\
\text { (1) }\end{array}$} & \multirow{2}{*}{$\begin{array}{c}\text { insolvency } \\
\text { rate } \\
\text { (2) }\end{array}$} & \multicolumn{3}{|c|}{$\begin{array}{l}\text { average equity of farms in the last } \\
\text { period (final value assets) }\end{array}$} & \multirow{2}{*}{$\begin{array}{l}\text { life } \\
\text { span } \\
\text { (6) }\end{array}$} \\
\hline & & $\underset{\text { (3) }}{\operatorname{determin} *}$ & $\begin{array}{l}\text { all farms } \\
\text { (4) }\end{array}$ & $\begin{array}{l}\text { survival } \\
\text { farms (5) }\end{array}$ & \\
\hline \multicolumn{6}{|c|}{ Model I: plant price triangle distributed, $\pm 10 \%$} \\
\hline reference & $5 \%(2$ & 2403711 & 2270758 & 2390538 & 38.2 \\
\hline diversification & $4 \% 1$ & 2122990 & 2029077 & 21137853 & 38.6 \\
\hline $\begin{array}{l}\text { risk discount: } \\
\text { pp } 10 \%\end{array}$ & $5 \%(2$ & 2353470 & 2236626 & 23546092 & 38.2 \\
\hline \multicolumn{6}{|c|}{ Model II: plant and animal price triangle distributed, $\pm 10 \%$} \\
\hline reference & $19 \%$ & 2411674 & 2015688 & 2484710 & 33.3 \\
\hline $\begin{array}{l}\text { diversification } \\
\text { risk discount: }\end{array}$ & $12 \%$ & 2122990 & 1903261 & 21593003 & 35.7 \\
\hline pp $10 \%$ & $18 \% 4$ & 2353470 & 1959650 & 24121732 & 33.3 \\
\hline ap $2.5 \%$ & $6 \%(2$ & 1969351 & 1830769 & 19513345 & 37.9 \\
\hline $\begin{array}{l}\text { pp } 10 \% \text {, ap } \\
2.5 \%\end{array}$ & $0.3 \%$ & 1997322 & 1946365 & 1952884 4 & 39.9 \\
\hline & & & & & \\
\hline
\end{tabular}

\section{Explanation:}

Since the alternatives strategies include an additional restriction, the profit generated must be lower than the profit using unrestricted profit maximisation, as shown in the table. In model I, only the plant price is random. The risk discount strategy is stochasticly dominated by profit maximisation. The diversification shows a trade-off between earnings and survivorship probability (and average life span).

With both prices are random (model II) grain price discounts nearly have no effect on the survivorship probability. Significantly less insolvencies occur if a farm makes a discount in animal prices. The larger effect of a discount in animal production is due to the fact, that the profit rate is much larger in plant than in animal production. Also diversification results in a significantly smaller rate of business failures. The rate of business failures expresses how many of the enterprises do not survive the $40^{\text {th }}$ period. A combination of risk discounts in plant and animal production almost ensure survivorship.

The numbers in the black circles describe the ranking of the strategies. In column 2, the referring criterion is the rate of failures (right span). This criterion leads to the same ranking than the average life span. In column 5, a second ranking is performed, now based on final net worth.

The two rankings differ. But two of the strategies are of no interest for risk-avers decision makers, since they are dominated (risk discounts only in animal production). Strategies which are not dominated are printed in italics. From this assemblage the manager has to take a decision under uncertainty, weighting more expectation value of profit against lower risk of failure. 


\section{What's the best strategy to sunive as a famer? A Monte-Carlo Simulation}

\section{Ludger Hinners-Tobrägel}

\section{"Profit maximisation is not always the best}

strategy for a farm to minimise ruin probability.

\section{Model Description}

- A very stylised farm model with plant and animal production on a polypolistic market. The farm is a price-taker and can only produce two products with linear technologies.

- The decision process: linear programming with rational expectations, rolling planning with a planning horizon of one period
- The environment: Prices of products are uncertain. To avoid the success of a strategy depending on the activities of its competitors each strategy is simulated in an identical environment.

- Steps of business planning: building of expectations, planning, realisation, surprise, new planning.
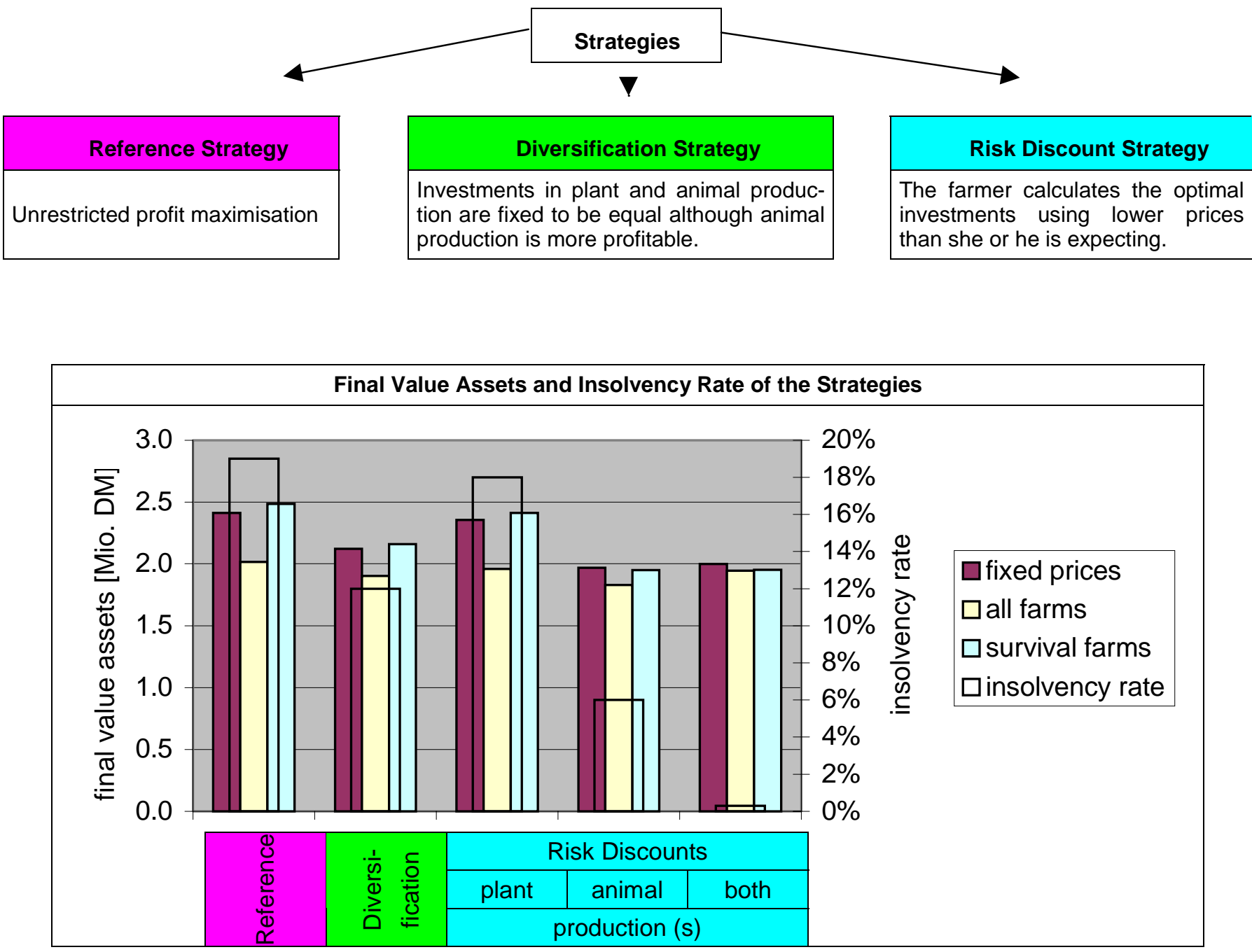



\section{Index}

$\cap 219$

$\mu$-Prinzip 100

Abbildung, adäquate 38 Siehe auch Modell

Abschreibungsmethode 60

Abwägungsbereich Siehe Unbestimmtheitsbereich

Agenten 259 Siehe auch Modell, Typen,

spieltheoretisches und agent based modeling;

Emergenzen

Aktivitäten 100, 114, 341

allgemeingültig 33, 36, 40, 43, 146

Altersbegrenzung 247

Altersverteilung 247

analytische Verfahren 31, 33, 44

Annuität 112, 115

Anschaffungsauszahlung 112

Anteilswert 37, 106, 296

Aufzinsungsfaktor 208, 210, 212, 252, 258

Ausscheideschwelle 279 Siehe auch Insolvenz, Ruin

Ausstattung 46, 89, 104, 277f, 283, 301f

Ausstiegskosten 80

Auszahlung 88, 97, 192f, 245, 247, 258, 263

Autokorrelation 190, 275, 276

Bayes

Anwendung der Bayes-Regel 196, 221, 331

Bayes-Prinzip 100

bedingte Wahrscheinlichkeit 131

Satz von 151

Betriebsentwicklungsmodell 19 Siehe Modell

Betriebsschließungen 17

Bilanz 279, 283f

black box 38

Bonini-Paradoxon 29

Bootstrap 106f

Buchführungsmodul 279

Chance Constrained Programming 104

Chaos 39f

charakteristisches Polynom 50f

Club of Rome 25, 42

Creditreform 17

Datenkranz 37, 300

Deduktion $31 \mathrm{f}$

density dependence theory 77
Dichtefunktion 130

Differenzengleichung 37, 40, 48f, 53, 55, 57, 191, 211f, 228, 263

diskreter Zeitablauf Siehe diskreter Zeitverlauf diskreter Zeitverlauf 34, 154, 190f, 214, 218

Diversifikation 77, 87, 103, 271

Drift 78

Dungeinheitengrenze 302

economies of scale 83

EinAusS $348 f$

Einzahlung 53ff

$\sim$ Svarianz 156

abhängige 194, 208

autonome 194

dichotome 147

dreieckverteilte 244

neutrale 147,190

Verteilungstyp der 190, 244

Einzahlungen, Kombinationen von 60

Einzahlungs-Auszahlungs-Differenz 63

Emergenzen 32

entgangene Zinsen 121, 125, 343, 357

Entnahmen 158, 277, 286, 292

Entscheidungsproblem 90

Entscheidungstheorie 90, 93

Entscheidungsvorbereitung 45

Entwicklungspfad 147, 227, 279

enumerativ $34,87,101 \mathrm{f}$

Erwartungen

autoregressive 274

rationale 275,283

subjektive 99

worst case 266

Erwartungsbildung $272 \mathrm{f}$

Erwartungswerte, Maximierung von 178

evolutorische Ökonomik 80

Mutation 259

natürliche Auslese 81, 158

Selektionsdruck 259

Extrapolation 82

Faktorfortschreibung 283

Fibonacci-Zahlen 49

Finanzierung 282 
Finanzmarkt 70

Fremdkapitalaufnahme 88, 112, 157, 262f, 270f, $278,281,333,352$

FRIEDMANsche Gewinnmaximierungshypothese $88,157,264$

Frühwarnindikatoren 81

Funktionslösung Siehe geschlossene Form

Fuzzy-Set-Theorie 104

genetische Algorithmen 259

geschlossene Form 40, 45, 49, 51, 58, 211, 228

Gewinn 357

Gewinnmaximierung 81, 97, 157f, 262, 270, 286

Goldene Bilanzregel 271

Grundmodell 286

GuV 284

Halbspanne 241

Handlungsmöglichkeit 90

Hazardrate 14, 79, 84, 154 Siehe auch

Überlebensquote, Insolvenzrate, Scheiterrate

Heaviside-Funktion 135

Hypothese 29, 31, 41, 105

iid Siehe Verteilung, iid

Illiquidität 279

Indifferenzkurve 91, 93

induktive Verfahren 32

Industriedichte 77

industrieökonomische Ansätze 79

Insolvenz 41, 81, 279

Insolvenzen 17

Insolvenzrate 75 Siehe auch Überlebensquote, Hazardrate, Scheiterrate

Insolvenzwahrscheinlichkeit 154 Siehe auch

Überlebenswahrscheinlichkeit

interne Verzinsung 72

Investition

〜sentscheidung 99, 115, 270, 290

aus Eigenmitteln 120, 125, 357

ganzzahlige 64

kalkulatorische Jahreskosten 115

Planung von Investitionen 281

Jackknife 106

k 195, 203, 228

Kalibrierung Siehe Modell

Kapazitätseffekt 47 Siehe auch Lohmann-RuchtiEffekt

Kapitaldienstgrenze 272
Kapitalmärkte, unvollkommene 71

Kapitalsaldo

kurzfristiger 347,359

totaler $287,348,356,360$

Kombinationseffekt 280

Korrekturverfahren 102

Korrelation 276

Kosten 112

Kostenrechnung 115

Kovarianz 276

Kreditaktivitäten 344

Kybernetik 25, 274, 285

Lebensdauer 78, 245, 247f, 252f, 255f, 258f, 261, 295,303

Lebenserwartung 247 Siehe auch Lebensdauer lerntheoretische Ansätze 79

liability of adolescence 76

liability of newness 75

liability of smallness 74, 83

lineare Programmierung 45, 100, 279

Fortschreibung des Tableaus auf die nächste

Periode 349

free-Restriktion 347

integer-Restriktion 347

Liquiditätsrestriktionen 345

Restriktionen, weitere 346

Strafkostenkonzept 104

upperbd-Restriktion 347

linksschief Siehe Verteilung, rechtssteile

Liquidationserlöse 17

Liquidität 41, 353

LOHMANN-RUCHTI-Effekt 46, 287, 290

Lösung

analytische 40,51

prodzedurale 40

Lotterie 178

M 145

MaInv 348

Maple 211f

Markovkette 214

Markovprozeß 214

Markteintrittsbarrieren 79

marktstrukturelle Ansätze 79

MARX-ENGELS-Effekt 47 Siehe LOHMANNRUCHTI-Effekt

Median 135 
Medianalter 247

Melin convolution 174

Modell 22, 262

Anforderungen 28

empirische Überprüfbarkeit 29

kiss 28

Plausibilität 29

positive economics 29

Betriebsentwicklungsmodell 46, 262, 269, 278

CEEPES 43

diskretes 191

Entscheidungsmodell 262

FLIPSIM 43

ganzzahliges 65

Implikationen 31

Kalibrierung 30

metrisch dynamische Funktionsmodelle 190

Modellanalyse 35

Modellandwirtin 279

random walk 78, 195, 214

Simulationsmodell 42, 64, 70, 104, 178, 236, 258, 261, 269, 333

Typen 22

agent based modeling 32

ex-ante vs. ex-post 27

geschlossenes vs. offenes 24

rekursives 24

spieltheoretisches 27

statisches vs. dynamisches 24

stochastisches vs. deterministisches 26

Überlebensmodell 84, 189, 277

Verweildauermodell 85

Überlebensmodell mit Auszahlung 206, 211

Überlebensmodell ohne Auszahlung 192

Validierung 30

Verifikation 30

Monte-Carlo-Methode 35 Siehe auch Monte-

Carlo-Simulation

Monte-Carlo-Simulation 104, 236, 253

Monte-Carlo-Studie 278

MOTAD 103

Multiagentensystem Siehe agent based modeling

Münchner Gründerstudie 85

natural selection Siehe evolutorische Ökonomik, natürliche Auslese

Nettostartkapital 207 neutrale Einzahlungen 14

nutzendivergierende Wirkungen 92

Nutzenfunktion 90

optimale Betriebsgröße 83

Optimum, lokales 38

Organisationsökologie 74

Organisationstheorie 73

Panelldaten 82

Parameter, stochastische 275

periodische Regressionslösung 49

Petersburger Paradoxon 178

Population 259

portfoliotheoretische Ansätze 103

positive Zahlen 148

Preisvarianz 303

pseudo-induktiv 32

Pseudozufallszahlen 253

$\mathrm{p}_{\mathrm{uil}}$ (2) 193

$\mathrm{p}_{\text {ül }}\left(K_{2}>0\right) 151$

$\mathrm{p}_{\mathrm{uil}}\left(K_{t}>0\right) 149$

$\mathrm{p}_{\text {ül }}(t) 149$

Punktprognose 267

Randomisation 105

range 92

Rationalität

begrenzte 97

erweiterte 98

Referenzstrategie 270

Reparaturen 56f

Reproduktionseigenschaft 138, 162

Restschulden 353

der langfristigen älteren Kredite 348

RHS 284

Risiko 92, 98

Risikoabschläge 271

Risikoanalyse 101f

Risikofunktion 84, 154

Robustheit 39

rollende Planung 279

Ruin 154

satisfizierendes Verhalten 271

Scheiterrate 77 Siehe auch Überlebensquote,

Hazardrate, Insolvenzrate

Schmetterlingseffekt 39

score function 87

Seed Siehe Startsequenz 
Sensitivitätsanalyse 27, 36, 102

Signifikanztest 104, 109, 110

Signum-Funktion 231

Simulation 32, 35, 44, 278 Siehe auch Modell,

Simulationsmodell;

Anwendungen 42

Einsatzgebiete 36

Nachteile 38

Signifikanzprüfung 41

Simulation oder Optimierung 44

stochastische 31,37

Vorteile 38

Weiterverwendung 42

Startkapital 52, 63, 67, 78, 86, 190, 243

beliebiges 192, 202

Startsequenz 253

StllInv 348

stochastische Dominanz 93, 144

Strategien

Diversifikation 289

Risikoabschläge 290

Vergleich von 107, 280, 288

Survivor-Bias 82,85

Survivorfunktion 154

Systeme

dynamische erster Ordnung 192

mathematisch-ökonomische 189

Systemforschung 130

theory of founding characteristics 77

Übergangswahrscheinlichkeit 215

Überlebensquote 154, 239 Siehe auch Hazardrate, Insolvenzrate, Scheiterrate

Überlebenswahrscheinlichkeit 84, 129, 154, 262, 278

ergebnisorientiert 148

verlaufsorientiert 148

von Unternehmen 73

überlegen 129, 133

Überschuldung 279

ül $(t) 149$

Umwelt 275

Unbestimmtheitsbereich 89, 93ff, 266

Ungenauigkeit 39

Ungewißheit 98

Unsicherheit 98f, 275

Unternehmensplanspiel 265
Validierung Siehe Modell

Varianz 155

des Maximums 254

Verhalten anderer Unternehmen 280

Verifikation Siehe Modell

Vermögensendwert 247

Verteilung

Dreieckverteilung 135f, 164, 294

Faltung 163

Gleichverteilung 162, 164f, 170ff, 230, 240

iid 133, 190, 193

multiplizierter Zufallsvariabler 159, 177

Normalverteilung 138, 160, 162ff, 166-77, 234

quadrierter Zufallsvariabler 159f, 167f, 170ff,

177

rechtssteile 135

$\chi^{2} 138,167 \mathrm{f}$

Verteilungsfunktion 130

Wachstumsparameter 78

Wahrscheinlichkeit

saussagen 36

$\sim$ sverteilung eines Produktes 210

bedingte $150 \mathrm{f}$

objektive 98

subjektive 99

weißes Rauschen 190

white noise Siehe weißes Rauschen

Wiener-Prozesse 191

Würfeln 131

zelluläre Automaten 226

Ziele 89, 269

Zielfunktion 44, 100, 115, 261, 341

Zielkonkurrenz 92, 262

Zinsen 355

Zinssaldo

kurzfristiger 348

totaler 348,356

Zufallsvariable Siehe Zufallsveränderliche

Zufallsveränderliche 130 Siehe auch Verteilung

Produkt 165, 173, 176

Quadrat 167

Summe 144, 160

$t$-dimensionale 149

zusammengesetzte 177

Zupacht 282 